\title{
Spent Nuclear Fuel Discharges from U.S. Reactors 1993
}

February 1995

Energy Information Administration

Office of Coal, Nuclear, Electric and Alternate Fuels

Survey Management Division

U.S. Department of Energy

Washington, DC 20585 


\section{DISCLAIMER}

This report was prepared as an account of work sponsored by an agency of the United States Government. Neither the United States Government nor any agency thereof, nor any of their employees, make any warranty, express or implied, or assumes any legal liability or responsibility for the accuracy, completeness, or usefulness of any information, apparatus, product, or process disclosed, or represents that its use would not infringe privately owned rights. Reference herein to any specific commercial product, process, or service by trade name, trademark, manufacturer, or otherwise does not necessarily constitute or imply its endorsement, recommendation, or favoring by the United States Government or any agency thereof. The views and opinions of authors expressed herein do not necessarily state or reflect those of the United States Government or any agency thereof. 


\section{DISCLAIMER}

Portions of this document may be illegible in electronic image products. Images are produced from the best available original document. 


\section{Preface}

Spent Nuclear Fuel Discharges from U.S. Reactors 1993 provides current statistical data on fuel assemblies irradiated at commercial nuclear reactors operating in the United States. This year's report provides data on the current inventories and storage capacities at these reactors. The report was prepared by the Energy Information Administration (EIA) under a Memorandum of Understanding with the Office of Civilian Radioactive Waste Management (OCRWM). The ELA is the independent agency for data collection and analysis within the U.S. Department of Energy (DOE).

Data are collected on Form RW-859, "Nuclear Fuel Data" survey, and provide a comprehensive statistical characterization of the industry's activities for the survey year and include some information about industry plans and commitments for the future. Detailed statistics on these data are presented in four chapters that highlight 1993 spent fuel discharges, storage capacities and inventories, canister and nonfuel component data, and assembly characteristics. Five appendices, a glossary, and bibliography are also included.

Chapter 1 provides detailed data on reactor spent fuel discharge and storage activity for 1993, nuclear power plant data, annual discharges, cladding, enrichment and burnup, and away-from-reactor storage facilities. Data on site capacities and inventories at utilities and storage facilities, as well as dry storage at utilities, are presented in Chapter 2. Data on canisters and their contents and nonfuel components for each storage pool site are presented in Chapter 3. Assembly type characteristics, their background, fabricator summary, assembly distribution, and assembly identifiers are presented in Chapter 4.

Appendix A provides information on the methodology used in the survey, including data collection procedures and data editing, analysis, and processing. The quantities and characteristics of each specific fuel assembly type discharged by each reactor are described in Appendix B. A series of pictograms showing storage site and pool configurations, capacities, and inventories, are shown in Appendix C. A description of the EIA Quality Assurance reports and a more detailed discussion of EIA Quality Assurance procedures are included in Appendix D. Appendix E contains technical notes providing further details on information contained in the report.

These data are used by a wide audience, including Congress, Federal and State agencies, the nuclear and electric industries, and the general public for assessing spent fuel storage requirements. They also constitute one of the inputs to the Characteristics Data Base (CDB) developed by Oak Ridge National Laboratory (ORNL) and maintained by TRW Environmental Safety Systems, Inc. The discharge dates, assembly types, burnups, and initial enrichments are used to calculate the gamma, neutron, and thermal source intensities. These radiological characteristics, along with reported fuel quantities and dimensions, are then used by the Civilian Radioactive Waste Management System (CRWMS) designers for shielding design, thermal design, and sizing of facilities and equipment.

The data are also used in the 1994 Acceptance Priority Ranking and Annual Capacity Report. The report details the order in which DOE will allocate Federal waste acceptance capacity. As required by the Standard Contract for Disposal of Spent Nuclear Fuel and/or High-Level Radioactive Waste (10 CFR 961), the ranking is based on the age of permanently discharged spent nuclear fuel, with the owners of the oldest spent nuclear fuel, on an industry-wide basis, given the highest priority.

The DOE estimates future discharges from U.S. commercial nuclear reactors and the characteristics of those discharges based on trends of historical spent nuclear fuel data provided by the respondents and on econometric projections. The need for additional spent fuel storage capacity is based on these estimated cumulative discharges and on the estimated maximum storage capacity of both at-reactor and away-fromreactor storage facilities. 
Questions regarding the contents of this report may be directed to:

Survey Management Division, EI-52

Energy Information Administration

U.S. Department of Energy

1000 Independence Ave., S.W.

Washington, DC 20585

Questions of a general nature should be directed to Howard Walton, Director of the Survey Management
Division (202/254-5500), Fred Mayes, Chief of the Renewable \& Financial Data Systems \& Outreach Branch (202/254-5482); or James Disbrow, Team Leader, Renewables \& Outreach (202/254-5558).

Questions of a detailed or technical nature should be directed to the Project Leader, Kathy Gibbard (202/254-5559, e-mail kgibbard@eia.doe.gov).

Additional copies may be obtained by returning the order form at the back of this report. 


\section{Contents}

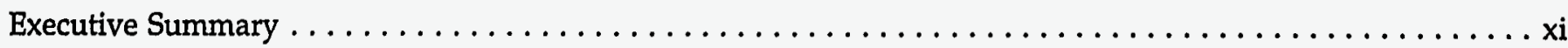

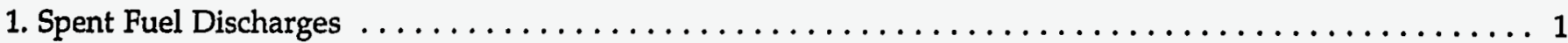

Reactor Spent Fuel Discharge and Storage Activity for $1993 \ldots \ldots \ldots \ldots \ldots \ldots \ldots \ldots \ldots \ldots \ldots \ldots$

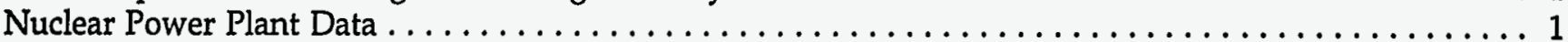

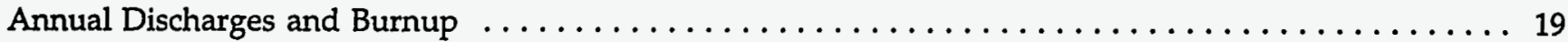

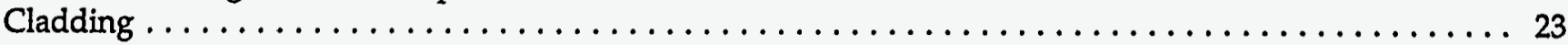

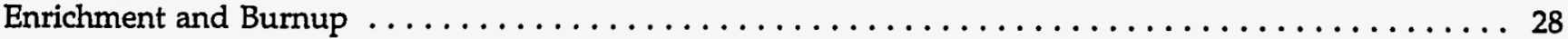

Utility Shipments to Away-from-reactor Storage Facilities $\ldots \ldots \ldots \ldots \ldots \ldots \ldots \ldots \ldots \ldots \ldots \ldots$

2. Site Capacities, Inventories, and Dry Storage at Utilities and Storage Facilities $\ldots \ldots \ldots \ldots \ldots \ldots \ldots$

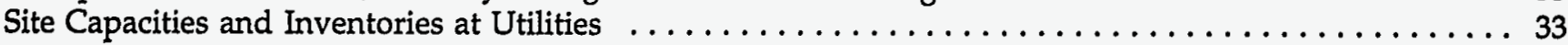

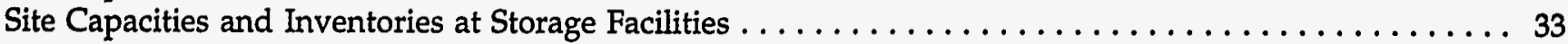

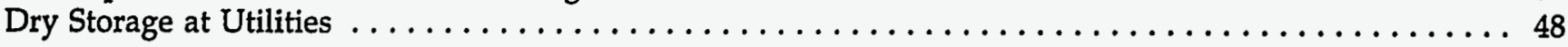

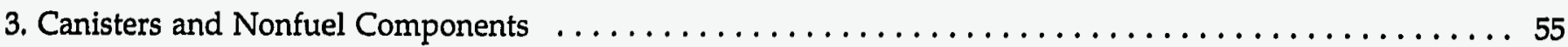

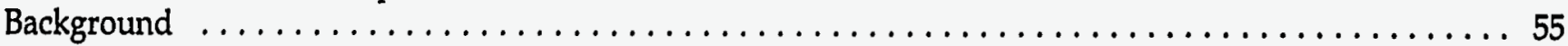

Canister and Nonfuel Component Data Through $1993 \ldots \ldots \ldots \ldots \ldots \ldots \ldots \ldots \ldots \ldots \ldots \ldots$

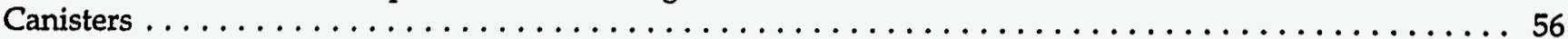

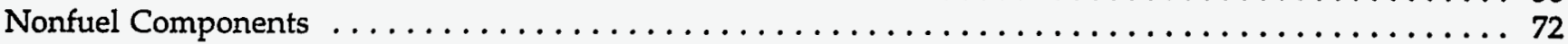

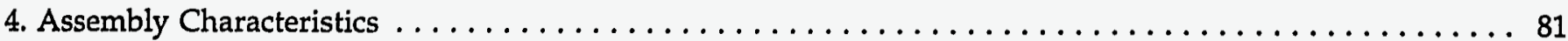

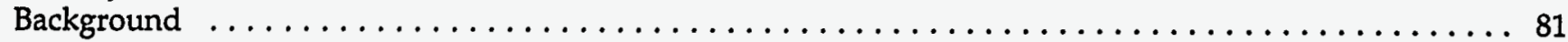

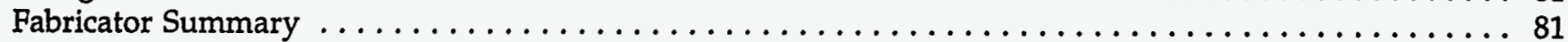

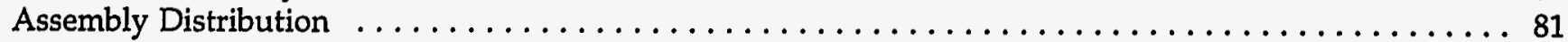

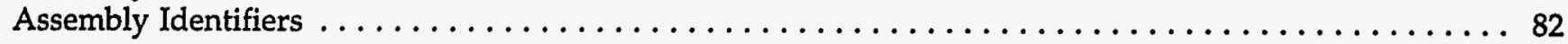

Appendices

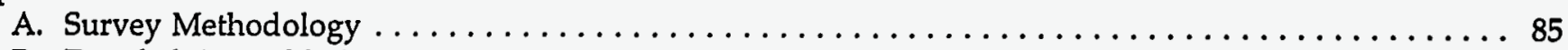

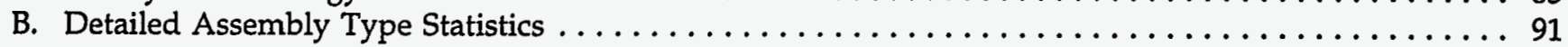

C. Pictograms Showing Reactor Storage Pool Configurations, Pool Capacities, and Inventories at Nuclear Power Plant Sites and

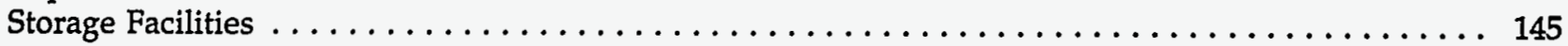

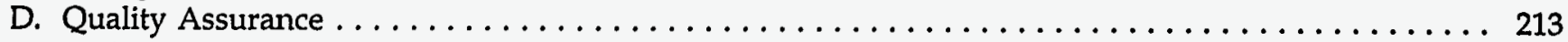

E. Technical Notes . . . . . . . . . . . . . . . . . . . . . . . . . . . . . . 219

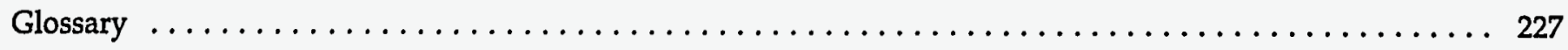

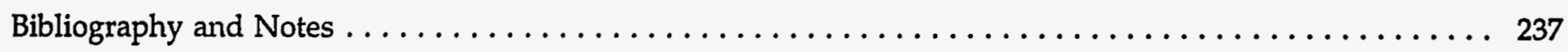




\section{Tables}

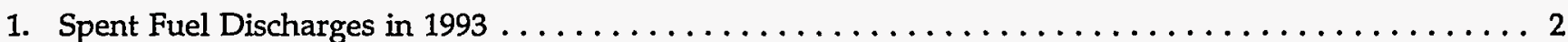

2. Reactor Spent Fuel Discharge and Storage Activity for $1993 \ldots \ldots \ldots$

3. Temporarily Discharged Assemblies . . . . . . . . . . . . . . . . . . . . . . 10

4. Nuclear Power Plant Data as of December $31,1993 \ldots \ldots \ldots \ldots$. . . . . . . . . . . . . . . . . 12

5. Annual Spent Fuel Discharges and Burnup, 1968-1993 . . . . . . . . . . . . . . . . . . . . . 20

6. Annual Spent Fuel Burnup (in Assemblies), 1968-1993 . . . . . . . . . . . . . . . . . . . . . . 24

7. Metric Tons of Uranium in Annual Spent Fuel Burnup, 1968-1993 . . . . . . . . . . . . . . . . 25

8. U.S. Burnup Distribution for All Discharged Assemblies . . . . . . . . . . . . . . . . . . . 26

9. Spent Fuel by Cladding and Reactor Type, 1968-1993 . . . . . . . . . . . . . . . . . . . . . . . 27

10. Reactors Discharging Stainless Steel Clad Assemblies . . . . . . . . . . . . . . . . . . . . . . . 27

11. Burnup Versus Enrichment by Cladding and Reactor Type (in Assemblies) . . . . . . . . . . . . 29

12. Fuel Stored in Away-from-reactor Facilities . . . . . . . . . . . . . . . . . . . . 31

13. Site Capacities and Inventories at Nuclear Power Plants as of December 31, 1993 . . . . . . . . . . . 34

14. Site Capacities and Inventories at Away-from-reactor Storage Facilities as of December 31, 1993 . . . 41

15. Spent Fuel in Storage by State and Storage Site . . . . . . . . . . . . . . . . . . . . . 42

16. Independent Spent Fuel Storage Installation (ISFSI) Data . . . . . . . . . . . . . . . . . . . 52

17. Dry Spent Fuel Storage Data and Projections . . . . . . . . . . . . . . . . . . . . . 54

18. Container Data as of December $31,1993 \ldots \ldots \ldots \ldots \ldots$

19. Canisters Containing Spent Fuel as of December $31,1993 \ldots \ldots \ldots$. . . . . . . . . . . . . . . 61

20. Canisters Containing Nonfuel Components as of December 31, 1993 . . . . . . . . . . . . . . 66

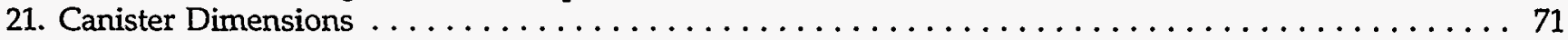

22. Nonfuel Assembly (NFA) Hardware from Babcock \& Wilcox Reactors . . . . . . . . . . . . . . . . 74

23. Nonfuel Assembly (NFA) Hardware from Combustion Engineering Reactors . . . . . . . . . . . . . 75

24. Nonfuel Assembly (NFA) Hardware from General Electric Reactors . . . . . . . . . . . . . . . . . . 76

25. Nonfuel Assembly (NFA) Hardware from Westinghouse Reactors . . . . . . . . . . . . . . . . . . . 77

26. Nonfuel Assembly (NFA) Hardware from Single-Reactor Assembly Class Reactors . . . . . . . . . . 79

27. Assemblies by Fuel Fabricating Company and Reactor Type . . . . . . . . . . . . . . . . . 82

28. Distribution of Assembly Transverse Dimensions . . . . . . . . . . . . . . . . . . 83

B1. Fabricator Identifiers . . . . . . . . . . . . . . . . . . . . . . . . . . 95

B2. Assembly Class and Design Type by Utility . . . . . . . . . . . . . . . . . . . . 96

B3. Reactors by Assembly Class and Design Type $\ldots \ldots \ldots \ldots \ldots \ldots$

B4. Descriptions of Assembly Types . . . . . . . . . . . . . . . . . . . . . . . 102

B5. Assembly Characteristics by Assembly Type . . . . . . . . . . . . . . . . . . . . 115

B6. Assembly Type Summary by Reactor . . . . . . . . . . . . . . . . . . . . . . . . 117

B7. Assembly Type Summary by Assembly Type . . . . . . . . . . . . . . . . . . . . . . . 128

B8. Spent Fuel Assemblies, by Type, Discharged by Year . . . . . . . . . . . . . . . . . . . 138

B9. Initial Uranium Content of Spent Fuel Assemblies, by Type, Discharged by Year . . . . . . . . . . . 140

B10. Burnup of Spent Fuel Assemblies, by Type, Discharged by Year . . . . . . . . . . . . . . . . . . 142

\section{Figures}

1. Loss of Ability to Operate Data as of December $31,1993 \ldots \ldots \ldots$

2. Projected Retirement Year Data as of December $31,1993 \ldots \ldots \ldots$

3. Annual Spent Fuel Discharges, $1968-1993 \ldots \ldots \ldots \ldots \ldots \ldots \ldots$

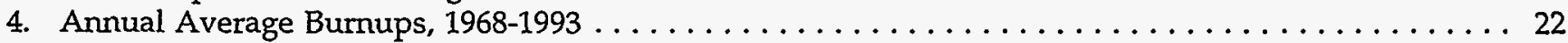

5. Spent Fuel Assemblies in Storage by State . . . . . . . . . . . . . . . . . . . . . 46

6. Initial Uranium Content of Spent Fuel Assemblies in Storage by State . . . . . . . . . . . . . . 47

D1. Energy Information Administration Quality Assurance Report . . . . . . . . . . . . . . . 216 


\section{Pictograms by Electric Utility}

Legend

Alabama Power Company

Arizona Public Service Company

Arkansas Power and Light Company

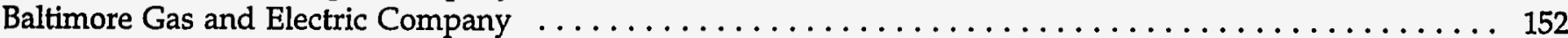

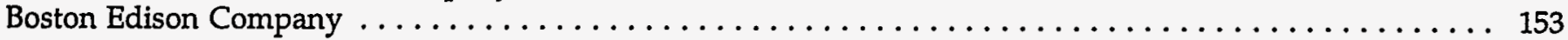

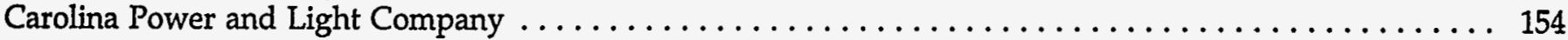

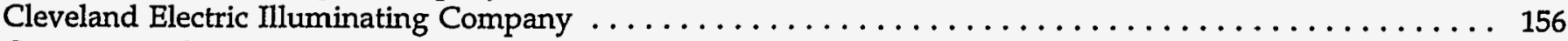

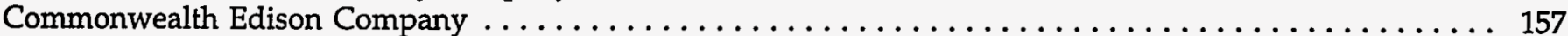

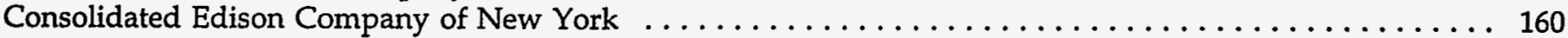

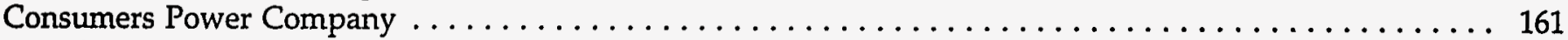

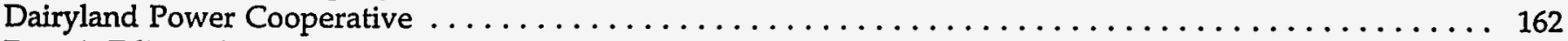

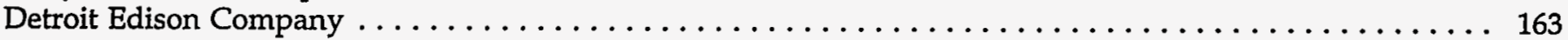

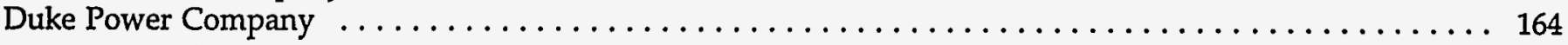

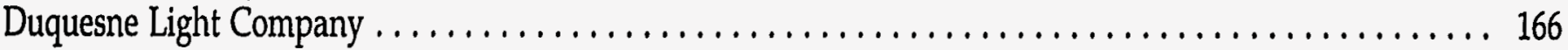

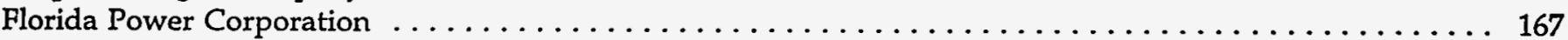

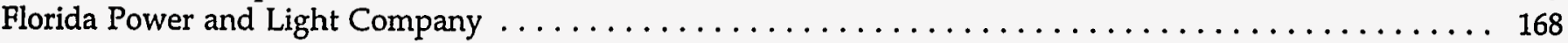

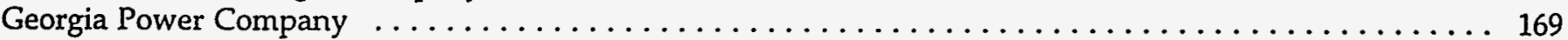

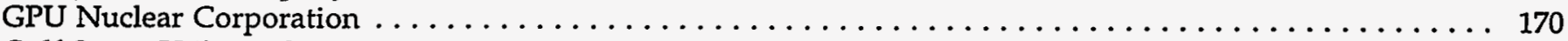

Gulf States Utilities Company $\ldots \ldots \ldots \ldots \ldots \ldots \ldots \ldots \ldots \ldots \ldots \ldots \ldots \ldots \ldots \ldots \ldots \ldots \ldots \ldots$

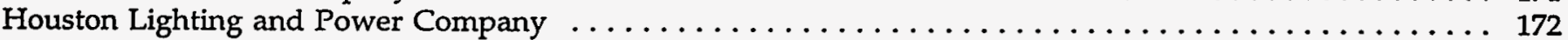

IES Utilities, Inc. (a.k.a., Iowa Electric Light and Power Company) $\ldots \ldots \ldots \ldots \ldots \ldots \ldots \ldots \ldots \ldots$

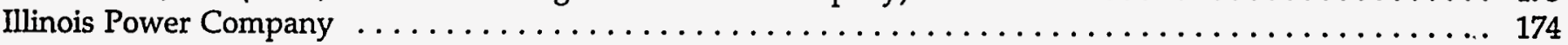

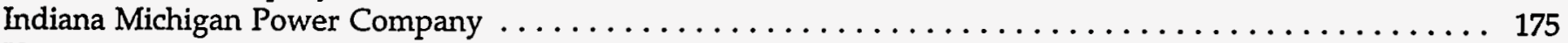

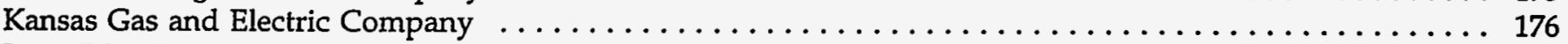

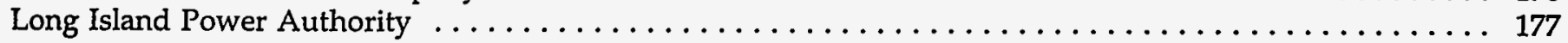

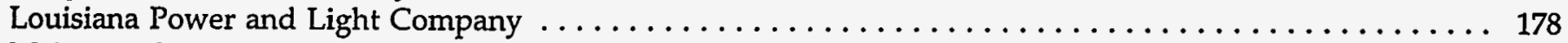

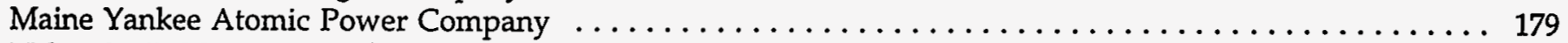

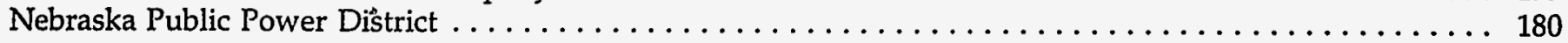

New York Power Authority (a.k.a., Power Authority of New York) $\ldots \ldots \ldots \ldots \ldots \ldots \ldots \ldots \ldots \ldots 1$

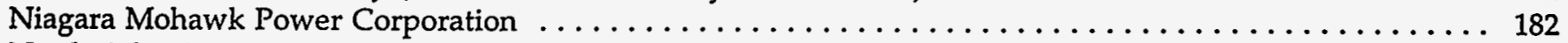

North Atlantic Energy Service Corporation $\ldots \ldots \ldots \ldots \ldots \ldots \ldots \ldots \ldots \ldots \ldots \ldots \ldots \ldots \ldots \ldots \ldots$

Northeast Utilities Service Company $\ldots \ldots \ldots \ldots \ldots \ldots \ldots \ldots \ldots \ldots \ldots \ldots \ldots \ldots \ldots \ldots \ldots \ldots$

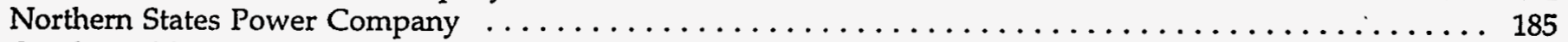

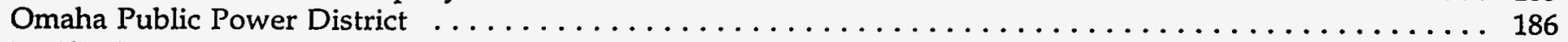

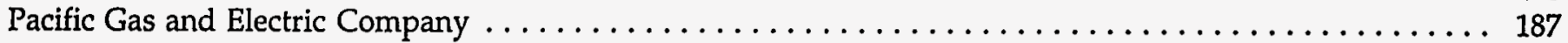

PECO Energy Company (a.k.a., Philadelphia Electric Company) $\ldots \ldots \ldots \ldots \ldots \ldots \ldots \ldots \ldots \ldots \ldots \ldots 188$

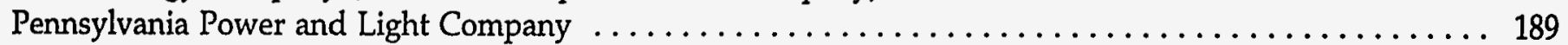

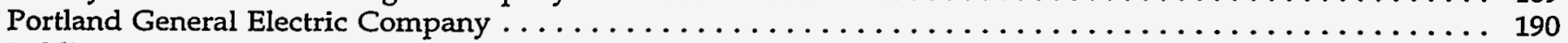

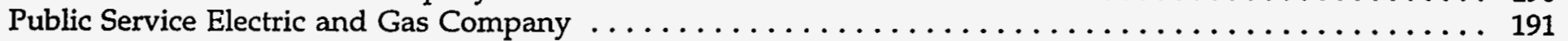

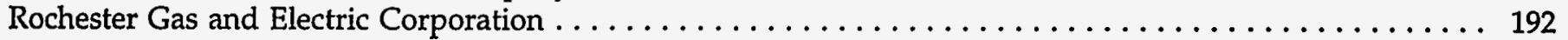

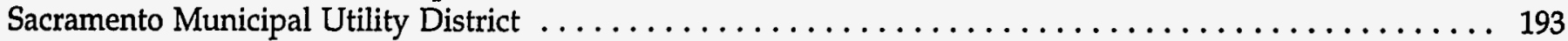

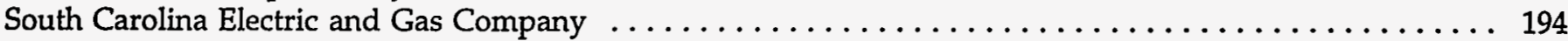

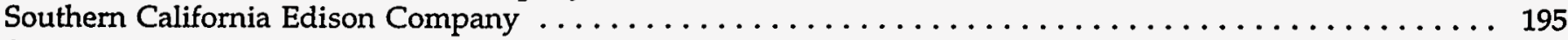

System Energy Resources, Inc. . . . . . . . . . . . . . . . . . . . . . . . . . . . . . 196

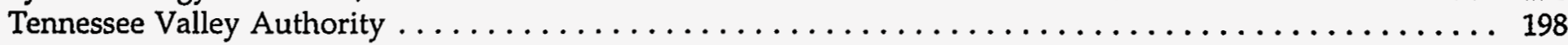

Toledo Edison Company . . . . . . . . . . . . . . . . . . . . . . . . . . . . . . 200

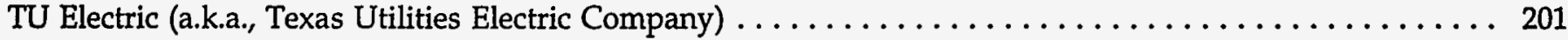

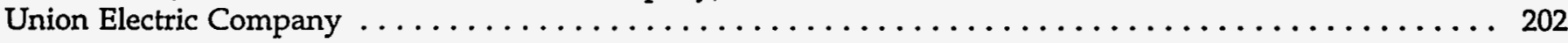


Vermont Yankee Nuclear Power Corporation . . . . . . . . . . . . . . . . . . . . . . . . 203

Virginia Power (a.k.a., Virginia Electric and Power Company) . . . . . . . . . . . . . . . . . . . 204

Washington Public Power Supply System . . . . . . . . . . . . . . . . . . . . . . . . . . . 206

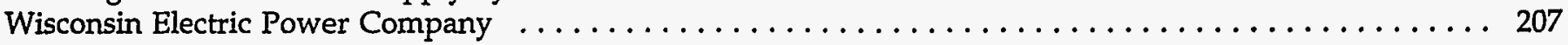

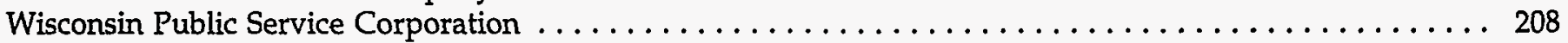

Yankee Atomic Electric Company . . . . . . . . . . . . . . . . . . . . . . . . . . . . . . . 209

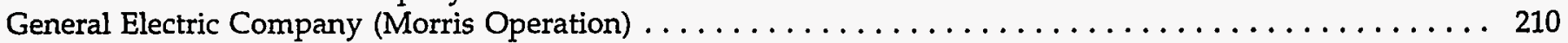

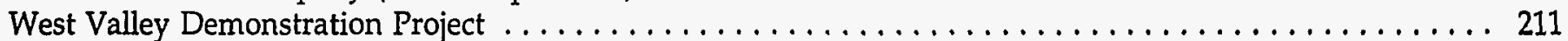

\section{Pictograms by Reactor}

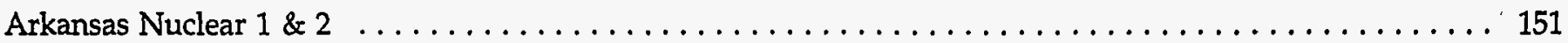

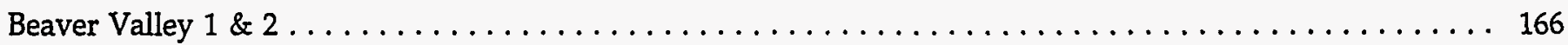

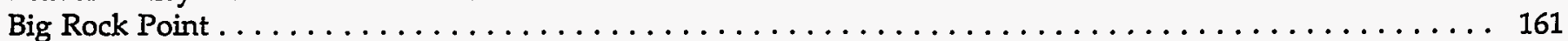

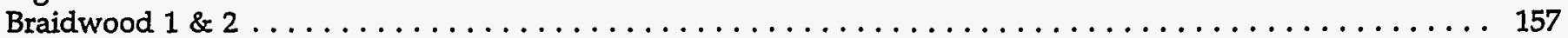

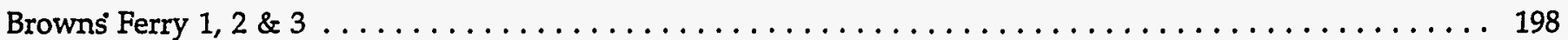

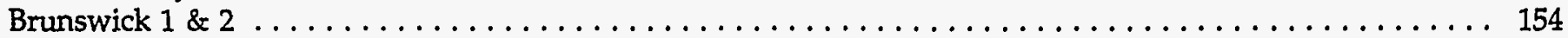

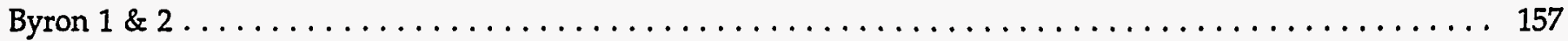

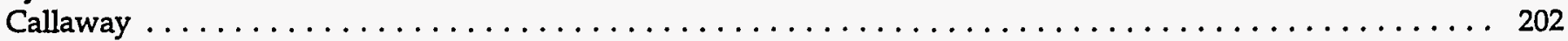

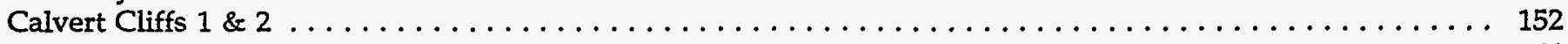

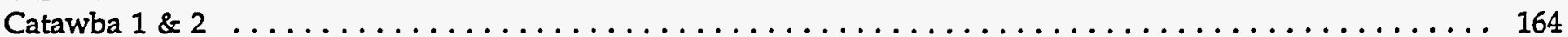

Clinton $1 \ldots \ldots \ldots \ldots \ldots \ldots \ldots \ldots \ldots \ldots \ldots \ldots \ldots \ldots \ldots \ldots \ldots \ldots \ldots \ldots \ldots \ldots$

Comanche Peak $1 \& 2 \ldots \ldots \ldots \ldots \ldots \ldots \ldots \ldots \ldots \ldots \ldots \ldots \ldots \ldots \ldots \ldots \ldots \ldots \ldots \ldots \ldots$

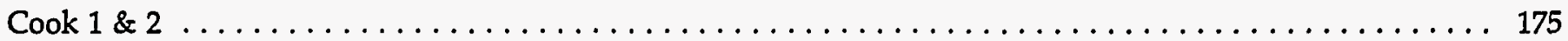

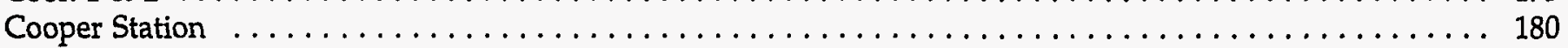

Crystal Rivër 3 . . . . . . . . . . . . . . . . . . . . . . . . . . . . . . . . . . . . . 167

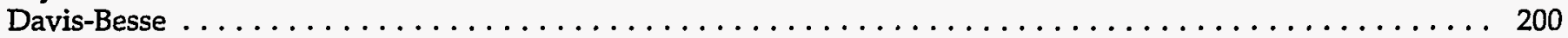

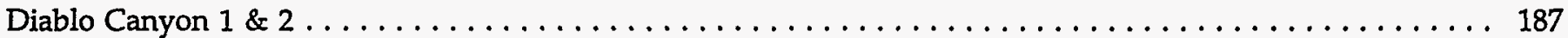

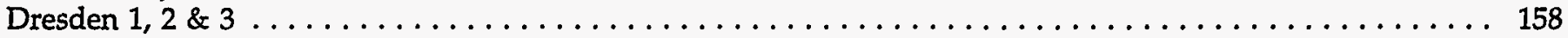

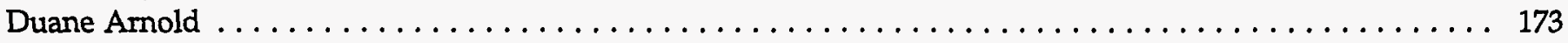

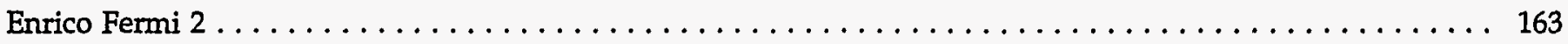

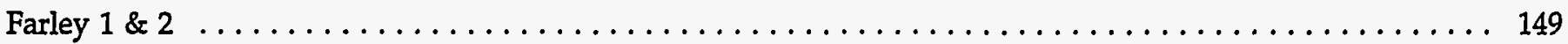

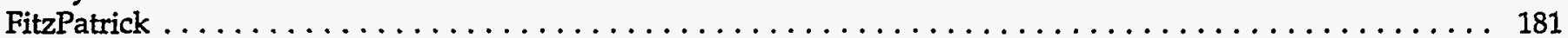

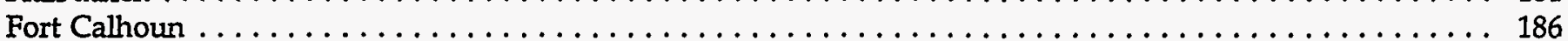

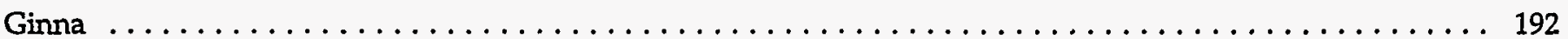

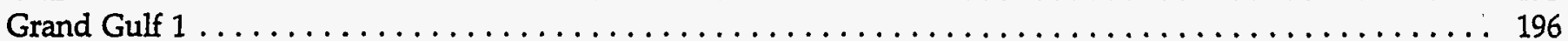

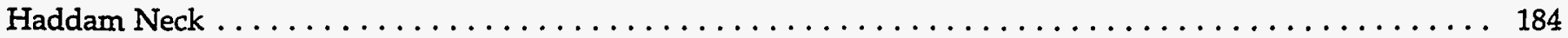

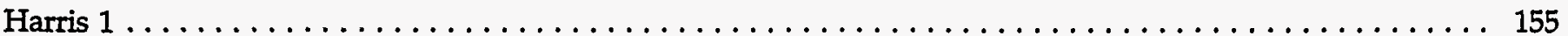

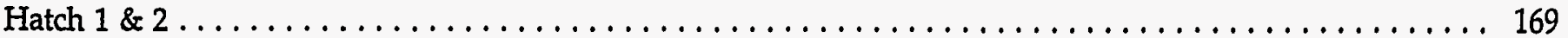

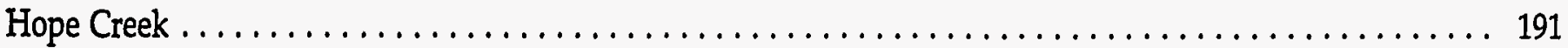

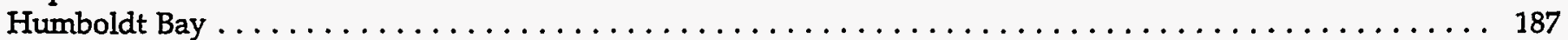

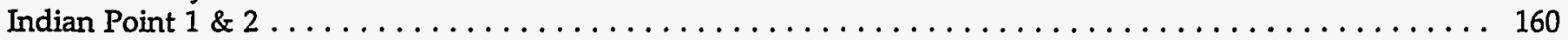

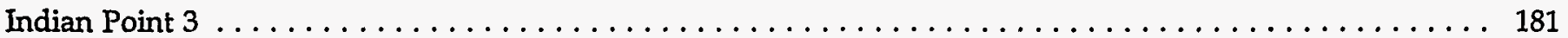

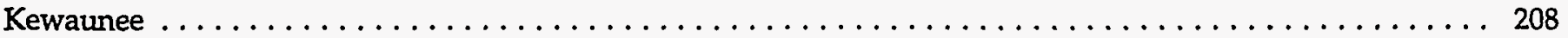

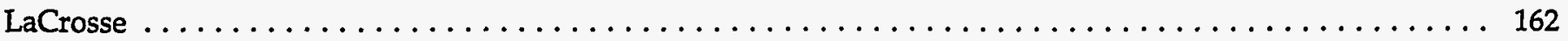

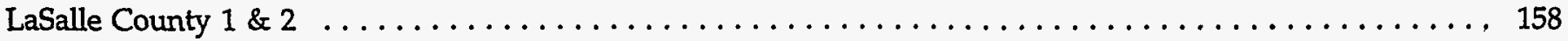

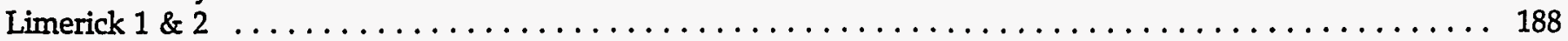

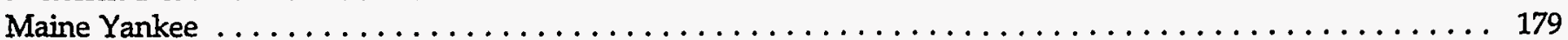

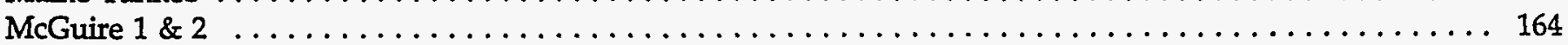

Millstone $1,2 \& 3 \ldots \ldots \ldots \ldots \ldots \ldots \ldots \ldots \ldots \ldots \ldots \ldots \ldots \ldots \ldots \ldots \ldots \ldots \ldots \ldots$ 


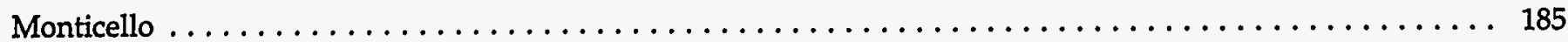

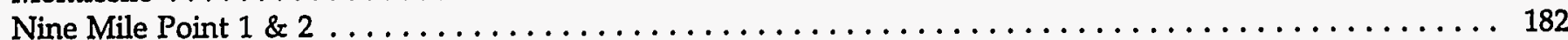

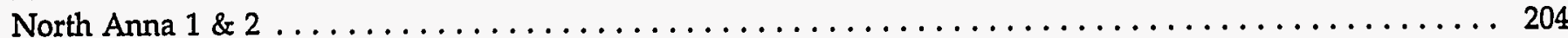

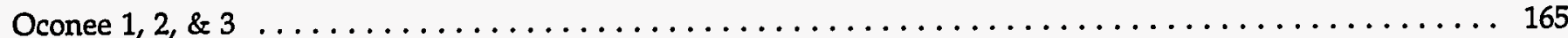

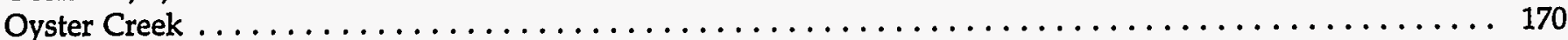

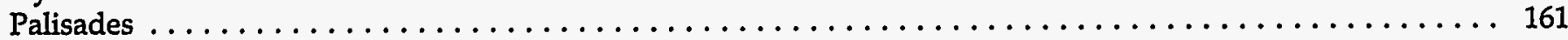

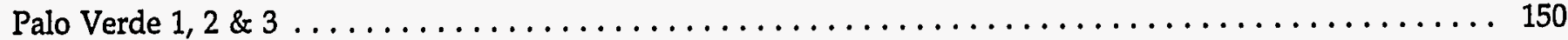

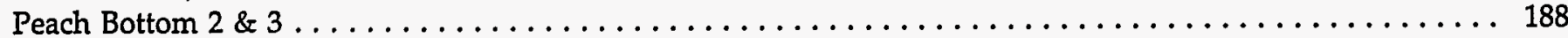

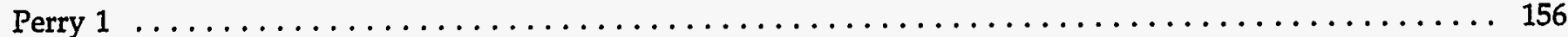

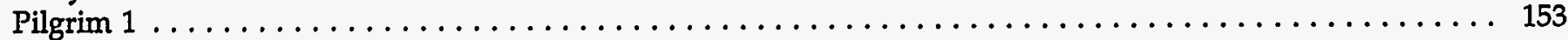

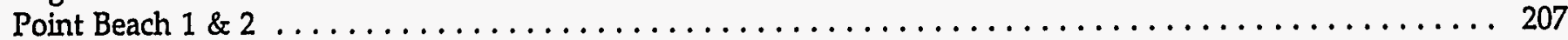

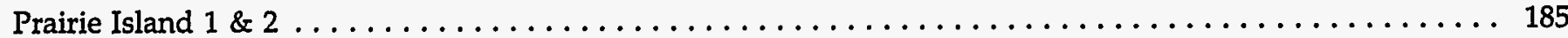

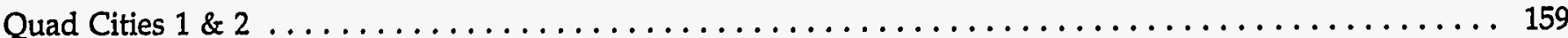

Rancho Seco ...................................................... 193

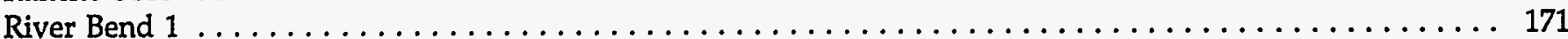

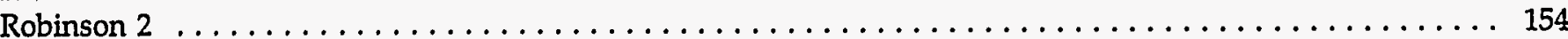

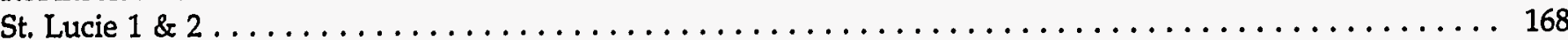

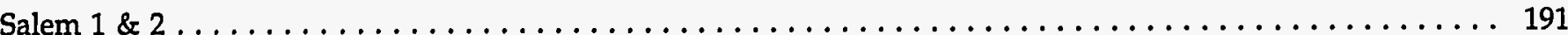

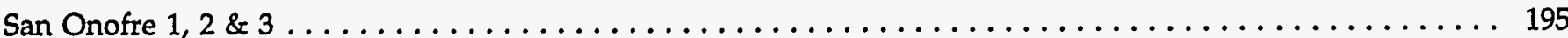

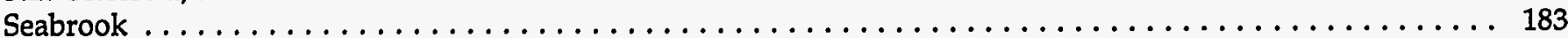

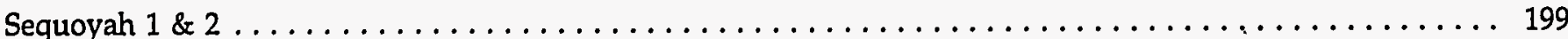

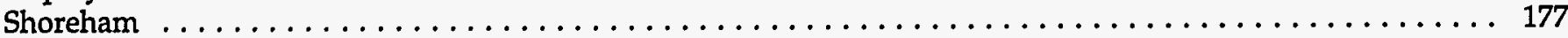

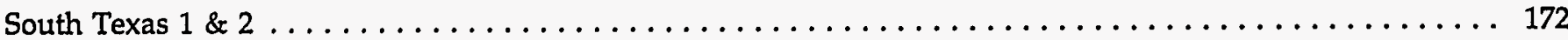

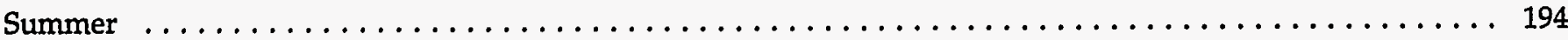

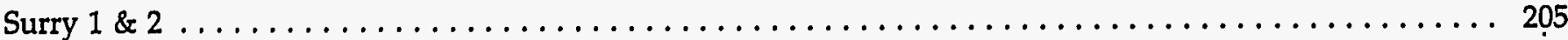

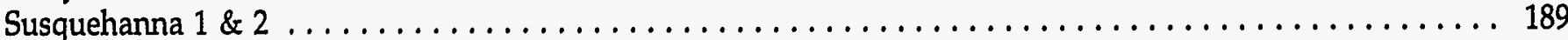

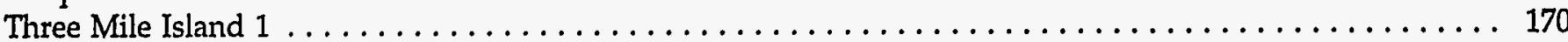

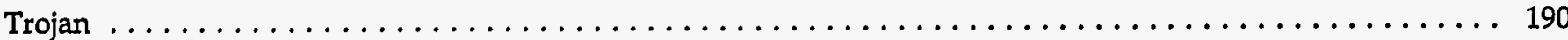

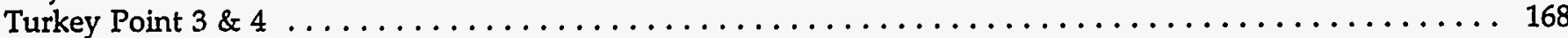

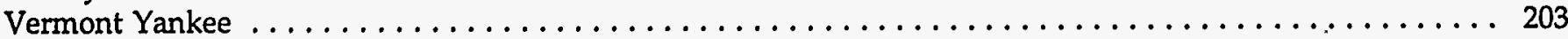

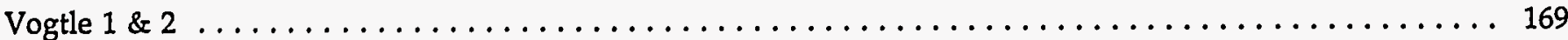

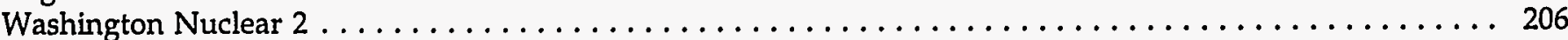

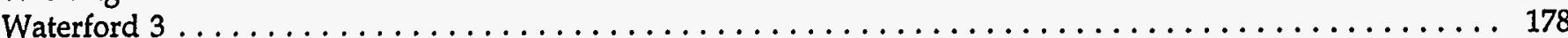

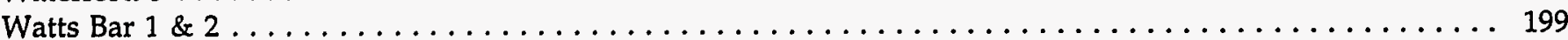

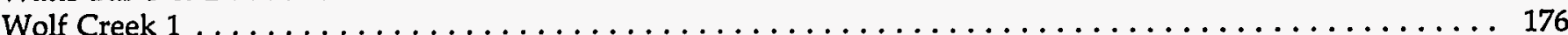

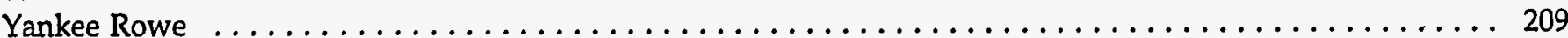

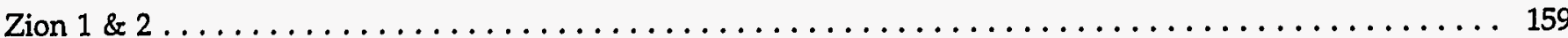




\section{Executive Summary}

The Energy Information Administration (EIA) of the U.S. Department of Energy (DOE) administers the Nuclear Fuel Data survey, Form RW-859. This form is used to collect data on fuel assemblies irradiated at commercial nuclear reactors operating in the United States, and the current inventories and storage capacities of those reactors. These data are important to the design and operation of the equipment and facilities that DOE will use for the future acceptance, transportation, and disposal of spent fuel. The information presented in this report summarizes the detailed data collected on Form RW-859 and identifies trends in burnup and enrichment in these data. This report focuses on commercial light-water reactor (LWR) spent nuclear fuel reported as discharged as of December 31, 1993.

\section{Spent Nuclear Fuel Discharges}

A total of 98,400 assemblies, with an initial loading weight of 28,136 metric tons of uranium (MTU) (Table ES1), have been discharged from 117 commercial
LWR's from 1968 through 1993. Electric utilities also reported 179 temporarily discharged assemblies at pressurized-water reactors (PWR's) and 431 temporarily discharged assemblies at boiling-water reactors (BWR's). Approximately 36 percent $(10,256.2$ MTU) of total discharges (by weight) are from BWR's; 64 percent $(17,879.9$ MTU) from PWR's. Reprocessed spent fuel, fuel from the damaged Three Mile Island Unit 2 reactor, and discharges from Fort St. Vrain [the only commercial high-temperature, gas-cooled reactor (HTGR) in the U.S.], are not within the scope of this report.

The characteristics of the permanently discharged spent fuel have changed over time. Prior to 1972, most spent commercial nuclear fuel discharged was reprocessed. Since that time, the annual average burnup for discharged BWR assemblies has shown a fairly steady increase to 30 gigawattdays thermal per metric ton of uranium (GWDt/MTU) in 1993. The average burnup has also continued to increase for PWR's, reaching a new high of almost 39 GWDt/MTU in 1993, the highest annual average yet attained.

Table ES1. Total U.S. Commercial Spent Nuclear Fuel Discharges, 1968-1993

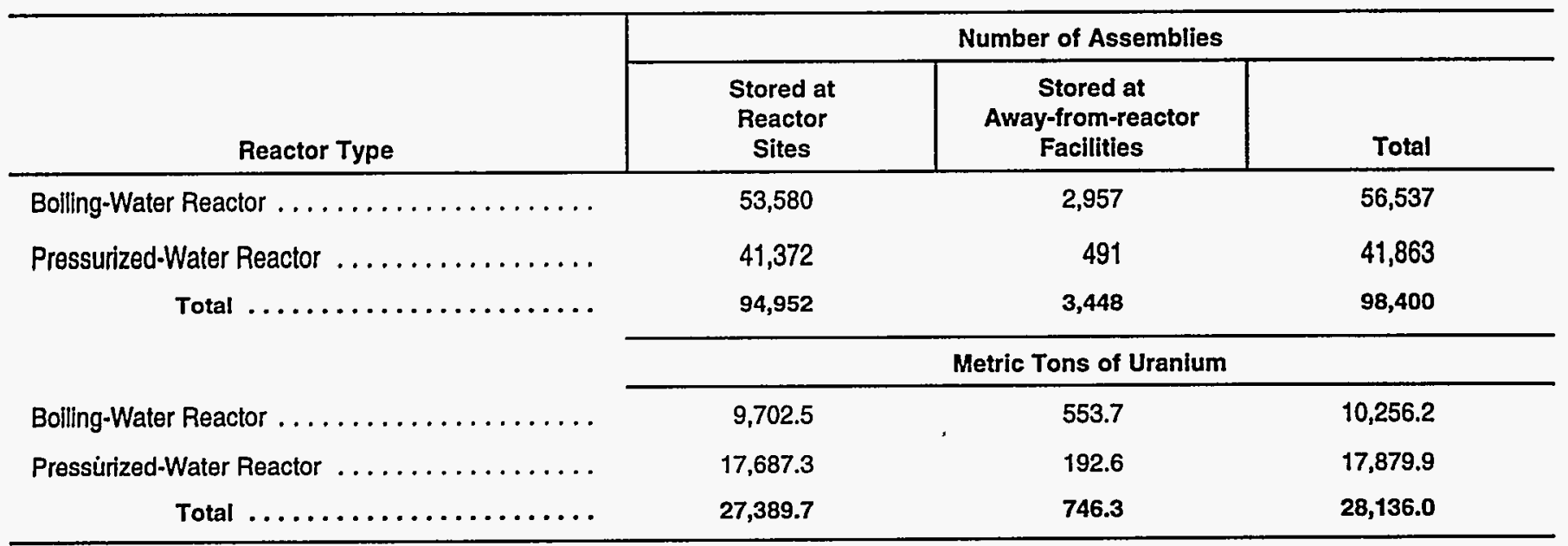

Notes: A number of assemblies discharged prior to 1972 were reprocessed and are not included in this table. See Table 12 and Technical Note 7 in Appendix $E$ for information on reprocessed assemblies. A total of 2,208 high-temperature, gas-cooled reactor (HTGR) fuel elements, with Initial uranium content equal to 24.2 metric tons of uranium (MTU), were discharged. These HTGR fuel elements are not included in the above table. See Technical Note 6 in Appendix E. Totals may not equal sum of components because of independent rounding. See Technical Note 11 in Appendix E.

Source: Energy Information Administration, Form RW-859, "Nuclear Fuel Data" (1993). 
At the end of 1993, a total of 3,448 of the assemblies covered by this report were in storage at six awayfrom-reactor storage facilities. Typically, assemblies were moved either to free up space in the discharging reactor's storage site or for use in a research program.

\section{Site Capacities and Inventories, 1993}

The total inventory of discharged LWR spent nuclear fuel in storage in the United States, as of December 31, 1993 , was 98,400 assemblies. The majority of spent nuclear fuel is stored in water filled pools, but 1,144 assemblies are in dry storage at Independent Spent Fuel Storage Installations (ISFSI's) at Virginia Power's Surry plant, Carolina Power and Light Company's Robinson 2 plant, Duke Power Company's Oconee plant, Consumers Power Company's Palisades plant, and Baltimore Gas and Electric Company's Calvert
Cliffs plant. This section includes a fold-out map showing the location of the commercial nuclear reactors as well as planned and existing ISFSI's. The current licensed storage capacity is 210,778 assemblies. The total maximum storage capacity of all storage sites, as reported by electric utilities and off-site storage, is 221,345 assemblies. However, the excess of total maximum capacity over current total inventory does not reflect the shortage of pool storage in many individual cases. Of the 111 reactors expected to be in operation by the year 2000, 14 reactors appear to require expansion above current pool maximums before 2000 .

The quantities of spent nuclear fuel in storage at nuclear power plants and away-from-reactor facilities are aggregated to the State level in Figures ES1 and ES2. These data account for all permanently and temporarily discharged assemblies from commercial nuclear reactors in the United States.

Figure ES1. Number of Assemblies of Spent Nuclear Fuel in Storage at U.S. Nuclear Power Plants and Away-from-reactor Facilities, by State

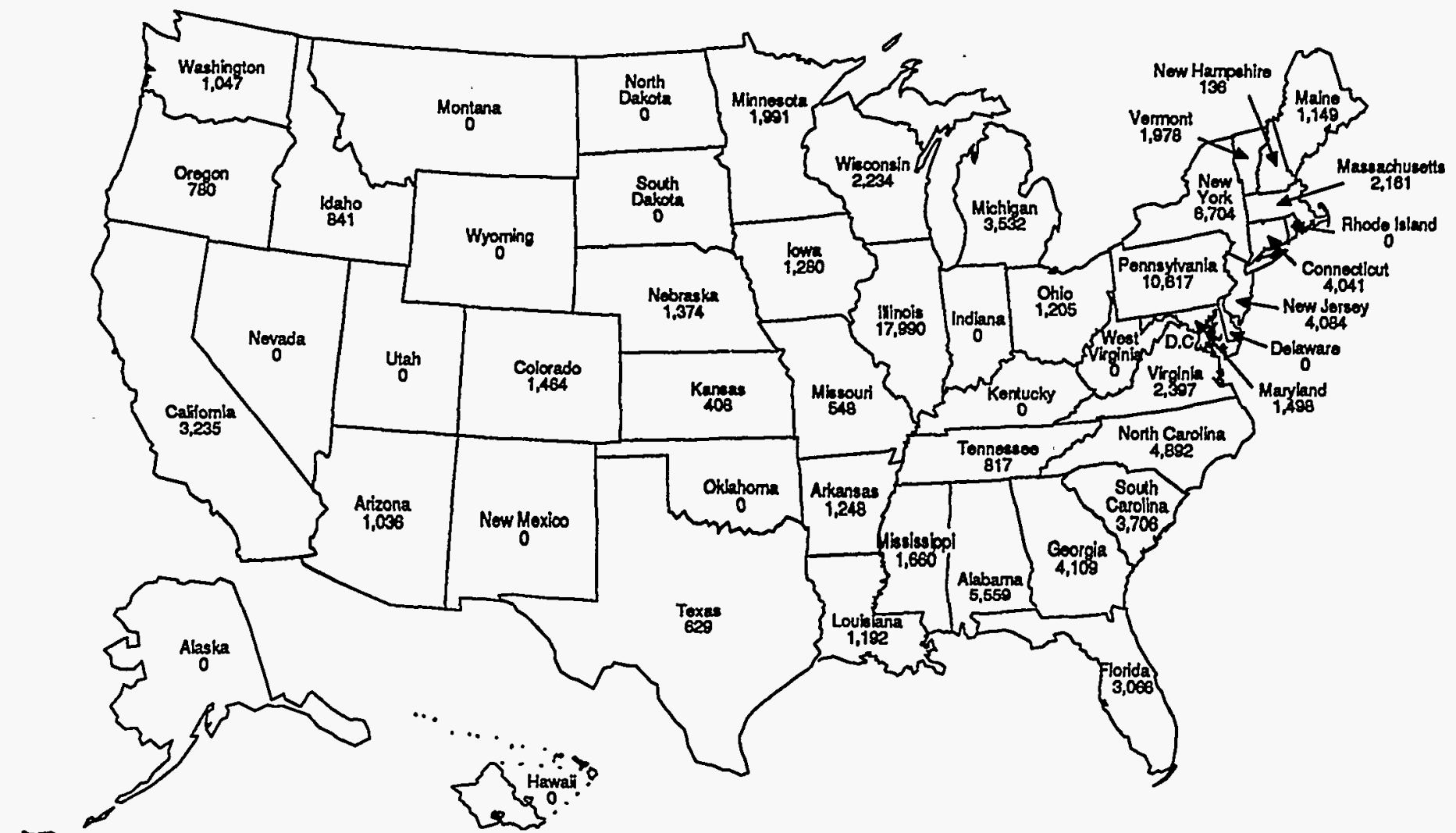

Notes: A total of 2,208 high-temperature, gas-cooled reactor (HTGR) fuel elements are in storage (744 in ldaho and 1,464 in Colorado). These HTGR fuel elements are reflected on this map but are not included in Table ES1. Numbers in the above map represent assemblies stored at nuclear power plants sites and away-from-reactor facilities, and include both permanently and temporarily discharged assemblies.

Source: Energy Information Administration, Form RW-859, "Nuclear Fuel Data" (1993). 
Figure ES2. Initial Uranium Content of Spent Nuclear Fuel in Storage at U.S. Nuclear Power Plants and Away-from-reactor Facilities, by State (Metric tons of uranium)

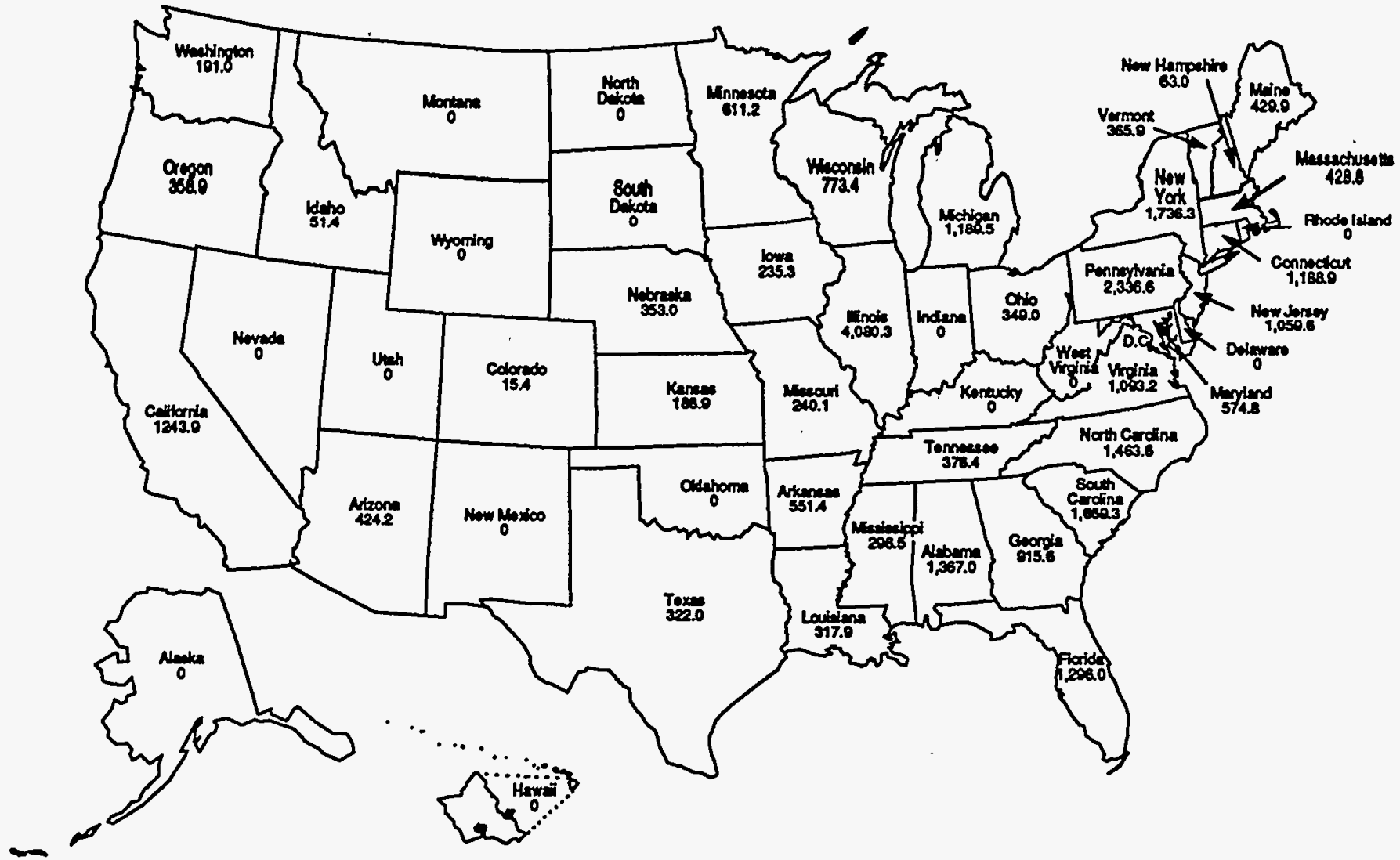

Notes: A total of 2,208 high-temperature, gas-cooled reactor (HTGR) fuel elements are in storage (744 with initial uranium content equal to 8.8 metric tons of uranium (MTU) stored in Idaho and 1,464 with initial uranium content equal 15.4 MTU stored in Colorado). These HTGR fuel elements are reflected on this map but are not included in Table ES1. Numbers in the above map represent the initial uranium content of assemblies stored at nuclear power plant sites and away-from-reactor facilities, and include both permanently and temporarily discharged assemblles.

Source: Energy Information Administration, Form RW-859, "Nuclear Fuel Data" (1993).

\section{Canisters and Nonfuel Components}

In 1991, Form RW-859 was revised to collect data on canisters and their contents and nonfuel assembly hardware for each storage pool site. The details of these inventories were first presented as a Feature Article in the report, Spent Nuclear Fuel Discharges from U.S. Reactors 1992. As of 1993, canister and nonfuel component data are included as a chapter in this report.

A total of 269 canisters were reported in storage pools as of December 31, 1993. This represents a decrease in 1993 of 17 canisters. The number of open baskets reported in pools, however, increased from 264 to 315 in 1993. Of the 269 canisters reported, 145 contain spent nuclear fuel, 96 contain nonfuel components, 15 contain both fuel and nonfuel, and 13 canisters are currently empty.

Nonfuel assembly (NFA) hardware data is collected on all generic and single-reactor assembly class reactors. In 1993, a total of 60,699 NFA hardware items were in storage at spent fuel pools. Of these items, 59,163 NFA items were in storage at 100 generic assembly class reactors, while the remaining 1,536 NFA items were in storage at 8 single-reactor assembly class reactors. No NFA data were reported by 9 generic and 3 singlereactor assembly class reactors. 


\section{Assembly Type Characteristics}

One hundred thirty-one reactor fuel assembly types have been identified for use in domestic commercial LWR's. These can be grouped by the two LWR types, BWR and PWR. Each assembly type has a unique combination of distinguishing characteristics such as envelope dimensions, rods per assembly, cladding type, and structural materials. Some types may fit in several different reactors. In some cases, a single reactor may use more than one assembly type. Initial enrichment and discharge burnup can vary significantly within a single assembly type.
Fifty BWR fuel assembly types have been discharged from 41 BWR's through 1993. However, five types make up 57 percent of the BWR assemblies discharged. General Electric (GE) fabricated about 90 percent of the discharged BWR assemblies. As fabricated, most are 5.44 inches wide, 176.2 inches long, and contain 183 to 195 kilograms $(\mathrm{kg})$ of uranium. Seventy-six PWR's have discharged 51 PWR fuel assembly types. Five types make up 55 percent of the PWR assemblies discharged. Westinghouse fabricated about 58 percent of the discharged PWR assemblies. As built, most are 8.44 inches wide, 159.8 inches long, and contain 425 to $460 \mathrm{~kg}$ of uranium.

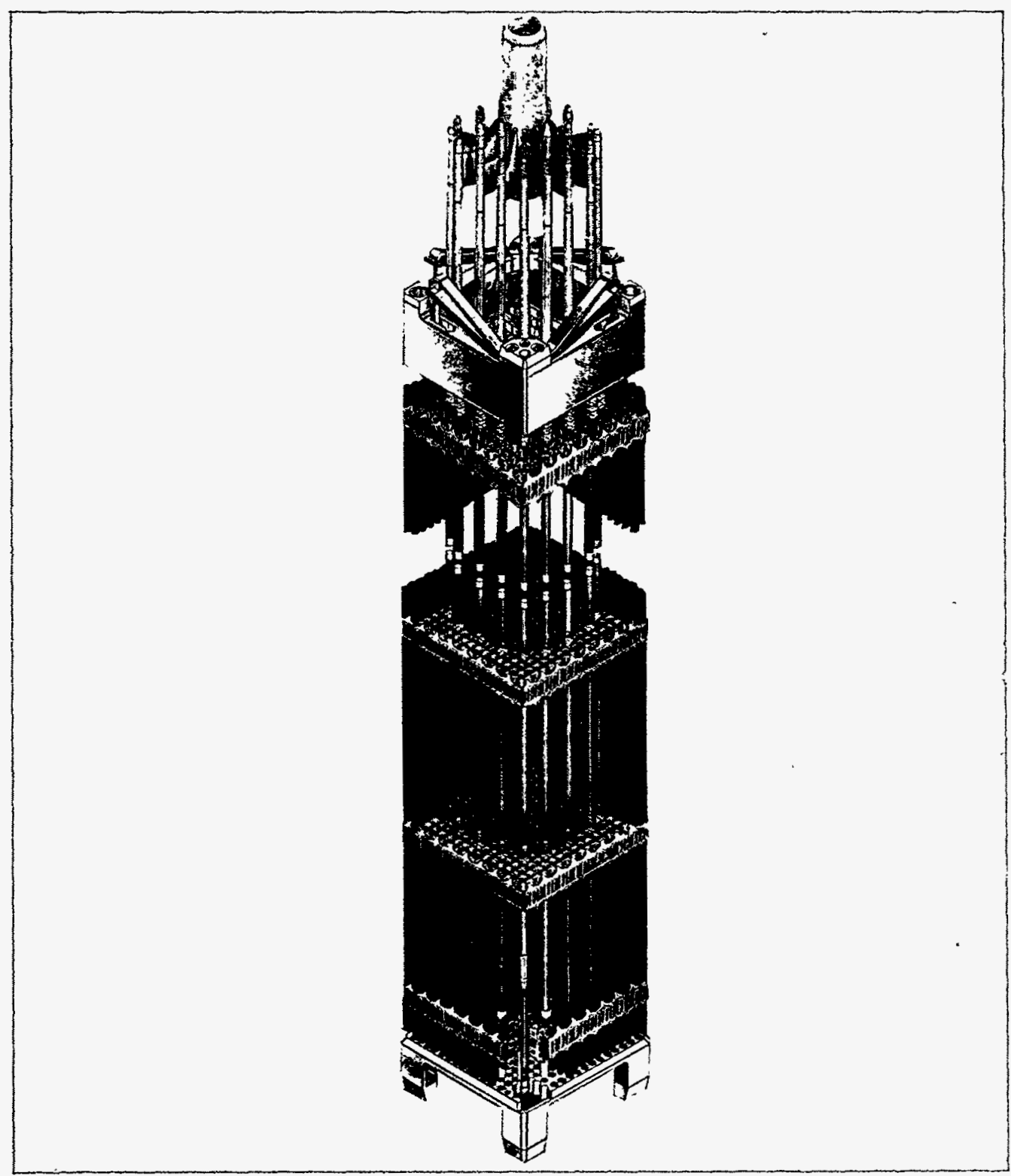

Westinghouse $17 x 17$ fuels assembly 


\section{Spent Fuel Discharges}

\section{Reactor Spent Fuel Discharge and Storage Activity for 1993}

A total of 117 U.S. commercial light-water reactors (LWR's) have discharged spent nuclear fuel as of December 31, 1993. No reactors discharged spent fuel for the first time in 1993. One reactor, Comanche Peak 2, began commercial operation in 1993. As no reactors were permanently shut down in 1993, the number of reactors in operation increased to 109.

A total of 71 reactors discharged 7,726 spent nuclear fuel assemblies in 1993 (Table 1). Of these, 7,532 were permanently discharged assemblies and 194 were classified as temporarily discharged assemblies. A total of 3,997 assemblies, containing 720.9 metric tons of uranium (MTU) were permanently discharged from 22 BWR's. A total of 3,535 assemblies, containing 1,533.6 MTU were permanently discharged from 49 PWR's. There were 120 BWR and 74 PWR temporarily discharged assemblies.

The cumulative inventory of LWR spent nuclear fuel in storage in the United States as of December 31, 1993, is 98,400 assemblies, containing 28,136.0 MTU (Table 2). These totals represent an increase from 1992 of 7,361 assemblies containing 2,178.3 MTU. The number of assemblies discharged in 1993 does not correspond with the increase in the number of assemblies stored, because of reinsertion of previously discharged assemblies, and revisions to historical survey data from Form RW-859. Transfers of assemblies between storage locations change the number of assemblies in storage at both locations but do not affect the overall number of assemblies in storage.

Temporarily discharged assemblies are assemblies that utilities have identified for future reinsertion. The cumulative number of discharged assemblies classified as temporarily discharged increased from 168 assemblies at the end of 1992 to 610 assemblies at the end of 1993 (Table 3). Of these temporarily discharged assemblies, 306 are assemblies from the Long Island Power Authority's Shoreham plant. In 1988, these assemblies were classified as permanently discharged when the Shoreham plant shut down. In 1993, these assemblies were reclassified as temporarily discharged as a result of the transfer of ownership to PECO Energy Company (formerly Philadelphia Electric Company). Some of these assemblies are slated for reinsertion into PECO's Limerick plant. (See Technical Note 14 in Appendix E.)

\section{Nuclear Power Plant Data}

All data from U.S. commercial LWR's which have discharged and/or are storing nuclear fuel assemblies as of December 31, 1993, are reported on Form RW-859. A total of 117 nuclear plants fall into this category: 41 BWR's and 76 PWR's. Three additional PWR's are expected to discharge spent fuel in the future. The data for U.S. commercial nuclear power reactors as of December 31, 1993 (Table 4) include:

- Electric utility name

- Reactor name

- State in which the reactor is located

- Reactor type

- Reactor vendor

- Summer capability rating (net) in megawatts electric (MWe)

- Number of assemblies in the reactor core during its operation (core size)

- Startup date (year in which electricity was first generated from the reactor)

- License expiration date

- Year projected by the utility for loss of the ability to operate absent pickup of spent fuel by the DOE

- Actual or projected retirement date

Figures 1 and 2 provide graphic representations of the loss of ability to operate and the projected retirement year for all U.S. reactors currently in operation.

The license expiration date and the electric utility's projected retirement date are generally the same for each reactor. All reactors that have shut down to date have done so before their license expiration dates. Some have shut down permanently and relinquished the operating licenses granted by the Nuclear Regulatory Commission. These are referred to as "retired" reactors. Others have shut down but have not 
surrendered their licenses. These reactors are in a "shut down mode."

Additional storage capacity is projected to be needed when a current storage facility is filled to capacity, based on an understanding of current storage conditions. Form RW-859 defines filled to capacity as the loss of ability to discharge spent fuel. EIA collects data based on the current plans for each commercial reactor for spent fuel storage. It also collects the estimated date by which the utility would discontinue reactor operation due to the lack of storage space for discharged fuel (without DOE pickup of spent fuel). This date may change in the future as utilities implement plans to rerack existing spent fuel storage pools, expand their storage capacities through licensed dry storage facilities, ship spent fuel, and modify fuel cycle management programs.

Table 1. Spent Fuel Discharges in 1993

\begin{tabular}{|c|c|c|c|c|c|c|c|c|}
\hline Electric Utility Name & Reactor Name & $\begin{array}{c}\text { Reactor } \\
\text { Type }\end{array}$ & $\underset{\text { ID }}{\text { Reactor }}$ & $\begin{array}{l}\text { Number of } \\
\text { Discharged } \\
\text { Assemblies }\end{array}$ & $\begin{array}{c}\text { Initial } \\
\text { Uranium } \\
\text { Content of } \\
\text { Discharged } \\
\text { Assemblies } \\
\text { (MTU) }\end{array}$ & \begin{tabular}{|} 
Number of \\
Temporarily \\
Discharged \\
Assemblies
\end{tabular} & \begin{tabular}{|} 
Initial \\
Uranium \\
Content \\
Temporarily \\
Discharged \\
Assemblies \\
(MTU)
\end{tabular} & \begin{tabular}{|l} 
\\
1993 \\
Reactor \\
Cycle \\
Shutdown \\
Date
\end{tabular} \\
\hline $\begin{array}{l}\text { Arizona Public Service } \\
\text { Company } \ldots \ldots \ldots \ldots\end{array}$ & $\begin{array}{l}\text { Palo Verde } 1 \\
\text { Palo Verde } 2\end{array}$ & $\begin{array}{l}\text { PWR } \\
\text { PWR }\end{array}$ & $\begin{array}{l}0301 \\
0302\end{array}$ & $\begin{array}{l}93 \\
97\end{array}$ & $\begin{array}{l}38.5 \\
38.8\end{array}$ & $\begin{array}{l}0 \\
0\end{array}$ & $\begin{array}{l}0.0 \\
0.0\end{array}$ & $\begin{array}{l}\text { Sep. } 04 \\
\text { Mar. } 19\end{array}$ \\
\hline $\begin{array}{l}\text { Arkansas Power and } \\
\text { Light Company } \ldots \ldots \ldots\end{array}$ & . Arkansas Nuclear 1 & PWR & 0401 & 60 & 27.8 & 0 & 0.0 & Sep. 06 \\
\hline $\begin{array}{l}\text { Carolina Power and Light } \\
\text { Company } \ldots \ldots \ldots \ldots\end{array}$ & $\begin{array}{l}\text { Brunswick } 1 \\
\text { Robinson } 2\end{array}$ & $\begin{array}{l}\text { BWR } \\
\text { PWR }\end{array}$ & $\begin{array}{l}0701 \\
0705\end{array}$ & $\begin{array}{r}124 \\
56\end{array}$ & $\begin{array}{l}22.9 \\
22.6\end{array}$ & $\begin{array}{l}0 \\
0\end{array}$ & $\begin{array}{l}0.0 \\
0.0\end{array}$ & $\begin{array}{l}\text { Apr. } 13 \\
\text { Sep. } 02\end{array}$ \\
\hline $\begin{array}{l}\text { Commonwealth Edison } \\
\text { Company } \ldots \ldots \ldots \ldots\end{array}$ & $\begin{array}{l}\text { Braidwood } 2 \\
\text { Byron } 1 \\
\text { Byron } 2 \\
\text { Dresden } 2 \\
\text { LaSalle County } 2 \\
\text { Quad Cities } 2 \\
\text { Zion } 1\end{array}$ & $\begin{array}{l}\text { PWR } \\
\text { PWR } \\
\text { PWR } \\
\text { BWR } \\
\text { BWR } \\
\text { BWR } \\
\text { PWR }\end{array}$ & $\begin{array}{l}1002 \\
1003 \\
1004 \\
1006 \\
1009 \\
1011 \\
1012\end{array}$ & $\begin{array}{r}84 \\
88 \\
88 \\
208 \\
232 \\
144 \\
76\end{array}$ & $\begin{array}{l}35.7 \\
37.3 \\
37.3 \\
35.9 \\
43.0 \\
25.3 \\
34.7\end{array}$ & $\begin{array}{l}0 \\
0 \\
0 \\
0 \\
0 \\
0 \\
0\end{array}$ & $\begin{array}{l}0.0 \\
0.0 \\
0.0 \\
0.0 \\
0.0 \\
0.0 \\
0.0\end{array}$ & $\begin{array}{l}\text { Mar. } 05 \\
\text { Feb. } 05 \\
\text { Sep. } 03 \\
\text { Jan. } 17 \\
\text { Sep. } 04 \\
\text { Mar. } 06 \\
\text { Oct. } 21\end{array}$ \\
\hline $\begin{array}{l}\text { Consolidated Edison } \\
\text { Company of New York ... }\end{array}$ & Indian Point 2 & PWR & 1102 & 89 & 41.1 & 1 & 0.5 & Jan. 29 \\
\hline $\begin{array}{l}\text { Consumers Power } \\
\text { Company } \ldots \ldots \ldots \ldots\end{array}$ & $\begin{array}{l}\text { Big Rock Point } \\
\text { Palisades }\end{array}$ & $\begin{array}{l}\text { BWR } \\
\text { PWR }\end{array}$ & $\begin{array}{l}1201 \\
1204\end{array}$ & $\begin{array}{l}22 \\
60\end{array}$ & $\begin{array}{r}2.9 \\
24.0\end{array}$ & $\begin{array}{l}0 \\
0\end{array}$ & $\begin{array}{l}0.0 \\
0.0\end{array}$ & $\begin{array}{l}\text { Jun. } 26 \\
\text { Jun. } 04\end{array}$ \\
\hline Detroit Edison Company .. & . Enrico Fermi 2 & BWR & 1402 & 320 & 57.0 & 40 & 6.8 & Dec. 25 \\
\hline
\end{tabular}

See footnotes at end of table. 


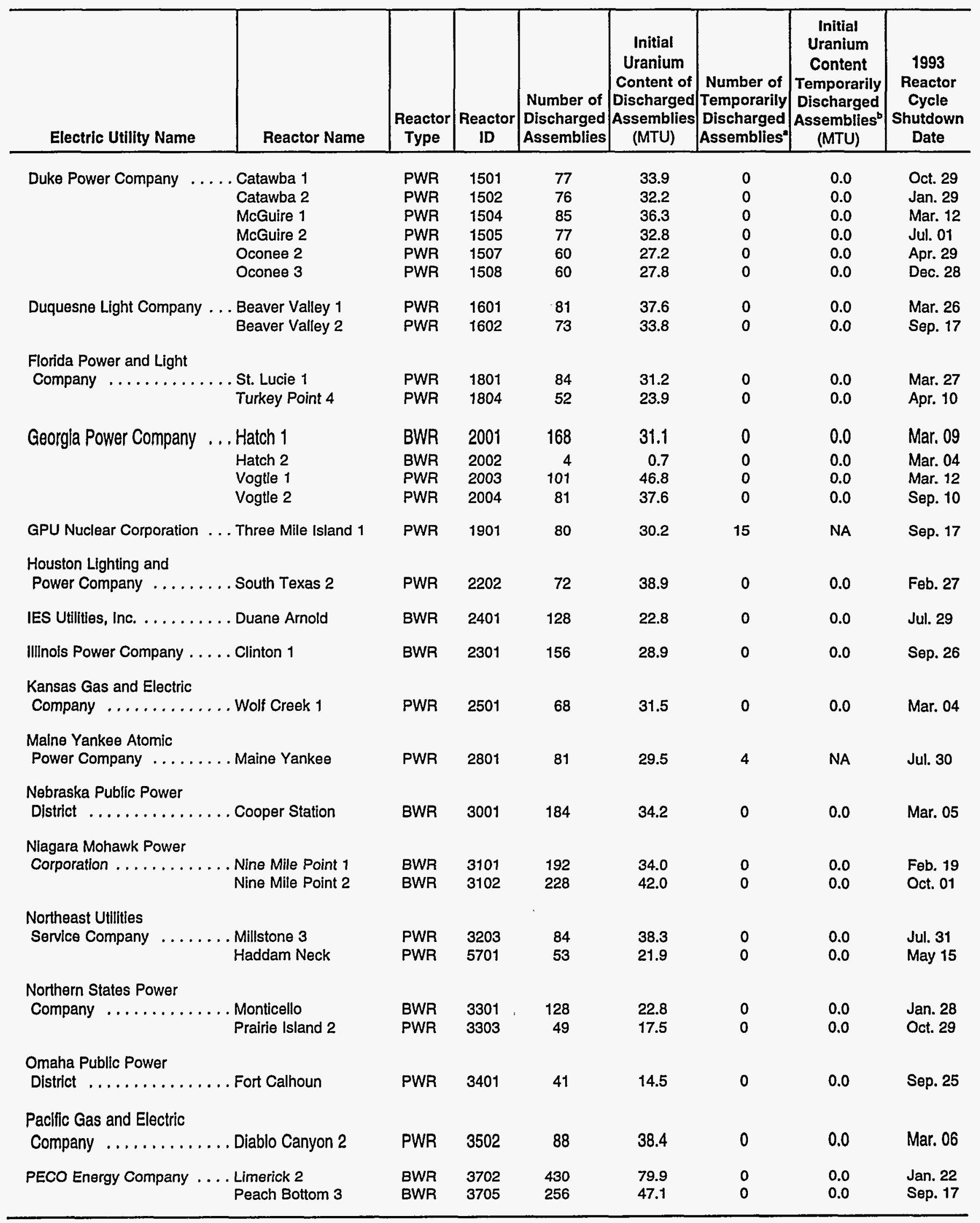

See footnotes at end of table. 


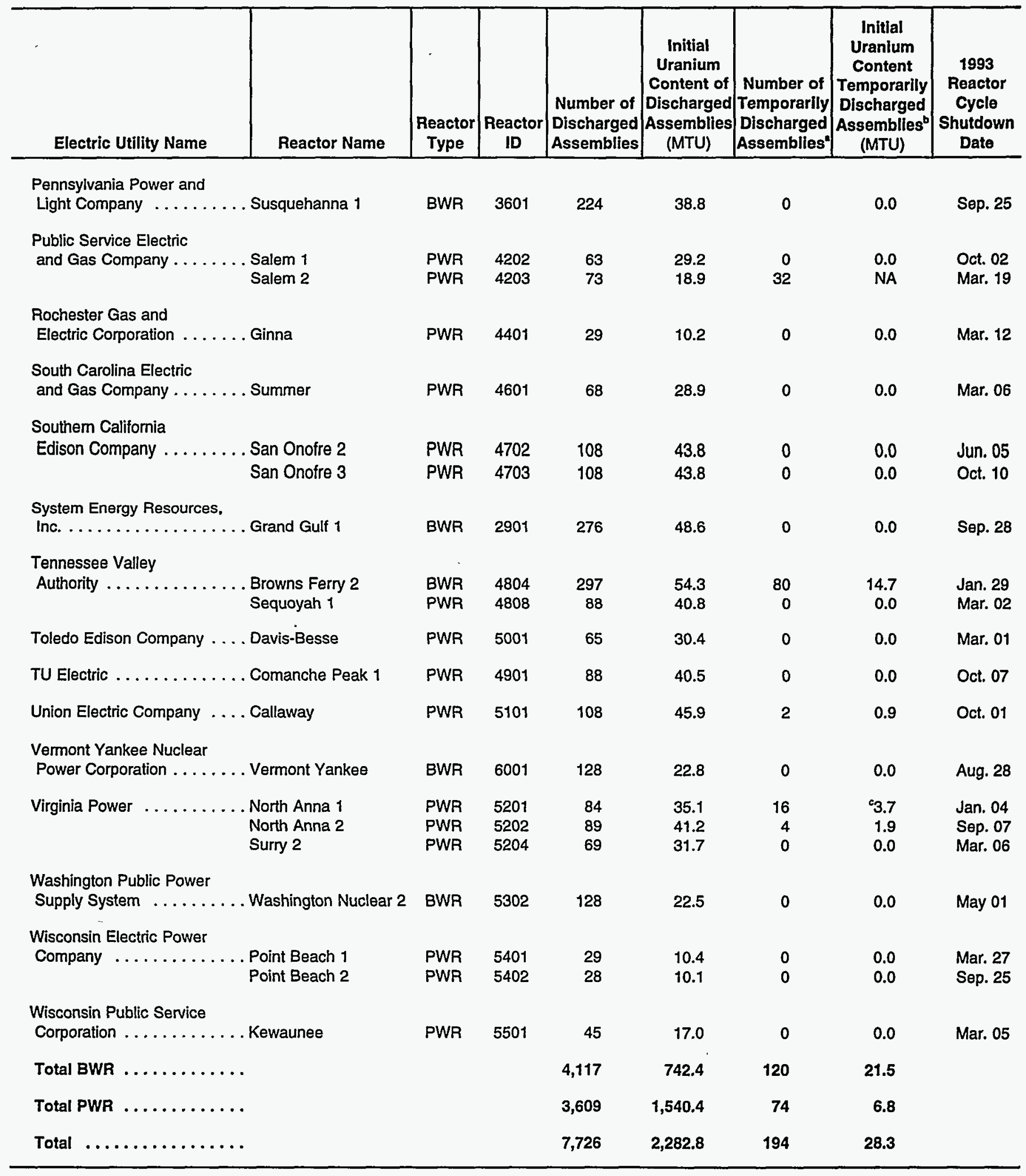

${ }^{a}$ These numbers are included in the Number of Discharged Assemblies column.

'These numbers are included in the Initial Uranium Content of Discharged Assemblies column.

No weight reported for 8 temporarily discharged assemblies.

NA = Not Available; MTU = Metric tons of uranium; PWR = Pressurized-water reactor; BWR = Boiling-water reactor.

Note: Totals may not equal sum of components because of independent rounding. See Technical Note 11 in Appendix E.

Source: Energy Information Administration, Form RW-859, "Nuclear Fuel Data" (1993). 
Table 2. Reactor Spent Fuel Discharge and Storage Activity for 1993

\begin{tabular}{|c|c|c|c|c|c|c|c|c|}
\hline Electric Utility Name & Reactor Name & $\begin{array}{l}\text { Reactor } \\
\text { Type }\end{array}$ & $\begin{array}{l}\text { Pool Site } \\
\text { ID }\end{array}$ & $\begin{array}{l}1992 \text { Assembly } \\
\text { Total }\end{array}$ & $\begin{array}{l}1993 \text { Assembly } \\
\text { Discharges }\end{array}$ & $\begin{array}{l}1993 \text { Assembly } \\
\text { Reinsertions }\end{array}$ & Other & $\begin{array}{l}1993 \text { Assembly } \\
\text { Total }\end{array}$ \\
\hline Alabama Power Company .... & $\begin{array}{l}\text { Farley } 1 \\
\text { Farley } 2\end{array}$ & $\begin{array}{l}\text { PWR } \\
\text { PWR }\end{array}$ & $\begin{array}{l}0101 \\
0102\end{array}$ & $\begin{array}{l}655 \\
498\end{array}$ & 60 & & & $\begin{array}{l}655 \\
558\end{array}$ \\
\hline $\begin{array}{l}\text { Arizona Public Service } \\
\text { Company } \ldots \ldots \ldots \ldots \ldots\end{array}$ & $\begin{array}{l}\text { Palo Verde } 1 \\
\text { Palo Verde } 2 \\
\text { Palo Verde } 3\end{array}$ & $\begin{array}{l}\text { PWR } \\
\text { PWR } \\
\text { PWR }\end{array}$ & $\begin{array}{l}0301 \\
0302 \\
0303\end{array}$ & $\begin{array}{l}276 \\
288 \\
284\end{array}$ & $\begin{array}{l}93 \\
97\end{array}$ & $\begin{array}{l}1 \\
1\end{array}$ & & $\begin{array}{l}368 \\
384 \\
284\end{array}$ \\
\hline $\begin{array}{l}\text { Arkansas Power and } \\
\text { Light Company } \ldots \ldots \ldots \ldots\end{array}$ & $\begin{array}{l}\text { Arkansas Nuclear } 1 \\
\text { Arkansas Nuclear } 2\end{array}$ & $\begin{array}{l}\text { PWR } \\
\text { PWR }\end{array}$ & $\begin{array}{l}0401 \\
0402\end{array}$ & $\begin{array}{l}624 \\
564\end{array}$ & 60 & & & $\begin{array}{l}684 \\
564\end{array}$ \\
\hline $\begin{array}{l}\text { Baltimore Gas and } \\
\text { Electric Company } \ldots \ldots \ldots\end{array}$ & $\begin{array}{l}\text { Calvert Cliffs } 1 \\
\text { Calvert Cliffs } 2 \\
\text { Dry Storage }\end{array}$ & $\begin{array}{l}\text { PWR } \\
\text { PWR } \\
\text { PWR }\end{array}$ & $\begin{array}{l}0501 \\
0501 D\end{array}$ & $\begin{array}{r}1,410 \\
0 \\
0\end{array}$ & 93 & 5 & $\begin{array}{l}a-48 \\
a 48\end{array}$ & $\begin{array}{r}1,450 \\
48\end{array}$ \\
\hline Boston Edison Company ..... & Pilgrim 1 & BWR & 0601 & 1,488 & 140 & & & 1,628 \\
\hline $\begin{array}{l}\text { Carolina Power and } \\
\text { Light Company } \ldots \ldots \ldots \ldots\end{array}$ & $\begin{array}{l}\text { Brunswick } 1 \\
\text { Brunswick } 1 \\
\text { Brunswick } 2 \\
\text { Brunswick } 2 \\
\text { Harris } 1 \\
\text { Harris } 1 \\
\text { Robinson } 2 \\
\text { Dry Storage }\end{array}$ & $\begin{array}{l}\text { BWR } \\
\text { PWR } \\
\text { BWR } \\
\text { PWR } \\
\text { PWR } \\
\text { BWR } \\
\text { PWR } \\
\text { PWR }\end{array}$ & $\begin{array}{l}0701 \\
0701 \\
0702 \\
0702 \\
0703 \\
0703 \\
0705 \\
0705 D\end{array}$ & $\begin{array}{r}1,090 \\
160 \\
1,113 \\
144 \\
420 \\
413 \\
212 \\
56\end{array}$ & $\begin{array}{r}124 \\
56\end{array}$ & $\cdot$ & $\begin{array}{l}b-68 \\
b^{b}-272 \\
c^{c} 28 \\
{ }^{0} 340 \\
c^{c}-28\end{array}$ & $\begin{array}{r}1.146 \\
160 \\
841 \\
144 \\
448 \\
753 \\
240 \\
56\end{array}$ \\
\hline $\begin{array}{l}\text { Cleveland Electric } \\
\text { Illuminating Company . . . . . . }\end{array}$ & Perry 1 & $\begin{array}{l}\text { BWR } \\
\text { BWR }\end{array}$ & $\begin{array}{l}0901 \\
0902\end{array}$ & 748 & & & & 748 \\
\hline $\begin{array}{l}\text { Commonwealth Edison } \\
\text { Company } \ldots \ldots \ldots\end{array}$ & $\begin{array}{l}\text { Braidwood } 1 \\
\text { Braidwood } 2 \\
\text { Byron 1 } \\
\text { Byron } 2 \\
\text { Dresden } 1 \\
\text { Dresden } 2 \\
\text { Dresden } 3 \\
\text { LaSalle County 1. } \\
\text { LaSalle County } 2 \\
\text { Quad Cities } 1 \\
\text { Quad Cities } 2 \\
\text { Zion } 1 \\
\text { Zion } 2\end{array}$ & $\begin{array}{l}\text { PWR } \\
\text { PWR } \\
\text { PWR } \\
\text { PWR } \\
\text { BWR } \\
\text { BWR } \\
\text { BWR } \\
\text { BWR } \\
\text { BWR } \\
\text { BWR } \\
\text { BWR } \\
\text { PWR } \\
\text { PWR }\end{array}$ & $\begin{array}{l}1001 \\
1003 \\
1005 \\
1006 \\
1007 \\
1008 \\
1010 \\
1012\end{array}$ & $\begin{array}{r}404 \\
596 \\
\\
683 \\
1,954 \\
1,968 \\
1,920 \\
0 \\
3,996 \\
0 \\
1,608\end{array}$ & $\begin{array}{r}84 \\
88 \\
88 \\
\\
\\
208\end{array}$ & & & $\begin{array}{r}488 \\
772 \\
683 \\
2,162 \\
1,968 \\
2,152 \\
4,140 \\
1,684\end{array}$ \\
\hline
\end{tabular}

See footnotes at end of table. 
Table 2. Reactor Spent Fuel Discharge and Storage Activity for 1993 (Continued)

\begin{tabular}{|c|c|c|c|c|c|c|c|c|}
\hline Electric Utility Name & Reactor Name & $\begin{array}{l}\text { Reactor } \\
\text { Type }\end{array}$ & $\begin{array}{l}\text { Pool Site } \\
\text { ID }\end{array}$ & $\begin{array}{l}1992 \text { Assembly } \\
\text { Total }\end{array}$ & $\begin{array}{l}1993 \text { Assembly } \\
\text { Discharges }\end{array}$ & $\begin{array}{l}1993 \text { Assembly } \\
\text { Reinsertions }\end{array}$ & Other & $\begin{array}{l}1993 \text { Assembly } \\
\text { Total }\end{array}$ \\
\hline $\begin{array}{l}\text { Consolidated Edison } \\
\text { Company of New York ...... }\end{array}$ & $\begin{array}{l}\text { Indian Point } 1 \\
\text { Indian Point } 2\end{array}$ & $\begin{array}{l}\text { PWR } \\
\text { PWR }\end{array}$ & $\begin{array}{l}1101 \\
1102\end{array}$ & $\begin{array}{l}160 \\
668\end{array}$ & 89 & 1 & & $\begin{array}{l}160 \\
756\end{array}$ \\
\hline $\begin{array}{l}\text { Consumers Power } \\
\text { Company } \ldots \ldots\end{array}$ & $\begin{array}{l}\text { Big Rock Point } \\
\text { Palisades } \\
\text { Dry Storage }\end{array}$ & $\begin{array}{l}\text { BWR } \\
\text { PWR } \\
\text { PWR }\end{array}$ & $\begin{array}{l}1201 \\
1204 \\
1204 D\end{array}$ & $\begin{array}{r}294 \\
733 \\
0\end{array}$ & $\begin{array}{l}22 \\
60\end{array}$ & & $\begin{array}{l}d \\
-48 \\
48\end{array}$ & $\begin{array}{r}316 \\
745 \\
48\end{array}$ \\
\hline $\begin{array}{l}\text { Dairyland Power } \\
\text { Cooperative } \ldots \ldots \ldots \ldots\end{array}$ & LaCrosse & BWR & 1301 & 333 & & & & 333 \\
\hline Detroit Edison Company ..... & Enrico Fermi 2 & BWR & 1402 & 672 & 320 & 92 & & 900 \\
\hline Duke Power Company ...... & $\begin{array}{l}\text { Catawba } 1 \\
\text { Catawba } 2 \\
\text { McGuire } 1 \\
\text { McGuire } 2 \\
\text { Oconee } 1 \\
\text { Oconee } 2 \\
\text { Oconee } 3 \\
\text { Dry Storage }\end{array}$ & $\begin{array}{l}\text { PWR } \\
\text { PWR } \\
\text { PWR } \\
\text { PWR } \\
\text { PWR } \\
\text { PWR } \\
\text { PWR } \\
\text { PWR }\end{array}$ & $\begin{array}{l}1501 \\
1502 \\
1504 \\
1505 \\
1506 \\
1508 \\
1506 D\end{array}$ & $\begin{array}{r}408 \\
280 \\
519 \\
741 \\
1,014 \\
\\
516 \\
480\end{array}$ & $\begin{array}{l}77 \\
76 \\
85 \\
77 \\
\\
60 \\
60\end{array}$ & $\begin{array}{r}1 \\
21 \\
1\end{array}$ & $\begin{array}{l}0-18 \\
-30 \\
-30 \\
-48 \\
096\end{array}$ & $\begin{array}{r}484 \\
356 \\
583 \\
817 \\
1,026 \\
\\
528 \\
576\end{array}$ \\
\hline Duquesne Light Company . . . . & $\begin{array}{l}\text { Beaver Valley } 1 \\
\text { Beaver Valley } 2\end{array}$ & $\begin{array}{l}\text { PWR } \\
\text { PWR }\end{array}$ & $\begin{array}{l}1601 \\
1602\end{array}$ & $\begin{array}{l}512 \\
192\end{array}$ & $\begin{array}{l}81 \\
73\end{array}$ & $\begin{array}{r}17 \\
5\end{array}$ & & $\begin{array}{l}576 \\
260\end{array}$ \\
\hline Florida Power Corporation . . . . & Crystal River 3 & PWR & 1701 & 536 & & & & 536 \\
\hline $\begin{array}{l}\text { Florida Power and Light } \\
\text { Company } \ldots \ldots \ldots \ldots \ldots\end{array}$ & $\begin{array}{l}\text { St. Lucie } 1 \\
\text { St. Lucie } 2 \\
\text { Turkey Point } 3 \\
\text { Turkey Point } 4\end{array}$ & $\begin{array}{l}\text { PWR } \\
\text { PWR } \\
\text { PWR } \\
\text { PẂR }\end{array}$ & $\begin{array}{l}1801 \\
1802 \\
1803 \\
1804\end{array}$ & $\begin{array}{l}796 \\
464 \\
588 \\
546\end{array}$ & $\begin{array}{l}84 \\
52\end{array}$ & & & $\begin{array}{l}880 \\
464 \\
588 \\
598\end{array}$ \\
\hline Georgia Power Company .... & $\begin{array}{l}\text { Hatch } 1 \\
\text { Hatch } 2 \\
\text { Vogtle } 1 \\
\text { Vogtle } 2\end{array}$ & $\begin{array}{l}\text { BWR } \\
\text { BWR } \\
\text { PWR } \\
\text { PWR }\end{array}$ & $\begin{array}{l}2001 \\
2003\end{array}$ & $\begin{array}{r}3,376 \\
0 \\
400 \\
0\end{array}$ & $\begin{array}{r}168 \\
4 \\
101 \\
81\end{array}$ & $\begin{array}{r}4 \\
17\end{array}$ & & $\begin{array}{r}3,544 \\
565\end{array}$ \\
\hline GPU Nuclear Corporation .... & $\begin{array}{l}\text { Three Mile Island } 1 \\
\text { Oyster Creek }\end{array}$ & $\begin{array}{l}\text { PWR } \\
\text { BWR }\end{array}$ & $\begin{array}{l}1901 \\
1903\end{array}$ & $\begin{array}{r}521 \\
1,876\end{array}$ & 80 & & & $\begin{array}{r}601 \\
1,876\end{array}$ \\
\hline $\begin{array}{l}\text { Gulf States Utilities } \\
\text { Company } \ldots \ldots \ldots \ldots \ldots\end{array}$ & River Bend 1 & BWR & 2101 & 764 & & & & 764 \\
\hline $\begin{array}{l}\text { Houston Lighting and } \\
\text { Power Company ... }\end{array}$ & $\begin{array}{l}\text { South Texas } 1 \\
\text { South Texas } 2\end{array}$ & $\begin{array}{l}\text { PWR } \\
\text { PWR }\end{array}$ & $\begin{array}{l}2201 \\
2202\end{array}$ & $\begin{array}{l}236 \\
116\end{array}$ & 72 & & & $\begin{array}{l}236 \\
188\end{array}$ \\
\hline
\end{tabular}

See footnotes at end of table. 
Table 2. Reactor Spent Fuel Discharge and Storage Activity for 1993 (Continued)

\begin{tabular}{|c|c|c|c|c|c|c|c|c|}
\hline Electric Utility Name & Reactor Name & $\begin{array}{l}\text { Reactor } \\
\text { Type }\end{array}$ & $\begin{array}{l}\text { Pool Site } \\
\text { ID }\end{array}$ & $\begin{array}{l}1992 \text { Assembly } \\
\text { Total }\end{array}$ & $\begin{array}{l}1993 \text { Assembly } \\
\text { Discharges }\end{array}$ & $\begin{array}{l}1993 \text { Assembly } \\
\text { Reinsertions }\end{array}$ & Other & $\begin{array}{l}1993 \text { Assembly } \\
\text { Total }\end{array}$ \\
\hline IES Utilities, Inc. . . . . . . . & Duane Amold & BWR & 2401 & 1,152 & 128 & & & 1,280 \\
\hline Illinois Power Company . . . . . . & Clinton 1 & BWR & 2301 & 568 & 156 & & & 724 \\
\hline $\begin{array}{l}\text { Indiana Michigan } \\
\text { Power Company }\end{array}$ & $\begin{array}{l}\text { Cook } 1 \\
\text { Cook } 2\end{array}$ & $\begin{array}{l}\text { PWR } \\
\text { PWR }\end{array}$ & 5801 & 1,523 & & & & 1,523 \\
\hline $\begin{array}{l}\text { Kansas Gas and Electric } \\
\text { Company } \ldots \ldots \ldots \ldots \ldots\end{array}$ & Wolf Creek 1 & PWR & 2501 & 340 & 68 & & & 408 \\
\hline $\begin{array}{l}\text { Long Island Power } \\
\text { Authority } \ldots \ldots \ldots \ldots \ldots\end{array}$ & Shoreham & BWR & 2601 & 560 & & & $9-306$ & 254 \\
\hline $\begin{array}{l}\text { Louisiana Power and } \\
\text { Light Company } \ldots \ldots \ldots \ldots\end{array}$ & Waterford 3 & PWR & 2701 & 428 & & & & 428 \\
\hline $\begin{array}{l}\text { Maine Yankee Atomic } \\
\text { Power Company } . . . . \ldots \ldots\end{array}$ & Maine Yankee & PWR & 2801 & 1,077 & 81 & 9 & & 1,149 \\
\hline $\begin{array}{l}\text { Nebraska Public Power } \\
\text { District } \ldots \ldots \ldots \ldots \ldots \ldots\end{array}$ & Cooper Station & BWR & 3001 & 620 & 184 & & & 804 \\
\hline $\begin{array}{l}\text { New York Power } \\
\text { Authority .............. }\end{array}$ & $\begin{array}{l}\text { FitzPatrick } \\
\text { Indian Point } 3\end{array}$ & $\begin{array}{l}\text { BWR } \\
\text { PWR }\end{array}$ & $\begin{array}{l}3901 \\
3902\end{array}$ & $\begin{array}{r}1,684 \\
584\end{array}$ & & & & $\begin{array}{r}1,684 \\
584\end{array}$ \\
\hline $\begin{array}{l}\text { Niagara Mohawk Power } \\
\text { Corporation ............. }\end{array}$ & $\begin{array}{l}\text { Nine Mile Point } 1 \\
\text { Nine Mile Point } 2\end{array}$ & $\begin{array}{l}\text { BWR } \\
\text { BWR }\end{array}$ & $\begin{array}{l}3101 \\
3102\end{array}$ & $\begin{array}{r}1,620 \\
444\end{array}$ & $\begin{array}{l}192 \\
228\end{array}$ & 32 & & $\begin{array}{r}1,812 \\
640\end{array}$ \\
\hline $\begin{array}{l}\text { North Atlantic Energy } \\
\text { Service Corporation ........ }\end{array}$ & Seabrook & PWR & 5901 & 136 & & & & 136 \\
\hline $\begin{array}{l}\text { Northeast Utilities } \\
\text { Service Company } \ldots \ldots \ldots \ldots\end{array}$ & $\begin{array}{l}\text { Millstone } 1 \\
\text { Millstone } 2 \\
\text { Millstone } 3 \\
\text { Haddam Neck }\end{array}$ & $\begin{array}{l}\text { BWR } \\
\text { PWR } \\
\text { PWR } \\
\text { PWR }\end{array}$ & $\begin{array}{l}3201 \\
3202 \\
3203 \\
5701\end{array}$ & $\begin{array}{r}2,116 \\
784 \\
248 \\
757\end{array}$ & $\begin{array}{l}84 \\
53\end{array}$ & 1 & & $\begin{array}{r}2,116 \\
784 \\
332 \\
809\end{array}$ \\
\hline $\begin{array}{l}\text { Northem States Power } \\
\text { Company } \ldots \ldots \ldots \ldots \ldots\end{array}$ & $\begin{array}{l}\text { Monticello } \\
\text { Prairie Island } 1 \\
\text { Prairie Island } 2\end{array}$ & $\begin{array}{l}\text { BWR } \\
\text { PWR } \\
\text { PWR }\end{array}$ & $\begin{array}{l}3301 \\
3302\end{array}$ & $\begin{array}{r}582 \\
1,233\end{array}$ & $\begin{array}{r}128 \\
49\end{array}$ & 1 & & $\begin{array}{r}710 \\
1,281\end{array}$ \\
\hline $\begin{array}{l}\text { Omaha Public Power } \\
\text { District } \ldots \ldots \ldots \ldots \ldots \ldots\end{array}$ & Fort Calhoun & PWR & 3401 & 529 & 41 & & & 570 \\
\hline
\end{tabular}

See footnotes at end of table. 
Table 2. Reactor Spent Fuel Discharge and Storage Activity for 1993 (Continued)

\begin{tabular}{|c|c|c|c|c|c|c|c|c|}
\hline Electric Utility Name & Reactor Name & $\begin{array}{l}\text { Reactor } \\
\text { Type }\end{array}$ & $\begin{array}{l}\text { Pool Site } \\
\text { ID }\end{array}$ & $\begin{array}{l}1992 \text { Assembly } \\
\text { Total }\end{array}$ & $\begin{array}{l}1993 \text { Assembly } \\
\text { Discharges }\end{array}$ & $\begin{array}{c}1993 \text { Assembly } \\
\text { Reinsertions }\end{array}$ & Other & $\begin{array}{l}1993 \text { Assembly } \\
\text { Total }\end{array}$ \\
\hline $\begin{array}{l}\text { Pacific Gas and Electric } \\
\text { Company } \ldots \ldots \ldots \ldots \ldots\end{array}$ & $\begin{array}{l}\text { Diablo Canyon } 1 \\
\text { Diablo Canyon } 2 \\
\text { Humboldt Bay }\end{array}$ & $\begin{array}{l}\text { PWR } \\
\text { PWR } \\
\text { BWR }\end{array}$ & $\begin{array}{l}3501 \\
3502 \\
3503\end{array}$ & $\begin{array}{l}376 \\
308 \\
390\end{array}$ & 88 & & & $\begin{array}{l}376 \\
396 \\
390\end{array}$ \\
\hline PECO Energy Company . . . . . & $\begin{array}{l}\text { Limerick } 1 \\
\text { Limerick } 2 \\
\text { Peach Bottom } 2 \\
\text { Peach Bottom } 3\end{array}$ & $\begin{array}{l}\text { BWR } \\
\text { BWR } \\
\text { BWR } \\
\text { BWR }\end{array}$ & $\begin{array}{l}3701 \\
3704 \\
3705\end{array}$ & $\begin{array}{r}1,140 \\
224 \\
2,162 \\
1,944\end{array}$ & $\begin{array}{r}0 \\
430 \\
256\end{array}$ & $n_{146}$ & 9306 & $\begin{array}{r}1,300 \\
654 \\
2,162 \\
2,200\end{array}$ \\
\hline $\begin{array}{l}\text { Pennsylvania Power and } \\
\text { Light Company } \ldots . . .\end{array}$ & $\begin{array}{l}\text { Susquehanna } 1 \\
\text { Susquehanna } 2\end{array}$ & $\begin{array}{l}\text { BWR } \\
\text { BWR }\end{array}$ & 3601 & $\begin{array}{l}1,404 \\
1,236\end{array}$ & 224 & & & $\begin{array}{l}1,628 \\
1,236\end{array}$ \\
\hline $\begin{array}{l}\text { Portland General Electric } \\
\text { Company } \ldots \ldots \ldots \ldots \ldots\end{array}$ & Trojan & PWR & 3801 & 780 & & & & 780 \\
\hline $\begin{array}{l}\text { Public Service Electric } \\
\text { and Gas Company ........ }\end{array}$ & $\begin{array}{l}\text { Hope Creek } \\
\text { Salem } 1 \\
\text { Salem } 2\end{array}$ & $\begin{array}{l}\text { BWR } \\
\text { PWR } \\
\text { PWR }\end{array}$ & $\begin{array}{l}4201 \\
4202 \\
4203\end{array}$ & $\begin{array}{r}1,008 \\
656 \\
436\end{array}$ & $\begin{array}{l}63 \\
73\end{array}$ & $\begin{array}{l}11 \\
17\end{array}$ & & $\begin{array}{r}1,008 \\
708 \\
492\end{array}$ \\
\hline $\begin{array}{l}\text { Rochester Gas and } \\
\text { Electric Corporation } \ldots \ldots \ldots\end{array}$ & Ginna & PWR & 4401 & 661 & 29 & 1 & & 689 \\
\hline $\begin{array}{l}\text { Sacramento Municipal } \\
\text { Utility District } \ldots \ldots \ldots \ldots\end{array}$ & Rancho Seco & PWR & 4501 & 493 & & & & 493 \\
\hline $\begin{array}{l}\text { South Carolina Electric } \\
\text { and Gas Company ......... }\end{array}$ & Summer & PWR & 4601 & 372 & 68 & & & 440 \\
\hline $\begin{array}{l}\text { Southem California } \\
\text { Edison Company ......... }\end{array}$ & $\begin{array}{l}\text { San Onofre } 1 \\
\text { San Onofre } 2 \\
\text { San Onofre } 3\end{array}$ & $\begin{array}{l}\text { PWR } \\
\text { PWR } \\
\text { PWR }\end{array}$ & $\begin{array}{l}4701 \\
4702 \\
4703\end{array}$ & $\begin{array}{l}207 \\
554 \\
602\end{array}$ & $\begin{array}{l}108 \\
108\end{array}$ & & & $\begin{array}{l}207 \\
662 \\
710\end{array}$ \\
\hline $\begin{array}{l}\text { System Energy Resources, } \\
\text { Inc. . . . . . . . . . . . . }\end{array}$ & Grand Gulf 1 & BWR & 2901 & 1,384 & 276 & & & 1,660 \\
\hline $\begin{array}{l}\text { Tennessee Valley } \\
\text { Authority } \ldots \ldots \ldots \ldots \ldots\end{array}$ & $\begin{array}{l}\text { Browns Ferry } 1 \\
\text { Browns Ferry } 2 \\
\text { Browns Ferry } 3 \\
\text { Sequoyah } 1 \\
\text { Sequoyah } 2 \\
\text { Watts Bar } 1 \\
\text { Watts Bar } 2\end{array}$ & $\begin{array}{l}\text { BWR } \\
\text { BWR } \\
\text { BWR } \\
\text { PWR } \\
\text { PWR } \\
\text { PWR } \\
\text { PWR }\end{array}$ & $\begin{array}{l}4803 \\
4805 \\
4808 \\
4810\end{array}$ & $\begin{array}{r}1,488 \\
1,440 \\
1,029 \\
737 \\
0\end{array}$ & $\begin{array}{r}297 \\
88\end{array}$ & $\begin{array}{l}1 \\
8\end{array}$ & $\begin{array}{l}192 \\
4\end{array}$ & $\begin{array}{r}1,580 \\
1,736 \\
1,030 \\
817 \\
0\end{array}$ \\
\hline Toledo Edison Company .... . . & Davis-Besse & PWR & 5001 & 392 & 65 & 1 & & 456 \\
\hline
\end{tabular}

\footnotetext{
See footnotes at end of table.
} 
Table 2. Reactor Spent Fuel Discharge and Storage Activity for 1993 (Continued)

\begin{tabular}{|c|c|c|c|c|c|c|c|c|}
\hline Electric Utility Name & Reactor Name & $\begin{array}{l}\text { Reactor } \\
\text { Type }\end{array}$ & $\begin{array}{l}\text { Pool Site } \\
\text { ID }\end{array}$ & $\begin{array}{l}1992 \text { Assembly } \\
\text { Total }\end{array}$ & $\begin{array}{l}1993 \text { Assembly } \\
\text { Discharges }\end{array}$ & $\begin{array}{l}1993 \text { Assembly } \\
\text { Reinsertions }\end{array}$ & Other & $\begin{array}{l}1993 \text { Assembly } \\
\text { Total }\end{array}$ \\
\hline TU Electric $\ldots \ldots \ldots \ldots \ldots$ & $\begin{array}{l}\text { Comanche Peak } 1 \\
\text { Comanche Peak } 2\end{array}$ & $\begin{array}{l}\text { PWR } \\
\text { PWR }\end{array}$ & 4901 & $\begin{array}{r}117 \\
0\end{array}$ & 88 & & & $\begin{array}{r}205 \\
0\end{array}$ \\
\hline Union Electric Company ..... & Callaway & PWR & 5101 & 452 & 108 & 12 & & 548 \\
\hline $\begin{array}{l}\text { Vermont Yankee Nuclear } \\
\text { Power Corporation ......... }\end{array}$ & Vermont Yankee & BWR & 6001 & 1,850 & 128 & & & 1,978 \\
\hline Virginia Power $\ldots \ldots \ldots \ldots$ & $\begin{array}{l}\text { North Anna } 1 \\
\text { North Anna } 2 \\
\text { Surry } 1 \\
\text { Surry } 2 \\
\text { Dry Storage }\end{array}$ & $\begin{array}{l}\text { PWR } \\
\text { PWR } \\
\text { PWR } \\
\text { PWR } \\
\text { PWR }\end{array}$ & $\begin{array}{l}5201 \\
5203 \\
5203 \mathrm{D}\end{array}$ & $\begin{array}{l}993 \\
837 \\
367\end{array}$ & $\begin{array}{l}84 \\
89 \\
69\end{array}$ & $\begin{array}{c}k_{20} \\
2^{2} \\
1 \\
1\end{array}$ & $\begin{array}{r}m_{-23} \\
m_{-26} \\
m_{49}\end{array}$ & $\begin{array}{r}1,125 \\
856 \\
416\end{array}$ \\
\hline $\begin{array}{l}\text { Washington Public Power } \\
\text { Supply System } \ldots \ldots \ldots \ldots\end{array}$ & Washington Nuclear 2 & BWR & 5302 & 912 & 128 & & & 1,040 \\
\hline $\begin{array}{l}\text { Wisconsin Electric Power } \\
\text { Company } \ldots \ldots \ldots \ldots \ldots\end{array}$ & $\begin{array}{l}\text { Point Beach } 1 \\
\text { Point Beach } 2\end{array}$ & $\begin{array}{l}\text { PWR } \\
\text { PWR }\end{array}$ & 5401 & 1,193 & $\begin{array}{l}29 \\
28\end{array}$ & 1 & & 1,249 \\
\hline $\begin{array}{l}\text { Wisconsin Public Service } \\
\text { Corporation ... . . . . . . }\end{array}$ & Kewaunee & PWR & 5501 & 616 & 45 & 9 & & 652 \\
\hline $\begin{array}{l}\text { Yankee Atomic Electric } \\
\text { Company } \ldots \ldots \ldots \ldots \ldots\end{array}$ & Yankee Rowe & PWR & 5601 & 533 & & & & 533 \\
\hline $\begin{array}{l}\text { Total BWR } \ldots \ldots \ldots \ldots \ldots \ldots \\
\text { Total PWR } \ldots \ldots \ldots \ldots \ldots \ldots\end{array}$ & $\ldots \ldots \ldots \ldots \ldots \ldots \ldots$ & $\cdots$ & $\ldots \cdots \cdots$ & $\begin{array}{l}49,645 \\
37,946\end{array}$ & $\begin{array}{l}4,117 \\
3,609\end{array}$ & $\begin{array}{l}275 \\
183\end{array}$ & $\begin{array}{r}93 \\
0\end{array}$ & $\begin{array}{l}53,580 \\
41,372\end{array}$ \\
\hline Total (Nuclear Power Plant Site & es Only) .......... & ... & $\ldots \ldots \ldots$ & 87,591 & 7,726 & 458 & 93 & 94,952 \\
\hline Storage Facilities (From Table & 14) $\ldots \ldots \ldots \ldots$ & ... & $\ldots \ldots \ldots$ & 3,448 & - & - & - & 3,448 \\
\hline Total (Including Storage Facil & & & & 91,039 & 7,726 & 458 & 93 & 98,400 \\
\hline
\end{tabular}

Transfer of 48 assemblies from Calvert Cliffs 1 to Calvert Cliffs dry storage.

Transfer of 340 assemblies from Brunswick storage pools to Harris 1 storage pools.

Transfer of 28 assemblies from Robinson 2 to Harris 1 storage pools.

Transfer of 48 assemblies from Palisades storage pools to Palisades dry storage.

-Transfer of 96 assemblies from Oconee storage pools to Oconee dry storage.

Total includes 8 assemblies from Vogtle 2 reinserted in core at Vogtle 1.

'Total of 306 assemblies shipped from Shoreham to Limerick 1. See Technical Note 14 in Appendix E.

"Total of 146 assemblies from Limerick 1 reinserted in core at Limerick 2.

'Total of 92 additional, twice burned fuel assemblies declared permanently discharged resulting in adjustment to historical inventory data.

'One additional assembly, missing an expansion ring, declared permanently discharged resulting in adjustment to historical inventory data.

kTotal includes 8 assemblies from North Anna 2 reinserted in core at North Anna 1.

'Total includes 1 assembly from North Anna 1 reinserted in core at North Anna 2.

mTransfer of $\mathbf{4 9}$ assemblies from Surry storage pools to Surry dry storage.

PWR = Pressurized-water reactor; BWR = Boiling-water reactor. - = Not applicable.

Source: Energy information Administration, Form RW-859, "Nuclear Fuel Data" (1993). 
Table 3. Temporarily Discharged Assemblies

\begin{tabular}{|c|c|c|c|c|c|c|}
\hline Electric Utility Name & Reactor Name & $\begin{array}{l}\text { Reactor } \\
\text { Type }\end{array}$ & $\begin{array}{l}\text { Pool } \\
\text { Site } \\
\text { ID }\end{array}$ & $\begin{array}{c}\text { Temporarily } \\
\text { Discharged } \\
\text { Assemblies } \\
\text { Through } \\
1993\end{array}$ & $\begin{array}{c}\text { Temporarily } \\
\text { Discharged } \\
\text { Assemblles } \\
\text { Through } \\
1992\end{array}$ & $\begin{array}{l}\text { Increase or } \\
\text { Reduction in } \\
\text { Temporarily } \\
\text { Discharged } \\
\text { Assemblies }\end{array}$ \\
\hline $\begin{array}{l}\text { Arizona Public Service } \\
\text { Company } \ldots \ldots \ldots \ldots \ldots \ldots\end{array}$ & Palo Verde 3 & PWR & 0303 & 1 & 1 & 0 \\
\hline $\begin{array}{l}\text { Carolina Power and Light } \\
\text { Company } \quad \ldots \ldots \ldots \ldots \ldots \ldots \ldots\end{array}$ & Brunswick 1 & BWR & 0701 & 1 & 1 & 0 \\
\hline $\begin{array}{l}\text { Cleveland Electric } \\
\text { llluminating Company } \ldots \ldots \ldots \ldots\end{array}$ & Perry 1 & BWR & 0901 & 4 & 4 & 0 \\
\hline $\begin{array}{l}\text { Consolidated Edison } \\
\text { Company of New York }\end{array}$ & Indian Point 2 & PWR & 1102 & 8 & 8 & 0 \\
\hline $\begin{array}{l}\text { Consumers Power } \\
\text { Company } \ldots \ldots \ldots \ldots \ldots \ldots\end{array}$ & Palisades & PWR & 1204 & 1 & 1 & 0 \\
\hline Detroit Edison Company ........ & Enrico Fermi 2 & BWR & 1402 & 40 & 0 & 40 \\
\hline Duquesne Light Company . . . . . . & $\begin{array}{l}\text { Beaver Valley } 1 \\
\text { Beaver Valley } 2\end{array}$ & $\begin{array}{l}\text { PWR } \\
\text { PWR }\end{array}$ & $\begin{array}{l}1601 \\
1602\end{array}$ & $\begin{array}{r}13 \\
0\end{array}$ & $\begin{array}{r}14 \\
5\end{array}$ & $\begin{array}{l}-1 \\
-5\end{array}$ \\
\hline Florida Power Corporation . . . . . . . & Crystal River 3 & PWR & 1701 & 5 & 5 & 0 \\
\hline GPU Nuclear Corporation $\ldots \ldots \ldots$ & Three Mile Island 1 & PWR & 1901 & 23 & 8 & 15 \\
\hline $\begin{array}{l}\text { Houston Lighting and } \\
\text { Power Company } \ldots \ldots \ldots \ldots \ldots\end{array}$ & South Texas 1 & PWR & 2201 & 7 & 7 & 0 \\
\hline $\begin{array}{l}\text { Maine Yankee Atomic } \\
\text { Power Company } \ldots \ldots \ldots \ldots \ldots\end{array}$ & Maine Yankee & PWR & 2801 & 18 & 23 & -5 \\
\hline $\begin{array}{l}\text { New York Power } \\
\text { Authority } \ldots \ldots \ldots \ldots \ldots \ldots \ldots\end{array}$ & Indian Point 3 & PWR & 3902 & 2 & 2 & 0 \\
\hline $\begin{array}{l}\text { Northeast Utilities } \\
\text { Service Company } \ldots \ldots \ldots \ldots \ldots\end{array}$ & Haddam Neck & PWR & 5701 & 0 & 1 & -1 \\
\hline PECO Energy Company . . . . . . . & Limerick 1 & BWR & 3701 & 306 & 0 & ${ }^{2} 306$ \\
\hline $\begin{array}{l}\text { Public Service Electric } \\
\text { and Gas Company.... }\end{array}$ & $\begin{array}{l}\text { Salem } 1 \\
\text { Salem } 2\end{array}$ & $\begin{array}{l}\text { PWR } \\
\text { PWR }\end{array}$ & $\begin{array}{l}4202 \\
4203\end{array}$ & $\begin{array}{l}23 \\
35\end{array}$ & $\begin{array}{r}25 \\
4\end{array}$ & $\begin{array}{l}-2 \\
31\end{array}$ \\
\hline $\begin{array}{l}\text { Tennessee Valley } \\
\text { Authority } \ldots \ldots \ldots \ldots \ldots \ldots \ldots\end{array}$ & Browns Ferry 2 & BWR & 4803 & 80 & 0 & 80 \\
\hline Toledo Edison Company . . . . . . . . & Davis-Besse & PWR & 5001 & 6 & 6 & 0 \\
\hline Union Electric Company . . . . . . . . & Callaway & PWR & 5101 & 2 & 0 & 2 \\
\hline
\end{tabular}

See footnotes at end of table. 
Table 3. Temporarily Discharged Assemblies (Continued)

\begin{tabular}{|c|c|c|c|c|c|c|}
\hline Electric Utility Name & Reactor Name & $\begin{array}{l}\text { Reactor } \\
\text { Type }\end{array}$ & $\begin{array}{c}\text { Pool } \\
\text { Site } \\
\text { ID }\end{array}$ & $\begin{array}{c}\text { Temporarily } \\
\text { Discharged } \\
\text { Assemblies } \\
\text { Through } \\
1993\end{array}$ & $\begin{array}{c}\text { Temporarily } \\
\text { Discharged } \\
\text { Assemblies } \\
\text { Through } \\
1992\end{array}$ & $\begin{array}{l}\text { Increase or } \\
\text { Reduction in } \\
\text { Temporarily } \\
\text { Discharged } \\
\text { Assemblies }\end{array}$ \\
\hline Virginia Power $\ldots \ldots \ldots \ldots \ldots$ & $\begin{array}{l}\text { North Anna } 1 \\
\text { Surry } 1\end{array}$ & $\begin{array}{l}\text { PWR } \\
\text { PWR }\end{array}$ & $\begin{array}{l}5201 \\
5203\end{array}$ & $\begin{array}{r}25 \\
6\end{array}$ & $\begin{array}{r}34 \\
7\end{array}$ & $\begin{array}{l}-9 \\
-1\end{array}$ \\
\hline $\begin{array}{l}\text { Wisconsin Public Service } \\
\text { Corporation ............. }\end{array}$ & Kewaunee & PWR & 5501 & 4 & 12 & -8 \\
\hline Total $\quad \ldots \ldots \ldots \ldots \ldots \ldots$ & & & & 610 & 168 & 442 \\
\hline
\end{tabular}

'A total of 306 temporarily discharged assemblies were shipped from Long Island Power Authority's Shoreham plant to Limerick 1. See Technical Note 14 in Appendix E.

PWR = Pressurized-water reactor; BWR = Boiling-water reactor.

Note: Changes in number of temporarily discharged assemblies are due to discharge of additional temporarily discharged assemblies, reinsertion of previously discharged assemblies, and/or change in status of previously discharged assemblies.

Source: Energy Information Administration, Form RW-859, "Nuclear Fuel Data" (1993). 


\begin{tabular}{|c|c|c|c|c|c|c|c|c|c|c|}
\hline Electric Utility Name & Reactor Name & State & $\begin{array}{l}\text { Reactor } \\
\text { Type }\end{array}$ & Vendor $^{\mathrm{a}}$ & $\begin{array}{c}\text { Net } \\
\text { Summer } \\
\text { Capability } \\
\text { (net MWe) }^{\mathrm{b}} \\
\end{array}$ & $\begin{array}{c}\text { Core Size } \\
\text { (number of } \\
\text { assemblies) }\end{array}$ & $\begin{array}{c}\text { Startup } \\
\text { (year) }^{c}\end{array}$ & $\begin{array}{c}\text { License } \\
\text { Expiration } \\
\text { (year) }^{d}\end{array}$ & $\begin{array}{c}\text { Loss of } \\
\text { Ability } \\
\text { to Operate } \\
\text { (year) }^{\circ}\end{array}$ & $\begin{array}{c}\text { Actual or } \\
\text { Projected } \\
\text { Retirement } \\
\text { (year) }\end{array}$ \\
\hline Alabama Power Company .. & $\begin{array}{l}\text { Fariey } 1 \\
\text { Fariey } 2\end{array}$ & $\begin{array}{l}\mathrm{AL} \\
\mathrm{AL}\end{array}$ & $\begin{array}{l}\text { PWR } \\
\text { PWR }\end{array}$ & $\begin{array}{l}\text { WE } \\
\text { WE }\end{array}$ & $\begin{array}{l}815 \\
825\end{array}$ & $\begin{array}{l}157 \\
157\end{array}$ & $\begin{array}{l}1977 \\
1981\end{array}$ & $\begin{array}{l}2017 \\
2021\end{array}$ & $\begin{array}{l}2010 \\
2013\end{array}$ & $\begin{array}{l}2017 \\
2021\end{array}$ \\
\hline $\begin{array}{l}\text { Arizona Public Service } \\
\text { Company } \ldots \ldots \ldots \ldots\end{array}$ & $\begin{array}{l}\text { Palo Verde } 1 \\
\text { Palo Verde } 2 \\
\text { Palo Verde } 3\end{array}$ & $\begin{array}{l}A Z \\
A Z \\
A Z\end{array}$ & $\begin{array}{l}\text { PWR } \\
\text { PWR } \\
\text { PWR }\end{array}$ & $\begin{array}{l}\mathrm{CE} \\
\mathrm{CE} \\
\mathrm{CE}\end{array}$ & $\begin{array}{l}1,270 \\
1,270 \\
1,270\end{array}$ & $\begin{array}{l}241 \\
241 \\
241\end{array}$ & $\begin{array}{l}1985 \\
1986 \\
1987\end{array}$ & $\begin{array}{l}2024 \\
2025 \\
2027\end{array}$ & $\begin{array}{l}2005 \\
2005 \\
2006\end{array}$ & $\begin{array}{l}2024 \\
2025 \\
2027\end{array}$ \\
\hline $\begin{array}{l}\text { Arkansas Power and } \\
\text { Light Company ........ }\end{array}$ & $\begin{array}{l}\text { Arkansas Nuclear } 1 \\
\text { Arkansas Nuclear } 2\end{array}$ & $\begin{array}{l}\text { AR } \\
\text { AR }\end{array}$ & $\begin{array}{l}\text { PWR } \\
\text { PWR }\end{array}$ & $\begin{array}{l}\text { B\&W } \\
\text { CE }\end{array}$ & $\begin{array}{l}836 \\
856\end{array}$ & $\begin{array}{l}177 \\
177\end{array}$ & $\begin{array}{l}1974 \\
1978\end{array}$ & $\begin{array}{l}2014 \\
2018\end{array}$ & $\begin{array}{l}1996 \\
1997\end{array}$ & $\begin{array}{l}2014 \\
2018\end{array}$ \\
\hline $\begin{array}{l}\text { Baltimore Gas and } \\
\text { Electric Company }\end{array}$ & $\begin{array}{l}\text { Calvert Cliffs } 1 \\
\text { Calvert Cliffs } 2\end{array}$ & $\begin{array}{l}\text { MD } \\
\text { MD }\end{array}$ & $\begin{array}{l}\text { PWR } \\
\text { PWR }\end{array}$ & $\begin{array}{l}\mathrm{CE} \\
\mathrm{CE}\end{array}$ & $\begin{array}{l}830 \\
830\end{array}$ & $\begin{array}{l}217 \\
217\end{array}$ & $\begin{array}{l}1974 \\
1976\end{array}$ & $\begin{array}{l}2014 \\
2016\end{array}$ & $\begin{array}{l}1994 \\
2002\end{array}$ & $\begin{array}{l}2016 \\
2016\end{array}$ \\
\hline Boston Edison Company ... & Pilgrim 1 & MA & BWR & GE & 665 & 580 & 1972 & 2012 & 2003 & 2012 \\
\hline $\begin{array}{l}\text { Carolina Power and Light } \\
\text { Company } \ldots \ldots \ldots \ldots .\end{array}$ & $\begin{array}{l}\text { Brunswick } 1 \\
\text { Brunswick } 2 \\
\text { Harris } 1 \\
\text { Robinson } 2\end{array}$ & $\begin{array}{l}\text { NC } \\
\text { NC } \\
\text { NC } \\
\text { SC }\end{array}$ & $\begin{array}{l}\text { BWR } \\
\text { BWR } \\
\text { PWR } \\
\text { PWR }\end{array}$ & $\begin{array}{l}\text { GE } \\
\text { GE } \\
\text { WE } \\
\text { WE }\end{array}$ & $\begin{array}{l}767 \\
754 \\
860 \\
683\end{array}$ & $\begin{array}{l}560 \\
560 \\
157 \\
157\end{array}$ & $\begin{array}{l}1976 \\
1975 \\
1987 \\
1970\end{array}$ & $\begin{array}{l}2016 \\
2014 \\
2026 \\
2010\end{array}$ & $\begin{array}{l}2001 \\
2003 \\
2018 \\
2004\end{array}$ & $\begin{array}{l}2016 \\
2014 \\
2026 \\
2010\end{array}$ \\
\hline $\begin{array}{l}\text { Cleveland Electric } \\
\text { Illuminating Company ..... }\end{array}$ & Perry 1 & $\mathrm{OH}$ & BWR & GE & 1,169 & 748 & 1986 & 2026 & 2009 & 2026 \\
\hline $\begin{array}{l}\text { Commonwealth Edison } \\
\text { Company } \ldots \ldots \ldots \ldots\end{array}$ & $\begin{array}{l}\text { Braidwood } 1 \\
\text { Braidwood } 2 \\
\text { Byron } 1 \\
\text { Byron } 2 \\
\text { Dresden } 1 \\
\text { Dresden } 2 \\
\text { Dresden } 3 \\
\text { LaSalle County } 1 \\
\text { LaSalle County } 2 \\
\text { Quad Cities } 1 \\
\text { Quad Cities } 2 \\
\text { Zion } 1 \\
\text { Zion } 2\end{array}$ & $\begin{array}{l}\text { IL } \\
\text { IL } \\
\text { IL } \\
\text { IL } \\
\text { IL } \\
\text { IL } \\
\text { IL } \\
\text { IL } \\
\text { IL } \\
\text { IL } \\
\text { IL } \\
\text { IL } \\
\text { IL }\end{array}$ & $\begin{array}{l}\text { PWR } \\
\text { PWR } \\
\text { PWR } \\
\text { PWR } \\
\text { BWR } \\
\text { BWR } \\
\text { BWR } \\
\text { BWR } \\
\text { BWR } \\
\text { BWR } \\
\text { BWR } \\
\text { PWR } \\
\text { PWR }\end{array}$ & $\begin{array}{l}\text { WE } \\
\text { WE } \\
\text { WE } \\
\text { WE } \\
\text { GE } \\
\text { GE } \\
\text { GE } \\
\text { GE } \\
\text { GE } \\
\text { GE } \\
\text { GE } \\
\text { WE } \\
\text { WE }\end{array}$ & $\begin{array}{r}1,090 \\
1,090 \\
1,120 \\
1,120 \\
200 \\
772 \\
773 \\
1,048 \\
1,048 \\
769 \\
769 \\
1,040 \\
1,040\end{array}$ & $\begin{array}{l}193 \\
193 \\
193 \\
193 \\
464 \\
724 \\
724 \\
764 \\
764 \\
724 \\
724 \\
193 \\
193\end{array}$ & $\begin{array}{l}1987 \\
1988 \\
1985 \\
1987 \\
1960 \\
1970 \\
1971 \\
1982 \\
1984 \\
1972 \\
1972 \\
1973 \\
1973\end{array}$ & $\begin{array}{l}2026 \\
2027 \\
2024 \\
2026 \\
1996 \\
2006 \\
2011 \\
2022 \\
2023 \\
2012 \\
2012 \\
2013 \\
2013\end{array}$ & $\begin{array}{r}2012 \\
2013 \\
2011 \\
2011 \\
\text { SD } \\
2000 \\
2001 \\
2013 \\
2015 \\
2007 \\
2006 \\
2006 \\
2006\end{array}$ & $\begin{array}{l}2028 \\
2028 \\
2025 \\
2027 \\
1984 \\
2010 \\
2013 \\
2024 \\
2024 \\
2013 \\
2013 \\
2013 \\
2014\end{array}$ \\
\hline
\end{tabular}

See footnotes at end of table. 
Table 4. Nuclear Power Plant Data as of December 31, 1993 (Continued)

\begin{tabular}{|c|c|c|c|c|c|c|c|c|c|c|}
\hline Electric Utility Name & Reactor Name & State & $\begin{array}{l}\text { Reactor } \\
\text { Type }\end{array}$ & Vendor ${ }^{a}$ & $\begin{array}{c}\text { Net } \\
\text { Summer } \\
\text { Capability } \\
\text { (net MWe) }^{\mathrm{b}}\end{array}$ & $\begin{array}{c}\text { Core Size } \\
\text { (number of } \\
\text { assemblies) }\end{array}$ & $\begin{array}{l}\text { Startup } \\
\text { (year) }^{c}\end{array}$ & $\begin{array}{c}\text { License } \\
\text { Expiration } \\
\text { (year) }^{d}\end{array}$ & $\begin{array}{c}\text { Loss of } \\
\text { Ability } \\
\text { to Operate } \\
\text { (year) }^{\circ}\end{array}$ & $\begin{array}{c}\text { Actual or } \\
\text { Projected } \\
\text { Retirement } \\
\text { (year) }\end{array}$ \\
\hline $\begin{array}{l}\text { Consolidated Edison } \\
\text { Company of New York .... }\end{array}$ & $\begin{array}{l}\text { Indian Point } 1 \\
\text { Indian Point } 2\end{array}$ & $\begin{array}{l}\text { NY } \\
\text { NY }\end{array}$ & $\begin{array}{l}\text { PWR } \\
\text { PWR }\end{array}$ & $\begin{array}{l}\text { B\&W } \\
\text { WE }\end{array}$ & $\begin{array}{l}265 \\
931\end{array}$ & $\begin{array}{l}120 \\
193\end{array}$ & $\begin{array}{l}1962 \\
1973\end{array}$ & $\begin{array}{l}1980 \\
2013\end{array}$ & $\begin{array}{r}R D \\
2003\end{array}$ & $\begin{array}{l}1980 \\
2013\end{array}$ \\
\hline $\begin{array}{l}\text { Consumers Power } \\
\text { Company } \quad \ldots \ldots \ldots \ldots\end{array}$ & $\begin{array}{l}\text { Big Rock Point } \\
\text { Palisades }\end{array}$ & $\begin{array}{l}\text { MI } \\
\text { MI }\end{array}$ & $\begin{array}{l}\text { BWR } \\
\text { PWR }\end{array}$ & $\begin{array}{l}\mathrm{GE} \\
\mathrm{CE}\end{array}$ & $\begin{array}{r}67 \\
755\end{array}$ & $\begin{array}{r}84 \\
204\end{array}$ & $\begin{array}{l}1962 \\
1971\end{array}$ & $\begin{array}{l}2000 \\
2007\end{array}$ & $\begin{array}{l}1999 \\
2007\end{array}$ & $\begin{array}{l}2000 \\
2011\end{array}$ \\
\hline $\begin{array}{l}\text { Dairyland Power } \\
\text { Cooperative } \ldots \ldots \ldots \ldots\end{array}$ & LaCrosse & WI & BWR & $A C$ & 50 & 72 & 1968 & 2031 & RD & 1987 \\
\hline Detroit Edison Company ... & Enrico Fermi 2 & MI & BWR & GE & 1,085 & 764 & 1985 & 2025 & 2006 & 2025 \\
\hline Duke Power Company .... & $\begin{array}{l}\text { Catawba } 1 \\
\text { Catawba } 2 \\
\text { McGuire } 1 \\
\text { McGuire } 2 \\
\text { Oconee } 1 \\
\text { Oconee } 2 \\
\text { Oconee } 3\end{array}$ & $\begin{array}{l}\text { SC } \\
\text { SC } \\
\text { NC } \\
\text { NC } \\
\text { SC } \\
\text { SC } \\
\text { SC }\end{array}$ & $\begin{array}{l}\text { PWR } \\
\text { PWR } \\
\text { PWR } \\
\text { PWR } \\
\text { PWR } \\
\text { PWR } \\
\text { PWR }\end{array}$ & $\begin{array}{l}\text { WE } \\
W E \\
W E \\
W E \\
B \& W \\
B \& W \\
B \& W\end{array}$ & $\begin{array}{r}1,129 \\
1,129 \\
1,129 \\
1,129 \\
846 \\
846 \\
846\end{array}$ & $\begin{array}{l}193 \\
193 \\
193 \\
193 \\
177 \\
177 \\
177\end{array}$ & $\begin{array}{l}1985 \\
1986 \\
1981 \\
1983 \\
1973 \\
1973 \\
1974\end{array}$ & $\begin{array}{l}2024 \\
2026 \\
2021 \\
2023 \\
2013 \\
2013 \\
2014\end{array}$ & $\begin{array}{l}2011 \\
2011 \\
2007 \\
2006 \\
2010 \\
2010 \\
2011\end{array}$ & $\begin{array}{l}2025 \\
2026 \\
2021 \\
2023 \\
2013 \\
2013 \\
2014\end{array}$ \\
\hline Duquesne Light Company . . & $\begin{array}{l}\text { Beaver Valley } 1 \\
\text { Beaver Valley } 2\end{array}$ & $\begin{array}{l}\text { PA } \\
\text { PA }\end{array}$ & $\begin{array}{l}\text { PWR } \\
\text { PWR }\end{array}$ & $\begin{array}{l}\text { WE } \\
\text { WE }\end{array}$ & $\begin{array}{l}810 \\
833\end{array}$ & $\begin{array}{l}157 \\
157\end{array}$ & $\begin{array}{l}1976 \\
1987\end{array}$ & $\begin{array}{l}2016 \\
2027\end{array}$ & $\begin{array}{l}2012 \\
2011\end{array}$ & $\begin{array}{l}2016 \\
2027\end{array}$ \\
\hline Florida Power Corporation . . & Crystal River 3 & FL & PWR & $B \& W$ & 816 & 177 & 1977 & 2016 & 2010 & 2016 \\
\hline $\begin{array}{l}\text { Florida Power and Light } \\
\text { Company } \quad \ldots \ldots \ldots \ldots\end{array}$ & $\begin{array}{l}\text { St. Lucie } 1 \\
\text { St. Lucie } 2 \\
\text { Turkey Point } 3 \\
\text { Turkey Point } 4\end{array}$ & $\begin{array}{l}\text { FL } \\
\text { FL } \\
\text { FL } \\
\text { FL }\end{array}$ & $\begin{array}{l}\text { PWR } \\
\text { PWR } \\
\text { PWR } \\
\text { PWR }\end{array}$ & $\begin{array}{l}\text { CE } \\
\text { CE } \\
\text { WE } \\
\text { WE }\end{array}$ & $\begin{array}{l}839 \\
839 \\
666 \\
666\end{array}$ & $\begin{array}{l}217 \\
217 \\
157 \\
157\end{array}$ & $\begin{array}{l}1976 \\
1983 \\
1972 \\
1973\end{array}$ & $\begin{array}{l}2016 \\
2023 \\
2007 \\
2007\end{array}$ & $\begin{array}{l}2007 \\
2001 \\
2012 \\
2013\end{array}$ & $\begin{array}{l}2016 \\
2023 \\
2007 \\
2013\end{array}$ \\
\hline Georgia Power Company .. & $\begin{array}{l}\text { Hatch } 1 \\
\text { Hatch } 2 \\
\text { Vogtle } 1 \\
\text { Vogtle } 2\end{array}$ & $\begin{array}{l}\text { GA } \\
\text { GA } \\
\text { GA } \\
\text { GA }\end{array}$ & $\begin{array}{l}\text { BWR } \\
\text { BWR } \\
\text { PWR } \\
\text { PWR }\end{array}$ & $\begin{array}{l}\text { GE } \\
\text { GE } \\
\text { WE } \\
\text { WE }\end{array}$ & $\begin{array}{r}740 \\
761 \\
1,162 \\
1,162\end{array}$ & $\begin{array}{l}560 \\
560 \\
193 \\
193\end{array}$ & $\begin{array}{l}1974 \\
1978 \\
1987 \\
1989\end{array}$ & $\begin{array}{l}2014 \\
2018 \\
2027 \\
2029\end{array}$ & $\begin{array}{l}2003 \\
2003 \\
2010 \\
2010\end{array}$ & $\begin{array}{l}2014 \\
2018 \\
2027 \\
2029\end{array}$ \\
\hline GPU Nuclear Corporation .. & $\begin{array}{l}\text { Three Mile Island } 1 \\
\text { Oyster Creek }\end{array}$ & $\begin{array}{l}\text { PA } \\
\text { NJ }\end{array}$ & $\begin{array}{l}\text { PWR } \\
\text { BWR }\end{array}$ & $\begin{array}{l}\text { B\&W } \\
\text { GE }\end{array}$ & $\begin{array}{l}786 \\
610\end{array}$ & $\begin{array}{l}177 \\
560\end{array}$ & $\begin{array}{l}1974 \\
1969\end{array}$ & $\begin{array}{l}2014 \\
2009\end{array}$ & $\begin{array}{l}2014 \\
2000\end{array}$ & $\begin{array}{l}2014 \\
2009\end{array}$ \\
\hline
\end{tabular}

See footnotes at end of table. 
Table 4. Nuclear Power Plant Data as of December 31, 1993 (Continued)

\begin{tabular}{|c|c|c|c|c|c|c|c|c|c|c|}
\hline Electric Utility Name & Reactor Name & State & $\begin{array}{c}\text { Reactor } \\
\text { Type }\end{array}$ & Vendor & $\begin{array}{c}\text { Net } \\
\text { Summer } \\
\text { Capability } \\
\text { (net MWe) }\end{array}$ & $\begin{array}{l}\text { Core Size } \\
\text { (number of } \\
\text { assemblies) }\end{array}$ & $\begin{array}{r}\text { Startup } \\
\text { (year) }^{c}\end{array}$ & $\begin{array}{c}\text { License } \\
\text { Expiration } \\
\text { (year) }^{d}\end{array}$ & $\begin{array}{c}\text { Loss of } \\
\text { Ability } \\
\text { to Operate } \\
\text { (year) }^{\circ}\end{array}$ & $\begin{array}{c}\text { Actual or } \\
\text { Projected } \\
\text { Retirement } \\
\text { (year) }\end{array}$ \\
\hline $\begin{array}{l}\text { Gulf States Utilities } \\
\text { Company } \ldots \ldots \ldots \ldots \ldots\end{array}$ & River Bend 1 & LA & BWR & GE & 931 & 624 & 1985 & 2025 & 2003 & 2025 \\
\hline $\begin{array}{l}\text { Houston Lighting and } \\
\text { Power Company } \ldots \ldots \ldots\end{array}$ & $\begin{array}{l}\text { South Texas } 1 \\
\text { South Texas } 2\end{array}$ & $\begin{array}{l}\text { TX } \\
\text { TX }\end{array}$ & $\begin{array}{l}\text { PWR } \\
\text { PWR }\end{array}$ & $\begin{array}{l}\text { WE } \\
\text { WE }\end{array}$ & $\begin{array}{l}1,241 \\
1,241\end{array}$ & $\begin{array}{l}193 \\
193\end{array}$ & $\begin{array}{l}1988 \\
1989\end{array}$ & $\begin{array}{l}2027 \\
2028\end{array}$ & $\begin{array}{l}2027 \\
2028\end{array}$ & $\begin{array}{l}2027 \\
2028\end{array}$ \\
\hline IES Utilities, Inc. . . . . . . . & Duane Amold & IA & BWR & GE & 515 & 368 & 1974 & 2014 & 1998 & 2014 \\
\hline Illinois Power Company . . . . . & Clinton 1 & IL & BWR & GE & 930 & 624 & 1987 & 2026 & 2008 & 2026 \\
\hline $\begin{array}{l}\text { Indiana Michigan } \\
\text { Power Company } \ldots \ldots \ldots \text {. }\end{array}$ & $\begin{array}{l}\text { Cook } 1 \\
\text { Cook } 2\end{array}$ & $\begin{array}{l}\text { MI } \\
\text { MI }\end{array}$ & $\begin{array}{l}\text { PWR } \\
\text { PWR }\end{array}$ & $\begin{array}{l}\text { WE } \\
\text { WE }\end{array}$ & $\begin{array}{l}1,000 \\
1,060\end{array}$ & $\begin{array}{l}193 \\
193\end{array}$ & $\begin{array}{l}1975 \\
1978\end{array}$ & $\begin{array}{l}2014 \\
2017\end{array}$ & $\begin{array}{l}2011 \\
2011\end{array}$ & $\begin{array}{l}2014 \\
2017\end{array}$ \\
\hline $\begin{array}{l}\text { Kansas Gas and Electric } \\
\text { Company } \ldots \ldots \ldots \ldots\end{array}$ & Wolf Creek 1 & KS & PWR & WE & 1,134 & 193 & 1985 & 2025 & 2006 & 2025 \\
\hline $\begin{array}{l}\text { Long Island Power } \\
\text { Authority } \ldots \ldots \ldots \ldots\end{array}$ & Shoreham & NY & BWR & GE & 849 & 560 & 1986 & 2013 & SD & 1987 \\
\hline $\begin{array}{l}\text { Louisiana Power and } \\
\text { Light Company } . . \ldots \ldots \ldots\end{array}$ & Waterford 3 & LA & PWR & CE & 1,075 & 217 & 1985 & 2024 & 2000 & 2024 \\
\hline $\begin{array}{l}\text { Maine Yankee Atomic } \\
\text { Power Company } \ldots \ldots \ldots\end{array}$ & Maine Yankee & ME & PWR & $\mathrm{CE}$ & 870 & 217 & 1972 & 2008 & 1999 & 2008 \\
\hline $\begin{array}{l}\text { Nebraska Public Power } \\
\text { District } \ldots \ldots \ldots \ldots \ldots\end{array}$ & Cooper Station & NE & BWR & $\mathrm{GE}$ & 778 & 548 & 1974 & 2014 & 2002 & 2014 \\
\hline $\begin{array}{l}\text { New York Power } \\
\text { Authority } \ldots \ldots \ldots \ldots \ldots\end{array}$ & $\begin{array}{l}\text { FitzPatrick } \\
\text { Indian Point } 3\end{array}$ & $\begin{array}{l}\text { NY } \\
\text { NY }\end{array}$ & $\begin{array}{l}\text { BWR } \\
\text { PWR }\end{array}$ & $\begin{array}{l}\text { GE } \\
\text { WE }\end{array}$ & $\begin{array}{l}800 \\
980\end{array}$ & $\begin{array}{l}560 \\
193\end{array}$ & $\begin{array}{l}1975 \\
1976\end{array}$ & $\begin{array}{l}2014 \\
2015\end{array}$ & $\begin{array}{l}2003 \\
2006\end{array}$ & $\begin{array}{l}2014 \\
2015\end{array}$ \\
\hline $\begin{array}{l}\text { Niagara Mohawk Power } \\
\text { Corporation } \ldots \ldots \ldots \ldots\end{array}$ & $\begin{array}{l}\text { Nine Mile Point } 1 \\
\text { Nine Mile Point } 2\end{array}$ & $\begin{array}{l}\text { NY } \\
\text { NY }\end{array}$ & $\begin{array}{l}\text { BWR } \\
\text { BWR }\end{array}$ & $\begin{array}{l}\text { GE } \\
\text { GE }\end{array}$ & $\begin{array}{r}605 \\
1,045\end{array}$ & $\begin{array}{l}532 \\
764\end{array}$ & $\begin{array}{l}1969 \\
1987\end{array}$ & $\begin{array}{l}2009 \\
2026\end{array}$ & $\begin{array}{l}2005 \\
2017\end{array}$ & $\begin{array}{l}2008 \\
2026\end{array}$ \\
\hline $\begin{array}{l}\text { North Atlantic Energy } \\
\text { Service Corporation . . . . . . }\end{array}$ & Seabrook & $\mathrm{NH}$ & PWR & WE & 1,150 & 193 & 1990 & 2026 & 2012 & 2030 \\
\hline
\end{tabular}

See footnotes at end of table. 
Table 4. Nuclear Power Plant Data as of December 31, 1993 (Continued)

\begin{tabular}{|c|c|c|c|c|c|c|c|c|c|c|}
\hline Electric Utility Name & Reactor Name & State & $\begin{array}{c}\text { Reactor } \\
\text { Type }\end{array}$ & Vendor ${ }^{\mathbf{a}}$ & $\begin{array}{c}\text { Net } \\
\text { Summer } \\
\text { Capability } \\
\text { (net MWe) }^{b}\end{array}$ & $\begin{array}{c}\text { Core Size } \\
\text { (number of } \\
\text { assemblies) }\end{array}$ & $\begin{array}{l}\text { Startup } \\
\text { (year) }^{c}\end{array}$ & $\begin{array}{c}\text { License } \\
\text { Expiration } \\
\text { (year) }^{\circ}\end{array}$ & $\begin{array}{c}\text { Loss of } \\
\text { Ability } \\
\text { to Operate } \\
\text { (year) }^{\circ}\end{array}$ & $\begin{array}{c}\text { Actual or } \\
\text { Projected } \\
\text { Retirement } \\
\text { (year) } \\
\end{array}$ \\
\hline $\begin{array}{l}\text { Northeast Utilities } \\
\text { Service Company } \ldots \ldots \ldots\end{array}$ & $\begin{array}{l}\text { Millstone } 1 \\
\text { Millstone } 2 \\
\text { Millstone } 3 \\
\text { Haddam Neck }\end{array}$ & $\begin{array}{l}\text { CT } \\
\text { CT } \\
\text { CT } \\
\text { CT }\end{array}$ & $\begin{array}{l}\text { BWR } \\
\text { PWR } \\
\text { PWR } \\
\text { PWR }\end{array}$ & $\begin{array}{l}\text { GE } \\
\text { CE } \\
\text { WE } \\
\text { WE }\end{array}$ & $\begin{array}{r}641 \\
873 \\
1,137 \\
560\end{array}$ & $\begin{array}{l}580 \\
217 \\
193 \\
157\end{array}$ & $\begin{array}{l}1970 \\
1975 \\
1986 \\
1967\end{array}$ & $\begin{array}{l}2010 \\
2015 \\
2025 \\
2007\end{array}$ & $\begin{array}{l}2004 \\
2004 \\
2003 \\
2002\end{array}$ & $\begin{array}{l}2010 \\
2015 \\
2025 \\
2007\end{array}$ \\
\hline $\begin{array}{l}\text { Northem States Power } \\
\text { Company } \ldots \ldots \ldots \ldots\end{array}$ & $\begin{array}{l}\text { Monticello } \\
\text { Prairie Island } 1 \\
\text { Prairie Island } 2\end{array}$ & $\begin{array}{l}M N \\
M N \\
M N\end{array}$ & $\begin{array}{l}\text { BWR } \\
\text { PWR } \\
\text { PWR }\end{array}$ & $\begin{array}{l}\text { GE } \\
\text { WE } \\
\text { WE }\end{array}$ & $\begin{array}{l}539 \\
510 \\
505\end{array}$ & $\begin{array}{l}484 \\
121 \\
121\end{array}$ & $\begin{array}{l}1971 \\
1973 \\
1974\end{array}$ & $\begin{array}{l}2010 \\
2013 \\
2014\end{array}$ & $\begin{array}{l}2004 \\
1995 \\
1995\end{array}$ & $\begin{array}{l}2010 \\
2013 \\
2014\end{array}$ \\
\hline $\begin{array}{l}\text { Omaha Public Power } \\
\text { District } \ldots \ldots \ldots \ldots \ldots\end{array}$ & Fort Calhoun & NE & PWR & CE & 476 & 133 & 1973 & 2013 & 2007 & 2013 \\
\hline $\begin{array}{l}\text { Pacific Gas and Electric } \\
\text { Company } \ldots \ldots \ldots \ldots\end{array}$ & $\begin{array}{l}\text { Diablo Canyon } 1 \\
\text { Diablo Canyon } 2 \\
\text { Humboldt Bay }\end{array}$ & $\begin{array}{l}\mathrm{CA} \\
\mathrm{CA} \\
\mathrm{CA}\end{array}$ & $\begin{array}{l}\text { PWR } \\
\text { PWR } \\
\text { BWR }\end{array}$ & $\begin{array}{l}\text { WE } \\
\text { WE } \\
\text { GE }\end{array}$ & $\begin{array}{r}1,073 \\
1,087 \\
65\end{array}$ & $\begin{array}{l}193 \\
193 \\
184\end{array}$ & $\begin{array}{l}1984 \\
1985 \\
1963\end{array}$ & $\begin{array}{l}2008 \\
2010 \\
2015\end{array}$ & $\begin{array}{r}2004 \\
2006 \\
S D\end{array}$ & $\begin{array}{l}2021 \\
2025 \\
1976\end{array}$ \\
\hline PECO Energy Company ... & $\begin{array}{l}\text { Limerick } 1 \\
\text { Limerick } 2 \\
\text { Peach Bottom } 2 \\
\text { Peach Bottom } 3\end{array}$ & $\begin{array}{l}\text { PA } \\
\text { PA } \\
\text { PA } \\
\text { PA }\end{array}$ & $\begin{array}{l}\text { BWR } \\
\text { BWR } \\
\text { BWR } \\
\text { BWR }\end{array}$ & $\begin{array}{l}\text { GE } \\
\text { GE } \\
\text { GE } \\
\text { GE }\end{array}$ & $\begin{array}{l}1,055 \\
1,055 \\
1,051 \\
1,035\end{array}$ & $\begin{array}{l}764 \\
764 \\
764 \\
764\end{array}$ & $\begin{array}{l}1985 \\
1989 \\
1974 \\
1974\end{array}$ & $\begin{array}{l}2024 \\
2029 \\
2008 \\
2008\end{array}$ & $\begin{array}{l}2000 \\
1998 \\
1998 \\
1999\end{array}$ & $\begin{array}{l}2024 \\
2029 \\
2008 \\
2008\end{array}$ \\
\hline $\begin{array}{l}\text { Pennsylvania Power and } \\
\text { Light Company } \ldots \ldots \ldots\end{array}$ & $\begin{array}{l}\text { Susquehanna } 1 \\
\text { Susquehanna } 2\end{array}$ & $\begin{array}{l}\text { PA } \\
\text { PA }\end{array}$ & $\begin{array}{l}\text { BWR } \\
\text { BWR }\end{array}$ & $\begin{array}{l}\text { GE } \\
\text { GE }\end{array}$ & $\begin{array}{l}1,040 \\
1,044\end{array}$ & $\begin{array}{l}764 \\
764\end{array}$ & $\begin{array}{l}1982 \\
1984\end{array}$ & $\begin{array}{l}2022 \\
2024\end{array}$ & $\begin{array}{l}2001 \\
2001\end{array}$ & $\begin{array}{l}2022 \\
2024\end{array}$ \\
\hline $\begin{array}{l}\text { Portland General Electric } \\
\text { Company } \ldots \ldots \ldots \ldots\end{array}$ & Trojan & OR & PWR & WE & 1,130 & 193 & 1975 & 2011 & $S D$ & 1992 \\
\hline $\begin{array}{l}\text { Public Service Electric } \\
\text { and Gas Company ....... }\end{array}$ & $\begin{array}{l}\text { Hope Creek } \\
\text { Salem } 1 \\
\text { Salem } 2\end{array}$ & $\begin{array}{l}\mathrm{NJ} \\
\mathrm{NJ} \\
\mathrm{NJ}\end{array}$ & $\begin{array}{l}\text { BWR } \\
\text { PWR } \\
\text { PWR }\end{array}$ & $\begin{array}{l}G E \\
W E \\
W E\end{array}$ & $\begin{array}{l}1,031 \\
1,106 \\
1,106\end{array}$ & $\begin{array}{l}764 \\
193 \\
193\end{array}$ & $\begin{array}{l}1986 \\
1976 \\
1981\end{array}$ & $\begin{array}{l}2026 \\
2016 \\
2020\end{array}$ & $\begin{array}{l}2010 \\
2002 \\
2008\end{array}$ & $\begin{array}{l}2026 \\
2016 \\
2020\end{array}$ \\
\hline $\begin{array}{l}\text { Rochester Gas and } \\
\text { Electric Corporation ...... }\end{array}$ & Ginna & NY & PWR & WE & 470 & 121 & 1969 & 2009 & 2003 & 2009 \\
\hline $\begin{array}{l}\text { Sacramento Municipal } \\
\text { Utility District . . . . . . . }\end{array}$ & Rancho Seco & $\mathrm{CA}$ & PWR & $B \& W$ & 918 & 177 & 1974 & 2008 & SD & 1989 \\
\hline
\end{tabular}


Table 4. Nuclear Power Plant Data as of December 31, 1993 (Continued)

\begin{tabular}{|c|c|c|c|c|c|c|c|c|c|c|}
\hline Electric Utility Name & Reactor Name & State & $\begin{array}{l}\text { Reactor } \\
\text { Type }\end{array}$ & Vendor ${ }^{a}$ & $\begin{array}{c}\text { Net } \\
\text { Summer } \\
\text { Capability } \\
\text { (net MWe) }^{\mathrm{b}}\end{array}$ & $\begin{array}{c}\text { Core Size } \\
\text { (number of } \\
\text { assemblies) }\end{array}$ & $\begin{array}{c}\text { Startup } \\
\text { (year) }^{c}\end{array}$ & $\begin{array}{c}\text { License } \\
\text { Expiration } \\
\text { (year) }^{d}\end{array}$ & $\begin{array}{c}\text { Loss of } \\
\text { Ability } \\
\text { to Operate } \\
\text { (year) }^{\circ}\end{array}$ & $\begin{array}{c}\text { Actual or } \\
\text { Projected } \\
\text { Retirement } \\
\text { (year) }\end{array}$ \\
\hline $\begin{array}{l}\text { South Carolina Electric } \\
\text { and Gas Company ....... }\end{array}$ & Summer & SC & PWR & WE & 885 & 157 & 1982 & 2022 & 2008 & 2035 \\
\hline $\begin{array}{l}\text { Southem California } \\
\text { Edison Company ....... }\end{array}$ & $\begin{array}{l}\text { San Onofre } 1 \\
\text { San Onofre } 2 \\
\text { San Onofre } 3\end{array}$ & $\begin{array}{l}C A \\
C A \\
C A\end{array}$ & $\begin{array}{l}\text { PWR } \\
\text { PWR } \\
\text { PWR }\end{array}$ & $\begin{array}{l}\text { WE } \\
\text { CE } \\
\text { CE }\end{array}$ & $\begin{array}{r}436 \\
1,070 \\
1,080\end{array}$ & $\begin{array}{l}157 \\
217 \\
217\end{array}$ & $\begin{array}{l}1967 \\
1982 \\
1983\end{array}$ & $\begin{array}{l}2004 \\
2013 \\
2013\end{array}$ & $\begin{array}{r}S D \\
2005 \\
2005\end{array}$ & $\begin{array}{l}1992 \\
2013 \\
2013\end{array}$ \\
\hline $\begin{array}{l}\text { System Energy Resources, } \\
\text { Inc. } \ldots \ldots \ldots \ldots \ldots \ldots \ldots\end{array}$ & Grand Gulf 1 & MS & BWR & GE & 1,143 & 800 & 1984 & 2022 & 2005 & 2022 \\
\hline $\begin{array}{l}\text { Tennessee Valley } \\
\text { Authority } . . . \ldots \ldots \ldots\end{array}$ & $\begin{array}{l}\text { Browns Ferry } 1 \\
\text { Browns Ferry } 2 \\
\text { Browns Ferry } 3 \\
\text { Sequoyah } 1 \\
\text { Sequoyah } 2 \\
\text { Watts Bar } 1 \\
\text { Watts Bar } 2\end{array}$ & $\begin{array}{l}A L \\
A L \\
A L \\
T N \\
T N \\
T N \\
T N\end{array}$ & $\begin{array}{l}\text { BWR } \\
\text { BWR } \\
\text { BWR } \\
\text { PWR } \\
\text { PWR } \\
\text { PWR } \\
\text { PWR }\end{array}$ & $\begin{array}{l}\text { GE } \\
\text { GE } \\
\text { GE } \\
\text { WE } \\
\text { WE } \\
\text { WE } \\
\text { WE }\end{array}$ & $\begin{array}{l}1,065 \\
1,065 \\
1,065 \\
1,111 \\
1,106 \\
1,165 \\
1,165\end{array}$ & $\begin{array}{l}764 \\
764 \\
764 \\
193 \\
193 \\
193 \\
193\end{array}$ & $\begin{array}{l}1973 \\
1974 \\
1976 \\
1980 \\
1981 \\
1994 \\
1996\end{array}$ & $\begin{array}{r}2013 \\
2014 \\
2016 \\
2020 \\
2021 \\
C \\
C\end{array}$ & $\begin{array}{l}2010 \\
2010 \\
2006 \\
2004 \\
2003 \\
2007 \\
2007\end{array}$ & $\begin{array}{l}2013 \\
2014 \\
2016 \\
2020 \\
2021 \\
2030 \\
2032\end{array}$ \\
\hline Toledo Edison Company ... & Davis-Besse & $\mathrm{OH}$ & PWR & $B \& W$ & 873 & 177 & 1977 & 2017 & 2017 & 2037 \\
\hline TU Electric $\ldots \ldots \ldots \ldots$ & $\begin{array}{l}\text { Comanche Peak } 1 \\
\text { Comanche Peak } 2\end{array}$ & $\begin{array}{l}\text { TX } \\
\text { TX }\end{array}$ & $\begin{array}{l}\text { PWR } \\
\text { PWR }\end{array}$ & $\begin{array}{l}\text { WE } \\
\text { WE }\end{array}$ & $\begin{array}{l}1,150 \\
1,150\end{array}$ & $\begin{array}{l}193 \\
193\end{array}$ & $\begin{array}{l}1990 \\
1993\end{array}$ & $\begin{array}{l}2030 \\
2033\end{array}$ & $\begin{array}{l}2019 \\
2021\end{array}$ & $\begin{array}{l}2030 \\
2033\end{array}$ \\
\hline Union Electric Company ... & Callaway & MO & PWR & WE & 1,125 & 193 & 1984 & 2024 & 2007 & 2024 \\
\hline $\begin{array}{l}\text { Vermont Yankee Nuclear } \\
\text { Power Corporation ....... }\end{array}$ & Vermont Yankee & VT & BWR & GE & 496 & 368 & 1972 & 2012 & 2004 & 2012 \\
\hline Virginia Power $\ldots \ldots \ldots$ & $\begin{array}{l}\text { North Anna } 1 \\
\text { North Anna } 2 \\
\text { Surry } 1 \\
\text { Surry } 2\end{array}$ & $\begin{array}{l}\text { VA } \\
\text { VA } \\
\text { VA } \\
\text { VA }\end{array}$ & $\begin{array}{l}\text { PWR } \\
\text { PWR } \\
\text { PWR } \\
\text { PWR }\end{array}$ & $\begin{array}{l}\text { WE } \\
\text { WE } \\
\text { WE } \\
\text { WE }\end{array}$ & $\begin{array}{l}900 \\
887 \\
781 \\
781\end{array}$ & $\begin{array}{l}157 \\
157 \\
157 \\
157\end{array}$ & $\begin{array}{l}1978 \\
1980 \\
1972 \\
1973\end{array}$ & $\begin{array}{l}2018 \\
2020 \\
2012 \\
2013\end{array}$ & $\begin{array}{l}2000 \\
2001 \\
2012 \\
2013\end{array}$ & $\begin{array}{l}2018 \\
2020 \\
2012 \\
2013\end{array}$ \\
\hline $\begin{array}{l}\text { Washington Public Power } \\
\text { Supply System ....... }\end{array}$ & Washington Nuclear 2 & WA & BWR & GE & 1,100 & 764 & 1984 & 2023 & 1999 & 2023 \\
\hline $\begin{array}{l}\text { Wisconsin Electric Power } \\
\text { Company } \ldots \ldots \ldots \ldots \ldots\end{array}$ & $\begin{array}{l}\text { Point Beach } 1 \\
\text { Point Beach } 2\end{array}$ & $\begin{array}{l}\text { WI } \\
\text { WI }\end{array}$ & $\begin{array}{l}\text { PWR } \\
\text { PWR }\end{array}$ & $\begin{array}{l}\text { WE } \\
\text { WE }\end{array}$ & $\begin{array}{l}492 \\
482\end{array}$ & $\begin{array}{l}121 \\
121\end{array}$ & $\begin{array}{l}1970 \\
1972\end{array}$ & $\begin{array}{l}2010 \\
2013\end{array}$ & $\begin{array}{l}1998 \\
1998\end{array}$ & $\begin{array}{l}2010 \\
2013\end{array}$ \\
\hline
\end{tabular}

See footnoles at end of table. 
Table 4. Nuclear Power Plant Data as of December 31, 1993 (Continued)

\begin{tabular}{|c|c|c|c|c|c|c|c|c|c|c|}
\hline Electric Utility Name & Reactor Name & State & $\begin{array}{l}\text { Reactor } \\
\text { Type }\end{array}$ & Vendor $^{\mathrm{a}}$ & $\begin{array}{c}\text { Net } \\
\text { Summer } \\
\text { Capability } \\
\text { (net MWe) }^{\mathrm{b}}\end{array}$ & $\begin{array}{l}\text { Core Size } \\
\text { (number of } \\
\text { assemblies) }\end{array}$ & $\begin{array}{l}\text { Startup } \\
\text { (year) }^{c}\end{array}$ & $\begin{array}{l}\text { License } \\
\text { Expiration } \\
\text { (year) }^{d}\end{array}$ & $\begin{array}{c}\text { Loss of } \\
\text { Ability } \\
\text { to Operate } \\
\text { (year) }^{\circ}\end{array}$ & $\begin{array}{c}\text { Actual or } \\
\text { Projected } \\
\text { Retirement } \\
\text { (year) }\end{array}$ \\
\hline $\begin{array}{l}\text { Wisconsin Public Service } \\
\text { Corporation ............. }\end{array}$ & Kewaunee & WI & PWR & WE & 522 & 121 & 1974 & 2013 & 2002 & 2014 \\
\hline $\begin{array}{l}\text { Yankee Atomic Electric } \\
\text { Company } \ldots \ldots \ldots \ldots \ldots\end{array}$ & Yankee Rowe & MA & PWR & WE & 175 & 76 & 1960 & 2000 & SD & 1992 \\
\hline
\end{tabular}

${ }^{2}$ Vendor codes are as follows: $\mathrm{AC}=$ Allis Chalmers; $\mathrm{B} \& \mathrm{~W}=$ Babcock \& Wilcox Company; $\mathrm{CE}=\mathrm{ABB}$ Combustion Engineering; $\mathrm{GE}=\mathrm{GE}$ Nuclear Energy; WE $=$ Westinghouse .

'Net Summer Capability (net MWe) data are not available on Form RW-859 data base. Data are from Energy Information Administration, World Nuclear Outlook 1994, (December 1994), Table C1.

'Startup Year not available for all reactors on Form RW-859 data base. Startup Year for Watts Bar 1 and 2 are from Energy Information Adminstration, World Nuclear Outlook 1994, (December 1994), Table D1.

'License Expiration Year not available for all reactors on Form RW-859 data base. License Expiration Year for Watts Bar 1 and 2 are from Energy Information Administration, World Nuclear Outlook 1994, (December 1994), Table D1. License status code: $C=$ Construction permit.

These data are compiled directly from question 2.3 on Form RW-859. It reads as follows: "What is the estimated date on which you would not continue reactor operation, because of a lack of storage space for discharged fuel absent spent fuel pickup by DOE?"

PWR = Pressurized-water reactor; BWR = Boiling-water reactor; $M W e=$ Megawatts electric; $S D=$ Shut down reactor; RD = Retired reactor.

Source: Energy Information Administration, Form RW-859, "Nuclear Fuel Data" (1993). 
Figure 1. Loss of Ability to Operate Data as of December 31, 1993

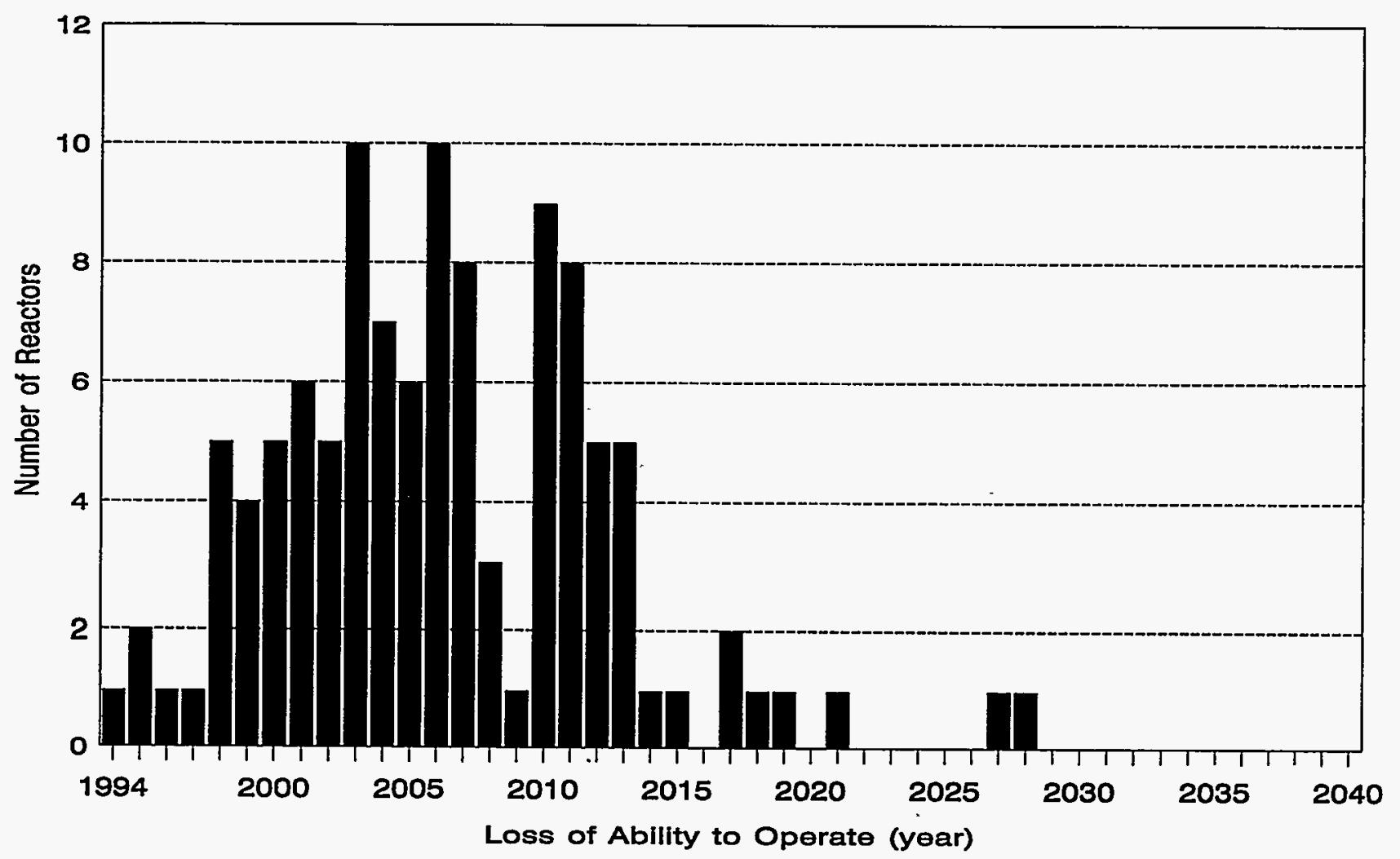

Figure 2. Projected Retirement Year Data as of December 31, 1993

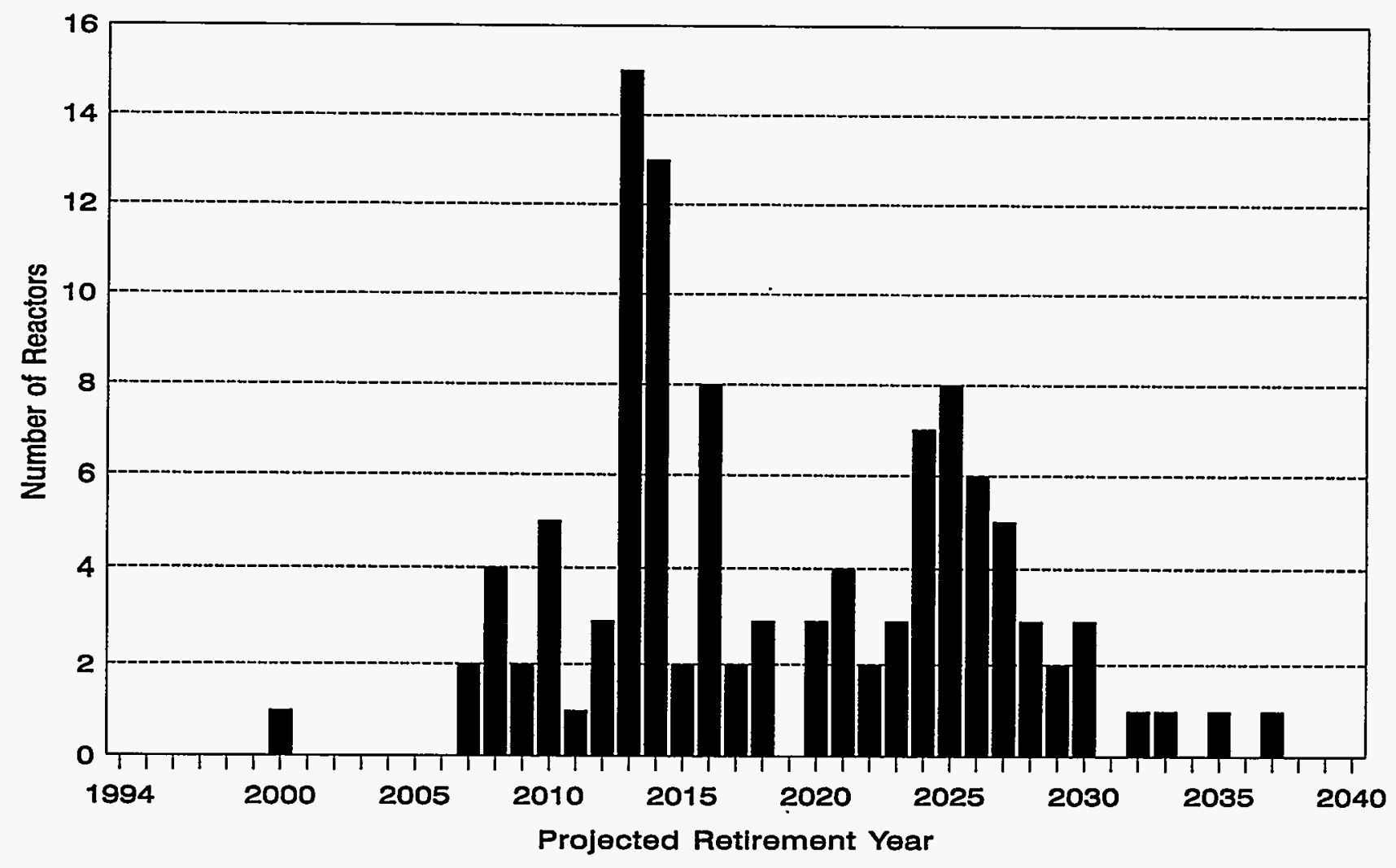




\section{Annual Discharges and Burnup}

A total of 98,400 spent nuclear fuel assemblies discharged through 1993 contained 28,136 MTU (Table 5 , Figure 3). By weight, approximately 36 percent of the spent nuclear fuel came from BWR's and 64 percent came from PWR's. In 1993, the number of PWR assemblies permanently discharged $(3,535)$ represents a decrease from the previous high of 3,711 in 1992. The number of BWR assemblies permanently discharged $(3,997)$ represents an increase from the previous high of 3,842 reported in 1992.

Annual spent fuel discharges and burnup values are summarized in Table 5. Permanently discharged spent fuel for BWR's and PWR's is shown by number of assemblies and metric tons of uranium in Figure 3. Both Table 5 and Figure 3 illustrate the number of assemblies and metric tons of uranium discharged in each year for BWR's and PWR's. The table also presents the annual average burnup in gigawattdays thermal per initial loading weight of uranium, in metric tons (GWDt/MTU), including assemblies discharged from nonequilibrium cycles.

Some electric utilities report plans to continue increasing burnup. This is accomplished by increasing the concentration of U-235 in the enriched uranium product and changing the design specifications of the fuel rods to allow for longer incore exposures. Electric utilities use higher fuel burnup to increase cycle lengths and/or to reduce spent fuel generation. Increasing cycle length allows the potential for reducing the relative outage time: for example, an annual cycle requires three refuelings in a 3-year period, while an 18-month cycle requires only two refuelings in the same time period. Thus, increased capacity factors can result from increased cycle length; however, increased lengths of fuel outages can offset this increase in capacity factors.
Starting in 1970, the annual average burnup for all discharged BWR assemblies have shown an increase to a high of $30.3 \mathrm{GWDt} / \mathrm{MTU}$ in 1993. Average burnups have fluctuated between 1984 and 1990 because of the number of reactors discharging first and second cycle assemblies. The average burnup for all PWR assemblies increased to $38.9 \mathrm{GWDt} / \mathrm{MTU}$ in 1993, the highest annual average yet attained. It had been fairly constant from 1980 through 1987 at approximately 30 GWDt/MTU.

The last two columns in Table 5 present the burnup of equilibrium cycle discharges for BWR's and PWR's. Equilibrium cycle is an analytical term that refers to fuel cycles occurring after the initial two cycles of a reactor's operation and prior to its final cycle. It is an assumed condition in which the spent nuclear fuel from a facility has a relatively constant composition from cycle to cycle. In a reactor, this condition typically begins with the third or fourth fuel loading, depending on the portion of the core being replaced. (For the purpose of this report, it is assumed to begin with the third fuel loading.) For example, the first fuel loading may have three sectors; one has an enrichment of 1.7 percent, the second an enrichment of 2.7 percent, and the third an enrichment of 3.7 percent. The first sector would be discharged at the end of the first cycle, with an average burnup of $10 \mathrm{GWDt} / \mathrm{MTU}$; the second sector would be discharged at the end of the second cycle, with a burnup of $20 \mathrm{GWDt} / \mathrm{MTU}$; the third sector would be the first equilibrium cycle discharge and would be discharged at the end of the third cycle.

For BWR's, the average equilibrium spent fuel discharge burnup in 1993 was 31.2 GWDt/MTU. For PWR's, the average equilibrium spent fuel discharge burnup in 1993 was 38.9 GWDt/MTU. The trends in achieved burnup levels for BWR and PWR spent fuel discharged to date (Figure 4) illustrate that PWR spent fuel has achieved increasingly higher burnup levels than BWR spent fuel. 


\begin{tabular}{|c|c|c|c|c|c|c|c|c|c|c|c|c|}
\hline \multirow{3}{*}{\multicolumn{2}{|c|}{ Year }} & \multirow{2}{*}{\multicolumn{3}{|c|}{ Number of Assemblies }} & \multirow{2}{*}{\multicolumn{3}{|c|}{ Metric Tons of Uranium }} & \multicolumn{4}{|c|}{ Average Burnup (GWDt/MTU) } & \\
\hline & & & & & & & & \multicolumn{2}{|c|}{ All Discharged Assemblies } & \multicolumn{2}{|c|}{ Equilibrium Cycle Discharges } & \\
\hline & & BWR & PWR & Total & BWR & PWR & Total & BWR & PWR & BWR & PWR & \\
\hline 1968 & $\ldots \ldots \ldots \ldots$ & 5 & 0 & 5 & 0.6 & 0.0 & 0.6 & 1.6 & 0.0 & 1.6 & 0.0 & \\
\hline 1969 & $\ldots \ldots \ldots \ldots$ & 96 & 0 & 96 & 9.8 & 0.0 & 9.8 & 15.2 & 0.0 & 15.2 & 0.0 & \\
\hline 1970 & $\ldots \ldots \ldots \ldots$ & 29 & 99 & 128 & 5.6 & 39.0 & 44.6 & 0.3 & 18.4 & 0.0 & 0.0 & \\
\hline 1971. & $\ldots \ldots \ldots \ldots$ & 413 & 113 & 526 & 64.7 & 44.5 & 109.2 & 5.8 & 23.9 & 16.3 & 0.0 & \\
\hline 1972 . & $\ldots \ldots \ldots \ldots$ & 801 & 282 & 1,083 & 145.6 & 99.9 & 245.5 & 6.6 & 21.9 & 14.9 & 28.0 & \\
\hline 1973. & $\ldots \ldots \ldots \ldots$ & 564 & 165 & 729 & 93.5 & 67.1 & 160.6 & 12.4 & 23.7 & 16.1 & 28.1 & \\
\hline 1974 . & $\ldots \ldots \ldots \ldots$ & 1,290 & 575 & 1,865 & 241.4 & 207.7 & 449.1 & 12.7 & 18.9 & 14.6 & 25.7 & \\
\hline 1975 . & $\ldots \ldots \ldots \ldots$ & 1,223 & 797 & 2,020 & 225.6 & 321.8 & 547.4 & 16.9 & 18.1 & 17.3 & 27.3 & \\
\hline 1976. & $\ldots \ldots \ldots \ldots$ & 1,666 & 931 & 2,597 & 297.3 & 401.0 & 698.3 & 13.4 & 22.2 & 16.7 & 25.8 & \\
\hline 1977 . & $\ldots \ldots \ldots \ldots$ & 2,047 & 1,107 & 3,154 & 382.9 & 466.9 & 849.8 & 16.6 & 25.1 & 19.0 & 27.8 & \\
\hline 1978 . & $\ldots \ldots \ldots \ldots$ & 2,239 & 1,666 & 3,905 & 383.2 & 699.1 & $1,082.3$ & 19.8 & 26.4 & 22.1 & 28.7 & \\
\hline 1979 . & $\ldots \ldots \ldots \ldots$ & 2,131 & 1,662 & 3,793 & 399.8 & 721.2 & $1,121.1$ & 22.4 & 27.0 & 23.7 & 30.2 & \\
\hline 1980 . & $\ldots \ldots \ldots \ldots$ & 3,330 & 1,456 & 4,786 & 619.8 & 618.1 & $1,237.9$ & 22.4 & 29.7 & 23.3 & 30.7 & , \\
\hline 1981. & $\ldots \ldots \ldots \ldots$ & 2,467 & 1,585 & 4,052 & 458.7 & 675.9 & $1,134.6$ & 23.9 & 30.2 & 23.9 & 30.9 & \\
\hline 1982 . & $\ldots \ldots \ldots \ldots$ & 1,951 & 1,491 & 3,442 & 357.2 & 640.4 & 997.6 & 24.7 & 29.7 & 24.9 & 32.3 & \\
\hline 1983 . & $\ldots \ldots \ldots \ldots$ & 2,698 & 1,776 & 4,474 & 491.3 & 771.3 & $1,262.6$ & 26.7 & 30.1 & 26.7 & 31.6 & \\
\hline 1984. & $\ldots \ldots \ldots \ldots$ & 2,735 & 1,937 & 4,672 & 497.9 & 841.3 & $1,339.2$ & 25.4 & 29.5 & 25.4 & 32.1 & \\
\hline 1985. & $\ldots \ldots \ldots \ldots$ & 2,928 & 2,036 & 4,964 & 531.6 & 860.9 & $1,392.5$ & 23.3 & 31.8 & 25.7 & 33.2 & \\
\hline 1986. & $\ldots \ldots \ldots \ldots$ & 2,551 & 2,291 & 4,842 & 458.3 & 996.2 & $1,454.5$ & 21.0 & 30.5 & 27.3 & 33.9 & \\
\hline 1987. & $\cdots \cdots \cdots \cdots$ & 3,570 & 2,594 & 6,164 & 643.5 & $1,109.2$ & $1,752.7$ & 20.8 & 31.3 & 27.0 & 34.4 & \\
\hline 1988. & $\ldots \ldots \ldots \ldots$ & 2,956 & 2,637 & 5,593 & 535.6 & $1,122.2$ & $1,657.8$ & 24.1 & 33.3 & 27.0 & 35.4 & \\
\hline 1989 . & $\ldots \ldots \ldots \ldots$ & 3,832 & 2,785 & 6,617 & 698.0 & $1,215.3$ & $1,913.3$ & 22.3 & 32.5 & 27.3 & 36.8 & \\
\hline 1990 . & $\ldots \ldots \ldots \ldots$ & 3,485 & 3,516 & 7,001 & 632.8 & $1,516.6$ & $2,149.4$ & 25.0 & 34.1 & 27.8 & 36.0 & \\
\hline 1991. & $\ldots \ldots \ldots \ldots$ & 3,260 & 2,937 & 6,197 & 588.0 & $1,282.6$ & $1,870.6$ & 28.2 & 35.2 & 30.3 & 37.4 & \\
\hline 1992. & $\ldots \ldots \ldots \ldots$ & 3,842 & 3,711 & 7,553 & 695.0 & $1,600.0$ & $2,295.0$ & 29.2 & 36.5 & 29.8 & 38.7 & \\
\hline $1993^{a}$. & & 3,997 & 3,535 & 7,532 & 720.9 & $1,533.6$ & $2,254.5$ & 30.3 & 38.9 & 31.2 & 38.9 & \\
\hline Temps & $s^{b} \ldots \ldots \ldots$ & 431 & 179 & 610 & 77.6 & 28.2 & 105.8 & 0.1 & 15.6 & 23.0 & 18.1 & \\
\hline Total . & $\ldots \ldots \ldots \ldots$ & 56,537 & 41,863 & 98,400 & $10,256.2$ & $17,879.9$ & $28,136.0$ & 22.8 & 31.6 & 26.1 & 34.6 & \\
\hline
\end{tabular}

aSome data for previous years have been revised. Current-year data may be revised in future publications. When utilities reinsert assemblies discharged in previous years, historical totals change. See Technical Note 12 in Appendix E.

'Temps are temporarily discharged assemblies, as of December 31, 1993.

PWR = Pressurized-water reactor; BWR = Boiling-water reactor; GWDt/MTU = Gigawattdays thermal per metric ton of uranium.

Notes: A total of 2,208 high-temperature, gas-cooled reactor (HTGR) fuel elements, with initial uranium content equal to $24.2 \mathrm{MTU}$, were discharged. These HTGR fuel elements are not included in the above table. See Technical Note 6 in Appendix E. Totals may not equal sum of components because of independent rounding. See Technical Note 11 in Appendix E. Source: Energy Information Administration, Form RW-859, "Nuclear Fuel Data" (1993). 
Figure 3. Annual Spent Fuel Discharges, 1968-1993
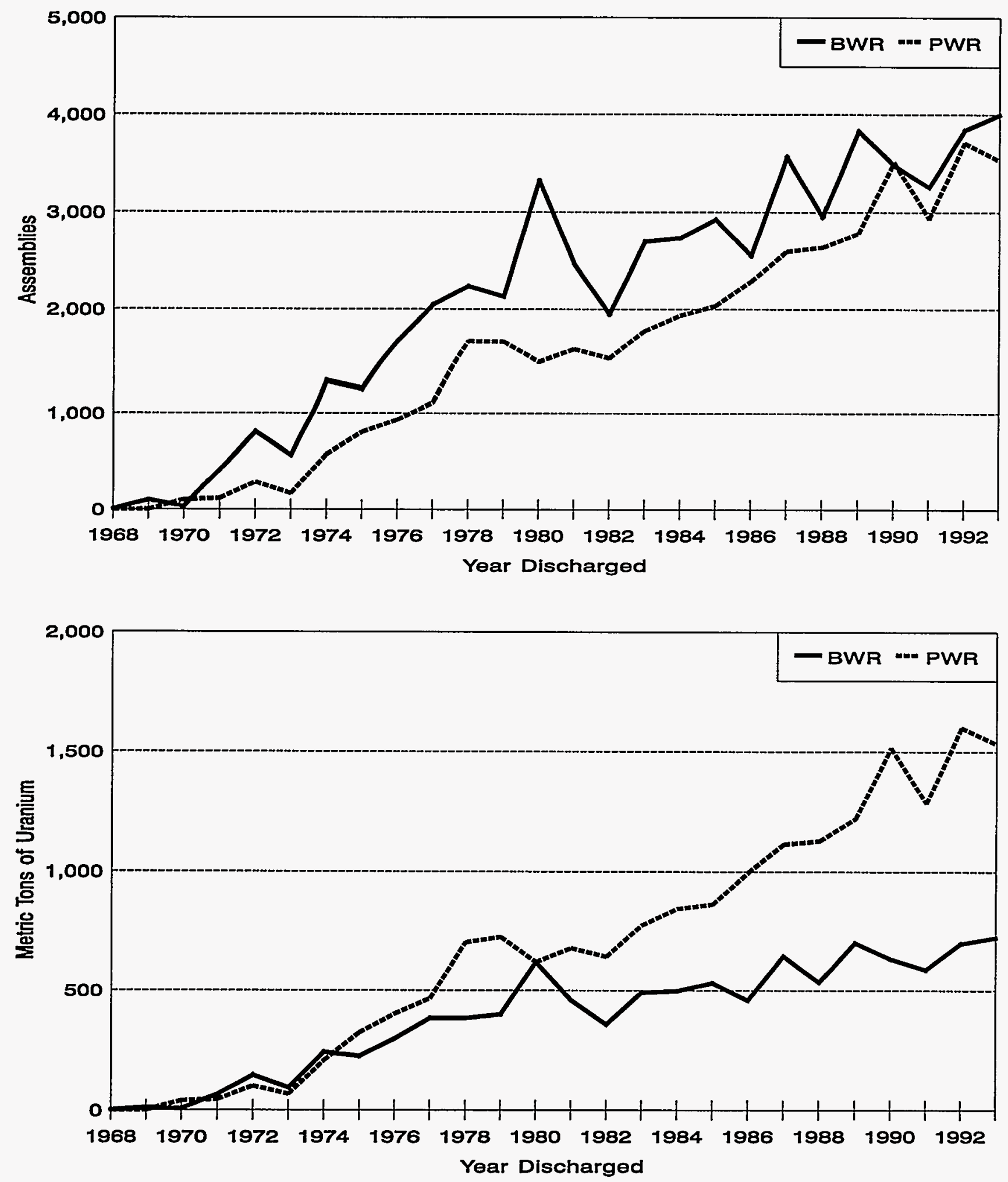

BWR = Bolling-water reactor; PWR = Pressurized-water reactor.

Notes: A number of assemblies discharged prior to 1972 were reprocessed and are not included in this figure. See Table 12 and Technical Note 7 in Appendix $E$ for information on reprocessed assemblies. Graphs do not include 2,208 high-temperature, gas-cooled reactor (HTGR) fuel elements and 610 temporarily discharged assemblies.

Source: Energy Information Administration, Form RW-859, "Nuclear Fuel Data" (1993). 
Figure 4. Annual Average Burnups, 1968-1993

All Discharged Assemblies

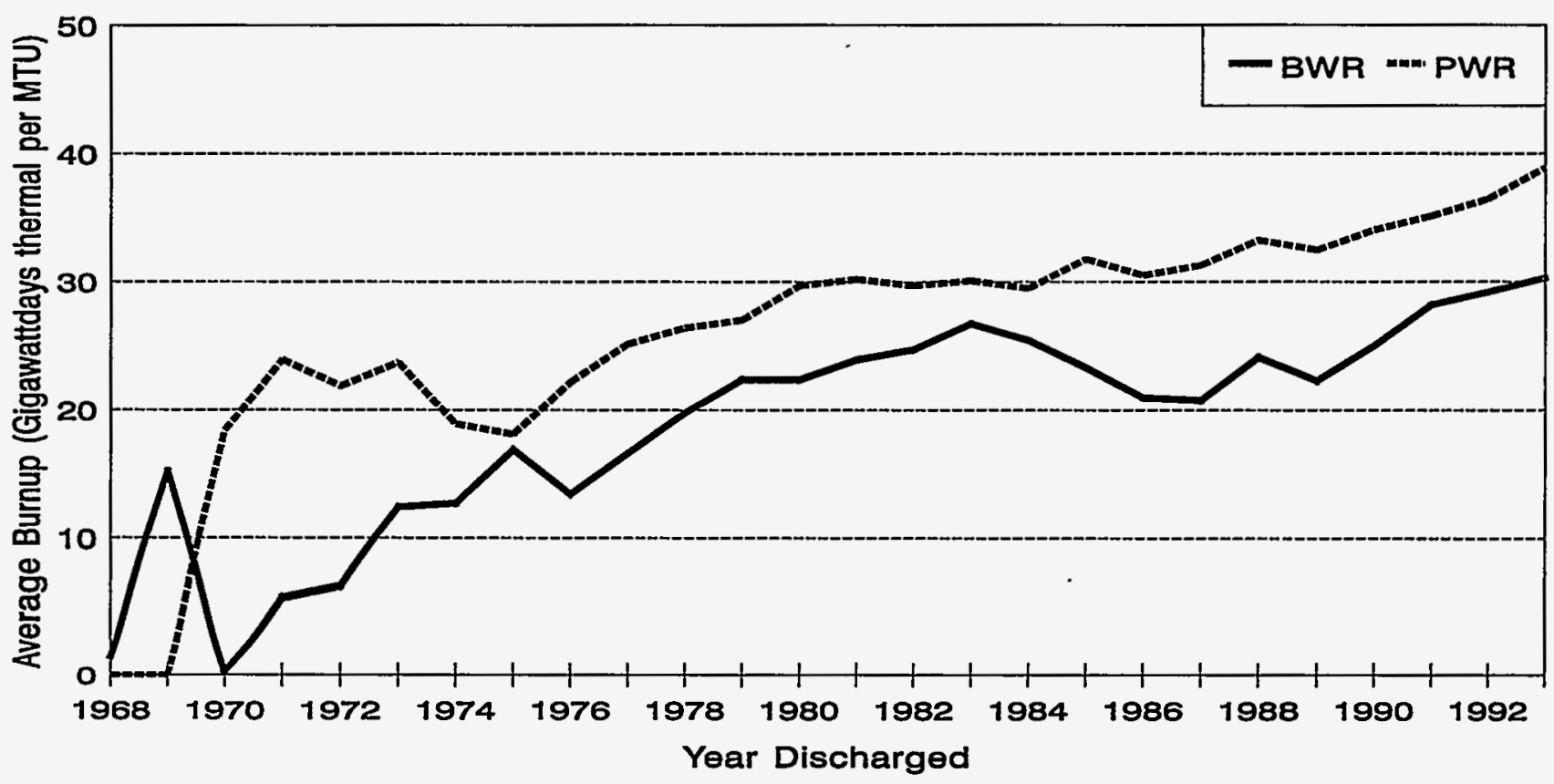

\section{Equilibrium Cycle Discharges}

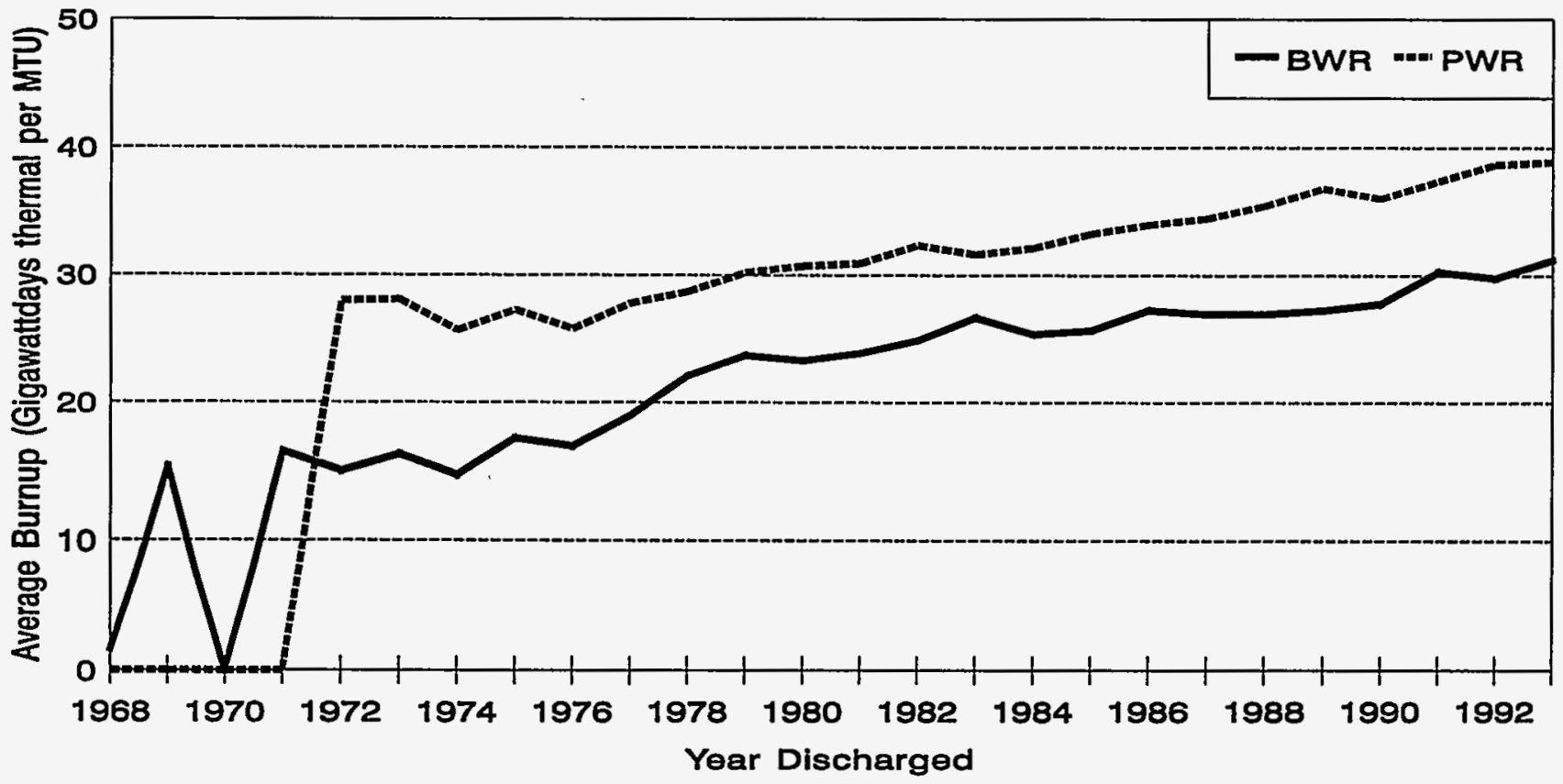

BWR = Boiling-water reactor; PWR = Pressurized-water reactor. $M T U=$ Metric tons of uranium.

Notes: A number of assemblies discharged prior to 1972 were reprocessed and are not included in this figure. See Table 12 and Technical Note 7 in Appendix $E$ for information on reprocessed assemblies. Graphs do not include 2,208 high-temperature, gas-cooled reactor (HTGR) fuel elements and 610 temporarily discharged assemblies. Equilibrium Cycle Discharges exclude discharge data for cycles 1 and 2 of each reactor. Source: Energy Information Administration, Form RW-859, "Nuclear Fuel Data" (1993). 
Annual spent fuel burnup distributions in number of BWR and PWR assemblies (Table 6) have increased over time, as indicated by the progression of larger concentrations of assemblies in higher burnup categories in most recent years. For example, 2,077 BWR assemblies discharged in 1993 (51.9 percent) range from 30 to 35 GWDt/MTU, the highest percentage for that range for any year. A total of 1,939 PWR assemblies (54.9 percent) had burnups greater than $40 \mathrm{GWDt} / \mathrm{MTU}$ in 1993. This total increased dramatically from the 1,499 PWR assemblies (40.4 percent) in 1992, thus continuing the pattern for larger concentrations of assemblies in higher burnup categories.

On the high end of the discharge burnups, only 7 BWR assemblies have exceeded $40 \mathrm{GWDt} / \mathrm{MTU}$, while 63 PWR assemblies have exceeded $50 \mathrm{GWDt} / \mathrm{MTU}$. As expected, the annual spent fuel burnup distribution by weight for BWR and PWR assemblies (Table 7) parallel the burnup distribution by assembly (Table 6). These increases show the effect of the improved fuel management practices being used to provide higher burnups.

Assembly weights, burnups, and enrichment data by burnup range for BWR's and PWR's are compared in Table 8. Examination of the data shows that BWR's have discharged over 57 percent of the assemblies, yet PWR's account for 63 percent of the uranium (by weight) that has been discharged; PWR assemblies contain substantially more uranium than BWR assemblies ( $450 \mathrm{~kg}$ versus $200 \mathrm{~kg}$, respectively). Of the discharged BWR assemblies, 66 percent (by initial loading weight of uranium) is concentrated in the 15 to $30 \mathrm{GWDt} / \mathrm{MTU}$ range. For PWR assemblies, 68 percent is concentrated in the 25 to 40 GWDt/MTU range (Table 8 ).

Data for reprocessed fuel assemblies have not been collected and, therefore, are not addressed in this report. Data for Fort St. Vrain have been omitted, since it is not a LWR. Three Mile Island Unit 2 ceased operation in 1979; the reactor core is now part of a DOE test and evaluation program. Because of this unique situation, the fuel from Three Mile Island Unit 2 is not included in this report.

\section{Cladding}

Early reactors used stainless steel cladding for their fuel assemblies. This was less than perfect because stainless steel is a neutron absorber or poison to the nuclear reaction. As a result, zircaloy-2 (for BWR's) and zircaloy-4 (for PWR's) have been used as the cladding material of choice since the early 1970s. For simplicity of data presentation, the designation zircaloy is used for both cladding types. Current LWR's use zircaloy cladding almost exclusively. Zircaloy cladding provides better neutron utilization, and is also more resistant to corrosion under the harsh physical and chemical conditions present in the reactor core. Only one reactor still has some stainless steel clad fuel rods in core.

Advances in the fabrication and treatment of zircaloy clad fuel have reduced fuel failures significantly. This has resulted in higher burnups and, to some extent, improved fuel economy. This trend is expected to continue as assemblies with the improved cladding are loaded with higher enrichments (over 4 percent enrichment), irradiated, and discharged. Zircaloy cladding is also usually much less radioactive after being discharged from the core than is stainless steel.

In an analysis of number of spent fuel assemblies and MTU of spent fuel discharged by year and by fuel rod cladding type (Table 9), it was found that most discharged spent fuel is zircaloy clad. Based on MTU, 99 percent of BWR fuel is zircaloy clad, and 96 percent of PWR fuel is zircaloy clad. Overall, only 2 percent of discharged fuel is stainless steel clad.

Only five reactors have discharged fuel rods with stainless steel cladding (Table 10). Of these, four reactors (Indian Point 1, LaCrosse, San Onofre 1, and Yankee Rowe) are permanently shut down. Yankee Rowe switched to zircaloy clad fuel in the mid-1970's. Haddam Neck is in the process of switching to zircaloy clad fuel. After Haddam Neck has completed its switch to zircaloy clad fuel, no U.S. commercial reactor will be using stainless steel clad fuel. 
Table 6. Annual Spent Fuel Burnup, 1968-1993

(Assemblies)

\begin{tabular}{|c|c|c|c|c|c|c|c|c|c|c|c|c|c|}
\hline \multirow[b]{2}{*}{ Year } & \multicolumn{13}{|c|}{$\begin{array}{c}\text { Burnup Ranges" } \\
\text { (GWDUMTU) }\end{array}$} \\
\hline & 0.5 & $5-10$ & $10-15$ & $15-20$ & $20-25$ & 25-30 & $30-35$ & $35-40$ & $40-45$ & $45-50$ & $50-55$ & $55-60$ & Total \\
\hline & \multicolumn{13}{|c|}{ Boiling-Water Reactors (BWR) } \\
\hline $\begin{array}{ll}1968 & \ldots \\
1969 & \ldots \\
1990 & \ldots \\
1971 & \ldots \\
1972 & \ldots \\
1973 & \ldots \\
1974 & \ldots \\
1975 & \ldots \\
1976 & \ldots \\
1977 & \ldots \\
1978 & \ldots \\
1979 & \ldots \\
1980 & \ldots \\
1981 & \ldots \\
1982 & \ldots \\
1983 & \ldots \\
1984 & \ldots \\
1985 & \ldots \\
1986 & \ldots \\
1987 & \ldots \\
1988 & \ldots \\
1989 & \ldots \\
1990 & \ldots \\
1991 & \ldots \\
1992 & \ldots \\
1993^{\circ} & \ldots \\
\text { Temps } & \end{array}$ & $\begin{array}{r}5 \\
0 \\
09 \\
216 \\
509 \\
51 \\
0 \\
2 \\
5 \\
5 \\
0 \\
66 \\
0 \\
76 \\
0 \\
0 \\
0 \\
0 \\
92 \\
276 \\
422 \\
92 \\
168 \\
92 \\
96 \\
0 \\
0 \\
0\end{array}$ & $\begin{array}{r}0 \\
11 \\
0 \\
47 \\
62 \\
91 \\
407 \\
15 \\
392 \\
251 \\
198 \\
0 \\
2 \\
1 \\
1 \\
0 \\
43 \\
232 \\
176 \\
204 \\
132 \\
92 \\
0 \\
132 \\
0 \\
0 \\
0\end{array}$ & $\begin{array}{r}0 \\
10 \\
0 \\
28 \\
172 \\
177 \\
597 \\
327 \\
599 \\
208 \\
106 \\
108 \\
3 \\
1 \\
26 \\
5 \\
237 \\
101 \\
232 \\
385 \\
10 \\
372 \\
184 \\
0 \\
41 \\
0 \\
0\end{array}$ & $\begin{array}{r}0 \\
72 \\
0 \\
106 \\
54 \\
232 \\
249 \\
735 \\
656 \\
1,250 \\
502 \\
582 \\
505 \\
314 \\
151 \\
25 \\
2 \\
226 \\
376 \\
232 \\
234 \\
388 \\
368 \\
40 \\
411 \\
167 \\
0\end{array}$ & $\begin{array}{r}0 \\
2 \\
0 \\
16 \\
4 \\
12 \\
37 \\
144 \\
14 \\
334 \\
1,283 \\
794 \\
2,210 \\
1,426 \\
748 \\
620 \\
742 \\
580 \\
243 \\
137 \\
929 \\
1,051 \\
582 \\
137 \\
340 \\
261 \\
1\end{array}$ & $\begin{array}{r}0 \\
1 \\
0 \\
0 \\
0 \\
1 \\
0 \\
0 \\
0 \\
4 \\
84 \\
645 \\
476 \\
717 \\
945 \\
1,852 \\
1,318 \\
1,640 \\
1,014 \\
1,950 \\
1,072 \\
1,263 \\
1,357 \\
1,183 \\
466 \\
793 \\
0\end{array}$ & $\begin{array}{r}0 \\
0 \\
0 \\
0 \\
0 \\
0 \\
0 \\
0 \\
0 \\
0 \\
0 \\
2 \\
58 \\
4 \\
75 \\
194 \\
391 \\
56 \\
232 \\
238 \\
487 \\
478 \\
893 \\
1,604 \\
1,992 \\
2,077 \\
0\end{array}$ & $\begin{array}{r}0 \\
0 \\
0 \\
0 \\
0 \\
0 \\
0 \\
0 \\
0 \\
0 \\
0 \\
0 \\
0 \\
4 \\
3 \\
2 \\
0 \\
0 \\
2 \\
0 \\
0 \\
20 \\
9 \\
68 \\
592 \\
699 \\
0\end{array}$ & $\begin{array}{l}0 \\
0 \\
0 \\
0 \\
0 \\
0 \\
0 \\
0 \\
0 \\
0 \\
0 \\
0 \\
0 \\
0 \\
2 \\
0 \\
2 \\
1 \\
0 \\
2 \\
0 \\
0 \\
0 \\
0 \\
0 \\
0 \\
0\end{array}$ & $\begin{array}{l}0 \\
0 \\
0 \\
0 \\
0 \\
0 \\
0 \\
0 \\
0 \\
0 \\
0 \\
0 \\
0 \\
0 \\
0 \\
0 \\
0 \\
0 \\
0 \\
0 \\
0 \\
0 \\
0 \\
0 \\
0 \\
0 \\
0\end{array}$ & $\begin{array}{l}0 \\
0 \\
0 \\
0 \\
0 \\
0 \\
0 \\
0 \\
0 \\
0 \\
0 \\
0 \\
0 \\
0 \\
0 \\
0 \\
0 \\
0 \\
0 \\
0 \\
0 \\
0 \\
0 \\
0 \\
0 \\
0 \\
0\end{array}$ & $\begin{array}{l}0 \\
0 \\
0 \\
0 \\
0 \\
0 \\
0 \\
0 \\
0 \\
0 \\
0 \\
0 \\
0 \\
0 \\
0 \\
0 \\
0 \\
0 \\
0 \\
0 \\
0 \\
0 \\
0 \\
0 \\
0 \\
0 \\
0\end{array}$ & $\begin{array}{r}5 \\
96 \\
29 \\
413 \\
801 \\
564 \\
1,290 \\
1,223 \\
1,666 \\
2,047 \\
2,239 \\
2,131 \\
3,330 \\
2,467 \\
1,951 \\
2,698 \\
2,735 \\
2,928 \\
2,551 \\
3,570 \\
2,956 \\
3,832 \\
3,485 \\
3,260 \\
3,842 \\
3,997 \\
\dot{d} 431\end{array}$ \\
\hline Total ... & 2,197 & 2,489 & 3,929 & 7,877 & 12,647 & 16,781 & 8,781 & 1,399 & 7 & 0 & 0 & 0 & 56,537 \\
\hline
\end{tabular}

Pressurized-Water Reactors (PWR)

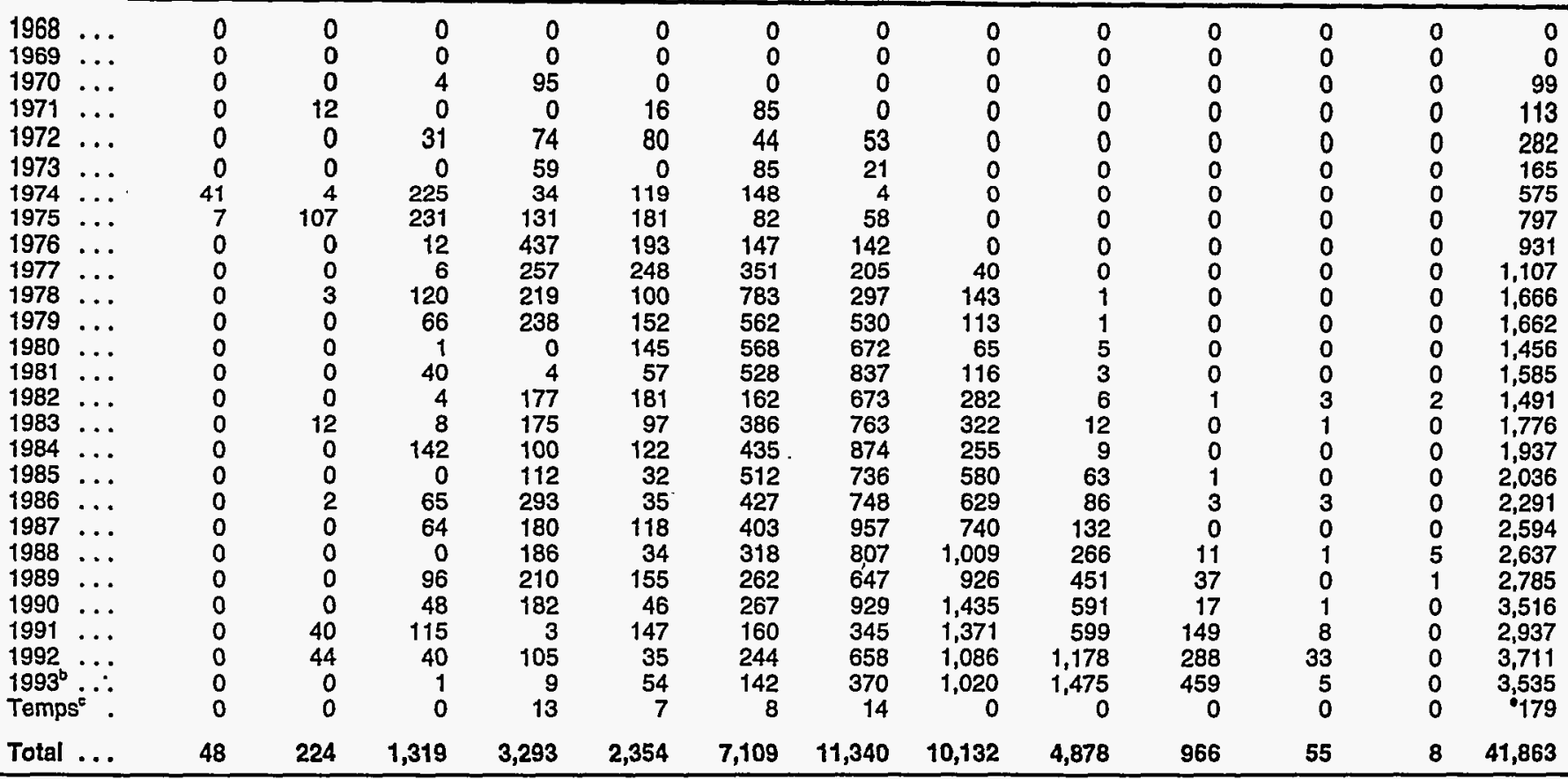

${ }^{2}$ Ranges do not overlap. The source data are in batch averages in gigawattdays thermal per metric ton of uranium (GWDt/MTU). Therefore, 0-5 GWDU/MTU means 0 through 4.999 GWDt/MTU; 5-10 GWDV/MTU means 5.000-9.999 GWDt/MTU; etc. See Technical Note 9 in Appendix E.

'Some data for previous years have been revised. Current-year data may be revised in future publications. When utilities reinsert assemblies discharged in previous years, historical totals change. See Technical Note 12 in Appendix E.

Temps are temporarily discharged assemblies, as of December 31, 1993, as reported on Form AW-859.

"Includes 430 temporarily discharged BWR assemblies with missing burnup data. See Technical Note 10 in Appendix E.

"includes 137 temporarily discharged PWR assemblies with missing bumup data. See Technical Note 10 in Appendix E.

Note: A total of 2,208 high-temperature, gas-cooled reactor (HTGR) fuel elements, with initial uranium content equal to $24.2 \mathrm{MTU}$, were discharged. These HTGR fuel elements are not included in the above table. See Technical Note 6 in Appendix $E$.

Source: Energy Information Administration, Form RW-859, "Nuclear Fuel Data" (1993). 
Table 7. Annual Spent Fuel Burnup, 1968-1993 (Metric tons of uranium)

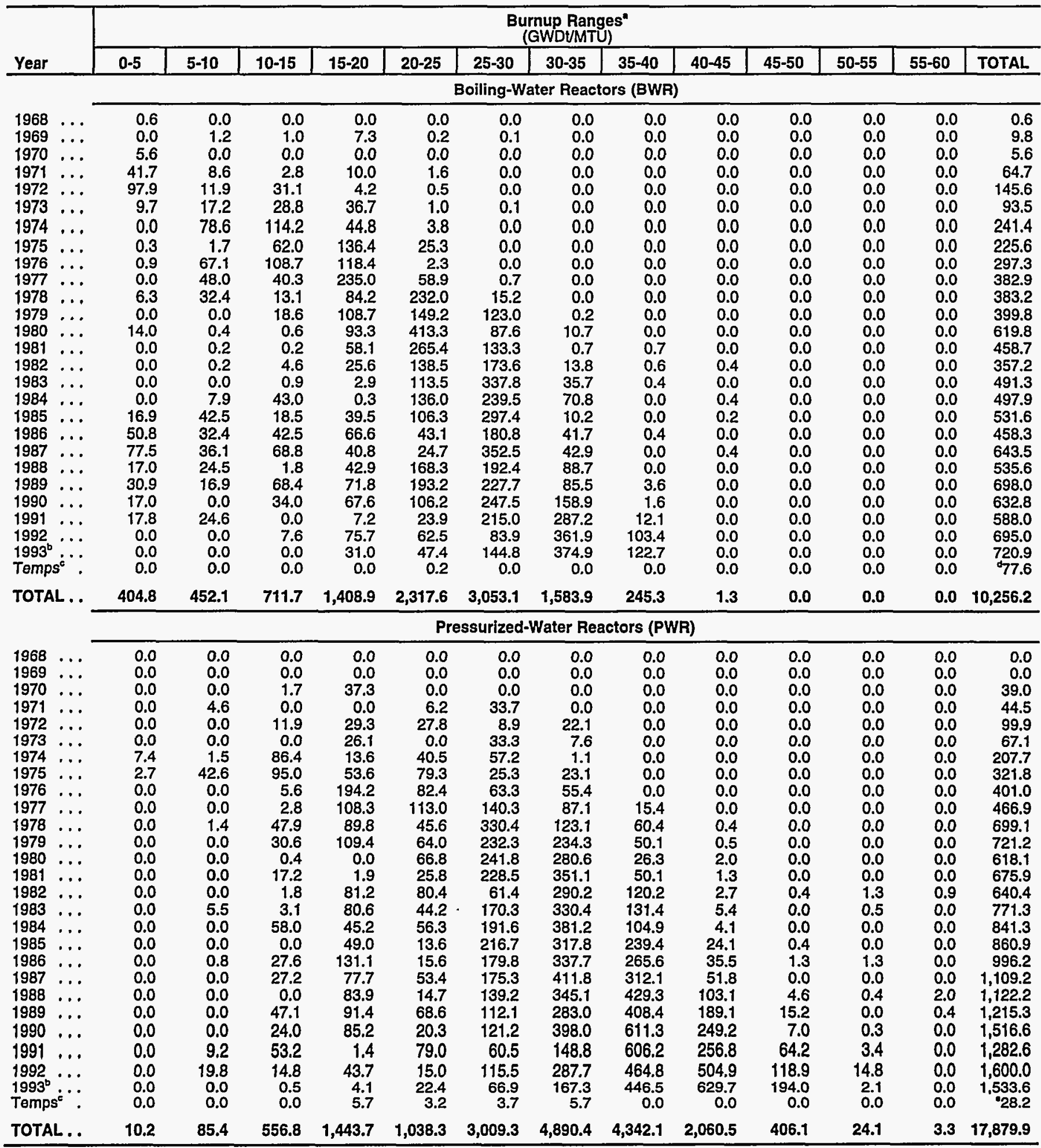

"Ranges do not overlap. The source data are in batch averages in gigawattdays thermal per metric ton of uranium (GWDtMTU). Therefore, $0-5$ GWDV/MTU means 0 through 4.999 GWDU/MTU; 5-10 GWDt/MTU means 5.000-9.999 GWDt/MTU; etc. See Technical Note 9 in Appendix E.

'Some data for previous years have been revised. Current-year data may be revised in future publications. When utilities reinsert assemblies discharged in previous years, historical totals change. See Technical Note 12 in Appendix E.

Temps are temporarily discharged assemblies, as of December 31, 1993, as reported on Form RW-859.

Includes 77.4 MTU for temporarily discharged BWR assemblies with missing burnup data. See Technical Note 10 in Appendix E.

Includes 9.9 MTU for temporarily discharged PWR assemblies with missing burnup data. See Technical Note 10 in Appendix E.

Note: A total of 2,208 high-temperature, gas-cooled reactor (HTGR) fuel elements, with initial uranium content equal to $24.2 \mathrm{MTU}$, were discharged.

These HTGR fuel elements are not included in the above table. See Technical Note 6 in Appendix E.

Source: Energy Information Administration, Form RW-859, "Nuclear Fuel Data" (1993). 
Table 8. U.S. Burnup Distribution for All Discharged Assemblies

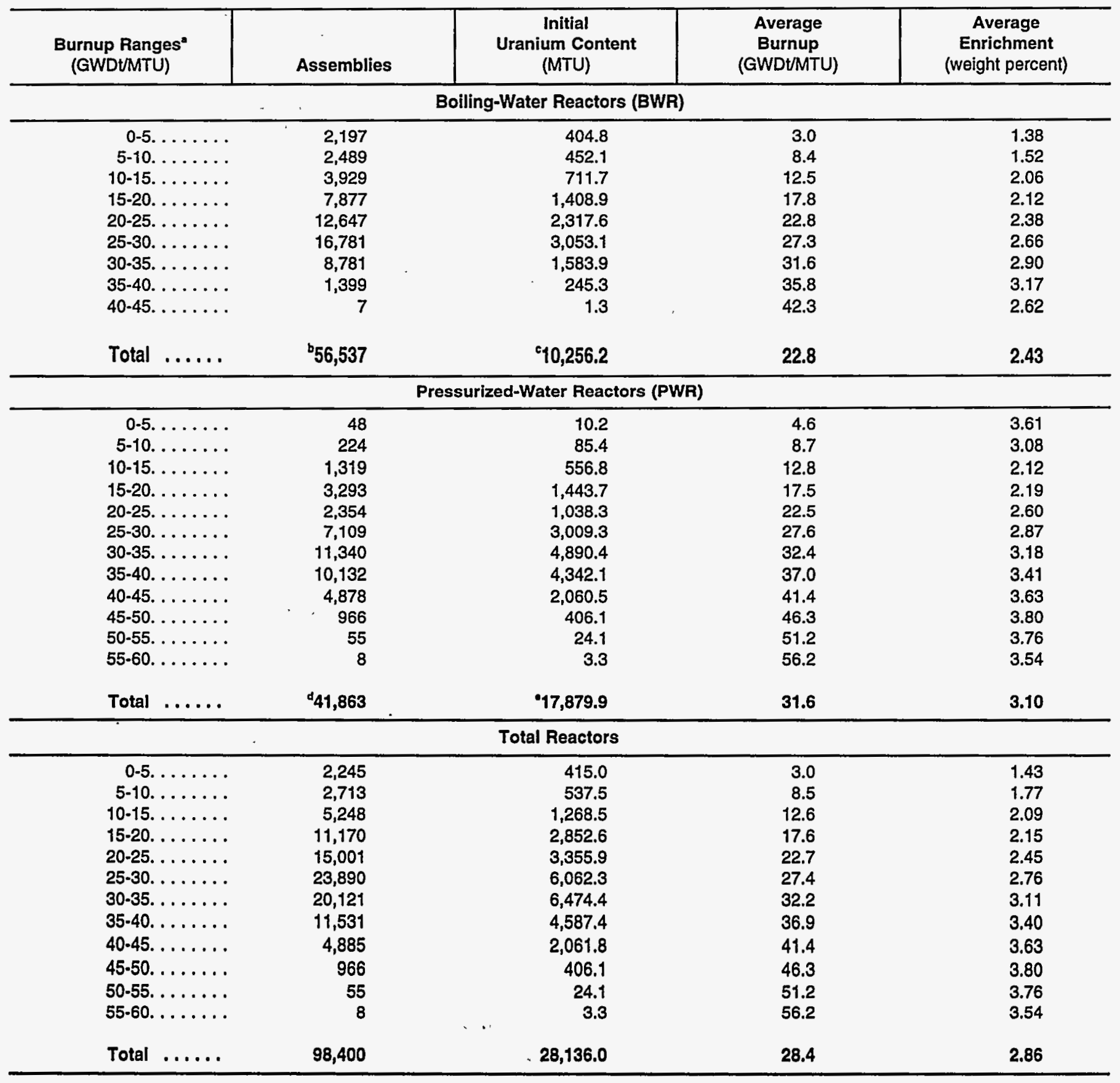

"Ranges do not overlap. The source data are in batch averages in gigawattdays thermal per metric ton of uranlum (GWDt/MTU). Therefore, 0.5 GWDU/MTU means 0 through 4.999 GWDUMTU; 5-10 GWDt/MTU means 5.000-9.999 GWDt/MTU; etc. See Technical Note 9 in Appendix E.

bo assigned bumup range for 430 temporarly discharged BWR asssemblies. These BWR assemblles are Included in the column total. See Technical Note 10 in Appendix E.

Includes 77.4 MTU for temporarily discharged BWR assemblles with no assigned burnup range. These weights are included in the column total. See Technical Note 10 in Appendix E.

No assigned burnup range for 137 temporarly discharged PWR assemblies. These PWR assemblies are included in the column total. Se日 Technical Note 10 in Appendix E.

Includes 9.9 MTU for temporarily discharged PWR assemblies with no assigned burnup range. These weights are included in the column total. See Technical Note 10 In Appendix E.

Notes: A total of 2,208 high-temperature, gas-cooled reactor (HTGR) fuel elements, with initial uranium content equal to $24.2 \mathrm{MTU}$, were discharged. These HTGR fuel elements are not included in the Total Reactors section of the table. See Technical Note 6 in Appendlx $E$. Totals may not equal sum of components because of independent rounding. See Technical Note 11 in Appendlx $E$.

Source: Energy Information Administration, Form RW-859, "Nuclear Fuel Data" (1993). 
Table 9. Spent Fuel by Cladding and Reactor Type, 1968-1993

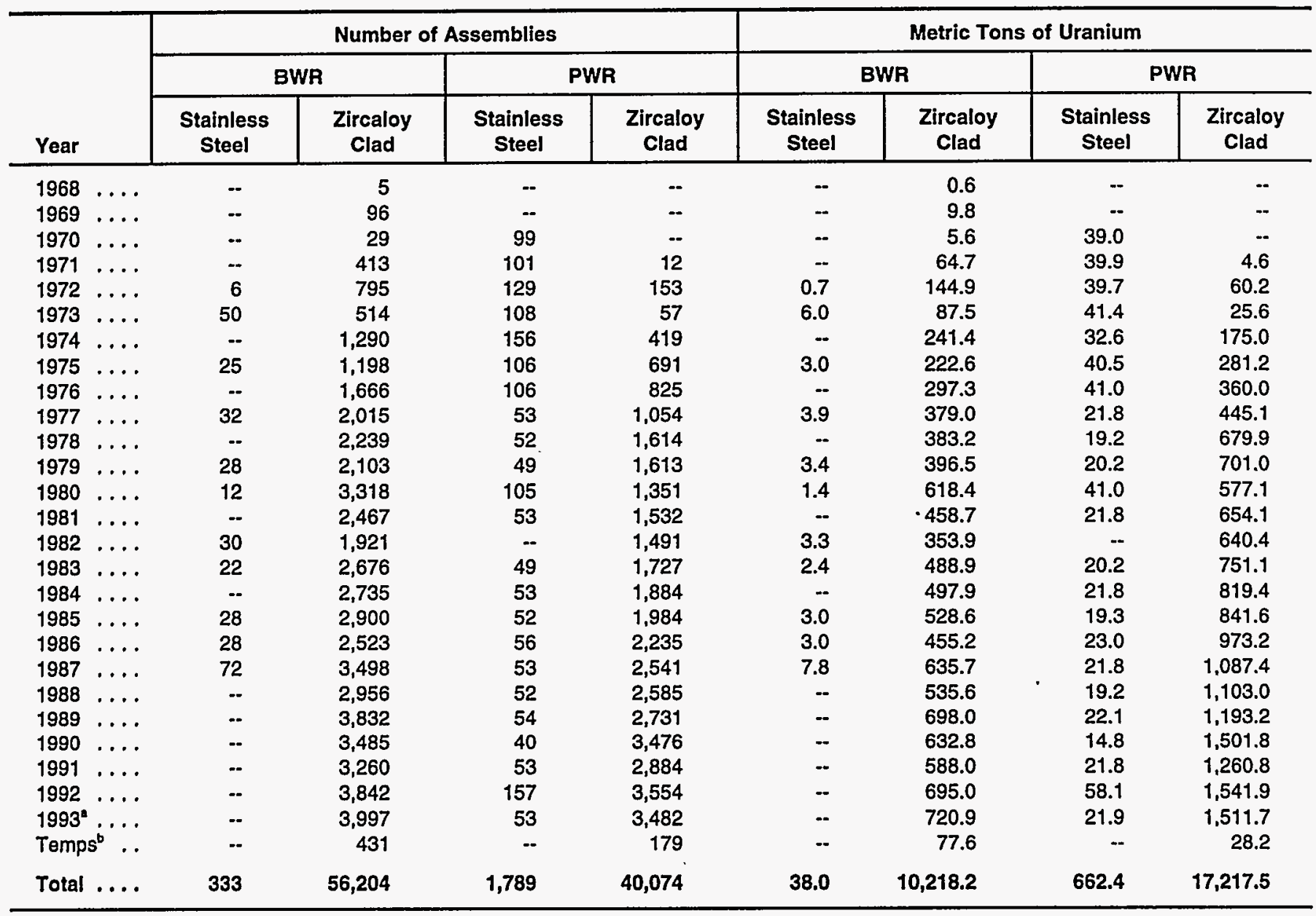

'Some data for previous years have been revised. Current-year data may be revised in future publications. When utilities reinsert assemblies discharged in previous years, historical totals change. See Technical Note 12 in Appendix E.

'Temps are temporarily discharged assemblies, as of December 31. 1993, as reported on Form RW-859.

.. = Not applicable. BWR = Bolling-water reactor; PWR $=$ Pressurized-water reactor.

Notes: A total of 2,208 high-temperature, gas-cooled reactor (HTGR) zircaloy clad fuel elements, with initial uranium content equal to 24.2 metric tons of uranium (MTU), were discharged. These HTGR fuel elements are not included in the above table. See Technical Note 6 in Appendix E. Totals may not equal sum of components because of independent rounding. See Technical Note 11 in Appendix $E$.

Source: Energy Information Administration, Form RW-859, "Nuclear Fuel Data" (1993).

Table 10. Reactors Discharging Stainless Steel Clad Assemblies

\begin{tabular}{|c|c|c|c|c|c|c|c|c|}
\hline \multirow[b]{3}{*}{ Reactor Name } & \multicolumn{4}{|c|}{ Number of Assemblies } & \multicolumn{4}{|c|}{ Metric Tons of Uranium } \\
\hline & \multicolumn{2}{|c|}{ BWR } & \multicolumn{2}{|c|}{ PWR } & \multicolumn{2}{|c|}{ BWR } & \multicolumn{2}{|c|}{ PWR } \\
\hline & $\begin{array}{l}\text { Stainless } \\
\text { Steel }\end{array}$ & $\begin{array}{l}\text { Zircaloy } \\
\text { Clad }\end{array}$ & $\begin{array}{c}\text { Stainless } \\
\text { Steel }\end{array}$ & $\begin{array}{l}\text { Zircaloy } \\
\text { Clad }\end{array}$ & $\begin{array}{c}\text { Stainless } \\
\text { Steel }\end{array}$ & $\begin{array}{l}\text { Zircaloy } \\
\text { Clad }\end{array}$ & $\begin{array}{c}\text { Stainless } \\
\text { Steel }\end{array}$ & $\begin{array}{l}\text { Zlrcaloy } \\
\text { Clad }\end{array}$ \\
\hline Haddam Neck & .. & .. & 888 & 4 & -- & -. & 366.7 & 1.5 \\
\hline Indlan Point 1 . & .. & .. & 160 & -. & -. & -- & 30.6 & .. \\
\hline LaCrosse .... & 333 & .. & .. & -- & 38.0 & -- & .. & -. \\
\hline San Onofre $1, \ldots$ & .. & .. & 665 & -- & -- & -- & 244.3 & -. \\
\hline Yankee Rowe ... & .. & .. & 76 & 457 & -- & .- & 20.8 & 106.5 \\
\hline Total ....... & 333 & - & 1,789 & 461 & 38.0 & - & 662.4 & 107.9 \\
\hline
\end{tabular}

. = Not applicable. BWR $=$ Bolling-water reactor; PWR $=$ Pressurlzed-water reactor.

Note: Totals may not equal sum of components because of Independent rounding. See Technical Note 11 in Appendix E.

Source: Energy Informatlon Administration, Form RW-859, "Nuclear Fuel Data" (1993). 


\section{Enrichment and Burnup}

The distribution of spent fuel assemblies discharged through 1993 by enrichment (weight percent) and burnup ranges (Table 11) are presented separately for each combination of reactor type (BWR and PWR) and cladding (zircaloy and stainless steel). Enrichment (weight percent) for stainless steel clad assemblies are higher than those for zircaloy clad assemblies due to the nature of stainless steel as a neutron poison. (Four PWR mixed-oxide assemblies were discharged from San Onofre 1. These assemblies are shown in the PWR stainless steel category in the 0.6 to 1.0 range, as the plutonium content of these assemblies is not reflected in the table.)

\section{Utility Shipments to Away-from-reactor Storage Facilities}

There are two types of spent fuel shipments, both of which are regulated by the U.S. Nuclear Regulatory Commission and monitored by State and local governments. The first is the transfer of spent fuel assemblies within an electric utility. These transfers allow the unused spent fuel storage capacity at one site to be used as additional storage for a reactor that may be running low on spent fuel storage capability. They also help utilities use available capacity efficiently and delay the need to build additional storage capabilities. These intrautility transfers are not covered in this report because there is no effect on the utility's storage capability. The second type of shipment is from a utility to an away-from-reactor storage facility (Table 12). There were no shipments of spent fuel from a utility to a storage facility in 1993. 
Table 11. Burnup Versus Enrichment by Cladding and Reactor Type (Assemblies)

\begin{tabular}{|c|c|c|c|c|c|c|c|c|c|c|c|c|c|}
\hline \multirow{2}{*}{$\begin{array}{l}\text { Enrichment } \\
\text { (weight percent) }^{\mathrm{a}}\end{array}$} & \multicolumn{13}{|c|}{ Burnup Ranges (GWDVMTU)" } \\
\hline & $0-5$ & $5-10$ & $10-15$ & $15-20$ & $20-25$ & $25-30$ & $30-35$ & $35-40$ & $40-45$ & $45-50$ & $50-55$ & $55-60$ & Total \\
\hline & \multicolumn{13}{|c|}{ Boiling-Water Reactor: Stainless Steel } \\
\hline $0.0-0.6 \ldots \ldots \ldots \ldots$ & - & - & - & - & -- & -- & -- & -- & -- & -- & - & - & -- \\
\hline $0.6-1.0 \ldots \ldots \ldots \ldots$ & - & - & -- & - & - & - & -- & -- & -- & -- & -- & -- & -- \\
\hline $1.0-1.4 \ldots \ldots \ldots \ldots$ & - & - & -- & - & -. & - & -- & - & -- & -. & - & -- & -- \\
\hline $1.4-1.8 \ldots \ldots \ldots \ldots$ & -- & -- & - & - & -- & $-\cdots$ & -- & -- & -- & -- & -- & -- & -- \\
\hline $1.8-2.2 \ldots \ldots \ldots \ldots$ & - & -- & - & -- & - & $\cdots$ & -- & - & -- & -- & -- & - & - \\
\hline $2.2-2.6 \ldots \ldots \ldots \ldots$ & - & -- & - & -- & - & - & -- & -- & -- & -- & -- & $\cdots$ & -- \\
\hline $2.6-3.0 \ldots \ldots \ldots \ldots$ & - & - & - & -- & -- &.- & - & - & -- & -- & -- & -- & -- \\
\hline $3.0-3.4 \ldots \ldots \ldots \ldots$ & - & - & 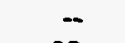 & -- & -- & $\cdots$ & -- & -- & - & -- & -- & -- & -- \\
\hline 3.4-3.8 $\ldots \ldots \ldots$ & -- & 25 & 88 & 143 & 4 & -- & -- & - & -- & -- & -- & -- & 260 \\
\hline $3.8-4.2 \ldots \ldots \ldots \ldots$ & -- & 2 & 32 & 39 & -- & $\cdots$ & -- & -- & -- & -. & -- & -- & 73 \\
\hline $4.2-4.6 \ldots \ldots \ldots \ldots$ & - & - & -- & - & -- & -- & - & -- & -- & -- & -. & -- & - \\
\hline $4.6-5.0 \ldots \ldots \ldots$ & - & - & - & -- & -- & $\because$ & -- & - & -- & -- & -- & -- & - \\
\hline \multirow[t]{2}{*}{ Total...$\ldots \ldots$} & - & 27 & 120 & 182 & 4 & - & - & - & - & - & - & - & 333 \\
\hline & \multicolumn{13}{|c|}{ Boiling-Water Reactor: Zircaloy Clad } \\
\hline $0.0-0.6 \ldots \ldots \ldots \ldots$ & - & -- & -- & - & - & -- & -- & -- & -- & -- & -. & -- & ${ }^{b} 430$ \\
\hline $0.6-1.0 \ldots \ldots \ldots$ & 1,060 & 536 & 140 & 52 & -- & -- & -- & -- & - & -- & $-\cdot$ & - & 1,788 \\
\hline $1.0-1.4 \ldots \ldots \ldots$ & - & 932 & 277 & -- & - & -- & -- & -- & -- & - & -- & - & 1,209 \\
\hline $1.4-1.8 \ldots \ldots \ldots \ldots$ & 258 & 172 & 188 & 548 & 37 & -- & -- & -- & -- & -- & -- & - & 1,203 \\
\hline $1.8-2.2 \ldots \ldots \ldots \ldots$ & 754 & 231 & 2,154 & 4,245 & 2,518 & 236 & -. & 1 & - & -- & -- & - & 10,139 \\
\hline $2.2-2.6 \ldots \ldots \ldots \ldots$ & 118 & 526 & 525 & 2,508 & 7,407 & 4,115 & 75 & 56 & - & -- & -. & - & 15,330 \\
\hline $2.6-3.0 \ldots \ldots \ldots \ldots$ & $=$ & 49 & 261 & 285 & 2,452 & 11,425 & 5,029 & 215 & 7 & -- & -- & $-\infty$ & 19,723 \\
\hline $3.0-3.4 \ldots \ldots \ldots \ldots$ & -- & - & 198 & 11 & 125 & 941 & 3,639 & 887 & -- & -- & -- & - & 5,801 \\
\hline $3.4-3.8 \ldots \ldots \ldots$ & 7 & 16 & 66 & 46 & 104 & 50 & 38 & 240 & -- & -- &.- & -- & 567 \\
\hline $3.8-4.2 \ldots \ldots \ldots \ldots$ & $\cdots$ & - & -- & - & -- & 14 & -- & -- & - & -- & -- & -- & 14 \\
\hline $4.2-4.6 \ldots \ldots \ldots \ldots$ & - & - & -- & -- & -- & -- & -- & -- & - & -- & -- & - & -. \\
\hline $4.6-5.0 \ldots \ldots \ldots$ & 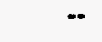 & - & -- & -- & -- & -- & -- & -- & $\cdots$ & -- & -- & -- & $-\cdot$ \\
\hline Total $\ldots . . . . .$. & 2,197 & 2,462 & 3,809 & 7,695 & 12,643 & 16,781 & 8,781 & 1,399 & 7 & - & - & - & 56,204 \\
\hline
\end{tabular}

See footnotes at end of table. 
Table 11. Burnup Versus Enrichment by Cladding and Reactor Type (Continued) (Assemblies)

\begin{tabular}{|c|c|c|c|c|c|c|c|c|c|c|c|c|c|}
\hline \multirow{2}{*}{$\begin{array}{l}\text { Enrichment } \\
\text { (weight percent) }^{\mathrm{a}}\end{array}$} & \multicolumn{13}{|c|}{ Burnup Ranges (GWDt/MTU) ${ }^{\mathfrak{a}}$} \\
\hline & $0-5$ & $5-10$ & 10-15 & $15-20$ & $20-25$ & $25-30$ & $30-35$ & $35-40$ & $40-45$ & $45-50$ & $50-55$ & $55-60$ & Total \\
\hline & \multicolumn{13}{|c|}{ Pressurized-Water Reactor: Stainless Steel } \\
\hline $0.0-0.6 \ldots \ldots \ldots$ & - & - & - & -- & -. & -. & -- & -. & -. & -- & .. & -- & -- \\
\hline $0.6-1.0 \ldots \ldots \ldots$ & - & - & -- & 4 & -- & -- & -- & -- & -- & -- & -- & -- & 4 \\
\hline $1.0-1.4 \ldots \ldots \ldots \ldots$ & - & - & - & -- & -- & - & $\ldots$ & -- & -- & - & -. & -- & - \\
\hline $1.4-1.8 \ldots \ldots \ldots \ldots$ & - & - & -- & - & -- & - & -. & -. & -- & - & -- & -- & -- \\
\hline $1.8-2.2 \ldots \ldots \ldots \ldots$ & - & - & -- & - & -- & -- & -- & - & -- & -- & -. & -- & - \\
\hline $2.2-2.6 \ldots \ldots \ldots \ldots$ & - & - & -- & - & -- & -- & -- & -- & -- & - & .- & -. &.- \\
\hline $2.6-3.0 \ldots \ldots \ldots \ldots$ & - & - & - & -. & .. & .- & -. & -. & -- & .- & .. & -- & .. \\
\hline $3.0-3.4 \ldots \ldots \ldots \ldots$ & - & - & - & 95 & 12 & 46 & -- & - & -- & $\ldots$ & .. & -- & 153 \\
\hline $3.4-3.8 \ldots \ldots \ldots \ldots$ & - & - & 4 & -- & 10 & 90 & 53 & 4 & -- & -- & -. & -- & 161 \\
\hline $3.8-4.2 \ldots \ldots \ldots \ldots$ & - & 2 & 85 & 52 & 40 & 197 & 835 & 144 & -- & -- & -- & -- & 1,355 \\
\hline $4.2-4.6 \ldots \ldots \ldots \ldots$ & 40 & - & - & -- & - & -- & -- & -- & - & - & -- & -- & 40 \\
\hline $4.6-5.0 \ldots \ldots \ldots$ & - & - & - & -- & 32 & 36 & 8 & - & - & -- & $-\cdot$ & $\cdots$ & 76 \\
\hline \multirow[t]{2}{*}{ Total ........... } & 40 & 2 & 89 & 151 & 94 & 369 & 896 & 148 & - & - & - & - & 1,789 \\
\hline & \multicolumn{13}{|c|}{ Pressurized-Water Reactor: Zircaloy Clad } \\
\hline $0.0-0.6 \ldots \ldots \ldots$ & - & - & - & -- & -- & - & - & -- & -- & - & -. & -- & $c_{137}$ \\
\hline $0.6-1.0 \ldots \ldots \ldots \ldots$ & -- & - & -- & -. & -- & -- & -- & .. & .- & -- & .. & .- & -- \\
\hline $1.0-1.4 \ldots \ldots \ldots$ & - & -- & - & - & 12 & - & -- & $\cdots$ & -- & - & -. & -- & 12 \\
\hline $1.4-1.8 \ldots \ldots \ldots \ldots$ & - & 25 & 418 & 78 & 7 & -- & -- & - & -. & - & -- & -. & 528 \\
\hline $1.8-2.2 \ldots \ldots \ldots \ldots$ & 7 & 3 & 571 & 2,114 & 255 & 98 & 1 & - & -. & - & -. & .. & 3,049 \\
\hline $2.2-2.6 \ldots \ldots \ldots \ldots$ & - & - & 69 & 796 & 948 & 992 & 5 & -. & 1 & - & -- & -- & 2,811 \\
\hline $2.6-3.0 \ldots \ldots \ldots \ldots$ & - & - & 4 & 57 & 621 & 3,532 & 2,606 & 182 & 83 & 5 & -. & -- & 7,090 \\
\hline $3.0-3.4 \ldots \ldots \ldots \ldots$ & - & 98 & 124 & 63 & 332 & 1,618 & 6,463 & 4,215 & 555 & 24 & 5 & 1 & 13,498 \\
\hline $3.4-3.8 \ldots \ldots \ldots$ & 1 & 56 & 35 & 16 & 58 & 368 & 1,259 & 4,795 & 2,519 & 325 & 11 & 6 & 9,449 \\
\hline $3.8-4.2 \ldots \ldots \ldots \ldots$ & - & 40 & 9 & 18 & 19 & 131 & 110 & 792 & 1,701 & 476 & 34 & 1 & 3,331 \\
\hline $4.2-4.6 \ldots \ldots \ldots$ & - & - & - & -. & 8 & 1 & -- & -- & 19 & 136 & 5 & -- & 169 \\
\hline $4.6-5.0 \ldots \ldots \ldots$ & - & - & - & -- & - & - & - & -- & - & - &.- & -- & -. \\
\hline Total .......... & 8 & 222 & 1,230 & 3,142 & 2,260 & 6,740 & 10,444 & 9,984 & 4,878 & 966 & 55 & 8 & 40,074 \\
\hline
\end{tabular}

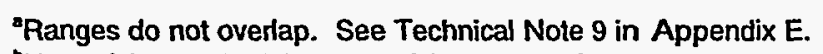

'No enrichment (weight percent) is reported for 430 temporarily discharged BWR assemblies. These BWR assemblies are included in the column total. See Technical Note 10 in Appendix E.

'No enrichment (weight percent) is reported for 137 temporarily discharged PWR assemblies. These PWR assemblies are included in the column total. See Technical Note 10 in Appendix E.

$-=$ Not applicable. GWDHMTU = Gigawattdays thermal per metric ton of uranium.

Note: A total of 2,208 high-temperature, gas-cooled reactor (HTGR) fuel elements, with initial uranium content equal to 24.2 MTU, were discharged. These HTGR fuel elements are not included in the above table. See Technical Note 6 in Appendix $E$

Source: Energy Information Administration, Form RW-859, "Nuclear Fuel Data" (1993). 
Table 12. Fuel Stored in Away-from-reactor Facilities

\begin{tabular}{|c|c|c|c|}
\hline Shipped From & \multicolumn{3}{|c|}{ Stored At } \\
\hline Reactor Name & Storage Facility Name & $\begin{array}{l}\text { Number of } \\
\text { Assemblies }\end{array}$ & $\begin{array}{l}\text { Initial Uranium } \\
\text { Content } \\
\text { (MTU) }\end{array}$ \\
\hline Calvert Cliffs $1 \ldots \ldots \ldots \ldots$ & Washington (Hanford) & 2 & 0.8 \\
\hline Dresden $1 \ldots \ldots \ldots \ldots \ldots$ & General Electric Company (Vallecitos Nuclear) & 1 & 0.1 \\
\hline Dresden $1 \ldots \ldots \ldots \ldots \ldots$ & Idaho National Engineering Laboratory & 2 & 0.2 \\
\hline Dresden $1 \ldots \ldots \ldots \ldots \ldots$ & South Carolina (Savannah River Site) & a 8 & 0.6 \\
\hline Dresden $1 \ldots \ldots \ldots \ldots \ldots$ & West Valley Demonstration Project & ${ }^{b} 683$ & 72.6 \\
\hline Dresden $2 \ldots$ & General Electric Company (Morris Operation) & 753 & 145.0 \\
\hline Blg Rock Point $\ldots \ldots \ldots \ldots \ldots$ & West Valley Demonstration Project & 85 & 11.5 \\
\hline LaCrosse ............ & South Carolina (Savannah River Site) & $a_{1}$ & 0.1 \\
\hline Turkey Point $3 \ldots \ldots \ldots \ldots$ & Idaho National Engineering Laboratory & 18 & 8.2 \\
\hline Cooper Station .... & General Electric Company (Morris Operation) & 1,054 & 198.0 \\
\hline Cooper Station $\ldots \ldots \ldots \ldots$ & Washington (Hanford) & 2 & 0.4 \\
\hline Monticello ................ & General Electric Company (Morris Operation) & 1,058 & 198.2 \\
\hline Humboldt Bay . . . . . . . . . & West Valley Demonstration Project & $c^{\circ} 270$ & 21.3 \\
\hline Peach Bottom 2 .. & Idaho National Engineering Laboratory & 2 & 0.4 \\
\hline Ginna ................... & West Valley Demonstration Project & 40 & 15.3 \\
\hline San Onofre $1 \ldots \ldots \ldots \ldots$ & General Electric Company (Morris Operation) & 270 & 98.4 \\
\hline Surry $1 \ldots \ldots \ldots \ldots \ldots$ & Idaho National Engineering Laboratory & 1 & 0.5 \\
\hline Surry $2 \ldots \ldots \ldots \ldots \ldots \ldots$ & Idaho National Engineering Laboratory & 68 & 31.0 \\
\hline Point Beach $1 \ldots \ldots \ldots \ldots \ldots$ & Idaho National Engineering Laboratory & 6 & 2.4 \\
\hline Point Beach $1 \ldots \ldots \ldots \ldots \ldots$ & Washington (Hanford) & 3 & 1.2 \\
\hline Haddam Neck $\ldots \ldots \ldots \ldots \ldots$ & General Electric Company (Morris Operation) & 82 & 34.5 \\
\hline Haddam Neck $\ldots \ldots \ldots \ldots \ldots$ & Ohio (Battelle) & 1 & 0.4 \\
\hline \multicolumn{2}{|c|}{ Total Assemblies Shipped from Utilities to Storage Facilities $\ldots \ldots \ldots \ldots \ldots \ldots$} & 4,410 & 841.0 \\
\hline \multicolumn{2}{|c|}{ Assemblles Reported on Form RW-859 as Reprocessed ${ }^{d} \ldots \ldots \ldots \ldots \ldots \ldots$} & 962 & 94.7 \\
\hline \multicolumn{2}{|c|}{ Total Fuel Stored in Away-from-reactor Facilities . . . . . . . . . . . . . . . . . . } & 3,448 & 746.3 \\
\hline
\end{tabular}

-All assemblies shipped to South Carolina (Savannah River Site) have been reprocessed.

'Assemblies shipped from Dresden 1 to West Valley Demonstration Project have since been reprocessed.

'Assemblies shipped from Humboldt Bay to West Valley Demonstration Project have since been reprocessed.

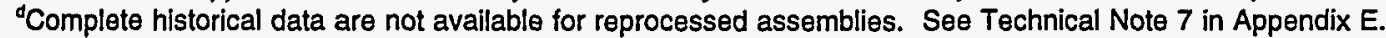

Notes: A total of 744 high-temperature, gas-cooled reactor (HTGR) fuel elements, with initial uranium content equal to 8.8 metric tons of uranium (MTU), were shipped from Fort St. Vrain to Idaho National Engineering Laboratory. These HTGR fuel elements are not included in the above table. See Technical Note 6 in Appendix E. Round-trip shipments are not included in this table. Total may not equal sum of components because of independent rounding. See Technical Note 11 in Appendix E.

Source: Energy Information Administration, Form RW-859, "Nuclear Fuel Data" (1993). 


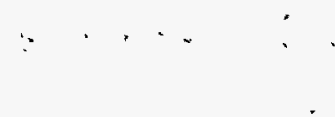




\section{Site Capacities, Inventories, and Dry Storage at Utilities and Storage Facilities}

\section{Site Capacities and Inventories at Utilities}

The total licensed capacity for storage of spent nuclear fuel in the United States as of December 31, 1993, was 210,778 slots (or cells) with each slot capable of holding one assembly (Table 13). The reported maximum capacity was 221,345 including 5,660 dry storage slots, 3,061 off-site slots, and 212,624 on-site slots in storage pools. These totals change slightly as the calculation assumptions change. Footnotes $h$ and $i$ of Table 13 explain the assumptions used in calculating this total.

Respondents to the Form RW-859 were asked to report their maximum established spent fuel storage capacity for each site (in number of assemblies). The definition used by EIA was "the maximum number of intact assemblies that will be able to be stored at some point in the future (between the reporting date and the reactor's end of life) considering any established or current studies or engineering evaluations, at the time of submittal for licensing approval from the U.S. Nuclear Regulatory Commission (NRC)" (see Technical Note 13). The maximum storage capacity can change from year to year as reflected in Table 13 and the pictograms in Appendix C.

Many electric utilities are in the process of reracking their storage pools to achieve a higher maximum capacity; some have inaccessible slots that lower their maximum capacity (Table 13). For example, Wisconsin Electric Company's Point Beach plant storage pool has 36 slots which are currently unusable. Of these slots, 19 contain fuel items which can be moved; 9 are damaged but can be repaired; 6 can be recovered by additional modification of the storage pool; and, 2 will never be used for spent fuel storage. Including the 34 slots which can be rendered useful for storing spent fuel, the Point Beach storage pool has a maximum established storage capacity of 1,500 assemblies. Northern States Power Company's Monticello plant storage pool has a maximum established storage capacity listed as 2,229 assemblies. This number does not include eight slots which will never be used for spent nuclear fuel.

The total initial uranium content of assemblies in storage is 28,136 metric tons of uranium (MTU). The total inventory of light-water reactor (LWR) spent nuclear fuel in storage in the United States as of December 31, 1993, is 98,400 assemblies. Of these, 94,952 assemblies are in storage at 118 reactors that have been or are discharging and/or storing nuclear fuel assemblies, including 1,144 assemblies in the Independent Spent Fuel Storage Installations (ISFSI's) at Virginia Power's Surry plant, Carolina Power and Light Company's Robinson 2 plant, Duke Power Company's Oconee plant, Consumers Power Company's Palisades plant, and Baltimore Gas and Electric Company's Calvert Cliffs plant. An additional 3,448 assemblies are stored at away-from-reactor storage facilities.

\section{Site Capacities and Inventories at Storage Facilities}

More than 99 percent of the assemblies at U.S. away-from-reactor storage facilities are located at General Electric Morris, DOE's Idaho National Engineering Laboratory, and the West Valley Demonstration Project (Table 14). There are 7 assemblies stored at Hanford (Washington), and 1 each at Battelle (Ohio), and Vallecitos (California). Table 15, Figure 5, and Figure 6 list all spent fuel in storage by State. LWR spent nuclear fuel is stored in 34 States. Additionally, high-temperature; gas-cooled reactor (HTGR) spent fuel elements are currently stored in Colorado and Idaho. The data in Table 15 include all permanently discharged assemblies and all temporarily discharged assemblies with uranium content that are scheduled to be reinserted at a later date. The away-from-reactor storage facilities are also included in the State totals. 
Table 13. Site Capacities and Inventories at Nuclear Power Plants as of December 31, 1993

\begin{tabular}{|c|c|c|c|c|c|c|c|c|}
\hline Electric Utility Name & $\begin{array}{l}\text { Reactor/Storage } \\
\text { Site Name }\end{array}$ & $\begin{array}{l}\text { Reactor } \\
\text { Type }\end{array}$ & $\begin{array}{l}\text { Pool } \\
\text { Site } \\
\text { ID }\end{array}$ & $\begin{array}{l}\text { Licensed } \\
\text { Storage } \\
\text { Capacity }\end{array}$ & $\begin{array}{l}\text { Maximum } \\
\text { Established } \\
\text { Storage } \\
\text { Capacity }^{\mathrm{b}}\end{array}$ & $\begin{array}{l}\text { Current } \\
\text { Inventory }\end{array}$ & $\begin{array}{c}\text { Initial Uranium } \\
\text { Content of } \\
\text { Pool Inventory } \\
\text { (MTU) }\end{array}$ & $\begin{array}{l}\text { Number of } \\
\text { Temporarily } \\
\text { Discharged } \\
\text { Assemblies }\end{array}$ \\
\hline Alabama Power Company ... & $\begin{array}{l}\text { Farley } 1 \\
\text { Farley } 2\end{array}$ & $\begin{array}{l}\text { PWR } \\
\text { PWR }\end{array}$ & $\begin{array}{l}0101 \\
0102\end{array}$ & $\begin{array}{l}1,407 \\
1,407\end{array}$ & $\begin{array}{l}1,407 \\
1,407\end{array}$ & $\begin{array}{l}655 \\
558\end{array}$ & $\begin{array}{l}301.8 \\
257.4\end{array}$ & $\begin{array}{l}0 \\
0\end{array}$ \\
\hline $\begin{array}{l}\text { Arizona Public Service } \\
\text { Company } \ldots \ldots \ldots \ldots . .\end{array}$ & $\begin{array}{l}\text { Palo Verde } 1 \\
\text { Palo Verde } 2 \\
\text { Palo Verde } 3\end{array}$ & $\begin{array}{l}\text { PWR } \\
\text { PWR } \\
\text { PWR }\end{array}$ & $\begin{array}{l}0301 \\
0302 \\
0303\end{array}$ & $\begin{array}{l}665 \\
665 \\
665\end{array}$ & $\begin{array}{l}1,323 \\
1,323 \\
1,322\end{array}$ & $\begin{array}{l}368 \\
384 \\
284\end{array}$ & $\begin{array}{l}151.7 \\
156.5 \\
115.9\end{array}$ & $\begin{array}{l}0 \\
0 \\
1\end{array}$ \\
\hline $\begin{array}{l}\text { Arkansas Power and } \\
\text { Light Company } \ldots \ldots \ldots\end{array}$ & $\begin{array}{l}\text { Arkansas Nuclear } 1 \\
\text { Arkansas Nuclear } 2\end{array}$ & $\begin{array}{l}\text { PWR } \\
\text { PWR }\end{array}$ & $\begin{array}{l}0401 \\
0402\end{array}$ & $\begin{array}{l}968 \\
988\end{array}$ & $\begin{array}{l}948 \\
933\end{array}$ & $\begin{array}{l}684 \\
564\end{array}$ & $\begin{array}{l}316.9 \\
234.5\end{array}$ & $\begin{array}{l}0 \\
0\end{array}$ \\
\hline $\begin{array}{l}\text { Baltimore Gas and } \\
\text { Electric Company } \ldots \ldots \ldots\end{array}$ & $\begin{array}{l}\text { Calvert Cliffs } 1 \\
\text { Calvert Cliffs } 2 \\
\text { Dry Storage }\end{array}$ & $\begin{array}{l}\text { PWR } \\
\text { PWR } \\
\text { PWR }\end{array}$ & $\begin{array}{l}0501 \\
0501 D\end{array}$ & $\begin{array}{r}1,830 \\
0 \\
2,880\end{array}$ & $\begin{array}{r}1,778 \\
0 \\
1,152\end{array}$ & $\begin{array}{r}1,450 \\
0 \\
48\end{array}$ & $\begin{array}{r}556.8 \\
0.0 \\
18.0\end{array}$ & $\begin{array}{l}0 \\
0 \\
0\end{array}$ \\
\hline Boston Edison Company .... & Pilgrim 1 & BWR & 0601 & 2,320 & 2,875 & 1,628 & 301.6 & 0 \\
\hline $\begin{array}{l}\text { Carolina Power and Light } \\
\text { Company } \ldots \ldots \ldots \ldots .\end{array}$ & $\begin{array}{l}\text { 'Brunswick } 1 \\
\text { Brunswick } 1 \\
\text { 'Brunswick } 2 \\
\text { Brunswick } 2 \\
\text { 'Harris } 1 \\
{ }^{9} \text { Harris } 1 \\
\text { Robinson } 2 \\
\text { Dry Storage }\end{array}$ & $\begin{array}{l}\text { BWR } \\
\text { 'PWR } \\
\text { BWR } \\
\text { 'PWR } \\
\text { PWR } \\
\text { BWR } \\
\text { PWR } \\
\text { PWR }\end{array}$ & $\begin{array}{l}0701 \\
0701 \\
0702 \\
0702 \\
0703 \\
0703 \\
0705 \\
0705 D\end{array}$ & $\begin{array}{r}1,803 \\
160 \\
1,839 \\
144 \\
4,184 \\
5,808 \\
544 \\
56\end{array}$ & $\begin{array}{r}1,803 \\
160 \\
1,839 \\
144 \\
1,832 \\
2,541 \\
537 \\
56\end{array}$ & $\begin{array}{r}1,146 \\
160 \\
841 \\
144 \\
448 \\
753 \\
240 \\
56\end{array}$ & $\begin{array}{r}211.8 \\
71.3 \\
156.1 \\
65.6 \\
199.3 \\
139.2 \\
102.0 \\
24.1\end{array}$ & $\begin{array}{l}1 \\
0 \\
0 \\
0 \\
0 \\
0 \\
0 \\
0\end{array}$ \\
\hline $\begin{array}{l}\text { Cleveland Electric } \\
\text { Illuminating Company . . . . . }\end{array}$ & Perry 1 & $\begin{array}{l}\text { BWR } \\
\text { BWA }\end{array}$ & $\begin{array}{l}0901 \\
0902\end{array}$ & $\begin{array}{l}2,400 \\
1,620\end{array}$ & $\begin{array}{l}2,400 \\
1,620\end{array}$ & $\begin{array}{r}748 \\
0\end{array}$ & $\begin{array}{r}137.1 \\
0.0\end{array}$ & $\begin{array}{l}4 \\
0\end{array}$ \\
\hline
\end{tabular}

See footnotes at end of table. 
Table 13. Site Capacities and Inventories at Nuclear Power Plants as of December 31, 1993 (Continued)

\begin{tabular}{|c|c|c|c|c|c|c|c|c|}
\hline Electric Utility Name & $\begin{array}{l}\text { Reactor/Storage } \\
\text { Site Name }\end{array}$ & $\begin{array}{l}\text { Reactor } \\
\text { Type }\end{array}$ & $\begin{array}{l}\text { Pool } \\
\text { Site } \\
\text { ID }\end{array}$ & $\begin{array}{l}\text { Licensed } \\
\text { Storage } \\
\text { Capacity }\end{array}$ & $\begin{array}{c}\text { Maximum } \\
\text { Established } \\
\text { Storage } \\
\text { Capacity }^{b}\end{array}$ & $\begin{array}{c}\text { Current } \\
\text { Inventory }\end{array}$ & $\mid \begin{array}{c}\text { Initial Uranium } \\
\text { Content of } \\
\text { Pool Inventory } \\
\text { (MTU) }\end{array}$ & $\begin{array}{l}\text { Number of } \\
\text { Temporarily } \\
\text { Discharged } \\
\text { Assemblies }^{d}\end{array}$ \\
\hline \multicolumn{9}{|l|}{ Commonwealth Edison } \\
\hline Company $\ldots . . .$. & $\begin{array}{l}\text { Braidwood } 1 \\
\text { Braidwood } 2\end{array}$ & $\begin{array}{l}\text { PWR } \\
\text { PWR }\end{array}$ & 1001 & 2,870 & 2,834 & 488 & 207.2 & 0 \\
\hline & $\begin{array}{l}\text { Byron } 1 \\
\text { Byron } 2\end{array}$ & $\begin{array}{l}\text { PWR } \\
\text { PWR }\end{array}$ & 1003 & 2,870 & 2,824 & 772 & 327.7 & 0 \\
\hline & Dresden 1 & BWR & 1005 & 720 & 718 & 683 & 69.5 & 0 \\
\hline & Dresden 2 & BWR & 1006 & 3,537 & 3,537 & 2,162 & 388.0 & 0 \\
\hline & Dresden 3 & BWR & 1007 & 3,537 & 3,537 & 1,968 & 355.5 & 0 \\
\hline & LaSalle County 1 & BWR & 1008 & 5,153 & 8,146 & 2,152 & 395.0 & 0 \\
\hline & LaSalle County 2 & BWR & & 0 & 0 & 0 & 0.0 & 0 \\
\hline & Quad Cities 1 & BWR & 1010 & 7,554 & 7,540 & 4,140 & 761.2 & 0 \\
\hline & Quad Cities 2 & BWR & & 0 & 0 & 0 & 0.0 & 0 \\
\hline & $\begin{array}{l}\text { Zion } 1 \\
\text { Zion } 2\end{array}$ & $\begin{array}{l}\text { PWR } \\
\text { PWR }\end{array}$ & 1012 & 2,112 & 3,012 & 1,684 & 768.9 & 0 \\
\hline \multicolumn{9}{|l|}{ Consolidated Edison } \\
\hline Company of New York ..... & Indian Point 1 & PWR & 1101 & 756 & 756 & 160 & 30.6 & 0 \\
\hline & Indian Point 2 & PWR & 1102 & 1,374 & 1,374 & 756 & 340.7 & 8 \\
\hline \multicolumn{9}{|l|}{ Consumers Power } \\
\hline Company $\ldots \ldots \ldots \ldots$ & Big Rock Point & BWR & 1201 & 441 & 441 & 316 & 41.4 & 0 \\
\hline & Palisades & PWR & 1204 & 896 & 888 & 745 & 297.9 & 1 \\
\hline & Dry Storage & PWR & $1204 \mathrm{D}$ & 600 & 576 & 48 & 18.6 & 0 \\
\hline \multicolumn{9}{|l|}{ Dairyland Power } \\
\hline Cooperative ....... & LaCrosse & BWR & 1301 & 440 & 440 & 333 & 38.0 & 0 \\
\hline Detroit Edison Company .... & Enrico Fermi 2 & BWR & 1402 & 2,383 & 2,383 & 900 & 161.9 & 40 \\
\hline \multirow[t]{8}{*}{ Duke Power Company } & Catawba 1 & PWR & 1501 & 1,419 & 2,615 & 484 & 206.6 & 0 \\
\hline & Catawba 2 & PWR & 1502 & 1,418 & 2,615 & 356 & 151.1 & 0 \\
\hline & McGuire 1 & PWR & 1504 & 1,463 & 1,581 & 583 & 255.9 & 0 \\
\hline & McGuire 2 & PWR & 1505 & 1,463 & 1,460 & 817 & 364.4 & 0 \\
\hline & Oconee 1 & PWR & 1506 & 1,312 & 1,311 & 1,026 & 474.7 & 0 \\
\hline & Oconee 2 & PWR & & & & & & \\
\hline & Oconee 3 & PWR & 1508 & 825 & 818 & 528 & 244.9 & 0 \\
\hline & Dry Storage & PWR & $1506 \mathrm{D}$ & 2,112 & 2,112 & 576 & 267.3 & 0 \\
\hline
\end{tabular}

See footnotes at end of table. 
Table 13. Site Capacities and Inventories at Nuclear Power Plants as of December 31, 1993 (Continued)

\begin{tabular}{|c|c|c|c|c|c|c|c|c|}
\hline Electric Utility Name & $\begin{array}{l}\text { Reactor/Storage } \\
\text { Site Name }\end{array}$ & $\begin{array}{l}\text { Reactor } \\
\text { Type }\end{array}$ & $\begin{array}{l}\text { Pool } \\
\text { Site } \\
\text { ID }\end{array}$ & $\begin{array}{l}\text { Licensed } \\
\text { Storage } \\
\text { Capacity }^{\mathrm{a}}\end{array}$ & $\begin{array}{l}\text { Maximum } \\
\text { Established } \\
\text { Storage } \\
\text { Capacity }^{\mathrm{b}}\end{array}$ & $\begin{array}{c}\text { Current } \\
\text { Inventory }\end{array}$ & $\left|\begin{array}{c}\text { Initial Uranium } \\
\text { Content of } \\
\text { Pool Inventory } \\
\text { (MTU) }\end{array}\right|$ & $\begin{array}{l}\text { Number of } \\
\text { Temporarily } \\
\text { Discharged } \\
\text { Assemblies }^{\mathrm{d}}\end{array}$ \\
\hline Duquesne Light Company ... & $\begin{array}{l}\text { Beaver Valley } 1 \\
\text { Beaver Valley } 2\end{array}$ & $\begin{array}{l}\text { PWR } \\
\text { PWR }\end{array}$ & $\begin{array}{l}1601 \\
1602\end{array}$ & $\begin{array}{r}833 \\
1,088\end{array}$ & $\begin{array}{l}1,621 \\
1,088\end{array}$ & $\begin{array}{l}576 \\
260\end{array}$ & $\begin{array}{l}265.6 \\
120.0\end{array}$ & $\begin{array}{r}13 \\
0\end{array}$ \\
\hline Florida Power Corporation ... & Crystal River 3 & PWR & 1701 & 1,357 & 1,357 & 536 & 248.8 & 5 \\
\hline $\begin{array}{l}\text { Florida Power and Light } \\
\text { Company } \ldots \ldots \ldots \ldots\end{array}$ & $\begin{array}{l}\text { St. Lucie } 1 \\
\text { St. Lucie } 2 \\
\text { Turkey Point } 3 \\
\text { Turkey Point } 4\end{array}$ & $\begin{array}{l}\text { PWR } \\
\text { PWR } \\
\text { PWR } \\
\text { PWR }\end{array}$ & $\begin{array}{l}1801 \\
1802 \\
1803 \\
1804\end{array}$ & $\begin{array}{l}1,706 \\
1,584 \\
1,404 \\
1,404\end{array}$ & $\begin{array}{l}1,705 \\
1,076 \\
1,376 \\
1,376\end{array}$ & $\begin{array}{l}880 \\
464 \\
588 \\
598\end{array}$ & $\begin{array}{l}331.5 \\
175.7 \\
267.7 \\
272.4\end{array}$ & $\begin{array}{l}0 \\
0 \\
0 \\
0\end{array}$ \\
\hline Georgia Power Company & $\begin{array}{l}\text { Hatch } 1 \\
\text { Hatch } 2 \\
\text { Vogtle } 1 \\
\text { Vogtle } 2\end{array}$ & $\begin{array}{l}\text { BWR } \\
\text { BWR } \\
\text { PWR } \\
\text { PWR }\end{array}$ & $\begin{array}{l}2001 \\
2003\end{array}$ & $\begin{array}{r}6,026 \\
0 \\
2,386 \\
0\end{array}$ & $\begin{array}{r}5,830 \\
0 \\
2,283 \\
0\end{array}$ & $\begin{array}{r}3,544 \\
0 \\
565 \\
0\end{array}$ & $\begin{array}{r}653.5 \\
0.0 \\
262.2 \\
0.0\end{array}$ & $\begin{array}{l}0 \\
0 \\
0 \\
0\end{array}$ \\
\hline GPU Nuclear Corporation ... & $\begin{array}{l}\text { Three Mile Island } 1 \\
\text { Oyster Creek }\end{array}$ & $\begin{array}{l}\text { PWR } \\
\text { BWR }\end{array}$ & $\begin{array}{l}1901 \\
1903\end{array}$ & $\begin{array}{r}752 \\
2,600\end{array}$ & $\begin{array}{l}1,284 \\
2,600\end{array}$ & $\begin{array}{r}601 \\
1,876\end{array}$ & $\begin{array}{l}268.2 \\
344.8\end{array}$ & $\begin{array}{r}23 \\
0\end{array}$ \\
\hline $\begin{array}{l}\text { Gulf States Utilities } \\
\text { Company } \ldots \ldots \ldots \ldots\end{array}$ & River Bend 1 & BWR & 2101 & 2,680 & 3,172 & 764 & 141.6 & 0 \\
\hline $\begin{array}{l}\text { Houston Lighting and } \\
\text { Power Company } \ldots \ldots \ldots\end{array}$ & $\begin{array}{l}\text { South Texas } 1 \\
\text { South Texas } 2\end{array}$ & $\begin{array}{l}\text { PWR } \\
\text { PWR }\end{array}$ & $\begin{array}{l}2201 \\
2202\end{array}$ & $\begin{array}{l}1,969 \\
1,969\end{array}$ & $\begin{array}{l}1,958 \\
1,958\end{array}$ & $\begin{array}{l}236 \\
188\end{array}$ & $\begin{array}{l}126.2 \\
101.5\end{array}$ & $\begin{array}{l}7 \\
0\end{array}$ \\
\hline IES Utilities, Inc. $\ldots \ldots \ldots \ldots$ & Duane Amold & BWR & 2401 & 2,050 & 1,898 & 1,280 & 235.3 & 0 \\
\hline Illinois Power Company ..... & Clinton 1 & BWR & 2301 & 2,512 & 2,512 & 724 & 133.2 & 0 \\
\hline $\begin{array}{l}\text { Indiana Michigan } \\
\text { Power Company } \ldots \ldots \ldots \text {. }\end{array}$ & $\begin{array}{l}\text { Cook } 1 \\
\text { Cook } 2\end{array}$ & $\begin{array}{l}\text { PWR } \\
\text { PWR }\end{array}$ & 5801 & 3,613 & 3,613 & 1,523 & 669.7 & 0 \\
\hline $\begin{array}{l}\text { Kansas Gas and Electric } \\
\text { Company } \ldots \ldots \ldots \ldots \ldots\end{array}$ & Wolf Creek 1 & PWR & 2501 & 1,340 & 1,327 & 408 & 188.9 & 0 \\
\hline
\end{tabular}

See footnotes at end of table. 
Table 13. Site Capacities and Inventories at Nuclear Power Plants as of December 31, 1993 (Continued)

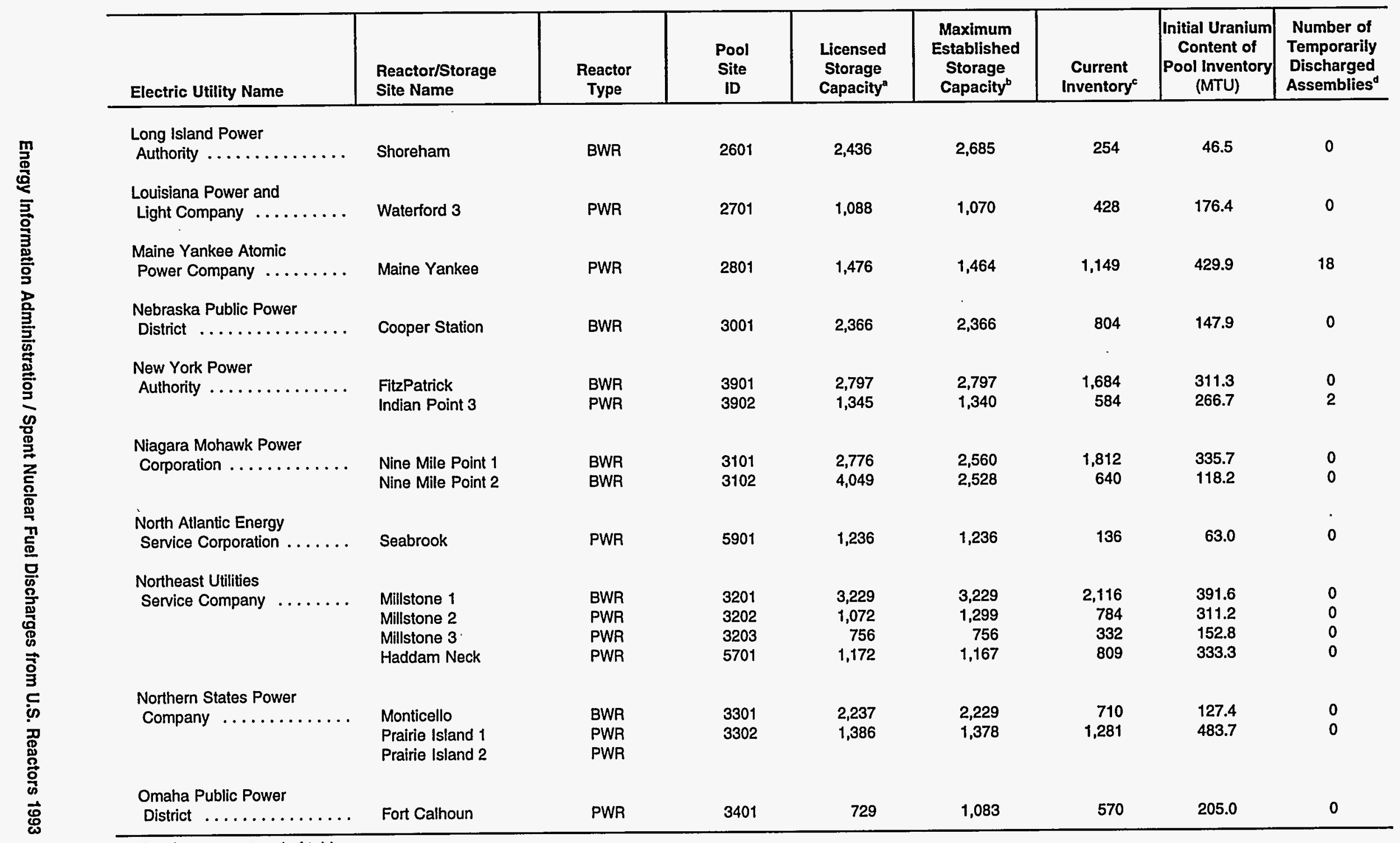

See footnotes at end of table. 
Table 13. Site Capacities and Inventories at Nuclear Power Plants as of December 31, 1993 (Continued)

\begin{tabular}{|c|c|c|c|c|c|c|c|c|}
\hline Electric Utility Name & $\begin{array}{l}\text { Reactor/Storage } \\
\text { Site Name }\end{array}$ & $\begin{array}{l}\text { Reactor } \\
\text { Type }\end{array}$ & $\begin{array}{l}\text { Pool } \\
\text { Site } \\
\text { ID }\end{array}$ & $\begin{array}{l}\text { Licensed } \\
\text { Storage } \\
\text { Capacity }\end{array}$ & $\begin{array}{l}\text { Maximum } \\
\text { Established } \\
\text { Storage } \\
\text { Capacity }\end{array}$ & $\begin{array}{l}\text { Current } \\
\text { Inventory }\end{array}$ & $\begin{array}{c}\text { Initial Uranium } \\
\text { Content of } \\
\text { Pool Inventory } \\
\text { (MTU) }\end{array}$ & $\begin{array}{l}\text { Number of } \\
\text { Temporarily } \\
\text { Discharged } \\
\text { Assemblies }^{d}\end{array}$ \\
\hline $\begin{array}{l}\text { Pacific Gas and Electric } \\
\text { Company } \ldots \ldots \ldots \ldots \ldots\end{array}$ & $\begin{array}{l}\text { Diablo Canyon } 1 \\
\text { Diablo Canyon } 2 \\
\text { Humboldt Bay }\end{array}$ & $\begin{array}{l}\text { PWR } \\
\text { PWR } \\
\text { BWR }\end{array}$ & $\begin{array}{l}3501 \\
3502 \\
3503\end{array}$ & $\begin{array}{r}1,324 \\
1,324 \\
486\end{array}$ & $\begin{array}{r}1,324 \\
1,317 \\
485\end{array}$ & $\begin{array}{l}376 \\
396 \\
390\end{array}$ & $\begin{array}{r}171.9 \\
180.3 \\
28.9\end{array}$ & $\begin{array}{l}0 \\
0 \\
0\end{array}$ \\
\hline PECO Energy Company .... & $\begin{array}{l}\text { Limerick } 1 \\
\text { Limerick } 2 \\
\text { Peach Bottom } 2 \\
\text { Peach Bottom } 3\end{array}$ & $\begin{array}{l}\text { BWR } \\
\text { BWR } \\
\text { BWR } \\
\text { BWR }\end{array}$ & $\begin{array}{l}3701 \\
3704 \\
3705\end{array}$ & $\begin{array}{l}2,040 \\
2,040 \\
3,819 \\
3,819\end{array}$ & $\begin{array}{l}2,766 \\
4,032 \\
3,819 \\
3,814\end{array}$ & $\begin{array}{r}1,300 \\
654 \\
2,162 \\
2,200\end{array}$ & $\begin{array}{l}239.8 \\
121.6 \\
400.4 \\
408.2\end{array}$ & $\begin{array}{r}1306 \\
0 \\
0 \\
0\end{array}$ \\
\hline $\begin{array}{l}\text { Pennsyivania Power and } \\
\text { Light Company } \ldots \ldots \ldots .\end{array}$ & $\begin{array}{l}\text { Susquehanna } 1 \\
\text { Susquehanna } 2\end{array}$ & $\begin{array}{l}\text { BWR } \\
\text { BWR }\end{array}$ & 3601 & $\begin{array}{l}2,840 \\
2,840\end{array}$ & $\begin{array}{l}2,840 \\
2,840\end{array}$ & $\begin{array}{l}1,628 \\
1,236\end{array}$ & $\begin{array}{l}291.0 \\
221.8\end{array}$ & $\begin{array}{l}0 \\
0\end{array}$ \\
\hline $\begin{array}{l}\text { Portland General Electric } \\
\text { Company } \ldots \ldots \ldots \ldots \ldots\end{array}$ & Trojan & PWR & 3801 & 1,408 & 1,395 & 780 & 358.9 & 0 \\
\hline $\begin{array}{l}\text { Public Service Electric } \\
\text { and Gas Company ........ }\end{array}$ & $\begin{array}{l}\text { Hope Creek } \\
\text { Salem } 1 \\
\text { Salem } 2\end{array}$ & $\begin{array}{l}\text { BWR } \\
\text { PWR } \\
\text { PWR }\end{array}$ & $\begin{array}{l}4201 \\
4202 \\
4203\end{array}$ & $\begin{array}{l}4,006 \\
1,170 \\
1,170\end{array}$ & $\begin{array}{l}3,998 \\
1,117 \\
1,139\end{array}$ & $\begin{array}{r}1,008 \\
708 \\
492\end{array}$ & $\begin{array}{l}186.6 \\
316.2 \\
212.0\end{array}$ & $\begin{array}{r}0 \\
23 \\
35\end{array}$ \\
\hline $\begin{array}{l}\text { Rochester Gas and } \\
\text { Electric Corporation ....... }\end{array}$ & Ginna & PWR & 4401 & 1,016 & 1,015 & 689 & 259.9 & 0 \\
\hline $\begin{array}{l}\text { Sacramento Municipal } \\
\text { Utility District } \ldots \ldots \ldots \ldots\end{array}$ & Pancho Seco & PWR & 4501 & 1,080 & 1,080 & 493 & 228.4 & 0 \\
\hline $\begin{array}{l}\text { South Carolina Electric } \\
\text { and Gas Company ........ }\end{array}$ & Summer & PWR & 4601 & 1,276 & 1,276 & 440 & 198.6 & 0 \\
\hline $\begin{array}{l}\text { Southem Califomia } \\
\text { Edison Company ......... }\end{array}$ & $\begin{array}{l}\text { San Onofre } 1 \\
\text { San Onofre } 2 \\
\text { San Onofre } 3\end{array}$ & $\begin{array}{l}\text { PWR } \\
\text { PWR } \\
\text { PWR }\end{array}$ & $\begin{array}{l}4701 \\
4702 \\
4703\end{array}$ & $\begin{array}{r}216 \\
1,542 \\
1,542\end{array}$ & $\begin{array}{r}216 \\
1,542 \\
1,542\end{array}$ & $\begin{array}{l}207 \\
662 \\
710\end{array}$ & $\begin{array}{r}76.4 \\
270.1 \\
287.8\end{array}$ & $\begin{array}{l}0 \\
0 \\
0\end{array}$ \\
\hline
\end{tabular}

See footnotes at end of table. 
Table 13. Site Capacities and Inventories at Nuclear Power Plants as of December 31, 1993 (Continued)

\begin{tabular}{|c|c|c|c|c|c|c|c|c|}
\hline Electric Utility Name & $\begin{array}{l}\text { Reactor/Storage } \\
\text { Site Name }\end{array}$ & $\begin{array}{l}\text { Reactor } \\
\text { Type }\end{array}$ & $\begin{array}{l}\text { Pool } \\
\text { Site } \\
\text { ID }\end{array}$ & $\begin{array}{l}\text { Licensed } \\
\text { Storage } \\
\text { Capacity }^{\mathrm{a}}\end{array}$ & $\begin{array}{l}\text { Maximum } \\
\text { Established } \\
\text { Storage } \\
\text { Capacity }^{b}\end{array}$ & $\begin{array}{c}\text { Current } \\
\text { Inventory }\end{array}$ & $\begin{array}{c}\text { Initial Uranium } \\
\text { Content of } \\
\text { Pool Inventory } \\
\text { (MTU) }\end{array}$ & $\begin{array}{l}\text { Number of } \\
\text { Temporarily } \\
\text { Discharged } \\
\text { Assemblies }^{d}\end{array}$ \\
\hline $\begin{array}{l}\text { System Energy Resources, } \\
\text { Inc. . . . . . . . . . . . . }\end{array}$ & Grand Gulf 1 & BWR & 2901 & 2,324 & 3,972 & 1,660 & 298.5 & 0 \\
\hline $\begin{array}{l}\text { Tennessee Valley } \\
\text { Authority } . \ldots \ldots \ldots \ldots \ldots\end{array}$ & $\begin{array}{l}\text { Browns Ferry } 1 \\
\text { Browns Ferry } 2 \\
\text { Browns Ferry } 3 \\
\text { Sequoyah } 1 \\
\text { Sequoyah } 2 \\
\text { Watts Bar } 1 \\
\text { Watts Bar } 2\end{array}$ & $\begin{array}{l}\text { BWR } \\
\text { BWR } \\
\text { BWR } \\
\text { PWR } \\
\text { PWR } \\
\text { PWR } \\
\text { PWR }\end{array}$ & $\begin{array}{l}4803 \\
4805 \\
4808 \\
\\
4810\end{array}$ & $\begin{array}{l}3,471 \\
3,471 \\
3,471 \\
1,386 \\
\\
1,312\end{array}$ & $\begin{array}{l}3,471 \\
3,471 \\
3,471 \\
2,091 \\
\\
1,294\end{array}$ & $\begin{array}{r}1,580 \\
1,736 \\
1,030 \\
817 \\
\\
0\end{array}$ & $\begin{array}{r}294.0 \\
322.3 \\
191.4 \\
376.4 \\
\\
0.0\end{array}$ & $\begin{array}{r}0 \\
80 \\
0 \\
0 \\
0\end{array}$ \\
\hline Toledo Edison Company .... & Davis-Besse & PWR & 5001 & 735 & 720 & 456 & 211.5 & 6 \\
\hline TU Electric $\ldots \ldots \ldots \ldots$ & $\begin{array}{l}\text { Comanche Peak } 1 \\
\text { Comanche Peak } 2\end{array}$ & $\begin{array}{l}\text { PWR } \\
\text { PWR }\end{array}$ & 4901 & $\begin{array}{l}556 \\
560\end{array}$ & $\begin{array}{l}1,693 \\
1,687\end{array}$ & $\begin{array}{r}205 \\
0\end{array}$ & $\begin{array}{r}94.2 \\
0.0\end{array}$ & $\begin{array}{l}0 \\
0\end{array}$ \\
\hline Union Electric Company .... & Callaway & PWR & 5101 & 1,340 & 1,340 & 548 & 240.1 & 2 \\
\hline $\begin{array}{l}\text { Vermont Yankee Nuclear } \\
\text { Power Corporation ........ }\end{array}$ & Vermont Yankee & BWR & 6001 & 2,870 & 2,860 & 1,978 & 365.9 & 0 \\
\hline Virginia Power $\ldots \ldots \ldots \ldots$ & $\begin{array}{l}\text { North Anna } 1 \\
\text { North Anna } 2 \\
\text { Surry } 1 \\
\text { Surry } 2 \\
\text { Dry Storage }\end{array}$ & $\begin{array}{l}\text { PWR } \\
\text { PWR } \\
\text { PWR } \\
\text { PWR } \\
\text { PWR }\end{array}$ & $\begin{array}{l}5201 \\
5203 \\
52030\end{array}$ & $\begin{array}{l}1,737 \\
1,044 \\
1,764\end{array}$ & $\begin{array}{l}1,677 \\
1,044 \\
1,764\end{array}$ & $\begin{array}{r}1,125 \\
856 \\
416\end{array}$ & $\begin{array}{l}513.4 \\
391.5 \\
188.3\end{array}$ & $\begin{array}{r}25 \\
6 \\
0\end{array}$ \\
\hline $\begin{array}{l}\text { Washington Public Power } \\
\text { Supply System } \ldots \ldots \ldots\end{array}$ & Washington Nuclear 2 & BWR & 5302 & 2,658 & 2,654 & 1,040 & 188.7 & 0 \\
\hline $\begin{array}{l}\text { Wisconsin Electric Power } \\
\text { Company } \ldots \ldots \ldots \ldots \ldots\end{array}$ & $\begin{array}{l}\text { Point Beach } 1 \\
\text { Point Beach } 2\end{array}$ & $\begin{array}{l}\text { PWR } \\
\text { PWR }\end{array}$ & 5401 & 1,502 & 1,500 & 1,249 & 485.7 & 0 \\
\hline
\end{tabular}

See footnotes at end of table. 
Table 13. Site Capacities and Inventories at Nuclear Power Plants as of December 31, 1993 (Continued)

\begin{tabular}{|c|c|c|c|c|c|c|c|c|}
\hline Electric Utility Name & $\begin{array}{l}\text { Reactor/Storage } \\
\text { Site Name }\end{array}$ & $\begin{array}{l}\text { Reactor } \\
\text { Type }\end{array}$ & $\begin{array}{l}\text { Pool } \\
\text { Site } \\
\text { ID }\end{array}$ & $\begin{array}{l}\text { Licensed } \\
\text { Storage } \\
\text { Capacity }\end{array}$ & $\begin{array}{l}\text { Maximum } \\
\text { Established } \\
\text { Storage } \\
\text { Capacity }^{\mathrm{b}}\end{array}$ & $\begin{array}{c}\text { Current } \\
\text { Inventoryc }\end{array}$ & $\begin{array}{c}\text { Initial Uranium } \\
\text { Content of } \\
\text { Pool Inventory } \\
\text { (MTU) }\end{array}$ & $\begin{array}{l}\text { Number of } \\
\text { Temporarily } \\
\text { Discharged } \\
\text { Assemblies }\end{array}$ \\
\hline \multicolumn{9}{|l|}{ Wisconsin Public Service } \\
\hline Corporation ........... & Kewaunee & PWR & 5501 & 990 & 990 & 652 & 249.8 & 4 \\
\hline \multicolumn{9}{|l|}{ Yankee Atomic Electric } \\
\hline Company $\ldots \ldots \ldots \ldots \ldots$ & Yankee Rowe & PWR & 5601 & 721 & 721 & 533 & 127.2 & 0 \\
\hline \multicolumn{4}{|c|}{ Total (Nuclear Power Plant Sites Only) $\ldots \ldots \ldots \ldots \ldots \ldots \ldots \ldots \ldots \ldots$} & $\mathrm{h}_{207,717}$ & $h_{218,284}$ & 94,952 & $27,389.7$ & 610 \\
\hline \multicolumn{4}{|c|}{ Away-from-reactor Storage Facilities (From Table 14) } & 3,061 & 3,061 & 3,448 & 746.3 & 0 \\
\hline \multicolumn{4}{|c|}{ Total (Including Away-From-Reactor Storage Facilities) $\ldots \ldots \ldots \ldots \ldots \ldots \ldots \ldots$} & 210,778 & '221,345 & 98,400 & $28,136.0$ & 610 \\
\hline
\end{tabular}

"Licensed Storage Capacity is the maximum number of spent nuclear fuel assemblies and canisters to be stored at a given site or nuclear fuel pool, as ficensed by the Nuclear Regulatory Commission.

'Maximum Established Storage Capacity is the maximum established spent fuel capacity for the site defined by the DOE as the maximum number of intact assemblies that will be able to be stored at some point in the future (between the reporting date and the reactor's end of life) taking into account any established or current studies or engineering evaluations, at the time of submittal for licensing approval from the NRC.

'Current Inventory is the number of spent nuclear fuel assemblies stored at a given site or spent nuclear fuel pool.

'Temporarily discharged assemblies are included in the current inventory column.

${ }^{\circ}$ Brunswick 1 and 2 are BWR reactors with the capability of storing both BWR and PWR fuel assemblies in their storage pools.

The current inventory of assemblies stored in the PWR portion of Brunswick pools came from the Robinson 2 reactor.

'Harris 1 is a PWR reactor with the capability of storing both PWR and BWR fuel assemblies in its storage pools, based on future needs. The pools are licensed to hold up to 4,184 PWR or 5,808 BWR assemblies.

'Licensed Storage Capacity total and Maximum Established Storage Capacity total assume that Carolina Power and Light Company's Harris 1 site (capable of storing PWR and BWR fuel assemblies), will store BWR assemblies only. Totals assuming PWR assemblies only are 206,093 licensed storage capacity and 217,575 maximum established storage capacity.

'Licensed Storage Capacity total and Maximum Established Storage Capacity total assume that Carolina Power and Light Company's Harris 1 site and General Electric Company's Morris Operation site (both capable of storing PWR and BWR fuel assemblies) will store BWR assemblies only. Totals assuming PWR assemblies only are 206,606 licensed storage capacity and 218,088 maximum established storage capacity.

'A total of 306 temporarily discharged assemblies were shipped from Long Island Power Authority's Shoreham plant to Limerick 1 . See Technical Note 14 in Appendix E.

MTU = Metric tons of uranium; PWR = Pressurized-water reactor; BWR $=$ Boiling-water reactor.

Note: Totals may not equal sum of components because of independent rounding. See Technical Note 11 in Appendix E.

Source: Energy Information Administration, Form RW-859, "Nuclear Fuel Data" (1993). 
Table 14. Site Capacities and Inventories at Away-from-reactor Storage Facilities as of December 31, 1993

\begin{tabular}{|c|c|c|c|c|c|c|c|c|}
\hline Storage Facility & $\begin{array}{c}\text { Contributing } \\
\text { Reactor }\end{array}$ & $\begin{array}{c}\text { Reactor } \\
\text { ID }\end{array}$ & $\begin{array}{c}\text { Reactor } \\
\text { Type }\end{array}$ & $\begin{array}{l}\text { Pool } \\
\text { Site } \\
\text { ID }\end{array}$ & $\begin{array}{l}\text { Licensed } \\
\text { Storage } \\
\text { Capacity }\end{array}$ & $\begin{array}{l}\text { Maximum } \\
\text { Established } \\
\text { Storage } \\
\text { Capacity" }\end{array}$ & $\begin{array}{c}\text { Current } \\
\text { Inventory" }\end{array}$ & $\begin{array}{c}\text { Initial Uranium } \\
\text { Content of } \\
\text { Pool Inventory } \\
\text { (MTU) } \\
\end{array}$ \\
\hline $\begin{array}{l}\text { Babcock \& Wilcox } \\
\text { Company (Lynchburg) ......... }\end{array}$ & & $\overline{-}$ & $\begin{array}{l}\text { PWR } \\
\text { BWR }\end{array}$ & 7101 & $\begin{array}{l}4 \\
4\end{array}$ & $\begin{array}{l}4 \\
4\end{array}$ & $\begin{array}{l}0 \\
0\end{array}$ & $\begin{array}{l}0.0 \\
0.0\end{array}$ \\
\hline $\begin{array}{l}\text { General Electric Company } \\
\text { (Morris Operation) } \ldots \ldots \ldots \ldots \ldots \\
\text { Subtotal } \ldots \ldots \ldots \ldots \ldots \ldots \ldots\end{array}$ & $\begin{array}{l}\text { San Onofre } 1 \\
\text { Haddam Neck } \\
\text { Dresden } 2 \\
\text { Cooper Station } \\
\text { Monticello }\end{array}$ & $\begin{array}{l}4701 \\
5701 \\
1006 \\
3001 \\
3301\end{array}$ & $\begin{array}{l}\text { PWR } \\
\text { PWR } \\
\text { BWR } \\
\text { BWR } \\
\text { BWR }\end{array}$ & 6601 & $\begin{array}{r}{ }^{3} 380 \\
{ }^{3}, 928\end{array}$ & $\begin{array}{r}380 \\
2,928\end{array}$ & $\begin{array}{r}270 \\
82 \\
753 \\
1,054 \\
1,058 \\
3,217\end{array}$ & $\begin{array}{r}98.4 \\
34.5 \\
145.0 \\
198.0 \\
198.2 \\
674.1\end{array}$ \\
\hline $\begin{array}{l}\text { General Electric Company } \\
\text { (Vallecitos Nuclear)..... }\end{array}$ & Dresden 1 & 1005 & BWR & 6201 & & & 1 & 0.1 \\
\hline $\begin{array}{l}\text { Idaho National } \\
\text { Engineering Laboratory }{ }^{c} \ldots \ldots \ldots \ldots \\
\text { Subtotal } \ldots \ldots \ldots \ldots \ldots \ldots \ldots\end{array}$ & $\begin{array}{l}\text { Turkey Point } 3 \\
\text { ¿ Surry } 1 \\
\text { dSurry } 2 \\
\text { Point Beach } 1 \\
\text { Dresden } 1 \\
\text { Peach Bottom } 2\end{array}$ & $\begin{array}{l}1803 \\
5203 \\
5204 \\
5401 \\
1005 \\
3704\end{array}$ & $\begin{array}{l}\text { PWR } \\
\text { PWR } \\
\text { PWR } \\
\text { PWR } \\
\text { BWR } \\
\text { BWR }\end{array}$ & 7002 & $\begin{array}{l}-: \\
- \\
- \\
-\end{array}$ & $\begin{array}{l}- \\
- \\
- \\
-\end{array}$ & $\begin{array}{r}18 \\
1 \\
68 \\
6 \\
2 \\
2 \\
97\end{array}$ & $\begin{array}{r}8.2 \\
0.5 \\
31.0 \\
2.4 \\
0.2 \\
0.4 \\
42.6\end{array}$ \\
\hline Ohio (Battelle) ${ }^{c} \ldots \ldots \ldots \ldots \ldots$ & Haddam Neck & 5701 & PWR & 7006 & -- & - & 1 & 0.4 \\
\hline $\begin{array}{l}\text { Washington }\left(\text { Hanford) }{ }^{c} \ldots \ldots \ldots \ldots\right. \\
\text { Subtotal } \ldots \ldots \ldots \ldots \ldots \ldots\end{array}$ & $\begin{array}{l}\text { Calvert Cliffs } 1 \\
\text { Point Beach } 1 \\
\text { Cooper Station }\end{array}$ & $\begin{array}{l}0501 \\
5401 \\
3001\end{array}$ & $\begin{array}{l}\text { PWR } \\
\text { PWR } \\
\text { BWR }\end{array}$ & 7007 & $\begin{array}{l}- \\
--\end{array}$ & $\begin{array}{l}-- \\
--\end{array}$ & $\begin{array}{l}2 \\
3 \\
2 \\
7\end{array}$ & $\begin{array}{l}0.8 \\
1.2 \\
0.4 \\
2.4\end{array}$ \\
\hline $\begin{array}{l}\text { West Valley } \\
\text { Demonstration Project } \ldots \ldots \ldots \ldots \\
\text { Subtotal } \ldots \ldots \ldots \ldots \ldots \ldots \ldots\end{array}$ & $\begin{array}{l}\text { Ginna } \\
\text { Big Rock Point }\end{array}$ & $\begin{array}{l}4401 \\
1201\end{array}$ & $\begin{array}{l}\text { PWR } \\
\text { BWR }\end{array}$ & 7005 & $\begin{array}{l}\circ 40 \\
\circ 85\end{array}$ & $\begin{array}{l}40 \\
85\end{array}$ & $\begin{array}{r}40 \\
85 \\
125\end{array}$ & $\begin{array}{l}15.3 \\
11.5 \\
26.8\end{array}$ \\
\hline Total $\ldots \ldots \ldots \ldots \ldots \ldots \ldots \ldots$ & $\ldots, \ldots, \cdots, \cdots$ & $\ldots \ldots$ & ...... & ........ & 3,061 & $' 3,061$ & 3,448 & 746.3 \\
\hline
\end{tabular}

aIn number of assemblies (see Glossary for definition).

'General Electric Company (Morris Operation) is not licensed to receive any more assemblies.

These storage facilities are not surveyed by Form RW-859. The current inventory data for these facilities are shipments to these facilities which were reported by the individual utilities on Form RW-859. See Technical Note 2 in Appendix E. Licensed and maximum established storage capacity data are not applicable to these facilities.

'Surry 1 and 2 spent fuel stored at Idaho National Engineering Laboratory is in dry storage.

West Valley Demonstration Project is not licensed to receive any more assemblies.

'Licensed Storage Capacity total and Maximum Established Storage Capacity total assume General Electric Company's Morris Operation site (capable of storing PWR and BWR fuel assemblies), will store BWR assemblies only. Totals assuming PWR assemblies only are 513 licensed storage capacity and 513 maximum established storage capacity.

MTU $=$ Metric tons of uranium. $\cdots=$ Not applicable. PWR $=$ Pressurized-water reactor; BWR $=$ Boiling-water reactor.

Note: Totals may not equal sum of components because of independent rounding. See Technical Note 11 in Appendix $E$.

Source: Energy Information Administration, Form RW-859, "Nuclear Fuel Data" (1993). 
Table 15. Spent Fuel in Storage by State and Storage Site

\begin{tabular}{|c|c|c|c|c|c|c|c|}
\hline State & Storage Site & $\begin{array}{c}\text { BWR } \\
\text { Assemblies } \\
\end{array}$ & $\begin{array}{c}\text { BWR } \\
\text { Initial Uranium } \\
\text { Content } \\
\text { (MTU) }\end{array}$ & $\begin{array}{c}\text { PWR } \\
\text { Assemblies }\end{array}$ & $\begin{array}{c}\text { PWR } \\
\text { Initial Uranium } \\
\text { Content } \\
\text { (MTU) }\end{array}$ & $\begin{array}{c}\text { Total } \\
\text { Stored } \\
\text { Assemblies }\end{array}$ & $\begin{array}{l}\text { Total } \\
\text { Initial Uranium } \\
\text { Content } \\
\text { (MTU) }\end{array}$ \\
\hline \multirow[t]{4}{*}{ Alabama ... } & Browns Ferry 1 & 3,316 & 616.3 & 0 & 0.0 & 3,316 & 616.3 \\
\hline & Browns Ferry 3 & 1,030 & 191.4 & 0 & 0.0 & 1,030 & 191.4 \\
\hline & Farley 1 & 0 & 0.0 & 655 & 301.8 & 655 & 301.8 \\
\hline & Farley 2 & 0 & 0.0 & 558 & 257.4 & 558 & 257.4 \\
\hline Alabama ..... & Total & 4,346 & 807.8 & 1,213 & 559.2 & 5,559 & $1,367.0$ \\
\hline \multirow[t]{3}{*}{ Arizona } & Palo Verde 1 & 0 & 0.0 & 368 & 151.7 & 368 & 151.7 \\
\hline & Palo Verde 2 & 0 & 0.0 & 384 & 156.5 & 384 & 156.5 \\
\hline & Palo Verde 3 & 0 & 0.0 & 284 & 115.9 & 284 & 115.9 \\
\hline Arizona ..... & Total & 0 & 0.0 & 1,036 & 424.2 & 1,036 & 424.2 \\
\hline \multirow[t]{2}{*}{ Arkansas } & Arkansas Nuclear 1 & 0 & 0.0 & 684 & 316.9 & 684 & 316.9 \\
\hline & Arkansas Nuclear 2 & 0 & 0.0 & 564 & 234.5 & 564 & 234.5 \\
\hline Arkansas ..... & Total & 0 & 0.0 & 1,248 & 551.4 & 1,248 & 551.4 \\
\hline \multirow[t]{8}{*}{ California } & Diablo Canyon 1 & 0 & 0.0 & 376 & 171.9 & 376 & 171.9 \\
\hline & Diablo Canyon 2 & 0 & 0.0 & 396 & 180.3 & 396 & 180.3 \\
\hline & Humboldt Bay & 390 & 28.9 & 0 & 0.0 & 390 & 28.9 \\
\hline & Rancho Seco & 0 & 0.0 & 493 & 228.4 & 493 & 228.4 \\
\hline & San Onofre 1 & 0 & 0.0 & 207 & 76.4 & 207 & 76.4 \\
\hline & San Onofre 2 & 0 & 0.0 & 662 & 270.1 & 662 & 270.1 \\
\hline & San Onofre 3 & 0 & 0.0 & 710 & 287.8 & 710 & 287.8 \\
\hline & Vallecitos & 1 & 0.1 & 0 & 0.0 & 1 & 0.1 \\
\hline California ..... & Total & 391 & 29.0 & 2,844 & $1,214.9$ & 3,235 & $1,243.9$ \\
\hline Colorado ...... & Fort St. Vrain & 0 & 0.0 & 0 & 0.0 & ${ }^{a} 1,464$ & ${ }^{\mathrm{a}} 15.4$ \\
\hline Colorado ..... & Total & 0 & 0.0 & 0 & 0.0 & 1,464 & 15.4 \\
\hline \multirow[t]{4}{*}{ Connecticut } & Haddam Neck & 0 & 0.0 & 809 & 333.3 & 809 & 333.3 \\
\hline & Millstone 1 & 2,116 & 391.6 & 0 & 0.0 & 2,116 & 391.6 \\
\hline & Millstone 2 & 0 & 0.0 & 784 & 311.2 & 784 & 311.2 \\
\hline & Millstone 3 & 0 & 0.0 & 332 & 152.8 & 332 & 152.8 \\
\hline Connecticut ... & Total & 2,116 & 391.6 & 1,925 & 797.3 & 4,041 & $1,188.9$ \\
\hline \multirow[t]{5}{*}{ Florida . } & Crystal River 3 & 0 & 0.0 & 536 & 248.8 & 536 & 248.8 \\
\hline & St. Lucie 1 & 0 & 0.0 & 880 & 331.5 & 880 & 331.5 \\
\hline & St. Lucie 2 & 0 & 0.0 & 464 & 175.7 & 464 & 175.7 \\
\hline & Turkey Point 3 & 0 & 0.0 & 588 & 267.7 & 588 & 267.7 \\
\hline & Turkey Point 4 & 0 & 0.0 & 598 & 272.4 & 598 & 272.4 \\
\hline Florida . . . . . & Total & 0 & 0.0 & 3,066 & $1,296.0$ & 3,066 & $1,296.0$ \\
\hline \multirow[t]{2}{*}{ Georgia . . } & Hatch 1 & 3,544 & 653.5 & 0 & 0.0 & 3,544 & 653.5 \\
\hline & Vogtle 1 & 0 & 0.0 & 565 & 262.2 & 565 & 262.2 \\
\hline Georgia ..... & Total & 3,544 & 653.5 & 565 & 262.2 & 4,109 & 915.6 \\
\hline \multirow{2}{*}{\multicolumn{2}{|c|}{$\begin{array}{l}\text { Idaho } \ldots \ldots \ldots \text { Idaho National } \\
\text { Idaho ....... Total }\end{array}$}} & 4 & 0.6 & 93 & 42.0 & '841 & $b_{51.4}$ \\
\hline & & 4 & 0.6 & 93 & 42.0 & '841 & $b_{51.4}$ \\
\hline
\end{tabular}

See footnotes at end of table. 
Table 15. Spent Fuel in Storage by State and Storage Site (Continued)

\begin{tabular}{|c|c|c|c|c|c|c|c|}
\hline State & Storage Site & $\begin{array}{c} \\
\text { BWR } \\
\text { Assemblies }\end{array}$ & $\begin{array}{c}\text { BWR } \\
\text { Initial Uranium } \\
\text { Content } \\
\text { (MTU) }\end{array}$ & $\begin{array}{c}\text { PWR } \\
\text { Assemblies }\end{array}$ & \begin{tabular}{|c|} 
PWR \\
Initial Uranium \\
Content \\
(MTU)
\end{tabular} & \begin{tabular}{|c|} 
Total \\
Stored \\
Assemblies
\end{tabular} & $\begin{array}{c}\text { Total } \\
\text { Initial Uranium } \\
\text { Content } \\
\text { (MTU) }\end{array}$ \\
\hline \multirow[t]{10}{*}{ Illinols } & Braidwood 1 & 0 & 0.0 & 488 & 207.2 & 488 & 207.2 \\
\hline & Byron 1 & 0 & 0.0 & 772 & 327.7 & 772 & 327.7 \\
\hline & Clinton 1 & 724 & 133.2 & 0 & 0.0 & 724 & 133.2 \\
\hline & Dresden 1 & 683 & 69.5 & 0 & 0.0 & 683 & 69.5 \\
\hline & Dresden 2 & 2,162 & 388.0 & 0 & 0.0 & 2,162 & 388.0 \\
\hline & Dresden 3 & 1,968 & 355.5 & 0 & 0.0 & 1,968 & 355.5 \\
\hline & LaSalle County 1 & 2,152 & 395.0 & 0 & 0.0 & 2,152 & 395.0 \\
\hline & Morris & 2,865 & 541.2 & 352 & 132.9 & 3,217 & 674.1 \\
\hline & Quad Cities 1 & 4,140 & 761.2 & 0 & 0.0 & 4,140 & 761.2 \\
\hline & Zion 1 & 0 & 0.0 & 1,684 & 768.9 & 1,684 & 768.9 \\
\hline Illinois ....... & Total & 14,694 & $2,643.6$ & 3,296 & $1,436.7$ & 17,990 & $4,080.3$ \\
\hline lowa $\ldots . . .$. & Duane Arnold & 1,280 & 235.3 & 0 & 0.0 & 1,280 & 235.3 \\
\hline lowa........ & Total & 1,280 & 235.3 & 0 & 0.0 & 1,280 & 235.3 \\
\hline Kansas ....... & Wolf Creek 1 & 0 & 0.0 & 408 & 188.9 & 408 & 188.9 \\
\hline Kansas ....... & Total & 0 & 0.0 & 408 & 188.9 & 408 & 188.9 \\
\hline \multirow[t]{2}{*}{ Louisiana ...... } & River Bend 1 & 764 & 141.6 & 0 & 0.0 & 764 & 141.6 \\
\hline & Waterford 3 & 0 & 0.0 & 428 & 176.4 & 428 & 176.4 \\
\hline Louisiana . . . . & Total & 764 & 141.6 & 428 & 176.4 & 1,192 & 317.9 \\
\hline \multirow{2}{*}{ 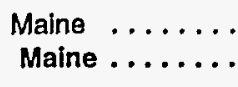 } & Maine Yankee & 0 & 0.0 & 1,149 & 429.9 & 1,149 & 429.9 \\
\hline & Total & 0 & 0.0 & 1,149 & 429.9 & 1,149 & 429.9 \\
\hline \multirow[t]{2}{*}{ Maryland } & Calvert Cliffs 1 & 0 & 0.0 & 1,450 & 556.8 & 1,450 & 556.8 \\
\hline & Calvert Cliffs 1 Dry & 0 & 0.0 & 48 & 18.0 & 48 & 18.0 \\
\hline Maryland .... & Total & 0 & 0.0 & 1,498 & 574.8 & 1,498 & 574.8 \\
\hline \multirow{2}{*}{ Massachusetts } & Pilgrim 1 & 1,628 & 301.6 & 0 & 0.0 & 1,628 & 301.6 \\
\hline & Yankee Rowe & 0 & 0.0 & 533 & 127.2 & 533 & 127.2 \\
\hline Massachusetts . & Total & 1,628 & 301.6 & 533 & 127.2 & 2,161 & 428.8 \\
\hline \multirow[t]{5}{*}{ Michigan } & Big Rock Point & 316 & 41.4 & 0 & 0.0 & 316 & 41.4 \\
\hline & Cook 1 & 0 & 0.0 & 1,523 & 669.7 & 1,523 & 669.7 \\
\hline & Enrico Fermi 2 & 900 & 161.9 & 0 & 0.0 & 900 & 161.9 \\
\hline & Palisades & 0 & 0.0 & 745 & 297.9 & 745 & 297.9 \\
\hline & Palisades Dry & 0 & 0.0 & 48 & 18.6 & 48 & 18.6 \\
\hline Michigan ..... & Total & 1,216 & 203.3 & 2,316 & 986.2 & 3,532 & $1,189.5$ \\
\hline \multirow[t]{2}{*}{ Minnesota } & Monticello & 710 & 127.4 & 0 & 0.0 & 710 & 127.4 \\
\hline & Prairie Island 1 & 0 & 0.0 & 1,281 & 483.7 & 1,281 & 483.7 \\
\hline Minnesota .... & Total & 710 & 127.4 & 1,281 & 483.7 & 1,991 & 611.2 \\
\hline \multirow{2}{*}{$\begin{array}{r}\text { Mississippl ..... } \\
\text { Mississippi . . . }\end{array}$} & Grand Gulf 1 & 1,660 & 298.5 & 0 & 0.0 & 1,660 & 298.5 \\
\hline & Total & 1,660 & 298.5 & 0 & 0.0 & 1,660 & 298.5 \\
\hline \multirow{2}{*}{$\begin{array}{l}\text { Missouri ... . . . } \\
\text { Missouri . . . . }\end{array}$} & Callaway & 0 & 0.0 & 548 & 240.1 & 548 & 240.1 \\
\hline & Total & 0 & 0.0 & 548 & 240.1 & 548 & 240.1 \\
\hline \multirow{2}{*}{ Nebraska ...... } & Cooper Station & 804 & 147.9 & 0 & 0.0 & 804 & 147.9 \\
\hline & Fort Calhoun & 0 & 0.0 & 570 & 205.0 & 570 & 205.0 \\
\hline Nebraska ..... & Total & 804 & 147.9 & 570 & 205.0 & 1,374 & 353.0 \\
\hline \multirow{2}{*}{$\begin{array}{l}\text { New Hampshire } \\
\text { New Hampshire }\end{array}$} & Seabrook & 0 & 0.0 & 136 & 63.0 & 136 & 63.0 \\
\hline & Total & 0 & 0.0 & 136 & 63.0 & 136 & 63.0 \\
\hline
\end{tabular}

See footnotes at end of table. 
Table 15. Spent Fuel in Storage by State and Storage Site (Continued)

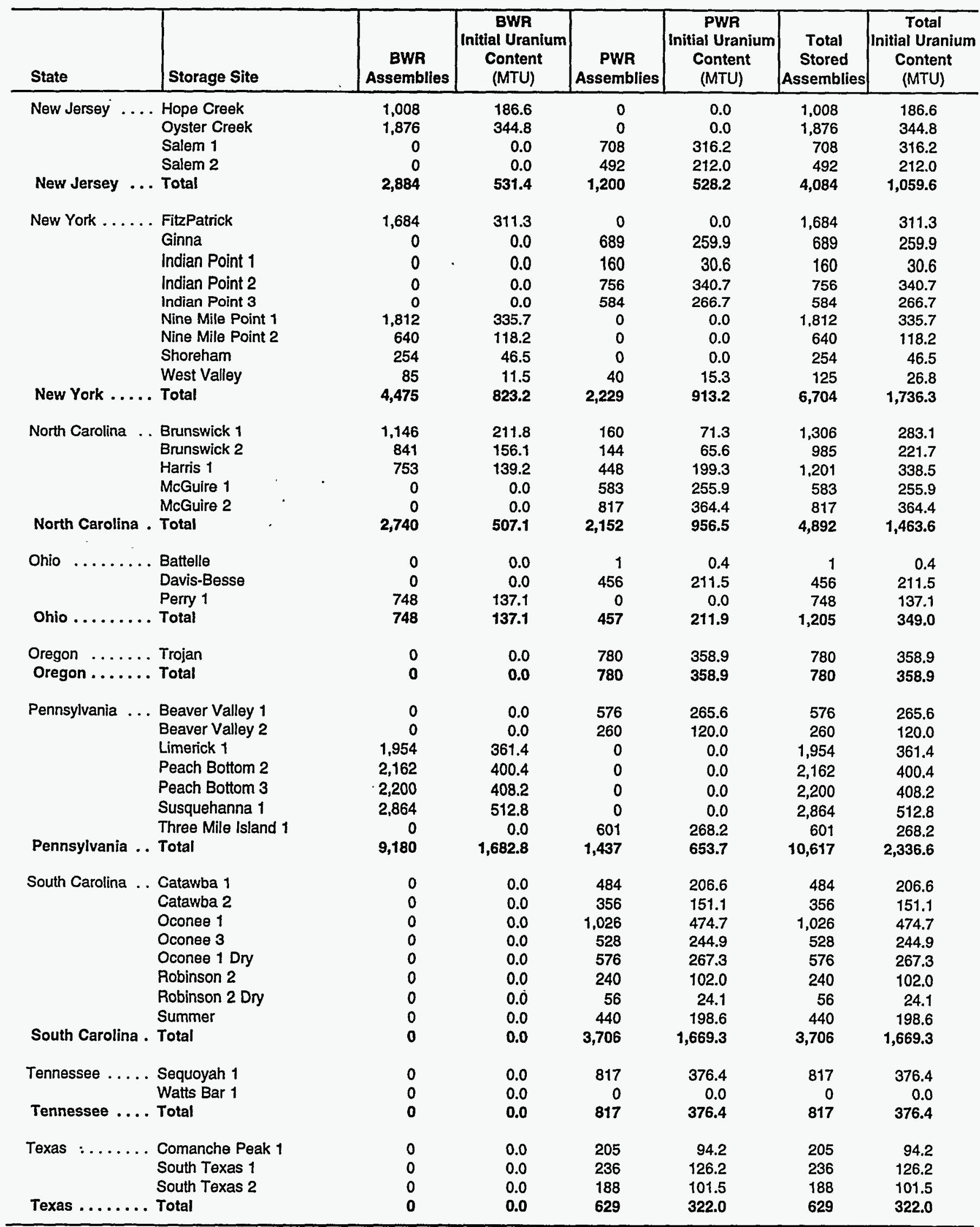

See footnotes at end of table. 
Table 15. Spent Fuel in Storage by State and Storage Site (Conntinued)

\begin{tabular}{|c|c|c|c|c|c|c|c|}
\hline State & Storage Site & $\begin{array}{c}\text { BWR } \\
\text { Assemblies }\end{array}$ & \begin{tabular}{|c|} 
BWR \\
Initial Uranium \\
Content \\
(MTU)
\end{tabular} & \begin{tabular}{|c|} 
PWR \\
Assemblies \\
\end{tabular} & $\begin{array}{c}\text { PWR } \\
\text { Initial Uranium } \\
\text { Content } \\
\text { (MTU) }\end{array}$ & $\begin{array}{c}\text { Total } \\
\text { Stored } \\
\text { Assemblies }\end{array}$ & $\begin{array}{l}\text { Total : } \\
\text { Initial Uranium } \\
\text { Content } \\
\text { (MTU) }\end{array}$ \\
\hline $\begin{array}{l}\text { Vermont } \ldots \ldots \\
\text { Vermont } \ldots \ldots\end{array}$ & $\begin{array}{l}\text { Vermont Yankee } \\
\text { Total }\end{array}$ & $\begin{array}{l}1,978 \\
1,978\end{array}$ & $\begin{array}{l}365.9 \\
365.9\end{array}$ & $\begin{array}{l}0 \\
0\end{array}$ & $\begin{array}{l}0.0 \\
0.0\end{array}$ & $\begin{array}{l}1,978 \\
1,978\end{array}$ & $\begin{array}{l}365.9 \\
365.9\end{array}$ \\
\hline $\begin{array}{l}\text { Virginia } \ldots \ldots \\
\text { Virginia } \ldots \ldots\end{array}$ & $\begin{array}{l}\text { North Anna } 1 \\
\text { Surry } 1 \\
\text { Surry } 1 \text { Dry } \\
\text { Total }\end{array}$ & $\begin{array}{l}0 \\
0 \\
0 \\
0\end{array}$ & $\begin{array}{l}0.0 \\
0.0 \\
0.0 \\
0.0\end{array}$ & $\begin{array}{r}1,125 \\
856 \\
416 \\
2,397\end{array}$ & $\begin{array}{r}513.4 \\
391.5 \\
188.3 \\
1,093.2\end{array}$ & $\begin{array}{r}1,125 \\
856 \\
.416 \\
23973\end{array}$ & $\begin{array}{r}513.4 \\
391.5 \\
188.3 \\
1,093.2\end{array}$ \\
\hline $\begin{array}{l}\text { Washington .... } \\
\text { Washington ... }\end{array}$ & $\begin{array}{l}\text { Hanford } \\
\text { Washington Nuclear } 2 \\
\text { Total }\end{array}$ & $\begin{array}{r}2 \\
1,040 \\
1,042\end{array}$ & $\begin{array}{r}0.4 \\
188.7 \\
189.1\end{array}$ & $\begin{array}{l}5 \\
0 \\
5\end{array}$ & $\begin{array}{l}2.0 \\
0.0 \\
2.0\end{array}$ & $\begin{array}{r}: 7 \\
1,040 \\
1,047\end{array}$ & $\begin{array}{r}2.4 \\
188.7 \\
191.0\end{array}$ \\
\hline $\begin{array}{l}\text { Wisconsin } \ldots . \\
\text { Wisconsin .... }\end{array}$ & $\begin{array}{l}\text { Kewaunee } \\
\text { LaCrosse } \\
\text { Point Beach } 1 \\
\text { Total }\end{array}$ & $\begin{array}{r}0 \\
333 \\
0 \\
333\end{array}$ & $\begin{array}{r}0.0 \\
38.0 \\
0.0 \\
38.0\end{array}$ & $\begin{array}{r}652 \\
0 \\
1,249 \\
1,901\end{array}$ & $\begin{array}{r}249.8 \\
0.0 \\
485.7 \\
735.5\end{array}$ & $\begin{array}{r}652 \\
333 \\
1,249 \\
2,234\end{array}$ & $\begin{array}{r}249.8 \\
38.0 \\
485.7 \\
773.4\end{array}$ \\
\hline Total ....... & & 56,537 & $10,256.2$ & 41,863 & $17,879.9$ & 98,400 & $28,136.0$ \\
\hline
\end{tabular}

These 1,464 high-temperature, gas-cooled reactor (HTGR) fuel elements are not included in the above table totals. See Technical Note- 6 in Appendix E.

${ }^{b} \mathrm{~A}$ total of $744 \mathrm{HTGR}$ fuel elements, with initial uranium content equal to 8.8 metric tons of uranium (MTU), were discharged. These HTGR fuel elements are not included in the above table totals. See Technical Note 6 in Appendix $E$.

BWR = Boiling-water reactor; PWR = Pressurized-water reactor; MTU = Metric tons of uranium.

Note: Totals may not equal sum of components because of independent rounding. See Technical Note 11 in Appendix E.

Source: Energy Information Administration, Form RW-859, "Nuclear Fuel Data" (1993). 
Figure 5. Spent Fuel Assemblies in Storage by State

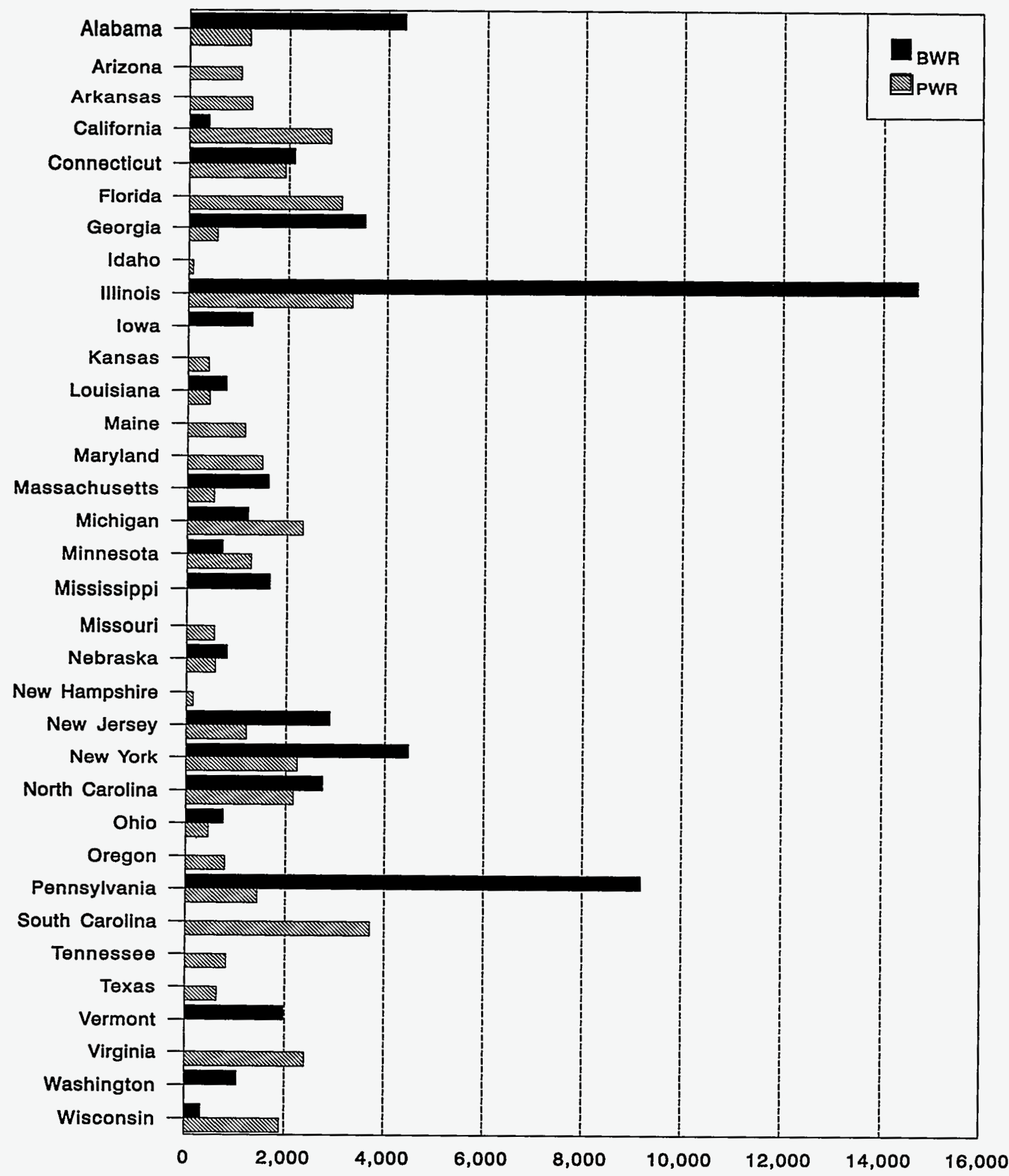

Assemblies

Notes: A total of 2,208 high-temperature, gas-cooled reactor (HTGR) fuel elements are in storage (744 stored in ldaho and 1,464 in Colorado). These HTGR fuel elements are not reflected on this graph. Numbers in the above graph represent assemblies stored at nuclear power plant sites and away-from-reactor facilities, and include both permanently and temporarily discharged assemblies.

Source: Energy Information Administration, Form RW-859, "Nuclear Fuel Data" (1993). 
Figure 6. Initial Uranium Content of Spent Fuel Assemblies in Storage by State (Metric tons of uranium)

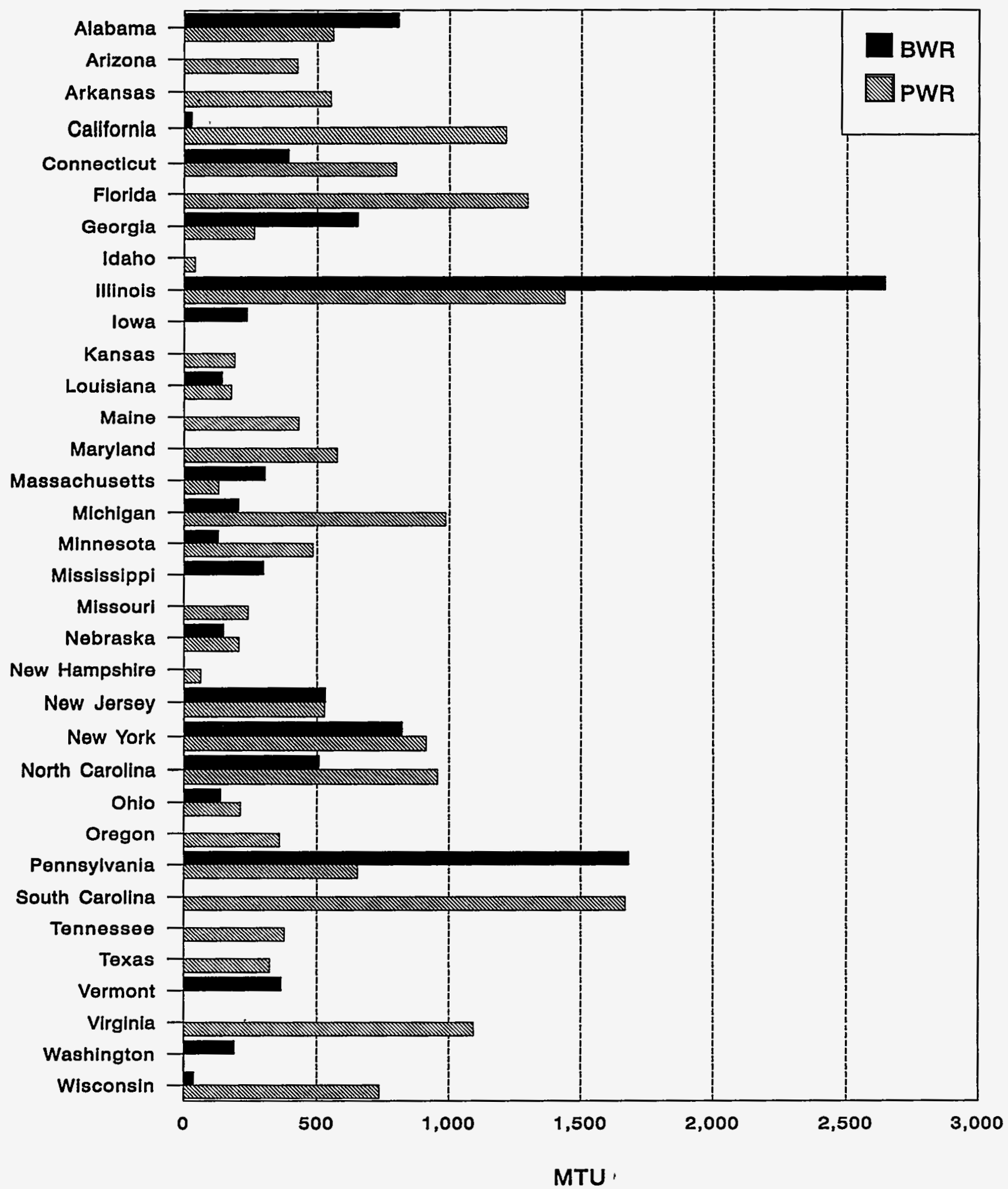

Notes: A total of 2,208 high-temperature, gas-cooled reactor (HTGR) fuel elements are in storage (744 with initial uranium content equal to 8.8 metric tons of uranium (MTU) stored in Idaho and 1,464 with initial uranium content equal to 15.4 MTU stored in Colorado). These HTGR fuel elements are not reflected on this graph. Numbers in the above graph represent the initial uranium content of assemblies stored at nuclear power plant sites and away-from-reactor facilities, and include both permanently and temporarily discharged assemblies.

Source: Energy Information Administration, Form RW-859, "Nuclear Fuel Data" (1993). 


\section{Dry Storage at Utilities}

In order to meet the demand for storage space, several utility companies have opted to increase their spent fuel storage capacity through the use of dry storage techniques. Many different dry storage techniques have been designed and are/will be licensed and used. These include metal storage casks, 'concrete storage casks, metal carịisters housed in concrete modules, and concrete storage vạults.

All U.S. commercial LWR's which are storing, or are planning to store nuclear fuel assemblies in an Independent Spent Fuel Storage Installation (ISFSI) as of December 31, 1993, are reported on Form RW-859. Several utilities have contributed supplementary dry storage information. Table 16 lists data for each of these utilities and affected reactors. Table 17 provides information on the current and projected number of assemblies in dry storage at these utilities.

As of November 1994, the NRC granted license certificates to eleven dry storage systems. The most widely used dry storage technology in the United States is the Nuxclear Horizontal Modular (NUHOMS) spent fuel storage system, designed by VECTRA Technologies, Inc. (formerly Pacific Nuclear). Three utilities currently use the NUHOMS design: Baltimore Gas and Electric Company, Carolinà Power and Light Company, and Duke Power Company. Four utilities plan to employ the NUHOMS system: GPU Nuclear Corporation, Pennsylvania Power and Light Company, Sacramento Municipal Utility District, and Toledo Edison Company. The NUHOMS system consists of three major components: a dry shielded canister (DSC), which provides a high integrity containment boundary and a controlled storage environment for the fuel; a reinforced concrete horizontal storage module (HSM), which houses the stored DSC and provides radiation shielding, protection against natural phenomena, and an efficient means for decay heat removal; and a transfer cask, which provides for the safe shielded transfer of the DSC from the plant spent fuel pool to the storage module. Rancho Seco will use the NUHOMS-MP187 multi-purpose cask for on-site transfer, off-site transfer, and contingency on-site storage. The NUHOMS system is designed and licensed to the requirements of 10 CFR 72 and American National Standards Institute/American National Standard (ANSI/ANS) 57.9 for ISFSI's.

Sierra Nuclear (formerly Pacific Sierra Nuclear Associates) designed the Ventilated Storage Cask (VSC) used at Consumers Power Company's Palisades plant. The VSC technology was the first concrete dry storage cask design to be granted a NRC certificate of compliance. This generic certificate allows the use of the cask at nuclear power plants without requiring a site-specific license.

The most recent cask technology licensed by the NRC is the TN-40 dry storage cask designed by Transnuclear, Inc., to store 40 assemblies. Other dry storage cask systems by Transnuclear allow for storage of 24 and 32 spent fuel assemblies. Although no sites currently have the TN-24, TN-32, or TN-40 casks in use, both Virginia Power's Surry plant and Northern States Power Company's Prairie Island plant have contractual agreements with Transnuclear for future dry storage systems.

\section{Arkansas Power and Light Company}

In 1994, the Arkansas Power and Light Company's Arkansas Nuclear 1 \& 2 plants finalized a contractual agreement with Sierra Nuclear for 14 VSC-24 casks. Because the VSC-24 has been granted a certificate of compliance by the NRC, Arkansas Power and Light will not be required to obtain a site-specific license.

The first cask is scheduled to be loaded and stored on a concrete pad located within the existing security protected area at the Arkansas Nuclear plants. The pad is designed to hold 26 casks, but can be expanded to provide space for an additional 50 casks. Existing rail lines and a new rail car specifically designed for the VSC will transport the casks from the plant's Auxiliary Building to the storage pad.

\section{Baltimore Gas and Electric Company}

The ISFSI at Baltimore Gas and Electric Company's Calvert Cliffs station is the NUHOMS-24P. The Calvert Cliffs ISFSI has been designed as a life-of-plant storage facility. The ISFSI will have the capacity to store all spent fuel discharged from Calvert Cliffs $1 \& 2$, beyond the spent fuel pool capacity, up to the 40 -year plant life, if necessary. The exact capacity needed is uncertain, and to limit capital investment until necessary, the ISFSI will be constructed in up to five phases. The ISFSI required the preparation of a 10 CFR 72 License Application, Safety Analysis Report, Environmental Report, and a Security Plan for NRC review and approval. The license material was prepared and submitted to the NRC in December 1989. Construction of the ISFSI west of the plant began in April 1991 after NRC approved the Environmental Report. The facility and its pre-operational testing 
were completed in October 1992. The ISFSI was licensed by the NRC on November 25, 1992.

On November 30,1993, the dry storage facility became fully operational with the successful loading of the first cask of fuel. The license allows Baltimore Gas and Electric Company to place as many as 2,880 pool-cooled assemblies in casks to be placed in ISFSI's. Each NUHOMS cask at Calvert Cliffs can hold 24 assemblies, and there are currently 120 planned storage modules. The license has a 20-year term, but the utility may seek to extend the term before it expires.

\section{Carolina Power and Light Company}

The ISFSI for Carolina Power and Light Company's Robinson 2 plant is composed of $8 \mathrm{HSMs}$, and is the NUHOMS-07P. Each HSM is a steel-reinforced concrete structure which holds 7 intact assemblies in each module. The ISFSI was licensed by the NRC in August 1986 to hold 56 assemblies. They are located inside the fenced area at the Robinson 2 plant site. The Carolina Power and Light Company also applied to the NRC for a license for an ISFSI to be built at its Brunswick plant. The ISFSI will be used only as a backup if shipping of spent nuclear fuel to the Harris plant is prohibited.

\section{Consumers Power Company}

In April 1993, the NRC approved the dry-cask storage of fuel that has already undergone pool cooling, and the use of the new VSC-24 cask by Sierra Nuclear Corporation at the Consumers Power Company's Palisades plant. The approval was challenged by the Michigan Attorney General and a citizen organization, the Lake Michigan Federation, on the grounds that the process should have entailed a full environmental impact statement, rather than the less elaborate environmental assessment.

In January 1995, the U.S. Circuit Court of Appeals declined to order the NRC to conduct a full hearing before allowing Consumers Power to use dry storage at the Palisades plant. The three judge panel said that the NRC had taken all the necessary steps to safeguard the environment, even though it had not prepared the site-specific analysis. The Appeal Court upheld the NRC's contention that the pad site was included in the environmental impact statement and that the pad site was acceptable for use.

The Palisades plant was the first to load spent fuel casks in its ISFSI under the NRC general licensing provisions. This ruling, under 10 CFR 72 subpart $K$, allows the use of certain cask/storage systems at licensed nuclear power plants without the need for further site-specific licensing. Plant personnel started loading casks on May 7, 1993, and by May 19, 1993, the job for the upcoming refueling was finished. Two casks each received 24 spent fuel assemblies, were welded shut and placed on the storage pad. By December 1994, 6 additional casks are expected to be loaded. The license amendment allows for as many as 25 casks to be used at the plant - enough to last Palisades through the end of its current licensed life, in 2007.

On August 1, 1994, Consumers Power Company notified the NRC of its plans to unload and replace 1 of the 7 dry storage casks in use at the Palisades plant. Although no leaks were detected, the utility found indications of minor flaws in the welds of the VSC-24 cask during its review of the manufacturer's quality assurance program. The utility stated that even though there was no actual health, engineering, or operational requirements, the cask was to.be replaced.

\section{Duke Power Company}

The Duke Power Company received its Oconee site license from the NRC in January 1990 to operate 88 modules of the NUHOMS-24P design. They are designed to store 24 pressurized-water reactor (PWR) assemblies per module; therefore, the maximum capacity is 2,112 assemblies. The first 20 modules were completed in 1990 and the second set of 20 were finished in 1992. The modules were loaded with fuel as follows: 4 modules in 1990, 9 modules in 1991, 7 modules in 1992, and 4 modules in 1993. Duke Power plans to load 5 more modules each year from 1994 to 1998.

\section{GPU Nuclear Corporation}

The GPU Nuclear Corporation contracted VECTRA Technologies, Inc., to engineer, license, and construct a spent fuel storage system for the Oyster Creek nuclear plant. The agreement includes the design and construction of concrete modules and stainless steel containers for use in storing the plant's spent fuel on-site. The facility will employ the NUHOMS-52B cask design. VECTRA Technologies will complete engineering and licensing work in 1994 and will begin delivering the fuel-storage equipment in 1995. Plans are for a total capacity of 20 storage modules $(1,040$ assemblies). This will be the first dry fuel storage project for BWR fuel in the U.S. 


\section{Northern States Power Company}

The decision by the Minnesota Public Utilities Commission to allow 17 containers for aboveground spent fuel storage at Northern States Power Company's Prairie Island site was granted June 26, 1991. The number of containers granted for use was considerably less than Northern States Power Company's request for 48 casks submitted in April 1991. The Commission found that in light of promising research and development in renewable energy resources, conservation, and load management, dry storage at the plant should be limited to 17 casks.

Without this additional storage, the spent fuel pool would have been full by the end of 1994 . The utility estimated that with no additional fuel storage space, the Prairie Island 2 reactor would shut down in May 1995 while the Prairie Island 1 reactor would shut down in January 1996. The 17 casks, although less than the utility requested, would enable the Prairie Island plant to remain in operation through the end of the century.

In August 1993, a Minnesota Supreme Court decision put on hold Northern States Power Company's plans for dry cask storage at Prairie Island. The State's highest court decided to let stand an Appeals Court decision that overturned the State regulators' approval of the on-site storage plan. The Court's decision was based on a Minnesota State law, enacted in the 1970's, requiring the State legislature's approval for "permanent" spent fuel storage within Minnesota. The Appeals Court concluded, and the State Supreme Court agreed, that Northern States' dry cask storage option constituted "permanent" storage, thus requiring State legislative approval.

Before the State Supreme Court ruling, Northern States finished all the necessary preparatory site work for the spent fuel casks, including construction of the concrete pads on which the casks would sit. The utility also contracted with Transnuclear, Inc., to purchase seven TN-40 casks; two of which were already fabricated. (Cask fabrication was suspended pending approval from the State legislature.) In October 1993, the NRC granted the utility a license for the Prairie Island site to store fuel in up to $48 \mathrm{TN}-40$ casks.

On May 6, 1994, the Minnesota State legislature approved a bill that authorized Northern States Power Company to store spent fuel in a total of 17 casks at the Prairie Island nuclear station. In a passing vote of 43-22 in the State Senate and 86-46 in the State House, the legislature allowed the utility to immediately store spent fuel in 5 casks. Storage in the remaining casks will be phased in until all 17 are in use by 1999. The provisions of the bill, however, require that the utility actively seek methods to produce wind generated energy as well as require the Prairie Island plant be shut down if its generating capacity drops below 55 percent for three consecutive years. The same bill passed by the Minnesota legislature also places a moratorium on the construction of new nuclear power plants throughout the state.

\section{Pennsylvania Power and Light Company}

In December 1994, Pennsylvania Power and Light Company selected the NUHOMS-52B dry fuel storage system $:$ for use at the Susquehanna plant. The Susquehanna ISFSI will store up to 15 concrete modules for a total of 780 BWR fuel assemblies.

\section{Public Service Company of Colorado}

The NRC licensed the ISFSI at Public Service Company of Colorado's closed Fort St. Vrain plant on November 4, 1991. The ISFSI is a Modular Vault Dry Storage System, made up of six air-cooled concrete vaults. The ISFSI will hold the fuel in dry storage until such time as DOE, under contract with the utility, takes possession of the material. The license authorizes storage in the ISFSI of up to 1,482 spent fuel elements, 37 reflector control rod elements, and 6 neutron source elements. In December 1991, the Public Service Company of Colorado transferred 18 elements to its ISFSI. In June 1992, 1,446 elements were transferred to the ISFSI, for a total of 1,464 which now reside in the ISFSI. The reflector elements and neutron source elements were disposed of off-site. The design lifetime of the facility is 40 years, although the current license is limited to 20 years. This facility is designed as a stand alone operation although it currently relies on a portion of the plant's security system.

\section{Sacramento Municipal Utility District}

The Sacramento Municipal Utility District (SMUD) Rancho Seco plant was permanently shut down in 1989 following a public referendum. As a part of their decommissioning strategy, SMUD chose to establish an on-site dry storage facility at the Rancho Seco plant.

SMUD first applied to the NRC for an ISFSI site license in October 1991. A revised application was submitted in October 1993. The ISFSI design will be similar to the Oconee and Calvert Cliffs plants: the 
NUHOMS-24P storage model. Twenty-two concrete storage modules are planned for the ISFSI facility with a total capacity of 493 assemblies. One storage canister is specially designed for failed fuel assemblies. The utility predicts that licensing of the ISFSI to store spent nuclear fuel will be issued by mid-1995. SMUD is also the first utility to apply for a transportable storage system license under 10 CFR 71. This license will allow for the transportation of casks to an off-site repository.

\section{Toledo Edison Company}

Toledo Edison Company chose to build an ISFSI at its Davis-Besse plant with an existing NRC-approved cask design. In the spring of 1993, Toledo Edison signed a contract with VECTRA Technologies, Inc., for the design and fabrication of NUHOMS casks at the DavisBesse plant. The contract will allow for additional onsite storage to support the plant through its current operating license term. The utility plans to load the first fuel into the casks in 1995. The ISFSI will store up to 32 casks with a total capacity of 768 fuel assemblies.

\section{Virginia Power}

Virginia Power was the first U.S. utility to use dry storage for spent nuclear fuel. The Virginia Power ISFSI, located at the Surry Power Station, is a design using metal storage casks. It was licensed by the NRC in July 1986, for use of the CASTOR V/21. Use of the MC-10, NAC-I28/ST, and CASTOR X/33 has also been approved by the NRC. Each cask is 16 feet high, 8 feet in diameter, and weighs 110 to 120 tons when loaded with fuel. These four cask designs hold between 21 and 33 fuel assemblies. The casks sit on a pad 230 feet long, 32 feet wide, and 3 feet thick (reinforced concrete). Each pad will hold 28 casks, and the facility license provides for 3 pads. The facility and casks have been evaluated for extreme temperatures $\left(-20^{\circ} \mathrm{F}\right.$ to $\left.115^{\circ} \mathrm{F}\right)$, extreme wind (105 m.p.h.), snow and ice, loss of electrical power, loss of cask radiation shielding, tornados ( 300 m.p.h.), gas pipeline explosions, and cask seal leakage and drops.

By the end of 1993, a total of 416 assemblies were stored in 12 casks. In 1994, 117 assemblies were projected to be stored in 5 additional casks; 4 casks holding 21 assemblies and 1 holding 33 assemblies. This will increase the total number of assemblies in dry storage at Surry to 533 by the end of 1994. Virginia Power projects the storage of 105 assemblies in 5 casks during 1995; 96 assemblies in 3 casks during 1996; 96 assemblies in 3 casks during 1997; and, 128 assemblies in 4 casks during 1998. The ISFSI has been licensed to hold up to 1,764 assemblies. For future dry storage needs, Virginia Power has ordered 10 Transnuclear TN-32 casks for delivery between 1996 and 1998.

\section{Wisconsin Electric Power Company}

On August 8, 1994, the final environmental impact statement (EIS) was completed for Wisconsin Electric Power Company's plans for dry storage at the Point Beach reactor site. The EIS, produced by the Public Service Commission of Wisconsin, supported the use of steel-reinforced concrete cylinders as a temporary dry storage system. The conclusion was consistent with internal analysis by Wisconsin Electric as well as with the NRC's storage system licensing process.

Wisconsin Electric has chosen Sierra Nuclear Corporation VSC-24 concrete cask design for dry storage use at the Point Beach Plant. Because the VSC-24 is a NRC certified cask, Wisconsin Electric will not be required to apply for a separate NRC sitespecific license. Although the utility does not have a contract with Sierra Nuclear for a specific number of casks, the facility is sized to accommodate 48 casks. The utility currently has plans to load 8 casks in 1995 and 4 casks in 1997. 


\begin{tabular}{|c|c|c|c|c|c|c|c|c|}
\hline \multirow[b]{2}{*}{ Electric Utility Name } & \multirow[b]{2}{*}{ Reactor } & \multirow{2}{*}{$\begin{array}{c}\text { Date } \\
\text { License } \\
\text { Issued/ } \\
\text { Submitted } \\
\end{array}$} & \multirow[b]{2}{*}{ Vendor } & \multirow[b]{2}{*}{$\begin{array}{l}\text { Storage } \\
\text { Type }\end{array}$} & \multirow[b]{2}{*}{ Model } & \multicolumn{3}{|c|}{ Planned Capacitya } \\
\hline & & & & & & $\begin{array}{l}\text { No. of } \\
\text { Modules }\end{array}$ & $\begin{array}{l}\text { Assemblies } \\
\text { per Module }\end{array}$ & Total \\
\hline $\begin{array}{l}\text { Arkansas Power and } \\
\text { Light Company } \ldots \ldots \ldots\end{array}$ & Arkansas Nuclear $1 \& 2$ & ${ }^{b}$ General & $\begin{array}{l}\text { Sierra Nuclear } \\
\text { Corporation }\end{array}$ & $\begin{array}{l}\text { Concrete } \\
\text { Cask }\end{array}$ & VSC-24 & 14 & 24 & 336 \\
\hline $\begin{array}{l}\text { Baltimore Gas and } \\
\text { Electric Company } \ldots \ldots \ldots\end{array}$ & Calvert Cliffs $1 \& 2$ & $11 / 92$ & $\begin{array}{l}\text { VECTRA } \\
\text { Technologies, Inc. }\end{array}$ & $\begin{array}{l}\text { Concrete } \\
\text { Module }\end{array}$ & NUHOMS-24P & 120 & 24 & 2,880 \\
\hline $\begin{array}{l}\text { Carolina Power and } \\
\text { Light Company } \ldots \ldots \ldots\end{array}$ & Robinson 2 & $08 / 86$ & $\begin{array}{l}\text { VECTRA } \\
\text { Technologies, Inc. }\end{array}$ & $\begin{array}{l}\text { Concrete } \\
\text { Module }\end{array}$ & NUHOMS-7P & 8 & 7 & 56 \\
\hline $\begin{array}{l}\text { Carolina Power and } \\
\text { Light Company } \ldots \ldots \ldots\end{array}$ & Brunswick 1 \& 2 & ${ }^{c} 05 / 89$ & $\begin{array}{l}\text { VECTRA } \\
\text { Technologies, Inc. }\end{array}$ & $\begin{array}{l}\text { Concrete } \\
\text { Module }\end{array}$ & NUHOMS-7P & 0 & 0 & 0 \\
\hline Consumers Power Company & Palisades & ${ }^{\circ} 03 / 90$ & $\begin{array}{l}\text { Sierra Nuclear } \\
\text { Corporation }\end{array}$ & $\begin{array}{l}\text { Concrete } \\
\text { Cask }\end{array}$ & VSC-24 & 24 & 24 & 576 \\
\hline Duke Power Company ..... & Oconee $1,2,3$ & $01 / 90$ & $\begin{array}{l}\text { VECTRA } \\
\text { Technologies, Inc. }\end{array}$ & $\begin{array}{l}\text { Concrete } \\
\text { Module }\end{array}$ & NUHOMS-24P & 88 & 24 & 2,112 \\
\hline GPU Nuclear Corporation ... & Oyster Creek & ${ }^{b}$ General & $\begin{array}{l}\text { VECTRA } \\
\text { Technologies, Inc. }\end{array}$ & $\begin{array}{l}\text { Concrete } \\
\text { Module }\end{array}$ & NUHOMS-52B & 20 & 52 & 1,040 \\
\hline $\begin{array}{l}\text { Northem States } \\
\text { Power Company } \ldots \ldots \ldots \text {. }\end{array}$ & Prairie Island 1 \& 2 & $10 / 93$ & Transnuclear, Inc. & Metal Cask & TN-40 & 7 & 40 & 280 \\
\hline $\begin{array}{l}\text { Pennsylvania Power and } \\
\text { Light Company } \ldots \ldots \ldots \ldots\end{array}$ & Susquehanna $1 \& 2$ & ${ }^{\circ}$ General & $\begin{array}{l}\text { VECTRA } \\
\text { Technologies, inc. }\end{array}$ & $\begin{array}{l}\text { Concrete } \\
\text { Module }\end{array}$ & NUHOMS-52B & 15 & 52 & 780 \\
\hline $\begin{array}{l}\text { Public Service Company } \\
\text { of Colorado ........... }\end{array}$ & Fort St. Vrain & $11 / 91$ & $\begin{array}{l}\text { Foster Wheeler } \\
\text { Energy Applications, } \\
\text { Inc. }\end{array}$ & $\begin{array}{l}\text { Concrete } \\
\text { Vault }\end{array}$ & $\begin{array}{l}\text { Modular Dry } \\
\text { Storage }\end{array}$ & 6 & 244 & 1,464 \\
\hline $\begin{array}{l}\text { Sacramento Municipal } \\
\text { Utility District ......... }\end{array}$ & Rancho Seco & ${ }^{\circ} 12 / 95$ & $\begin{array}{l}\text { VECTRA } \\
\text { Technologies, Inc. }\end{array}$ & $\begin{array}{l}\text { Concrete } \\
\text { Module }\end{array}$ & NUHOMS-24P & 21 & 24 & 504 \\
\hline
\end{tabular}

\footnotetext{
See footnotes at end of table.
} 
Table 16. Independent Spent Fuel Storage Installation (ISFSI) Data (Continued)

\begin{tabular}{|c|c|c|c|c|c|c|c|c|}
\hline \multirow[b]{2}{*}{ Electric Utility Name } & \multirow[b]{2}{*}{ Reactor } & \multirow{2}{*}{$\begin{array}{c}\text { Date } \\
\text { License } \\
\text { Issued/ } \\
\text { Submitted }\end{array}$} & \multirow[b]{2}{*}{ Vendor } & \multirow[b]{2}{*}{$\begin{array}{c}\text { Storage } \\
\text { Type }\end{array}$} & \multirow[b]{2}{*}{ Model } & \multicolumn{3}{|c|}{ Planned Capacity" } \\
\hline & & & & & & $\begin{array}{c}\text { No. of } \\
\text { Modules }\end{array}$ & $\begin{array}{l}\text { Assemblies } \\
\text { per Module }\end{array}$ & Total \\
\hline Toledo Edison Company .... & Davis-Besse & 'General & $\begin{array}{l}\text { VECTRA } \\
\text { Technologies, Inc. }\end{array}$ & $\begin{array}{l}\text { Concrete } \\
\text { Module }\end{array}$ & NUHOMS-24P & 32 & 24 & 768 \\
\hline Virginia Power & Surry $1 \& 2$ & $07 / 86$ & General Nuclear & Metal Cask & CASTOR V/21 & 25 & 21 & 525 \\
\hline & & & $\begin{array}{l}\text { General Nuclear } \\
\text { Systems, Inc. }\end{array}$ & Metal Cask & CASTOR X/33 & 1 & 33 & 33 \\
\hline & & & $\begin{array}{l}\text { Westinghouse } \\
\text { Nuclear Assurance }\end{array}$ & $\begin{array}{l}\text { Metal Cask } \\
\text { Metal Cask }\end{array}$ & $\begin{array}{c}\text { MC-10 } \\
\text { NAC-128 S/T }\end{array}$ & $\begin{array}{l}1 \\
2\end{array}$ & $\begin{array}{l}24 \\
28\end{array}$ & $\begin{array}{l}24 \\
56\end{array}$ \\
\hline & & & $\begin{array}{l}\text { Corporation } \\
\text { Transnuclear }\end{array}$ & & & 10 & 32 & 320 \\
\hline & & & Total Vendors & & & 39 & & 958 \\
\hline
\end{tabular}

aplanned Capacity is based on contractual agreement with vendors.

'The model of dry storage employed by the utilities has been granted a certificate of compliance by the Nuclear Regulatory Commission (NRC) thus eliminating the requirement for a site-specific license.

"Date license application was submitted to the NRC.

'Estimated date for Part 71 (transportation) and Part 72 (storage) license.

Source: Energy Information Administration, Form RW-859, "Nuclear Fuel Data" (1993). 


\begin{tabular}{|c|c|c|c|c|c|c|c|c|c|c|c|c|c|}
\hline \multirow[b]{2}{*}{ Electric Utility Name } & \multirow[b]{2}{*}{ Reactor } & \multicolumn{6}{|c|}{ Assemblies Stored } & \multirow{2}{*}{$\begin{array}{l}\text { Total } \\
\text { MTU }^{a}\end{array}$} & \multicolumn{5}{|c|}{ Projected Assemblies Stored } \\
\hline & & 1989 & 1990 & 1991 & 1992 & 1993 & Total & & 1994 & 1995 & 1996 & 1997 & 1998 \\
\hline $\begin{array}{l}\text { Arkansas Power and } \\
\text { Light Company } \ldots \ldots \ldots \ldots\end{array}$ & Arkansas Nuclear $1 \& 2$ & .- & -- & -- & -- & -. & -- & -- & & 72 & 72 & 96 & 96 \\
\hline $\begin{array}{l}\text { Baltimore Gas and } \\
\text { Electric Company } \ldots \ldots \ldots\end{array}$ & Calvert Cliffs $1 \& 2$ &.. & -- & - & -- & 48 & 48 & 18.0 & 144 & 144 & 144 & 144 & 144 \\
\hline $\begin{array}{l}\text { Carolina Power and } \\
\text { Light Company } \ldots \ldots \ldots \ldots\end{array}$ & Robinson 2 & ${ }^{5} 56$ & -- & - & -- & -- & 56 & 24.1 & & & & & \\
\hline $\begin{array}{l}\text { Carolina Power } \\
\text { and Light Company ........ }\end{array}$ & Brunswick $1 \& 2$ & -. & - & -- & - & -- & -. & . & & & & & \\
\hline Consumers Power Company .. & Palisades & -- & - & - & -- & 48 & 48 & 18.6 & 144 & 120 & 72 & & \\
\hline Duke Power Company ...... & Oconee $1,2,3$ & .. & 96 & 216 & 168 & 96 & 576 & 267.3 & 120 & 120 & 120 & 120 & 120 \\
\hline GPU Nuclear Corporation .... & Oyster Creek & -- & - & -. & - & -- & -. & -- & & & 416 & & \\
\hline $\begin{array}{l}\text { Northern States } \\
\text { Power Company } \ldots \ldots \ldots \ldots\end{array}$ & Prairie Island 1 \& 2 & -- & -- & -- & -- & -- & -- & - & & 200 & & 160 & \\
\hline $\begin{array}{l}\text { Pennsylvania Power and } \\
\text { Light Company } \ldots \ldots \ldots \ldots\end{array}$ & Susquehanna $1 \& 2$ & - & - & -- & -- & -- & - & -- & & & & 48 & 48 \\
\hline $\begin{array}{l}\text { Public Service Company } \\
\text { of Colorado } \ldots \ldots \ldots \ldots \ldots\end{array}$ & Fort St. Vrain & - & -- & $c_{18}$ & $c_{1,446}$ & $\cdots$ & ${ }^{c} 1,464$ & ${ }^{c_{15}} 15.4$ & & & & & \\
\hline $\begin{array}{l}\text { Sacramento Municipal } \\
\text { Utility District . . . . . }\end{array}$ & Rancho Seco & - & - & -- & -- & -- & -- & -- & & & & & \\
\hline Toledo Edison Company ..... . & Davis-Besse & - & - & -- & -- & -- & -- & -- & & & & & \\
\hline Virginia Power $\ldots . \ldots \ldots$ & Surry $1 \& 2$ & ${ }^{b} 168$ & 84 & 52 & 63 & 49 & ${ }^{d} 416$ & $d_{188.3}$ & 117 & 105 & 96 & 96 & 128 \\
\hline $\begin{array}{l}\text { Wisconsin Electric } \\
\text { Power Company } \ldots \ldots \ldots \text {. }\end{array}$ & Point Beach $1 \& 2$ & -- & -- & -- & - & - & - & -- & & 192 & & 96 & \\
\hline Total $\ldots \ldots \ldots \ldots \ldots \ldots$ & & 224 & 180 & 268 & 231 & 241 & 1,144 & 516.3 & 525 & 953 & 920 & 760 & 536 \\
\hline
\end{tabular}

anitial uranium content in metric tons of uranium (MTU) of assemblies in dry storage as of December 31, 1993.

'Assemblies in dry storage through 1989.

'A total of 1,464 high-temperature, gas-cooled reactor (HTGR) fuel elements, with initial uranium content equal to 15.4 MTU are not included in the table totals. See Technical Note 6 in Appendix $E$.

Total does not include 69 assemblies, with initial uranium content equal to $31.5 \mathrm{MTU}$, in dry storage at Idaho National Engineering Laboratory.

$-=$ Not applicable.

Source: Energy Information Administration, Form RW-859, "Nuclear Fuel Data" (1993). 


\section{Canisters and Nonfuel Components}

\section{Background}

The storage of nonfuel assembly (NFA) hardware and its subsequent impact on a Federal Waste Management System has been the subject of much discussion because of the effect on waste acceptance procedures and transportation and disposal requirements. NFA hardware, if integral to a fuel assembly, could represent an addition to the total weight of each shipment. This additional weight may have impacts on the ability of fully loaded transportation casks to meet legal weight limits.

The Form RW-859 "Nuclear Fuel Data" survey was revised in 1991 to collect approximately 30 data elements on canisters and their contents, and nonfuel components (NFC) for each storage pool site. For the past 3 years, the Energy Information Administration (EIA) has collected these data as part of this survey. This chapter presents the most current data on canisters and NFC stored at these facilities as of December 31, 1993. The results obtained thus far in the area of canisters and nonfuel components present a framework from which further data may be collected.

Because the nonfuel components section of the Form RW-859 is a recent addition to the survey, respondent answers were not always consistent even though the reactors are similar. This is particularly apparent with respect to the number of fuel channels reported at BWR reactors. While some of these reactors report a number of fuel channels which are roughly equivalent to the number of discharged fuel assemblies, other reactors report quantities which are significantly lower and appear to represent the number of fuel channels which are not attached to assemblies. These differences in reporting must be understood and eventually reconciled in order to accurately estimate quantities which might be designated for disposal.

When analyzing canister data provided by utilities on Form RW-859, a number of errors occurred due to differences in the utility and EIA definition of canisters and the misinterpretation of questions. These errors were particularly apparent in the assessment of the total number of baskets reported by each utility in which the basket total may have been already accounted for in the total number of canisters for each utility.

The Form RW-859 survey is currently being reviewed so that more thorough and consistent data can be collected in these areas. The need for further information will depend on the level of detail required by designers and systems engineers to plan for the handling and disposition of these components.

\section{Canister and Nonfuel Component Data Through 1993}

As of December 31, 1993, at total of 269 canisters were reported in storage pools. Because all but one Form RW-859 respondent reported the number of canisters on the 1991, 1992, or 1993 survey (the one that did not report had no canisters in earlier years), these 269 canisters should represent the total in pools as of the above date. Inventories were not reported for 8 of these canisters, representing 3 percent of the total. An additional 321 open baskets containing miscellaneous spent nuclear fuel (SNF) and nonfuel materials were also reported. All of the 269 canisters meet the dimensional envelope requirements specified for disposal of "standard fuel" under the Standard Contract for Disposal of Spent Nuclear Fuel and/or High-Level Radioactive Waste (10 CFR 961) while most of the baskets do not.

A total of 60,699 pieces of NFA hardware were reported in storage at reactor sites. This total represents the NFA hardware reported by the owners of 100 of the 109 reactors in generic assembly classes (e.g., Babcock \& Wilcox (B\&W) $15 \times 15$, Westinghouse (WE) $17 \times 17$, General Electric (GE) 4, 5, 6, Combustion Engineering (CE) System 80, etc.). The remaining 9 reactors either did not report nonfuel components data or reported no NFA hardware components. This chapter addresses the nonfuel components (including NFA hardware) reported by owners of reactors in single-reactor assembly classes (e.g., Big Rock Point, Dresden 1, Palisades, LaCrosse, etc.) separately at the end of the chapter. 


\section{Canisters}

A canister is a container which may hold spent nuclear fuel, high-level radioactive waste, or nonfuel components. A canister may contain an intact spent fuel assembly, consolidated spent fuel assemblies, spent fuel rods or pieces, NFA hardware, nonfuel disassembly hardware, or other fuel and nonfuel material. These canisters are temporarily stored in spent fuel pools at reactor sites and occupy one or more slots in the pool. When full, a canister is typically closed with a lid and sealed shut. Waste may also be placed in rodlet or garbage and debris baskets, which are also stored in the spent fuel pools, but not necessarily in the pool racks.

Because many utilities face spent fuel storage constraints, the number of canisters stored at these facilities and the contents of these canisters are important concerns. A few utilities have used fuel consolidation campaigns as a means of preserving fuel assemblies and placing them in a canister in a grid storage pool space. Fuel is consolidated by removing rods from fuel assemblies and placing them in a canister in a grid with spacing closer than that of an intact assembly. In addition to maximizing density and lowering criticality, heat transfer characteristics are also improved.

A summary of data on canisters, baskets, and their contents as reported on the Form RW-859 survey are presented by storage pool site (Table 18). Due to different reporting schedules, not all respondents were required to report on the 1993 Form RW-859 survey, so data for 12 storage pool sites have been carried over from 1992. In addition, one site did not report canister data for the past three survey years. Of the 269 canisters reported, 145 contain only spent fuel (intact assemblies, consolidated assemblies, or fuel rods/pieces), 96 contain only nonfuel components, 15 contain both fuel and nonfuel, and 13 canisters are currently empty. The specific contents of 8 canisters were not reported so these tables should not be considered representative of the complete enumeration of these data. A total of 321 open baskets were also reported, containing miscellaneous fuel and nonfuel materials, garbage and debris.

Data on the contents of canisters containing spent nuclear fuel (intact assemblies, rods and/or pieces, or consolidated assemblies) are summarized in Table 19. The 160 canisters containing SNF can be further subdivided by whether the fuel was irradiated in a boiling-water reactor (BWR) or pressurized-water reactor (PWR). Of the 48 canisters containing BWR spent nuclear fuel, 24 contain intact spent fuel assemblies, and 24 contain fuel rods. No fuel was consolidated at any BWR. Of the 112 canisters containing PWR spent nuclear fuel, 16 contain intact assemblies, 58 contain fuel rods, and 36 canisters contain a total of 78 consolidated assemblies. These consolidation campaigns took place at Palisades, Oconee 1 \& 2, Millstone 2, Prairie Island 1 \& 2, and Ginna. The contents of 2 canisters at PWR's were not reported.

Of the 111 canisters containing nonfuel components (Table 20), a majority contained nonfuel assembly hardware ( 60 canisters). The contents of 6 nonfuel canisters were not reported so this table should not be considered representative of the complete enumeration of these data. It should be noted that nonfuel canisters may include more than one class of components.

Table 21 presents a summary of the dimensions of all reported canisters, in inches, and corresponding assembly dimensions found in the fuel canisters as reported on the Form RW-859 survey. The most

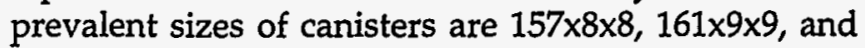
$180 \times 10 \times 10$. No canisters were reported as having a length greater than the longest PWR assembly, which is 199 inches. Five canisters were reported containing partial length fuel rods used in a DOE fuel performance improvement program in the late 1970's. With the exception of these five canisters, containers with a reported length of less than 84 inches are classified as baskets. Some respondents submitted data on the contents of a particular canister, but did not submit the canister's dimensions.

In most cases, the dimensions of the most prominent assembly were less than or equal to the dimensions of the canister. In some cases (i.e., canister dimensions $144 \times 9 \times 9$ ), the dimensions of the assembly were larger than the canister. For each of these canisters, the corresponding canister contained only assembly rods and pieces, with dimensions less than an intact assembly. 
Table 18. Container Data as of December 31, 1993

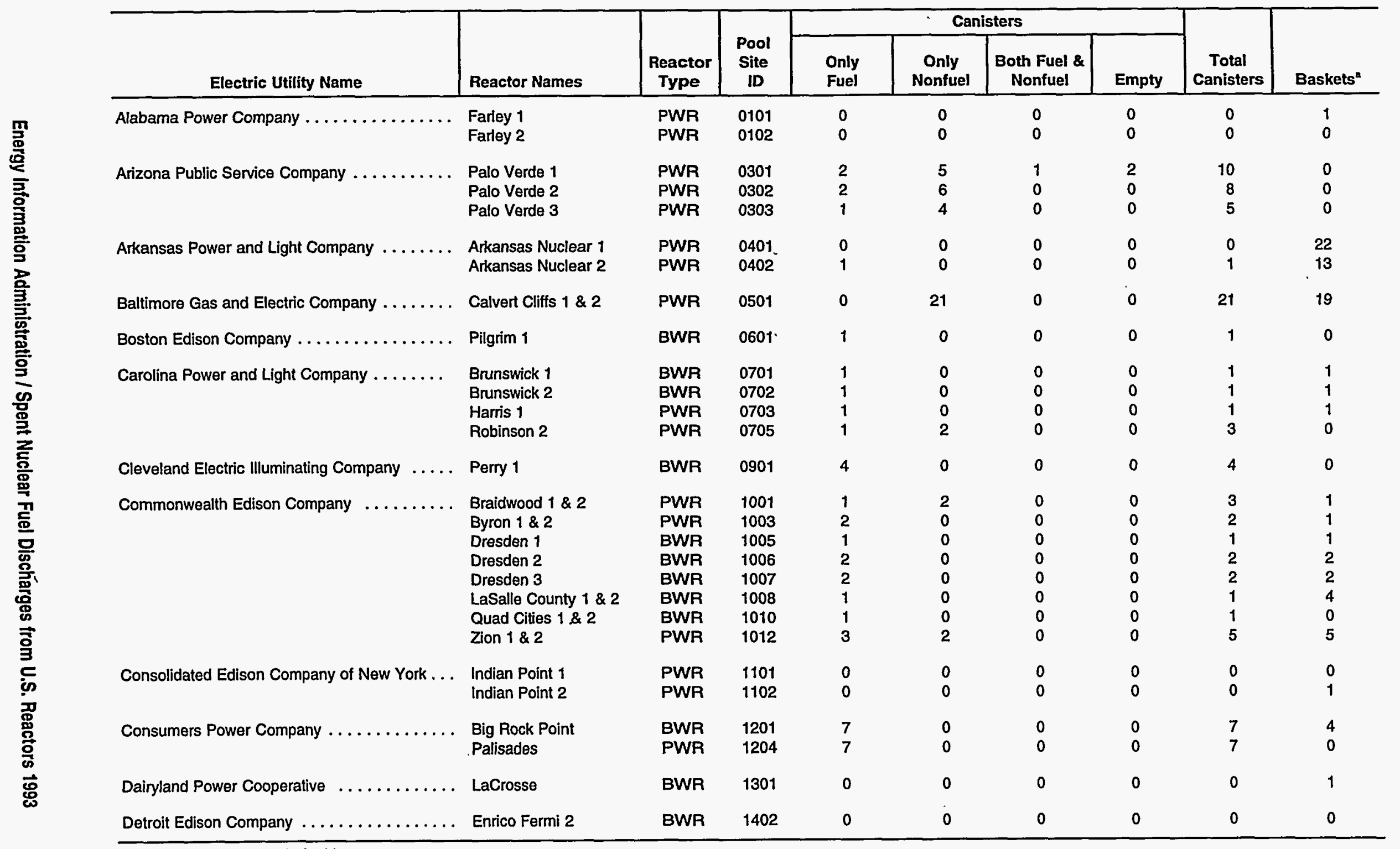

See footnotes at end of table. 


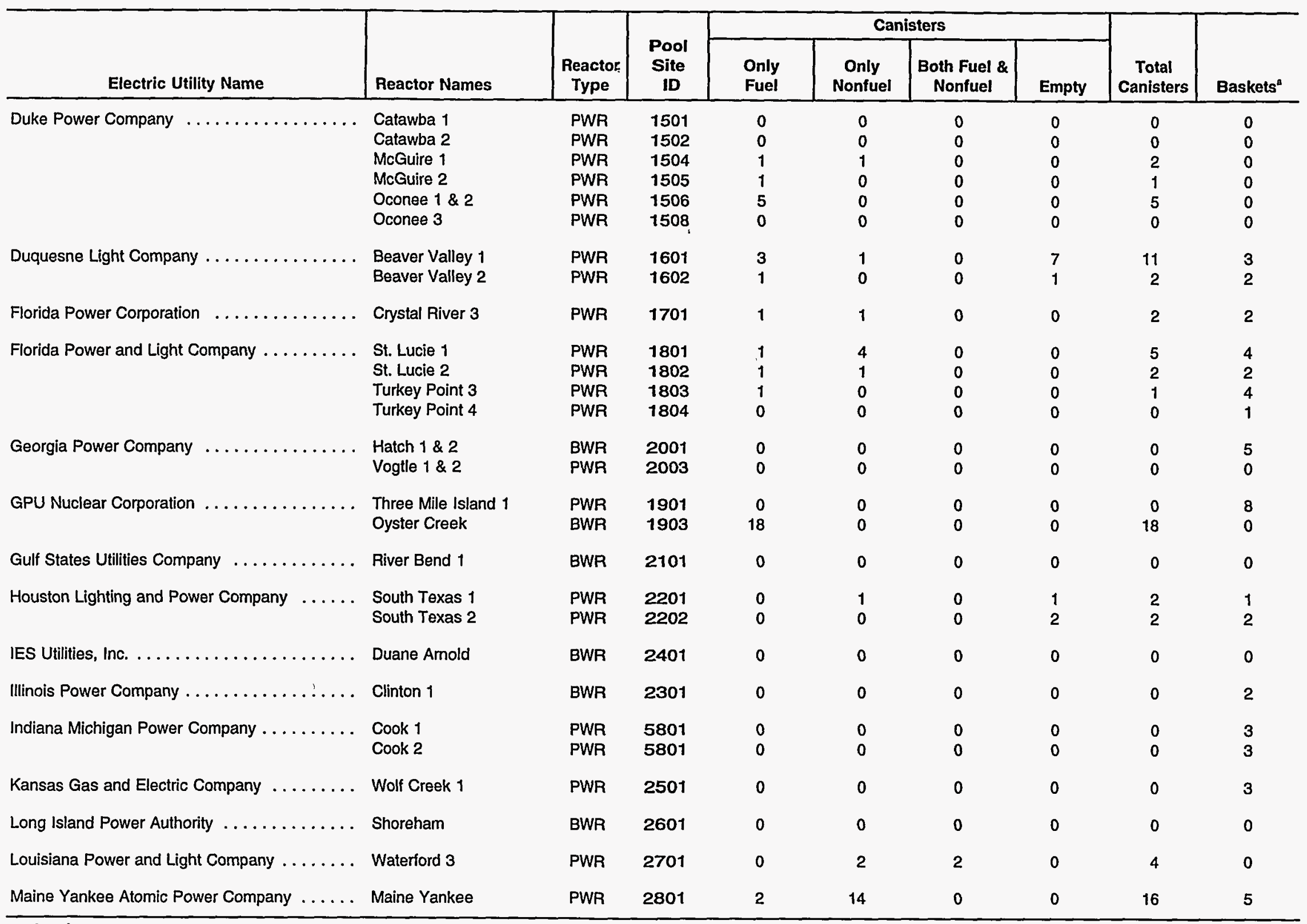

See footnotes at end of table. 
Table 18. Container Data as of December 31, 1993 (Continued)

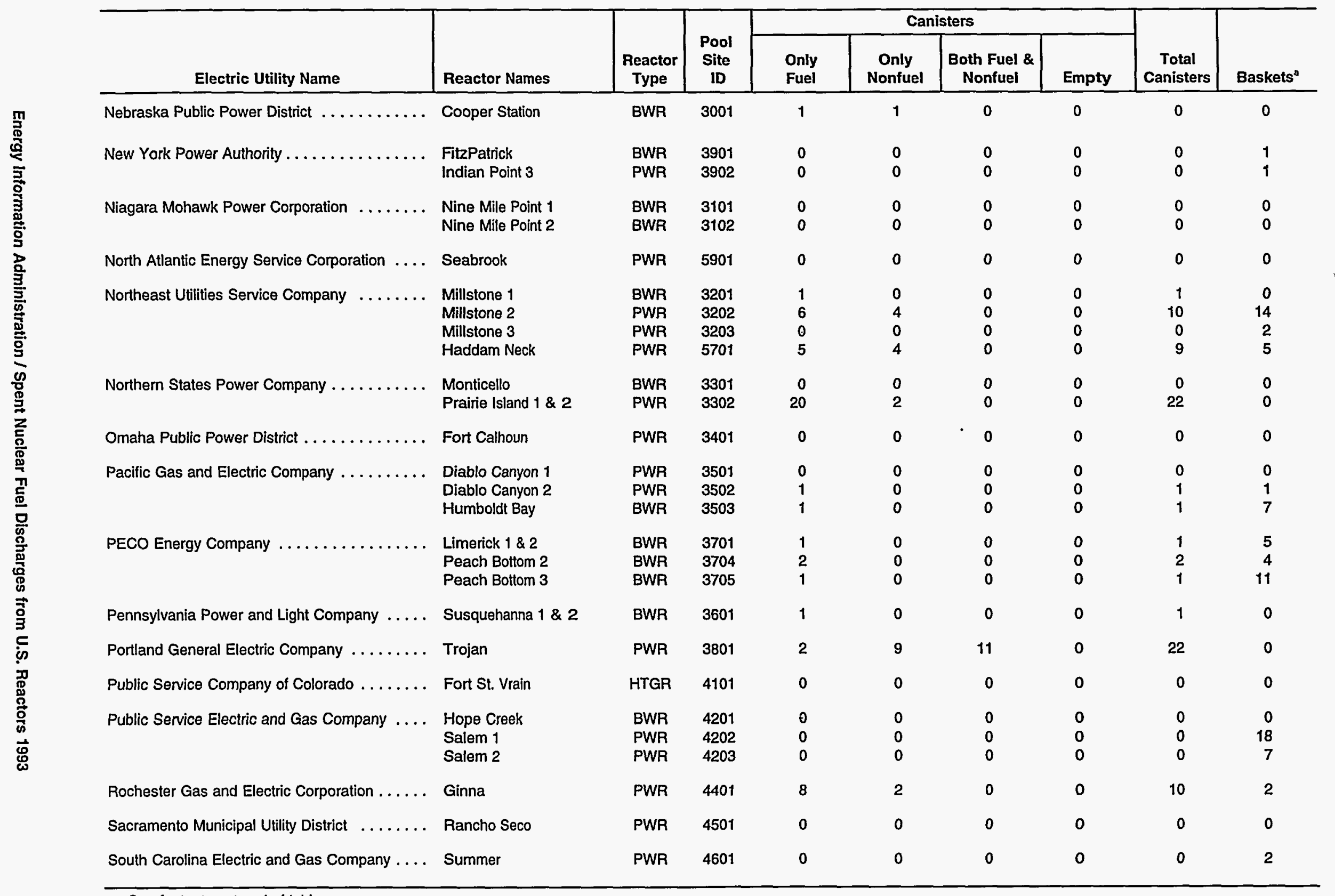


Table 18. Container Data as of December 31, 1993 (Continued)

\begin{tabular}{|c|c|c|c|c|c|c|c|c|c|}
\hline \multirow[b]{2}{*}{ Electric Utility Name } & \multirow[b]{2}{*}{ Reactor Names } & \multirow[b]{2}{*}{$\begin{array}{c}\text { Reactor } \\
\text { Type }\end{array}$} & \multirow{2}{*}{$\begin{array}{c}\text { Pool } \\
\text { Site } \\
\text { ID }\end{array}$} & \multicolumn{4}{|c|}{ Canisters } & \multirow[b]{2}{*}{$\begin{array}{c}\text { Total } \\
\text { Canisters }\end{array}$} & \multirow[b]{2}{*}{ Baskets $^{\circ}$} \\
\hline & & & & $\begin{array}{l}\text { Only } \\
\text { Fuel }\end{array}$ & $\begin{array}{c}\text { Only } \\
\text { Nonfuel }\end{array}$ & $\begin{array}{c}\text { Both Fuel \& } \\
\text { Nonfuel }\end{array}$ & Empty & & \\
\hline Southern California Edison Company ........ & $\begin{array}{l}\text { San Onofre } 1 \\
\text { San Onofre } 2 \\
\text { San Onofre } 3\end{array}$ & $\begin{array}{l}\text { PWR } \\
\text { PWR } \\
\text { PWR }\end{array}$ & $\begin{array}{l}4701 \\
4702 \\
4703\end{array}$ & $\begin{array}{l}0 \\
0 \\
0\end{array}$ & $\begin{array}{l}0 \\
0 \\
0\end{array}$ & $\begin{array}{l}0 \\
0 \\
0\end{array}$ & $\begin{array}{l}0 \\
0 \\
0\end{array}$ & $\begin{array}{l}0 \\
0 \\
0\end{array}$ & $\begin{array}{r}4 \\
9 \\
10\end{array}$ \\
\hline System Energy Resources, Inc. . . . . . . . . . & Grand Gulf 1 & BWR & 2901 & 1 & 0 & 0 & 0 & 1 & 18 \\
\hline Tennessee Valley Authority..$\ldots \ldots \ldots \ldots$ & $\begin{array}{l}\text { Bellefonte } 1 \\
\text { Bellefonte } 2 \\
\text { Browns Ferry } 1 \& 2 \\
\text { Browns Ferry } 3 \\
\text { Sequoyah } 1 \text { \& } 2 \\
\text { Watts Bar } 1 \& 2\end{array}$ & $\begin{array}{l}\text { PWR } \\
\text { PWR } \\
\text { BWR } \\
\text { BWR } \\
\text { PWR } \\
\text { PWR }\end{array}$ & $\begin{array}{l}4801 \\
4802 \\
4803 \\
4805 \\
4808 \\
4810\end{array}$ & $\begin{array}{l}0 \\
0 \\
1 \\
0 \\
0 \\
0\end{array}$ & $\begin{array}{l}0 \\
0 \\
0 \\
0 \\
0 \\
0\end{array}$ & $\begin{array}{l}0 \\
0 \\
0 \\
0 \\
0 \\
0\end{array}$ & $\begin{array}{l}0 \\
0 \\
0 \\
0 \\
0 \\
0\end{array}$ & $\begin{array}{l}0 \\
0 \\
1 \\
0 \\
0 \\
0\end{array}$ & $\begin{array}{l}0 \\
0 \\
0 \\
0 \\
0 \\
0\end{array}$ \\
\hline Toledo Edison Company .............. & Davis-Besse & PWR & 5001 & 1 & 3 & 0 & 0 & 4 & 4 \\
\hline TU Electric $\ldots \ldots \ldots \ldots \ldots \ldots \ldots$ & Comanche Peak $1 \& 2$ & PWR & 4901 & 0 & 0 & 0 & 0 & 0 & 37 \\
\hline Union Electric Company $\ldots \ldots \ldots \ldots \ldots$ & Callaway & PWR & 5101 & 1 & 0 & 0 & 0 & 1 & 1 \\
\hline Vermont Yankee Nuclear Power Corporation & Vermont Yankee & BWR & 6001 & 0 & 0 & 0 & 0 & 0 & 0 \\
\hline Virginia Power...$\ldots \ldots \ldots \ldots \ldots \ldots$ & $\begin{array}{l}\text { North Anna } 1 \& 2 \\
\text { Surry } 1 \& 2\end{array}$ & $\begin{array}{l}\text { PWR } \\
\text { PWR }\end{array}$ & $\begin{array}{l}5201 \\
5203\end{array}$ & $\begin{array}{l}1 \\
1\end{array}$ & $\begin{array}{l}2 \\
0\end{array}$ & $\begin{array}{l}0 \\
1\end{array}$ & $\begin{array}{l}0 \\
0\end{array}$ & $\begin{array}{l}3 \\
2\end{array}$ & $\begin{array}{l}3 \\
8\end{array}$ \\
\hline Washington Public Power Supply System ... & Washington Nuclear 2 & BWR & 5302 & 0 & 0 & 0 & 0 & 0 & 1 \\
\hline Wisconsin Electric Power Company ........ & Point Beach $1 \& 2$ & PWR & 5401 & 13 & 3 & 0 & 0 & 16 & 16 \\
\hline Wisconsin Public Service Corporation ...... & Kewaunee & PWR & 5501 & 0 & 0 & 0 & 0 & 0 & 0 \\
\hline Yankee Atomic Electric Company $\ldots . . . \ldots$ & Yankee Rowe & PWR & 5601 & 0 & 0 & 0 & 0 & 0 & 0 \\
\hline Total $\ldots \ldots \ldots \ldots \ldots \ldots \ldots \ldots \ldots \ldots$ & & & & 145 & 96 & 15 & 13 & 269 & 321 \\
\hline
\end{tabular}

"Baskets column represents the number of open baskets, including rodlet or garbage and debris baskets. Due to reporting errors, the number of baskets reported may be duplicated in canister data.

PWR = Pressurized-water reactor; BWR = Boiling-water reactor; HTGR = High-temperature, gas-cooled reactor.

Source: Energy Information Administration, Form RW-859, "Nuclear Fuel Data" (1993). 


\section{Table 19. Canisters Containing Spent Fuel as of December 31, 1993}

\begin{tabular}{|c|c|c|c|c|c|c|c|c|c|}
\hline \multirow[b]{2}{*}{ Electric Utility Name } & \multirow[b]{2}{*}{ Reactor Names } & \multirow[b]{2}{*}{$\begin{array}{l}\text { Reactor } \\
\text { Type }\end{array}$} & \multirow[b]{2}{*}{$\begin{array}{l}\text { Pool } \\
\text { Site } \\
\text { ID }\end{array}$} & \multicolumn{5}{|c|}{ Canisters Fuel } & \multirow[b]{2}{*}{$\begin{array}{c}\text { Total } \\
\text { Canisters } \\
\text { Fuel }^{\mathrm{C}}\end{array}$} \\
\hline & & & & $\begin{array}{c}\text { Intact } \\
\text { Assemblies }\end{array}$ & $\begin{array}{l}\text { Rods and } \\
\text { Pieces }^{2}\end{array}$ & $\begin{array}{c}\text { Consolidated } \\
\text { Assemblies }\end{array}$ & $\begin{array}{c}\text { Number of } \\
\text { Assemblies } \\
\text { Consolidated }\end{array}$ & $\begin{array}{l}\text { Unknown } \\
\text { Contents }^{b}\end{array}$ & \\
\hline \multirow{2}{*}{ Alabama Power Company ...... } & Farley 1 & PWR & 0101 & 0 & 0 & 0 & 0 & 0 & 0 \\
\hline & Farley 2 & PWR & 0102 & 0 & $\mathbf{0}$ & 0 & 0 & 0 & 0 \\
\hline \multirow[t]{3}{*}{ Arizona Public Service Company } & Palo Verde 1 & PWR & 0301 & 0 & 3 & 0 & 0 & 0 & 3 \\
\hline & Palo Verde 2 & PWR & 0302 & 0 & 2 & 0 & 0 & 0 & 2 \\
\hline & Palo Verde 3 & PWR & 0303 & 0 & 1 & 0 & 0 & 0 & 1 \\
\hline \multirow[t]{2}{*}{ Arkansas Power and Light Company } & Arkansas Nuclear 1 & PWR & 0401 & 0 & 0 & 0 & 0 & 0 & 0 \\
\hline & Arkansas Nuclear 2 & PWR & 0402 & 0 & 1 & 0 & 0 & 0 & 1 \\
\hline Baltimore Gas and Electric Company . & Calvert Cliffs $1 \& 2$ & PWR & 0501 & 0 & 0 & 0 & 0 & 0 & 0 \\
\hline Boston Edison Company . . . . . . . . & Pilgrim 1 & BWR & 0601 & 1 & 0 & 0 & 0 & 0 & 1 \\
\hline \multirow[t]{4}{*}{ Carolina Power and Light Company } & Brunswick 1 & BWR & 0701 & 0 & 1 & 0 & 0 & 0 & 1 \\
\hline & Brunswick 2 & BWR & 0702 & 0 & 1 & 0 & 0 & 0 & 1 \\
\hline & Harris 1 & PWR & 0703 & 0 & 1 & 0 & 0 & 0 & 1 \\
\hline & Robinson 2 & PWR & 0705 & 0 & 1 & 0 & 0 & 0 & 1 \\
\hline \multicolumn{10}{|l|}{ Cleveland Electric Illuminating } \\
\hline Company $\ldots \ldots \ldots \ldots \ldots \ldots$ & Perry 1 & BWR & 0901 & 3 & 1 & 0 & 0 & 0 & 4 \\
\hline \multirow[t]{8}{*}{ Commonwealth Edison Company } & Braidwood 1 \& 2 & PWR & 1001 & 0 & 1 & 0 & 0 & 0 & 1 \\
\hline & Byron $1 \& 2$ & PWR & 1003 & 2 & 0 & 0 & 0 & 0 & 2 \\
\hline & Dresden 1 & BWR & 1005 & 0 & 1 & 0 & 0 & 0 & 1 \\
\hline & Dresden 2 & BWR & 1006 & 0 & 2 & 0 & 0 & 0 & 2 \\
\hline & Dresden 3 & BWR & 1007 & 0 & 2 & 0 & 0 & 0 & 2 \\
\hline & LaSalle County $1 \& 2$ & BWR & 1008 & 0 & 1 & 0 & 0 & 0 & 1 \\
\hline & Quad Cities 1 \& 2 & BWR & 1010 & 0 & 1 & 0 & 0 & 0 & 1 \\
\hline & Zion $1 \& 2$ & PWR & 1012 & 0 & 3 & 0 & 0 & 0 & 3 \\
\hline \multicolumn{10}{|l|}{ Consolidated Edison Company } \\
\hline \multirow[t]{2}{*}{ 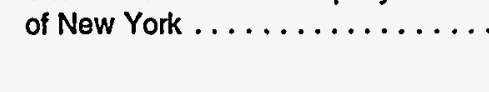 } & Indian Point 1 & PWR & 1101 & 0 & 0 & 0 & 0 & 0 & 0 \\
\hline & Indian Point 2 & PWR & 1102 & 0 & 0 & 0 & 0 & 0 & 0 \\
\hline \multirow[t]{2}{*}{ Consumers Power Company ....... } & Big Rock Point & BWR & 1201 & 0 & 7 & 0 & 0 & 0 & 7 \\
\hline & Palisades & PWR & 1204 & 0 & 2 & 5 & 17 & 0 & 7 \\
\hline Dairyland Power Cooperative $\ldots \ldots$ & LaCrosse & BWR & 1301 & 0 & 0 & 0 & 0 & 0 & 0 \\
\hline Detroit Edison Company ......... & Enrico Fermi 2 & BWR & 1402 & 0 & 0 & 0 & 0 & 0 & 0 \\
\hline
\end{tabular}

See footnotes at end of table. 
Table 19. Canisters Containing Spent Fuel as of December 31, 1993 (Continued)

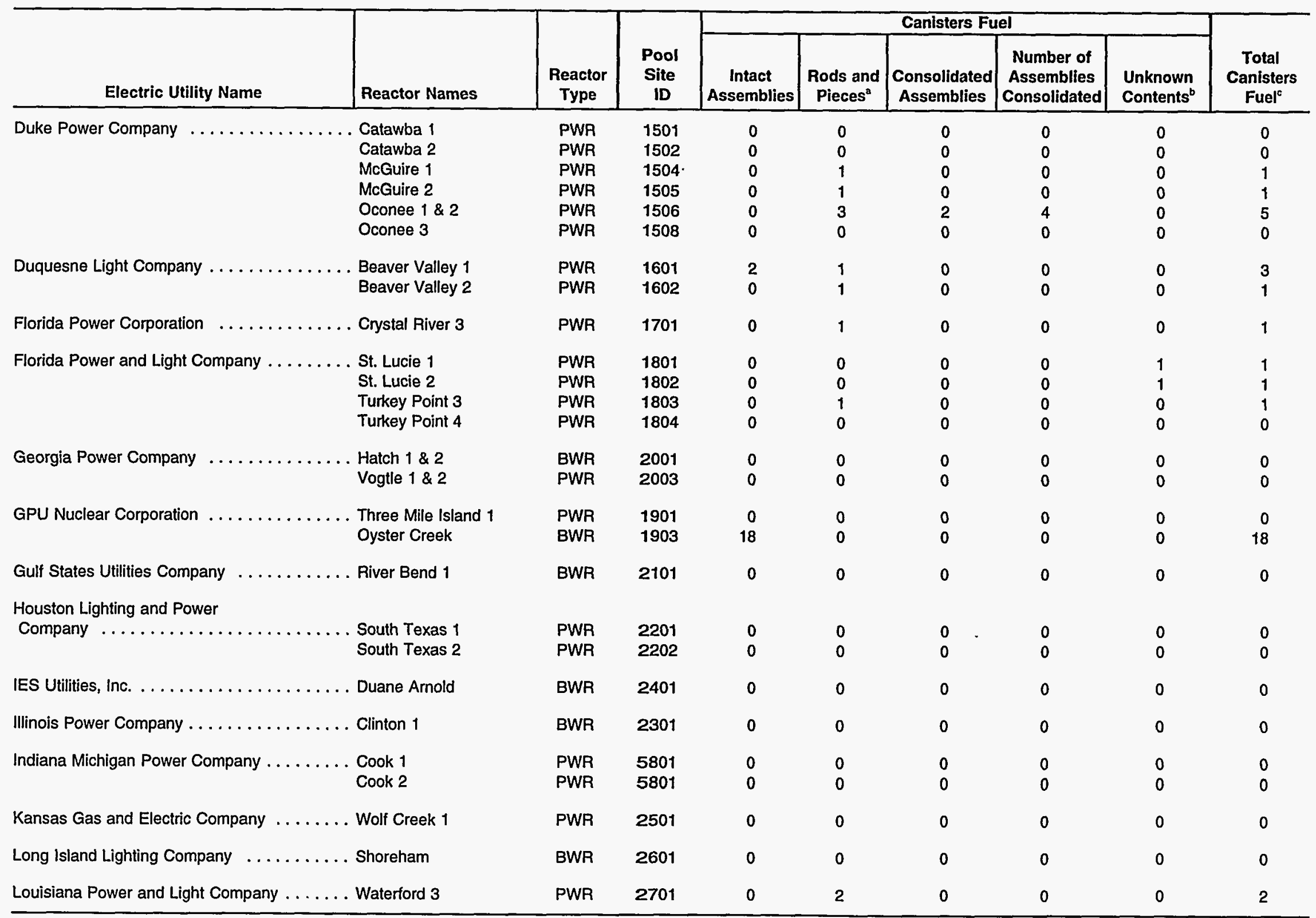

See footnotes at end of table. 
Table 19. Canisters Containing Spent Fuel as of December 31, 1993 (Continued)

\begin{tabular}{|c|c|c|c|c|c|c|c|c|c|}
\hline \multirow[b]{2}{*}{ Electric Utility Name } & \multirow[b]{2}{*}{ Reactor Names } & \multirow[b]{2}{*}{$\begin{array}{c}\text { Reactor } \\
\text { Type }\end{array}$} & \multirow[b]{2}{*}{$\begin{array}{c}\text { Pool } \\
\text { Site } \\
\text { ID }\end{array}$} & \multicolumn{5}{|c|}{ Canisters Fuel } & \multirow[b]{2}{*}{$\begin{array}{l}\text { Total } \\
\text { Canisters }^{\text {Fuel }} \\
\end{array}$} \\
\hline & & & & $\begin{array}{c}\text { Intact } \\
\text { Assemblies }\end{array}$ & $\begin{array}{c}\text { Rods and } \\
\text { Pieces }^{\mathrm{a}}\end{array}$ & \begin{tabular}{c|} 
Consolidated \\
Assemblies
\end{tabular} & $\begin{array}{c}\text { Number of } \\
\text { Assemblies } \\
\text { Consolidated }\end{array}$ & $\begin{array}{l}\text { Unknown } \\
\text { Contents }^{\mathrm{b}}\end{array}$ & \\
\hline $\begin{array}{l}\text { Maine Yankee Atomic Power } \\
\text { Company } \ldots \ldots \ldots \ldots \ldots \ldots \ldots\end{array}$ & Maine Yankee & PWR & 2801 & 0 & 2 & 0 & 0 & 0 & 2 \\
\hline Nebraska Public Power District .... & Cooper Station & BWR & 3001 & 0 & 0 & 0 & 0 & 0 & 0 \\
\hline New York Power Authority... & $\begin{array}{l}\text { FitzPatrick } \\
\text { Indian Point } 3\end{array}$ & $\begin{array}{l}\text { BWR } \\
\text { PWR }\end{array}$ & $\begin{array}{l}3901 \\
3902\end{array}$ & $\begin{array}{l}0 \\
0\end{array}$ & $\begin{array}{l}0 \\
0\end{array}$ & $\begin{array}{l}0 \\
0\end{array}$ & $\begin{array}{l}0 \\
0\end{array}$ & $\begin{array}{l}0 \\
0\end{array}$ & $\begin{array}{l}0 \\
0\end{array}$ \\
\hline Niagara Mohawk Power Corporation & $\begin{array}{l}\text { Nine Mile Point } 1 \\
\text { Nine Mile Point } 2\end{array}$ & $\begin{array}{l}\text { BWR } \\
\text { BWR }\end{array}$ & $\begin{array}{l}3101 \\
3102\end{array}$ & $\begin{array}{l}0 \\
0\end{array}$ & $\begin{array}{l}0 \\
0\end{array}$ & $\begin{array}{l}0 \\
0\end{array}$ & $\begin{array}{l}0 \\
0\end{array}$ & $\begin{array}{l}0 \\
0\end{array}$ & $\begin{array}{l}0 \\
0\end{array}$ \\
\hline $\begin{array}{l}\text { North Atlantic Energy Service } \\
\text { Corporation ............. }\end{array}$ & Seabrook & PWR & 5901 & 0 & 0 & 0 & 0 & 0 & 0 \\
\hline Northeast Utilities Service Company & $\begin{array}{l}\text { Millstone } 1 \\
\text { Millstone } 2 \\
\text { Millstone } 3 \\
\text { Haddam Neck }\end{array}$ & $\begin{array}{l}\text { BWR } \\
\text { PWR } \\
\text { PWR } \\
\text { PWR }\end{array}$ & $\begin{array}{l}3201 \\
3202 \\
3203 \\
5701\end{array}$ & $\begin{array}{l}0 \\
0 \\
0 \\
0\end{array}$ & $\begin{array}{l}1 \\
3 \\
0 \\
5\end{array}$ & $\begin{array}{l}0 \\
3 \\
0 \\
0\end{array}$ & $\begin{array}{l}0 \\
6 \\
0 \\
0\end{array}$ & $\begin{array}{l}0 \\
0 \\
0 \\
0\end{array}$ & $\begin{array}{l}1 \\
6 \\
0 \\
5\end{array}$ \\
\hline Northern States Power Company ... & $\begin{array}{l}\text { Monticello } \\
\text { Prairie Island } 1 \text { \& } 2\end{array}$ & $\begin{array}{l}\text { BWR } \\
\text { PWR }\end{array}$ & $\begin{array}{l}3301 \\
3302\end{array}$ & $\begin{array}{l}0 \\
0\end{array}$ & $\begin{array}{l}0 \\
2\end{array}$ & $\begin{array}{r}0 \\
18\end{array}$ & $\begin{array}{r}0 \\
36\end{array}$ & $\begin{array}{l}0 \\
0\end{array}$ & $\begin{array}{r}0 \\
20\end{array}$ \\
\hline Omaha Public Power District . & Fort Calhoun & PWR & 3401 & 0 & 0 & 0 & 0 & 0 & 0 \\
\hline Pacific Gas and Electric Company . & $\begin{array}{l}\text { Diablo Canyon } 1 \\
\text { Diablo Canyon } 2 \\
\text { Humboldt Bay }\end{array}$ & $\begin{array}{l}\text { PWR } \\
\text { PWR } \\
\text { BWR }\end{array}$ & $\begin{array}{l}3501 \\
3502 \\
3503\end{array}$ & $\begin{array}{l}0 \\
0 \\
1\end{array}$ & $\begin{array}{l}0 \\
1 \\
0\end{array}$ & $\begin{array}{l}0 \\
0 \\
0\end{array}$ & $\begin{array}{l}0 \\
0 \\
0\end{array}$ & $\begin{array}{l}0 \\
0 \\
0\end{array}$ & $\begin{array}{l}0 \\
1 \\
1\end{array}$ \\
\hline PECO Energy Company ........ & $\begin{array}{l}\text { Limerick } 1 \& 2 \\
\text { Peach Bottom } 2 \\
\text { Peach Bottom } 3\end{array}$ & $\begin{array}{l}\text { BWR } \\
\text { BWR } \\
\text { BWR }\end{array}$ & $\begin{array}{l}3701 \\
3704 \\
3705\end{array}$ & $\begin{array}{l}0 \\
0 \\
0\end{array}$ & $\begin{array}{l}1 \\
2 \\
1\end{array}$ & $\begin{array}{l}0 \\
0 \\
0\end{array}$ & $\begin{array}{l}0 \\
0 \\
0\end{array}$ & $\begin{array}{l}0 \\
0 \\
0\end{array}$ & $\begin{array}{l}1 \\
2 \\
1\end{array}$ \\
\hline $\begin{array}{l}\text { Pennsylvania Power and Light } \\
\text { Company } \ldots \ldots \ldots \ldots \ldots \ldots\end{array}$ & Susquehanna $1 \& 2$ & BWR & 3601 & 0 & 1 & 0 & 0 & 0 & 1 \\
\hline Portland General Electric Company & Trojan & PWR & 3801 & 0 & 13 & 0 & 0 & 0 & 13 \\
\hline Public Service Company of Colorado & Fort St. Vrain & HTGR & 4101 & 0 & 0 & 0 & 0 & 0 & 0 \\
\hline
\end{tabular}

\footnotetext{
See footnotes at end of table.
} 
Table 19. Canisters Containing Spent Fuel as of December 31, 1993 (Continued)

\begin{tabular}{|c|c|c|c|c|c|c|c|c|c|}
\hline \multirow[b]{2}{*}{ Electric Utility Name } & \multirow[b]{2}{*}{ Reactor Names } & \multirow[b]{2}{*}{$\begin{array}{c}\text { Reactor } \\
\text { Type }\end{array}$} & \multirow[b]{2}{*}{$\begin{array}{l}\text { Pool } \\
\text { Site } \\
\text { ID }\end{array}$} & \multicolumn{5}{|c|}{ Canisters Fuel } & \multirow[b]{2}{*}{$\begin{array}{c}\text { Total } \\
\text { Canisters } \\
\text { Fuel }^{6}\end{array}$} \\
\hline & & & & $\begin{array}{c}\text { Intact } \\
\text { Assemblies }\end{array}$ & $\begin{array}{c}\text { Rods and } \\
\text { Pieces }^{\mathrm{a}}\end{array}$ & $\begin{array}{c}\text { Consolidated } \\
\text { Assemblies }\end{array}$ & $\begin{array}{c}\text { Number of } \\
\text { Assemblies } \\
\text { Consolidated }\end{array}$ & $\begin{array}{l}\text { Unknown } \\
\text { Contents }^{b}\end{array}$ & \\
\hline \multicolumn{10}{|l|}{ Public Service Electric and Gas } \\
\hline Company . . . . . . . . . . & Hope Creek & BWA & 4201 & 0 & 0 & 0 & 0 & 0 & 0 \\
\hline & Salem 1 & PWR & 4202 & 0 & 0 & 0 & 0 & 0 & 0 \\
\hline & Salem 2 & PWR & 4203 & 0 & 0 & 0 & 0 & 0 & 0 \\
\hline \multicolumn{10}{|l|}{ Rochester Gas and Electric } \\
\hline Corporation $\ldots \ldots \ldots \ldots \ldots$ & Ginna & PWR & 4401 & 0 & 0 & 8 & 11 & 0 & 8 \\
\hline Sacramento Municipal Utility District & Rancho Seco & PWR & 4501 & 0 & 0 & 0 & 0 & 0 & 0 \\
\hline \multicolumn{10}{|l|}{ South Carolina Electric and } \\
\hline Gas Company $\ldots \ldots \ldots \ldots \ldots$ & Summer & PWR & 4601 & 0 & 0 & 0 & 0 & 0 & 0 \\
\hline \multirow[t]{3}{*}{ Southern California Edison Company } & San Onofre 1 & PWR & 4701 & 0 & 0 & 0 & 0 & 0 & 0 \\
\hline & San Onofre 2 & PWR & 4702 & 0 & 0 & 0 & 0 & 0 & 0 \\
\hline & San Onofre 3 & PWR & 4703 & 0 & 0 & 0 & 0 & 0 & 0 \\
\hline System Energy Resources, Inc. . . . . & Grand Gulf 1 & BWR & 2901 & 0 & 1 & 0 & 0 & 0 & 1 \\
\hline \multirow[t]{6}{*}{ Tennessee Valley Authority. } & Bellefonte 1 & PWR & 4801 & 0 & 0 & 0 & 0 & 0 & 0 \\
\hline & Bellefonte 2 & PWR & 4802 & 0 & 0 & 0 & 0 & 0 & 0 \\
\hline & Browns Ferry $1 \& 2$ & BWR & 4803 & 1 & 0 & 0 & 0 & 0 & 1 \\
\hline & Browns Ferry 3 & BWR & 4805 & 0 & 0 & 0 & $>9$ & 0 & 0 \\
\hline & Sequoyah 1 \& 2 & PWR & 4808 & 0 & 0 & 0 & 0 & 0 & 0 \\
\hline & Watts Bar $1 \& 2$ & PWR & 4810 & 0 & 0 & 0 & 0 & 0 & 0 \\
\hline Toledo Edison Company ........ & Davis-Besse & PWR & 5001 & 0 & 1 & 0 & 0 & 0 & 1 \\
\hline TU Electric $\ldots \ldots \ldots \ldots \ldots$ & Comanche Peak $1 \& 2$ & PWR & 4901 & 0 & 0 & 0 & 0 & 0 & 0 \\
\hline Union Electric Company $\ldots \ldots \ldots$ & Callaway & PWR & 5101 & 0 & 1 & 0 & 0 & 0 & 1 \\
\hline \multicolumn{10}{|l|}{ Vermont Yankee Nuclear Power } \\
\hline Corporation $\ldots \ldots \ldots \ldots \ldots$ & Vermont Yankee & BWR & 6001 & 0 & 0 & 0 & 0 & 0 & 0 \\
\hline \multirow[t]{2}{*}{ Virginia Power } & North Anna $1 \& 2$ & PWR & 5201 & 0 & 1 & 0 & 0 & 0 & 1 \\
\hline & Surry $1 \& 2$ & PWR & 5203 & 0 & 2 & 0 & 0 & 0 & 2 \\
\hline \multicolumn{10}{|l|}{ Washington Public Power Supply } \\
\hline System $\ldots \ldots \ldots \ldots \ldots \ldots$ & Washington Nuclear 2 & BWR & 5302 & 0 & 0 & 0 & 0 & 0 & 0 \\
\hline
\end{tabular}

See footnotes at end of table. 
Table 19. Canisters Containing Spent Fuel as of December 31, 1993 (Continued)

\begin{tabular}{|c|c|c|c|c|c|c|c|c|c|}
\hline \multirow[b]{2}{*}{ Electric Utility Name } & \multirow[b]{2}{*}{ Reactor Names } & \multirow[b]{2}{*}{$\begin{array}{c}\text { Reactor } \\
\text { Type }\end{array}$} & \multirow[b]{2}{*}{$\begin{array}{l}\text { Pool } \\
\text { Site } \\
\text { ID }\end{array}$} & \multicolumn{5}{|c|}{ Canisters Fuel } & \multirow[b]{2}{*}{$\begin{array}{c}\text { Total } \\
\text { Canisters } \\
\text { Fuel }^{c}\end{array}$} \\
\hline & & & & $\begin{array}{c}\text { Intact } \\
\text { Assemblies }\end{array}$ & $\begin{array}{l}\text { Rods and } \\
\text { Pieces }^{\mathrm{a}}\end{array}$ & \begin{tabular}{l|} 
Consolidated \\
Assemblies
\end{tabular} & $\begin{array}{c}\text { Number of } \\
\text { Assemblies } \\
\text { Consolidated }\end{array}$ & $\begin{array}{l}\text { Unknown } \\
\text { Contents }^{\circ}\end{array}$ & \\
\hline \multicolumn{2}{|c|}{ Wisconsin Electric Power Company ....... Point Beach $1 \& 2$} & PWR & 5401 & 12 & 1 & 0 & 0 & 0 & 13 \\
\hline \multicolumn{2}{|c|}{ Wisconsin Public Service Corporation ...... Kewaunee } & PWR & 5501 & 0 & 0 & 0 & 0 & 0 & 0 \\
\hline \multicolumn{2}{|l|}{ Yankee Atomic Electric Company } & PWR & 5601 & 0 & 0 & 0 & 0 & 0 & 0 \\
\hline \multirow{3}{*}{\multicolumn{4}{|c|}{ 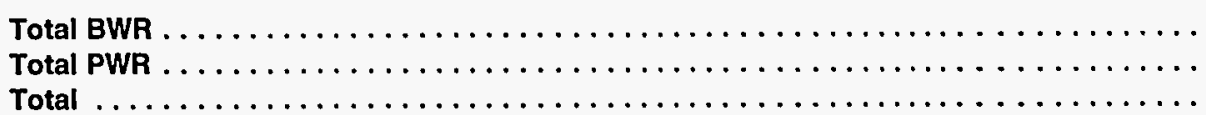 }} & 24 & 24 & 0 & 0 & 0 & 48 \\
\hline & & & & 16 & 58 & 36 & 78 & 2 & 112 \\
\hline & & & & 40 & 82 & 36 & 78 & 2 & 160 \\
\hline
\end{tabular}

a Canisters with Rods and Pieces column represents the number of canisters containing intact fuel rods from consolidation, reconstitution, or reconstruction.

bUnknown Contents column represents the number of canisters containing fuel for which the specific contents of the canister were not reported.

Total includes 145 canisters containing only fuel and 15 canisters containing both fuel and nonfuel items.

PWR = Pressurized-water reactor; BWR = Boiling-water reactor; HTGR = High-temperature, gas-cooled reactor.

Source: Energy Information Administration, Form RW-859, "Nuclear Fuel Data" (1993). 


\begin{tabular}{|c|c|c|c|c|c|c|c|c|c|}
\hline \multirow[b]{2}{*}{ Electric Utility Name } & \multirow[b]{2}{*}{ Reactor Names } & \multirow[b]{2}{*}{$\begin{array}{c}\text { Reactor } \\
\text { Type }\end{array}$} & \multirow[b]{2}{*}{$\begin{array}{l}\text { Pool } \\
\text { Site } \\
\text { ID }\end{array}$} & \multicolumn{5}{|c|}{ Canister Nonfuel } & \multirow[b]{2}{*}{$\begin{array}{c}\text { Total } \\
\text { Nonfuel } \\
\text { Canisters }^{\circ}\end{array}$} \\
\hline & & & & Debris $^{a}$ & \begin{tabular}{|c|} 
Nonfuel \\
Disassembly \\
Hardware
\end{tabular} & $\begin{array}{c}\text { Nonfuel } \\
\text { Components }^{b}\end{array}$ & Other $^{c}$ & $\begin{array}{l}\text { Unknown } \\
\text { Contents }^{d}\end{array}$ & \\
\hline \multirow[t]{2}{*}{ Alabama Power Company. } & Farley 1 & PWR & 0101 & 0 & 0 & 0 & 0 & 0 & 0 \\
\hline & Farley 2 & PWR & 0102 & 0 & 0 & 0 & 0 & 0 & 0 \\
\hline \multirow[t]{3}{*}{ Arizona Public Service Company } & Palo Verde 1 & PWR & 0301 & 1 & 2 & 4 & 0 & 0 & 5 \\
\hline & Palo Verde 2 & PWR & 0302 & 0 & 4 & 4 & 0 & 0 & 6 \\
\hline & Palo Verde 3 & PWR & 0303 & 0 & 1 & 3 & 0 & 0 & 4 \\
\hline \multirow[t]{2}{*}{ Arkansas Power and Light Company ..... } & Arkansas Nuclear 1 & PWR & 0401 & 0 & 0 & 0 & 0 & 0 & 0 \\
\hline & Arkansas Nuclear 2 & PWR & 0402 & 0 & 0 & 0 & 0 & 0 & 0 \\
\hline Baltimore Gas and Electric Company ..... & Calvert Cliffs 1 \& 2 & PWR & 0501 & 0 & 4 & 7 & 10 & 0 & 21 \\
\hline Boston Edison Company ............. & Pilgrim 1 & BWR & 0601 & 0 & 0 & 0 & 0 & 0 & 0 \\
\hline \multirow[t]{4}{*}{ Carolina Power and Light Company ...... } & Brunswick 1 & BWR & 0701 & 0 & 0 & 0 & 0 & 0 & 0 \\
\hline & Brunswick 2 & BWR & 0702 & 0 & 0 & 0 & 0 & 0 & 0 \\
\hline & Harris 1 & PWR & 0703 & 0 & 0 & 0 & 0 & 0 & 0 \\
\hline & Robinson 2 & PWR & 0705 & 0 & 0 & 0 & 2 & 0 & 2 \\
\hline \multicolumn{10}{|l|}{ Cleveland Electric Illuminating } \\
\hline Company $\ldots \ldots \ldots \ldots \ldots \ldots$ & Perry 1 & BWR & 0901 & 0 & 0 & 0 & 0 & 0 & 0 \\
\hline \multirow[t]{8}{*}{ Commonwealth Edison Company } & Braidwood $1 \& 2$ & PWR & 1001 & 0 & 0 & 0 & 2 & 0 & 2 \\
\hline & Byron 1 \& 2 & PWR & 1003 & 0 & 0 & 0 & 0 & 0 & 0 \\
\hline & Dresden 1 & BWR & 1005 & 0 & 0 & 0 & 0 & 0 & 0 \\
\hline & Dresden 2 & BWR & 1006 & 0 & 0 & 0 & 0 & 0 & 0 \\
\hline & Dresden 3 & BWR & 1007 & 0 & 0 & 0 & 0 & 0 & 0 \\
\hline & LaSalle County $1 \& 2$ & BWR & 1008 & 0 & 0 & 0 & 0 & 0 & 0 \\
\hline & Quad Cities 1 \& 2 & BWR & 1010 & 0 & 0 & 0 & 0 & 0 & 0 \\
\hline & Zion $1 \& 2$ & PWR & 1012 & 0 & 0 & 0 & 2 & 0 & 2 \\
\hline \multirow[t]{2}{*}{ Consolidated Edison Company of New York } & Indian Point 1 & PWR & 1101 & 0 & 0 & 0 & 0 & 0 & 0 \\
\hline & Indian Point 2 & PWR & 1102 & 0 & 0 & 0 & 0 & 0 & 0 \\
\hline \multirow[t]{2}{*}{ Consumers Power Company ... } & Big Rock Point & BWR & 1201 & 0 & 0 & 0 & 0 & 0 & 0 \\
\hline & Palisades & PWR & 1204 & 0 & 0 & 0 & 0 & 0 & 0 \\
\hline Dairyland Power Cooperative $\ldots . . .$. & LaCrosse & BWR & 1301 & 0 & 0 & 0 & 0 & 0 & 0 \\
\hline Detroit Edison Company .............. & Enrico Fermi 2 & BWR & 1402 & 0 & 0 & 0 & 0 & 0 & 0 \\
\hline
\end{tabular}

\footnotetext{
See footnotes at end of table.
} 
Table 20. Canisters Containing Nonfuel Components as of December 31, 1993 (Continued)

\begin{tabular}{|c|c|c|c|c|c|c|c|c|c|}
\hline \multirow[b]{2}{*}{ Electric Utility Name } & \multirow[b]{2}{*}{ Reactor Names } & \multirow[b]{2}{*}{$\begin{array}{c}\text { Reactor } \\
\text { Type }\end{array}$} & \multirow[b]{2}{*}{$\begin{array}{l}\text { Pool } \\
\text { Site } \\
\text { ID } \\
\end{array}$} & \multicolumn{5}{|c|}{ Canister Nonfuel } & \multirow[b]{2}{*}{$\begin{array}{c}\text { Total } \\
\text { Nonfuel } \\
\text { Canisters }\end{array}$} \\
\hline & & & & Debris $^{\mathrm{a}}$ & $\begin{array}{c}\text { Nonfuel } \\
\text { Disassembly } \\
\text { Hardware }\end{array}$ & $\begin{array}{c}\begin{array}{c}\text { Nonfuel } \\
\text { Components }\end{array} \\
\end{array}$ & Other ${ }^{c}$ & $\begin{array}{l}\text { Unknown } \\
\text { Contents }^{d}\end{array}$ & \\
\hline \multirow[t]{6}{*}{ Duke Power Company } & Catawba 1 & PWR & 1501 & 0 & 0 & 0 & 0 & 0 & 0 \\
\hline & Catawba 2 & PWR & 1502 & 0 & 0 & 0 & 0 & 0 & 0 \\
\hline & McGuire 1 & PWR & 1504 & 1 & 0 & 0 & 0 & 0 & 1 \\
\hline & McGuire 2 & PWR & 1505 & 0 & 0 & 0 & 0 & 0 & 0 \\
\hline & Oconee $1 \& 2$ & PWR & 1506 & 0 & 0 & 0 & 0 & 0 & 0 \\
\hline & Oconee 3 & PWR & 1508 & 0 & 0 & 0 & 0 & 0 & 0 \\
\hline \multirow{2}{*}{ Duquesne Light Company . } & Beaver Valley 1 & PWR & 1601 & 0 & 1 & 0 & 1 & 0 & 1 \\
\hline & Beaver Valley 2 & PWR & 1602 & 0 & 0 & 1 & 0 & 0 & 0 \\
\hline Florida Power Corporation & Crystal River 3 & PWR & 1701 & 0 & 1 & 1 & 1 & 0 & 1 \\
\hline \multirow[t]{4}{*}{ Florida Power and Light Company . } & St. Lucie 1 & PWR & 1801 & 0 & 0 & 0 & 0 & 4 & 4 \\
\hline & St. Lucie 2 & PWR & 1802 & 0 & 0 & 0 & 0 & 1 & 1 \\
\hline & Turkey Point 3 & PWR & 1803 & 0 & 0 & 0 & 0 & 0 & 0 \\
\hline & Turkey Point 4 & PWR & 1804 & 0 & 0 & 0 & 0 & 0 & 0 \\
\hline \multirow[t]{2}{*}{ Georgia Power Company } & Hatch $1 \& 2$ & BWR & 2001 & 0 & 0 & 0 & 0 & 0 & 0 \\
\hline & Vogtle $1 \& 2$ & PWR & 2003 & 0 & 0 & 0 & 0 & 0 & 0 \\
\hline \multirow[t]{2}{*}{ GPU Nuclear Corporation } & Three Mile Island 1 & PWR & 1901 & 0 & 0 & 0 & 0 & 0 & 0 \\
\hline & Oyster Creek & BWR & 1903 & 0 & 0 & 0 & 0 & 0 & 0 \\
\hline Gulf States Utilities Company & River Bend 1 & BWR & 2101 & 0 & 0 & 0 & 0 & 0 & 0 \\
\hline \multirow[t]{2}{*}{ Houston Lighting and Power Company } & South Texas 1 & PWR & 2201 & 0 & 0 & 1 & 0 & 0 & 1 \\
\hline & South Texas 2 & PWR & 2202 & 0 & 0 & 0 & 0 & 0 & 0 \\
\hline IES Utilitities, Inc. . . . . . . . . . & Duane Arnold & BWR & 2401 & 0 & 0 & 0 & 0 & 0 & 0 \\
\hline Illinois Power Company ........... & Clinton 1 & BWR & 2301 & 0 & 0 & 0 & 0 & 0 & 0 \\
\hline \multirow[t]{2}{*}{ Indiana Michigan Power Company .. } & Cook 1 & PWR & 5801 & 0 & 0 & 0 & 0 & 0 & 0 \\
\hline & Cook 2 & PWR & 5801 & 0 & 0 & 0 & 0 & 0 & 0 \\
\hline Kansas Gas and Electric Company . & Wolf Creek 1 & PWR & 2501 & 0 & 0 & 0 & 0 & 0 & 0 \\
\hline Long Island Lighting Company $\ldots$ & Shoreham & BWR & 2601 & 0 & 0 & 0 & 0 & 0 & 0 \\
\hline Louisiana Power and Light Company .. & Waterford 3 & PWR & 2701 & 2 & 4 & 4 & 0 & 0 & 4 \\
\hline
\end{tabular}

\footnotetext{
See footnotes at end of table.
} 
Table 20. Canisters Containing Nonfuel Components as of December 31, 1993 (Continued)

\begin{tabular}{|c|c|c|c|c|c|c|c|c|c|}
\hline \multirow[b]{2}{*}{ Electric Utility Name } & \multirow[b]{2}{*}{ Reactor Names } & \multirow[b]{2}{*}{$\begin{array}{c}\text { Reactor } \\
\text { Type }\end{array}$} & \multirow[b]{2}{*}{$\begin{array}{l}\text { Pool } \\
\text { Site } \\
\text { ID }\end{array}$} & \multicolumn{5}{|c|}{ Canister Nonfuel } & \multirow[b]{2}{*}{$\begin{array}{c}\text { Total } \\
\text { Nonfuel } \\
\text { Canisters }^{\circ}\end{array}$} \\
\hline & & & & Debris $^{a}$ & \begin{tabular}{|c|} 
Nonfuel \\
Disassembly \\
Hardware \\
\end{tabular} & $\begin{array}{c}\text { Nonfuel } \\
\text { Components }\end{array}$ & Other & $\begin{array}{l}\text { Unknown } \\
\text { Contents }^{d}\end{array}$ & \\
\hline Maine Yankee Atomic Power Company & Maine Yankee & PWR & 2801 & 0 & 0 & 5 & 10 & 0 & 14 \\
\hline Nebraska Public Power District ........ & Cooper Station & BWR & 3001 & 0 & 0 & 0 & 0 & 0 & 0 \\
\hline New York Power Authority ............ & $\begin{array}{l}\text { FitzPatrick } \\
\text { Indian Point } 3\end{array}$ & $\begin{array}{l}\text { BWR } \\
\text { PWR }\end{array}$ & $\begin{array}{l}3901 \\
3902\end{array}$ & $\begin{array}{l}0 \\
0\end{array}$ & $\begin{array}{l}0 \\
0\end{array}$ & $\begin{array}{l}0 \\
0\end{array}$ & $\begin{array}{l}0 \\
0\end{array}$ & $\begin{array}{l}0 \\
0\end{array}$ & $\begin{array}{l}0 \\
0\end{array}$ \\
\hline Niagara Mohawk Power Corporation .... & $\begin{array}{l}\text { Nine Mile Point } 1 \\
\text { Nine Mile Point } 2\end{array}$ & $\begin{array}{l}\text { BWR } \\
\text { BWR }\end{array}$ & $\begin{array}{l}3101 \\
3102\end{array}$ & $\begin{array}{l}0 \\
0\end{array}$ & $\begin{array}{l}0 \\
0\end{array}$ & $\begin{array}{l}0 \\
0\end{array}$ & $\begin{array}{l}0 \\
0\end{array}$ & $\begin{array}{l}0 \\
0\end{array}$ & $\begin{array}{l}0 \\
0\end{array}$ \\
\hline North Atlantic Energy Service Corporation & Seabrook & PWR & 5901 & 0 & 0 & 0 & 0 & 0 & 0 \\
\hline Northeast Utilities Service Company & $\begin{array}{l}\text { Millstone } 1 \\
\text { Millstone } 2 \\
\text { Millstone } 3 \\
\text { Haddam Neck }\end{array}$ & $\begin{array}{l}\text { BWR } \\
\text { PWR } \\
\text { PWR } \\
\text { PWR }\end{array}$ & $\begin{array}{l}3201 \\
3202 \\
3203 \\
5701\end{array}$ & $\begin{array}{l}0 \\
0 \\
0 \\
0\end{array}$ & $\begin{array}{l}0 \\
1 \\
0 \\
0\end{array}$ & $\begin{array}{l}0 \\
3 \\
0 \\
1\end{array}$ & $\begin{array}{l}0 \\
0 \\
0 \\
3\end{array}$ & $\begin{array}{l}0 \\
0 \\
0 \\
0\end{array}$ & $\begin{array}{l}0 \\
4 \\
0 \\
4\end{array}$ \\
\hline Northern States Power Company ....... & $\begin{array}{l}\text { Monticello } \\
\text { Prairie Island } 1 \text { \& } 2\end{array}$ & $\begin{array}{l}\text { BWR } \\
\text { PWR }\end{array}$ & $\begin{array}{l}3301 \\
3302\end{array}$ & $\begin{array}{l}0 \\
0\end{array}$ & $\begin{array}{l}0 \\
0\end{array}$ & $\begin{array}{l}0 \\
2\end{array}$ & $\begin{array}{l}0 \\
0\end{array}$ & $\begin{array}{l}0 \\
0\end{array}$ & $\begin{array}{l}0 \\
2\end{array}$ \\
\hline Omaha Public Power District ........ & Fort Calhoun & PWR & 3401 & 0 & 0 & 0 & 0 & 0 & 0 \\
\hline Pacific Gas and Electric Company ... & $\begin{array}{l}\text { Diablo Canyon } 1 \\
\text { Diablo Canyon } 2 \\
\text { Humboldt Bay }\end{array}$ & $\begin{array}{l}\text { PWR } \\
\text { PWR } \\
\text { BWR }\end{array}$ & $\begin{array}{l}3501 \\
3502 \\
3503\end{array}$ & $\begin{array}{l}0 \\
0 \\
0\end{array}$ & $\begin{array}{l}0 \\
0 \\
0\end{array}$ & $\begin{array}{l}0 \\
0 \\
0\end{array}$ & $\begin{array}{l}0 \\
0 \\
0\end{array}$ & $\begin{array}{l}0 \\
0 \\
0\end{array}$ & $\begin{array}{l}0 \\
0 \\
0\end{array}$ \\
\hline PECO Energy Company ...... & $\begin{array}{l}\text { Limerick } 1 \text { \& } 2 \\
\text { Peach Bottom } 2 \\
\text { Peach Bottom } 3\end{array}$ & $\begin{array}{l}\text { BWR } \\
\text { BWR } \\
\text { BWR }\end{array}$ & $\begin{array}{l}3701 \\
3704 \\
3705\end{array}$ & $\begin{array}{l}0 \\
0 \\
0\end{array}$ & $\begin{array}{l}0 \\
0 \\
0\end{array}$ & $\begin{array}{l}0 \\
0 \\
0\end{array}$ & $\begin{array}{l}0 \\
0 \\
0\end{array}$ & $\begin{array}{l}0 \\
0 \\
0\end{array}$ & $\begin{array}{l}0 \\
0 \\
0\end{array}$ \\
\hline Pennsylvania Power and Light Company & Susquehanna $1 \& 2$ & BWR & 3601 & 0 & 0 & 0 & 0 & 0 & 0 \\
\hline Portland General Electric Company ..... & Trojan & PWR & 3801 & 4 & 6 & 20 & 0 & 0 & 20 \\
\hline Public Service Company of Colorado .... & Fort St. Vrain & HTGR & 4101 & 0 & 0 & 0 & 0 & 0 & 0 \\
\hline Public Service Electric and Gas Company & $\begin{array}{l}\text { Hope Creek } \\
\text { Salem } 1 \\
\text { Salem } 2\end{array}$ & $\begin{array}{l}\text { BWR } \\
\text { PWR } \\
\text { PWR }\end{array}$ & $\begin{array}{l}4201 \\
4202 \\
4203\end{array}$ & $\begin{array}{l}0 \\
0 \\
0\end{array}$ & $\begin{array}{l}0 \\
0 \\
0\end{array}$ & $\begin{array}{l}0 \\
0 \\
0\end{array}$ & $\begin{array}{l}0 \\
0 \\
0\end{array}$ & $\begin{array}{l}0 \\
0 \\
0\end{array}$ & $\begin{array}{l}0 \\
0 \\
0\end{array}$ \\
\hline
\end{tabular}

See footnotes at end of table. 
Table 20. Canisters Containing Nonfuel Components as of December 31, 1993 (Continued)

\begin{tabular}{|c|c|c|c|c|c|c|c|c|c|}
\hline \multirow[b]{2}{*}{ Electric Utility Name } & \multirow[b]{2}{*}{ Reactor Names } & \multirow[b]{2}{*}{$\begin{array}{c}\text { Reactor } \\
\text { Type }\end{array}$} & \multirow[b]{2}{*}{$\begin{array}{l}\text { Pool } \\
\text { Site } \\
\text { ID } \\
\end{array}$} & \multicolumn{5}{|c|}{ Canister Nonfuel } & \multirow[b]{2}{*}{$\begin{array}{c}\text { Total } \\
\text { Nonfuel } \\
\text { Canisters }^{\circ}\end{array}$} \\
\hline & & & & Debris $^{a}$ & $\begin{array}{c}\text { Nonfuel } \\
\text { Disassembly } \\
\text { Hardware }\end{array}$ & $\begin{array}{c}\text { Nonfuel } \\
\text { Components }\end{array}$ & Other $^{e}$ & $\begin{array}{l}\text { Unknown } \\
\text { Contents }^{d}\end{array}$ & \\
\hline Rochester Gas and Electric Corporation .. & Ginna & PWR & 4401 & 0 & 2 & 0 & 0 & 0 & 2 \\
\hline Sacramento Municipal Utility District $\ldots \ldots$ & Rancho Seco & PWR & 4501 & 0 & 0 & 0 & 0 & 0 & 0 \\
\hline South Carolina Electric and Gas Company . & Summer & PWR & 4601 & 0 & 0 & 0 & 0 & 0 & 0 \\
\hline Southem California Edison Company ..... & $\begin{array}{l}\text { San Onofre } 1 \\
\text { San Onofre } 2 \\
\text { San Onofre } 3\end{array}$ & $\begin{array}{l}\text { PWR } \\
\text { PWR } \\
\text { PWR }\end{array}$ & $\begin{array}{l}4701 \\
4702 \\
4703\end{array}$ & $\begin{array}{l}0 \\
0 \\
0\end{array}$ & $\begin{array}{l}0 \\
0 \\
0\end{array}$ & $\begin{array}{l}0 \\
0 \\
0\end{array}$ & $\begin{array}{l}0 \\
0 \\
0\end{array}$ & $\begin{array}{l}0 \\
0 \\
0\end{array}$ & $\begin{array}{l}0 \\
0 \\
0\end{array}$ \\
\hline System Energy Resources, Inc. . & Grand Gulf 1 & BWR & 2901 & 0 & 0 & 0 & 0 & 0 & 0 \\
\hline Tennessee Valley Authority ... & $\begin{array}{l}\text { Bellefonte } 1 \\
\text { Bellefonte } 2 \\
\text { Browns Ferry } 1 \& 2 \\
\text { Browns Ferry } 3 \\
\text { Sequoyah } 1 \& 2 \\
\text { Watts Bar } 1 \& 2\end{array}$ & $\begin{array}{l}\text { PWR } \\
\text { PWR } \\
\text { BWR } \\
\text { BWR } \\
\text { PWR } \\
\text { PWR }\end{array}$ & $\begin{array}{l}4801 \\
4802 \\
4803 \\
4805 \\
4808 \\
4810\end{array}$ & $\begin{array}{l}0 \\
0 \\
0 \\
0 \\
0 \\
0\end{array}$ & $\begin{array}{l}0 \\
0 \\
0 \\
0 \\
0 \\
0\end{array}$ & $\begin{array}{l}0 \\
0 \\
0 \\
0 \\
0 \\
0\end{array}$ & $\begin{array}{l}0 \\
0 \\
0 \\
0 \\
0 \\
0\end{array}$ & $\begin{array}{l}0 \\
0 \\
0 \\
0 \\
0 \\
0\end{array}$ & $\begin{array}{l}0 \\
0 \\
0 \\
0 \\
0 \\
0\end{array}$ \\
\hline Toledo Edison Company ............ & Davis-Besse & PWR & 5001 & 0 & 2 & 1 & 0 & 0 & 3 \\
\hline TU Electric & Comanche Peak $1 \& 2$ & PWR & 4901 & 0 & 0 & 0 & 0 & 0 & 0 \\
\hline Union Electric Company . & Callaway & PWR & 5101 & 0 & 0 & 0 & 0 & 0 & 0 \\
\hline $\begin{array}{l}\text { Vermont Yankee Nuclear Power } \\
\text { Corporation } \ldots \ldots \ldots \ldots \ldots . . . .\end{array}$ & Vermont Yankee & BWR & 6001 & 0 & 0 & 0 & 0 & 0 & 0 \\
\hline Virginia Power . & $\begin{array}{l}\text { North Anna } 1 \& 2 \\
\text { Surry } 1 \& 2\end{array}$ & $\begin{array}{l}\text { PWR } \\
\text { PWR }\end{array}$ & $\begin{array}{l}5201 \\
5203\end{array}$ & $\begin{array}{l}0 \\
0\end{array}$ & $\begin{array}{l}0 \\
0\end{array}$ & $\begin{array}{l}1 \\
1\end{array}$ & $\begin{array}{l}0 \\
0\end{array}$ & $\begin{array}{l}1 \\
0\end{array}$ & $\begin{array}{l}2 \\
1\end{array}$ \\
\hline Washington Public Power Supply System & Washington Nuclear 2 & BWR & 5302 & 0 & 0 & 0 & 0 & 0 & 0 \\
\hline Wisconsin Electric Power Company . . . . . . & Point Beach $1 \& 2$ & PWR & 5401 & 1 & 0 & 2 & 0 & 0 & 3 \\
\hline
\end{tabular}




\begin{tabular}{|c|c|c|c|c|c|c|c|c|c|}
\hline \multirow[b]{2}{*}{ Electric Utility Name } & \multirow[b]{2}{*}{ Reactor Names } & \multirow[b]{2}{*}{$\begin{array}{c}\text { Reactor } \\
\text { Type }\end{array}$} & \multirow[b]{2}{*}{$\begin{array}{l}\text { Pool } \\
\text { Site } \\
\text { ID }\end{array}$} & \multicolumn{5}{|c|}{ Canister Nonfuel } & \multirow[b]{2}{*}{$\begin{array}{c}\text { Total } \\
\text { Nonfuel } \\
\text { Canisters }\end{array}$} \\
\hline & & & & Debris $^{a}$ & $\begin{array}{c}\text { Nonfuel } \\
\text { Disassembly } \\
\text { Hardware }\end{array}$ & $\begin{array}{c}\text { Nonfuel } \\
\text { Components }^{b}\end{array}$ & Other $^{\mathrm{C}}$ & $\begin{array}{l}\text { Unknown } \\
\text { Contents }\end{array}$ & \\
\hline Wisconsin Public Service Corporation & Kewaunee & PWR & 5501 & 0 & 0 & 0 & $\mathbf{0}$ & 0 & 0 \\
\hline Yankee Atomic Electric Company & Yankee Rowe & PWR & 5601 & 0 & 0 & 0 & 0 & 0 & 0 \\
\hline Total $\ldots . . . . . . . . . . .$. & $\cdots \ldots \ldots \ldots$ & & $\ldots$. & 98 & 28 & 60 & 31 & 6 & 111 \\
\hline
\end{tabular}

aDebris column represents canisters containing fuel rod pieces, fuel pellets, etc.

'Nonfuel Components column represents canisters containing nonfuel assembly hardware.

'Other column represents canisters containing other nonfuel material.

dUnknown Contents column represents the number of canisters containing nonfuel components for which specific data on the contents of the canister were not reported on the Form RW-859 survey.

'Total includes 96 cansiters containing only nonfuel and 15 canisters containing both fuel and nonfuel items. Total Nonfuel Canisters column may not equal the sum of individual canister contents columns since canisters may contain more than one category of nonfuel items.

PWR = Pressurized-water reactor; BWR = Boiling-water reactor; HTGR = High-temperature, gas-cooled reactor

Source: Energy Information Administration, Form RW-859, "Nuclear Fuel Data" (1993). 


\begin{tabular}{|c|c|c|c|c|c|}
\hline \multirow[b]{3}{*}{$\begin{array}{l}\text { Length } \\
\text { (inches) }\end{array}$} & \multicolumn{4}{|c|}{ Canister Dimensions } & \multirow{3}{*}{$\begin{array}{l}\text { Most Prominent } \\
\text { Assembly Size" } \\
\text { (inches) }\end{array}$} \\
\hline & \multicolumn{2}{|c|}{ Most Prominent } & \multicolumn{2}{|c|}{ Other } & \\
\hline & $\begin{array}{c}\text { Width } \\
\text { (inches) }\end{array}$ & $\begin{array}{c}\text { Number } \\
\text { of Canisters }\end{array}$ & $\begin{array}{c}\text { Width } \\
\text { (inches) }\end{array}$ & $\begin{array}{c}\text { Number } \\
\text { of Canisters }\end{array}$ & \\
\hline$\ldots \ldots \ldots \ldots$ & $12 \times 12$ & 5 & -- & -. & $84 \times 7 \times 7$ \\
\hline $84, \ldots \ldots \ldots$ & $6 \times 6$ & 2 & $12 \times 12$ & 1 & $84 \times 7 \times 7$ \\
\hline $100 \ldots \ldots \ldots \ldots$ & $6 \times 6$ & 1 & -- & - & -- \\
\hline $138 \ldots \ldots \ldots \ldots$ & $8 \times 8$ & 9 & - & - & $137 \times 8 \times 8$ \\
\hline $144 \ldots \ldots \ldots \ldots$ & $9 \times 9$ & 7 & $6 \times 6$ & 1 & $148 \times 8 \times 8$ \\
\hline $152 \ldots \ldots \ldots \ldots$ & $6 \times 6$ & 1 & - & - & $173 \times 5 \times 5$ \\
\hline $153 \ldots \ldots \ldots$ & $8 \times 8$ & 1 & - & - & $157 \times 8 \times 8$ \\
\hline $155 \ldots \ldots \ldots$ & $8 \times 8$ & 3 & - & - & - \\
\hline $156 \ldots \ldots \ldots$ & $8 \times 8$ & 16 & $9 \times 9$ & 9 & $157 \times 8 \times 8$ \\
\hline $157 \ldots \ldots \ldots$ & $8 \times 8$ & 24 & - & - & - \\
\hline $158 \ldots \ldots \ldots$ & $9 \times 9$ & 4 & $\cdots$ & - & $157 \times 8 \times 8$ \\
\hline $159 \ldots \ldots \ldots \ldots$ & $8 \times 8$ & 9 & $\begin{array}{l}5 \times 5 \\
9 \times 9\end{array}$ & $\begin{array}{l}2 \\
1\end{array}$ & $160 \times 8 \times 8$ \\
\hline $160 \ldots \ldots \ldots \ldots$ & $8 \times 8$ & 4 & $9 \times 9$ & 3 & $160 \times 8 \times 8$ \\
\hline $161 \ldots \ldots \ldots \ldots$ & $9 \times 9$ & 22 & $8 \times 8$ & 19 & $160 \times 8 \times 8$ \\
\hline $163 \ldots \ldots \ldots$ & $8 \times 8$ & 2 & $6 \times 6$ & 1 & $176 \times 5 \times 5$ \\
\hline $166 \ldots \ldots \ldots$ & $8 \times 8$ & 1 & - & -- & $166 \times 9 \times 9$ \\
\hline $168 \ldots \ldots \ldots$ & $8 \times 8$ & 10 & - & -- & $160 \times 8 \times 8$ \\
\hline $170 \ldots \ldots \ldots$ & $10 \times 10$ & 5 & $\begin{array}{l}16 \times 16 \\
19 \times 19\end{array}$ & $\begin{array}{l}2 \\
2\end{array}$ & $166 \times 9 \times 9$ \\
\hline $171 \ldots \ldots \ldots$ & $9 \times 9$ & 3 & $3 \times 3$ & 2 & $157 \times 8 \times 8$ \\
\hline $174 \ldots \ldots \ldots$ & $5 \times 5$ & 1 & -- & - & $176 \times 5 \times 5$ \\
\hline $176 \ldots \ldots \ldots$ & $6 \times 6$ & 6 & $16 \times 16$ & 1 & $176 \times 5 \times 5$ \\
\hline $180 \ldots \ldots \ldots$ & $9 \times 9$ & 22 & $\begin{array}{c}1 \times 1 \\
6 \times 6 \\
8 \times 8 \\
12 \times 12\end{array}$ & $\begin{array}{r}2 \\
18 \\
16 \\
1\end{array}$ & $178 \times 8 \times 8$ \\
\hline $182 \ldots \ldots \ldots \ldots$ & $8 \times 8$ & 4 & -- & -- & $176 \times 5 \times 5$ \\
\hline $186 \ldots \ldots \ldots$ & $8 \times 8$ & 1 & - & - & - \\
\hline $189 \ldots \ldots \ldots$ & $8 \times 8$ & 3 & $\cdots$ & $\cdots$ & $\cdots$ \\
\hline $192 \ldots \ldots \ldots \ldots$ & $10 \times 10$ & 2 & 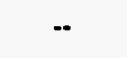 & - & $166 \times 9 \times 9$ \\
\hline Total .......... & & 168 & & ${ }^{9} 101$ & \\
\hline
\end{tabular}

"Most Prominent Assembly Size column represents the dimensions of the assembly (rounded to the nearest inch) found in the most prominent fuel canister at each length.

'Canisters with length less than 84 inches contain partial length fuel rods.

'Total includes 20 canisters with missing or incomplete dimensions.

-. = Not applicable.

Source: Energy Information Administration, Form RW-859, "Nuclear Fuel Data" (1993). 


\section{Nonfuel Components}

The term "nonfuel components" may be defined broadly to cover all non-fuel-bearing components which receive radiation during power generation. Generally included are:

- The components used to initiate, control, and monitor the chain reaction in the core, often called NFA hardware (neutron sources, control elements, burnable absorbers, incore instrumentation, etc.)

- The nonfuel portion of a fuel assembly, often called disassembly hardware (guide tubes, water rods, grids, nozzles, etc.)

- Miscellaneous hardware used in the reactor core which is not a part of fuel assemblies (dummy assemblies, coupon trees, etc.).

The Standard Contract has a more limited definition of nonfuel components which includes most NFA hardware. The contract does not explicitly identify BWR control assemblies, located between, rather than within, the SNF assemblies. Nor does it mention disassembly hardware and miscellaneous hardware.

In terms of location of each piece of hardware, a noncanistered nonfuel component can also be distinguished by whether it is integral or nonintegral with an assembly. Nonfuel components may be included as an integral part of the SNF assemblies delivered for disposal. Nonfuel components are generally used within or between assemblies but are not necessarily permanently attached to assemblies. These nonfuel components are usually retired from service on a schedule that is different from that of fuel assemblies.

Because all nonfuel components listed in the Standard Contract fall into the NFA hardware grouping, NFA hardware has been the focus of the EIA data collection efforts on nonfuel components. Some utilities have reported nonfuel components which are either disassembly hardware or miscellaneous hardware. However, the reporting and analysis which follow deal primarily with NFA hardware.

\section{Nonfuel Components - NFA Categories by Vendor}

Tables 22, 23, 24, and 25 summarize the responses of the utilities to the Form RW-859 survey section on discharged NFA hardware for the generic reactor assembly classes. These tables do not include other nonfuel components reported by these utilities. The nonfuel components (including NFA hardware) reported by owners of reactors in single-reactor assembly classes (i.e., Dresden 1, Palisades, LaCrosse, etc.) are presented in Table 26. Of the 109 generic assembly class reactors, 9 reactors either did not report nonfuel components data or reported no NFA hardware components. Of the 11 single-reactor assembly class reactors, 3 did not report NFA data. The components of NFA hardware used at various reactors vary significantly with vendors of the Nuclear Steam Supply System (NSSS). Each of the first four tables presents the NFA hardware reported for one of the major NSSS vendors (Babcock \& Wilcox, Combustion Engineering, General Electric, and Westinghouse).

\section{Storage of Integral NFA Hardware}

The Form RW-859 survey also requested the quantities of NFA hardware which are stored in or attached to assemblies and the dimensions of those NFA hardware components. NFA hardware may have been integral to an assembly, noncanistered in baskets, destined for disposal as low-level radioactive waste, or stored in canisters. Approximately 89 percent of the 60,699 pieces of NFA hardware reported were integral to an assembly. Reactor-by-reactor percentages of NFA hardware stored in or attached to assemblies are presented in Tables 22 through 26.

\section{NFA Hardware Discharge Rates}

The following factors may affect NFA hardware discharge rates:

- Changes in fuel management strategies

- Changeout of infrequently replaced items (control rods, etc)

- Early changeout of hafnium control rod assemblies.

\section{Changes in Fuel Management Strategies}

Changes in fuel management strategies have impacted discharge rates of NFA hardware, particularly burnable absorber assemblies. Reactors built by Westinghouse and Babcock \& Wilcox used one set of burnable absorber assemblies with the initial reactor fuel. The use of burnable absorber assemblies was resumed in later cycles to control the excess reactivity associated with higher initial fuel enrichments. The effect on quantities of NFA hardware was that reactors built by Westinghouse and Babcock \& Wilcox typically began to use significantly larger quantities of NFA hardware 
each cycle; preliminary results indicate nearly a one-toone ratio of burnable absorber assemblies with fresh fuel assemblies for both vendors.

Newer features, such as the use of integral fuel burnable absorbers by Westinghouse, are likely to once again reduce quantities of NFA hardware discharged by reactors.

\section{Changeout of Infrequently Replaced Items}

Many estimates of NFA hardware discharges are related to discharged fuel assemblies because the number of discharged fuel assemblies gives a simple and quantitative measure of a reactor's use. However, NFA hardware is discharged on one schedule, fuel assemblies on another. This may distort estimates of NFA hardware discharges, especially when dealing with infrequently replaced components, such as control element assemblies. For example, operators of reactors in the $C E 14 \times 14$ assembly class report having discharged 611 control element assemblies and nearly 4,295 fuel assemblies. Operators of reactors in the CE $16 \times 16$ assembly class report having discharged only 3 control element assemblies and nearly 2,176 fuel assemblies. For CE $14 \times 14$ reactors, the number of control element assemblies is approximately 14.0 percent of the number of discharged fuel assemblies; for $C E 16 \times 16$ reactors, the number of control element assemblies is approximately 0.1 percent of the discharged fuel assemblies. The explanation is that the older reactors (CE $14 \times 14$ ) have completed a changeout of their control element assemblies, whereas the newer reactors (CE $16 \times 16$ ) have only discharged defective or damaged control element assemblies. This effect can also be observed between the older and newer Westinghouse assembly classes (e.g., WE $14 \times 14$ and WE $15 \times 15$ versus WE $17 \times 17$ ). The effect in Westinghouse assembly classes is somewhat obscured by the early changeout of hafnium control rod assemblies from some WE $17 \times 17$ plants.

\section{Early Changeout of Hafnium Control Rod Assemblies}

Several of the WE $17 \times 17$ plants using hafnium control rod assemblies experienced unexpected hydrating and subsequent control rod swelling. As a result, these assemblies have been replaced at all or nearly all of the plants originally using them, sometimes after as little as a single cycle of use. This development means that the number of control rod assemblies at WE $17 \times 17$ plants will not be directly correlated to the number of discharged fuel assemblies.

\section{Disposal of NFA Hardware as Low-Level Radioactive Waste}

When NFA hardware has reached the end of its useful life, it may exceed the Class $C$ limits for disposal established in 10 CFR 61 for low-level radioactive waste. Hardware which exceeds these limits is referred to as Greater-Than-Class-C waste. NFA hardware which does not exceed these limits may be disposed as a low-level waste. Some utilities have disposed of NFA hardware in this manner, but the EIA does not collect these data on the Form RW-859 survey.

\section{NFA Hardware from Single-Reactor Assembly Classes}

The single-reactor assembly class consists of reactors which are not included in the generic assembly class reactors. Table 26 summarizes the response of utilities which own these non-generic reactors to the Form RW-859 survey section on discharged NFA hardware. As of December 31, 1993, there were 4,829 permanently discharged fuel assemblies from the 8 single-reactor assembly class reactors that reported the data. Three single-reactor assembly class reactors did not report. There were 1,536 pieces of NFA hardware reported, of which 509 (35 percent), were integral to an assembly. 
Table 22. Nonfuel Assembly (NFA) Hardware from Babcock \& Wilcox Reactors

\begin{tabular}{|c|c|c|c|c|c|c|c|c|c|}
\hline \multirow[b]{2}{*}{ Reactor Names ${ }^{a}$} & \multirow{2}{*}{$\begin{array}{c}\text { Permanently } \\
\text { Discharged } \\
\text { Fuel } \\
\text { Assemblies } \\
\end{array}$} & \multicolumn{5}{|c|}{ NFA Hardware } & \multirow[b]{2}{*}{$\begin{array}{c}\text { Total Reported } \\
\text { NFA Hardware } \\
\text { Items }\end{array}$} & \multirow{2}{*}{$\begin{array}{c}\text { NFA Hardware } \\
\text { Items Reported } \\
\text { In/Attached to } \\
\text { Fuel } \\
\text { Assemblies }\end{array}$} & \multirow{2}{*}{$\begin{array}{c}\text { NFA Hardwar } \\
\text { Items } \\
\text { In/Attached to } \\
\text { Fuel } \\
\text { Assemblies } \\
\text { (percent) }\end{array}$} \\
\hline & & \begin{tabular}{|c|} 
Burnable \\
Poison Rod \\
Assemblies
\end{tabular} & $\begin{array}{l}\text { Axial Power } \\
\text { Shaping } \\
\text { Assemblies }\end{array}$ & $\begin{array}{l}\text { Control Rod } \\
\text { Assemblies }\end{array}$ & $\begin{array}{l}\text { Neutron } \\
\text { Sources }\end{array}$ & $\begin{array}{c}\text { Orifice } \\
\text { Rod } \\
\text { Assemblies }\end{array}$ & & & \\
\hline Arkansas Nuclear $1 \ldots \ldots \ldots \ldots$ & 684 & 464 & 16 & 61 & 2 & 68 & 611 & 543 & 89 \\
\hline Crystal River $3 \ldots \ldots \ldots \ldots \ldots \ldots$ & 531 & 232 & 8 & 61 & 2 & 2 & 305 & 305 & 100 \\
\hline Davis-Besse $\ldots \ldots \ldots \ldots \ldots \ldots$ & 450 & 228 & 8 & 8 & 4 & 2 & 250 & 250 & 100 \\
\hline Oconee $1 \& 2 \ldots \ldots \ldots \ldots \ldots$ & $b_{1,622}$ & $c_{907}$ & $c_{31}$ & $c_{178}$ & $c_{4}$ & $c_{22}$ & 1,142 & 1,141 & 100 \\
\hline Oconee $3 \ldots \ldots \ldots \ldots \ldots \ldots$ & 808 & 404 & 13 & 6 & 2 & 7 & 432 & 429 & 99 \\
\hline Rancho Seco ................ & 493 & 272 & $₫ 16$ & 61 & 4 & 62 & 415 & 0 & 0 \\
\hline Three Mile island $1 \ldots \ldots \ldots \ldots$ & 578 & 268 & 8 & 13 & 4 & 77 & 370 & 366 & 99 \\
\hline $\begin{array}{l}\text { Total From Reporting Reactors ... } \\
\text { Ratio to Permanently Discharged }\end{array}$ & 5,166 & 2,775 & 100 & 388 & 22 & 240 & 3,525 & 3,034 & 86 \\
\hline Fuel Assemblies (percent) $\quad \ldots \ldots \ldots$ & & 53.72 & 1.94 & 7.51 & 0.43 & 4.65 & & & \\
\hline
\end{tabular}

Includes all Babcock \& Wilcox reactors.

'Number of fuel assemblies in pool represents fuel discharged from two reactors into one pool.

'Number of nonfuel components in pool represents components discharged from two reactors into one pool.

Includes 8 axial power shaping assemblies and 8 gray axial power shaping assemblies.

Source: Energy Information Administration, Form RW-859, "Nuclear Fuel Data" (1993). 
Table 23. Nonfuel Assembly (NFA) Hardware from Combustion Engineering Reactors

\begin{tabular}{|c|c|c|c|c|c|c|c|}
\hline \multirow[b]{2}{*}{ Reactor Names ${ }^{a}$} & \multirow[b]{2}{*}{$\begin{array}{c}\text { Permanently } \\
\text { Discharged } \\
\text { Fuel } \\
\text { Assemblies }\end{array}$} & \multicolumn{3}{|c|}{ NFA Hardware } & \multirow[b]{2}{*}{$\begin{array}{c}\text { Total Reported } \\
\text { NFA Hardware } \\
\text { Items }\end{array}$} & \multirow[b]{2}{*}{$\begin{array}{l}\text { NFA Hardware } \\
\text { Items Reported } \\
\text { In/Attached to } \\
\text { Fuel Assemblies }\end{array}$} & \multirow{2}{*}{$\begin{array}{c}\text { NFA Hardware } \\
\text { Items } \\
\text { In/Attached to } \\
\text { Fuel Assemblies } \\
\text { (percent) }\end{array}$} \\
\hline & & $\begin{array}{c}\text { Control } \\
\text { Element } \\
\text { Assemblies }\end{array}$ & $\begin{array}{l}\text { Neutron } \\
\text { Sources }\end{array}$ & $\begin{array}{c}\text { Control Element } \\
\text { Assembly } \\
\text { Flow Plugs }\end{array}$ & & & \\
\hline Arkansas Nuclear $2 \quad \ldots \ldots \ldots \ldots \ldots$ & 564 & 0 & 2 & 0 & 2 & 2 & 100 \\
\hline Calvert Cliffs $1 \& 2 \ldots \ldots \ldots \ldots \ldots$ & $b_{1,500}$ & $c_{353}$ & $c^{c}$ & 0 & $35 \overline{7}$ & 355 & 99 \\
\hline 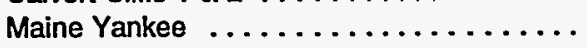 & 1,131 & 92 & 1 & 0 & 93 & 93 & 100 \\
\hline Millstone $2 \ldots \ldots \ldots \ldots \ldots \ldots$ & 784 & 82 & 4 & 8 & 94 & 91 & 97 \\
\hline St. Lucie $1 \ldots \ldots \ldots \ldots \ldots \ldots \ldots$ & 880 & 84 & 2 & 0 & 86 & 86 & 100 \\
\hline San Onofre $2 \ldots \ldots \ldots \ldots \ldots \ldots$ & 592 & 1 & 0 & 0 & 1 & 1 & 100 \\
\hline San Onofre $3 \ldots \ldots \ldots \ldots \ldots \ldots$ & 592 & 1 & 0 & 0 & 1 & 1 & 100 \\
\hline Waterford $3 \ldots \ldots \ldots \ldots \ldots \ldots$ & 428 & 1 & 0 & 0 & 1 & 1 & 100 \\
\hline $\begin{array}{l}\text { Total From Reporting Reactors } \ldots \ldots \ldots \\
\text { Ratio to Permanently Discharged }\end{array}$ & 6,471 & 614 & 13 & 8 & 635 & 630 & 99 \\
\hline Fuel Assemblies (percent) . . . . . . . . & & 9.49 & 0.20 & 0.12 & & & \\
\hline
\end{tabular}

ancludes all Combustion Engineering reactors except Palo Verde 1, Palo Verde 2, and Palo Verde 3 which did not report these data.

Number of fuel assemblies in pool represents fuel discharged from two reactors into separate pools joined by a transfer canal.

cNumber of nonfuel components in pool represents components discharged from two reactors into separate pools joined by a transfer canal.

Source: Energy Information Administration, Form RW-859, "Nuclear Fuel Data" (1993). 


\begin{tabular}{|c|c|c|c|c|c|c|c|c|c|}
\hline \multirow[b]{2}{*}{ Reactor Names ${ }^{a}$} & \multirow{2}{*}{$\begin{array}{c}\text { Permanently } \\
\text { Discharged } \\
\text { Fuel } \\
\text { Assemblies }\end{array}$} & \multicolumn{5}{|c|}{ NFA Hardware } & \multirow[b]{2}{*}{$\begin{array}{c}\text { Total Reported } \\
\text { NFA Hardware } \\
\text { Items }\end{array}$} & \multirow{2}{*}{$\begin{array}{c}\text { NFA Hardware } \\
\text { Items Reported } \\
\text { In/Attached to } \\
\text { Fuel } \\
\text { Assemblies }\end{array}$} & \multirow{2}{*}{$\begin{array}{l}\text { NFA Hardware } \\
\text { Items } \\
\text { In/Attached to } \\
\text { Fuel } \\
\text { Assemblies } \\
\text { (percent) }\end{array}$} \\
\hline & & $\begin{array}{c}\text { Fuel } \\
\text { Channels }\end{array}$ & $\begin{array}{l}\text { Control } \\
\text { Blades }\end{array}$ & $\begin{array}{l}\text { Neutron } \\
\text { Sources }\end{array}$ & $\begin{array}{c}\text { Local Power } \\
\text { Range } \\
\text { Monitors }\end{array}$ & $\begin{array}{c}\text { Other } \\
\text { Instrumentation }\end{array}$ & & & \\
\hline Browns Ferry $1 \ldots \ldots \ldots \ldots \ldots \ldots$ & 1,580 & 1,555 & 0 & 0 & 0 & 0 & 1,555 & 1,555 & 100 \\
\hline Browns Ferry $2 \ldots \ldots \ldots \ldots \ldots \ldots$ & 1,656 & 1,625 & 0 & 0 & 0 & 1 & 1,626 & 1,625 & 100 \\
\hline Browns Ferry $3 \ldots \ldots \ldots \ldots \ldots$ & 1,030 & 1,018 & 0 & 0 & 0 & 0 & 1,018 & 1,004 & 99 \\
\hline Brunswick $1 \ldots \ldots \ldots \ldots \ldots$ & 1,339 & 11 & 25 & 0 & 0 & 0 & 36 & 0 & 0 \\
\hline Brunswick $2 \ldots \ldots \ldots \ldots \ldots$ & 1,400 & 6 & 0 & 0 & 0 & 0 & 6 & 0 & 0 \\
\hline Clinton $1 \ldots \ldots \ldots \ldots \ldots \ldots$ & 724 & 724 & 0 & 5 & 17 & 11 & 757 & 724 & 96 \\
\hline Cooper Station . ............. & 1,860 & 4 & 4 & 0 & 3 & 25 & 36 & 0 & 0 \\
\hline Dresden $2 \ldots \ldots \ldots \ldots \ldots$ & 2,813 & 23 & 6 & 0 & 5 & 20 & 54 & 0 & 0 \\
\hline Dresden 3 ................. & 1,864 & 70 & 56 & 0 & 5 & 0 & 131 & 0 & 0 \\
\hline Enrico Fermi $2 \ldots \ldots \ldots \ldots \ldots$ & 860 & 860 & 165 & 0 & 6 & 0 & 1,031 & 858 & 83 \\
\hline FitzPatrick $\ldots \ldots \ldots \ldots \ldots \ldots \ldots$ & 1,684 & 1,684 & 51 & 0 & 15 & 0 & 1,750 & 1,684 & 96 \\
\hline Grand Gulf $1, \ldots \ldots \ldots \ldots \ldots \ldots$ & 1,660 & 1,660 & 0 & 7 & 71 & 18 & 1,756 & 1,660 & 95 \\
\hline Hatch $1 \& 2 \ldots \ldots \ldots \ldots \ldots \ldots$ & 3,544 & $c^{c} 3,549$ & 0 & 0 & 0 & 0 & 3,549 & 3,549 & 100 \\
\hline Hope Creek ................ & 1,008 & 1,008 & 27 & 1 & 8 & 0 & 1,044 & 1,008 & 97 \\
\hline LaSalle County $1 \& 2 \ldots \ldots \ldots \ldots$ & b 2,152 & ${ }^{c} 1,512$ & ${ }^{c} 9$ & $c_{14}$ & ${ }^{c} 47$ & $c_{34}$ & 1,616 & 1,472 & 91 \\
\hline Limerick $1 \ldots \ldots \ldots \ldots \ldots \ldots$ & 994 & 994 & 0 & 6 & 0 & 0 & 1,000 & 994 & 99 \\
\hline Limerick $2 \ldots \ldots \ldots \ldots \ldots \ldots \ldots$ & 654 & 654 & 0 & 3 & 0 & 0 & 657 & 654 & 100 \\
\hline Millstone $1 \ldots \ldots \ldots \ldots \ldots \ldots$ & 2,116 & 1,800 & 0 & 0 & 0 & 0 & 1,800 & 1,800 & 100 \\
\hline Monticello ................. & 1,768 & 625 & 20 & 0 & 6 & 0 & 651 & 620 & 95 \\
\hline Nine Mile Point $1 \ldots \ldots \ldots \ldots$ & 1,812 & 1,620 & 17 & 0 & 14 & 0 & 1,651 & 0 & 0 \\
\hline Nine Mile Point $2 \ldots \ldots \ldots \ldots \ldots$ & 640 & 444 & 33 & 4 & 13 & 0 & 494 & 0 & 0 \\
\hline Oyster Creek ................ & 1,876 & 1,389 & 13 & 2 & 0 & 0 & 1,404 & 1,389 & 99 \\
\hline Peach Bottom $2 \ldots \ldots \ldots \ldots \ldots$ & 2,164 & 2,162 & 0 & 0 & 0 & 0 & 2,162 & 2,157 & 100 \\
\hline Peach Bottom $3 . \ldots \ldots \ldots \ldots$ & 2,200 & 2,200 & 41 & 1 & 0 & 0 & 2,242 & 2,200 & 98 \\
\hline Perry $1 \ldots \ldots \ldots \ldots \ldots \ldots \ldots$ & 744 & 744 & 24 & 11 & 43 & 0 & 822 & 743 & 90 \\
\hline Pilgrim $1, \ldots \ldots \ldots \ldots \ldots \ldots \ldots$ & 1,628 & 1,629 & 55 & 1 & 112 & 44 & 1,841 & 1,654 & 90 \\
\hline Quad Cities $1 \& 2 \ldots \ldots \ldots \ldots \ldots$ & 8,140 & 370 & ${ }^{\circ} 9$ & 0 & ${ }^{c} 12$ & $c_{15}$ & 406 & 369 & 91 \\
\hline River Bend $1 \ldots \ldots \ldots \ldots \ldots$ & 764 & 764 & 24 & 5 & 18 & 6 & 817 & 764 & 94 \\
\hline Susquehanna $1 \ldots \ldots \ldots \ldots \ldots$ & 1,628 & 1,628 & 0 & 0 & 0 & 0 & 1,628 & 1,628 & 100 \\
\hline Susquehanna $2 \ldots \ldots \ldots \ldots$ & 1,236 & 1,236 & 0 & 0 & 0 & 0 & 1,236 & 1,236 & 100 \\
\hline Vermont Yanker ............... & 1,978 & 1,494 & 3 & 0 & 3 & 0 & 1,500 & 1,494 & 100 \\
\hline Washington Nuclear $2 \ldots \ldots \ldots \ldots$ & 1,040 & 824 & 0 & 0 & 0 & 0 & 824 & 781 & 95 \\
\hline $\begin{array}{l}\text { Total From Reporting Reactors } \ldots . . \\
\text { Ratio to Permanently Discharged }\end{array}$ & 52,556 & 35,886 & 582 & 60 & 398 & 174 & 37,100 & 33,622 & 91 \\
\hline Fuel Assemblies (percent) . ........ & & 68.28 & 1.11 & 0.11 & 0.76 & 0.33 & & & \\
\hline
\end{tabular}

"Includes all General Electric reactors except Duane Arnold and Shoreham which did not report these data.

'Number of fuel assemblies in pool represents fuel discharged from two reactors into separate pools joined by a transfer canal.

"Number of nonfuel components in pool represents components discharged from two reactors into separate pools joined by a transfer canal.

Source: Energy Information Administration, Form RW-859, "Nuclear Fuel Data" (1993). 
Table 25. Nonfuel Assembly (NFA) Hardware from Westinghouse Reactors

\begin{tabular}{|c|c|c|c|c|c|c|c|c|c|c|}
\hline \multirow{2}{*}{\multicolumn{2}{|c|}{ m $\quad$ Reactor Names ${ }^{a}$}} & \multirow[b]{2}{*}{$\begin{array}{c}\text { Permanently } \\
\text { Discharged } \\
\text { Fuel } \\
\text { Assemblies }\end{array}$} & \multicolumn{5}{|c|}{ NFA Hardware } & \multirow[b]{2}{*}{$\begin{array}{c}\text { Total Reported } \\
\text { NFA Hardware } \\
\text { Items }\end{array}$} & \multirow{2}{*}{$\begin{array}{c}\text { NFA Hardware } \\
\text { Items Reported } \\
\text { In/Attached to } \\
\text { Fuel } \\
\text { Assemblies }\end{array}$} & \multirow{2}{*}{$\begin{array}{l}\text { NFA Hardware } \\
\text { Items } \\
\text { In/Attached to } \\
\text { Fuel } \\
\text { Assemblies } \\
\text { (percent) }\end{array}$} \\
\hline & & & $\begin{array}{c}\text { Burnable } \\
\text { Poison Rod } \\
\text { Assemblies }\end{array}$ & \begin{tabular}{|l|} 
Thimble Plug \\
Assemblies
\end{tabular} & $\begin{array}{c}\text { Rod Cluster } \\
\text { Control } \\
\text { Assemblies }\end{array}$ & $\begin{array}{l}\text { Neutron } \\
\text { Sources }\end{array}$ & $\begin{array}{c}\text { Incore } \\
\text { Instrumentation }\end{array}$ & & & \\
\hline 毫 & Beaver Valley $1 \ldots \ldots \ldots \ldots \ldots$ & 563 & 223 & 103 & 5 & 4 & 0 & 335 & 334 & 100 \\
\hline 5 & Beaver Valley $2 \ldots \ldots \ldots \ldots$ & 260 & 66 & b140 & 0 & 4 & 0 & 210 & 194 & 92 \\
\hline के & Braidwood $1 \& 2 \ldots \ldots \ldots \ldots \ldots$ & $c^{c} 488$ & ${ }^{d} 360$ & $d_{31}$ & ${ }^{d 12}$ & 4 & $d_{3}$ & 510 & 338 & 66 \\
\hline $\bar{\exists}$ & Byron $1 \& 2 \ldots \ldots \ldots \ldots \ldots$ & ${ }^{\circ} 772$ & $d_{512}$ & 57 & ${ }^{d} 107$ & $\mathrm{~d}^{\mathrm{d}}$ & 0 & 680 & 476 & 70 \\
\hline$\stackrel{\mathscr{0}}{\stackrel{*}{*}}$ & 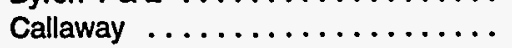 & 546 & 230 & 28 & 55 & 2 & 0 & 315 & 259 & 82 \\
\hline 号 & 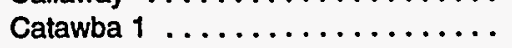 & 484 & 382 & 35 & 8 & 2 & 0 & 427 & 402 & 94 \\
\hline D & Catawba $2 \ldots \ldots \ldots \ldots \ldots$ & 356 & 282 & 34 & 5 & 2 & 0 & 323 & 288 & 89 \\
\hline 毕 & Comanche Peak $1 \& 2 \ldots \ldots \ldots$ & 205 & 166 & 49 & 0 & $1 / 4$ & 15 & 224 & 157 & 70 \\
\hline & Cook $1 \& 2 \ldots \ldots \ldots \ldots \ldots \ldots$ & $c 1,523$ & ${ }^{d} 876$ & ${ }^{d} 149$ & d22 & ${ }^{d 8}$ & 0 & 1,055 & 1,045 & 99 \\
\hline & Diablo Canyon $1 \ldots \ldots \ldots \ldots \ldots$ & 376 & 188 & 94 & 0 & 3 & 10 & 295 & 261 & 88 \\
\hline & Diablo Canyon $2 \ldots \ldots \ldots \ldots \ldots$ & 396 & 136 & 0 & 0 & 3 & 6 & 145 & 122 & 84 \\
\hline & Farley $1 \ldots \ldots \ldots \ldots \ldots \ldots$ & 655 & 431 & 177 & 5 & 4 & 102 & 719 & 617 & 86 \\
\hline$\infty$ & Farley $2 \ldots \ldots \ldots \ldots \ldots \ldots$ & 558 & 386 & 106 & 0 & 4 & 102 & 598 & 496 & 83 \\
\hline (D) & 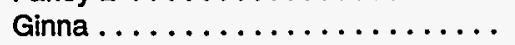 & 729 & 47 & 175 & 36 & 10 & 0 & 268 & 267 & 100 \\
\hline 罵 & Harris $1 \ldots \ldots \ldots \ldots \ldots \ldots$ & 224 & 110 & 7 & 57 & 3 & 14 & 191 & 177 & 93 \\
\hline$z$ & Indian Point $2 \ldots \ldots \ldots \ldots \ldots$ & 748 & 458 & 219 & 1 & 10 & 0 & 688 & 687 & 100 \\
\hline$\frac{5}{9}$ & Indian Point $3 \ldots \ldots \ldots \ldots \ldots$ & 582 & 0 & 1 & 10 & 0 & 0 & 11 & 0 & 0 \\
\hline$\stackrel{\mathbb{M}}{9}$ & Kewaunee ................. & 648 & 278 & 102 & 68 & 7 & 72 & 527 & 453 & 86 \\
\hline $\boldsymbol{n}$ & McGuire $1 \ldots \ldots \ldots \ldots \ldots \ldots$ & 548 & 487 & 48 & 8 & 2 & 0 & 545 & 512 & 94 \\
\hline 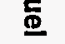 & McGuire $2 \ldots \ldots \ldots \ldots \ldots$ & 552 & 596 & 68 & 13 & 1 & 0 & 678 & 670 & 99 \\
\hline 물 & Millstone $3 \ldots \ldots \ldots \ldots \ldots$ & 332 & 140 & 91 & 40 & 2 & 2 & 275 & 236 & 86 \\
\hline 营 & North Anna $1 \& 2 \ldots \ldots \ldots \ldots$ & $c^{c}, 100$ & d960 & ${ }^{d} 201$ & ${ }^{\circ} 6$ & $d_{12}$ & 0 & 1,179 & 1,179 & 100 \\
\hline$\underline{\mathbf{w}}$ & 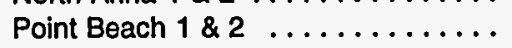 & $c_{1,258}$ & d361 & ${ }^{d} 328$ & ${ }^{\mathrm{d}} 66$ & ${ }^{14}$ & 0 & 769 & 762 & 99 \\
\hline $\bar{D}$ & Robinson $2 \ldots \ldots \ldots \ldots \ldots \ldots$ & 824 & 4 & 2 & 6 & 5 & 33 & 50 & 15 & 30 \\
\hline$\Rightarrow$ & Salem $1 \ldots \ldots \ldots \ldots \ldots \ldots$ & 685 & 505 & 135 & 22 & 5 & 0 & 667 & 594 & 89 \\
\hline $\overrightarrow{\overrightarrow{0}}$ & Salem $2 \ldots \ldots \ldots \ldots \ldots \ldots$ & 457 & 282 & 64 & 0 & 2 & 0 & 348 & 348 & 100 \\
\hline & Seabrook ................ & 136 & 90 & 2 & 7 & 2 & 0 & 101 & 60 & 59 \\
\hline$\sum_{\infty}$ & Sequoyah $1 \& 2 \ldots \ldots \ldots \ldots$ & '817 & ${ }^{\circ} 495$ & ${ }^{d} 87$ & ${ }^{d} 12$ & d9 & 0 & 603 & 603 & 100 \\
\hline (ب) & South Texas $1 \ldots \ldots \ldots \ldots$ & 229 & 189 & 3 & 57 & 2 & 0 & 251 & 251 & 100 \\
\hline$\underset{\mathbf{D}}{\mathbf{D}}$ & South Texas $2 \ldots \ldots \ldots \ldots$ & 188 & 98 & 0 & 57 & 2 & 0 & 157 & 157 & 100 \\
\hline 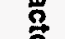 & 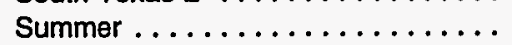 & 440 & 222 & 76 & 0 & 4 & 0 & 302 & 276 & 91 \\
\hline है & Surry $1 \& 2 \ldots \ldots \ldots \ldots \ldots$ & ${ }^{c} 1,335$ & ${ }^{5} 533$ & ${ }^{\circ} 69$ & ${ }^{d} 109$ & 7 & 0 & 718 & 718 & 100 \\
\hline$\vec{\omega}$ & 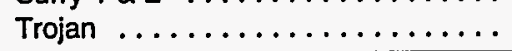 & 780 & 92 & 140 & 8 & 4 & 0 & 244 & 244 & 100 \\
\hline
\end{tabular}

See footnotes at end of table. 


\begin{tabular}{|c|c|c|c|c|c|c|c|c|c|}
\hline \multirow[b]{2}{*}{ Reactor Names ${ }^{a}$} & \multirow{2}{*}{$\begin{array}{c}\text { Permanently } \\
\text { Discharged } \\
\text { Fuel } \\
\text { Assemblies }\end{array}$} & \multicolumn{5}{|c|}{ NFA Hardware } & \multirow[b]{2}{*}{$\begin{array}{c}\text { Total Reported } \\
\text { NFA Hardware } \\
\text { Items }\end{array}$} & \multirow{2}{*}{$\begin{array}{l}\text { NFA Hardware } \\
\text { Items Reported } \\
\text { In/Attached to } \\
\text { Fuel } \\
\text { Assemblies }\end{array}$} & \multirow{2}{*}{$\begin{array}{l}\text { NFA Hardware } \\
\text { Items } \\
\text { In/Attached to } \\
\text { Fuel } \\
\text { Assemblies } \\
\text { (percent) }\end{array}$} \\
\hline & & $\begin{array}{c}\text { Burnable } \\
\text { Poison Rod } \\
\text { Assemblies }\end{array}$ & $\begin{array}{l}\text { Thimble Plug } \\
\text { Assemblies }\end{array}$ & $\begin{array}{c}\text { Rod Cluster } \\
\text { Ćontrol } \\
\text { Assemblies }\end{array}$ & $\begin{array}{l}\text { Neutron } \\
\text { Sources }\end{array}$ & $\begin{array}{c}\text { Incore } \\
\text { Instrumentation }\end{array}$ & & & \\
\hline Turkey Point $3 \ldots \ldots \ldots \ldots \ldots$ & 605 & 274 & 191 & 10 & 2 & 0 & 477 & 476 & 100 \\
\hline Turkey Point $4 \ldots \ldots \ldots \ldots \ldots$ & 599 & 316 & 115 & 9 & 2 & 0 & 442 & 442 & 100 \\
\hline Vogtle $1 \& 2 \ldots \ldots \ldots \ldots \ldots \ldots$ & ${ }^{\circ} 565$ & 430 & $i_{1}$ & $' 106$ & 12 & 123 & 562 & 537 & 96 \\
\hline Wolf Creek $1 \ldots \ldots \ldots \ldots \ldots$ & 408 & 346 & 131 & 108 & 2 & 12 & 599 & 408 & 68 \\
\hline Zion $1 \& 2 \ldots \ldots \ldots \ldots \ldots$ & ${ }^{c} 1,684$ & d862 & ${ }^{d} 442$ & ¿95 & 8 & $\mathrm{~d} 8$ & 1,415 & 1,399 & 99 \\
\hline $\begin{array}{l}\text { Total From Reporting Reactors ... } \\
\text { Ratio to Permanently Discharged }\end{array}$ & 23,655 & 12,413 & $\mathbf{3 , 7 0 1}$ & 1,230 & 167 & 392 & 17,903 & 16,460 & 92 \\
\hline Fuel Assemblies (percent) . . . . . . . & • & 52.48 & 15.65 & 5.20 & 0.71 & 1.66 & & & \\
\hline
\end{tabular}

Includes all Westinghouse reactors except Prairie Island 1 \& 2 and Watts Bar 1 \& 2 which did not report these data.

'Quantity includes 32 water displacement assemblies.

'Number of fuel assemblies in pool represents fuel discharged from two reactors into one pool.

NNumber of nonfuel components in pool represents components discharged from two reactors into one pool.

"Number of fuel assemblies in pool represents fuel discharged from two reactors into separate pools joined by a transfer canal.

'Number of nonfuel components in pool represents components discharged from two reactors into separate pools joined by a transfer canal.

Source: Energy Information Administration, Form RW-859, "Nuclear Fuel Data" (1993). 
Table 26. Nonfuel Assembly (NFA) Hardware from Single-Reactor Assembly Class Reactors

\begin{tabular}{|c|c|c|c|c|c|c|c|c|c|c|}
\hline \multirow[b]{2}{*}{ Reactor Names } & \multirow{2}{*}{$\begin{array}{l}\text { Permanently } \\
\text { Discharged } \\
\text { Fuel } \\
\text { Assemblies }\end{array}$} & \multicolumn{6}{|c|}{ NFA Hardware } & \multirow{2}{*}{$\begin{array}{c}\text { Total } \\
\text { Reported } \\
\text { NFA } \\
\text { Hardware } \\
\text { Items }\end{array}$} & \multirow{2}{*}{$\begin{array}{c}\text { NFA } \\
\text { Hardware } \\
\text { Items } \\
\text { Reported } \\
\text { In/Attached } \\
\text { to Fuel } \\
\text { Assemblies }\end{array}$} & \multirow{2}{*}{$\begin{array}{c}\text { NFA } \\
\text { Hardware } \\
\text { Items } \\
\text { In/Attached } \\
\text { to Fuel } \\
\text { Assemblies } \\
\text { (percent) } \\
\end{array}$} \\
\hline & & $\begin{array}{c}\text { Burnable } \\
\text { Absorbers }\end{array}$ & $\begin{array}{c}\text { Plug } \\
\text { Assemblies }\end{array}$ & $\begin{array}{c}\text { Control } \\
\text { Assemblies }^{\mathrm{b}}\end{array}$ & $\begin{array}{l}\text { Neutron } \\
\text { Sources }\end{array}$ & $\begin{array}{c}\text { Incore } \\
\text { Instrumentation }\end{array}$ & $\begin{array}{c}\text { Fuel } \\
\text { Channels }\end{array}$ & & & \\
\hline Big Rock Point & 401 & 0 & 0 & 52 & 8 & 41 & 100 & 201 & 2 & 1 \\
\hline Dresden $1 \ldots$ & 892 & 0 & 0 & 0 & 4 & 0 & 512 & 516 & 0 & 0 \\
\hline Haddam Neck..$\ldots \ldots \ldots \ldots$ & 892 & 0 & 2 & 50 & 12 & 19 & 0 & 83 & 63 & 76 \\
\hline Humboldt Bay... & 390 & 0 & 0 & 0 & 2 & 0 & 389 & 391 & 389 & 99 \\
\hline LaCrosse ..... & 333 & 0 & 0 & 39 & 2 & 0 & 77 & 118 & 0 & 0 \\
\hline Palisades ..... & 792 & 0 & 16 & 1 & 6 & 153 & 0 & 176 & 6 & 3 \\
\hline St. Lucie $2 \ldots .$. & 464 & 0 & $\mathbf{0}$ & 39 & 2 & 0 & 0 & 41 & 41 & 100 \\
\hline San Onofre $1 \ldots .$. & 665 & 0 & 8 & 2 & 0 & 0 & 0 & 10 & 8 & 80 \\
\hline Total From Reporting Reactors & 4,829 & $\mathbf{0}$ & 26 & 183 & 36 & 213 & 1,078 & 1,536 & 509 & 35 \\
\hline Fuel Assemblies (percent) ..... & & 0.00 & 0.54 & 3.79 & 0.75 & 4.41 & 22.32 & & & \\
\hline
\end{tabular}

"Includes all single-reactor assembly class reactor except Fort Calhoun, Indian Point 1, and Yankee Rowe which did not report these data.

bincludes control rod blades and control rod assemblies.

Source: Energy Information Administration, Form RW-859, "Nuclear Fuel Data" (1993). 
(- 


\section{Assembly Characteristics}

\section{Background}

A light-water reactor (LWR) fuel assembly (or bundle) is a configuration of fuel rods assembled together for insertion into a reactor core. Each fuel rod consists essentially of a stack of uranium dioxide $\left(\mathrm{UO}_{2}\right)$ pellets encapsulated within a metal tube that is sealed at both ends. Current LWR's use zircaloy cladding almost exclusively because of its lower neutron absorption relative to stainless steel.

Generally, the assembly configurations are square arrays of many different sizes. For example, one boiling-water reactor (BWR) design uses an array of 6 rods by 6 rods. BWR designs have had $6 \times 6,7 \times 7,8 \times 8$, $9 \times 9,10 \times 10$, and $11 \times 11$ fuel rod arrays. Pressurized-

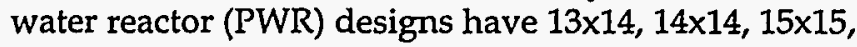
$15 \times 16,16 \times 16,17 \times 17$, and $17 \times 18$ fuel rod arrays. Virtually all assemblies are stamped with a unique identifier.

The EIA uses a categorization of assembly classes and assembly types within each class for spent fuel. This categorization, described in Appendix B, is based on the rod array configuration, fuel vendor, and other distinguishing characteristics, such as assembly length and width and type of fuel cladding. The eightcharacter assembly type codes listed in Appendix B are the codes used on Form RW-859. Neither enrichment nor discharge burnup affects the assembly type classification.

Some assembly types have had very limited use. For example, the Big Rock Point reactor has had 84 -inch-long assemblies loaded with fuel rods in $7 \times 7$, $8 \times 8,9 \times 9$, and $11 \times 11$ array configurations. These assemblies had a range of initial loading weights of uranium and were fabricated by either General Electric, Nuclear Fuel Services, or Siemens Nuclear Fuel Company (formerly Advanced Nuclear Fuel Corporation, Exxon Nuclear Corporation, and Jersey Nuclear). These assemblies were manufactured exclusively for the Big Rock Point reactor.

Appendix B provides an excerpt from Form RW-859 instructions, titled "Descriptions of Assembly Types" (Table B4). This table defines the assembly type codes used in this report, and was the basis for developing
Tables 27 and 28. Assembly types are listed by reactor type (BWR, PWR) and are then further identified by fabricator, cladding material, initial uranium content, size, and number discharged (Appendix B, Table B5). There are 131 different reactor fuel assembly types which have been fabricated for nuclear power reactors (Table B5). BWR fuel assemblies range from 4.28 to 6.52 inches in width, 84.0 to 176.2 inches in length, and have a uranium component of 70 to 195 kilograms $(\mathrm{kg})$. PWR fuel assemblies range from 6.14 to 8.54 inches in width, from 111.8 to 199.0 inches in length, and have a uranium component of 152 to $542 \mathrm{~kg}$. Assembly type summaries by reactor unit (Table B6) and by assembly type (Table B7) are also presented.

The Characteristics Data Base (CDB) contains a more complete physical description of each type of assembly. The CDB includes the characteristics of the fuel pellets in each assembly, the material composition of each component of the hardware, and related information. It also contains detailed mechanical drawings for some assembly types.

\section{Fabricator Summary}

The percentage of BWR and PWR assemblies produced by each fabricator of spent fuel assemblies is presented in Table 27. General Electric (GE) fabricated 90 percent of the BWR discharged fuel assemblies, most of which are 176.2 inches long. Approximately 58 percent of the discharged PWR fuel assemblies were manufactured by Westinghouse. The majority of Westinghouse PWR fuel assemblies have a length of 159.8 inches.

\section{Assembly Distribution}

The distribution of assembly transverse dimensions (Table 28) vary from 84.0 to 176.2 inches long for assemblies utilized in BWR's. However, 96 percent of all these assemblies are greater than or equal to 171.2 inches in length. Assembly lengths used in PWR's range from 111.8 to 199.0 inches; 80 percent of all PWR assemblies are greater than or equal to 159.8 inches in length. Assemblies used in BWR's vary in maximum "as built" assembly width from 4.28 to 6.52 inches. 
Maximum as built assembly widths used in PWR's are from 6.27 to 8.54 inches. More than 97 percent of the BWR assemblies are greater than or equal to 5.44 inches in width, and approximately 62 percent of PWR assemblies are greater than or equal to 8.44 inches in width.

\section{Assembly Identifiers}

Domestic LWR fuel assemblies are identified by a serial number that is stamped on each assembly. These serial numbers serve as identifiers throughout the life of the fuel. The uniqueness of assembly serial numbers is important in determining their effectiveness as unambiguous identifiers. The Oak Ridge National Laboratory (ORNL) report, Analysis of Assembly Serial Number Usage in Domestic Light-Water Reactors (ORNL/TM11841, May 1991), studied serial numbering schemes, the effectiveness of these schemes, and how many duplicate serial numbers occur on domestic LWR fuel assemblies. The serial numbering scheme adopted by the American National Standards Institute (ANSI) ensures uniqueness of assembly serial numbers. Westinghouse, Babcock \& Wilcox, and Siemens use the
ANSI Standard for serial numbers. Combustion Engineering and General Electric both use serial numbers which are unique but do not follow the ANSI Standard. Some assemblies fabricated prior to the establishment of the ANSI Standard in 1978 (ANSI/ANS 57.8 - 1978, reaffirmed in 1987) may have their own serial number schemes which are unique only at the reactor where they were originally irradiated.

Most of the numbering schemes used by electric utilities are not inherently unique, and errors within such schemes account for all of the identified serial number duplication except for three isolated instances. Three duplicate serial numbers were found when analysis focused on duplication within the individual fuel inventory at each reactor site, but these were traced back to data response errors. There were also three instances where the serial numbers used to identify assemblies used for hot cell studies differed from the serial numbers reported to EIA. As a result of the study, EIA is requesting that utilities provide the stamped serial number on the assembly when reporting these data on the Form RW-859 survey.

Table 27. Assemblies by Fuel Fabricating Company and Reactor Type

\begin{tabular}{|c|c|c|}
\hline Fabricating Company & Total Assemblies & $\begin{array}{c}\text { Fabricating Company } \\
\text { (Percent) }\end{array}$ \\
\hline \multicolumn{3}{|l|}{ BWR Assemblies } \\
\hline Allis Chalmers ........... & 155 & 0.27 \\
\hline GE Nuclear Energy $\ldots \ldots \ldots \ldots \ldots \ldots \ldots \ldots$ & 51,119 & 90.42 \\
\hline Nuclear Fuel Services $\ldots \ldots \ldots \ldots \ldots \ldots \ldots \ldots$ & 8 & 0.01 \\
\hline Siemens Nuclear Fuel Company $\ldots \ldots \ldots \ldots \ldots$ & 4,789 & 8.47 \\
\hline United Nuclear Corporation . . . . . . . . . . . & 458 & 0.81 \\
\hline Westinghouse Electric $\ldots \ldots \ldots \ldots \ldots \ldots \ldots$ & 4 & 0.01 \\
\hline Undetermined $^{\mathrm{a}} \ldots \ldots \ldots \ldots \ldots \ldots \ldots$ & 4 & 0.01 \\
\hline BWR Subtotal $\ldots \ldots \ldots \ldots \ldots \ldots \ldots$ & 56,537 & 100.00 \\
\hline \multicolumn{3}{|l|}{ PWR Assemblies } \\
\hline ABB Combustion Engineering $\ldots \ldots \ldots \ldots \ldots$ & 7,763 & 18.54 \\
\hline Babcock \& Wilcox Company . . . . . . . . . . . . . & 5,841 & 13.95 \\
\hline General Atomics $\ldots \ldots \ldots \ldots \ldots \ldots \ldots \ldots$ & 3 & 0.01 \\
\hline Nuclear Materials and Equipment Corporation ... & 4 & 0.01 \\
\hline Siemens Nuclear Fuel Company . . . . . . . . . & 3,786 & 9.04 \\
\hline United Nuclear Corporation $\ldots \ldots \ldots \ldots \ldots \ldots$ & 73 & 0.17 \\
\hline Westinghouse Electric . . . . . . . . . . . . & 24,289 & 58.02 \\
\hline Undetermined $^{\mathrm{a}} \ldots \ldots \ldots \ldots \ldots \ldots \ldots \ldots$ & 104 & 0.25 \\
\hline PWR Subtotal $\ldots \ldots \ldots \ldots \ldots \ldots \ldots \ldots$ & 41,863 & 100.00 \\
\hline Total $\quad \ldots \ldots \ldots \ldots \ldots \ldots \ldots \ldots \ldots$ & 98,400 & 100.00 \\
\hline
\end{tabular}

A total of 4 BWR and 104 PWR temporarily discharged assemblies do not have an assigned assembly type code. Without an assembly type code, the fuel fabricating company cannot be identified. See Technical Note 10 in Appendix E.

BWR $=$ Boiling-water reactor; $P W R=$ Pressurized-water reactor.

Note: A total of 2,208 high-temperature, gas-cooled reactor (HTGR) fuel elements, with initial uranium content equal to 24.2 metric tons of uranium (MTU), were discharged. These HTGR fuel elements are not included in the above table. See Technical Note 6 in Appendix E.

Source: Energy Information Administration, Form RW-859, "Nuclear Fuel Data" (1993). 
Table 28. Distribution of Assembly Transverse Dimensions

\begin{tabular}{|c|c|c|c|}
\hline $\begin{array}{l}\text { Assembly } \\
\text { Length } \\
\text { (inches) }\end{array}$ & $\begin{array}{c}\text { Assembly } \\
\text { Width } \\
\text { (inches) }\end{array}$ & $\begin{array}{c}\text { Number } \\
\text { of } \\
\text { Assemblies }\end{array}$ & $\begin{array}{c}\text { Assembly } \\
\text { (Percent) }\end{array}$ \\
\hline \multicolumn{4}{|c|}{ Boiling-Water Reactors (BWR) } \\
\hline $\begin{array}{r}84.0 \\
95.0 \\
102.5 \\
134.4 \\
171.2 \\
176.2\end{array}$ & $\begin{array}{l}6.52 \\
4.67 \\
5.62 \\
4.28 \\
5.44 \\
5.44\end{array}$ & $\begin{array}{r}401 \\
390 \\
333 \\
892 \\
18,017 \\
36,500\end{array}$ & $\begin{array}{r}0.71 \\
0.69 \\
0.59 \\
1.58 \\
31.87 \\
64.56\end{array}$ \\
\hline Total & & $\bullet 56,537$ & ${ }^{2} 100.00$ \\
\hline \multicolumn{4}{|c|}{ Pressurized-Water Reactors (PWR) } \\
\hline 111.8 & 7.62 & 533 & 1.28 \\
\hline 137,1 & 7.76 & 665 & 1.59 \\
\hline 137.1 & 8.42 & 892 & 2.14 \\
\hline 138.8 & 6.27 & 160 & 0.38 \\
\hline 146.0 & 8.10 & 570 & 1.36 \\
\hline 147.5 & 8.20 & 793 & 1.90 \\
\hline 157.0 & 8.10 & 4,309 & 10.32 \\
\hline 158.2 & 8.10 & 464 & 1.11 \\
\hline 159.8 & 7.76 & 3,916 & 9.38 \\
\hline 159.8 & 8.44 & 20,645 & 49.44 \\
\hline 165.7 & 8.54 & 5,179 & 12.40 \\
\hline 176.8 & 8.10 & 2,176 & 5.21 \\
\hline 178.3 & 8.10 & 1,036 & 2.48 \\
\hline 199.0 & 8.43 & 421 & 1.01 \\
\hline Total & & ' 41,863 & ${ }^{b} 100.00$ \\
\hline
\end{tabular}

A total of 4 temporarily discharged BWR assemblies that have not been assigned an assembly type code are included in the column total. See Technical Note 10 in Appendix $E$.

'A total of 104 temporarily discharged PWR assemblies that have not been assigned an assembly type code are included in the column total. See Technical Note 10 in Appendix E.

Source: Energy Information Administration, Form RW-859, "Nuclear Fuel Data" (1993). 

Appendix A

\section{Survey Methodology}

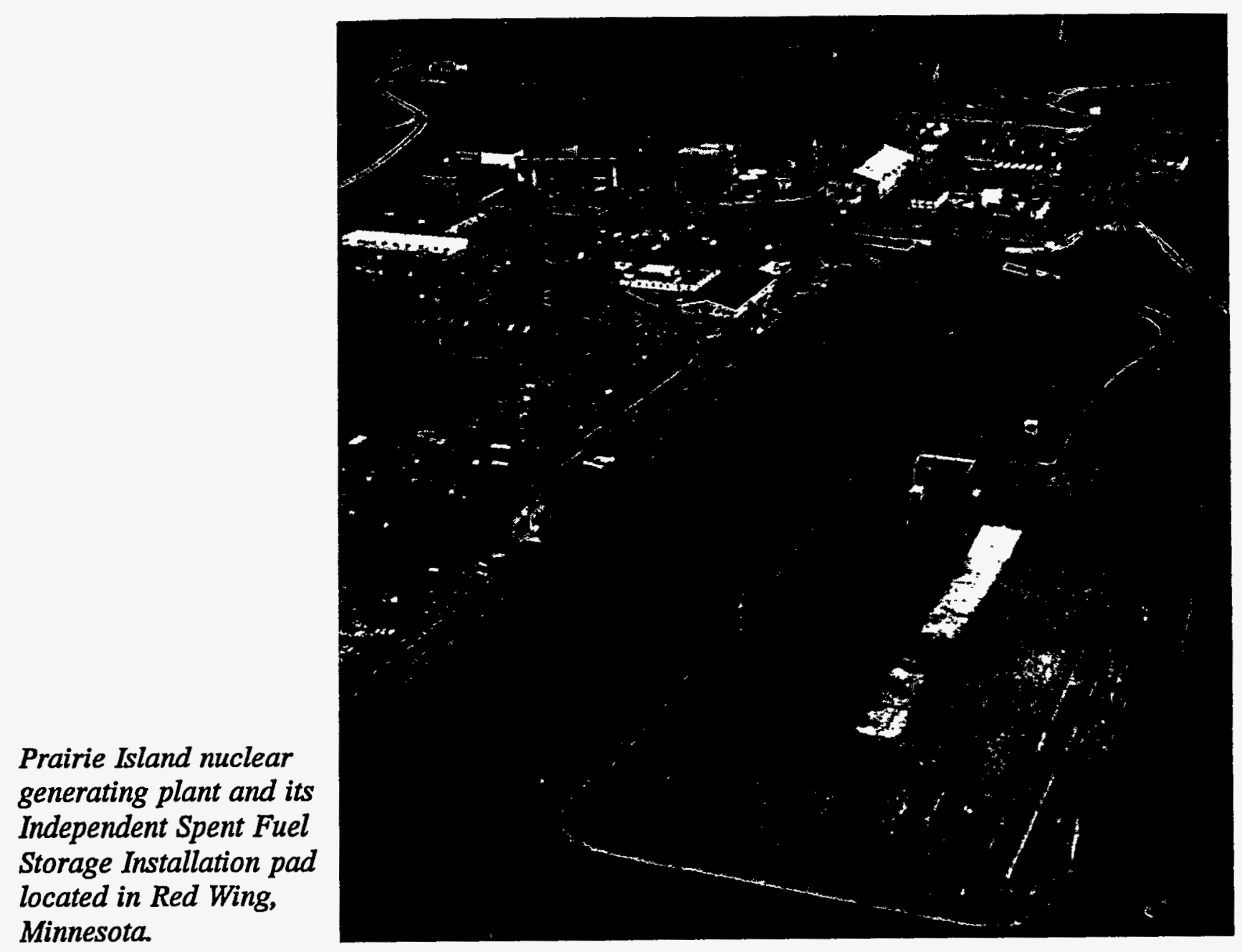




\section{Survey Methodology}

\section{Survey Design}

The Energy Information Administration (EIA) administers the Nuclear Fuel Data survey, Form RW-859, under a Memorandum of Understanding with the Office of Civilian Radioactive Waste Management (OCRWM). Form RW-859 is used to collect data from all commercial utilities that operate nuclear power plants and from all other owners of commercial spent nuclear fuel. Form RW-859 is a mandatory data collection form authorized under the Federal Energy Administration Act of 1974 (15 USC 761 et seq.) and the Nuclear Waste Policy Act of 1982, as amended (42 USC 10101 et seq.).

Respondents to the Nuclear Fuel Data survey are required to report data on every fuel assembly discharged from domestic commercial nuclear reactors, spent fuel projected to be discharged, and spent fuel storage pool inventories and capacities. Form RW-859 respondents list all assemblies discharged at the end of the reporting cycle, assemblies to be inserted during the next cycle, previously irradiated assemblies to be inserted in the next cycle, and assemblies shipped to another storage site or to dry storage.

For all assemblies discharged during the most recent refueling interval, the utilities submit the following data on Form RW-859:

- Discharge cycle

- End-of-cycle date

- Average initial loading weight for each batch of assemblies, in kilograms of uranium $(\mathrm{kg})$

- Average nominal initial enrichment assay for each batch of assemblies, in percent weight

- Average discharge burnup for each batch of assemblies, in gigawattdays thermal per metric ton of uranium (GWDt/MTU)

- Assembly type (Table B4) and rod array configuration for each batch of assemblies

- Current storage location of each assembly

- Standard disposal contract number applicable to each assembly

- Number of assemblies in each batch.
The above spent fuel discharge data are reported at the batch level. A batch is characterized by the number of assemblies in it, the nominal initial fuel enrichment, and the batch average discharge burnup. The utilities also provide spent fuel discharge projections for their next five fueling cycles, along with the projected cycle shut down dates and the cycle burnup in terms of effective full-power days. Spent fuel storage data include the amount of fuel stored at each pool site, or in dry storage, current, licensed and maximum storage site capacities, data on planned increases in pool storage capacity, maximum cask weights that can be handled at each site, and scheduled shipments of assemblies to other locations.

\section{Survey Universe and Frame}

Form RW-859 is a total universe survey of all owners of commercial spent nuclear fuel. The Form RW-859 survey frame, therefore, consists of all commercial electric utility nuclear power plants and all storage facilities possessing irradiated nuclear fuel from commercial power plants. The respondent population for Form RW-859 is stable. The frame contains operating reactors, storage facilities, reactors that have been permanently shut down, and reactors that have been constructed but are not yet operating. Shut down reactors are maintained on the survey frame for their historical data. Currently, 54 utilities report for 120 reactors. Four storage facilities are also required to report.

\section{Data Collection Procedures}

EIA sends survey packages in December to an official of each company, via certified mail to ensure receipt and to ascertain the correct mailing address. Survey packages include a diskette containing current data, a software diskette, a paper copy of the survey form, a set of instructions, a pictogram showing current inventory and storage data, a cover letter, and a return envelope. Most Form RW-859 respondents submit their data on diskette. 
The microcomputer software system used to collect From RW-859 data is referred to as the Automated Nuclear Fuel Data Collection System (ANFDCS). This is a Clipper-compiled program with a dBASE file structure. The microcomputer software is designed to run on any IBM-compatible microcomputer.

To reduce respondent burden, EIA provides the utilities with their current data and requests updated data using the ANFDCS and the instructions provided. EIA provides assistance in completing the survey by telephone when requested. Most respondents successfully and accurately update data diskettes with little or no assistance and tend to file their completed surveys by the requested deadlines.

\section{Data Editing, Analysis, and Processing}

As EIA receives survey packages from Form RW-859 respondents, they are logged in and reviewed. A preliminary review is conducted to check for obvious errors or omissions and to verify respondent name, address, and contact information. Data from the completed Form RW-859 surveys are then uploaded to the EIA mainframe computer and maintained in two files. The first is an assembly-level SAS file, which contains data on all individual assemblies at all reactors. These detailed assembly data are aggregated to the batch level. Batch level data are maintained in the second file, a partitioned data set of flat transaction files, with one member for each reactor.

Computer programs are utilized to review individual assembly data and report the data in organized formats that are useful to both analysts and management. A number of the tables and graphs presented in this report were produced from these programs. These tables are also available through the Nuclear Data Information System (NUCDIS), a microcomputer-based, menu-driven information retrieval system. Additional tables, which may be of interest to analysts requiring greater detail, are available upon request from OCRWM or EIA.

In addition to updating the historical Form RW-859 data files, EIA also produces spent fuel projections, using a set of assumptions concerning the future operation of the Nation's nuclear power plants. While the projected data submitted by the utilities generally forecast a more optimistic operating environment, in aggregate, than has actually been achieved, both EIA and utility forecasts track each other reasonably well in the short term. EIA developed the Disaggregate Forecasting System to bring the aggregated EIA forecasts in line with comparable utility projections. This process preserves, as much as possible, the information that the reactor operators provide in their spent fuel projections. The disaggregated forecasts are published annually in EIA's World Nuclear Outlook report.

The historical data submitted on Form RW-859, aggregated to the batch-level, along with the projections of spent fuel discharges from EIA's disaggregated forecasts, constitute the official DOE spent fuel data base. EIA distributes this data base in computer files on magnetic tape to the OCRWM and its contractors, and to the DOE national laboratories for their review and analysis.

\section{Response Rates}

Respondents to Form RW-859 are required to submit forms under one of two options, chosen by the utility. Option A requires a utility to submit its data within 90 days of cycle startup after refueling. Data reflect the utility's status at cycle startup. Option B requires a utility to submit data once each year by February 15 . Data reflect the utility's status as of December 31 of the previous year. Storage-only. facilities are required to report annually by February 15 for the previous year.

A total of 34 Option A reactors submitted data reflecting a cycle startup after refueling in 1993. Data for the remaining 12 Option $A$ reactors were simply "rolled over" from the previous year. All 74 Option B reactors and 4 storage facilities also reported. The data presented in this report, therefore, represent an accurate account as of December 31, 1993.

\section{Missing Data and Imputation}

EIA encourages utilities to complete all elements on Form RW-859. There is no imputing of missing values. If there are omissions, or if incorrect or inconsistent data are reported, the EIA Survey Manager telephones the utility to obtain missing or corrected data.

A limited number of data items were identified as missing or omitted from Form RW-859 data files. Most missing items represented data not available to the utilities in their data files. Respondents were telephoned regarding the missing items to verify that these items could not be reported. Only confirmed 
company-reported data are contained in the data base and included in this report.

\section{EIA Quality Assurance Procedures}

To ensure the quality and accuracy of data collected on Form RW-859, EIA has developed extensive quality assurance (QA) procedures as part of the Form RW-859 survey processing system. At the core of the process is a $Q A$ program that produces a matrix for each reactor identifying possible errors throughout the processing cycle. The program ensures that data submitted on diskettes have been correctly uploaded from the PC to the mainframe. After the Survey Manager and other data users have reviewed the EIA QA matrix for a particular reactor, data are corrected and an iterative process of running additional reports is performed until all data for that reactor are clean. A description of the EIA QA reports and a more detailed discussion of EIA QA procedures is included in Appendix D of this report.

The accuracy of spent fuel assembly weights and discharge dates has been verified through reconciliation with two separate but related efforts: the spent fuel fee paid by utilities for assemblies discharged prior to April 7, 1983; and, the acceptance priority ranking process which designates the order in which spent fuel will be picked up by DOE on an oldest-fuel-first basis.

\section{Proposed Changes to Survey Form}

OCRWM is currently undertaking an extensive effort to consolidate the range of data sources and data bases into a single unified system. This Unified DataBase (UDB) system is being designed to integrate information throughout the Civilian Radioactive Waste Management System (CRWMS), to eliminate duplication of data and effort within OCRWM, and to expand the current information base available to data users. Preliminary plans are that the Form RW-859, Nuclear Fuel Data survey be used as the annual UDB data collection vehicle.

EIA normally revises the Nuclear Fuel Data survey form every three years. The most recent revision, for the 1991 survey year, included additional questions on dry storage, assembly identifier codes, canisters, and nonfuel components. Due to the UDB design effort, the revised version of the Form RW-859 survey planned for the 1994 survey year was not implemented. The Office of Management and Budget (OMB) has granted EIA a three year extension of the expiration date of the current form to allow for UDB design and implementation. 
Appendix B

\section{Detailed Assembly Type Statistics}

Calvert Cliffs nuclear power plant and its Independent Spent Fuel Storage Installation located in Lusby, Maryland.

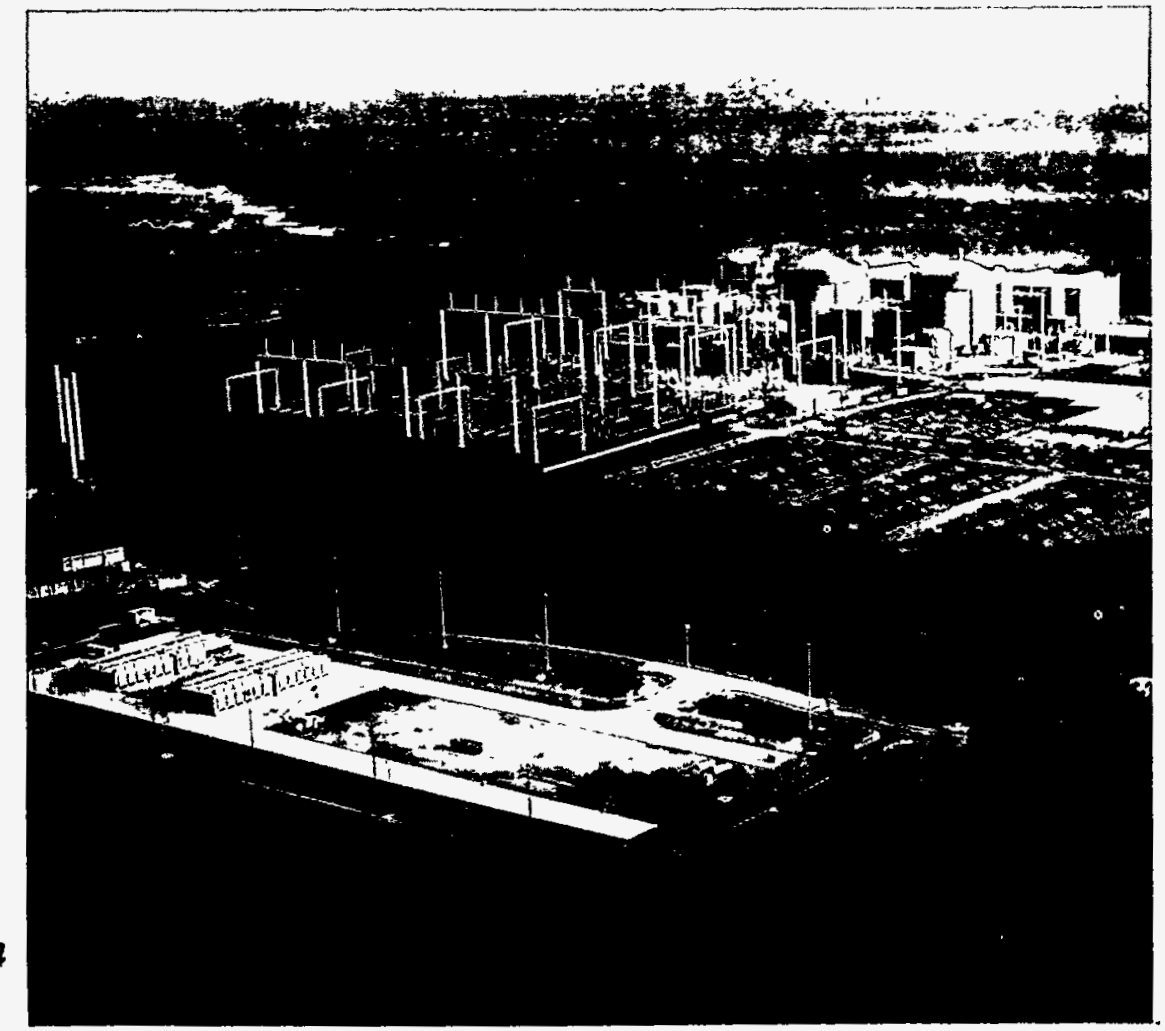





\section{Appendix B}

\section{Detailed Assembly Type Statistics}

The spent fuel Characteristics Data Base (CDB) developed by Oak Ridge National Laboratory (ORNL) includes detailed information on physical, radiological, and quantitative characteristics of spent nuclear fuel. The physical characteristics are organized by assembly class and, within each assembly class, by assembly type codes (developed jointly by ORNL and EIA) to provide detailed information about each type. Included in this information are the length and width of the overall assembly and the individual fuel rods, the number of fuel rods, the type of material used in the cladding and other parts of the assembly, and detailed assembly drawings. An eight-character assembly type code links Form RW-859 survey data with the CDB.

The characteristics of fuel assemblies are important in many areas of radioactive waste management. Assembly dimensions are clearly a major concern for shipping cask and waste package designers. If the fuel is to be consolidated, knowledge of the individual assembly types is essential, from the rod array configuration to the location of the bolts and welds for disassembly. The composition of the assembly hardware and cladding is also important. For example, cobalt-60, one of the major activation products, is more concentrated in stainless steel than in zircaloy. Assembly type characteristics are summarized by assembly type and reactor and are further amplified by a CDB module referred to as "LWR Fuel Assemblies Data Base," as described in the four-volume publication, Characteristics of Potential Repository Wastes (DOE/RW-0184-RI).

Whereas the physical characteristics of assemblies are identified through the assembly type codes, the radiological data in the $C D B$ are calculated based on the reactor type, discharge burnup, initial enrichment, and age from time of discharge. All these data are obtainable from Form RW-859. The radiological characteristics of the discharged assemblies are time-dependent and generally subject to the laws of exponential decay. The radiological characteristics in the CDB include grams, curies, and watts for more than 350 isotopes; photons for 18 gamma energy ranges; and neutrons.
This appendix provides detailed information on assembly type characteristics that is not included in the body of the report. These data are presented in table format and include the following:

- Fabricator Identifiers (Table B1)

- Assembly Class and Design Type by Utility (Table B2)

- Reactors by Assembly Class and Design Type (Table B3)

- Descriptions of Assembly Types (Table B4)

- Assembly Characteristics by Assembly Type (Table B5)

- Assembly Type Summary by Reactor (Table B6)

- Assembly Type Summary by Assembly Type (Table B7)

- Spent Fuel Assemblies, by Type, Discharged by Year (Table B8)

- Initial Uranium Content of Spent Fuel Assemblies, by Type, Discharged by Year (Table B9)

- Burnup of Spent Fuel Assemblies, by Type, Discharged by Year (Table B10).

The fuel assembly types throughout Appendix B have been listed in the following manner:

- The primary ordering criterion is assembly class - only assembly types within a reactor's particular assembly class are used at that reactor

- Within an assembly class, the secondary ordering criterion is fabricator. All fuel assembly types fabricated by the primary fuel fabricator (typically the reactor vendor) are listed first, followed by other fabricators in a generally alphabetical manner

- Within a fabricator in an assembly class, the primary ordering criterion is chronology. Earlier versions of fuels are listed first, more recent versions are listed later. This ordering is not absolute, but is based on the progression of designs over time. 
The detailed assembly type table statistics presented in Appendix B use the eight-character assembly type code. The eight-character code is used exclusively for Form RW-859 submissions and in the CDB.

The code is presented as follows: 8-Character Code W1414ATR

The first three characters of the code identify the assembly class and are fully compatible with the CDB scheme. The W14 in the example indicates Westing- house (WE) $14 \times 14$ assembly class. All reactor-specific assembly type codes have an " $X$ " as the first characteristic of the eight-character code (i.e., XHN indicates Haddam Neck). The next two characters identify the array size $(14=14 \times 14)$. The sixth character represents the fuel vendor [i.e., A for Advanced Nuclear Fuel (formerly Exxon Nuclear)]. The last two characters are a discriminator code that is designed to distinguish two fuel assembly types that are otherwise the same. The TR in the example indicates a top rod version.

Examples of discriminator codes that are used as the last two characters are:

Discriminator

- $\mathrm{L}$

- $\mathrm{O}$

- 5

- $P$
Meaning

Low parasitic fuel (LOPAR), zircaloy guide tubes

Optimized Fuel Assembly (OFA) zircaloy spacers, smaller fuel rods

GE-5 fuel design (original retrofit fuel)

Prepressurized BWR fuel rods
Assembly Type Code

W1414WL W1515WL

W1414WO

W1515WO

W1717WO

G2308G5

G4608G5

G2308GP

G4608GP 
A fuel rod, used in a nuclear power reactor, consists of uranium dioxide pellets encapsulated in a metal tube that is sealed at both ends. A fuel assembly is fabricated from a number of individual fuel rods arranged in arrays. The arrays are generally square, and have been made in many different dimensions. There are 131 reactor fuel assembly types which have been, or will be used. Each assembly type has certain distinguishing characteristics, such as the number of rods per assembly, fuel rod diameter, cladding type, materials used in fabrication, and other design features. The fuel assembly fabricator, the specific reactor and fuel models are characteristics often used to describe or name assembly types.

Assembly classes generally refer to generic groupings of assembly types designed for a particular class of reactor (for example, WE $14 \times 14$ class reactors). Assembly classes can also be reactor-specific, because in some cases fuel assembly arrays are configured to fit only one reactor (i.e., the Big Rock Point reactor). The fabricator of an assembly for a given reactor is not necessarily related to the reactor vendor or to the original fuel fabricator.

Table B1. Fabricator Identifiers

\begin{tabular}{|c|c|c|c|}
\hline Current Fabricator Name & Other Names Used & Short Name & Code \\
\hline ABB Combustion Engineering .... & $\begin{array}{l}\text { Combustion Engineering } \\
\text { ABB Atom (ABB) (BWR Only) }\end{array}$ & $\mathrm{CE}$ & C \\
\hline Allis Chalmers $\ldots \ldots \ldots \ldots \ldots \ldots$ & & $A C$ & L \\
\hline Babcock \& Wilcox Company & & $B \& W$ & B \\
\hline GE Nuclear Energy $\ldots \ldots \ldots \ldots \ldots \ldots \ldots \ldots$ & General Electric & GE & G \\
\hline General Atomics ... & $\begin{array}{l}\text { Gulf General Atomics (GGA) } \\
\text { Gulf/United Nuclear Fuels (GULF) }\end{array}$ & GA & $\mathrm{H}$ \\
\hline Nuclear Fuel Services $\ldots \ldots \ldots \ldots \ldots \ldots \ldots$ & & NFS & $N$ \\
\hline Nuclear Materials and Equipment Corporation ....... & NUMEC & NU & M \\
\hline Slemens Nuclear Corporation & $\begin{array}{l}\text { Advanced Nuclear Fuel Corporation (ANF) } \\
\text { Exxon Nuclear Corporation (EXA) } \\
\text { Jersey Nuclear }\end{array}$ & SIEM & A \\
\hline United Nuclear Corporation $\ldots \ldots \ldots \ldots \ldots \ldots$ & & UNC & $u$ \\
\hline Westinghouse Electric $\ldots \ldots \ldots \ldots \ldots \ldots \ldots \ldots$ & & WE & w \\
\hline
\end{tabular}

BWR $=$ Boiling-water reactor

Source: Energy Information Administration, Form RW-859, "Nuclear Fuel Data" (1993). 
Table B2. Assembly Class and Design Type by Utility

\begin{tabular}{|c|c|c|c|c|c|}
\hline Electric Utility Name & Reactor Name & $\begin{array}{l}\text { Reactor } \\
\text { ID }\end{array}$ & $\begin{array}{l}\text { Assembly } \\
\text { Class }\end{array}$ & $\begin{array}{l}\text { Nuclear Steam } \\
\text { System Supplier }\end{array}$ & Design Typo ${ }^{b}$ \\
\hline Alabama Power Company ...... & $\begin{array}{l}\text { Farley } 1 \\
\text { Farley } 2\end{array}$ & $\begin{array}{l}0101 \\
0102\end{array}$ & $\begin{array}{l}\text { WE } 17 \times 17 \\
\text { WE } 17 \times 17\end{array}$ & $\begin{array}{l}\text { WE } \\
\text { WE }\end{array}$ & $\begin{array}{l}\text { 3LP } \\
\text { 3LP }\end{array}$ \\
\hline $\begin{array}{l}\text { Arizona Public Service } \\
\text { Company .......... }\end{array}$ & $\begin{array}{l}\text { Palo Verde } 1 \\
\text { Palo Verde } 2 \\
\text { Palo Verde } 3\end{array}$ & $\begin{array}{l}0301 \\
0302 \\
0303\end{array}$ & $\begin{array}{l}\text { CE System } 80 \\
\text { CE System } 80 \\
\text { CE System } 80\end{array}$ & $\begin{array}{l}\mathrm{CE} \\
\mathrm{CE} \\
\mathrm{CE}\end{array}$ & $\begin{array}{l}\text { CE80 } \\
\text { CE80 } \\
\text { CE80 }\end{array}$ \\
\hline $\begin{array}{l}\text { Arkansas Power and } \\
\text { Light Company ..... }\end{array}$ & $\begin{array}{l}\text { Arkansas Nuclear } 1 \\
\text { Arkansas Nuclear } 2\end{array}$ & $\begin{array}{l}0401 \\
0402\end{array}$ & $\begin{array}{l}\text { BW } 15 \times 15 \\
\text { CE } 16 \times 16\end{array}$ & $\begin{array}{l}\mathrm{B \& W} \\
\mathrm{CE}\end{array}$ & $\begin{array}{l}\text { LLP } \\
\text { CE }\end{array}$ \\
\hline $\begin{array}{l}\text { Baltimore Gas and } \\
\text { Electric Company } \ldots \ldots \ldots \ldots\end{array}$ & $\begin{array}{l}\text { Calvert Cliffs } 1 \\
\text { Calvert Cliffs } 2\end{array}$ & $\begin{array}{l}0501 \\
0502\end{array}$ & $\begin{array}{l}\text { CE } 14 \times 14 \\
\text { CE } 14 \times 14\end{array}$ & $\begin{array}{l}C E \\
C E\end{array}$ & $\begin{array}{l}\mathrm{CE} \\
\mathrm{CE}\end{array}$ \\
\hline Boston Edison Company ...... & Pilgrim 1 & 0601 & GE BWR/2,3 & GE & 3 \\
\hline $\begin{array}{l}\text { Carolina Power and Light } \\
\text { Company ........... }\end{array}$ & $\begin{array}{l}\text { Brunswick } 1 \\
\text { Brunswick } 2 \\
\text { Harris } 1 \\
\text { Robinson } 2\end{array}$ & $\begin{array}{l}0701 \\
0702 \\
0703 \\
0705\end{array}$ & $\begin{array}{l}\text { GE BWR/4-6 } \\
\text { GE BWR/4-6 } \\
\text { WE } 17 \times 17 \\
\text { WE } 15 \times 15\end{array}$ & $\begin{array}{l}\text { GE } \\
G E \\
W E \\
W E\end{array}$ & $\begin{array}{l}4 \\
4 \\
3 L P \\
3 L P\end{array}$ \\
\hline $\begin{array}{l}\text { Cleveland Electric } \\
\text { Illuminating Company . }\end{array}$ & Perry 1 & 0901 & GE BWR/4-6 & GE & 6 \\
\hline $\begin{array}{l}\text { Commonwealth Edison } \\
\text { Company .......... }\end{array}$ & $\begin{array}{l}\text { Braidwood } 1 \\
\text { Braidwood } 2 \\
\text { Byron } 1 \\
\text { Byron } 2 \\
\text { Dresden } 1 \\
\text { Dresden } 2 \\
\text { Dresden } 3 \\
\text { LaSalle County } 1 \\
\text { LaSalle County } 1 \\
\text { Quad Cities } 1 \\
\text { Quad Cities } 2 \\
\text { Zion } 1 \\
\text { Zion } 2\end{array}$ & $\begin{array}{l}1001 \\
1002 \\
1003 \\
1004 \\
1005 \\
1006 \\
1007 \\
1008 \\
1009 \\
1010 \\
1011 \\
1012 \\
1013 \\
\end{array}$ & $\begin{array}{l}\text { WE } 17 \times 17 \\
\text { WE } 17 \times 17 \\
\text { WE } 17 \times 17 \\
\text { WE } 17 \times 17 \\
\text { Dresden } 1 \\
\text { GE BWR/2,3 } \\
\text { GE BWR/2,3 } \\
\text { GE BWR/4-6 } \\
\text { GE BWR/4-6 } \\
\text { GE BWR/2,3 } \\
\text { GE BWR/2,3 } \\
\text { WE } 15 \times 15 \\
\text { WE } 15 \times 15\end{array}$ & $\begin{array}{l}\text { WE } \\
W E \\
W E \\
W E \\
\text { GE } \\
G E \\
G E \\
G E \\
G E \\
G E \\
G E \\
W E \\
W E\end{array}$ & $\begin{array}{l}4 L P \\
4 L P \\
4 L P \\
4 L P \\
1 \\
3 \\
3 \\
5 \\
5 \\
3 \\
3 \\
4 L P \\
4 L P\end{array}$ \\
\hline $\begin{array}{l}\text { Consolidated Edison } \\
\text { Company of New York ...... }\end{array}$ & $\begin{array}{l}\text { Indian Point } 1 \\
\text { Indian Point } 2\end{array}$ & $\begin{array}{l}1101 \\
1102\end{array}$ & $\begin{array}{l}\text { Indian Point } 1 \\
\text { WE } 15 \times 15\end{array}$ & $\begin{array}{l}\text { B\&W } \\
\text { WE }\end{array}$ & $\ddot{4 L P}$ \\
\hline $\begin{array}{l}\text { Consumers Power } \\
\text { Company } \ldots \ldots \ldots \ldots \ldots\end{array}$ & $\begin{array}{l}\text { Big Rock Point } \\
\text { Palisades }\end{array}$ & $\begin{array}{l}1201 \\
1204\end{array}$ & $\begin{array}{l}\text { Big Rock Point } \\
\text { Palisades }\end{array}$ & $\begin{array}{l}\text { GE } \\
\text { CE }\end{array}$ & $\begin{array}{l}1 \\
\mathrm{CE}\end{array}$ \\
\hline $\begin{array}{l}\text { Dairyland Power } \\
\text { Cooperative } \ldots \ldots \ldots \ldots \ldots\end{array}$ & LaCrosse & 1301 & LaCrosse & $A C$ & .- \\
\hline Detroit Edison Company ...... & Enrico Fermi 2 & 1402 & GE BWR/4-6 & GE & 4 \\
\hline
\end{tabular}

See footnotes at end of table. 
Table B2. Assembly Class and Design Type by Utility (Continued)

\begin{tabular}{|c|c|c|c|c|c|}
\hline Electric Utility Name & Reactor Name & $\begin{array}{l}\text { Reactor } \\
\text { ID }\end{array}$ & $\begin{array}{l}\text { Assembly } \\
\text { Class }\end{array}$ & $\begin{array}{l}\text { Nuclear Steam } \\
\text { System Supplier" }\end{array}$ & Design Type ${ }^{D}$ \\
\hline Duke Power Company $\ldots \ldots$. & $\begin{array}{l}\text { Catawba } 1 \\
\text { Catawba } 2 \\
\text { McGuire } 1 \\
\text { McGuire } 2 \\
\text { Oconee } 1 \\
\text { Oconee } 2 \\
\text { Oconee } 3\end{array}$ & $\begin{array}{l}1501 \\
1502 \\
1504 \\
1505 \\
1506 \\
1507 \\
1508 \\
\end{array}$ & $\begin{array}{l}\text { WE } 17 \times 17 \\
\text { WE } 17 \times 17 \\
\text { WE } 17 \times 17 \\
\text { WE } 17 \times 17 \\
\text { BW } 15 \times 15 \\
\text { BW } 15 \times 15 \\
\text { BW } 15 \times 15\end{array}$ & $\begin{array}{l}\text { WE } \\
W E \\
W E \\
W E \\
\text { B\&W } \\
\text { B\&W } \\
\text { B\&W }\end{array}$ & $\begin{array}{l}4 L P \\
4 L P \\
4 L P \\
4 L P \\
\text { LLP } \\
\text { LLP } \\
\text { LLP }\end{array}$ \\
\hline Duquesne Light Company ..... & $\begin{array}{l}\text { Beaver Valley } 1 \\
\text { Beaver Valley } 2\end{array}$ & $\begin{array}{l}1601 \\
1602\end{array}$ & $\begin{array}{l}\text { WE } 17 \times 17 \\
\text { WE } 17 \times 17\end{array}$ & $\begin{array}{l}\text { WE } \\
\text { WE }\end{array}$ & $\begin{array}{l}\text { 3LP } \\
\text { 3LP }\end{array}$ \\
\hline Florida Power Corporation ..... & Crystal River 3 & 1701 & $B W 15 \times 15$ & B\&W & LLP \\
\hline $\begin{array}{l}\text { Florida Power and Light } \\
\text { Company } \ldots \ldots \ldots \ldots \ldots\end{array}$ & $\begin{array}{l}\text { St. Lucie } 1 \\
\text { St. Lucie } 2 \\
\text { Turkey Point } 3 \\
\text { Turkey Point } 4\end{array}$ & $\begin{array}{l}1801 \\
1802 \\
1803 \\
1804\end{array}$ & $\begin{array}{l}\text { CE } 14 \times 14 \\
\text { St. Lucie } 2 \\
\text { WE } 15 \times 15 \\
\text { WE } 15 \times 15\end{array}$ & $\begin{array}{l}\text { CE } \\
C E \\
\text { WE } \\
\text { WE }\end{array}$ & $\begin{array}{l}\text { CE } \\
\text { CE } \\
\text { 3LP } \\
\text { 3LP }\end{array}$ \\
\hline Georgla Power Company & $\begin{array}{l}\text { Hatch } 1 \\
\text { Hatch } 2 \\
\text { Vogtle } 1 \\
\text { Vogtle } 2\end{array}$ & $\begin{array}{l}2001 \\
2002 \\
2003 \\
2004\end{array}$ & $\begin{array}{l}\text { GE BWR/4-6 } \\
\text { GE BWR/4-6 } \\
\text { WE } 17 \times 17 \\
\text { WE } 17 \times 17\end{array}$ & $\begin{array}{l}\mathrm{GE} \\
\mathrm{GE} \\
\mathrm{WE} \\
\text { WE }\end{array}$ & $\begin{array}{l}4 \\
4 \\
4 L P \\
4 L P\end{array}$ \\
\hline GPU Nuclear Corporation ..... & $\begin{array}{l}\text { Three Mile Island } 1 \\
\text { Oyster Creek }\end{array}$ & $\begin{array}{l}1901 \\
1903\end{array}$ & $\begin{array}{l}\text { BW } 15 \times 15 \\
\text { GE BWR/2,3 }\end{array}$ & $\begin{array}{l}\text { B\&W } \\
\mathrm{GE}\end{array}$ & $\begin{array}{l}\text { LLP } \\
2\end{array}$ \\
\hline $\begin{array}{l}\text { Gulf States Utilities } \\
\text { Company } \ldots \ldots \ldots\end{array}$ & River Bend 1 & 2101 & GE BWR/4-6 & GE & 6 \\
\hline $\begin{array}{l}\text { Houston Lighting and } \\
\text { Power Company ... }\end{array}$ & $\begin{array}{l}\text { South Texas } 1 \\
\text { South Texas } 2\end{array}$ & $\begin{array}{l}2201 \\
2202\end{array}$ & $\begin{array}{l}\text { South Texas } \\
\text { South Texas }\end{array}$ & $\begin{array}{l}\text { WE } \\
\text { WE }\end{array}$ & $\begin{array}{l}\text { 4LP } \\
\text { 4LP }\end{array}$ \\
\hline IES Utilities, Inc. . . . . . . . . & Duane Amold & 2401 & GE BWR/4-6 & GE & 4 \\
\hline Illinols Power Company ....... & Clinton 1 & 2301 & GE BWR/4-6 & GE & 6 \\
\hline $\begin{array}{l}\text { Indiana Michigan } \\
\text { Power Company .......... }\end{array}$ & $\begin{array}{l}\text { Cook } 1 \\
\text { Cook } 2\end{array}$ & $\begin{array}{l}5801 \\
5802\end{array}$ & $\begin{array}{l}\text { WE } 15 \times 15 \\
\text { WE } 17 \times 17\end{array}$ & $\begin{array}{l}\text { WE } \\
\text { WE }\end{array}$ & $\begin{array}{l}\text { 4LP } \\
\text { 4LP }\end{array}$ \\
\hline $\begin{array}{l}\text { Kansas Gas and Electric } \\
\text { Company } \ldots \ldots \ldots \ldots \ldots \ldots\end{array}$ & Wolf Creek 1 & 2501 & WE $17 \times 17$ & WE & $4 L P$ \\
\hline $\begin{array}{l}\text { Long Island Power } \\
\text { Authority } \ldots \ldots \ldots\end{array}$ & Shoreham & 2601 & GE BWR/4-6 & GE & 4 \\
\hline $\begin{array}{l}\text { Louisiana Power and } \\
\text { Light Company ........... }\end{array}$ & Waterford 3 & 2701 & CE $16 \times 16$ & CE & CE \\
\hline $\begin{array}{c}\text { Maine Yankee Atomic } \\
\text { Power Company .... }\end{array}$ & Maine Yankee & 2801 & CE $14 \times 14$ & CE & $\mathrm{CE}$ \\
\hline
\end{tabular}

See footnotes at end of table. 
Table B2. Assembly Class and Design Type by Utility (Continued)

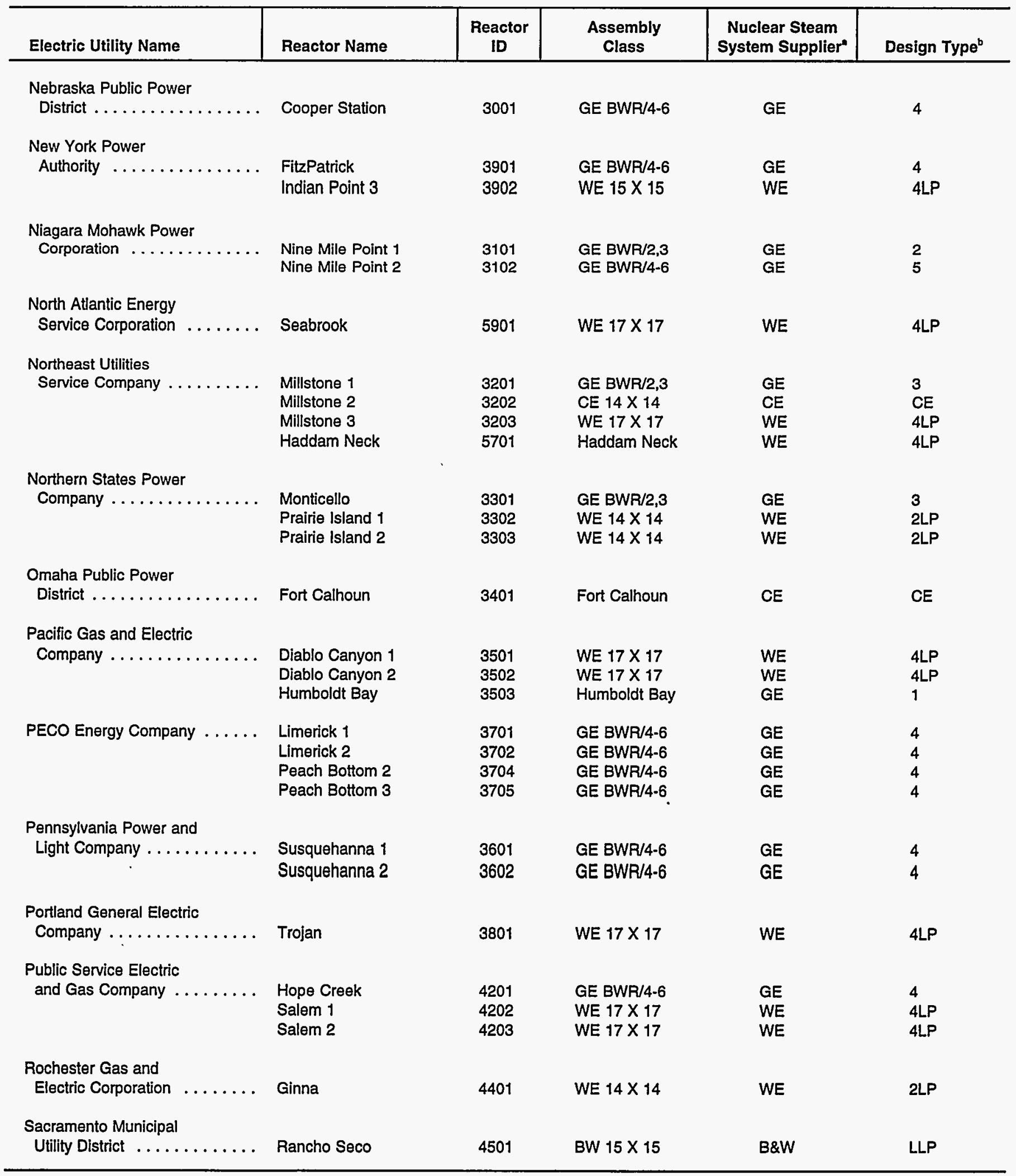

See footnotes at end of table. 
Table B2. Assembly Class and Design Type by Utility (Continued)

\begin{tabular}{|c|c|c|c|c|c|}
\hline Electric Utillty Name & Reactor Name & $\begin{array}{l}\text { Reactor } \\
\text { ID }\end{array}$ & $\begin{array}{l}\text { Assembly } \\
\text { Class }\end{array}$ & $\begin{array}{l}\text { Nuclear Steam } \\
\text { System Supplier }\end{array}$ & Design Type \\
\hline $\begin{array}{l}\text { South Carolina Electric } \\
\text { and Gas Company ......... }\end{array}$ & Summer & 4601 & WE $17 \times 17$ & WE & 3LP \\
\hline $\begin{array}{l}\text { Southem California } \\
\text { Edison Company } . . \ldots \ldots \ldots\end{array}$ & $\begin{array}{l}\text { San Onofre } 1 \\
\text { San Onofre } 2 \\
\text { San Onofre } 3\end{array}$ & $\begin{array}{l}4701 \\
4702 \\
4703\end{array}$ & $\begin{array}{l}\text { San Onofre } 1 \\
\text { CE } 16 \times 16 \\
\text { CE } 16 \times 16\end{array}$ & $\begin{array}{l}\text { WE } \\
C E \\
C E\end{array}$ & $\begin{array}{l}3 L P \\
\mathrm{CE} \\
\mathrm{CE}\end{array}$ \\
\hline $\begin{array}{l}\text { System Energy Resources, } \\
\text { Inc. } \ldots \ldots \ldots \ldots \ldots \ldots \ldots \ldots\end{array}$ & Grand Gulf 1 & 2901 & GE BWR/4-6 & GE & 6 \\
\hline $\begin{array}{l}\text { Tennesses Valley } \\
\text { Authority } \ldots \ldots \ldots \ldots \ldots\end{array}$ & $\begin{array}{l}\text { Browns Ferry } 1 \\
\text { Browns Ferry } 2 \\
\text { Browns Ferry } 3 \\
\text { Sequoyah } 1 \\
\text { Sequoyah } 2 \\
\text { Watts Bar } 1 \\
\text { Watts Bar } 2\end{array}$ & $\begin{array}{l}4803 \\
4804 \\
4805 \\
4808 \\
4809 \\
4810 \\
4811\end{array}$ & $\begin{array}{l}\text { GE BWR/4-6 } \\
\text { GE BWR/4-6 } \\
\text { GE BWR/4-6 } \\
\text { WE } 17 \times 17 \\
\text { WE } 17 \times 17 \\
\text { WE } 17 \times 17 \\
\text { WE } 17 \times 17\end{array}$ & $\begin{array}{l}\text { GE } \\
\text { GE } \\
\text { GE } \\
\text { WE } \\
\text { WE } \\
\text { WE } \\
\text { WE }\end{array}$ & $\begin{array}{l}4 \\
4 \\
4 \\
4 \mathrm{LP} \\
4 \mathrm{LP} \\
4 \mathrm{LP} \\
4 \mathrm{LP}\end{array}$ \\
\hline Toledo Edison Company . . . . . . & Davis-Besse & 5001 & $\mathrm{BW} 15 \times 15$ & B\&W & LLP \\
\hline TU Electric . ............ & $\begin{array}{l}\text { Comanche Peak } 1 \\
\text { Comanche Peak } 2\end{array}$ & $\begin{array}{l}4901 \\
4902\end{array}$ & $\begin{array}{l}\text { WE } 17 \times 17 \\
\text { WE } 17 \times 17\end{array}$ & $\begin{array}{l}\text { WE } \\
\text { WE }\end{array}$ & $\begin{array}{l}\text { 4LP } \\
4 L P\end{array}$ \\
\hline Unlon Electric Company $\ldots \ldots$ & Callaway & 5101 & WE $17 \times 17$ & WE & 4LP \\
\hline $\begin{array}{l}\text { Vermont Yankee Nuclear } \\
\text { Power Corporation ........ }\end{array}$ & Vermont Yankee & 6001 & GE BWR/4-6 & GE & 4 \\
\hline $\begin{array}{l}\text { Wisconsin Electric Power } \\
\text { Company ............. }\end{array}$ & $\begin{array}{l}\text { Point Beach } 1 \\
\text { Point Beach } 2\end{array}$ & $\begin{array}{l}5401 \\
5402\end{array}$ & $\begin{array}{l}\text { WE } 14 \times 14 \\
\text { WE } 14 \times 14\end{array}$ & $\begin{array}{l}\text { WE } \\
\text { WE }\end{array}$ & $\begin{array}{l}2 L P \\
2 L P\end{array}$ \\
\hline $\begin{array}{l}\text { Wisconsin Publlc Service } \\
\text { Corporation } \ldots \ldots \ldots \ldots \ldots\end{array}$ & Kewaunee & 5501 & WE $14 \times 14$ & WE & 2LP \\
\hline $\begin{array}{l}\text { Yankee Atomic Electric } \\
\text { Company } \ldots \ldots \ldots \ldots \ldots \ldots\end{array}$ & Yankee Rowe & 5601 & Yankee Rowe & WE & 4LP \\
\hline
\end{tabular}

"Nuclear Steam System Supplier (NSSS) codes are as follows: AC = Allis Chalmers; B\&W = Babcock \& Wilcox Company; CE = Combustion Engineering; GE = GE Nuclear Energy; WE = Westinghouse Electric. Data obtained from Nuclear Regulatory Commission, Information Digest 1994, (March 1994), Appendix A.

Design types are as follows: $1=$ GE Type $1 ; 2=$ GE Type 2; $3=$ GE Type 3; $4=$ GE Type 4; $5=$ GE Type 5; $6=$ GE Type 6; 2LP = Westinghouse Two-Loop; 3LP = Westinghouse Three-Loop; 4LP = Westinghouse Four-Loop; CE = Combustion Engineering; CE80 = CE Standard Design; LLP = B\&W Lowered Loop. Data obtained from Nuclear Regulatory Commission publication Information Digest 1994, (March 1994), Appendlx A, NSSS column.

$\ldots=$ Not appllcable.

Source: Energy Information Administration, Form RW-859, "Nuclear Fuel Data" (1993). 
Table B3. Reactors by Assembly Class and Design Type

B\&W $15 \times 15$ Assembly Class

Arkansas Nuclear 1 (LLP)

Oconee 1 (LLP)

Rancho Seco (LLP)*

B\&W $17 \times 17$ Assembly Class

(4 test assemblies irradiated at Oconee 1, 2, \& 3)

CE $14 \times 14$ Assembly Class

Calvert Cliffs 1 (CE)

Millstone 2 (CE)

Calvert Cliffs 2 (CE)

St. Lucie 1 (CE)

CE $16 \times 16$ Assembly Class

Arkansas Nuclear 2 (CE)

Waterford 3 (CE)

CE $16 \times 16$ System 80 Assembly Class

Palo Verde 1 (CE80)

Palo Verde 2 (CE80)

Palo Verde 3 (CE80)

GE BWR/2,3 Assembly Class

Dresden 2 (3)

Monticello (3)

Pilgrim 1 (3)

Dresden 3 (3)

Nine Mile Point 1 (2)

Quad Cities 1 (3)

GE BWR/4-6 Assembly Class

Browns Ferry 1 (4)

Brunswick 1 (4)

Cooper Station (4)

FitzPatrick (4)

Hatch 2 (4)

LaSalle County 2 (5)

Nine Mile Point 2 (5)

Perry 1 (6)

Susquehanna 1 (4)

Washington Nuclear 2 (5)

WE $14 \times 14$ Assembly Class

Ginna (2LP)

Point Beach 2 (2LP)

WE $15 \times 15$ Assembly Class

Cook 1 (4LP)

Robinson 2 (3LP)

Turkey Point 3 (3LP)

Zion 2 (4LP)
Crystal River 3 (LLP)

Davis-Besse (LLP)

Oconee 3 (LLP)
Maine Yankee (CE)

San Onofre 3 (CE)

San Onofre 2 (CE)

Millstone 1 (3)

Oyster Creek (2)

Quad Cities 2 (3)

Browns Ferry 2 (4)

Brunswick 2 (4)

Duane Arnold (4)

Grand Gulf 1 (6)

Hope Creek (4)

Limerick 1 (4)

Peach Bottom 2 (4)

River Bend 1 (6)

Susquehanna 2 (4)

Browns Ferry 3 (4)

Clinton 1 . (6)

Enrico Fermi 2 (4)

Hatch 1 (4)

LaSalle County 1 (5)

Limerick 2 (4)

Peach Bottom 3 (4)

Shoreham (4)*

Vermont Yankee (4)

Kewaunee (2LP)

Prairie Island 1 (2LP)

Point Beach 1 (2LP)

Prairie Island 2 (2LP)

Indian Point 3 (4LP)

Surry 2 (3LP)

Zion 1 (4LP)

See footnotes at end of table. 
WE $17 \times 17$ Assembly Class

Beaver Valley 1 (3LP)

Braidwood 2 (4LP)

Callaway (4LP)

Comanche Peak 1 (4LP)

Diablo Canyon 1 (4LP)

Farley 2 (3LP)

McGuire 2 (4LP)

North Anna 2 (3LP)

Seabrook (4LP)

Summer (3LP)

Vogtle 2 (4LP)

Wolf Creek 1 (4LP)

South Texas Assembly Class

South Texas 1 (4LP)

Single Reactor Assembly Classes

Big Rock Point (1)

Humboldt Bay (1)*

LaCrosse (--)*

San Onofre $1(3 \mathrm{LP})^{*}$
Beaver Valley 2 (3LP)

Byron 1 (4LP)

Catawba 1 (4LP)

Comanche Peak 2 (4LP)

Diablo Canyon 2 (4LP)

Harris 1 (3LP)

Millstone 3 (4LP)

Salem 1 (4LP)

Sequoyah 1 (4LP)

Trojan (4LP)*

Watts Bar 1 (4LP)

South Texas 2 (4LP)

Dresden 1 (1)*

Haddam Neck (4LP)

Palisades (CE)

Yankee Rowe (4LP)*
Braidwood 1 (4LP)

Byron 2 (4LP)

Catawba 2 (4LP)

Cook 2 (4LP)

Farley 1 (3LP)

McGuire 1 (4LP)

North Anna 1 (3LP)

Salem 2 (4LP)

Sequoyah 2 (4LP)

Vogtle 1 (4LP)

Watts Bar 2 (4LP)

-- = Not applicable. " = Reactors are permanently shut down.

Notes: Design types are as follows: $1=$ GE Type 1; $2=$ GE Type 2; $3=$ GE Type 3; $4=$ GE Type 4; $5=$ GE Type 5; $6=$ GE Type 6; 2LP = Westinghouse Two-Loop; 3LP = Westinghouse Three-Loop; 4LP = Westinghouse Four-Loop; CE = Combustion Engineering; CE80 = CE Standard Design; LLP = B\&W Lowered Loop. Data obtained from Nuclear Regulatory Commission, Information Digest 1994, (March 1994), Appendix A.

Source: Energy Information Administration, Form RW-859, "Nuclear Fuel Data" (1993). 
Table B4. Descriptions of Assembly Types

\begin{tabular}{l|c|c}
\hline Assembly Type & Description & $\begin{array}{c}\text { Assembly } \\
\text { Type Code }\end{array}$ \\
\hline
\end{tabular}

B\&W 15 X 15 Assembly Class

(Reactor Type: PWR / Length: 165.7 in. / Width: 8.54 in.)

B\&W 15 X 15 B\&W Mark B2

$B \& W 15 \times 15$ B\&W Mark B3

B\&W 15 X 15 B\&W Mark B4

$B \& W 15 \times 15$ B\&W Mark B4Z

$B \& W 15 \times 15$ B\&W Mark B5

$B \& W 15 \times 15$ B\&W Mark B5Z

B\&W $15 \times 15$ B\&W Mark B6

$B \& W 15 \times 15$ B\&W Mark B7

B\&W $15 \times 15$ B\&W Mark B8

B\&W 15 X 15 B\&W Mark B9
B\&W-manufactured fuel for B\&W $15 \times 15$ reactors; Mark B2 fuel uses a corrugated flexible grid spacer and a zirconium dioxide solid spacer between the fuel column and the fuel rod end plug; $\sim 664 \mathrm{~kg} \mathrm{U}$.

$B \& W$-manufactured fuel for B\&W $15 \times 15$ reactors; Mark B3 characteristics are not well defined because it is an early fuel design; $\sim 464 \mathrm{~kg} \mathrm{U}$.

$B \& W$-manufactured fuel for B\&W $15 \times 15$ reactors; standard fuel from B\&W for many years; Inconel spacer grids; $-464 \mathrm{~kg} \mathrm{U}$.

$B \& W$-manufactured fuel for B\&W $15 \times 15$ reactors; features 6 zircaloy grid spacers in the core zone; $464 \mathrm{~kg} \mathrm{U}$.

$B \& W$-manufactured fuel for B\&W $15 \times 15$ reactors; redesigned upper end fitting eliminates retainers for Burnable Poison Rod Assembly holddown; redesigned holddown spring made of Inconel 718 rather than Inconel $X-750 ; \sim 464 \mathrm{~kg} \mathrm{U}$.

$B \& W$-manufactured fuel for $B \& W 15 \times 15$ reactors; assemblies feature 6 zircaloy acid spacers in the core zone, a redesigned upper end fitting which eliminates the retainers for Burnable Poison Rod Assembly holddown, and a redesigned holddown spring made of Inconel 718 rather than Inconel X-750; $464 \mathrm{~kg} \mathrm{U.}$

B\&W-manufactured fuel for B\&W $15 \times 15$ reactors; assemblies feature 6 zircaloy grid spacers in the core zone and a skirtless and removable upper end fitting; $\sim 464 \mathrm{~kg} \mathrm{U}$.

$B \& W$-manufactured fuel for B\&W $15 \times 15$ reactors; in addition to the Mark B6 fuel, the Mark B7 features slightly longer fuel rods and a shorter lower end fitting; these features increase the plenum volume and fuel rod-to-nozzle gap, allowing for increased discharge burnups; $464 \mathrm{~kg} \mathrm{U}$.

$B \& W$-manufactured fuel for B\&W $15 \times 15$ reactors; in addition to the features of Mark B7 fuel, the Mark B8 fuel has a debris fretting resistant fuel rod design; $\sim 464 \mathrm{~kg} \mathrm{U}$.

$B \& W$-manufactured fuel for $B \& W 15 \times 15$ reactors; Mark $B 8$ features plus slightly increased pellet diameter and reduced B1515B9 stack length; $-464 \mathrm{~kg} \mathrm{U.}$ 
Table B4. Descriptions of Assembly Types (Continued)

\begin{tabular}{|c|c|c|}
\hline Assembly Type & Description & $\begin{array}{l}\text { Assembly } \\
\text { Type Code }\end{array}$ \\
\hline \multicolumn{3}{|c|}{$\begin{array}{l}\text { B\&W } 15 \text { X } 15 \text { Assembly Class (Continued) } \\
\text { (Reactor Type: PWR / Length: } 165.7 \text { in. / Width: } 8.54 \text { in.) }\end{array}$} \\
\hline B\&W 15 X 15 B\&W Mark B10 & $\begin{array}{l}\text { B\&W-manufactured fuel for B\&W } 15 \times 15 \text { reactors; Mark B9 } \\
\text { features; redesigned upper end fitting (helical holddown } \\
\text { springs replaced by cruciform leaf-spring design); } \\
\text { zone-loaded fuel enrichment variations; } \sim 464 \mathrm{~kg} \mathrm{U} \text {. }\end{array}$ & B1515B10 \\
\hline B\&W 15 X 15 B\&W Mark B & $\begin{array}{l}\mathrm{B} \& W \text {-manufactured fuel for } B \& W 15 \times 15 \text { reactors; Mark } B \\
\text { is a generic designation and is used when the specific } \\
\text { assembly type is unknown; } \sim 464 \mathrm{~kg} \mathrm{U} \text {. }\end{array}$ & B1515B \\
\hline B\&W 15 X 15 B\&W Zirc Grids & $\begin{array}{l}\text { B\&W-manufactured fuel for B\&W } 15 \times 15 \text { reactors; generic } \\
\text { designation for fuels with zircaloy grid spaces which } \\
\text { encompasses Mark B4Z, B5Z, B6, B7 and B8 fuels when } \\
\text { specific assembly type has not been determined; } \\
\sim 464 \mathrm{~kg} \mathrm{U} \text {. }\end{array}$ & $\mathrm{B} 1515 \mathrm{BZ}$ \\
\hline B\&W $15 \times 15$ B\&W Mark BEB & $\begin{array}{l}\text { B\&W-manufactured fuel for B\&W } 15 \times 15 \text { reactors; } \\
\text { designed to test extended burnup features, this fuel had a } \\
\text { shorter active fuel length and was used only as lead test } \\
\text { assemblies at Arkansas Nuclear } 1 ; \sim 464 \mathrm{~kg} \mathrm{U} \text {. }\end{array}$ & B1515BEB \\
\hline B\&W $15 \times 15$ B\&W Mark BGd & $\begin{array}{l}\text { B\&W-manufactured fuel for } B \& W 15 \times 15 \text { reactors; used as } \\
\text { lead test assemblies at Oconee } 1 \text {; this fuel utilized gadolinia } \\
\text { in the fuel rods as a neutron absorber; apparently had } \\
\text { removable upper end fittings, the forerunner of the skirtless } \\
\text { (Mark B6) upper end fitting; } \sim 430 \mathrm{~kg} \mathrm{U} \text {. }\end{array}$ & B1515BGD' \\
\hline$B \& W 15 \times 15$ WE & $\begin{array}{l}\text { WE-manufactured fuel for B\&W } 15 \times 15 \text { reactors; } \\
\text { scheduled for lead test assembly use at Three Mile } \\
\text { Island 1; } 462 \mathrm{~kg} \mathrm{U} \text {. }\end{array}$ & B1515W \\
\hline \multicolumn{3}{|c|}{$\begin{array}{l}\text { B\&W } 17 \times 17 \text { Assembly Class } \\
\text { (Reactor Type: PWR / Length: } 165.7 \text { in. / Width: } 8.54 \text { in.) }\end{array}$} \\
\hline B\&W 17 X 17 B\&W Mark C & $\begin{array}{l}\text { B\&W-manufactured fuel for } B \& W 17 \times 17 \text { reactors; test } \\
\text { assemblies used at Oconee } 1,2, \& 3 ;-456 \mathrm{~kg} \mathrm{U} \text {. } \\
\text { CE } 14 \times 14 \text { Assembly Class } \\
\text { ype: PWR / Length: } 157 \text { in. / Width: } 8.1 \text { in.) }\end{array}$ & $\mathrm{B} 1717 \mathrm{~B}$ \\
\hline CE $14 \times 14 \mathrm{CE}$ & CE-manufactured fuel for CE $14 \times 14$ reactors; $~ 382 \mathrm{~kg} \mathrm{U}$. & C1414C \\
\hline CE $14 \times 14$ ANF & ANF-manufactured fuel for CE $14 \times 14$ reactors; $\sim 370 \mathrm{~kg} \mathrm{U}$. & C1414A \\
\hline CE $14 \times 14$ WE & WE-manufactured fuel for CE $14 \times 14$ reactors; $\sim 407 \mathrm{~kg} \mathrm{U}$. & C1414W \\
\hline
\end{tabular}


Table B4. Descriptions of Assembly Types (Continued)

\begin{tabular}{|c|c|c|}
\hline Assembly Type & Description & $\begin{array}{l}\text { Assembly } \\
\text { Type Code }\end{array}$ \\
\hline \multicolumn{3}{|c|}{$\begin{array}{l}\text { CE } 16 \text { X } 16 \text { Assembly Class } \\
\text { (Reactor Type: PWR / Length: 176.8 in. / Width: } 8.1 \text { in.) }\end{array}$} \\
\hline \multicolumn{3}{|c|}{$\begin{array}{l}\text { CE } 16 \text { X } 16 \text { System } 80 \text { Assembly Class } \\
\text { (Reactor Type: PWR / Length: } 178.3 \text { in. / Width: } 8.1 \text { in.) }\end{array}$} \\
\hline CE $16 \times 16$ CE System 80 & $\begin{array}{l}\text { CE-manufactured fuel for CE System } 80 \text { reactors; } \\
\sim 413 \mathrm{~kg} \mathrm{U} \text {. }\end{array}$ & C8016C \\
\hline \multicolumn{3}{|c|}{$\begin{array}{l}\text { GE BWR/2,3 Assembly Class } \\
\text { (Reactor Type: BWR / Length: } 171.2 \text { in. / Width: } 5.44 \text { in.) }\end{array}$} \\
\hline GE BWR/2,3 7 X 7 GE-2a & $\begin{array}{l}\text { GE-manufactured fuel for GE BWR/2,3 reactors; original } \\
\text { core fuel at Oyster Creek, Nine Mile Point } 1 \text {, and } \\
\text { Millstone 1; fuel rod diameter of } 0.570^{\prime \prime} ; \sim 195 \mathrm{~kg} \mathrm{U} \text {. }\end{array}$ & G2307G2A \\
\hline GE BWR/2;3 $7 \times 7$ GE-2b & $\begin{array}{l}\text { GE-manufactured fuel for GE BWR/2,3 reactors; original } \\
\text { core fuel at other reactors; fuel rod diameter of } 0.563^{\prime \prime} \text {; } \\
\text { cladding thickness of } 0.032^{\prime \prime} ; \sim 193 \mathrm{~kg} \mathrm{U} \text {. }\end{array}$ & G2307G2B \\
\hline GE BWR/2,3 $7 \times 7$ GE-3 & $\begin{array}{l}\text { GE-manufactured fuel for GE BWR/2,3 reactors; "Improved" } \\
\text { fuel; cladding thickness of } 0.037 \text { "; hydrogen getter } \\
\text { introduced; } \sim 188 \mathrm{~kg} \mathrm{U} \text {. }\end{array}$ & G2307G3 \\
\hline GE BWR/2,3 8 X 8 GE-4 & $\begin{array}{l}\text { GE-manufactured fuel for GE BWR/2,3 reactors; first } 8 \times 8 \\
\text { fuel; } 1 \text { water rod; } \sim 184 \mathrm{~kg} \mathrm{U} \text {. }\end{array}$ & G2308G4 \\
\hline GE BWR/2,3 8 X 8 GE-5 & $\begin{array}{l}\text { GE-manufactured fuel for GE BWR/2,3 reactors; "retrofit" } \\
\text { fuel; } 2 \text { water rods; natural uranium axial blankets; } \\
\sim 177 \mathrm{~kg} \mathrm{U.}\end{array}$ & G2308G5 \\
\hline GE BWR/2,3 8 X 8 Prepres. & $\begin{array}{l}\text { GE-manufactured fuel for GE BWR/2,3 reactors; fuel rods } \\
\text { prepressurized to } 3 \text { atmospheres (atm) He; } 2 \text { water rods; } \\
\sim 177 \mathrm{~kg} \mathrm{U} \text {. }\end{array}$ & G2308GP. \\
\hline GE BWR/2,3 8 X 8 Barrier & $\begin{array}{l}\text { GE-manufactured fuel for GE BWR/2,3 reactors; pure } \\
\text { zirconium "barrier" on inside of cladding to reduce } \\
\text { pellet-clad interaction; } \sim 177 \mathrm{~kg} \mathrm{U} \text {. }\end{array}$ & G2308GB \\
\hline GE BWR/2,3 8 X 8 GE-8a & $\begin{array}{l}\text { GE-manufactured fuel for GE BWR/2,3 reactors; features of } \\
\text { GE BWR/2,3 } 8 \times 8 \text { fuel with only } 2 \text { water rods; } \sim 177 \mathrm{~kg} \mathrm{U} \text {. }\end{array}$ & G2308G8A \\
\hline GE BWR/2,3 8 X 8 GE-8b & $\begin{array}{l}\text { GE-manufactured fuel for GE BWR/2,3 reactors; } 4 \text { water } \\
\text { rods; introduces axially zoned enrichments and burnable } \\
\text { absorbers; fuel rod prepressurization increased to } 5 \text { atm; } \\
\sim 172 \mathrm{~kg} \mathrm{U} \text {. }\end{array}$ & G2308G8B \\
\hline
\end{tabular}




\begin{tabular}{|c|c|c|}
\hline Assembly Type & Description & $\begin{array}{l}\text { Assembly } \\
\text { Type Code }\end{array}$ \\
\hline \multicolumn{3}{|c|}{$\begin{array}{l}\text { GE BWR/2,3 Assembly Class (Continued) } \\
\text { (Reactor Type: BWR / Length: } 171.2 \text { in. / Width: } 5.44 \text { in.) }\end{array}$} \\
\hline GE BWR/2,3 8 X 8 GE-9 & $\begin{array}{l}\text { GE-manufactured fuel for GE BWR/2,3 reactors; } \\
\text { ferrule-type spacer grids; large diameter water rod which } \\
\text { displaced } 4 \text { fuel rod positions; axially zoned enrichment and } \\
\text { burnable absorbers; fuel rod prepressurization of } 5 \text { atm; } \\
\text { "barrier" cladding; }-172 \mathrm{~kg} \mathrm{U.}\end{array}$ & G2308G9 \\
\hline GE BWR/2,3 8 X 8 GE-10 & $\begin{array}{l}\text { GE-manufactured fuel for GE BWR/2,3 reactors; fuel } \\
\text { channel is } 100 \text {-mils thick at corners and } 65 \text {-mils thick on } \\
\text { sides reducing the parasitic material in core; uses flow } \\
\text { directors on the inside of the channel thus redirecting the } \\
\text { flow of water away from the channel wall towards the center } \\
\text { of the fuel bundle; estimated } 172 \mathrm{~kg} \mathrm{U.}\end{array}$ & G2308G10 \\
\hline GE BWR/2,3 9 X 9 GE-11 & $\begin{array}{l}\text { GE-manufactured fuel for GE BWR/ } 2,3 \text { reactors; uses } 2 \\
\text { medium diameter water rods which replace } 7 \text { fuel rods; } 8 \text { of } \\
\text { the remaining fuel rods extend only to the top of the fifth } \\
\text { spacer grid; no weight available. }\end{array}$ & G2309G11 \\
\hline GE BWR/2,3 $10 \times 10$ GE-12 & $\begin{array}{l}\text { GE-manufactured fuel for GE BWR/2,3 reactors; } 10 \times 10 \\
\text { fuel rod array; corresponding lower linear heat generation } \\
\text { rate, part-length fuel rods, low pressure drop spacer; no } \\
\text { weight available. }\end{array}$ & G2310G12 \\
\hline GE BWR/2,3 9 X 9 GE-13 & $\begin{array}{l}\text { GE-manufactured fuel for GE BWR/2,3 reactors; } 9 \times 9 \text { fuel } \\
\text { rod array; ferrule spacer, increased bundle weight relative } \\
\text { to GE-11 fuel, choice of active fuel lengths; offers critical } \\
\text { power capability improvements; no weight available. }\end{array}$ & G2309G13 \\
\hline GE BWR/2,3 $7 \times 7$ ANF & $\begin{array}{l}\text { ANF-manufactured fuel for GE BWR/2,3 reactors; } 7 \times 7 \text { fuel } \\
\text { rod array; used only at Oyster Creek; } \sim 182 \mathrm{~kg} \mathrm{U.}\end{array}$ & G2307A \\
\hline GE BWR/2,3 $8 \times 8$ ANF & $\begin{array}{l}\text { ANF-manufactured fuel for GE BWR/2,3 reactors; } 8 \times 8 \text { fuel } \\
\text { rod array; } 1 \text { water rod; estimated } 175 \mathrm{~kg} \text { U. }\end{array}$ & G2308A \\
\hline GE BWR/2,3 8 X 8 ANF Prepres. & $\begin{array}{l}\text { ANF-manufactured fuel for GE BWR/2,3 reactors; rods } \\
\text { prepressurized with He to several atms; } \sim 175 \mathrm{~kg} \mathrm{U} \text {. }\end{array}$ & G2308AP \\
\hline GE BWR/2,3 $9 \times 9$ ANF & $\begin{array}{l}\text { ANF-manufactured fuel for GE BWR/2,3 reactors; } 9 \times 9 \text { fuel } \\
\text { rod array; } 2 \text { water rods; } \sim 168 \mathrm{~kg} \mathrm{U.}\end{array}$ & G2309A \\
\hline GE BWR/2,3 $9 \times 9$ ANF 9-5 & $\begin{array}{l}\text { ANF-manufactured fuel for GE BWR/2,3 reactors; } 5 \text { water } \\
\text { rods per assembly; estimated } 161 \mathrm{~kg} \mathrm{U} \text {. }\end{array}$ & G2309A5 \\
\hline GE BWR/2,3 $9 \times 9$ ANF $9 \times$ & $\begin{array}{l}\text { ANF-manufactured fuel for GE BWR } / 2,3 \text { reactors; uses a } \\
\text { central water channel that replaces } 9 \text { water rods; estimated } \\
153 \mathrm{~kg} \mathrm{U.}\end{array}$ & G2309A9X \\
\hline
\end{tabular}




\begin{tabular}{c|c|c}
\hline Assembly Type & Description & $\begin{array}{c}\text { Assembly } \\
\text { Type Code }\end{array}$ \\
\hline $\begin{array}{c}\text { GE BWR/2,3 Assembly Class (Continued) } \\
\text { (Reactor Type: BWR / Length: } 171.2 \text { in. / Width: } 5.44 \text { in.) }\end{array}$
\end{tabular}

GE BWR/2,3 $9 \times 9$ ANF IX

ANF-manufactured fuel for GE BWR/2,3 reactors; uses a G2309AIX central water channel that replaces 9 rods; the regular fuel rods in the IX version utilize an internal cladding liner of pure zirconium; estimated $153 \mathrm{~kg} \mathrm{U}$.

\section{GE BWR/4-6 Assembly Class}

(Reactor Type: BWR / Length: 176.2 in. / Width: 5.44 in.)

GE BWR/4-6 $7 \times 7$ GE-2

GE BWR/4-6 $7 \times 7$ GE-3a

GE BWR/4-6 7 X 7 GE-3b

GE BWR/4-6 8 X 8 GE-4a

GE BWR/4- $68 \times 8$ GE-4b

GE BWR/4-6 8 X 8 GE-5

GE BWR/4-6 8 X 8 GE Prepres.

GE BWR/4-6 8 X 8 Barrier

GE BWR/4-6 $8 \times 8$ GE-8

GE BWR/4-6 8 X 8 GE-9
GE-manufactured fuel for GE BWR/4-6 reactors; original core fuel for several BWR/4 plants; high failure rate instigated introduction of GE-3 and GE-4 fuels; 195 kg U.

GE-manufactured fuel for GE BWR/4-6 reactors; "Improved" fuel; cladding thickness of $0.037^{\prime \prime}$; hydrogen getter introduced; 144 inch active fuel length; $-187 \mathrm{~kg} \mathrm{U}$.

GE-manufactured fuel for GE BWR/4-6 reactors; "Improved" fuel; cladding thickness of $0.037^{\prime \prime}$; hydrogen getter introduced; 146 active length; $\sim 190 \mathrm{~kg} \mathrm{U}$.

GE-manufactured fuel for GE BWR/4-6 reactors; first $8 \times 8$ fuel; 1 water rod; 144 inch active fuel length; $184 \mathrm{~kg} \mathrm{U.}$

GE-manufactured fuel for GE BWR/4-6 reactors; first $8 \times 8$ fuel; 1 water rod; 146 inch active fuel length; $\sim 187 \mathrm{~kg} \mathrm{U}$.

GE-manufactured fuel for GE BWR/4-6 reactors; "retrofit" fuel; 2 water rods; natural uranium axial blankets; $\sim 183 \mathrm{~kg} \mathrm{U}$.

GE-manufactured fuel for GE BWR/4-6 reactors; fuel rods prepressurized to $3 \mathrm{~atm} \mathrm{He} 2$ water rods; $\sim 183 \mathrm{~kg} \mathrm{U}$.

GE-manufactured fuel for GE BWR/4-6 reactors; pure zirconium "barrier" on inside of cladding to reduce pellet-clad interaction; $185 \mathrm{~kg} \mathrm{U}$.

GE-manufactured fuel for GE BWR/4-6 reactors; 4 water rods; axially zoned enrichment and burnable absorbers; fuel rod prepressurization increased to $5 \mathrm{~atm}$; other "barrier" fuel features; $\sim 179 \mathrm{~kg} \mathrm{U}$.

GE-manufactured fuel for GE BWR/4-6 reactors; ferrule-type spacer grids; large diameter water rod which displaced 4 fuel rod positions; axially zoned enrichment and burnable absorbers; fuel rod prepressurization of $5 \mathrm{~atm}$; "barrier" cladding; $172 \mathrm{~kg} \mathrm{U}$. 


\begin{tabular}{l|l|l}
\hline Assembly Type & Description & $\begin{array}{c}\text { Assembly } \\
\text { Type Code }\end{array}$ \\
\hline
\end{tabular}

\section{GE BWR/4-6 Assembly Class (Continued)}

(Reactor Type: BWR / Length: 176.2 in. / Width: 5.44 in.)

GE BWR/4-6 8 X 8 GE-10

GE BWR/4-6 $9 \times 9$ GE-11

GE BWR/4-6 $10 \times 10$ GE-12

GE BWR/4-6 $9 \times 9$ GE-13

GE BWR/4-6 $8 \times 8$ ANF

GE BWR/4-6 8 X 8 ANF Prepres.

GE BWR/4-6 $9 \times 9$ ANF

GE BWR/4-6 $9 \times 9$ ANF 9-5

GE BWR/4-6 9 X 9 ANF 9X

GE BWR/4-6 $9 \times 9$ ANF IX

GE BWR/4-6 9 X 9 ANF IX+
GE-manufactured fuel for GE BWR/4-6 reactors; fuel channel is 100 mils thick at the corners and 65 mils thick on G4608G10 the sides, reducing the parasitic material in core; uses flow directors on the inside of the channel thus redirecting the flow of water away from the channel wall towards the center of the fuel bundle; estimated $177 \mathrm{~kg} \mathrm{U}$.

GE-manufactured fuel for GE BWR/4-6 reactors; uses 2 medium diameter water rods which replace 7 fuel rods; 8 of the rods remaining extend to only the fifth spacer grid; estimated $170 \mathrm{~kg} \mathrm{U}$.

GE-manufactured fuel for GE BWR/4-6 reactors; $10 \times 10$ fuel rod array; corresponding lower linear heat generation rate, part-length fuel rods, low pressure drop spacer; no weight available.

GE-manufactured fuel for GE BWR/4-6 reactors; $9 \times 9$ fuel rod array; ferrule spacer, increased bundle weight relative to GE-11 fuel, choice of active fuel lengths; offers critical power capability improvements; no weight available.

ANF-manufactured fuel for GE BWR/4-6 reactors; $8 \times 8$ fuel rod array; 1 water rod; $-176 \mathrm{~kg} \mathrm{U}$.

ANF-manufactured fuel for GE BWR/4-6 reactors; prepressurized fuel rods; $\sim 176 \mathrm{~kg} \mathrm{U}$.

ANF-manufactured fuel for GE BWR/4-6 reactors; $9 \times 9$ fuel rod array; 2 water rods; $\sim 173 \mathrm{~kg} \mathrm{U}$.

ANF-manufactured fuel for GE BWR/4-6 reactors; 5 water rods per assembly; estimated $168 \mathrm{~kg} \mathrm{U}$.

ANF-manufactured fuel for GE BWR/4-6 reactors; central water channel replaces 9 fuel rods; estimated $168 \mathrm{~kg} \mathrm{U}$.

ANF-manufactured fuel for GE BWR/4-6 reactors; central water channel replaces 9 fuel rods; regular fuel rods utilize an internal cladding liner of pure zirconium; estimated $168 \mathrm{~kg} \mathrm{U}$.

ANF-manufactured fuel for GE BWR/4-6 reactors; central water channel replaces 9 fuel rods; high-performance thermal spacers; estimated $168 \mathrm{~kg} \mathrm{U}$.
G4608AP

G4609G11

G4610G12

G4609G13

G4608A

G4609A

G4609A5

G4609A9X

G4609AIX

G4609AX+ 
Table B4. Descriptions of Assembly Types (Continued)

\begin{tabular}{c|c|c}
\hline Assembly Type & Description & $\begin{array}{c}\text { Assembly } \\
\text { Type Code }\end{array}$ \\
\hline
\end{tabular}

GE BWR/4-6 Assembly Class (Continued)

(Reactor Type: BWR / Length: 176.2 in. / Width: 5.44 in.)

GE BWR/4-6 $8 \times 8$ WE

GE BWR/4-6 $10 \times 10 \mathrm{ABB}$

WE $14 \times 14$ WE OFA

WE $14 \times 14$ ANF

WE $14 \times 14$ ANF Top Rod

WE $14 \times 14$ B\&W

WE $14 \times 14$ WE Standard

WE $14 \times 14$ WE LOPAR
WE-manufactured fuel for GE BWR/4-6 reactors; reactors; "QUAD + fuel"; design of fuel is lead test assembly use only; a licensed version of ABB Atom water cross fuel; $\sim 174 \mathrm{~kg} \mathrm{U}$.

ABB Atom-manufactured fuel for GE BWR/4-6 reactors; fuel has $4,5 \times 5$ minibundles, each with 24 fuel rods; fuel bundles are separated by a water cross and a centralized water channel; estimated $176 \mathrm{~kg} \mathrm{U}$.

\section{WE $14 \times 14$ Assembly Class}

(Reactor Type: PWR / Length: 159.8 in. / Width: 7.76 in.)
WE $14 \times 14$ ANF Top Rod ard
WE-manufactured fuel for WE $14 \times 14$ reactors; zircaloy cladding; stainless steel guide tubes; $\sim 394 \mathrm{~kg} \mathrm{U}$.

WE-manufactured fuel for WE $14 \times 14$ reactors; low parasitic (LOPAR) fuel; zircaloy guide tubes; often referred to as "Standard" fuel; $\sim 399 \mathrm{~kg} \mathrm{U}$.

WE-manufactured fuel for WE $14 \times 14$ reactors; Optimized Fuel Assembly; zircaloy spacer grids; smaller fuel rod diameter; $\sim 358 \mathrm{~kg} \mathrm{U}$.

ANF-manufactured fuel for WE $14 \times 14$ reactors; shorter, larger diameter fuel rod than WE $14 \times 14$ ANF Top Rod fuel; $\sim 377 \mathrm{~kg} \mathrm{U}$.

ANF-manufactured fuel for WE $14 \times 14$ reactors; longer, smaller diameter fuel rod than WE $14 \times 14$ ANF fuel; $\sim 361 \mathrm{~kg} \mathrm{U}$.

B\&W-manufactured fuel for WE $14 \times 14$ reactors; only 2 lead test assemblies used at Ginna; $-383 \mathrm{~kg} \mathrm{U}$.

\section{WE $15 \times 15$ Assembly Class}

(Reactor Type: PWR / Length: 159.8 in. / Width: 8.44 in.)

WE $15 \times 15$ WE Standard

WE $15 \times 15$ WE LOPAR

WE $15 \times 15$ WE OFA
WE-manufactured fuel for WE $15 \times 15$ reactors; zircaloy cladding; stainless steel guide tubes; $454 \mathrm{~kg} \mathrm{U}$.

WE-manufactured fuel for WE $15 \times 15$ reactors; low to as "Standard" fuel; $\sim 455 \mathrm{~kg} \mathrm{U}$.

WE-manufactured fuel for WE $15 \times 15$ reactors; Optimized Fuel Assembly; zircaloy spacer grids; $\sim 460 \mathrm{~kg} \mathrm{U}$. parasitic (LOPAR) fuel; zircaloy guide tubes; often referred
G4608W

G4610C

W1414W

W1414WL

W1414WO

W1414A

W1414ATR

W1414B

W1515W

W1515WL

W1515WO 
Table B4. Descriptions of Assembly Types (Continued)

\begin{tabular}{c|c|c}
\hline Assembly Type & Description & $\begin{array}{c}\text { Assembly } \\
\text { Type Code }\end{array}$ \\
\hline $\begin{array}{c}\text { WE 15 X } 15 \text { Assembly Class (Continued) } \\
\text { (Reactor Type: PWR / Length: } 159.8 \text { in. / Width: } 8.44 \text { in.) }\end{array}$
\end{tabular}

WE $15 \times 15$ WE Vantage 5

WE $15 \times 15$ ANF

WE $15 \times 15$ ANF Part Length

WE $15 \times 15$ B\&W Mark BW
WE-manufactured fuel for WE $15 \times 15$ reactors; integral zirconium dioboride neutron absorbers in fuel; natural uranium axial blankets, intermediated flow mixers, removable top nozzle, increased discharged burnup; other Optimized Fuel Assembly features; $461 \mathrm{~kg} \mathrm{U}$.

ANF-manufactured fuel for WE $15 \times 15$ reactors; $\sim 429 \mathrm{~kg} \mathrm{U}$.

ANF-manufactured fuel for WE $15 \times 15$ reactors; bottom 42 inches of fuel rods replaced by inserts made from stainless steel 304 ; assemblies placed in positions on the core periphery to provide shielding for core support structure; estimated $305 \mathrm{~kg} \mathrm{U}$.

B\&W-manufactured fuel for WE $15 \times 15$ reactors; no weight available.

\section{WE $17 \times 17$ Assembly Class}

(Reactor Type: PWR / Length: 159.8 in. / Width: 8.44 in.)
WE-manufactured fuel for WE $17 \times 17$ reactors; low parasitic (LOPAR) fuel; zircaloy guide tubes; Inconel spacer grids; often referred to as "Standard" fuel; $\sim 460 \mathrm{~kg} \mathrm{U}$.

WE $17 \times 17$ WE OFA

WE $17 \times 17$ WE Vantage 5

WE $17 \times 17$ WE Vantage +

WE $17 \times 17$ WE Vantage $5 \mathrm{H}$

WE $17 \times 17$ ANF
WE-manufactured fuel for WE $17 \times 17$ reactors; Optimized Fuel Assembly; zircaloy spacer grids; $425 \mathrm{~kg} \mathrm{U}$.

WE-manufactured fuel for WE $17 \times 17$ reactors; integral zirconium dioboride neutron absorbers in fuel; natural uranium axial blankets, intermediated flow mixers, removable top nozzle, increased discharged burnup; other Optimized Fuel Assembly features; $\sim 426 \mathrm{~kg} \mathrm{U}$.

WE-manufactured fuel for WE $17 \times 17$ reactors; combines a new cladding material - ZIRLO (a zirconium-niobium alloy) with the advanced neutronic characteristics of Vantage 5 fuel; estimated $426 \mathrm{~kg} \mathrm{U}$.

WE-manufactured fuel for WE $17 \times 17$ reactors; hybrid fuel combining the advanced neutronic characteristics of Vantage 5 fuel with the larger fuel rod diameter associated with low parasitic (LOPAR) fuel; $\sim 464 \mathrm{~kg} \mathrm{U}$.

ANF-manufactured fuel for WE $17 \times 17$ reactors; $\sim 402 \mathrm{~kg} \mathrm{U}$.
W1515WV5

W1515A

W1515APL

W1515B

W1717WL

W1717WO

W1717WV5

$W 1717 W V_{+}$

W1717WVH

W1717A 
Table B4. Descriptions of Assembly Types (Continued)

\begin{tabular}{c|c|c}
\hline Assembly Type & Description & $\begin{array}{c}\text { Assembly } \\
\text { Type Code }\end{array}$ \\
\hline
\end{tabular}

WE 17 X 17 Assembly Class (Continued)

(Reactor Type: PWR / Length: 159.8 in. / Width: 8.44 in.)

WE $17 \times 17$ B\&W Mark BW $\quad$ B\&W-manufactured fuel for WE $17 \times 17$ reactors; $\sim 455 \mathrm{~kg} \mathrm{U}$.

W1717B

\section{South Texas Assembly Class}

(Reactor Type: PWR / Length: 199 in. / Width: 8.43 in.)

South Texas $17 \times 17$ WE

WE-manufactured fuel for use at South Texas reactors; initial core fuel; $\sim 542 \mathrm{~kg} \mathrm{U}$.

WST17W

\section{Big Rock Point Assembly Class}

(Reactor Type: BWR / Length: 84 in. / Width: 6.52 in.)

Big Rock Point $12 \times 12$ GE

Big Rock Point $11 \times 11 \mathrm{GE}$

Big Rock Point $7 \times 7$ GE

Big Rock Point 8 X 8 GE

Big Rock Point $9 \times 9$ GE

Big Rock Point $9 \times 9$ ANF

Big Rock Point $11 \times 11$ ANF

Big Rock Point $11 \times 11$ NFS
GE-manufactured fuel for use at Big Rock Point; stainless steel clad fuel; all assemblies reprocessed at West Valley.

GE-manufactured fuel for use at Big Rock Point; zircaloy cladding; generic designation which encompasses " $\mathrm{B}$, " $\mathrm{C}$," "D1," and "D2" assembly types; most assemblies reprocessed at West Valley; $\sim 124 \mathrm{~kg} \mathrm{U}$.

GE-manufactured fuel for use at Big Rock Point developmental center-melt fuel; test assembly use only; $\sim 131 \mathrm{~kg} \mathrm{U}$.

GE-manufactured fuel for use at Big Rock Point developmental center-melt fuel; test assembly use only; $\sim 112 \mathrm{~kg} \mathrm{U}$.

GE-manufactured fuel for use at Big Rock Point; zircaloy cladding; generic designation which encompasses "E," "EG," "F," "MEG," and "PEG" assembly types; 137 kg U.

ANF-manufactured fuel for use at Big Rock Point; lead test assembly use only; $\sim 127 \mathrm{~kg} \mathrm{U}$.

ANF-manufactured fuel for use at Big Rock Point; $\sim 128 \mathrm{~kg} \mathrm{U}$.

NFS-manufactured fuel for use at Big Rock Point; lead test assembly use only; $129 \mathrm{~kg} \mathrm{U}$.
XBR07G

XBR08G

XBR12G

XBR11G

XBR09G

XBRO9A

XBR11A

XBR11N 
Table B4. Descriptions of Assembly Types (Continued)

\begin{tabular}{c|c|c}
\hline Assembly Type & Description & $\begin{array}{c}\text { Assembly } \\
\text { Type Code }\end{array}$ \\
\hline \multicolumn{2}{c}{ Dresden 1 Assembly Class }
\end{tabular}

(Reactor Type: BWR / Length: 134.4 in. / Width: 4.28 in.)

Dresden $16 \times 6$ GE Type 1

Dresden $17 \times 7 \mathrm{GE}$

Dresden 16 X 6 GE Type III-B

Dresden 16 X 6 GE Type III-F

Dresden $16 \times 6$ GE Type V

Dresden $17 \times 7$ GE SA-1

Dresden $18 \times 8$ GE PF Fuels

Dresden $16 \times 6$ UNC

Dresden $16 \times 6$ ANF
GE-manufactured fuel for use at Dresden 1; all but one assembly reprocessed at West Valley; $\sim 111 \mathrm{~kg} \mathrm{U}$.

GE-manufactured fuel for use at Dresden 1; stainless steel clad fuel; 9 thorium oxide corner rods; all reprocessed at West Valley except for the corner rods, which were shipped to the Savannah River Site.

GE-manufactured fuel for use at Dresden 1; erbium oxide as burnable absorber in all 36 fuel rods; some assemblies reprocessed at West Valley; $\sim 102 \mathrm{~kg} \mathrm{U}$.

GE-manufactured fuel for use at Dresden 1; gadolinium oxide as a burnable absorber in a single, nonfueled rod; some assemblies reprocessed at West Valley; $\sim 102 \mathrm{~kg} \mathrm{U}$.

GE-manufactured fuel for use at Dresden 1; gadolinium oxide as a burnable absorber in selected fuel rods; $\sim 106 \mathrm{~kg} \mathrm{U}$.

GE-manufactured fuel for use at Dresden 1; a single prototype fuel assembly manufactured and owned by GE.

GE-manufactured fuel for use at Dresden 1; prototype fuel assemblies with $6 \times 6,7 \times 7$, and $8 \times 8$ fuel rod arrays; all have been reprocessed except for one $8 \times 8$ assembly; $\sim 100 \mathrm{~kg} \mathrm{U}$.

UNC-manufactured fuel for use at Dresden $1 ;-102 \mathrm{~kg} \mathrm{U}$.

ANF-manufactured fuel for use at Dresden $1 ; \sim 95 \mathrm{~kg} \mathrm{U}$.
XDR06G

XDR07G

XDR06G3B

XDR06G3F

XDR06G5

XDR07GS

XDR08G

XDR06U

XDR06A

\section{Fort Calhoun Assembly Class}

(Reactor Type: PWR / Length: 146 in. / Width: 8.1 in.)
Fort Calhoun $14 \times 14 \mathrm{CE}$

Fort Calhoun $14 \times 14$ ANF

Fort Calhoun $14 \times 14$ WE
CE-manufactured fuel for use at Fort Calhoun; similar to $C E$ $14 \times 14$ class fuels except for shorter length; $\sim 366 \mathrm{~kg} \mathrm{U}$.

ANF-manufactured fuel for use at Fort Calhoun; similar to CE $14 \times 14$ class fuels except for shorter length; $\sim 353 \mathrm{~kg} \mathrm{U}$.

WE-manufactured fuel for use at Fort Calhoun; similar to CE $14 \times 14$ class fuels except for shorter length; $\sim 374 \mathrm{~kg} \mathrm{U}$.
$\mathrm{XFC14C}$

XFC14A

XFC14W 
Table B4. Descriptions of Assembly Types (Continued)

\begin{tabular}{l|l|l}
\hline Assembly Type & Description & $\begin{array}{l}\text { Assembly } \\
\text { Type Code }\end{array}$ \\
\hline
\end{tabular}

Humboldt Bay Assembly Class

(Reactor Type: BWR / Length: 95 in. / Width: 4.67 in.)

Humboldt Bay $7 \times 7$ GE Type I

Humboldt Bay 7 X 7 GE Type II

Humboldt Bay 6 X 6 GE

Humboldt Bay $6 \times 6$ ANF

\section{WE}

Haddam Neck $15 \times 15$ WE

Haddam Neck $15 \times 15$ NUMEC SS

Haddam Neck $15 \times 15$ NUMEC Zr

Haddam Neck $15 \times 15$ GULF SS

Haddam Neck 15 X 15 GULF Zr

Haddam Neck $15 \times 15$ B\&W SS

Haddam Neck $15 \times 15$ B\&W Zr
GE-manufactured fuel for use at Humboldt Bay; stainless steel clad fuel; all assemblies reprocessed at West Valley; $\sim 80 \mathrm{~kg} \mathrm{U}$.

GE-manufactured fuel for use at Humboldt Bay; zircaloy cladding; some assemblies reprocessed at West Valley; $\sim 76 \mathrm{~kg} \mathrm{U}$.

GE-manufactured fuel for use at Humboldt Bay; zircaloy cladding; $\sim 76 \mathrm{~kg} \mathrm{U}$.

ANF-manufactured fuel for use at Humboldt Bay; zircaloy cladding; $\sim 70 \mathrm{~kg} \mathrm{U}$.

XHB07G

XHB07G2

Haddam Neck Assembly Class

Reactor Type: PWR / Length: 137.1 in. / Width: 8.42 in.)

WE-manufactured fuel for use at Haddam Neck; stainless steel clad; $\sim 416 \mathrm{~kg} \mathrm{U}$.

XHN15W

NU-manufactured fuel for use at Haddam Neck; stainless steel clad; lead test assembly use only; $\sim 406 \mathrm{~kg} \mathrm{U}$.

NU-manufactured fuel for use at Haddam Neck; zircaloy clad; lead test assembly use only; $\sim 371 \mathrm{~kg} \mathrm{U}$.

GULF-manufactured fuel for use at Haddam Neck; stainless steel clad; lead test assembly use only; $\sim 406 \mathrm{~kg} \mathrm{U}$.

GULF-manufactured fuel for use at Haddam Neck; zircaloy clad; lead test assembly use only; $363 \mathrm{~kg} \mathrm{U}$.

B\&W-manufactured fuel for use at Haddam Neck; stainless steel clad; $\sim 412 \mathrm{~kg} \mathrm{U}$.

XHN15B

B\&W-manufactured fuel for use at Haddam Neck; zircaloy clad; $364 \mathrm{~kg} \mathrm{U}$.

XHN15MS

XHN15MZ

XHN15HS

$\mathrm{XHN15HZ}$

XHN15BZ 


\begin{tabular}{|c|c|c|}
\hline Assembly Type & Description & $\begin{array}{l}\text { Assembly } \\
\text { Type Code }\end{array}$ \\
\hline \multicolumn{3}{|c|}{$\begin{array}{l}\text { Indian Point } 1 \text { Assembly Class } \\
\text { (Reactor Type: PWR / Length: } 138.8 \text { in. / Width: } 6.27 \text { in.) }\end{array}$} \\
\hline Indian Point 114 X 15 B\&W & $\begin{array}{l}\text { B\&W-manufactured fuel for use at Indian Point } 1 \text {; a } \\
\text { thorium/uranium oxide fuel pellet; stainless steel clad; } \\
\text { assemblies were used with the initial core configuration and } \\
\text { were not the same assembly dimensions as later fuels; all } \\
\text { assemblies reprocessed at West Valley; } \sim 152 \mathrm{~kg} \mathrm{U} \text {, } \\
\text { thorium/uranium. }\end{array}$ & XIP14B \\
\hline Indian Point $113 \times 14$ WE & $\begin{array}{l}\text { WE-manufactured fuel for use at Indian Point } 1 \text {; stainless } \\
\text { steel clad; dimensions are as specified above for Indian } \\
\text { Point } 1 \text { assembly class; some assemblies reprocessed at } \\
\text { West Valley; } \sim 191 \mathrm{~kg} \mathrm{U} \text {. }\end{array}$ & XIP14W \\
\hline \multicolumn{3}{|c|}{$\begin{array}{l}\text { LaCrosse Assembly Class } \\
\text { (Reactor Type: BWR / Length: } 102.5 \text { in. / Width: } 5.62 \text { in.) }\end{array}$} \\
\hline LaCrosse $10 \times 10 \mathrm{AC}$ & $\begin{array}{l}\text { AC-manufactured fuel for use at LaCrosse; stainless steel } \\
\text { cladding; } \sim 120 \mathrm{~kg} \mathrm{U} \text {. }\end{array}$ & XLC10L \\
\hline LaCrosse $10 \times 10$ ANF & $\begin{array}{l}\text { ANF-manufactured fuel for use at LaCrosse; stainless steel } \\
\text { cladding; } \sim 109 \mathrm{~kg} \mathrm{U.}\end{array}$ & XLC10A \\
\hline \multicolumn{3}{|c|}{$\begin{array}{l}\text { Palisades Assembly Class } \\
\text { (Reactor Type: PWR / Length: } 147.5 \text { in. / Width: } 8.2 \text { in.) }\end{array}$} \\
\hline Palisades $15 \times 15 \mathrm{CE}$ & CE-manufactured fuel for use at Palisades; $\sim 412 \mathrm{~kg} \mathrm{U.}$ & XPA15C \\
\hline \multicolumn{3}{|c|}{$\begin{array}{l}\text { St. Lucie } 2 \text { Assembly Class } \\
\text { (Reactor Type: PWR / Length: } 158.2 \text { in. / Width: } 8.1 \text { in.) }\end{array}$} \\
\hline \multicolumn{3}{|c|}{$\begin{array}{l}\text { San Onofre } 1 \text { Assembly Class } \\
\text { (Reactor Type: PWR / Length: } 137.1 \text { in. / Width: } 7.76 \text { in.) }\end{array}$} \\
\hline San Onofre $114 \times 14$ WE & $\begin{array}{l}\text { WE-manufactured fuel for use at San Onofre 1; stainless } \\
\text { steel clad; } \sim 366 \mathrm{~kg} \mathrm{U} \text {. }\end{array}$ & XSO14W \\
\hline San Onofre $114 \times 14$ WE Zr & $\begin{array}{l}\text { WE-manufactured fuel for use at San Onofre 1; zircaloy } \\
\text { clad demonstration fuel; no weight available. }\end{array}$ & XSO14WZ \\
\hline
\end{tabular}


Table B4. Descriptions of Assembly Types (Continued)

\begin{tabular}{l|c|c}
\hline Assembly Type & Description & $\begin{array}{c}\text { Assembly } \\
\text { Type Code }\end{array}$ \\
\hline
\end{tabular}

Yankee Rowe Assembly Class

(Reactor Type: PWR / Length: 111.8 in. / Width: 7.62 in.)

Yankee Rowe $17 \times 18$ WE

WE-manufactured fuel for use at Yankee Rowe; stainless

XYR18W

steel clad; nonsquare array designed to allow space for

cruciform control blades; most assemblies reprocessed at

West Valley; $\sim 273 \mathrm{~kg} \mathrm{U}$.

Yankee Rowe $15 \times 16$ UNC

UNC-manufactured fuel for use at Yankee Rowe;

XYR16U

nonsquare array designed to allow for space for cruciform control blades; $239 \mathrm{~kg} \mathrm{U}$.

Yankee Rowe $15 \times 16$ ANF

ANF-manufactured fuel for use at Yankee Rowe; nonsquare XYR16A array designed to allow space for cruciform control blades; $\sim 234 \mathrm{~kg} \mathrm{U}$.

Yankee Rowe $15 \times 16$ CE

CE-manufactured fuel for use at Yankee Rowe; nonsquare array designed to allow space for cruciform control blades;

XYR16C $\sim 229 \mathrm{~kg} \mathrm{U}$.

Source: Energy Information Administration, Form RW-859, "Nuclear Fuel Data" (1993). 
Table B5. Assembly Characteristics by Assembly Type

\begin{tabular}{|c|c|c|c|c|c|c|c|c|c|}
\hline \multicolumn{7}{|c|}{ Assemblies } & \multicolumn{3}{|c|}{ Rods } \\
\hline $\begin{array}{c}\text { Assembly } \\
\text { Type } \\
\text { Code }\end{array}$ & $\begin{array}{l}\text { Current } \\
\text { Fabricator }\end{array}$ & Cladding & $\begin{array}{c}\text { Avg. Initial } \\
\text { Loading } \\
\text { Weight } \\
\text { of Uranium } \\
\text { (kg) }\end{array}$ & $\begin{array}{l}\text { Width } \\
\text { as Built } \\
\text { (inches) }\end{array}$ & $\begin{array}{l}\text { Length } \\
\text { as Built } \\
\text { (inches) }\end{array}$ & $\begin{array}{c}\text { Number } \\
\text { Discharged }\end{array}$ & $\begin{array}{l}\text { Length } \\
\text { (inches) }\end{array}$ & $\begin{array}{l}\text { Number of } \\
\text { Positions }\end{array}$ & $\begin{array}{l}\text { Number of } \\
\text { Fuel Rods }\end{array}$ \\
\hline \multicolumn{10}{|c|}{ Boiling-Water Reactors (BWR) } \\
\hline $\begin{array}{l}\text { G2307G2A } \\
\text { G2307G2B } \\
\text { G2307G3 } \\
\text { G2308G4 } \\
\text { G2308G5 } \\
\text { G2308GP } \\
\text { G2308GB } \\
\text { G2308G8A } \\
\text { G2308G8B } \\
\text { G2308G9 } \\
\text { G2308G10 } \\
\text { G2309G11 } \\
\text { G2310G12 } \\
\text { G2309G13 } \\
\text { G2307A } \\
\text { G2308A } \\
\text { G2308AP } \\
\text { G2309A } \\
\text { G2309A5 } \\
\text { G2309A9X } \\
\text { G2309AIX } \\
\text { G4607G2 } \\
\text { G4607G3A } \\
\text { G4607G3B } \\
\text { G4608G4A } \\
\text { G4608G4B } \\
\text { G4608G5 } \\
\text { G4608GP } \\
\text { G4608GB } \\
\text { G4608G8 } \\
\text { G4608G9 } \\
\text { G4608G10 } \\
\text { G4609G11 } \\
\text { G4610G12 } \\
\text { G4609G13 } \\
\text { G4608A } \\
\text { G4608AP } \\
\text { G4609A } \\
\text { G4609A5 } \\
\text { G4609A9X } \\
\text { G4609AIX } \\
\text { G4609AX+ } \\
\text { G4608W } \\
\text { G4610C } \\
\text { XBR12G } \\
\text { XBR11G } \\
\text { XBR07G } \\
\text { XBR08G } \\
\text { XBR09G } \\
\text { XBR09A } \\
\text { XBR11A } \\
\text { XBR11N } \\
\text { XDR06G } \\
\text { XDR07G } \\
\text { XDR06G3B } \\
\text { XDR06G3F } \\
\text { XDR06G55 } \\
\text { XDR07GSS } \\
\text { XDR08G } \\
\text { XDR06U } \\
\text { XDR06A } \\
\text { XHB07G } \\
\text { XHB07G2 } \\
\text { XHB06G } \\
\text { XHB06A } \\
\text { XLC10L } \\
\text { XLC10A }\end{array}$ & 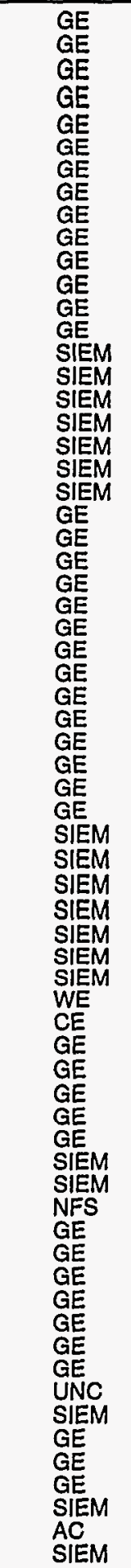 & 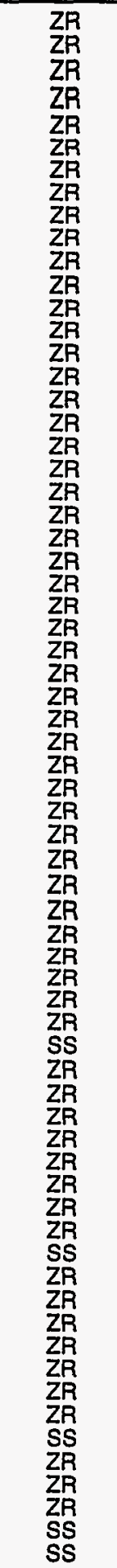 & 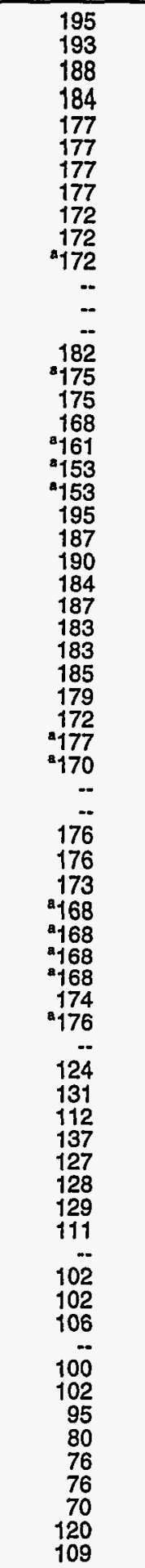 & $\begin{array}{l}5.44 \\
5.44 \\
5.44 \\
5.44 \\
5.44 \\
5.44 \\
5.44 \\
5.44 \\
5.44 \\
5.44 \\
5.44 \\
5.44 \\
5.44 \\
5.44 \\
5.44 \\
5.44 \\
5.44 \\
5.44 \\
5.44 \\
5.44 \\
5.44 \\
5.44 \\
5.44 \\
5.44 \\
5.44 \\
5.44 \\
5.44 \\
5.44 \\
5.44 \\
5.44 \\
5.44 \\
5.44 \\
5.44 \\
5.44 \\
5.44 \\
5.44 \\
5.44 \\
5.44 \\
5.44 \\
5.44 \\
5.44 \\
5.44 \\
5.44 \\
5.44 \\
6.52 \\
6.52 \\
6.52 \\
6.52 \\
6.52 \\
6.52 \\
6.52 \\
6.52 \\
4.28 \\
4.28 \\
4.28 \\
4.28 \\
4.28 \\
4.28 \\
4.28 \\
4.28 \\
4.28 \\
4.67 \\
4.67 \\
4.67 \\
4.67 \\
5.62 \\
5.62 \\
\end{array}$ & $\begin{array}{r}171.2 \\
171.2 \\
171.2 \\
171.2 \\
171.2 \\
171.2 \\
171.2 \\
171.2 \\
171.2 \\
171.2 \\
171.2 \\
171.2 \\
171.2 \\
171.2 \\
171.2 \\
171.2 \\
171.2 \\
171.2 \\
171.2 \\
171.2 \\
171.2 \\
176.2 \\
176.2 \\
176.2 \\
176.2 \\
176.2 \\
176.2 \\
176.2 \\
176.2 \\
176.2 \\
176.2 \\
176.2 \\
176.2 \\
176.2 \\
176.2 \\
176.2 \\
176.2 \\
176.2 \\
176.2 \\
176.2 \\
176.2 \\
176.2 \\
176.2 \\
176.2 \\
84.0 \\
85.0 \\
95.0 \\
95.0 \\
95.0 \\
102.5 \\
102.5 \\
84.0 \\
84.0 \\
84.0 \\
84.0 \\
84.0 \\
84.0 \\
134.4 \\
134.4 \\
134.4 \\
134.4 \\
134.4 \\
134.4 \\
134.4 \\
134.4 \\
134.4 \\
\end{array}$ & $\begin{array}{r}1,672 \\
5,047 \\
394 \\
3,876 \\
879 \\
2,832 \\
1,280 \\
312 \\
28 \\
- \\
-0 \\
-- \\
- \\
- \\
260 \\
1,409 \\
-38 \\
28 \\
- \\
- \\
-142 \\
1,142 \\
3,752 \\
1,184 \\
1,785 \\
1,787 \\
4,380 \\
11,520 \\
7,544 \\
852 \\
22 \\
4 \\
40 \\
-- \\
-0 \\
1,336 \\
248 \\
860 \\
36 \\
-- \\
178 \\
126 \\
155 \\
178\end{array}$ & $\begin{array}{r}158.9 \\
158.9 \\
158.9 \\
158.9 \\
158.9 \\
158.9 \\
158.9 \\
158.9 \\
158.9 \\
158.9 \\
158.9 \\
158.9 \\
158.9 \\
158.9 \\
158.9 \\
158.9 \\
158.9 \\
158.9 \\
158.9 \\
158.9 \\
158.9 \\
163.8 \\
163.8 \\
163.8 \\
163.8 \\
163.8 \\
163.8 \\
163.8 \\
163.8 \\
163.8 \\
163.8 \\
163.8 \\
b 163.8 \\
163.8 \\
163.8 \\
163.8 \\
163.8 \\
163.8 \\
163.8 \\
163.8 \\
163.8 \\
163.8 \\
163.8 \\
163.8 \\
N \\
75.0 \\
75.0 \\
75.0 \\
75.0 \\
75.0 \\
75.0 \\
75.0 \\
117.0 \\
117.0 \\
117.0 \\
117.0 \\
117.0 \\
117.0 \\
117.0 \\
117.0 \\
117.0 \\
N A \\
83.2 \\
79.0 \\
79.0 \\
90.0 \\
90.0 \\
\end{array}$ & $\begin{array}{r}49 \\
49 \\
49 \\
64 \\
64 \\
64 \\
64 \\
64 \\
64 \\
64 \\
64 \\
64 \\
64 \\
81 \\
100 \\
81 \\
0\end{array}$ & 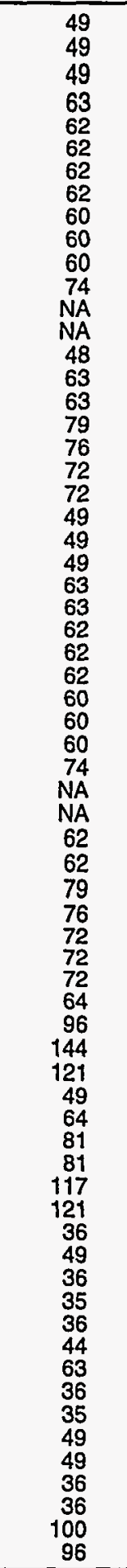 \\
\hline
\end{tabular}

See footnotes at end of table. 
Table B5. Assembly Characteristics by Assembly Type (Continued)

\begin{tabular}{|c|c|c|c|c|c|c|c|c|c|}
\hline \multicolumn{7}{|c|}{ Assemblies } & \multicolumn{3}{|c|}{ Rods } \\
\hline $\begin{array}{c}\text { Assembly } \\
\text { Type } \\
\text { Code }\end{array}$ & $\begin{array}{c}\text { Current } \\
\text { Fabricator }\end{array}$ & Cladding & $\begin{array}{c}\text { Avg. Initial } \\
\text { Loading } \\
\text { Weight } \\
\text { of Uranium } \\
\text { (kg) }\end{array}$ & $\begin{array}{l}\text { Width } \\
\text { as Built } \\
\text { (inches) }\end{array}$ & $\begin{array}{l}\text { Length } \\
\text { as Built } \\
\text { (inches) }\end{array}$ & $\begin{array}{c}\text { Number } \\
\text { Discharged }\end{array}$ & $\begin{array}{l}\text { Length } \\
\text { (inches) }\end{array}$ & $\begin{array}{c}\text { Number of } \\
\text { Positions }\end{array}$ & $\begin{array}{l}\text { Number of } \\
\text { Fuel Rods }\end{array}$ \\
\hline \multicolumn{10}{|c|}{ Pressurized-Water Reactors (PWR) } \\
\hline $\begin{array}{l}\text { B1515B2 } \\
\text { B1515B3 } \\
\text { B1515B4 } \\
\text { B1515B4Z } \\
\text { B1515B5 } \\
\text { B1515B5Z } \\
\text { B1515B6 } \\
\text { B1515B7 } \\
\text { B1515B8 } \\
\text { B1515B9 } \\
\text { B1515B10 } \\
\text { B1515B } \\
\text { B1515BZ } \\
\text { B1515BEB } \\
\text { B1515BGD } \\
\text { B1515W } \\
\text { B1717B } \\
\text { C1414C } \\
\text { C1414A } \\
\text { C1414W } \\
\text { C1616CSD } \\
\text { C8016C } \\
\text { W1414W } \\
\text { W1414WL } \\
\text { W1414WO } \\
\text { W1414A } \\
\text { W1414ATR } \\
\text { W1414B } \\
\text { W1515W } \\
\text { W1515WL } \\
\text { W1515WO } \\
\text { W1515WV5 } \\
\text { W1515A } \\
\text { W1515APL } \\
\text { W1515B } \\
\text { W1717WL } \\
\text { W1717WO } \\
\text { W1717WN5 } \\
\text { W1717WV+ } \\
\text { W1717WWH } \\
\text { W1717A } \\
\text { W1717B } \\
\text { WST17W } \\
\text { XFC14C } \\
\text { XFC14A } \\
\text { XFC14W } \\
\text { XHN15W } \\
\text { XHN15MS } \\
\text { XHN15MZ } \\
\text { XHN15HS } \\
\text { XHN15HZ } \\
\text { XHN15B } \\
\text { XHN15BZ } \\
\text { XIP14B } \\
\text { XIP14W } \\
\text { XPA15C } \\
\text { XPA15A } \\
\text { XSL16C } \\
\text { XS014W } \\
\text { XS014WZ } \\
\text { XYR18W } \\
\text { XYR16U } \\
\text { XYR16A } \\
\text { XYR16C }\end{array}$ & 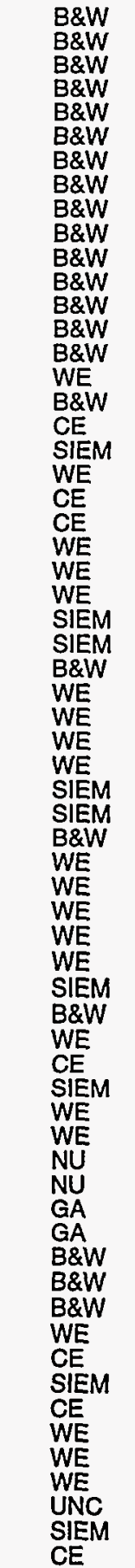 & 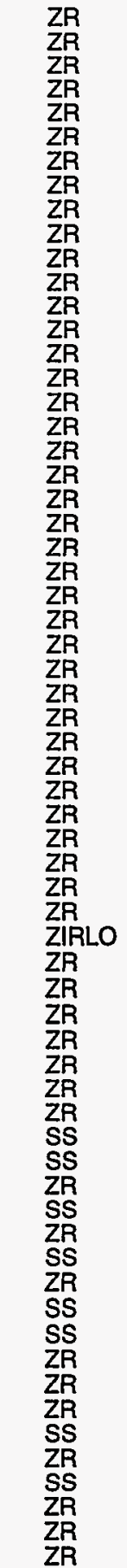 & 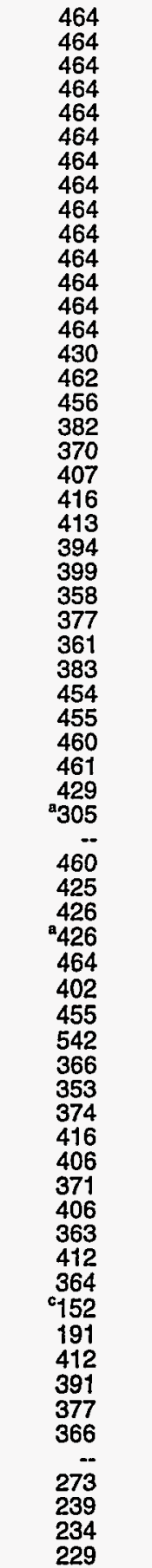 & $\begin{array}{l}8.54 \\
8.54 \\
8.54 \\
8.54 \\
8.54 \\
8.54 \\
8.54 \\
8.54 \\
8.54 \\
8.54 \\
8.54 \\
8.54 \\
8.54 \\
8.54 \\
8.54 \\
8.54 \\
8.54 \\
8.10 \\
8.10 \\
8.10 \\
8.10 \\
8.10 \\
7.76 \\
7.76 \\
7.76 \\
7.76 \\
7.76 \\
7.76 \\
8.44 \\
8.44 \\
8.44 \\
8.44 \\
8.44 \\
8.44 \\
8.44 \\
8.44 \\
8.44 \\
8.44 \\
8.44 \\
8.44 \\
8.44 \\
8.44 \\
8.43 \\
8.10 \\
8.10 \\
8.10 \\
8.42 \\
8.42 \\
8.42 \\
8.42 \\
8.42 \\
8.42 \\
8.42 \\
6.14 \\
6.27 \\
7.60 \\
7.62\end{array}$ & 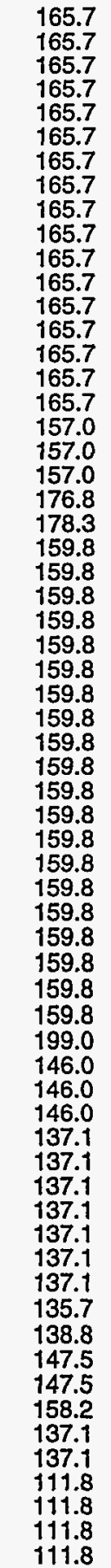 & 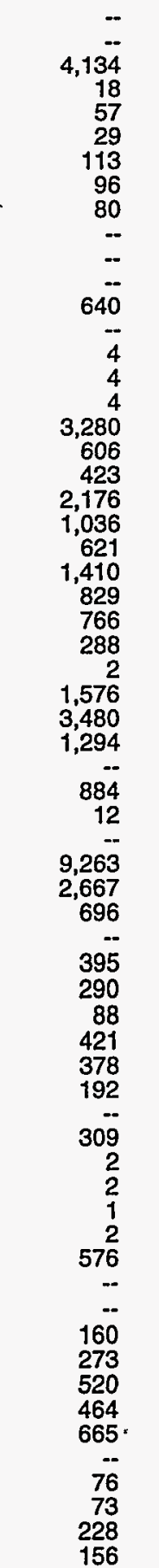 & 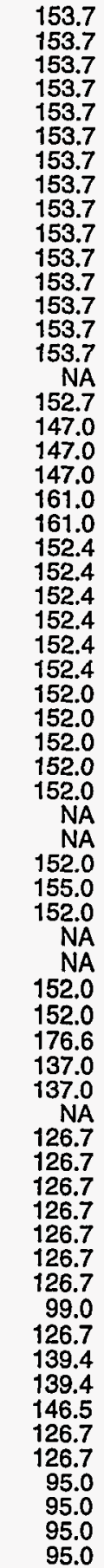 & $\begin{array}{l}225 \\
225 \\
225 \\
225 \\
225 \\
225 \\
225 \\
225 \\
225 \\
225 \\
225 \\
225 \\
225 \\
225 \\
225 \\
225 \\
289 \\
176 \\
176 \\
176 \\
236 \\
236 \\
196 \\
196 \\
196 \\
196 \\
196 \\
196 \\
225 \\
225 \\
225 \\
225 \\
225 \\
225 \\
225 \\
289 \\
289 \\
289 \\
225 \\
236 \\
196 \\
196 \\
305 \\
240 \\
240 \\
240\end{array}$ & 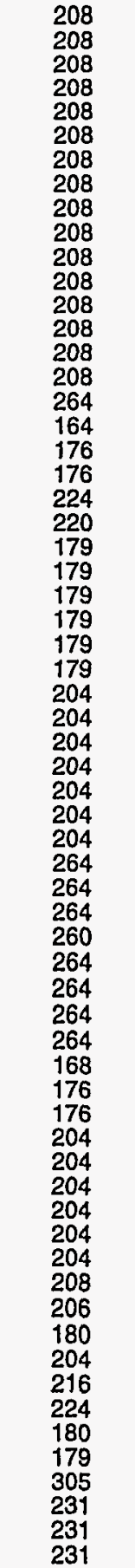 \\
\hline
\end{tabular}

Estimated weight based on most current data.

Eight part-length fuel rods.

"Average initial loading weight of thorium/uranium (kg).

$-=$ Not applicable. NA $=$ Not available.

Note: There are 4 temporarily discharged BWR assemblies and 104 temporarily discharged PWR assemblies that have not been assigned an assembly type code. These assemblies are not included in the above table. See Technical Note 10 in Appendix $E$.

Source: Energy Information Administration, Form RW-859, "Nuclear Fuel Data" (1993). 
Table B6. Assembly Type Summary by Reactor

\begin{tabular}{|c|c|c|c|c|c|}
\hline Reactor Name & $\begin{array}{l}\text { Assembly } \\
\text { Type } \\
\text { Code }\end{array}$ & $\begin{array}{l}\text { Number of } \\
\text { Assemblies }\end{array}$ & $\begin{array}{l}\text { Initial Uranium } \\
\text { Content } \\
\text { (MTU) }\end{array}$ & $\begin{array}{l}\text { Average } \\
\text { Burnup } \\
\text { (GWDtMTU) }\end{array}$ & $\begin{array}{c}\text { Average } \\
\text { Enrichment } \\
\text { (weight percent) }\end{array}$ \\
\hline $\begin{array}{l}\text { Arkansas Nuclear } 1 \ldots \ldots \ldots \ldots \\
\text { Subtotal Arkansas Nuclear } 1 \ldots\end{array}$ & $\begin{array}{l}\mathrm{B} 1515 \mathrm{~B} 4 \\
\mathrm{~B} 1515 \mathrm{BZ}\end{array}$ & $\begin{array}{r}624 \\
60 \\
684\end{array}$ & $\begin{array}{r}289.1 \\
27.8 \\
316.9\end{array}$ & $\begin{array}{l}30.8 \\
38.2\end{array}$ & $\begin{array}{l}2.97 \\
3.45\end{array}$ \\
\hline $\begin{array}{l}\text { Beaver Valley } 1 \ldots \ldots \ldots \\
\text { Subtotal Beaver Valley } 1 \ldots \ldots\end{array}$ & $\begin{array}{l}\text { W1717WL } \\
\text { W1717WO } \\
\text { W1717WVH }\end{array}$ & $\begin{array}{r}454 \\
2 \\
120 \\
576\end{array}$ & $\begin{array}{r}209.0 \\
0.8 \\
55.7 \\
265.6\end{array}$ & $\begin{array}{l}29.9 \\
35.8 \\
35.4\end{array}$ & $\begin{array}{l}2.99 \\
3.20 \\
3.56\end{array}$ \\
\hline Braidwood $1, \ldots \ldots \ldots \ldots \ldots$ & W1717WO & 236 & 100.4 & 29.0 & 2.79 \\
\hline $\begin{array}{l}\text { Braidwood } 2 \ldots \ldots \ldots \ldots \ldots \ldots \\
\text { Subtotal Braidwood } 2 \ldots \ldots \ldots\end{array}$ & $\begin{array}{l}\text { W1717WO } \\
\text { W1717WV5 }\end{array}$ & $\begin{array}{r}193 \\
59 \\
252\end{array}$ & $\begin{array}{r}81.6 \\
25.1 \\
106.7\end{array}$ & $\begin{array}{l}27.7 \\
36.8\end{array}$ & $\begin{array}{l}2.60 \\
3.68\end{array}$ \\
\hline Subtotal Browns Ferry $2 \ldots \ldots$ & $\begin{array}{l}\text { G4607G2 } \\
\text { G4607G3A } \\
\text { G4608G4A } \\
\text { G4608G5 } \\
\text { G4608GP }\end{array}$ & $\begin{array}{r}168 \\
596 \\
168 \\
232 \\
572 \\
1,736\end{array}$ & $\begin{array}{r}32.9 \\
111.5 \\
30.9 \\
42.3 \\
104.7 \\
322.3\end{array}$ & $\begin{array}{r}9.8 \\
23.0 \\
28.2 \\
28.9 \\
17.3\end{array}$ & $\begin{array}{l}1.10 \\
2.51 \\
2.73 \\
2.87 \\
2.82\end{array}$ \\
\hline Subtotal Browns Ferry $3 \ldots \ldots$ & $\begin{array}{l}\text { G4608G4B } \\
\text { G4608G5 } \\
\text { G4608GP }\end{array}$ & $\begin{array}{r}764 \\
208 \\
58 \\
1,030\end{array}$ & $\begin{array}{r}142.8 \\
38.1 \\
10.6 \\
191.4\end{array}$ & $\begin{array}{l}20.7 \\
28.0 \\
25.5\end{array}$ & $\begin{array}{l}2.20 \\
2.65 \\
2.66\end{array}$ \\
\hline Subtotal Brunswick $1 \ldots \ldots \ldots$ & $\begin{array}{l}\text { G4608G4B } \\
\text { G4608G5 } \\
\text { G4608GP } \\
\text { G4608GB }\end{array}$ & $\begin{array}{r}560 \\
360 \\
153 \\
267 \\
1,340\end{array}$ & $\begin{array}{r}104.4 \\
65.9 \\
28.0 \\
49.3 \\
247.6\end{array}$ & $\begin{array}{l}18.3 \\
28.9 \\
29.4 \\
33.0\end{array}$ & $\begin{array}{l}2.11 \\
2.78 \\
2.84 \\
2.99\end{array}$ \\
\hline
\end{tabular}

See footnotes at end of table. 
Table B6. Assembly Type Summary by Reactor (Continued)

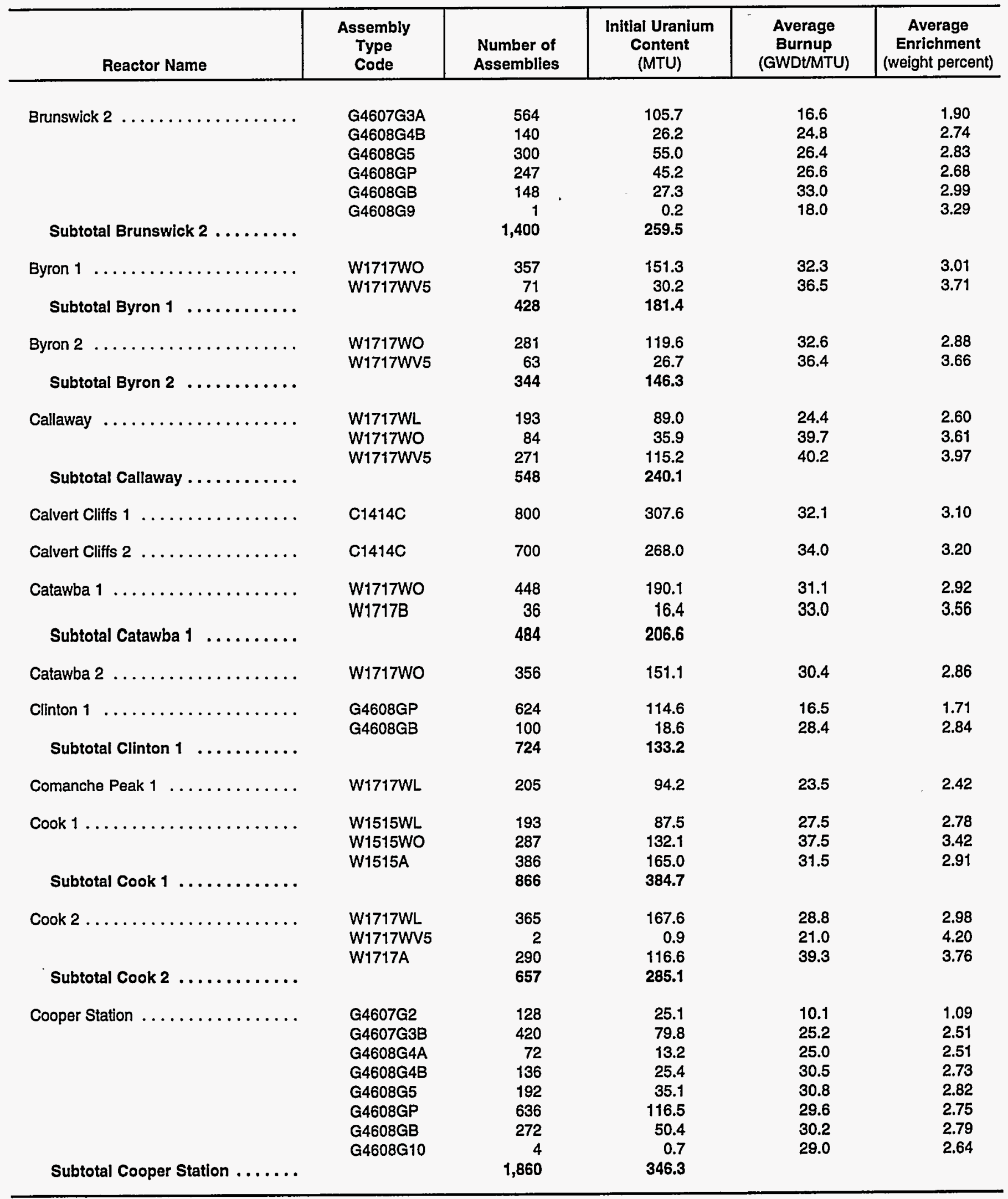

See footnotes at end of table. 
Table B6. Assembly Type Summary by Reactor (Continued)

\begin{tabular}{|c|c|c|c|c|c|}
\hline Reactor Name & $\begin{array}{l}\text { Assembly } \\
\text { Type } \\
\text { Code }\end{array}$ & $\begin{array}{l}\text { Number of } \\
\text { Assemblies }\end{array}$ & $\begin{array}{l}\text { Initial Uranium } \\
\text { Content } \\
\text { (MTU) }\end{array}$ & $\begin{array}{c}\text { Average } \\
\text { Burnup } \\
\text { (GWDt/MTU) }\end{array}$ & $\begin{array}{c}\text { Average } \\
\text { Enrichment } \\
\text { (weight percent) }\end{array}$ \\
\hline $\begin{array}{l}\text { Crystal River } 3 \ldots \ldots \ldots \ldots \ldots \ldots \\
\text { Subtotal Crystal River } 3 \ldots \ldots \ldots\end{array}$ & $\begin{array}{l}\text { B1515B4 } \\
\text { B1515BZ }\end{array}$ & $\begin{array}{r}496 \\
40 \\
536\end{array}$ & $\begin{array}{r}230.3 \\
18.5 \\
248.8\end{array}$ & $\begin{array}{l}26.7 \\
38.0\end{array}$ & $\begin{array}{l}2.77 \\
3.85\end{array}$ \\
\hline Subtotal Davis-Besse ........ & $\begin{array}{l}\text { B1515B4 } \\
\text { B1515B5 } \\
\text { B1515B8 } \\
\text { 'Temps }\end{array}$ & $\begin{array}{r}385 \\
57 \\
8 \\
6 \\
456\end{array}$ & $\begin{array}{r}181.0 \\
26.7 \\
3.7 \\
0.0 \\
211.5\end{array}$ & $\begin{array}{l}30.0 \\
38.0 \\
35.0\end{array}$ & $\begin{array}{l}2.82 \\
3.13 \\
3.38\end{array}$ \\
\hline $\begin{array}{l}\text { Diablo Canyon } 1 \ldots \ldots \ldots \ldots \ldots \\
\text { Subtotal Diablo Canyon } 1 \ldots \ldots\end{array}$ & $\begin{array}{l}\text { W1717WL } \\
\text { W1717WV5 }\end{array}$ & $\begin{array}{r}337 \\
39 \\
376\end{array}$ & $\begin{array}{r}155.4 \\
16.6 \\
171.9\end{array}$ & $\begin{array}{l}33.5 \\
39.7\end{array}$ & $\begin{array}{l}3.06 \\
4.01\end{array}$ \\
\hline $\begin{array}{l}\text { Diablo Canyon } 2 \ldots \ldots \ldots \ldots \ldots \\
\text { Subtotal Dlablo Canyon } 2 \ldots \ldots \ldots\end{array}$ & $\begin{array}{l}\text { W1717WL } \\
\text { W1717WW5 }\end{array}$ & $\begin{array}{r}337 \\
59 \\
396\end{array}$ & $\begin{array}{r}155.2 \\
25.1 \\
180.3\end{array}$ & $\begin{array}{l}31.0 \\
44.9\end{array}$ & $\begin{array}{l}2.97 \\
4.24\end{array}$ \\
\hline Dresden $1 \ldots \ldots \ldots \ldots \ldots \cdots$ & $\begin{array}{l}\text { XDR06G } \\
\text { XDR06G3B } \\
\text { XDR06G3F } \\
\text { XDR06G5 } \\
\text { XDR07GS } \\
\text { XDR08G } \\
\text { XDR06U } \\
\text { XDR06A }\end{array}$ & $\begin{array}{r}1 \\
163 \\
96 \\
106 \\
1 \\
1 \\
1 \\
458 \\
66 \\
892\end{array}$ & $\begin{array}{r}0.1 \\
16.6 \\
9.8 \\
11.2 \\
0.1 \\
0.1 \\
46.7 \\
6.3 \\
60.9 \\
90.9\end{array}$ & \begin{tabular}{r|}
21.3 \\
16.9 \\
20.0 \\
19.0 \\
29.0 \\
23.3 \\
16.1 \\
4.5
\end{tabular} & $\begin{array}{l}1.47 \\
1.83 \\
2.25 \\
2.26 \\
3.10 \\
1.95 \\
2.24 \\
2.23\end{array}$ \\
\hline Subtotal Dresden $2 \ldots \ldots \ldots \ldots$ & $\begin{array}{l}\text { G2307G2B } \\
\text { G2307G3 } \\
\text { G2308G4 } \\
\text { G2308G5 } \\
\text { G2308GP } \\
\text { G2308A } \\
\text { G2309A }\end{array}$ & $\begin{array}{r}1,477 \\
32 \\
476 \\
160 \\
228 \\
412 \\
28 \\
2,813\end{array}$ & $\begin{array}{r}284.1 \\
6.0 \\
87.8 \\
28.3 \\
40.4 \\
71.3 \\
4.7 \\
522.6\end{array}$ & $\begin{array}{l}10.4 \\
23.7 \\
25.1 \\
28.7 \\
28.4 \\
31.8 \\
35.4\end{array}$ & $\begin{array}{l}2.08 \\
2.29 \\
2.52 \\
2.67 \\
2.64 \\
2.82 \\
3.08\end{array}$ \\
\hline $\begin{array}{l}\text { Dresden } 3 \ldots \ldots \ldots \ldots \ldots \ldots \\
\text { Subtotal Dresden } 3 \ldots \ldots \ldots \ldots\end{array}$ & $\begin{array}{l}\text { G2307G2B } \\
\text { G2307G3 } \\
\text { G2308G4 } \\
\text { G2308GP } \\
\text { G2308A }\end{array}$ & $\begin{array}{r}724 \\
52 \\
508 \\
200 \\
380 \\
1,864\end{array}$ & $\begin{array}{r}140.2 \\
9.8 \\
93.4 \\
35.4 \\
66.0 \\
344.8\end{array}$ & $\begin{array}{l}17.3 \\
24.0 \\
25.7 \\
28.0 \\
31.9\end{array}$ & $\begin{array}{l}2.13 \\
2.30 \\
2.56 \\
2.65 \\
2.74\end{array}$ \\
\hline Subtotal Duane Arnold ........ & $\begin{array}{l}\text { G4607G3A } \\
\text { G4608G4A } \\
\text { G4608GP } \\
\text { G4608GB } \\
\text { G4608G8 }\end{array}$ & $\begin{array}{r}372 \\
185 \\
388 \\
119 \\
216 \\
1,280\end{array}$ & $\begin{array}{r}69.8 \\
34.1 \\
71.0 \\
21.9 \\
38.5 \\
235.3\end{array}$ & $\begin{array}{l}15.9 \\
26.3 \\
28.6 \\
28.0 \\
33.9\end{array}$ & $\begin{array}{l}1.90 \\
2.74 \\
2.86 \\
3.01 \\
3.06\end{array}$ \\
\hline $\begin{array}{l}\text { Enrico Ferml } 2 \ldots \ldots \ldots \ldots \\
\text { Subtotal Enrlco Fermi } 2 \ldots \ldots\end{array}$ & $\begin{array}{l}\text { G4608GP } \\
\text { G4608G8 } \\
\text { G4609G11 }\end{array}$ & $\begin{array}{r}672 \\
188 \\
40 \\
900\end{array}$ & $\begin{array}{r}121.5 \\
33.7 \\
6.8 \\
161.9\end{array}$ & $\begin{array}{l}20.6 \\
31.0 \\
{ }^{\circ} 0.0\end{array}$ & $\begin{array}{l}1.91 \\
3.19 \\
3.31\end{array}$ \\
\hline
\end{tabular}

See footnotes at end of table. 
Table B6. Assembly Type Summary by Reactor (Continued)

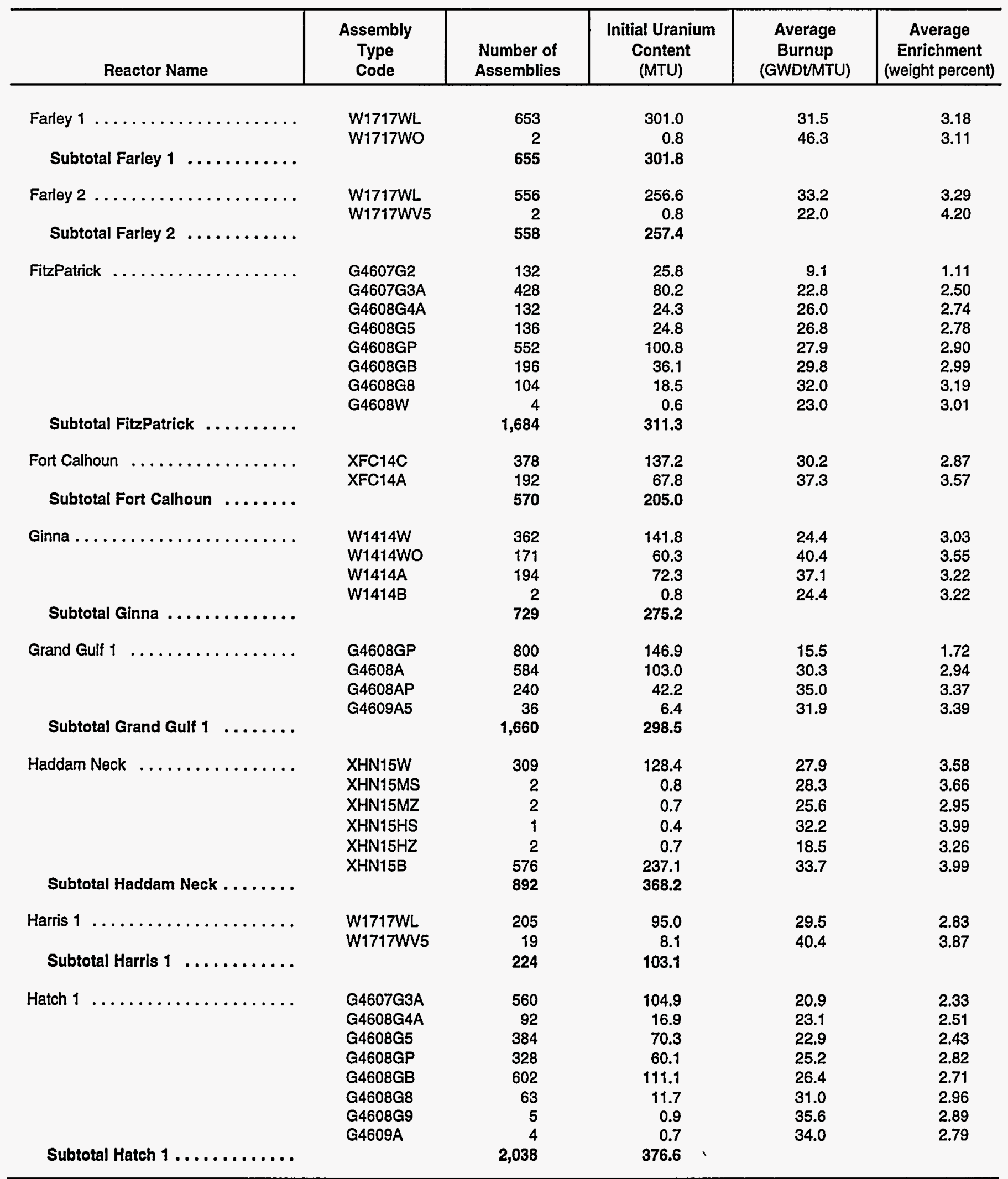

See footnotes at end of table. 
Table B6. Assembly Type Summary by Reactor (Continued)

\begin{tabular}{|c|c|c|c|c|c|}
\hline Reactor Name & $\begin{array}{l}\text { Assembly } \\
\text { Type } \\
\text { Code } \\
\end{array}$ & $\begin{array}{l}\text { Number of } \\
\text { Assemblies }\end{array}$ & $\begin{array}{l}\text { Initial Uranium } \\
\text { Content } \\
\text { (MTU) }\end{array}$ & $\begin{array}{c}\text { Average } \\
\text { Burnup } \\
\text { (GWDt/MTU) }\end{array}$ & $\begin{array}{c}\text { Average } \\
\text { Enrichment } \\
\text { (weight percent) }\end{array}$ \\
\hline Hatch $2 \ldots \ldots \ldots \ldots \ldots \ldots$ & $\begin{array}{l}\text { G4608G5 } \\
\text { G4608GP } \\
\text { G4608GB } \\
\text { G4608G9 } \\
\text { G4609A }\end{array}$ & $\begin{array}{r}509 \\
407 \\
582 \\
4 \\
4 \\
1,506\end{array}$ & $\begin{array}{r}93.4 \\
74.5 \\
107.6 \\
0.7 \\
0.7 \\
276.9\end{array}$ & $\begin{array}{l}19.2 \\
25.0 \\
27.0 \\
16.3 \\
36.0\end{array}$ & $\begin{array}{l}1.97 \\
2.74 \\
2.74 \\
3.14 \\
2.79\end{array}$ \\
\hline $\begin{array}{l}\text { Hope Creek } \ldots \ldots \ldots \ldots \ldots \ldots \\
\text { Subtotal Hope Creek } \ldots \ldots \ldots \ldots\end{array}$ & $\begin{array}{l}\text { G4608GP } \\
\text { G4608GB }\end{array}$ & $\begin{array}{r}92 \\
916 \\
1,008\end{array}$ & $\begin{array}{r}17.0 \\
169.5 \\
186.6\end{array}$ & $\begin{array}{r}3.0 \\
22.7\end{array}$ & $\begin{array}{l}0.73 \\
2.27\end{array}$ \\
\hline Humboldt Bay.$\ldots \ldots \ldots \ldots \ldots$ & $\begin{array}{l}\text { XHB07G2 } \\
\text { XHB06G } \\
\text { XHB06A }\end{array}$ & $\begin{array}{r}88 \\
176 \\
126 \\
390\end{array}$ & $\begin{array}{r}6.7 \\
13.4 \\
8.8 \\
28.9\end{array}$ & $\begin{array}{r}18.2 \\
17.2 \\
9.0\end{array}$ & $\begin{array}{l}2.11 \\
2.43 \\
2.40\end{array}$ \\
\hline Indian Point $1 \ldots \ldots \ldots \ldots \ldots$ & $X I P 14 W$ & 160 & 30.6 & 16.7 & 4.11 \\
\hline $\begin{array}{l}\text { Indian Point } 2 \ldots \ldots \ldots \ldots \ldots \ldots \\
\text { Subtotal Indian Point } 2 \ldots \ldots \ldots\end{array}$ & $\begin{array}{l}\text { W1515W } \\
\text { W1515WL } \\
\text { W1515WO } \\
\text { "Temps }\end{array}$ & $\begin{array}{r}393 \\
332 \\
24 \\
7 \\
756\end{array}$ & $\begin{array}{r}177.3 \\
152.4 \\
11.0 \\
0.0 \\
340.7\end{array}$ & $\begin{array}{l}29.3 \\
36.8 \\
34.5\end{array}$ & $\begin{array}{l}3.00 \\
3.37 \\
3.63\end{array}$ \\
\hline $\begin{array}{l}\text { Indian Point } 3 \ldots \ldots \ldots \ldots \ldots \\
\text { Subtotal Indlan Point } 3 \ldots \ldots \ldots\end{array}$ & $\begin{array}{l}\text { W1515WL } \\
\text { W1515WO } \\
\text { aTemps }\end{array}$ & $\begin{array}{r}409 \\
173 \\
2 \\
584\end{array}$ & $\begin{array}{r}186.8 \\
79.9 \\
0.0 \\
266.7\end{array}$ & $\begin{array}{l}31.5 \\
36.6\end{array}$ & $\begin{array}{l}3.03 \\
3.35\end{array}$ \\
\hline $\begin{array}{l}\text { Kewaunee } \ldots \ldots \ldots \ldots \ldots \ldots \\
\text { Subtotal Kewaunee } \ldots \ldots \ldots \ldots\end{array}$ & $\begin{array}{l}\text { W1414W } \\
\text { W1414A } \\
\text { aTemps }\end{array}$ & $\begin{array}{r}241 \\
407 \\
4 \\
652\end{array}$ & $\begin{array}{r}95.7 \\
154.1 \\
0.0 \\
249.8\end{array}$ & $\begin{array}{l}31.7 \\
35.4\end{array}$ & $\begin{array}{l}3.06 \\
3.29\end{array}$ \\
\hline $\begin{array}{l}\text { LaCrosse } \ldots \ldots \ldots \ldots \ldots \ldots \ldots \\
\text { Subtotal LaCrosse } \ldots \ldots \ldots \ldots\end{array}$ & $\begin{array}{l}\text { XLC10L } \\
\text { XLC10A }\end{array}$ & $\begin{array}{l}155 \\
178 \\
333\end{array}$ & $\begin{array}{l}18.6 \\
19.3 \\
38.0\end{array}$ & $\begin{array}{l}14.5 \\
14.9\end{array}$ & $\begin{array}{l}3.77 \\
3.69\end{array}$ \\
\hline LaSalle County $1 \ldots \ldots \ldots \ldots$ & $\begin{array}{l}\text { G4608G5 } \\
\text { G4608GP } \\
\text { G4608GB }\end{array}$ & $\begin{array}{r}627 \\
192 \\
201 \\
1,020\end{array}$ & $\begin{array}{r}114.9 \\
35.0 \\
37.2 \\
187.1\end{array}$ & $\begin{array}{l}17.9 \\
28.9 \\
33.5\end{array}$ & $\begin{array}{l}1.81 \\
2.42 \\
3.04\end{array}$ \\
\hline Subtotal LaSalle County $2 \ldots$. & $\begin{array}{l}\text { G4608G5 } \\
\text { G4608GB } \\
\text { G4608G8 }\end{array}$ & $\begin{array}{r}764 \\
224 \\
144 \\
1,132\end{array}$ & $\begin{array}{r}139.6 \\
41.7 \\
26.6 \\
207.9\end{array}$ & $\begin{array}{l}19.7 \\
33.0 \\
31.4\end{array}$ & $\begin{array}{l}1.89 \\
3.00 \\
3.12\end{array}$ \\
\hline $\begin{array}{l}\text { Limerick } 1, \ldots \ldots \ldots \ldots \ldots \ldots \\
\text { Subtotal Limerick } 1 \ldots \ldots \ldots \ldots\end{array}$ & $\begin{array}{l}\text { G4608GP } \\
\text { G4608GB }\end{array}$ & $\begin{array}{r}434 \\
866 \\
1,300\end{array}$ & $\begin{array}{r}79.7 \\
160.0 \\
239.7\end{array}$ & $\begin{array}{r}3.1 \\
17.5\end{array}$ & $\begin{array}{l}1.71 \\
2.23\end{array}$ \\
\hline Limerick $2, \ldots \ldots \ldots \ldots \ldots \ldots$ & G4608GB & 654 & 121.6 & 17.2 & 1.71 \\
\hline
\end{tabular}

See footnotes at end of table. 
Table B6. Assembly Type Summary by Reactor (Continued)

\begin{tabular}{|c|c|c|c|c|c|}
\hline Reactor Name & $\begin{array}{l}\text { Assembly } \\
\text { Type } \\
\text { Code }\end{array}$ & $\begin{array}{l}\text { Number of } \\
\text { Assemblies }\end{array}$ & $\begin{array}{l}\text { Initial Uranium } \\
\text { Content } \\
\text { (MTU) }\end{array}$ & $\begin{array}{l}\text { Average } \\
\text { Burnup } \\
\text { (GWDIMTU) }\end{array}$ & $\begin{array}{c}\text { Average } \\
\text { Enrichment } \\
\text { (weight percent) }\end{array}$ \\
\hline $\begin{array}{l}\text { Maine Yankee } \ldots \ldots \ldots \ldots \ldots \\
\text { Subtotal Maine Yankee } . . . \ldots \ldots\end{array}$ & $\begin{array}{l}\text { C1414C } \\
\text { C1414A } \\
\text { aTemps }\end{array}$ & $\begin{array}{r}922 \\
223 \\
4 \\
1,149\end{array}$ & $\begin{array}{r}347.4 \\
82.5 \\
0.0 \\
429.9\end{array}$ & $\begin{array}{l}27.1 \\
36.9\end{array}$ & $\begin{array}{l}2.75 \\
3.20\end{array}$ \\
\hline $\begin{array}{l}\text { McGuire } 1, \ldots \ldots \ldots \ldots \ldots \ldots \\
\text { Subtotal McGuire } 1 \ldots \ldots \ldots \ldots\end{array}$ & $\begin{array}{l}\text { W1717WL } \\
\text { W1717WO } \\
\text { W1717B }\end{array}$ & $\begin{array}{r}193 \\
347 \\
8 \\
548\end{array}$ & $\begin{array}{r}88.7 \\
147.3 \\
3.5 \\
239.5\end{array}$ & $\begin{array}{l}27.5 \\
35.7 \\
29.6\end{array}$ & $\begin{array}{l}2.60 \\
3.40 \\
3.34\end{array}$ \\
\hline $\begin{array}{l}\text { McGuire } 2 \ldots \ldots \ldots \ldots \ldots \ldots \\
\text { Subtotal McGuire } 2 \ldots \ldots \ldots \ldots\end{array}$ & $\begin{array}{l}\text { W1717WL } \\
\text { W1717WO }\end{array}$ & $\begin{array}{l}193 \\
359 \\
552\end{array}$ & $\begin{array}{r}88.8 \\
152.6 \\
241.4\end{array}$ & $\begin{array}{l}26.1 \\
36.1\end{array}$ & $\begin{array}{l}2.58 \\
3.32\end{array}$ \\
\hline Millstone $1 \ldots \ldots \ldots \ldots \ldots \ldots$ & $\begin{array}{l}\text { G2307G2A } \\
\text { G2307G2B } \\
\text { G2307G3 } \\
\text { G2308G4 } \\
\text { G2308G5 } \\
\text { G2308GP } \\
\text { G2308GB } \\
\text { G2308G8A }\end{array}$ & $\begin{array}{r}580 \\
82 \\
154 \\
392 \\
148 \\
360 \\
212 \\
188 \\
2,116\end{array}$ & \begin{tabular}{r|}
113.4 \\
15.9 \\
28.9 \\
72.2 \\
26.2 \\
63.8 \\
37.7 \\
33.5 \\
391.6
\end{tabular} & $\begin{array}{l}16.8 \\
21.0 \\
24.1 \\
26.9 \\
26.4 \\
27.9 \\
27.8 \\
32.0\end{array}$ & $\begin{array}{l}2.08 \\
2.30 \\
2.45 \\
2.69 \\
2.66 \\
2.79 \\
3.00 \\
2.98\end{array}$ \\
\hline $\begin{array}{l}\text { Millstone } 2 \ldots \ldots \ldots \ldots \ldots \\
\text { Subtotal Millstone } 2 \ldots \ldots \ldots\end{array}$ & $\begin{array}{l}\text { C1414C } \\
\text { C1414W }\end{array}$ & $\begin{array}{l}361 \\
423 \\
784\end{array}$ & $\begin{array}{l}138.9 \\
172.3 \\
311.2\end{array}$ & $\begin{array}{l}28.6 \\
32.9\end{array}$ & $\begin{array}{l}2.61 \\
3.05\end{array}$ \\
\hline Millstone $3 . . . \ldots \ldots \ldots \ldots \ldots$ & W1717WL & 332 & 152.8 & 33.3 & 3.25 \\
\hline Monticello................. & $\begin{array}{l}\text { G2307G2B } \\
\text { G2307G3 } \\
\text { G2308G4 } \\
\text { G2308G5 } \\
\text { G2308GP } \\
\text { G2308G8A } \\
\text { G2308G8B }\end{array}$ & $\begin{array}{r}484 \\
20 \\
596 \\
112 \\
404 \\
124 \\
28 \\
1,768\end{array}$ & $\begin{array}{r}93.7 \\
3.7 \\
109.7 \\
19.8 \\
71.6 \\
22.2 \\
4.8 \\
325.6\end{array}$ & $\begin{array}{l}15.5 \\
23.8 \\
24.6 \\
30.8 \\
32.2 \\
34.2 \\
38.4\end{array}$ & $\begin{array}{l}2.25 \\
2.30 \\
2.41 \\
2.74 \\
2.72 \\
2.98 \\
3.19\end{array}$ \\
\hline Subtotal Nine Mlle Point $1 \ldots$. & $\begin{array}{l}\text { G2307G2A } \\
\text { G2307G2B } \\
\text { G2307G3 } \\
\text { G2308G4 } \\
\text { G2308G5 } \\
\text { G2308GP }\end{array}$ & $\begin{array}{r}532 \\
96 \\
108 \\
456 \\
184 \\
436 \\
1,812\end{array}$ & \begin{tabular}{r|r}
103.2 & \\
18.6 \\
20.2 \\
83.9 \\
32.5 \\
77.2 \\
335.7
\end{tabular} & $\begin{array}{l}16.2 \\
20.1 \\
26.0 \\
25.7 \\
27.9 \\
26.4\end{array}$ & $\begin{array}{l}2.11 \\
2.42 \\
2.50 \\
2.60 \\
2.77 \\
2.79\end{array}$ \\
\hline $\begin{array}{l}\text { Nine Mile Point } 2 \ldots \ldots \ldots \ldots \ldots \\
\text { Subtotal Nine Mlle Point } 2 \ldots \ldots\end{array}$ & $\begin{array}{l}\text { G4608GP } \\
\text { G4608GB }\end{array}$ & $\begin{array}{l}332 \\
308 \\
640\end{array}$ & $\begin{array}{r}61.4 \\
56.8 \\
118.2\end{array}$ & $\begin{array}{l}12.5 \\
23.5\end{array}$ & $\begin{array}{l}1.47 \\
2.19\end{array}$ \\
\hline $\begin{array}{l}\text { North Anna } 1 \ldots \ldots \ldots \ldots \ldots \\
\text { Subtotal North Anna } 1 \ldots \ldots \ldots\end{array}$ & $\begin{array}{l}\text { W1717WL } \\
\text { aTemps }\end{array}$ & $\begin{array}{r}549 \\
8 \\
557\end{array}$ & $\begin{array}{r}252.4 \\
0.0 \\
252.4\end{array}$ & 33.1 & 3.32 \\
\hline
\end{tabular}

See footnotes at end of table. 
Table B6. Assembly Type Summary by Reactor (Continued)

\begin{tabular}{|c|c|c|c|c|c|}
\hline Reactor Name & $\begin{array}{l}\text { Assembly } \\
\text { Type } \\
\text { Code }\end{array}$ & $\begin{array}{l}\text { Number of } \\
\text { Assemblies }\end{array}$ & $\begin{array}{l}\text { Initial Uranium } \\
\text { Content } \\
\text { (MTU) }\end{array}$ & $\begin{array}{l}\text { Average } \\
\text { Burnup } \\
\text { (GWDtMTU) }\end{array}$ & $\begin{array}{c}\text { Average } \\
\text { Enrichment } \\
\text { (weight percent) }\end{array}$ \\
\hline $\begin{array}{l}\text { North Anna } 2 \ldots \ldots \ldots \ldots \ldots \\
\text { Subtotal North Anna } 2 \ldots \ldots \ldots\end{array}$ & $\begin{array}{l}\text { W1717WL } \\
\text { W1717WVH } \\
\text { aTemps }\end{array}$ & $\begin{array}{r}512 \\
55 \\
1 \\
568\end{array}$ & $\begin{array}{r}235.5 \\
25.5 \\
\mathrm{~b} 0.0 \\
261.0\end{array}$ & $\begin{array}{l}35.2 \\
39.8\end{array}$ & $\begin{array}{l}3.35 \\
4.10\end{array}$ \\
\hline Oconee $1 \ldots \ldots \ldots \ldots \ldots \ldots \ldots$ & $\begin{array}{l}\text { B1515B4 } \\
\text { B1515B4Z } \\
\text { B1515B6 } \\
\text { B1515BZ } \\
\text { B1515BGD }\end{array}$ & $\begin{array}{r}569 \\
4 \\
43 \\
206 \\
4 \\
826\end{array}$ & $\begin{array}{r}264.5 \\
1.9 \\
19.9 \\
95.5 \\
1.7 \\
383.5\end{array}$ & $\begin{array}{l}26.7 \\
42.0 \\
42.6 \\
37.9 \\
43.0\end{array}$ & $\begin{array}{l}2.75 \\
3.32 \\
3.59 \\
3.33 \\
3.92\end{array}$ \\
\hline Subtotal Oconee $2 \ldots \ldots \ldots$. & $\begin{array}{l}\text { B1515B4 } \\
\text { B1515B4Z } \\
\text { B1515B5Z } \\
\text { B1515B6 } \\
\text { B1515B7 } \\
\text { B1515B8 } \\
\text { B1515BZ } \\
\text { B1717B }\end{array}$ & $\begin{array}{r}568 \\
8 \\
29 \\
57 \\
36 \\
3 \\
91 \\
4 \\
496\end{array}$ & \begin{tabular}{r|}
263.5 \\
3.7 \\
13.5 \\
26.3 \\
16.2 \\
1.4 \\
42.2 \\
1.8 \\
368.5
\end{tabular} & $\begin{array}{l}29.1 \\
36.0 \\
36.0 \\
41.7 \\
43.0 \\
35.0 \\
36.2 \\
29.5\end{array}$ & $\begin{array}{l}2.89 \\
3.22 \\
3.21 \\
3.40 \\
3.55 \\
3.88 \\
3.22 \\
2.84\end{array}$ \\
\hline Subtotal Oconee $3 . \ldots \ldots \ldots$. & $\begin{array}{l}\text { B1515B4 } \\
\text { B1515B6 } \\
\text { B1515B7 } \\
\text { B1515B8 } \\
\text { B1515BZ }\end{array}$ & $\begin{array}{r}505 \\
13 \\
60 \\
43 \\
187 \\
808\end{array}$ & $\begin{array}{r}233.9 \\
6.0 \\
27.8 \\
20.0 \\
86.7 \\
374.3\end{array}$ & $\begin{array}{l}28.3 \\
37.0 \\
41.0 \\
44.6 \\
34.1\end{array}$ & $\begin{array}{l}2.83 \\
3.20 \\
3.50 \\
3.80 \\
3.24\end{array}$ \\
\hline Oyster Creek $\ldots \ldots \ldots \ldots \ldots \ldots$ & $\begin{array}{l}\text { G2307G2A } \\
\text { G2307G2B } \\
\text { G2308G5 } \\
\text { G2308GP } \\
\text { G2308GB } \\
\text { G2307A } \\
\text { G2308A }\end{array}$ & $\begin{array}{r}560 \\
156 \\
87 \\
156 \\
40 \\
260 \\
617 \\
1,876\end{array}$ & $\begin{array}{r}109.3 \\
30.2 \\
15.4 \\
27.5 \\
7.1 \\
47.4 \\
108.0 \\
344.8\end{array}$ & $\begin{array}{l}17.3 \\
22.7 \\
26.0 \\
29.2 \\
25.6 \\
23.5 \\
25.0\end{array}$ & $\begin{array}{l}2.11 \\
2.62 \\
2.39 \\
2.84 \\
2.68 \\
2.64 \\
2.50\end{array}$ \\
\hline $\begin{array}{l}\text { Palisades } \ldots \ldots \ldots \ldots \ldots \ldots \ldots \\
\text { Subtotal Palisades } \ldots \ldots \ldots \ldots\end{array}$ & $\begin{array}{l}\text { XPA15C } \\
\text { XPA15A }\end{array}$ & $\begin{array}{l}273 \\
520 \\
793\end{array}$ & $\begin{array}{l}112.6 \\
203.9 \\
316.5\end{array}$ & $\begin{array}{l}16.0 \\
30.8\end{array}$ & $\begin{array}{l}2.47 \\
2.95\end{array}$ \\
\hline Palo Verde $1, \ldots \ldots \ldots \ldots \ldots$ & C8016C & 368 & 151.7 & 32.2 & 3.00 \\
\hline Palo Verde $2 \ldots \ldots \ldots \ldots \ldots$ & C8016C & 384 & 156.5 & 31.5 & 3.03 \\
\hline Palo Verde $3 \ldots \ldots \ldots \ldots$ & C8016C & 284 & 115.9 & 27.2 & 2.79 \\
\hline Subtotal Peach Bottom $2 \ldots .$. & $\begin{array}{l}\text { G4607G2 } \\
\text { G4607G3A } \\
\text { G4608G4A } \\
\text { G4608G5 } \\
\text { G4608GP } \\
\text { G4608GB }\end{array}$ & $\begin{array}{r}168 \\
596 \\
360 \\
260 \\
552 \\
228 \\
2,164\end{array}$ & $\begin{array}{r}32.9 \\
111.7 \\
66.3 \\
47.5 \\
100.8 \\
41.7 \\
400.8\end{array}$ & $\begin{array}{r}9.5 \\
22.7 \\
25.5 \\
29.2 \\
28.0 \\
31.4\end{array}$ & $\begin{array}{l}1.10 \\
2.51 \\
2.74 \\
2.86 \\
2.89 \\
2.99\end{array}$ \\
\hline
\end{tabular}

See footnotes at end of table. 
Table B6. Assembly Type Summary by Reactor (Continued)

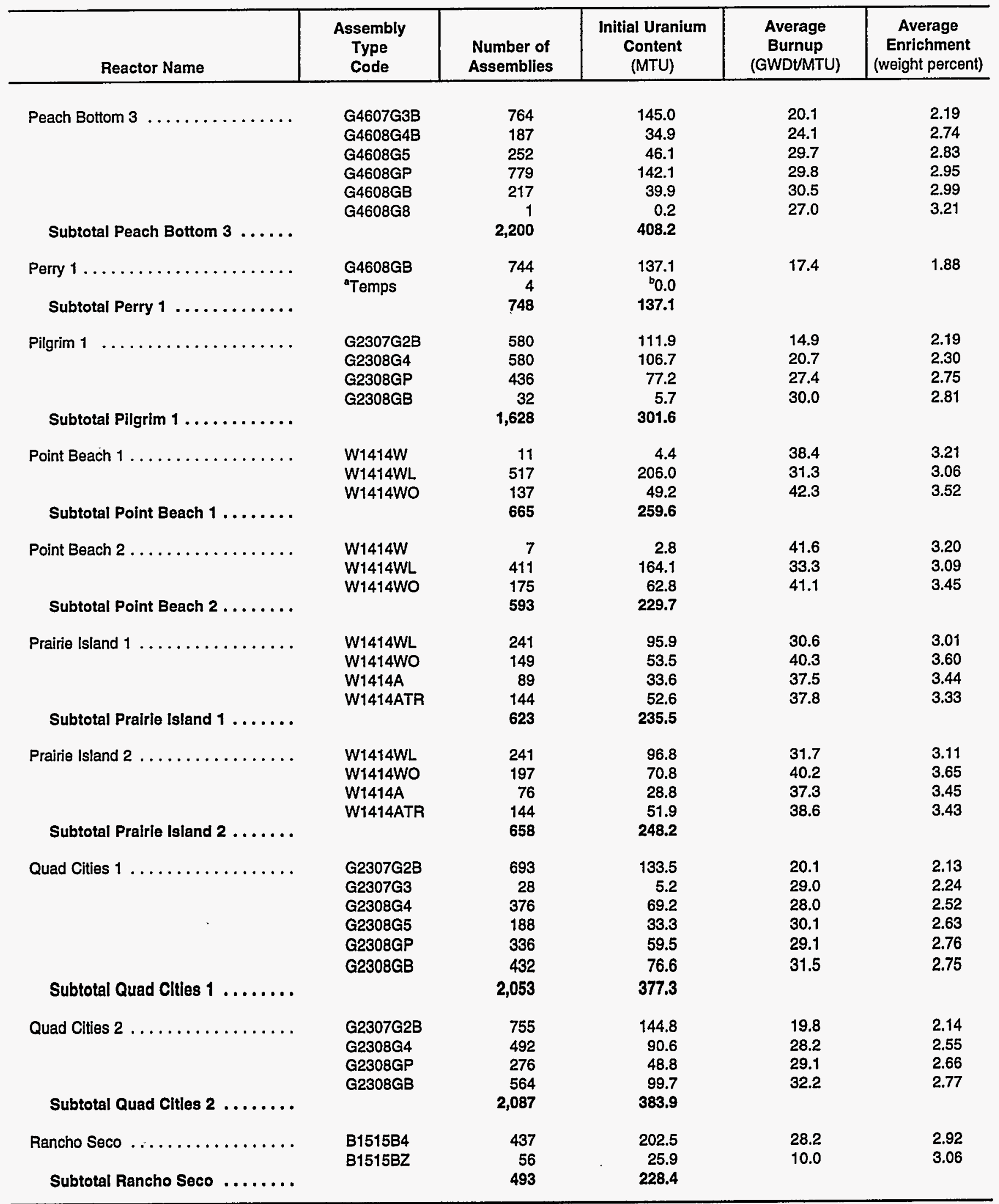

See footnotes at end of table. 
Table B6. Assembly Type Summary by Reactor (Continued)

\begin{tabular}{|c|c|c|c|c|c|}
\hline Reactor Name & $\begin{array}{l}\text { Assembly } \\
\text { Type } \\
\text { Code } \\
\end{array}$ & $\begin{array}{l}\text { Number of } \\
\text { Assemblies }\end{array}$ & $\begin{array}{l}\text { Initial Uranium } \\
\text { Content } \\
\text { (MTU) }\end{array}$ & $\begin{array}{c}\text { Average } \\
\text { Burnup } \\
\text { (GWDt/MTU) }\end{array}$ & $\begin{array}{c}\text { Average } \\
\text { Enrichment } \\
\text { (weight percent) }\end{array}$ \\
\hline River Bend $1 \ldots \ldots \ldots \ldots \ldots$ & G4608GB & 764 & 141.6 & 20.8 & 2.07 \\
\hline \multirow[t]{3}{*}{ Robinson $2 \ldots \ldots \ldots \ldots \ldots$} & W1515WL & 314 & 141.5 & 23.7 & 2.63 \\
\hline & W1515A & 498 & 214.1 & 34.0 & 3.05 \\
\hline & W1515APL & 12 & 3.7 & 22.0 & 1.21 \\
\hline Subtotal Robinson $2 \ldots \ldots \ldots$ & & 824 & 359.2 & & \\
\hline \multirow[t]{2}{*}{ St. Lucie $1 \ldots \ldots \ldots \ldots \ldots \ldots$} & C1414C & 497 & 189.6 & 28.9 & 2.81 \\
\hline & C1414A & 383 & 141.9 & 37.7 & 3.50 \\
\hline Subtotal St. Lucie 1 ......... & & 880 & 331.5 & & \\
\hline St. Lucie $2 \ldots$ & XSL16C & 464 & 175.7 & 32.1 & 2.93 \\
\hline \multirow[t]{4}{*}{ Salem $1 \ldots \ldots \ldots \ldots \ldots \ldots$} & W1717WL & 624 & 287.5 & 32.8 & 3.23 \\
\hline & W1717wo & 2 & 0.8 & 34.7 & 2.79 \\
\hline & W1717WVH & 60 & 27.9 & 38.3 & 3.76 \\
\hline & “Temps & 22 & $\begin{aligned} 0.0 \\
8\end{aligned}$ & & \\
\hline \multicolumn{2}{|l|}{ Subtotal Salem $1 \quad \ldots \ldots \ldots \ldots$} & 708 & 316.2 & & \\
\hline \multirow{2}{*}{$\begin{array}{l}\text { Salem } 2 \ldots \ldots \ldots \ldots \ldots \ldots \\
\qquad \text { Subtotal Salem } 2 \ldots \ldots \ldots \ldots\end{array}$} & $\begin{array}{l}\text { W1717WL } \\
\text { "Temps }\end{array}$ & $\begin{array}{r}460 \\
32\end{array}$ & $\begin{array}{r}212.0 \\
0.0\end{array}$ & 32.2 & 3.25 \\
\hline & & 492 & 212.0 & & \\
\hline San Onofre $1 \ldots \ldots \ldots \ldots \ldots$ & XS014W & 665 & 244.3 & 27.3 & 3.86 \\
\hline San Onofre $2 \ldots \ldots \ldots \ldots \ldots$ & C1616CSD & 592 & 244.2 & 32.1 & 3.26 \\
\hline San Onofre $3 \ldots \ldots \ldots \ldots \ldots$ & C1616CSD & 592 & 244.1 & 32.3 & 3.27 \\
\hline Seabrook $\ldots \ldots \ldots \ldots \ldots \ldots$ & W1717WL & 136 & 63.0 & 20.2 & 2.06 \\
\hline \multirow{2}{*}{ Sequoyah $1, \ldots \ldots \ldots \ldots \ldots$} & W1717WL & 382 & 175.8 & 32.3 & 3.11 \\
\hline & W1717WVH & 56 & 26.0 & 32.2 & 3.65 \\
\hline Subtotal Sequoyah $1 \ldots \ldots \ldots$ & & 438 & 201.8 & & \\
\hline Sequoyah $2 \ldots \ldots \ldots \ldots \ldots$ & W1717WL & 379 & 174.5 & 31.6 & 3.05 \\
\hline Shoreham $\ldots \ldots \ldots \ldots \ldots \ldots$ & G4608GP & 254 & 46.5 & 0.5 & 1.70 \\
\hline \multirow{2}{*}{$\begin{array}{l}\text { South Texas } 1 \ldots \ldots \ldots \ldots \ldots \\
\text { Subtotal South Texas } 1 \ldots \ldots \ldots\end{array}$} & WST17W & 233 & 126.2 & 21.3 & 2.37 \\
\hline & Temps & $\begin{array}{r}3 \\
236\end{array}$ & $\begin{array}{r}0.0 \\
126.2\end{array}$ & & \\
\hline South Texas $2 \ldots \ldots \ldots \ldots \ldots$ & WST17W & 188 & 101.5 & 24.2 & 2.53 \\
\hline \multirow[t]{2}{*}{ Summer $\ldots \ldots \ldots \ldots \ldots \ldots \ldots$} & W1717WL & 329 & 151.5 & 31.6 & 3.16 \\
\hline & W1717WV5 & 111 & 47.1 & 39.3 & 3.67 \\
\hline Subtotal Summer $\ldots \ldots \ldots \ldots$ & & 440 & 198.6 & & \\
\hline \multirow{4}{*}{ Surry $1, \ldots \ldots \ldots \ldots \ldots \ldots$} & W1515W & 613 & 279.0 & 29.6 & 2.99 \\
\hline & W1515WL & 57 & 26.2 & 43.6 & 3.64 \\
\hline & W1515WO & 8 & 3.7 & 37.0 & 3.80 \\
\hline & W1717WL & 2 & 0.9 & 16.1 & 1.86 \\
\hline \multicolumn{2}{|l|}{ Subtotal Surry $1 \ldots \ldots \ldots \ldots$} & 680 & 309.8 & & \\
\hline
\end{tabular}

See footnotes at end of table. 
Table B6. Assembly Type Summary by Reactor (Continued)

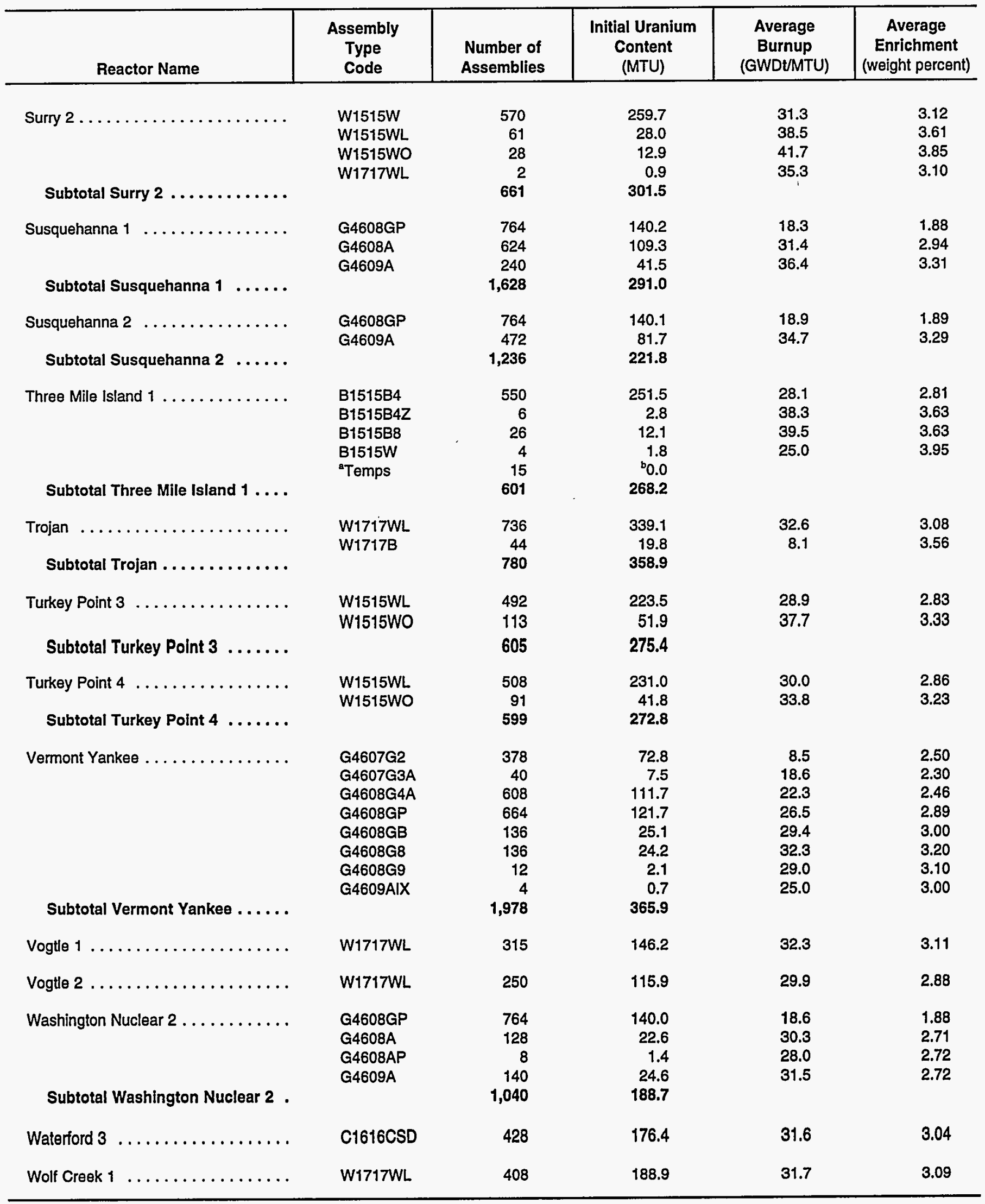

See footnotes at end of table. 
Table B6. Assembly Type Summary by Reactor (Continued)

\begin{tabular}{|c|c|c|c|c|c|}
\hline Reactor Name & $\begin{array}{c}\text { Assembly } \\
\text { Type } \\
\text { Code }\end{array}$ & $\begin{array}{l}\text { Number of } \\
\text { Assemblies }\end{array}$ & $\begin{array}{l}\text { Initial Uranium } \\
\text { Content } \\
\text { (MTU) }\end{array}$ & $\begin{array}{c}\text { Average } \\
\text { Burnup } \\
\text { (GWDtMTU) }\end{array}$ & $\begin{array}{c}\text { Average } \\
\text { Enrichment } \\
\text { (weight percent) }\end{array}$ \\
\hline \multirow[t]{4}{*}{ Yankee Rowe $\ldots \ldots \ldots \ldots \ldots$} & XYR18W & 76 & 20.8 & 25.5 & 4.94 \\
\hline & XYR16U & 73 & 17.4 & 27.5 & 3.83 \\
\hline & XYR16A & 228 & 53.2 & 29.0 & 3.70 \\
\hline & XYR16C & 156 & 35.8 & 24.3 & 3.83 \\
\hline Subtotal Yankee Rowe . . . . . . . . & & 533 & 127.2 & & \\
\hline \multirow[t]{2}{*}{ Zlon $1 \ldots \ldots \ldots \ldots \ldots \ldots$} & W1515WL & 567 & 258.4 & 31.5 & 2.93 \\
\hline & W1515WO & 311 & 142.8 & 35.2 & 3.19 \\
\hline Subtotal Zion $1 \ldots \ldots \ldots \ldots$. . & & 878 & 401.3 & & \\
\hline \multirow{2}{*}{ Zion $2 \ldots \ldots \ldots \ldots \ldots \ldots \ldots$} & W1515WL & 547 & 248.7 & 31.9 & 2.95 \\
\hline & W1515WO & 259 & 118.9 & 35.1 & 3.25 \\
\hline Subtotal Zlon $2 \ldots \ldots \ldots \ldots$. . . & & 806 & 367.7 & & \\
\hline Total $\ldots \ldots \ldots \ldots \ldots \ldots \ldots$ & & 98,400 & $28,136.0$ & & \\
\hline
\end{tabular}

"Temps represent temporarily discharged assemblies with no assigned assembly type. See Technical Note 10 in Appendix E.

"No welght reported for temporarily discharged assemblies with no assigned assembly type. See Technical Note 10 in Appendix E. 'No bumup reported for temporarily discharged assemblies. See Technical Note 10 in Appendix E.

MTU = Metric tons of uranium; GWDt/MTU = Gigawattdays thermal per metric ton of uranium.

Note: Totals may not equal sum of components because of independent rounding. See Technical Note 11 in Appendix E.

Source: Energy Information Administration, Form RW-859, "Nuclear Fuel Data" (1993). 
Table B7. Assembly Type Summary by Assembly Type

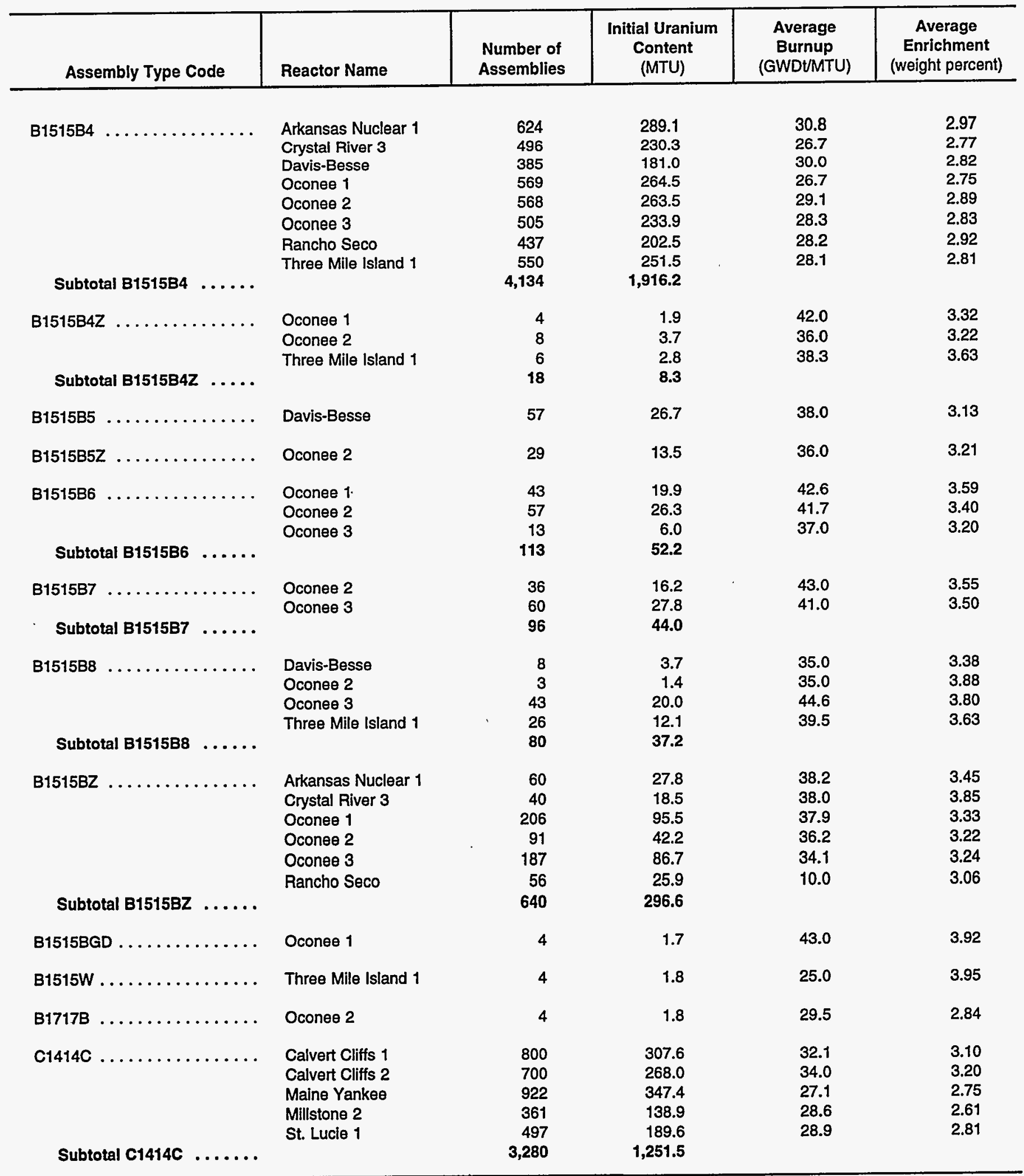

See footnotes at end of table. 
Table B7. Assembly Type Summary by Assembly Type (Continued)

\begin{tabular}{|c|c|c|c|c|c|}
\hline Assembly Type Code & Reactor Name & $\begin{array}{l}\text { Number of } \\
\text { Assemblies }\end{array}$ & $\begin{array}{l}\text { Initial Uranium } \\
\text { Content } \\
\text { (MTU) }\end{array}$ & $\begin{array}{c}\text { Average } \\
\text { Burnup } \\
\text { (GWDtMTU) }\end{array}$ & $\begin{array}{c}\text { Average } \\
\text { Enrichment } \\
\text { (weight percent) }\end{array}$ \\
\hline $\begin{array}{r}\text { C1414A } \ldots \ldots \ldots \ldots \ldots \\
\text { Subtotal C1414A } \ldots \ldots\end{array}$ & $\begin{array}{l}\text { Maine Yankee } \\
\text { St. Lucie } 1\end{array}$ & $\begin{array}{l}223 \\
383 \\
606\end{array}$ & $\begin{array}{r}82.5 \\
141.9 \\
224.4\end{array}$ & $\begin{array}{l}36.9 \\
37.7\end{array}$ & $\begin{array}{l}3.20 \\
3.50\end{array}$ \\
\hline C1414W .............. & Millstone 2 & 423 & 172.3 & 32.9 & 3.05 \\
\hline $\begin{array}{l}\text { C1616CSD } \ldots \ldots \ldots \ldots \ldots \\
\text { Subtotal C1616CSD } \ldots \ldots\end{array}$ & $\begin{array}{l}\text { Arkansas Nuclear } 2 \\
\text { San Onofre } 2 \\
\text { San Onofre } 3 \\
\text { Waterford } 3\end{array}$ & $\begin{array}{r}564 \\
592 \\
592 \\
428 \\
2,176\end{array}$ & $\begin{array}{l}234.5 \\
244.2 \\
244.1 \\
176.4 \\
899.2\end{array}$ & $\begin{array}{l}34.7 \\
32.1 \\
32.3 \\
31.6\end{array}$ & $\begin{array}{l}3.25 \\
3.26 \\
3.27 \\
3.04\end{array}$ \\
\hline $\begin{array}{l}\text { C8016C } \ldots \ldots \ldots \ldots \ldots \\
\text { Subtotal C8016C } \ldots \ldots \ldots\end{array}$ & $\begin{array}{l}\text { Palo Verde } 1 \\
\text { Palo Verde } 2 \\
\text { Palo Verde } 3\end{array}$ & $\begin{array}{r}368 \\
384 \\
284 \\
1,036\end{array}$ & $\begin{array}{l}151.7 \\
156.5 \\
115.9 \\
424.2\end{array}$ & $\begin{array}{l}32.2 \\
31.5 \\
27.2\end{array}$ & $\begin{array}{l}3.00 \\
3.03 \\
2.79\end{array}$ \\
\hline $\begin{array}{r}\text { G2307G2A } \ldots \ldots \ldots \ldots \ldots \\
\text { Subtotal G2307G2A } \ldots \ldots\end{array}$ & $\begin{array}{l}\text { Millstone } 1 \\
\text { Nine Mile Point } 1 \\
\text { Oyster Creek }\end{array}$ & $\begin{array}{r}580 \\
532 \\
560 \\
1,672\end{array}$ & $\begin{array}{l}113.4 \\
103.2 \\
109.3 \\
325.9\end{array}$ & $\begin{array}{l}16.8 \\
16.2 \\
17.3\end{array}$ & $\begin{array}{l}2.08 \\
2.11 \\
2.11\end{array}$ \\
\hline Subtotal G2307G2B . . . . . & $\begin{array}{l}\text { Dresden } 2 \\
\text { Dresden } 3 \\
\text { Millstone 1 } \\
\text { Monticello } \\
\text { Nine Mile Point } 1 \\
\text { Oyster Creek } \\
\text { Pilgrim 1 } \\
\text { Quad Cities } 1 \\
\text { Quad Cities } 2\end{array}$ & $\begin{array}{r}1,477 \\
724 \\
82 \\
484 \\
96 \\
156 \\
580 \\
693 \\
755 \\
5,047\end{array}$ & $\begin{array}{r}284.1 \\
140.2 \\
15.9 \\
93.7 \\
18.6 \\
30.2 \\
111.9 \\
133.5 \\
144.8 \\
972.9\end{array}$ & $\begin{array}{l}10.4 \\
17.3 \\
21.0 \\
15.5 \\
20.1 \\
22.7 \\
14.9 \\
20.1 \\
19.8\end{array}$ & $\begin{array}{l}2.08 \\
2.13 \\
2.30 \\
2.25 \\
2.42 \\
2.62 \\
2.19 \\
2.13 \\
2.14\end{array}$ \\
\hline Subtotal G2307G3 ..... & $\begin{array}{l}\text { Dresden } 2 \\
\text { Dresden } 3 \\
\text { Millstone } 1 \\
\text { Monticello } \\
\text { Nine Mile Point } 1 \\
\text { Quad Cities } 1\end{array}$ & $\begin{array}{r}32 \\
52 \\
154 \\
20 \\
108 \\
28 \\
394\end{array}$ & $\begin{array}{r}6.0 \\
9.8 \\
28.9 \\
3.7 \\
20.2 \\
5.2 \\
73.9\end{array}$ & $\begin{array}{l}23.7 \\
24.0 \\
24.1 \\
23.8 \\
26.0 \\
29.0\end{array}$ & $\begin{array}{l}2.29 \\
2.30 \\
2.45 \\
2.30 \\
2.50 \\
2.24\end{array}$ \\
\hline Subtotal G2308G4 . . . . . & $\begin{array}{l}\text { Dresden } 2 \\
\text { Dresden } 3 \\
\text { Millstone } 1 \\
\text { Monticello } \\
\text { Nine Mile Point } 1 \\
\text { Pilgrim } 1 \\
\text { Quad Cities } 1 \\
\text { Quad Cities } 2\end{array}$ & $\begin{array}{r}476 \\
508 \\
392 \\
596 \\
456 \\
580 \\
376 \\
492 \\
3,876\end{array}$ & $\begin{array}{r}87.8 \\
93.4 \\
72.2 \\
109.7 \\
83.9 \\
106.7 \\
69.2 \\
90.6 \\
713.6\end{array}$ & $\begin{array}{l}25.1 \\
25.7 \\
26.9 \\
24.6 \\
25.7 \\
20.7 \\
28.0 \\
28.2\end{array}$ & $\begin{array}{l}2.52 \\
2.56 \\
2.69 \\
2.41 \\
2.60 \\
2.30 \\
2.52 \\
2.55\end{array}$ \\
\hline
\end{tabular}

See footnotes at end of table. 
Table B7. Assembly Type Summary by Assembly Type (Continued)

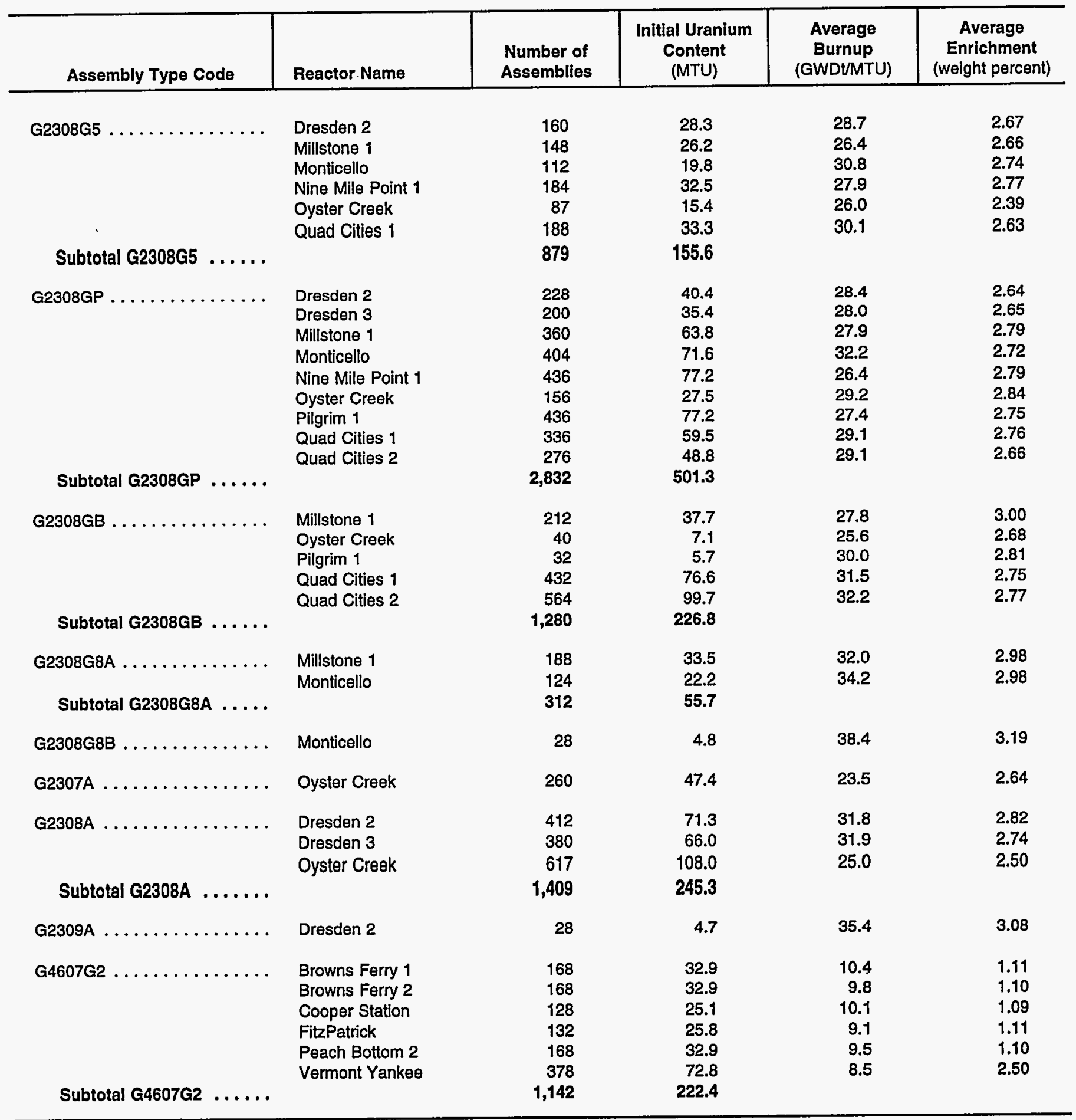

See footnotes at end of table. 
Table B7. Assembly Type Summary by Assembly Type (Continued)

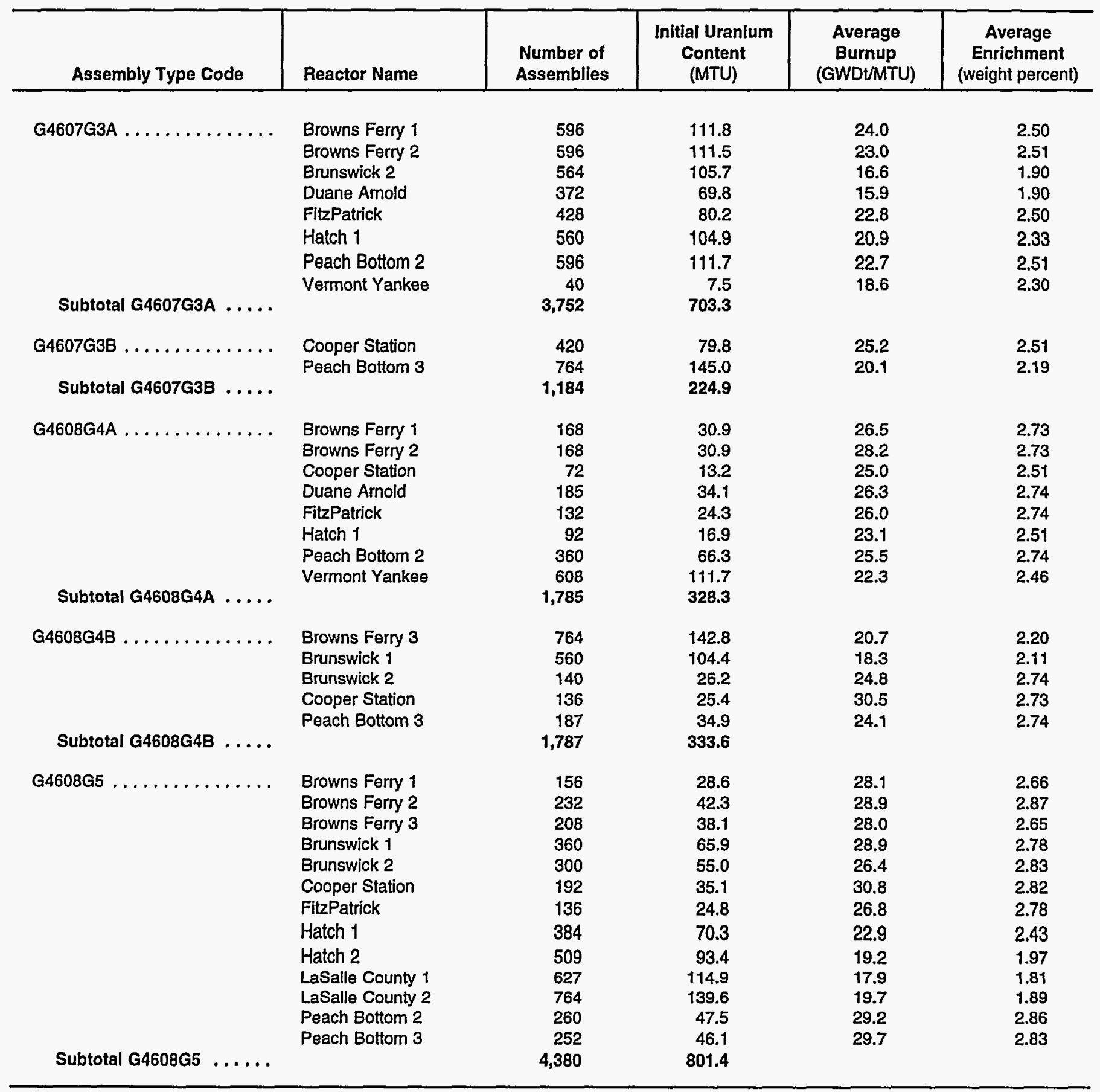

See footnotes at end of table. 
Table B7. Assembly Type Summary by Assembly Type (Continued)

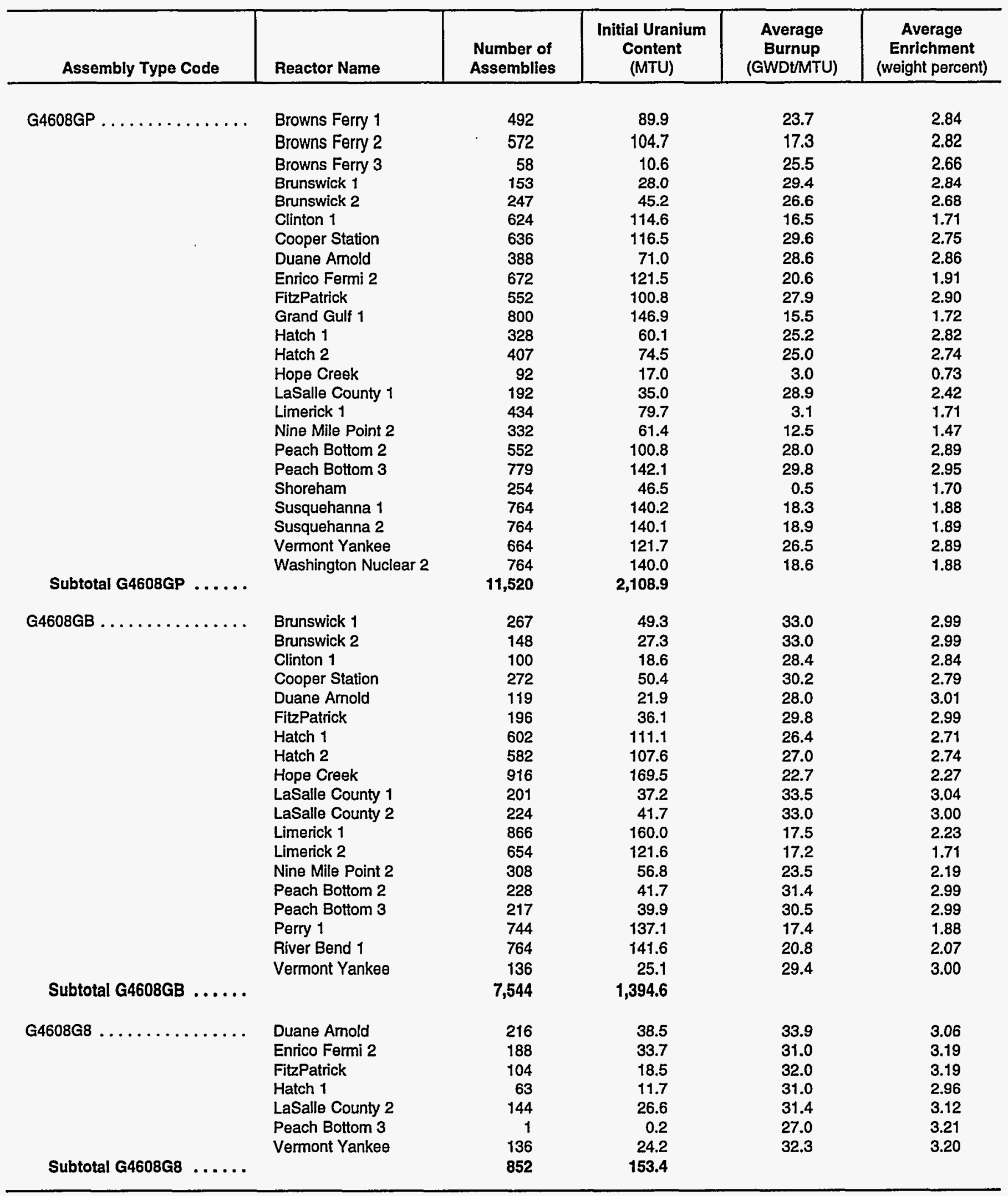

See footnotes at end of table. 
Table B7. Assembly Type Summary by Assembly Type (Continued)

\begin{tabular}{|c|c|c|c|c|c|}
\hline Assembly Type Code & Reactor Name & $\begin{array}{l}\text { Number of } \\
\text { Assemblies }\end{array}$ & $\begin{array}{l}\text { Initial Uranium } \\
\text { Content } \\
\text { (MTU) }\end{array}$ & $\begin{array}{l}\text { Average } \\
\text { Burnup } \\
\text { (GWDt/MTU) }\end{array}$ & $\begin{array}{c}\text { Average } \\
\text { Enrichment } \\
\text { (weight percent) }\end{array}$ \\
\hline \multirow[t]{4}{*}{ G4608G9 } & Brunswick 2 & 1 & 0.2 & 18.0 & 3.29 \\
\hline & Hatch 1 & 5 & 0.9 & 35.6 & 2.89 \\
\hline & Hatch 2 & 4 & 0.7 & 16.3 & 3.14 \\
\hline & Vermont Yankee & 12 & 2.1 & 29.0 & 3.10 \\
\hline Subtotal G4608G9 ...... & & 22 & 3.9 & & \\
\hline G4608G10 $\ldots \ldots \ldots \ldots \ldots$ & Cooper Station & 4 & 0.7 & 29.0 & 2.64 \\
\hline G4609G11 $\ldots \ldots \ldots \ldots \ldots$ & Enrico Fermi 2 & 40 & 6.8 & 0.0 & 3.31 \\
\hline \multirow[t]{3}{*}{ G4608A $\ldots \ldots \ldots \ldots \ldots$} & Grand Gulf 1 & 584 & 103.0 & 30.3 & 2.94 \\
\hline & Susquehanna 1 & 624 & 109.3 & 31.4 & 2.94 \\
\hline & Washington Nuclear 2 & 128 & 22.6 & 30.3 & 2.71 \\
\hline Subtotal G4608A ....... & & 1,336 & 234.9 & & \\
\hline \multirow[t]{2}{*}{ G4608AP } & Grand Gulf 1 & 240 & 42.2 & 35.0 & 3.37 \\
\hline & Washington Nuclear 2 & 8 & 1.4 & 28.0 & 2.72 \\
\hline Subtotal G4608AP ...... & & 248 & 43.6 & & \\
\hline \multirow[t]{5}{*}{ G4609A $\ldots \ldots \ldots \ldots \ldots$} & Hatch 1 & 4 & 0.7 & 34.0 & 2.79 \\
\hline & Hatch 2 & 4 & 0.7 & 36.0 & 2.79 \\
\hline & Susquehanna 1 & 240 & 41.5 & 36.4 & 3.31 \\
\hline & Susquehanna 2 & 472 & 81.7 & 34.7 & 3.29 \\
\hline & Washington Nuclear 2 & 140 & 24.6 & 31.5 & 2.72 \\
\hline Subtotal G4609A ....... & & 860 & 149.2 & & \\
\hline G4609A5 . & Grand Gulf 1 & 36 & 6.4 & 31.9 & 3.39 \\
\hline G4609AIX $\ldots \ldots \ldots \ldots \ldots$ & Vermont Yankee & 4 & 0.7 & 25.0 & 3.00 \\
\hline G4608W & FitzPatrick & 4 & 0.6 & 23.0 & 3.01 \\
\hline \multirow[t]{4}{*}{$W_{1414 W} \ldots \ldots \ldots \ldots \ldots$} & Ginna & 362 & 141.8 & 24.4 & 3.03 \\
\hline & Kewaunee & 241 & 95.7 & 31.7 & 3.06 \\
\hline & Point Beach 1 & 11 & 4.4 & 38.4 & 3.21 \\
\hline & Point Beach 2 & 7 & 2.8 & 41.6 & 3.20 \\
\hline Subtotal W1414W ...... & & 621 & 244.7 & & \\
\hline \multirow[t]{4}{*}{ W1414WL $\ldots \ldots \ldots \ldots \ldots$} & Point Beach 1 & 517 & 206.0 & 31.3 & 3.06 \\
\hline & Point Beach 2 & 411 & 164.1 & 33.3 & 3.09 \\
\hline & Prairie Island 1 & 241 & 95.9 & 30.6 & 3.01 \\
\hline & Prairie Island 2 & 241 & 96.8 & 31.7 & 3.11 \\
\hline Subtotal W1414WL ...... & & 1,410 & 562.7 & & \\
\hline \multirow[t]{5}{*}{ W1414WO } & Ginna & 171 & 60.3 & 40.4 & 3.55 \\
\hline & Point Beach 1 & 137 & 49.2 & 42.3 & 3.52 \\
\hline & Point Beach 2 & 175 & 62.8 & 41.1 & 3.45 \\
\hline & Prairie Island 1 & 149 & 53.5 & 40.3 & 3.60 \\
\hline & Prairie Island 2 & 197 & 70.8 & 40.2 & 3.65 \\
\hline Subtotal W1414Wo ..... & & 829 & 296.6 & & \\
\hline \multirow[t]{4}{*}{$W_{1414 A} \ldots \ldots \ldots \ldots$} & Ginna & 194 & 72.3 & 37.1 & 3.22 \\
\hline & Kewaunee & 407 & 154.1 & 35.4 & 3.29 \\
\hline & Prairie Island 1 & 89 & 33.6 & 37.5 & 3.44 \\
\hline & Prairie Island 2 & 76 & 28.8 & 37.3 & 3.45 \\
\hline Subtotal W1414A ....... & & 766 & 288.7 & & \\
\hline
\end{tabular}

See footnotes at end of table. 
Table B7. Assembly Type Summary by Assembly Type (Continued)

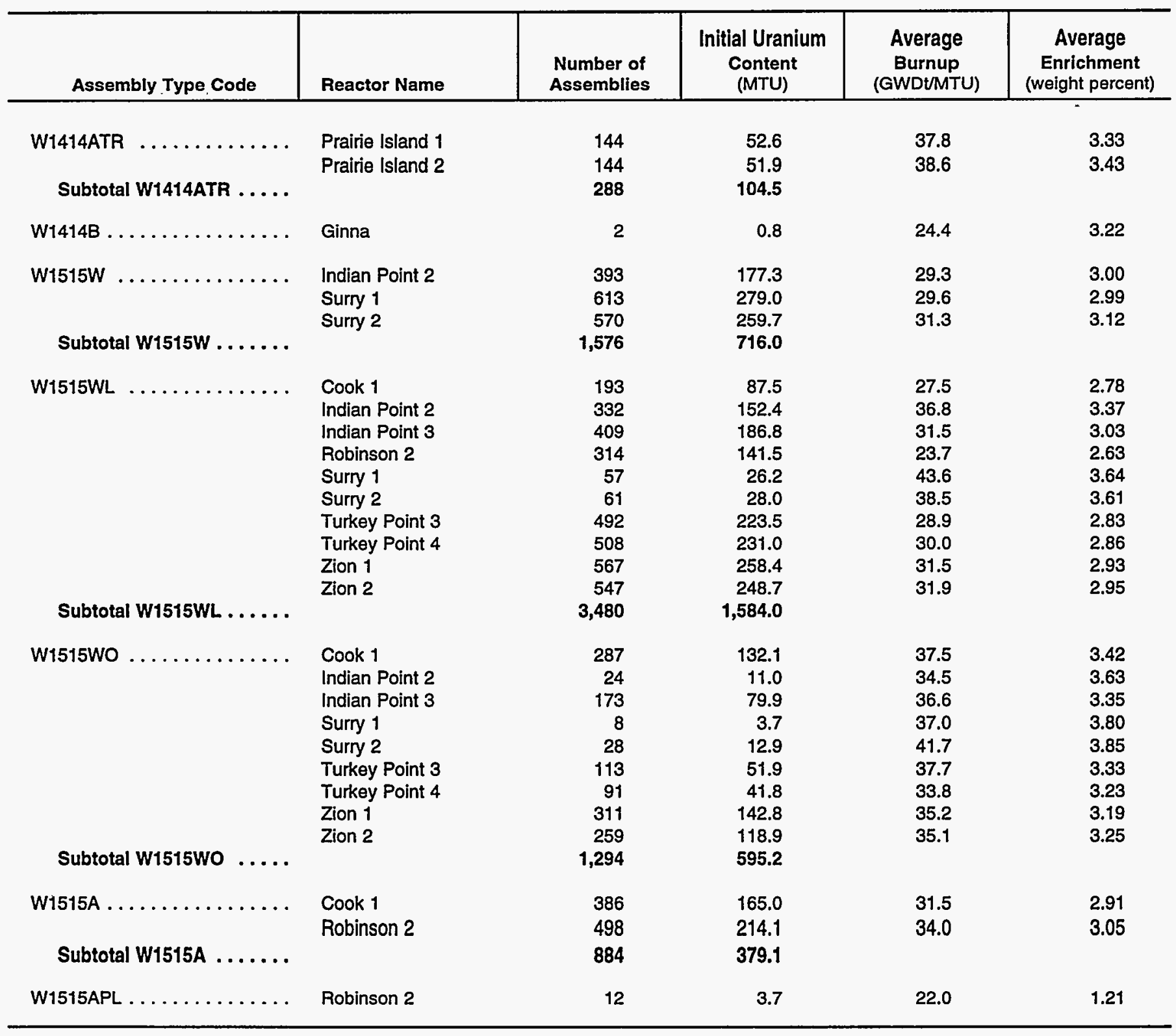

See footnotes at end of table. 
Table B7. Assembly Type Summary by Assembly Type (Continued)

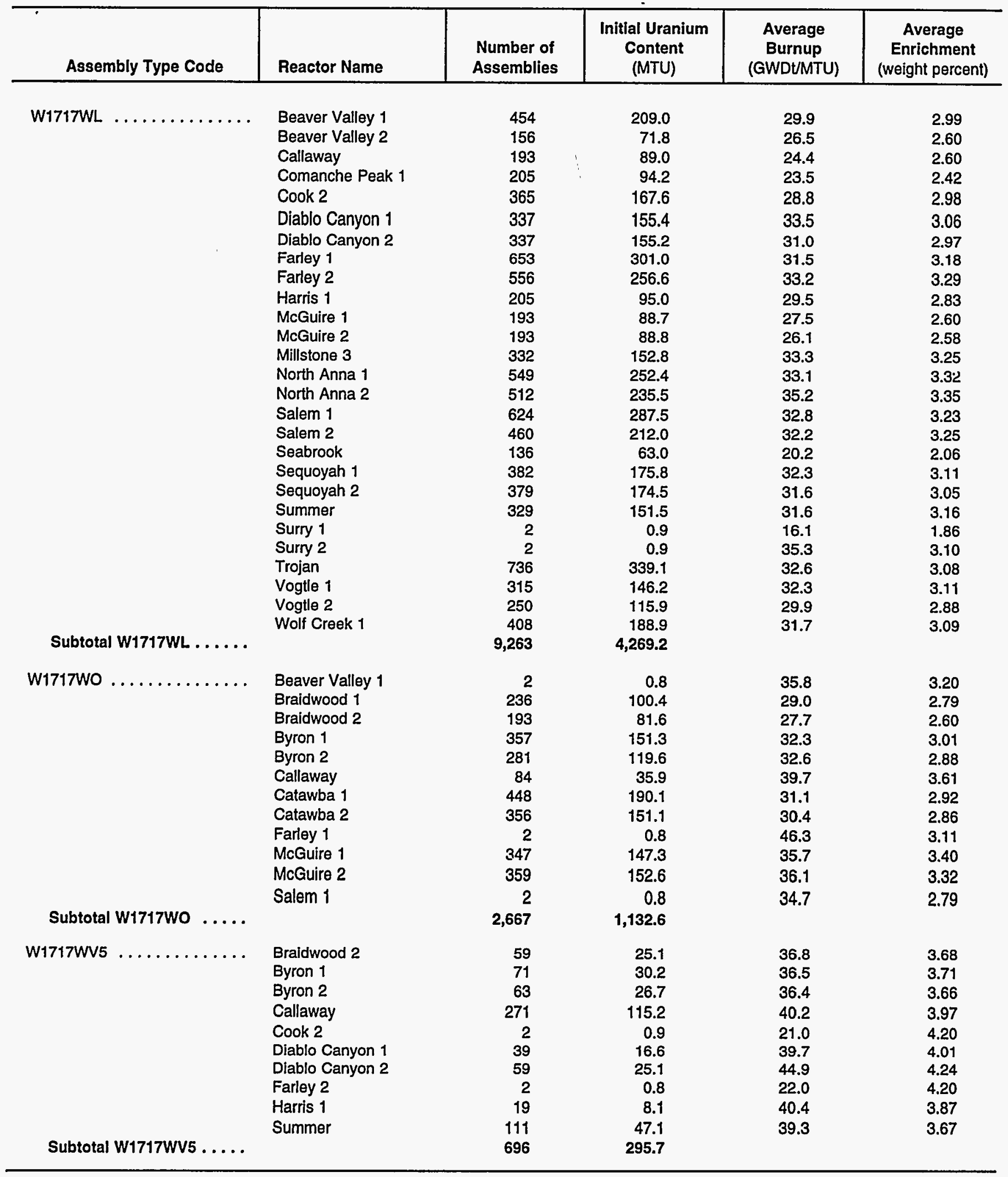

See footnotes at end of table. 
Table B7. Assembly Type Summary by Assembly Type (Continued)

\begin{tabular}{|c|c|c|c|c|c|}
\hline Assembly Type Code & Reactor Name & $\begin{array}{l}\text { Number of } \\
\text { Assemblies }\end{array}$ & $\begin{array}{l}\text { Initial Uranium } \\
\text { Content } \\
\text { (MTU) }\end{array}$ & $\begin{array}{c}\text { Average } \\
\text { Burnup } \\
\text { (GWDt/MTU) }\end{array}$ & $\begin{array}{c}\text { Average } \\
\text { Enrichment } \\
\text { (weight percent) }\end{array}$ \\
\hline \multirow[t]{3}{*}{ W1717WVH $\ldots \ldots \ldots \ldots$} & Beaver Valley 1 & 120 & 55.7 & 35.4 & 3.56 \\
\hline & Beaver Valley 2 & 104 & 48.2 & 37.4 & 3.54 \\
\hline & North Anna 2 & 55 & 25.5 & 39.8 & 4.10 \\
\hline Subtotal W1717WVH $\ldots$ & & 395 & 183.3 & & \\
\hline$w 1717 A \ldots \ldots \ldots \ldots$ & Cook 2 & 290 & 116.6 & 39.3 & 3.76 \\
\hline \multirow{2}{*}{$w 1717 B \ldots \ldots \ldots \ldots$} & Catawba 1 & 36 & 16.4 & 33.0 & 3.56 \\
\hline & McGuire 1 & 8 & 3.5 & 29.6 & 3.34 \\
\hline Subtotal WST17W ..... & & 421 & 227.8 & & \\
\hline$\ldots \ldots \ldots \ldots \ldots$ & Big Rock Point & 6 & 0.7 & 19.8 & 3.63 \\
\hline XBR07G $\ldots \ldots \ldots \ldots \ldots$ & Big Rock Point & 4 & 0.5 & 1.5 & 3.63 \\
\hline XBR08G $\ldots \ldots \ldots \ldots \ldots$ & Big Rock Point & 2 & 0.2 & 4.1 & 3.63 \\
\hline XBR09G $\ldots \ldots \ldots \ldots \ldots$ & Big Rock Point & 143 & 19.6 & 14.5 & 3.59 \\
\hline XBR09A $\ldots \ldots \ldots \ldots \ldots$ & Big Rock Point & 4 & 0.5 & 20.9 & 3.48 \\
\hline XDR06G5 $\ldots \ldots \ldots \ldots$ & Dresden 1 & 106 & 11.2 & 19.0 & 2.26 \\
\hline XDR07GS $\ldots \ldots \ldots \ldots$ & Dresden 1 & 1 & 0.1 & 29.0 & 3.10 \\
\hline XDR08G $\ldots \ldots \ldots \ldots \ldots$ & Dresden 1 & 1 & 0.1 & 23.3 & 1.95 \\
\hline XDRO6U $\ldots \ldots \ldots \ldots \ldots$ & Dresden 1 & 458 & 46.7 & 16.1 & 2.24 \\
\hline XDR06A $\ldots \ldots \ldots \ldots \ldots$ & Dresden 1 & 66 & 6.3 & 4.5 & 2.23 \\
\hline XFC14C $\ldots \ldots \ldots \ldots \ldots$ & Fort Calhoun & 378 & 137.2 & 30.2 & 2.87 \\
\hline XFC14A $\ldots \ldots \ldots \ldots \ldots$ & Fort Calhoun & 192 & 67.8 & 37.3 & 3.57 \\
\hline XHB07G2 $\ldots \ldots \ldots \ldots$ & Humboldt Bay & 88 & 6.7 & 18.2 & 2.11 \\
\hline XHBO6G $\ldots \ldots \ldots \ldots \ldots$ & Humboldt Bay & 176 & 13.4 & 17.2 & 2.43 \\
\hline XHB06A ............ & Humboldt Bay & 126 & 8.8 & 9.0 & 2.40 \\
\hline
\end{tabular}

See footnotes at end of table. 
Table B7. Assembly Type Summary by Assembly Type (Continued)

\begin{tabular}{|c|c|c|c|c|c|}
\hline Assembly Type Code & Reactor Name & $\begin{array}{l}\text { Number of } \\
\text { Assemblies }\end{array}$ & $\begin{array}{l}\text { Initial Uranium } \\
\text { Content } \\
\text { (MTU) }\end{array}$ & $\begin{array}{c}\text { Average } \\
\text { Burnup } \\
\text { (GWDt/MTU) }\end{array}$ & $\begin{array}{c}\text { Average } \\
\text { Enrichment } \\
\text { (weight percent) }\end{array}$ \\
\hline XHN15W $\ldots \ldots \ldots \ldots \ldots$ & Haddam Neck & 309 & 128.4 & 27.9 & 3.58 \\
\hline XHN15MS $\ldots \ldots \ldots \ldots \ldots$ & Haddam Neck & 2 & 0.8 & 28.3 & 3.66 \\
\hline$X H N 15 M Z \ldots \ldots \ldots \ldots$ & Haddam Neck & 2 & 0.7 & 25.6 & 2.95 \\
\hline XHN15HS $\ldots \ldots \ldots \ldots \ldots$ & Haddam Neck & 1 & 0.4 & 32.2 & 3.99 \\
\hline$X H N 15 H Z \ldots \ldots \ldots \ldots$ & Haddam Neck & 2 & 0.7 & 18.5 & 3.26 \\
\hline XHN15B ............. & Haddam Neck & 576 & 237.1 & 33.7 & 3.99 \\
\hline XIP14W $\ldots \ldots \ldots \ldots \ldots$ & Indian Point 1 & 160 & 30.6 & 16.7 & 4.11 \\
\hline XLC10L $\ldots \ldots \ldots \ldots \ldots$ & LaCrosse & 155 & 18.6 & 14.5 & 3.77 \\
\hline XLC10A $\ldots \ldots \ldots \ldots \ldots$ & LaCrosse & 178 & 19.3 & 14.9 & 3.69 \\
\hline XPA15C $\ldots \ldots \ldots \ldots \ldots$ & Palisades & 273 & 112.6 & 16.0 & 2.47 \\
\hline XPA15A $\ldots \ldots \ldots \ldots \ldots$ & Palisades & 520 & 203.9 & 30.8 & 2.95 \\
\hline$x S L 16 C \ldots \ldots \ldots \ldots$ & St. Lucie 2 & 464 & 175.7 & 32.1 & 2.93 \\
\hline xs014W $\ldots \ldots \ldots \ldots \ldots$ & San Onofre 1 & 665 & 244.3 & 27.3 & 3.86 \\
\hline XYA18W ............... & Yankee Rowe & 76 & 20.8 & 25.5 & 4.94 \\
\hline XYR16U $\ldots \ldots \ldots \ldots \ldots$ & Yankee Rowe & 73 & 17.4 & 27.5 & 3.83 \\
\hline XYR16A $\ldots \ldots \ldots \ldots \ldots$ & Yankee Rowe & 228 & 53.2 & 29.0 & 3.70 \\
\hline XYR16C $\ldots \ldots \ldots \ldots \ldots$ & Yankee Rowe & 156 & 35.8 & 24.3 & 3.83 \\
\hline Total ................ & & b98,400 & $28,136.0$ & & \\
\hline
\end{tabular}

"No burnup reported for temporarily discharged assemblies. See Technical Note 10 in Appendix E.

'Includes 4 temporarily discharged boiling-water reactor (BWR) assemblies and 104 temporarily discharged pressurized-water reactor (PWR) assemblies with no assigned assembly type. See Technical Note 10 in Appendix E.

GWDt/MTU = Gigawattdays thermal per metric ton of uranium.

Note: Totals may not equal sum of components because of independent rounding. See Technical Note 11 in Appendix E.

Source: Energy Information Administration, Form RW-859, "Nuclear Fuel Data" (1993). 
Table B8. Spent Fuel Assemblies, by Type, Discharged by Year

\begin{tabular}{|c|c|c|c|c|c|c|c|c|c|c|c|c|c|c|c|}
\hline $\begin{array}{l}\text { Assembly } \\
\text { Type } \\
\text { Code }\end{array}$ & $\begin{array}{c}1968- \\
1969\end{array}$ & $\begin{array}{l}1970- \\
1971\end{array}$ & $\begin{array}{c}1972- \\
1973\end{array}$ & $\begin{array}{l}1974- \\
1975\end{array}$ & $\begin{array}{l}1976- \\
1977\end{array}$ & $\begin{array}{l}1978- \\
1979\end{array}$ & $\begin{array}{l}1980- \\
1981\end{array}$ & $\begin{array}{l}1982- \\
1983\end{array}$ & $\begin{array}{l}1984- \\
1985\end{array}$ & $\begin{array}{l}1986- \\
1987\end{array}$ & $\begin{array}{l}1988- \\
1989\end{array}$ & $\begin{array}{l}1990- \\
1991\end{array}$ & $\begin{array}{l}1992- \\
1993^{b}\end{array}$ & Temps $^{c}$ & Total \\
\hline \multicolumn{16}{|c|}{ Boiling-Water Reactors (BWR) } \\
\hline G2307G2A & - & 41 & 447 & 917 & 174 & 93 & -. & -- & - & -- & -- & -- & -- & -- & 1,672 \\
\hline G2307G2B & -- & 244 & 593 & 1,045 & 1,765 & 993 & 407 & -- & -- & - & -- & -- & -- & $\cdots$ & 5,047 \\
\hline G2307G3 & - & - & - & 2 & 52 & 191 & 119 & 30 & -- & -- & $\cdots$ & -- & -- & $\cdots$ & 394 \\
\hline G2308G4 & - & - & - & -- & -- & 102 & 1,268 & 1,636 & 730 & 140 & - & -. & -- & -- & 3,876 \\
\hline G2308G5 & -- & - & - & -- & -- & -. & -- & 2 & 518 & 272 & -- & 87 & -- & -- & 879 \\
\hline G2308GP & -. & -- & - & -- & -- & -- & -. & -- & 300 & 1,184 & 544 & 348 & 456 & -. & 2,832 \\
\hline G2308GB & $\cdots$ & - & - & -- & -- & -. & -- & -- & -- & 164 & 348 & 288 & 480 & -. & 1,280 \\
\hline G2308G8A & - & -- & -- & - & -. & - & - & - & - & -. & -- & 212 & 100 & -- & 312 \\
\hline G2308G8B & $\cdots$ & -- & -- & -- & - & -. & -- & -- & - & -. & -. & -- & 28 & - & 28 \\
\hline G2307A & - & - & - & - & 4 & 124 & 108 & 24 & - & -- & -- & -. & -- & -- & 260 \\
\hline G2308A & -- & - & -- & -- & -- & -- & 45 & 183 & -- & 184 & 408 & 393 & 196 & -- & 1,409 \\
\hline G2309A & -- & - & -- & -- & -- & -. & -- & - & - & -. & -- & 4 & 24 & -- & 28 \\
\hline G4607G2 & -- & - & 50 & 328 & 589 & 175 & -- & -- & -- & -- & -- & -- & -. & -- & 1,142 \\
\hline G4607G3A & -- & -- & - & 2 & 501 & 1,037 & 1,723 & 349 & 140 & .- & -- & -- & -- & - & 3,752 \\
\hline G4607G3B & - & -- & - & - & 192 & 747 & 209 & 36 & -- & -. & -- & -- & -. & -- & 1,184 \\
\hline G4608G4A & - & -. & - & - & 112 & 250 & 647 & 571 & 204 & -. & -- & -- & 1 & - & 1,785 \\
\hline G4608G4B & -- & - & -- &.- & -- & 140 & 1,100 & 353 & 194 & -- & -- & -- & -- & -- & 1.787 \\
\hline G4608G5 & - & - & -- & - & -- & -- & 111 & 1,170 & 1,426 & 312 & 987 & 216 & 158 & -- & 4,380 \\
\hline G4608GP & -- & -- & $\cdots$ & -- & -- & -. & 26 & 199 & 2,086 & 3,508 & 2,500 & 1,749 & 1,066 & 386 & 11,520 \\
\hline G4608GB & -- & -- & -- & -- & -- & -. & -. & -- & 1 & 233 & 1,738 & 2,436 & 3,135 & 1 & 7,544 \\
\hline G4608G8 & - & - & -- & -- & -. & .. & - & - & - & -. & -. & 104 & 748 & -- & 852 \\
\hline G4608G9 & - & -- & - & -- & - & -- & - & - & -. & -- & $-\cdot$ & 1 & 21 & - & 22 \\
\hline G4608G10 & - & -- & - & - & - & -- & -- & -- & -- & -- & -- & -- & 4 & -- & 4 \\
\hline G4609G11 & -. & -- & -- & -. & - &.- & -- & -- & -- & -. & -- & -- & -. & 40 & 40 \\
\hline G4608A & -- & -. & .- & -- &.- & .. & -. & -. & -. & -. & 220 & 632 & 484 & .- & 1,336 \\
\hline G4608AP & - & -. & -. & -. & -. & .. & -. & -. & -. & .. & .. & .. & 248 & -. & 248 \\
\hline G4609A & -- & -- & -- & - & -- & -. & -- & -- & -- & -- & 1 & 231 & 628 & -- & 860 \\
\hline G4609A5 & -- & -- & -- & -- & -- & -- & -- & -- & -- & -. & -. & -. & 36 & -- & 36 \\
\hline G4609AIX & -- & -- & -. & -- & -- & -- & -- & - & -- & -. & -. & -. & 4 & -- & 4 \\
\hline G4608W & - & -- & -- & -- & .. & -- & -- & - & -- & -- & -- & 4 & - & -- & 4 \\
\hline XBR11G & -- & -- & 6 & -- &.- & -. & -- & - & - & -. & -. & .. & -. & - & 6 \\
\hline XBR07G & 4 & -- & -. & -- & -. & .- & -- & .. & $\ldots$ & 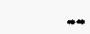 & .. & .. & .. & .. & 4 \\
\hline XBROBG & 2 & -- & - & -- & -. & -- & - & - & -. & -- & -. & -. & -. & -- & 2 \\
\hline XBR09G & - & 5 & 55 & 29 & 32 & 18 & 4 & -- & -- & -- & -. & -- & -. & -- & 143 \\
\hline XBR09A & -- & -- & -. & -- & 4 & -- & -- & -. & -. & $-\infty$ & -. & -- & -- & -- & 4 \\
\hline XBR11A & -. & -. & -- & -. & .. & 8 & 18 & 44 & 36 & 24 & 42 & 40 & 22 & -- & 234 \\
\hline XBR11N & -- & -- & -- & 2 & 6 & -. & -- & -- & -- & -- & -- & -- & -- & -- & 8 \\
\hline XDR06G & 1 & -- & - & -- & -- & -- & -- & -- & -. & -- & -- & -- & -. & - & 1 \\
\hline XDR06G3B & 58 & 69 & 5 & 22 & 9 & -- & -- & -. & .. & -. & $\cdots$ & -. & -. & - & 163 \\
\hline XDR06G3F & 24 & 20 & 3 & 36 & 13 & -- & -- & -. & -- & -. & -- & -. & .- & - & 96 \\
\hline XDR06G5 & 9 & 15 & 11 & 2 & 15 & 54 & -. & -- & -. & -. & -- & -- & -. & -- & 106 \\
\hline XDR07GS & 1 & -- & -- & -- & -. & -- & -- & -- & .- & -. & -. & -. & -. & - & 1 \\
\hline XDR08G & 1 & -- & -- & -- & -- & -- & -- & -- & -- & -. & -. & -- & -- & - & 1 \\
\hline XDR06U & 1 & 8 & 33 & 43 & 29 & 344 & .. & .. & -- & -- & -- & -- & -- & -- & 458 \\
\hline XDROGA & 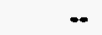 &.- & -- & -- & -. & 66 & -- & -- & -- & -- & -- & -- & -. & -- & 66 \\
\hline XHB07G2 & - & 33 & 55 & -. & .. & .. & $\ldots$ & -- & -- & -- & -- & -- & -. & -- & 88 \\
\hline XHB06G & -- & 7 & 51 & 56 & 62 & -- & - & -- & -. & -- & -- & -- & -- & -- & 176 \\
\hline XHB06A & .. & -- & -- & 4 & 122 & .. & .. & -- & .- & -- & -- & -- & -- & -- & 126 \\
\hline XLC1OL & -- & -. & 56 & 25 & 32 & 28 & 12 & 2 & -. & -. & -- & -. & -. & -. & 155 \\
\hline XLC10A & $\cdots$ & -- & -- & -- & -- & -. & -- & 50 & 28 & 100 & -. & -- & .. & - & 178 \\
\hline Total BWR & 101 & 442 & 1,365 & 2,513 & 3,713 & 4,370 & 5,797 & 4,649 & 5,663 & 6,121 & 6,788 & 6,745 & 7,839 & 431 & ${ }^{d} 56,537$ \\
\hline
\end{tabular}

See footnotes at end of table. 
Table B8. Spent Fuel Assemblies, by Type, Discharged by Year (Continued)

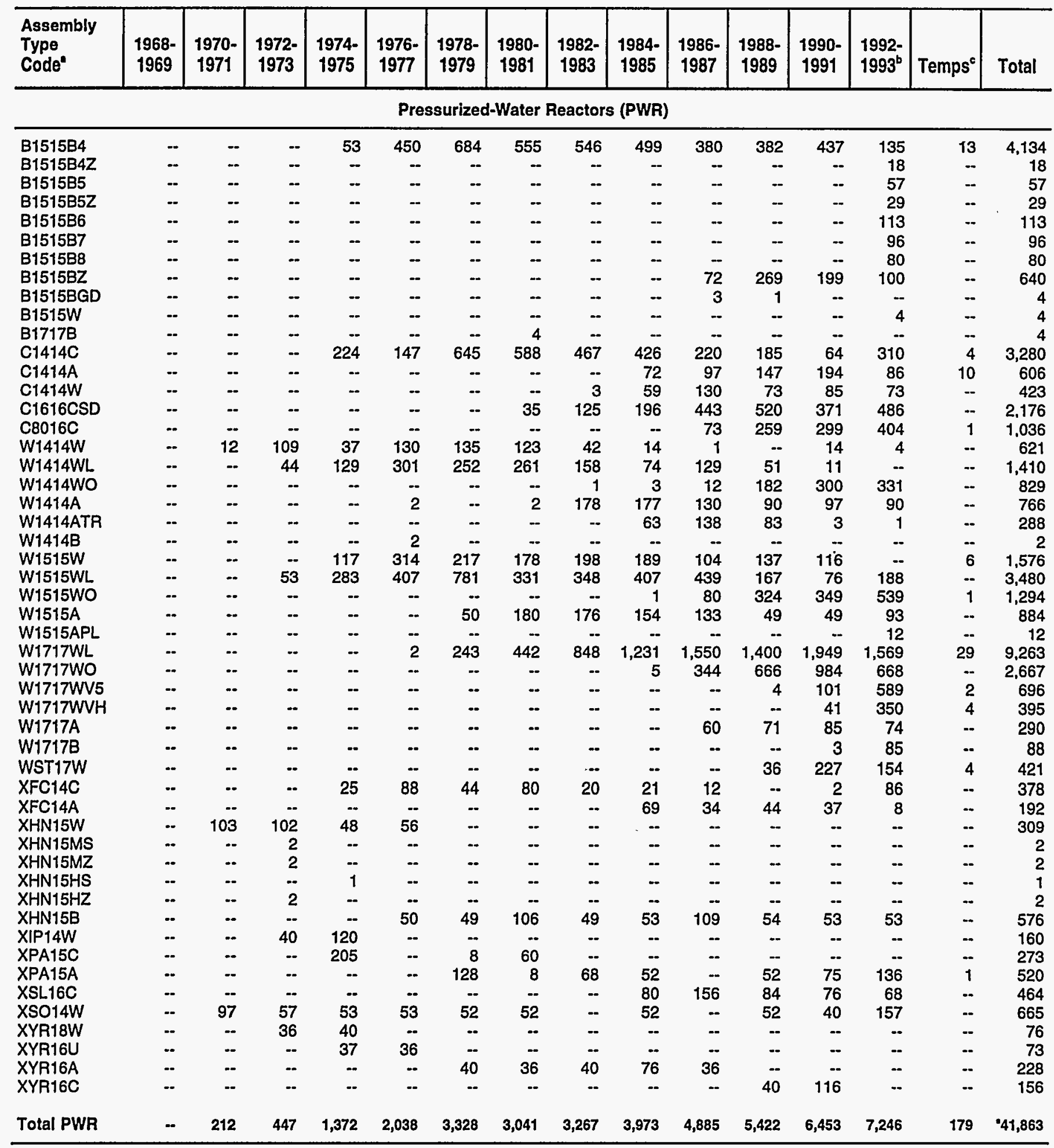

Assembly types are defined in Table B4.

'Some data for previous years have been revised. Current-year data may be revised in future publications. When utilities reinsert assemblies discharged in previous years, historical totals change. See Technical Note 12 in Appendix $E$.

'Temps are temporarily discharged assemblies, as of December 31, 1993, as reported on Form RW-859.

'No assigned assembly type for 4 temporarily discharged BWR assemblies. These BWR assemblies are included in the column totals. See Technical Note 10 in Appendix $E$.

'No assigned assembly type for 104 temporarily discharged PWR assemblies. These PWR assemblies are included in the column totals. See Technical Note 10 in Appendix E.

-. = Not applicable.

Source: Energy Information Administration, Form RW-859, "Nuclear Fuel Data" (1993). 
Table B9. Initial Uranium Content of Spent Fuel Assemblies, by Type, Discharged by Year (Metric tons of uranium)

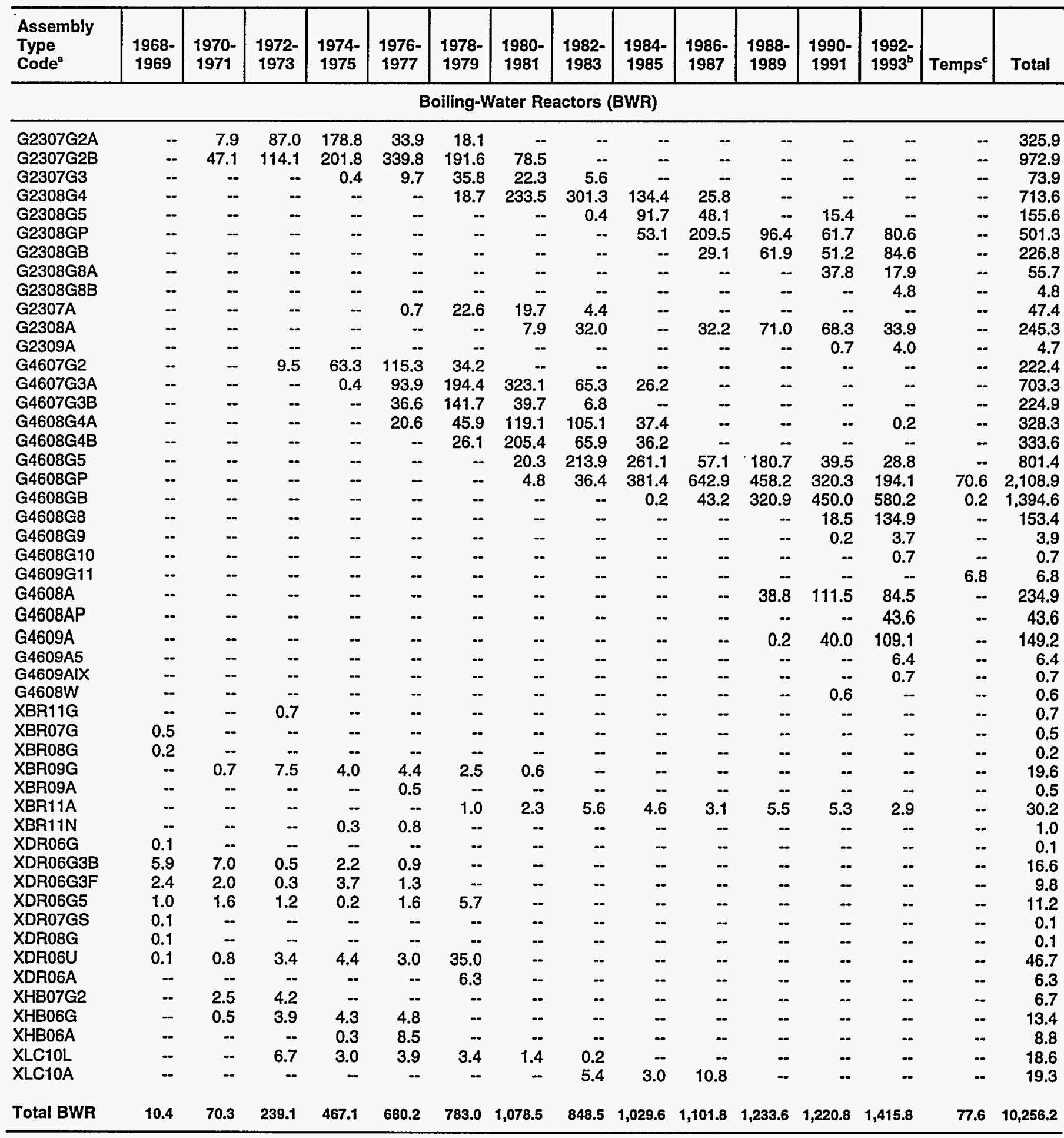

See footnotes at end of table. 
Table B9. Initial Uranium Content of Spent Fuel Assemblies, by Type, Discharged by Year (Continued) (Metric tons of uranium)

\begin{tabular}{|c|c|c|c|c|c|c|c|c|c|c|c|c|c|c|c|}
\hline $\begin{array}{l}\text { Assembly } \\
\text { Type } \\
\text { Code }\end{array}$ & $\begin{array}{c}1968- \\
1969\end{array}$ & $\begin{array}{l}1970- \\
1971\end{array}$ & $\begin{array}{l}1972- \\
1973\end{array}$ & $\begin{array}{c}1974- \\
1975\end{array}$ & $\begin{array}{l}1976- \\
1977\end{array}$ & $\begin{array}{l}1978- \\
1979\end{array}$ & $\begin{array}{l}1980- \\
1981\end{array}$ & $\begin{array}{l}1982- \\
1983\end{array}$ & $\begin{array}{c}1984- \\
1985\end{array}$ & $\begin{array}{c}1986- \\
1987\end{array}$ & $\begin{array}{l}1988- \\
1989\end{array}$ & $\begin{array}{l}1990- \\
1991\end{array}$ & $\begin{array}{l}1992- \\
1993^{\mathrm{b}}\end{array}$ & Temps $^{c}$ & Total \\
\hline \multicolumn{16}{|c|}{ Pressurized-Water Reactors (PWR) } \\
\hline B1515B4 & -. & .- & -- & 24.8 & 209.2 & 317.0 & 257.2 & 254.8 & 231.7 & 176.0 & 177.4 & 203.3 & 62.5 & 2.3 & $1,916.2$ \\
\hline B1515B4Z & -. & $\therefore$ & -. & -. & -. & - & .. & .. & -. & -- & - & - & 8.3 & -. & 8.3 \\
\hline B1515B5 & -- & -- & -- & -. & -- & -- & -- & -- & -- & -- & - & -- & 26.7 & -- & 26.7 \\
\hline B1515B5Z & .. & .. & .. & .. & .- & .- & .. & .. & -- & -- & -- & -- & 13.5 & .- & 13.5 \\
\hline B1515B6 & .. & .. & .. & .. & -. & -. & .- & .- & .- & -- & -- & .. & 52.2 & .- & 52.2 \\
\hline B1515B7 & -- & -. & .. & .- & -- & - & -- & -. & - & - & - & -- & 44.0 & -- & 44.0 \\
\hline B1515B8 & -. & -. & -. & -. & -- & -- & -- & -. & -. & - & -- & -- & 37.2 & -- & 37.2 \\
\hline B1515BZ & -- & -- & .. & -. & -. & - & -. & -- & - & 33.4 & 124.6 & 92.3 & 46.4 & .- & 296.6 \\
\hline B1515BGD & -- & .. & -- & -- & -- & -- & - & -- & .- & 1.3 & 0.4 & - & - & -- & 1.7 \\
\hline B1515W & -. & -. & -. & -- & -- & -- & -- & .- & -- & - & -- & - & 1.8 & .- & 1.8 \\
\hline B1717B & -. & -- & -- & -- & -- & -- & 1.8 & -. & -- & -. & -- & - & .- & -. & 1.8 \\
\hline C1414C & .. & .- & .- & 84.2 & 57.6 & 245.1 & 224.3 & 179.6 & 162.1 & 84.4 & 71.9 & 24.0 & 118.4 & 0.0 & $1,251.5$ \\
\hline C1414A & .- & -. & -- & -. & - & - & -. & .. & 27.0 & 35.0 & 54.3 & 72.5 & 31.9 & 3.7 & 224.4 \\
\hline C1414W & -- & -. & -- & -- & -- & -- & -- & 1.2 & 24.0 & 52.8 & 29.8 & 34.7 & 29.8 & -. & 172.3 \\
\hline C1616CSD & -- & -. & .- & .- & -- & -- & 14.9 & 51.3 & 83.7 & 182.8 & 216.1 & 152.8 & 197.6 & -. & 899.2 \\
\hline C8016C & .. & -- & - & -- & - & - & - & - & -- & 31.1 & 107.0 & 120.5 & 165.2 & 0.4 & 424.2 \\
\hline W1414W & -- & 4.6 & 42.7 & 14.4 & 51.1 & 53.0 & 48.9 & 16.8 & 5.6 & 0.4 & - & 5.6 & 1.6 & -- & 244.7 \\
\hline W1414WL & .- & .. & 17.5 & 50.5 & 119.4 & 100.6 & 104.8 & 63.5 & 29.7 & 51.8 & 20.5 & 4.4 & .- & .- & 562.7 \\
\hline W1414WO & -. & -- & .. & -. & -- & - & -. & 0.4 & 1.1 & 4.3 & 65.1 & 107.5 & 118.3 & -. & 296.6 \\
\hline W1414A & .. & -. & -- & -- & 0.7 & - & 0.7 & 67.3 & 66.7 & 48.8 & 33.9 & 36.4 & 34.1 & -- & 288.7 \\
\hline W1414ATR & -- & .- & .. & -- & -- & -- & .. & -. & 23.0 & 50.3 & 29.7 & 1.1 & 0.4 & -- & 104.5 \\
\hline W1414 & -- & -- & -. & -- & 0.8 & - & -- & -. & - & & & & - & -- & 0.8 \\
\hline & -- & .. & -. & 53.0 & 141.2 & 98.3 & 81.1 & 90.0 & 86.0 & 47.6 & 62.8 & 53.3 & -- & 2.7 & 716.0 \\
\hline W1515WL & -- & -- & 24.2 & 126.8 & 183.9 & 354.2 & 151.3 & 159.0 & 186.0 & 200.6 & 76.5 & 35.0 & 86.6 & $\ldots$ & $1,584.0$ \\
\hline W1515WO & -- & -- & -. & -- & -- & - & -. & -. & 0.5 & 36.8 & 149.0 & 160.6 & 247.8 & 0.5 & 595.2 \\
\hline W1515A & .. & .. & -. & -. & -- & 21.4 & 77.1 & 75.3 & 66.0 & 57.2 & 21.1 & 21.2 & 39.8 & .. & 379.1 \\
\hline W1515APL & .- & .- & .. & .- & -- & 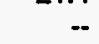 & - & - & .. & -- & 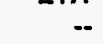 & 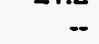 & 3.7 & -- & 3.7 \\
\hline W1717WL & -. & -. & -. & -- & 0.9 & 111.7 & 203.2 & 389.3 & 564.9 & 713.5 & 646.4 & 900.7 & 725.2 & 13.3 & 4.269 .2 \\
\hline W1717WO & .- & .- & -- & -- & -- & -- & -. & -. & 2.1 & 145.9 & 283.3 & 417.5 & 283.8 & .- & $1,132.6$ \\
\hline W1717WV5 & .- & -. & .- & .- & -- & -. & - & -- & -. & -- & 1.7 & 42.9 & 250.2 & 0.9 & 295.7 \\
\hline W1717WVH & .- & .. & .- & .- & .- & -- & -- & -- & -- & - & - & 19.1 & 162.4 & 1.9 & 183.3 \\
\hline W1717A & .. & .- & -. & -- & -- & -- & .. & .- & .. & 24.1 & 28.5 & 34.2 & 29.8 & -- & 116.6 \\
\hline W1717B & .- & -- & .. & .- & -- & -- & -. & -- & .- & - & - & 1.3 & 38.5 & -- & 39.8 \\
\hline WST17W & -. & -. & -. & -- & -- & -. & .- & -- & -- & -- & 19.6 & 122.7 & 83.3 & 2.2 & 227.8 \\
\hline $\mathrm{XFC} 14 \mathrm{C}$ & -. & -- & -- & 9.3 & 31.7 & 16.4 & 29.3 & 7.3 & 7.6 & 4.4 & - & 0.7 & 30.5 & - & 137.2 \\
\hline XFC $14 A$ & .- & -- & .- & -. & -. & - & -. & -- & 24.7 & 12.1 & 15.2 & 13.0 & 2.9 & -- & 67.8 \\
\hline XHN15W & -. & 43.3 & 42.3 & 19.8 & 23.0 & -- & -. & .. & -. & -- & -- & -. & - & .- & 128.4 \\
\hline XHN15MS & -. & -. & 0.8 & -- & -- & -- & .. & -. & -- & -- & -- & - & -- & -. & 0.8 \\
\hline & -- & -- & 0.7 & -- & -- & -- & -- & .- & -. & -- & - & -- & - & -- & 0.7 \\
\hline XHN15HS & -- & .. & .. & 0.4 & -- & -- & -- & -. & -. & -- & -- & -- & - & -- & 0.4 \\
\hline XHN15HZ & -- & .. & 0.7 & .. & - & -- & -- & -- & -- & - & -. & -- & - & -. & 0.7 \\
\hline XHN15B & .- & -. & -- & -- & 20.6 & 20.2 & 43.6 & 20.2 & 21.8 & 44.9 & 22.1 & 21.8 & 21.9 & .- & 237.1 \\
\hline XIP14W & -. & .. & 7.8 & 22.8 & -. & -- & .. & .- & -- & -- & -- & -- & .- & -- & 30.6 \\
\hline XPA15C & -. & .. & -- & 84.3 & - & 3.3 & 24.9 & . & -- & -- & - & & & & 112.6 \\
\hline & .- & -. & -- & - & -- & 50.5 & 3.1 & 26.3 & 20.2 & - & 20.4 & 29.3 & 53.7 & 0.4 & 203.9 \\
\hline & -- & & -- & -- & -- & & & -. & 31.0 & 57.9 & 31.8 & 29.3 & 25.8 & -- & 175.7 \\
\hline XSO14W & -- & 35.6 & 20.4 & 19.3 & 19.2 & 19.2 & 19.2 & -- & 19.3 & -. & 19.2 & 14.8 & 58.1 & -- & 244.3 \\
\hline XYR18W & -. & .. & 9.8 & 10.9 & - & - & -- & -- & -- & -- & - & - & -- & -- & 20.8 \\
\hline XYR16U & -. & -- & -- & 8.8 & 8.6 & -- & -- & -- & -- & -- & -- & -. & -- & -- & 17.4 \\
\hline XYR16A & .. & .. & .- & -- & - & 9.4 & 8.5 & 9.4 & 17.7 & 8.3 & -- & .. & -- & .- & 53.2 \\
\hline XYR16C & -- & - & -. & -- & - & -. & -. & .. & -. & - & 9.2 & 26.6 & -- & -- & 35.8 \\
\hline Total PWR & -. & 83.5 & 167.0 & 529.4 & 867.9 & $1,420.3$ & $1,294.0$ & $1,411.7$ & $1,702.2$ & $2,105.4$ & $2,337.5$ & $2,799.2$ & $3,133.6$ & 28.2 & $17,879.9$ \\
\hline
\end{tabular}

Assembly types are identified in Table B4.

bome data for previous years have been revised. Current-year data may be revised in future publications. When utilities reinsert assemblies discharged in previous years, historical totals change. See Technical Note 12 in Appendix E.

Temps are temporarily discharged assemblies, as of December 31, 1993, as reported on Form RW-859.

-. = Not applicable.

Note: Tolals may not equal sum of components because of independent rounding. See Technical Note 11 in Appendix E.

Source: Energy Information Administration, Form RW-859, "Nuclear Fuel Data" (1993). 
Table B10. Burnup of Spent Fuel Assemblies, by Type, Discharged by Year

(Gigawattdays thermal per metric ton of uranium)

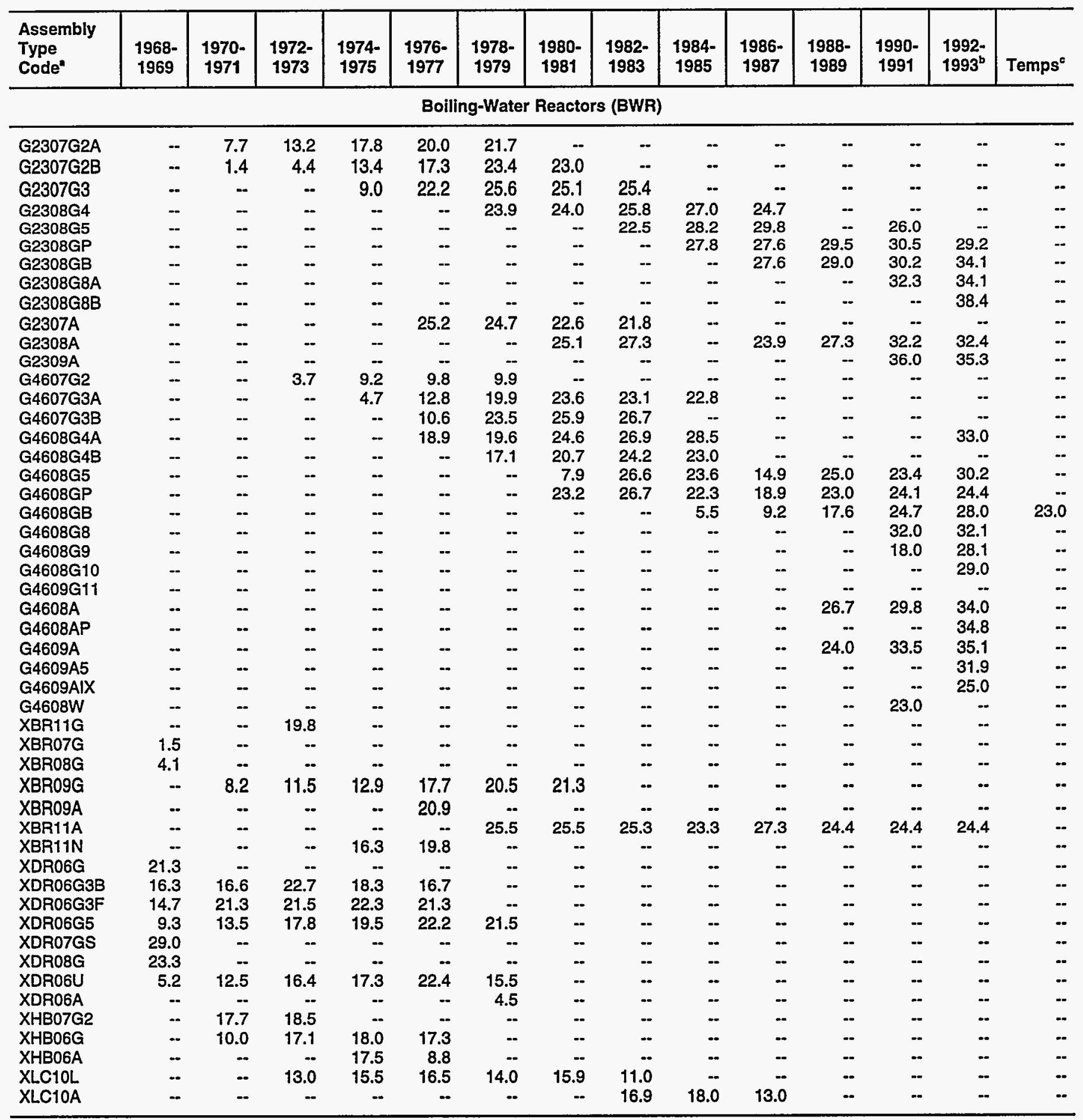

See footnotes at end of table. 
Table B10. Burnup of Spent Fuel Assemblies, by Type, Discharged by Year (Continued) (Gigawattdays thermal per metric ton of uranium)

\begin{tabular}{|c|c|c|c|c|c|c|c|c|c|c|c|c|c|c|}
\hline $\begin{array}{l}\text { Assembly } \\
\text { Type } \\
\text { Code" }\end{array}$ & $\begin{array}{l}1968- \\
1969\end{array}$ & $\begin{array}{l}1970- \\
1971\end{array}$ & $\begin{array}{l}1972- \\
1973\end{array}$ & $\begin{array}{l}1974- \\
1975\end{array}$ & $\begin{array}{c}1976- \\
1977\end{array}$ & $\begin{array}{l}1978- \\
1979\end{array}$ & $\begin{array}{l}1980- \\
1981\end{array}$ & $\begin{array}{l}1982- \\
1983\end{array}$ & $\begin{array}{l}1984- \\
1985\end{array}$ & $\begin{array}{l}1986- \\
1987\end{array}$ & $\begin{array}{l}1988- \\
1989\end{array}$ & $\begin{array}{l}1990- \\
1991\end{array}$ & $\begin{array}{l}1992- \\
1993^{\circ}\end{array}$ & Temps $^{c}$ \\
\hline \multicolumn{15}{|c|}{ Pressurized-Water Reactors (PWR) } \\
\hline B151584 & -- & -- & - & 11.6 & 21.1 & 25.7 & 27.7 & 28.3 & 31.0 & 32.2 & 31.7 & 32.9 & 35.6 & 20.8 \\
\hline B1515B4Z & -- & -- & -- & -. & - & - & - & -. & -- & - & .. & -- & 38.1 & -. \\
\hline $\mathrm{B} 1515 \mathrm{~B} 5$ & $\cdots$ & - & - & $\cdots$ & - & -- & - & -- & - & - & -- & -- & 38.0 & .. \\
\hline B1515B5Z & -- & -- & -. & -- & -- & -- & - & -- & -- & -- & .- & -- & 36.0 & .- \\
\hline $\mathrm{B} 1515 \mathrm{~B} 6$ & -- & -- & $\cdots$ & -. & - & $\cdots$ & -- & -- & -- & - & -. & -. & 41.5 & -- \\
\hline B1515B7 & -- & -- & - & -- & -- & -- & -- & -- & - & - & -- & -- & 41.7 & -. \\
\hline B1515B8 & .. & -- & - & .- & - & -. & -- & $\cdots$ & -- & -. & -. & -- & 41.6 & -- \\
\hline $\mathrm{B} 1515 \mathrm{BZ}$ & -. & -- & - & -- & -- & -. & -. & -. & -- & 34.9 & 28.9 & 38.9 & 38.1 & .. \\
\hline B1515BGD & .. & .. & -. & .. & -- & -- & - & -- & -- & 38.0 & 58.0 & .. & .. & .. \\
\hline $\mathrm{B} 1515 \mathrm{~W}$ & -- & -. & - & -- & - & - & - & -. & -- & -. & -- & - & 25.0 & - \\
\hline B1717B & -. & -- & - & -- & -- & -- & 29.5 & - & -- & -- & -. & -- & -. & -- \\
\hline C1414C & -. & -. & $\cdots$ & 12.9 & 17.4 & 22.8 & 30.8 & 32.2 & 34.3 & 37.6 & 39.2 & 40.8 & 42.1 & -. \\
\hline C1414A & -. & - & - & -- & -. & -. & -- & -. & 35.7 & 37.0 & 38.6 & 36.6 & 39.9 & 27.9 \\
\hline C1414W & -- & -- & $\cdots$ & -- & -- & $\ldots$ & -- & 22.1 & 33.8 & 33.7 & 32.3 & 31.3 & 33.6 & -. \\
\hline C1616CSD & .. & -- & -- & .- & -. & .- & 13.6 & 26.9 & 21.0 & 25.8 & 33.4 & 39.4 & 41.2 & -- \\
\hline C8016C & .. & -. & -- & -. & -- & -- & -- & - & - & 17.1 & 21.1 & 32.6 & 37.8 & 18.0 \\
\hline W1414W & $\cdots$ & 7.7 & 17.6 & 24.4 & 26.6 & 31.0 & 32.1 & 34.3 & 35.2 & 30.5 & -- & 39.5 & 40.0 & -. \\
\hline W1414WL & -. & -- & 18.4 & 24.3 & 27.2 & 32.9 & 34.3 & 35.5 & 36.2 & 36.4 & 40.0 & 43.0 & -. & $\cdots$ \\
\hline W1414WO & -- & - & -- & -. & -. & -. & -- & 19.4 & 40.7 & 36.0 & 39.6 & 39.7 & 42.7 & -. \\
\hline W1414A & -. & .. & -- & -- & 25.3 & -- & 28.6 & 36.2 & 35.2 & 37.2 & 35.9 & 36.9 & 37.3 & -. \\
\hline W1414ATR & - & -- & - & -- & -. & $=$ & - & -- & 37.6 & 38.0 & 38.5 & 45.0 & 44.0 & -- \\
\hline$W 1414 B$ & .. & -- & -- & -. & 24.4 & -- & -. & -- & - & -- & -- & -- & -- & .. \\
\hline W1515W & .. & -- & -- & 19.3 & 21.2 & 30.7 & 29.0 & 33.1 & 35.2 & 35.5 & 37.2 & 39.2 & -- & 27.3 \\
\hline W1515WL & -- & - & 15.9 & 21.1 & 24.3 & 30.2 & 31.5 & 33.3 & 32.9 & 35.0 & 35.4 & 36.4 & 41.0 & -. \\
\hline W1515WO & -. & -- & -- & .. & -. & -. & -- & .. & 18.3 & 32.1 & 35.9 & 35.6 & 37.3 & .. \\
\hline W1515A & 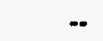 & -- & -- & .- & - & 30.3 & 31.6 & 31.4 & 30.6 & 32.6 & 35.7 & 38.0 & 39.7 & - \\
\hline W1515APL & -- & .. & -- & -- & -- & -. & -- & -. & -. & -- & -. & - & 22.0 & .. \\
\hline W1717WL & & -. & - & -- & 16.1 & 17.0 & 28.0 & 25.2 & 28.4 & 29.6 & 32.7 & 34.6 & 36.4 & 14.8 \\
\hline W1717WO & -- & $\cdots$ & -- & -- & -- & -. & -- & -. & 36.1 & 20.4 & 30.2 & 34.0 & 38.6 & -. \\
\hline W1717WV5 & -- & -- & -. & -. & -- & .- & -. & -- & -- & .. & 46.0 & 38.2 & 39.6 & -- \\
\hline W1717WVH & -- & $\ldots$ & -- & -- & -. & -. & -- & -. & - & -- & -. & 35.3 & 37.1 & -. \\
\hline W1717A & & .. & -- & -- & -- & -. & -- & -- & -- & 37.0 & 38.6 & 38.9 & 42.1 & -. \\
\hline W1717 & & - & -. & - & - & $\cdots$ & -- & -- & 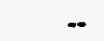 & -- & - & 42.0 & 19.6 & -. \\
\hline WST17W & & .. & -. & -- & -. & -- & -- & .- & -- & -- & 12.0 & 19.4 & 30.5 & -- \\
\hline XFC14C & & - & -- & 8.6 & 25.5 & 24.0 & 30.9 & 36.1 & 39.2 & 39.0 & -- & 31.0 & 39.4 & -- \\
\hline XFC14A & & -- & -- & -. & -. & -. & .. & -- & 34.8 & 36.3 & 38.9 & 41.0 & 38.0 & -. \\
\hline XHN15W & -- & 22.5 & 29.3 & 32.8 & 31.2 & -. & .- & -- & .- & -. & -- & -- & .- & .. \\
\hline XHN15MS & - & -. & 28.3 & -- & .. & - & -- & -- & -- & - & - & -- & -. & $\cdots$ \\
\hline XHN15MZ & -. & - & 25.6 & -- & -- & - & -- & -- & -- & -- & - & - & -- & -. \\
\hline XHN15HS & -. & -- & .. & 32.2 & -- & - & -- & -- & -. & -- & - & - & -- & -. \\
\hline XHN15HZ & -- & -. & 18.5 & -- & -. & -- & -- & -- & -- & -. & -- & - & -- & -- \\
\hline XHN15B & -- & -- & -. & -- & 33.5 & 32.7 & 33.8 & 33.8 & 35.2 & 33.9 & 32.0 & 34.3 & 33.2 & -- \\
\hline XIP14W & -. & -- & 25.2 & 13.8 & -. & -. & -- & -- & -. & -- & -. & -- & -- & -- \\
\hline XPA15C & -- & -- & .. & 11.3 & -- & 26.7 & 30.3 & -. & -. & - & -. & -- & -- & -- \\
\hline XPA15A & -- & -- & -- & -. & -- & 20.2 & 35.3 & 33.3 & 34.8 & -- & 35.0 & 36.7 & 33.2 & 23.5 \\
\hline XSL16C & -- & -. & -. & -- & -. & -- & .. & -- & 12.6 & 29.5 & 39.1 & 39.8 & 43.6 & .. \\
\hline XS014W & $=$ & 21.7 & 29.0 & 28.9 & 31.9 & 31.9 & 30.5 & -- & 29.7 & -- & 34.9 & 30.0 & 21.7 & -- \\
\hline XYR18W & -. & .. & 23.9 & 27.0 & .. & -- & .. & -- & -. & -- & -- & -- & -. & -- \\
\hline XYR16U & -. & -- & -. & 26.9 & 28.2 & -- & -. & -- & -. & -- & - & -- & - & -- \\
\hline XYR16A & $\cdots$ & -. & -. & -. & -. & 27.3 & 29.9 & 29.0 & 28.5 & 31.0 & - & -- & - & -. \\
\hline XYR16C & -- & -. & $\ldots$ & -- & - & -- & -. & -. & - & -- & 32.0 & 21.6 & -- & -. \\
\hline
\end{tabular}

\footnotetext{
"Assembly types are identified in Table B4.

"Some data for previous years have been revised. Current-year data may be revised in future publications. When utilities reinsert assemblies discharged in previous years, historical totals change. See Technical Note 12 in Appendix $E$.

Temps are temporarily discharged assemblies, as of December 31, 1993, as reported on Form RW-859.

-. = Not applicable.

Source: Energy Information Administration, Form RW-859, "Nuclear Fuel Data" (1993).
} 
Appendix C

\section{Pictograms Showing Reactor Storage Pool Configurations, Pool Capacities, and Inventories at Nuclear Power Plant Sites and Storage Facilities}

Spent fuel storage pool at General Electric Company's Morris Operation.

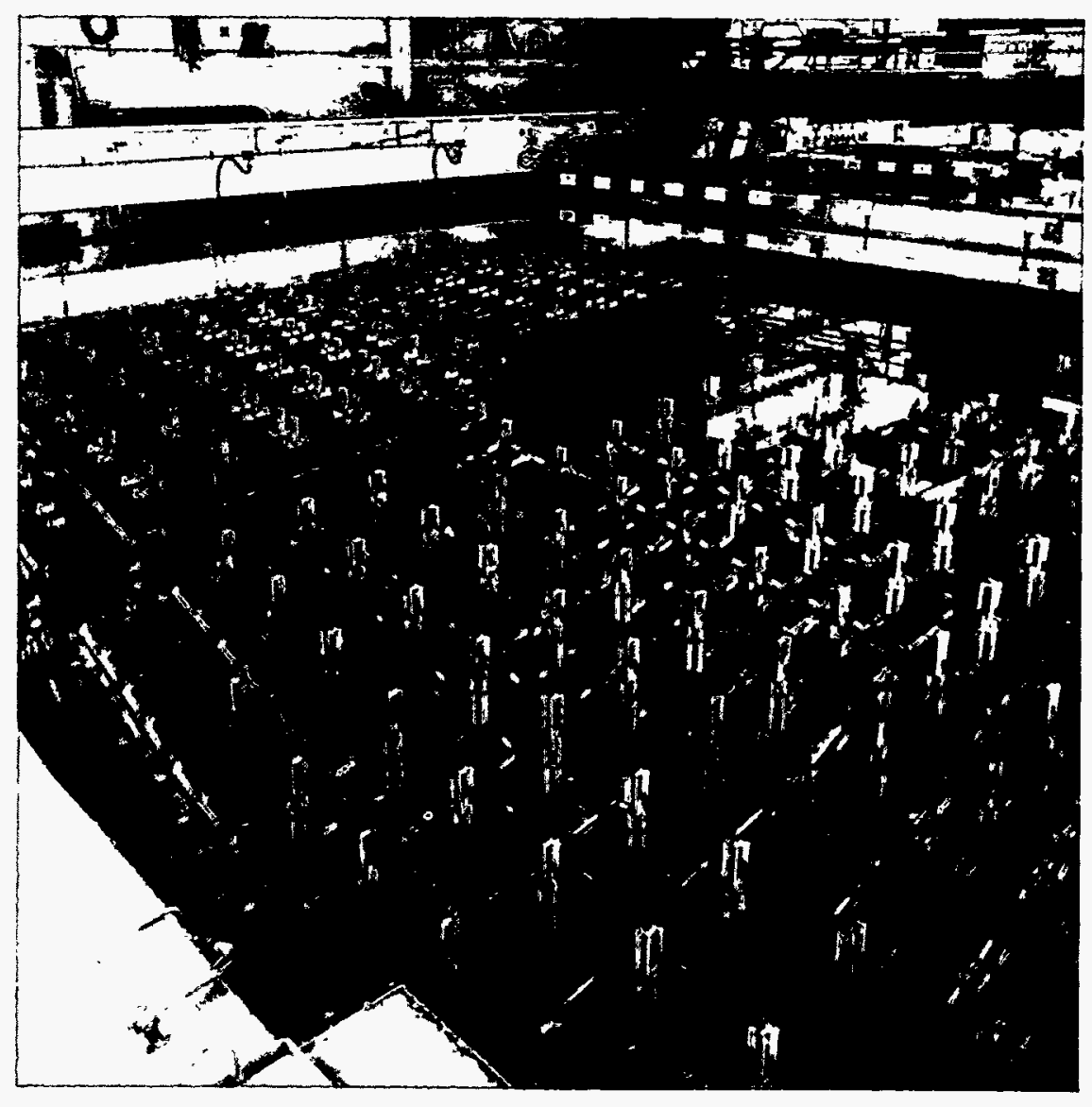




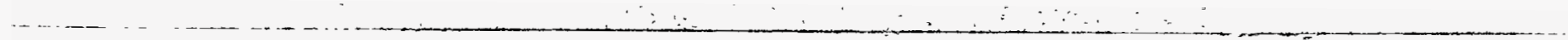




\section{Pictograms Showing Reactor Storage Pool Configurations, Pool Capacities, and Inventories at Nuclear Power Plant Sites and Storage Facilities}

The pool site pictograms contained in this appendix graphically compare the maximum storage capacity of each spent fuel storage pool site with its inventory as of December 31, 1993. (A pool site is a configuration of pools having a single cask loading area.) EIA defines maximum established spent fuel capacity for the site as the number of intact assemblies that will be able to be stored at some point in the future (between the reporting date and the reactor's end of life) considering any established or current studies or engineering evaluations, at the time of submittal for licensing approval from the U.S. Nuclear Regulatory Commission. (See Technical Note 13.)
The pictograms are ordered by utility and highlight the various reactor/pool site configurations, such as the absence or presence of transfer canals, multiple pools servicing single reactors, and reactors sharing one pool site. Multiple storage pools linked by transfer canals have one pool site ID number because they share a common cask loading dock. In the pictograms, temporarily discharged assemblies are included in the present inventory totals. The movement of fuel between the storage pools and the Independent Spent Fuel Storage Installations (ISFSI's) at an individual site is represented by arrows. Two different designs of ISFSI's are also shown. Additional information on the ISFSI's is reported in Chapter 2. 


\section{Legend}

In Appendix $C$ the pictograms show selected data from Tables 13 and 14. Data in the pictograms include Utility and Reactor Names, Pool Site ID Number, Maximum Established Storage Capacity, and Current Inventory.

Each pictogram illustrates reactor/pool site configurations, such as the absence or presence of transfer canals, multiple pools servicing single reactors, and reactors sharing one pool site. Multiple storage pools linked by transfer canals have one pool site ID number. The location of each reactor is identified on a State map that includes the city and county of each reactor site. Two different designs of Independent Spent Fuel Storage Installations (ISFSI's) are depicted. A pie chart indicating the reactor's percentage of storage capacity remaining is shown in each pictogram.

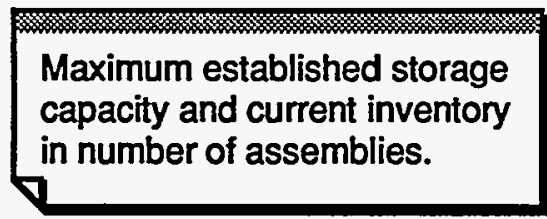

\section{Utility Name Reactor Name POOL SITE ID \#}

The arrow depicts movement of discharged assemblies from a reactor to a pool or storage module, or from one pool to another.
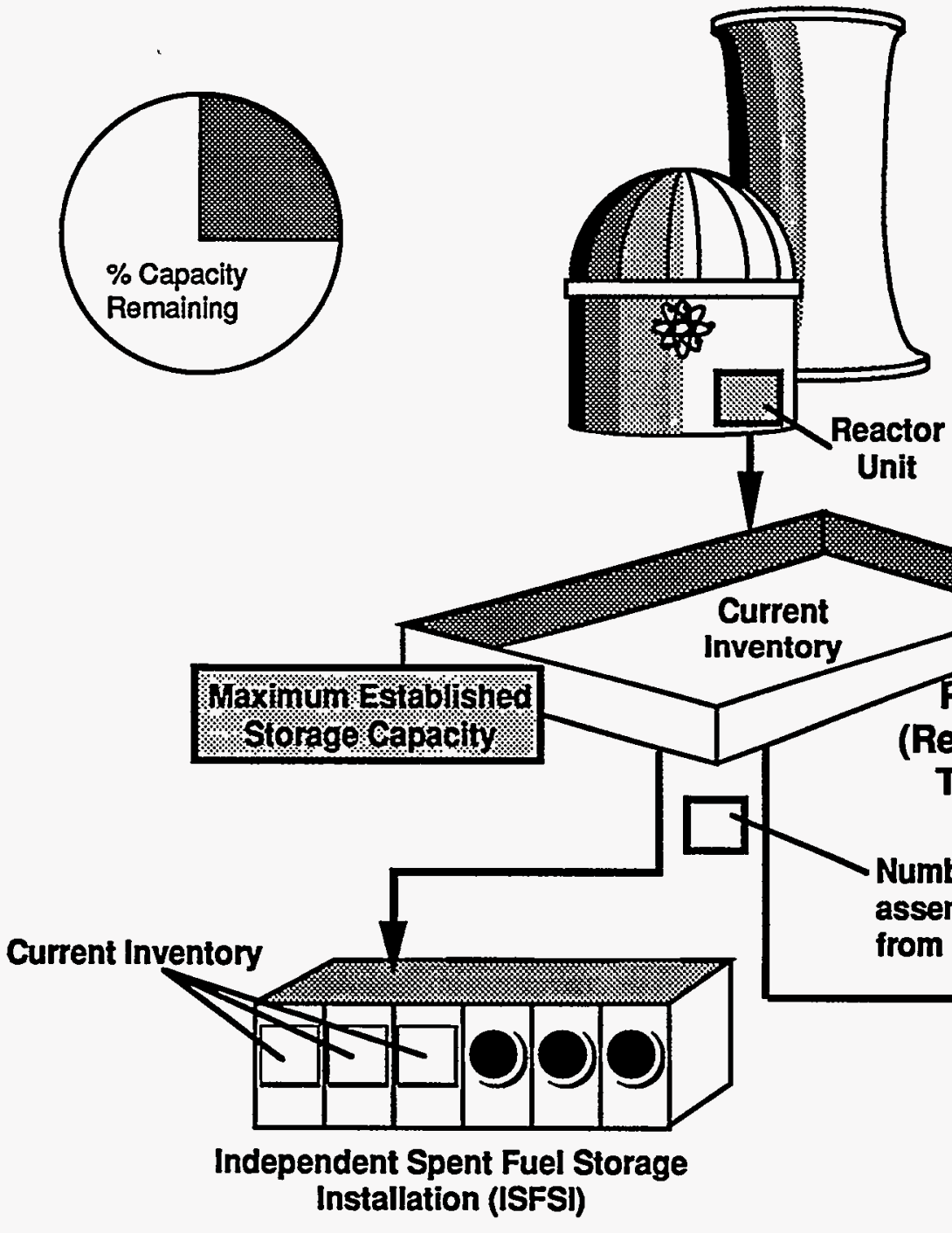

State Map

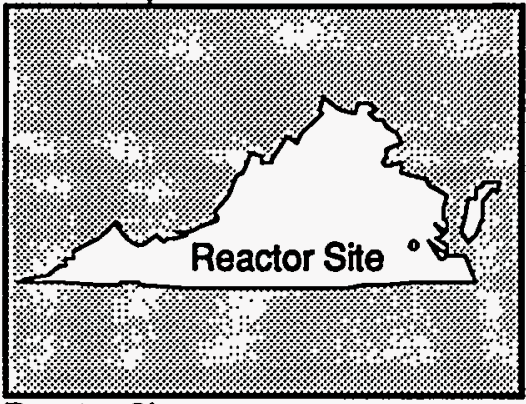

Reactor City

Reactor County

Unit

Transfer Canal

Current
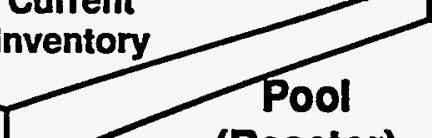

(Reactor)

Type
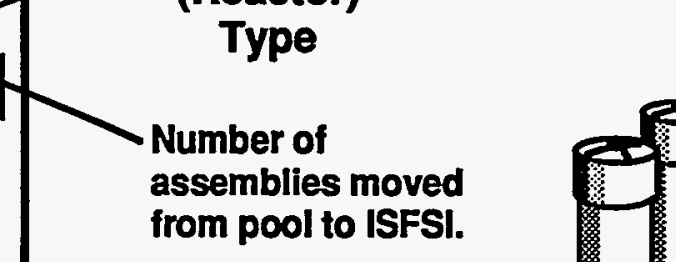

\section{.}




\section{Alabama Power Company Farley}
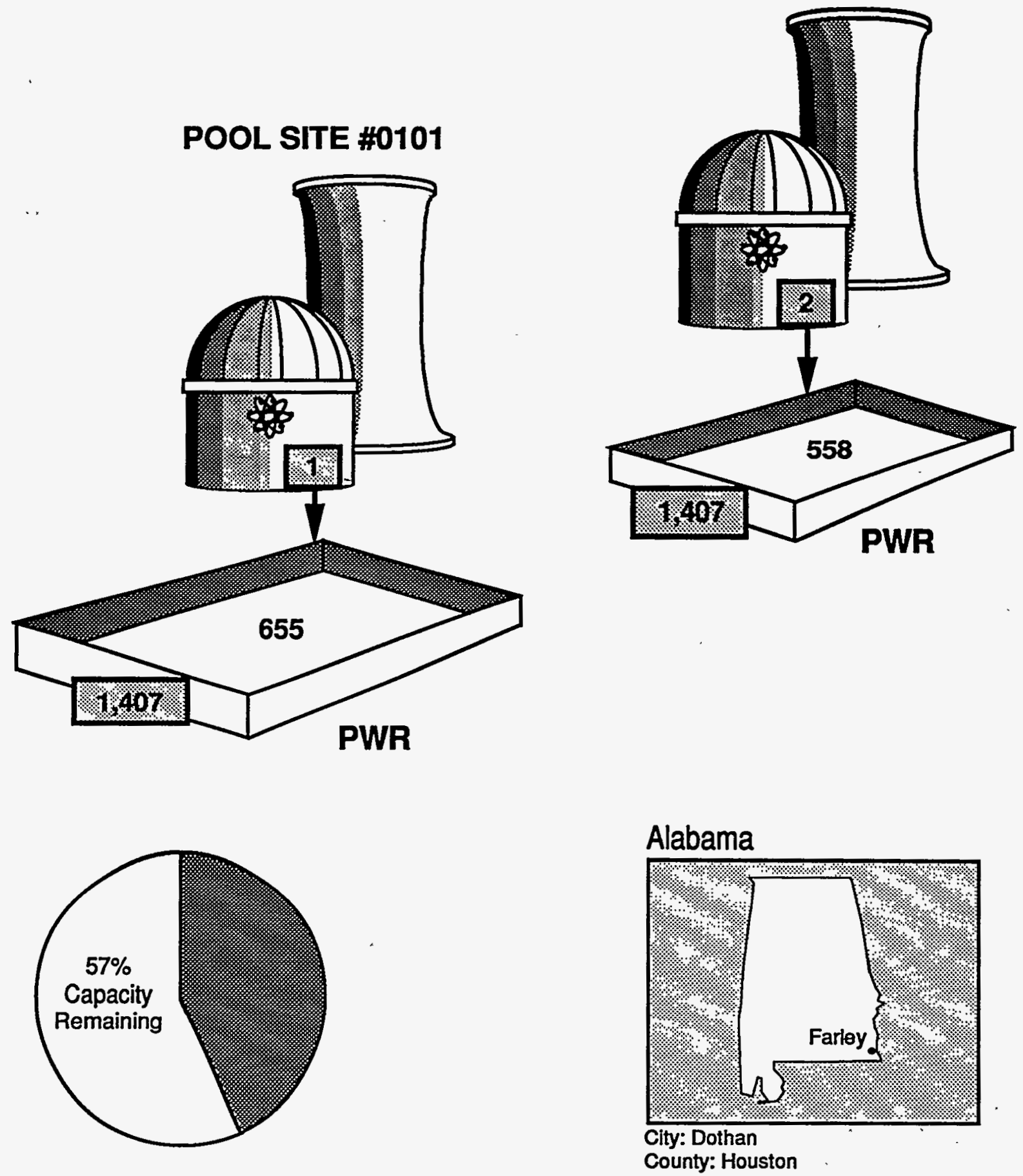


\section{Arizona Public Service Company Palo Verde}
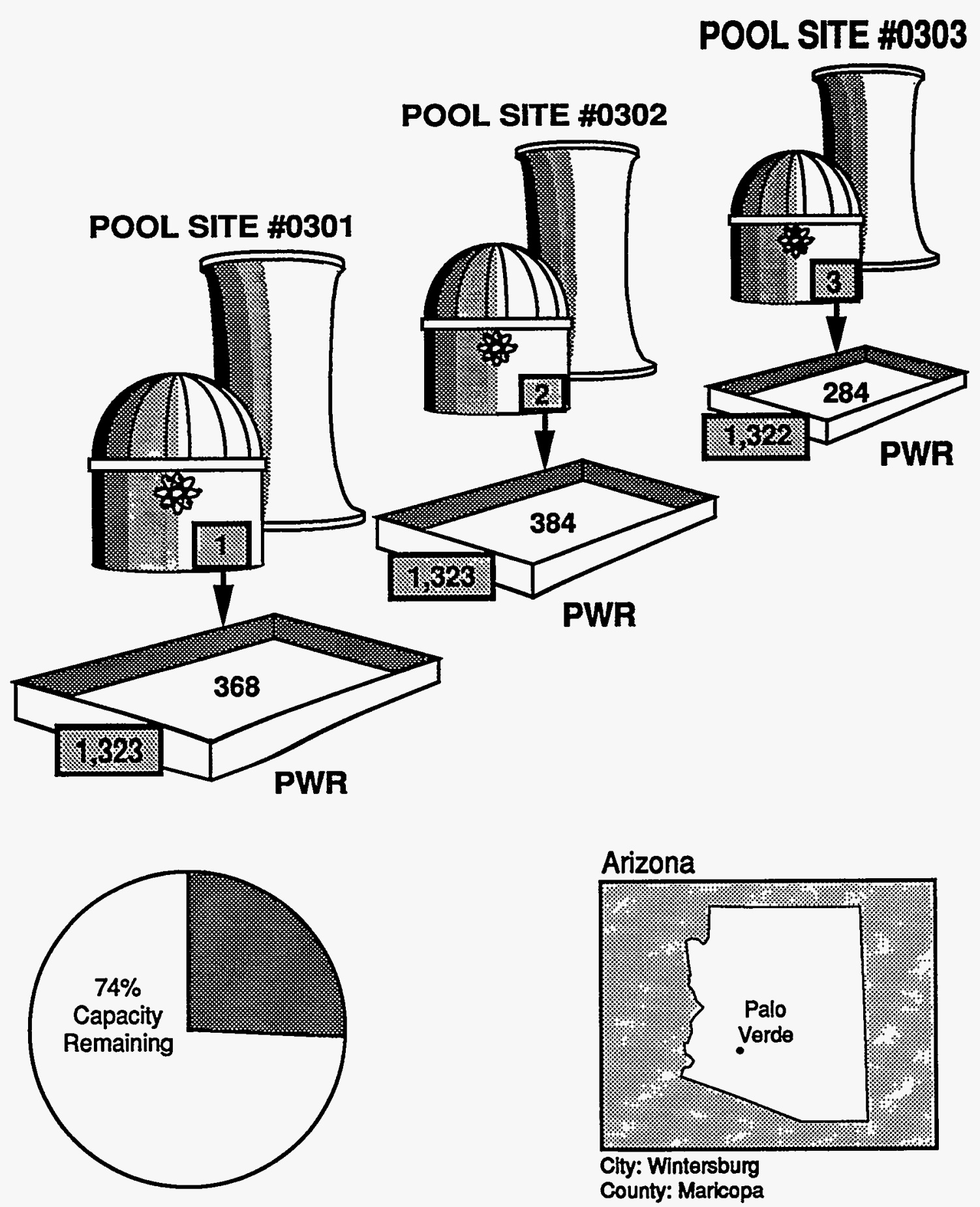


\section{Arkansas Power and Light Company Arkansas Nuclear}

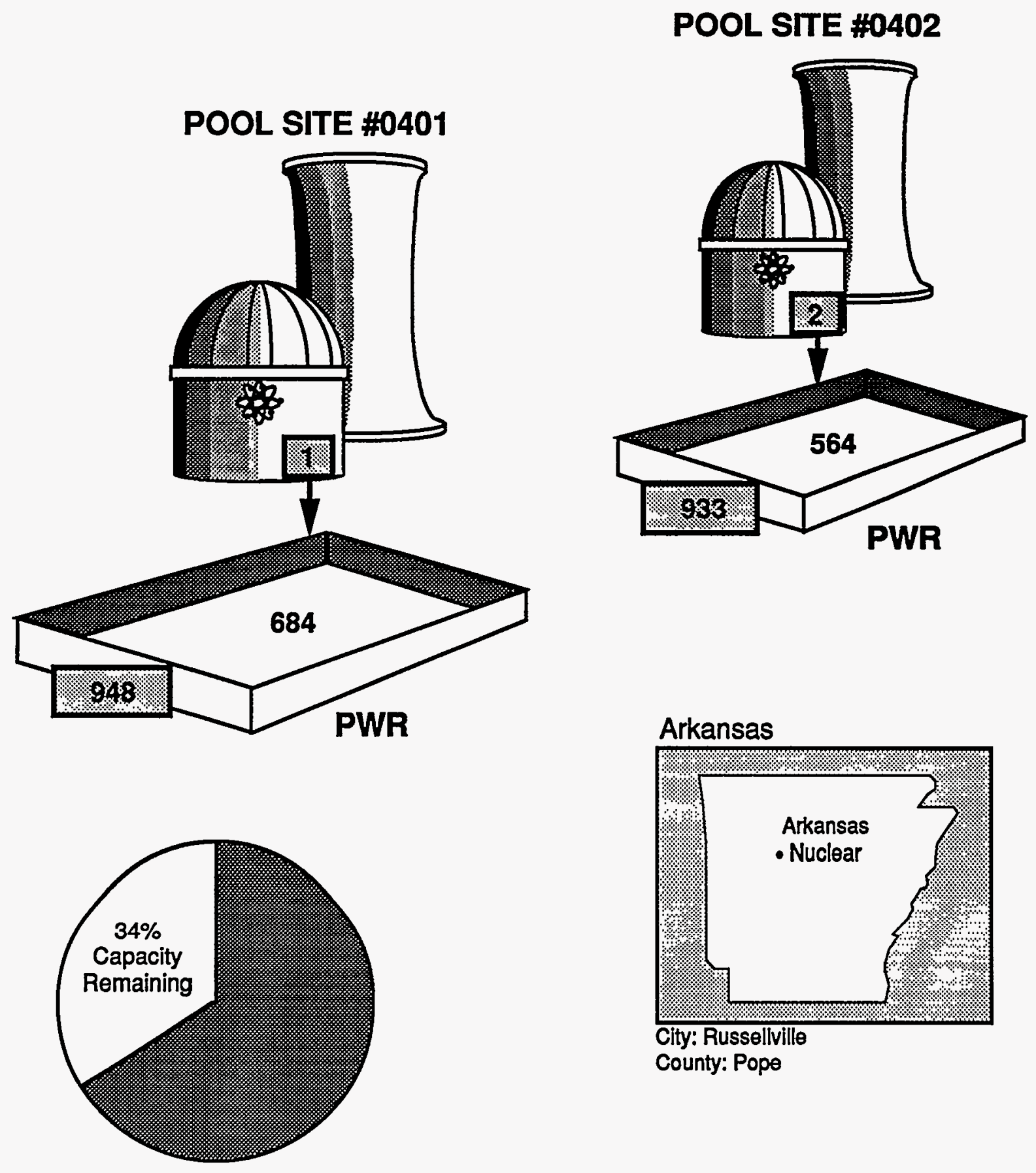




\section{Baltimore Gas and Electric Company Calvert Cliffs}

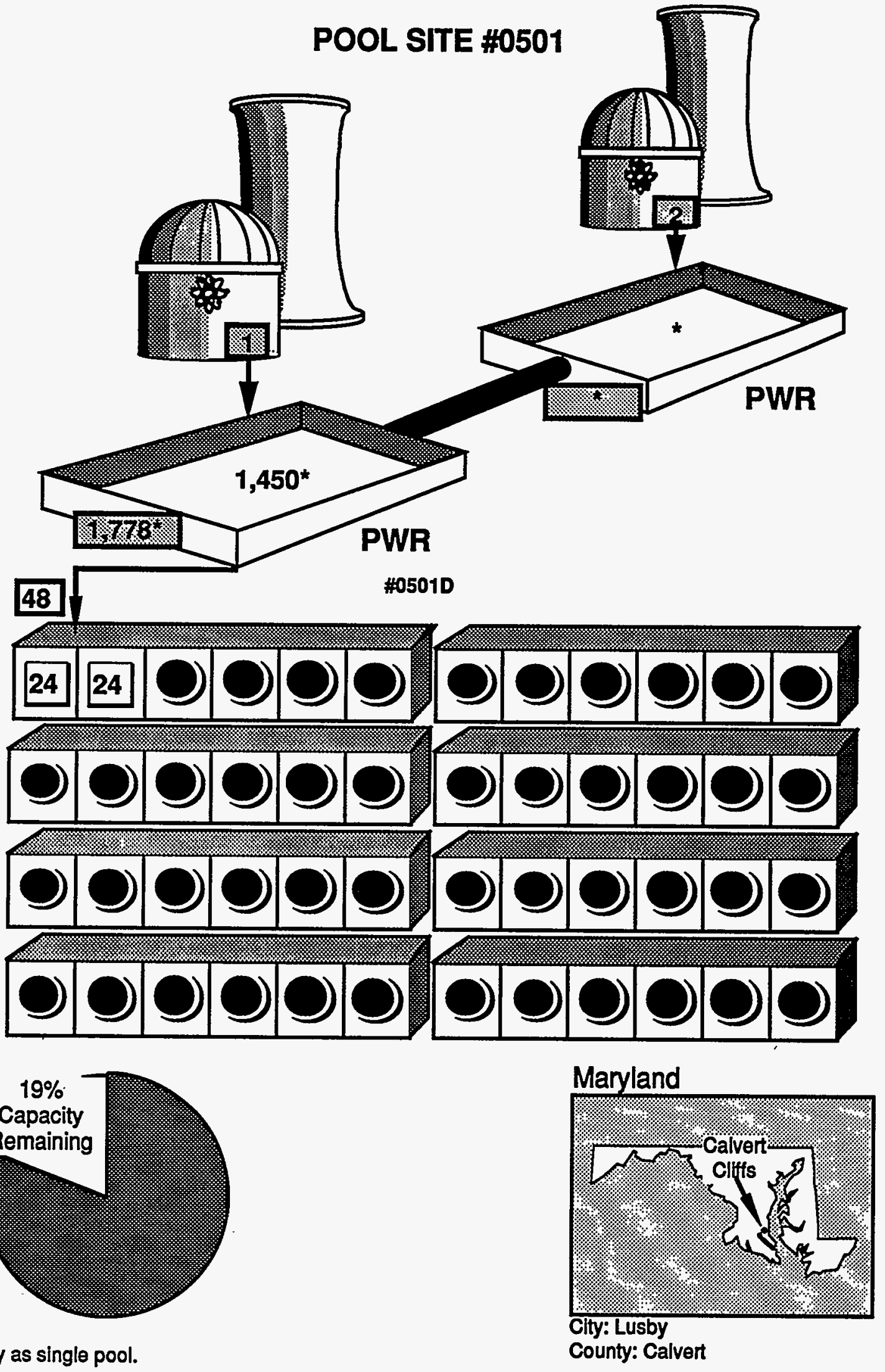




\section{Boston Edison Company Pilgrim}
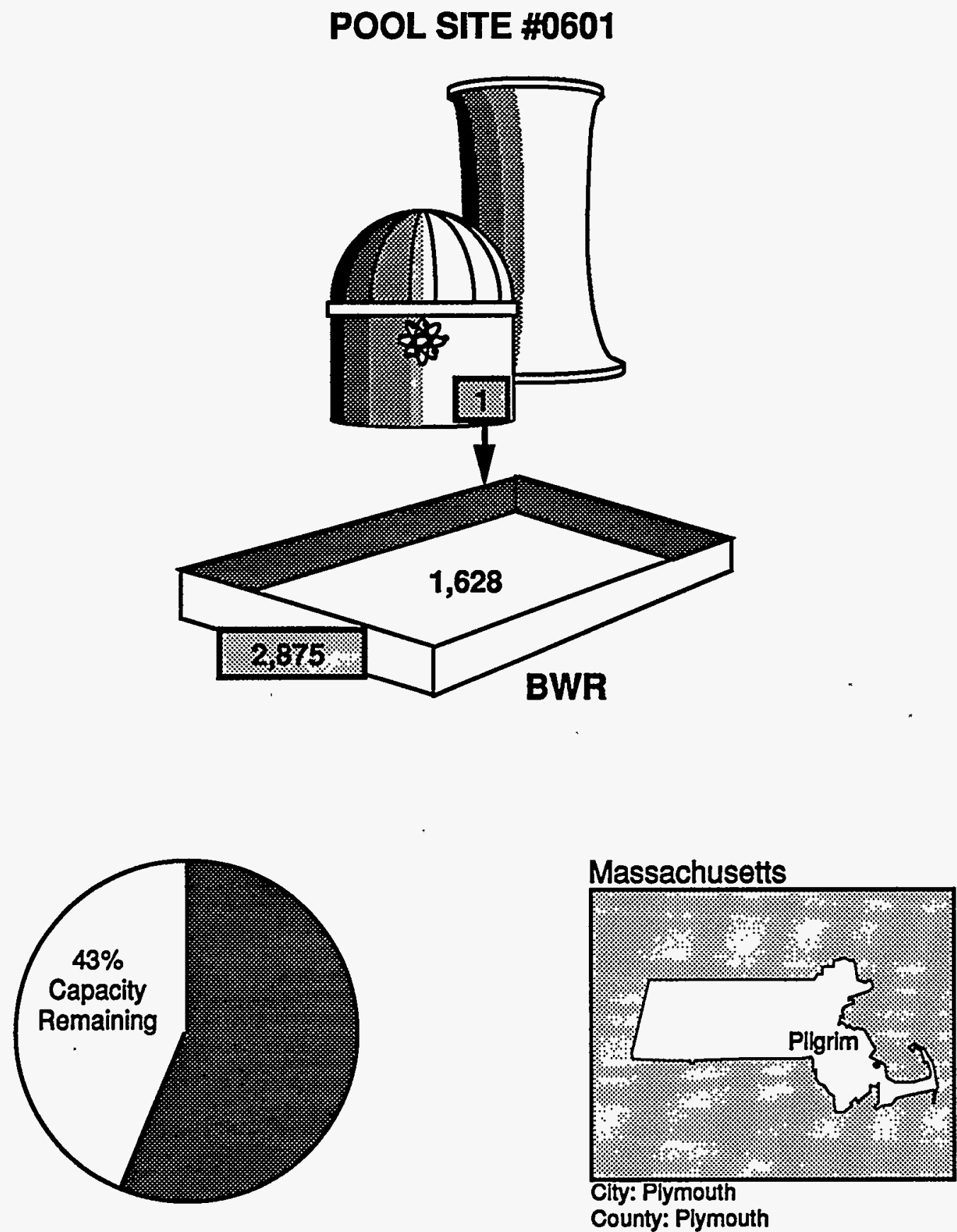


\section{Carolina Power and Light Company Brunswick}

\section{POOL SITE \#0702}

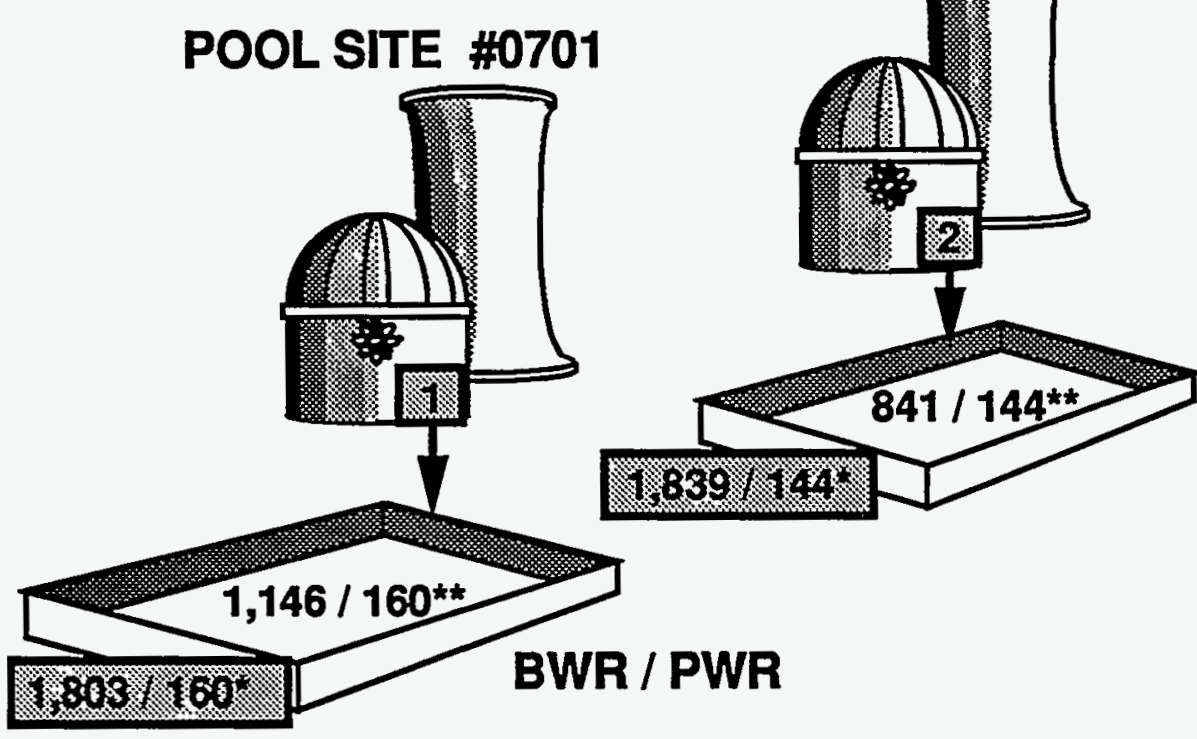

BWR / PWR

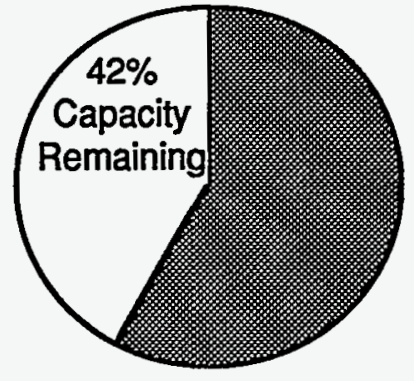

\section{Robinson}

POOL SITE \#0705

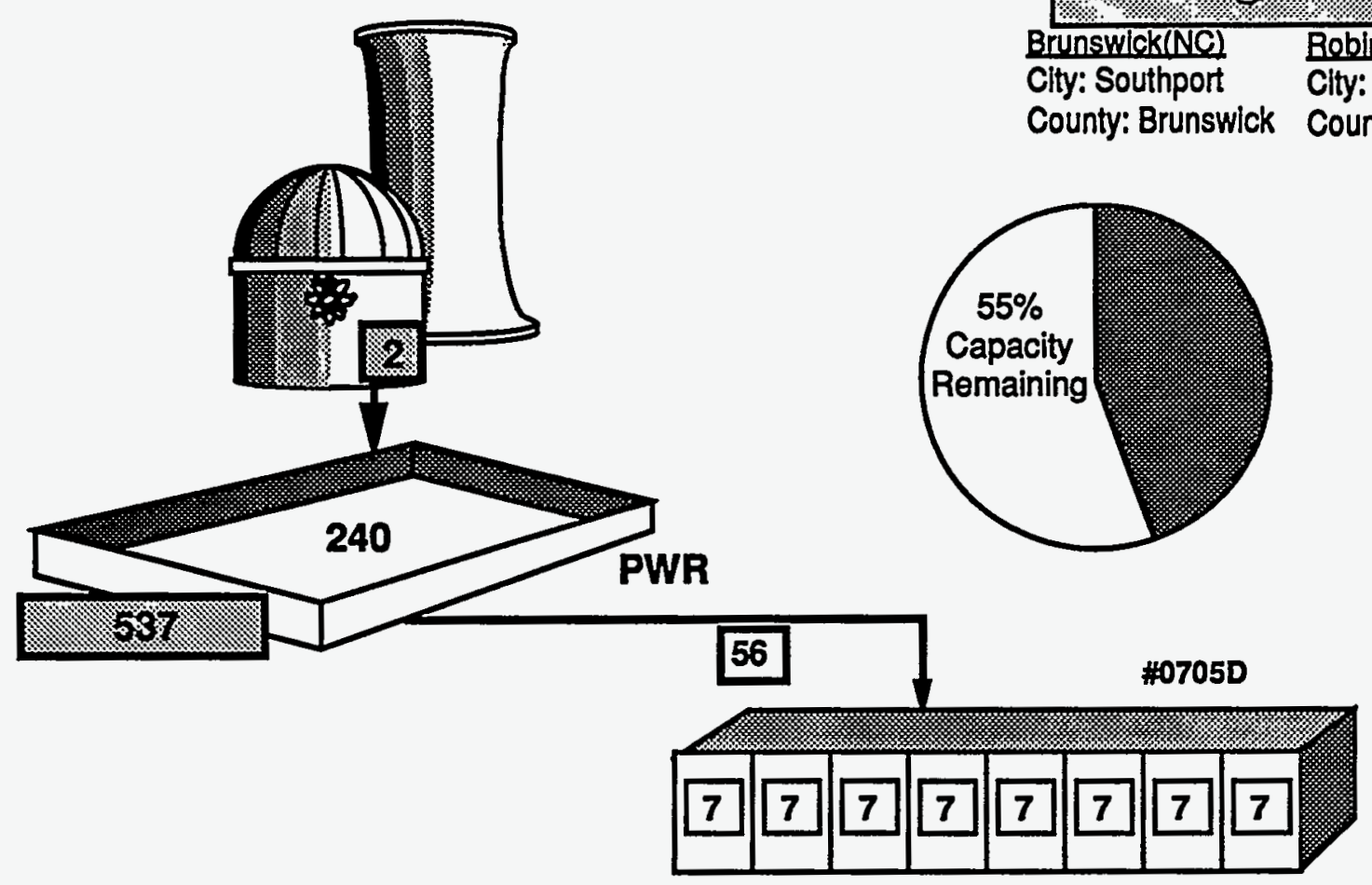

North Carolina/ South Carolina

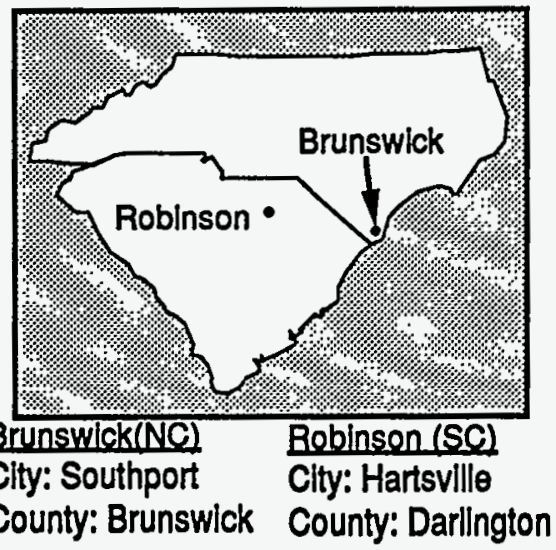

- The first number in the ratio denotes the maximum established storage capacity for assemblies in the BWR portion of the Brunswick pools. The second number is the maximum established storage capacity for assemblies in the PWR portion of the Brunswick pools.

*: The first number in the ratio denotes the current inventory of assemblies stored in the BWR portion of the Brunswick pools. The second number is the current inventory of assemblies stored in the PWR portion of the Brunswick pools. 


\section{Carolina Power and Light Company \\ (Continued)}

\section{Harris}
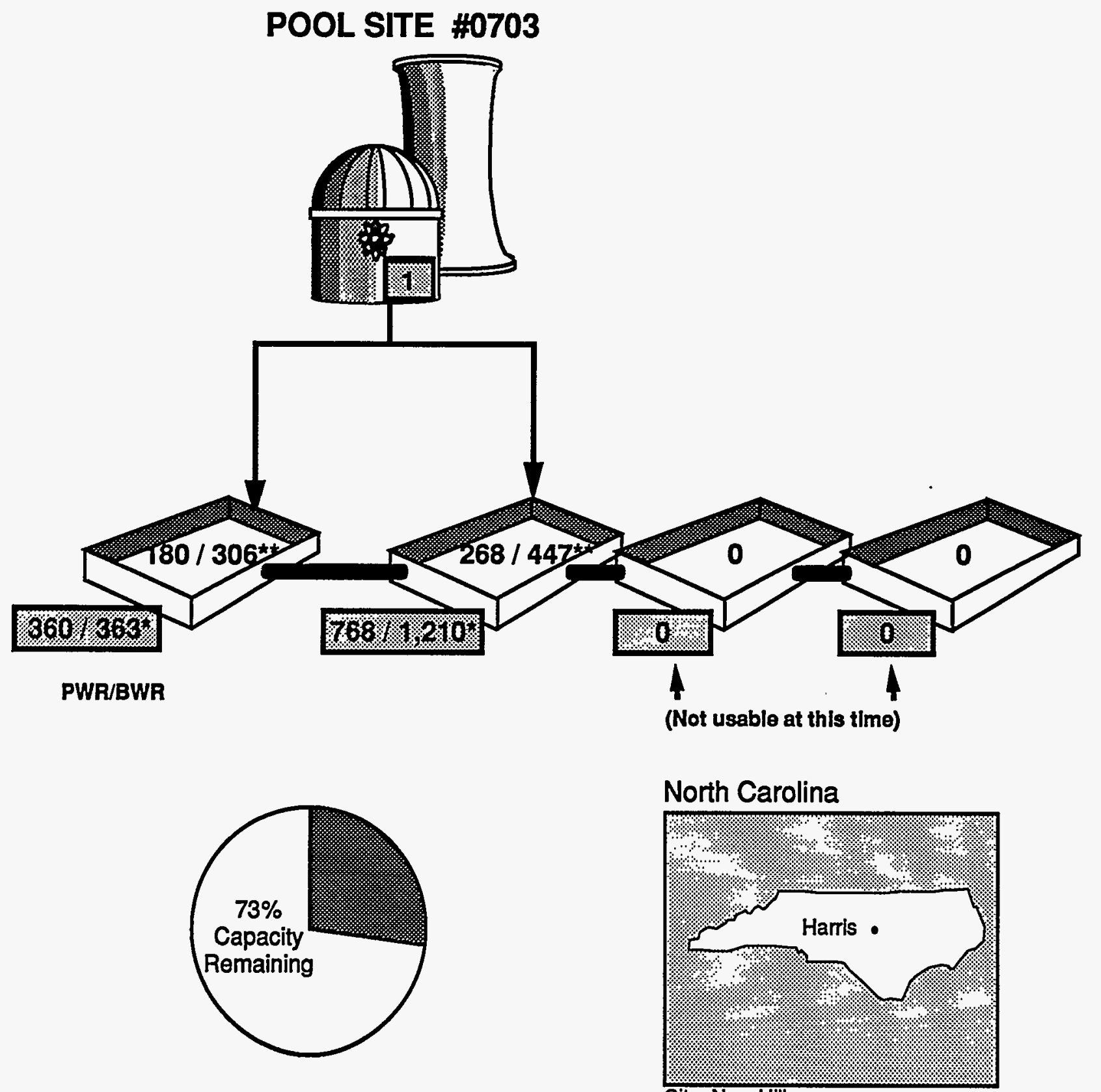

City: New Hill

County: Wake

The first number in the ratio denotes the maximum established storage capacity for assemblies in the PWR portion of the Harris pool. The second number is the maximum established storage capacity for assemblies in the BWR portion of the Harris pool.

"* The first number in the ratio denotes the current inventory of assemblies stored in the PWR portion of the Harris pool. The second number is the current inventory of assemlbles stored in the BWR portion of the Harris pool.

Notes: The Harris pools are licensed to hold up to 4,184 PWR or 5,808 BWR assemblies or a combination of each. In addition to the capacities shown, 704 PWR and 968 BWh racks are avallable as needed from site storage. These capacities reflect the maximum storage until additional storage racks are purchased. 


\section{Cleveland Electric Illuminating Company \\ Perry}

\section{POOL SITES \#0901 and \#0902}
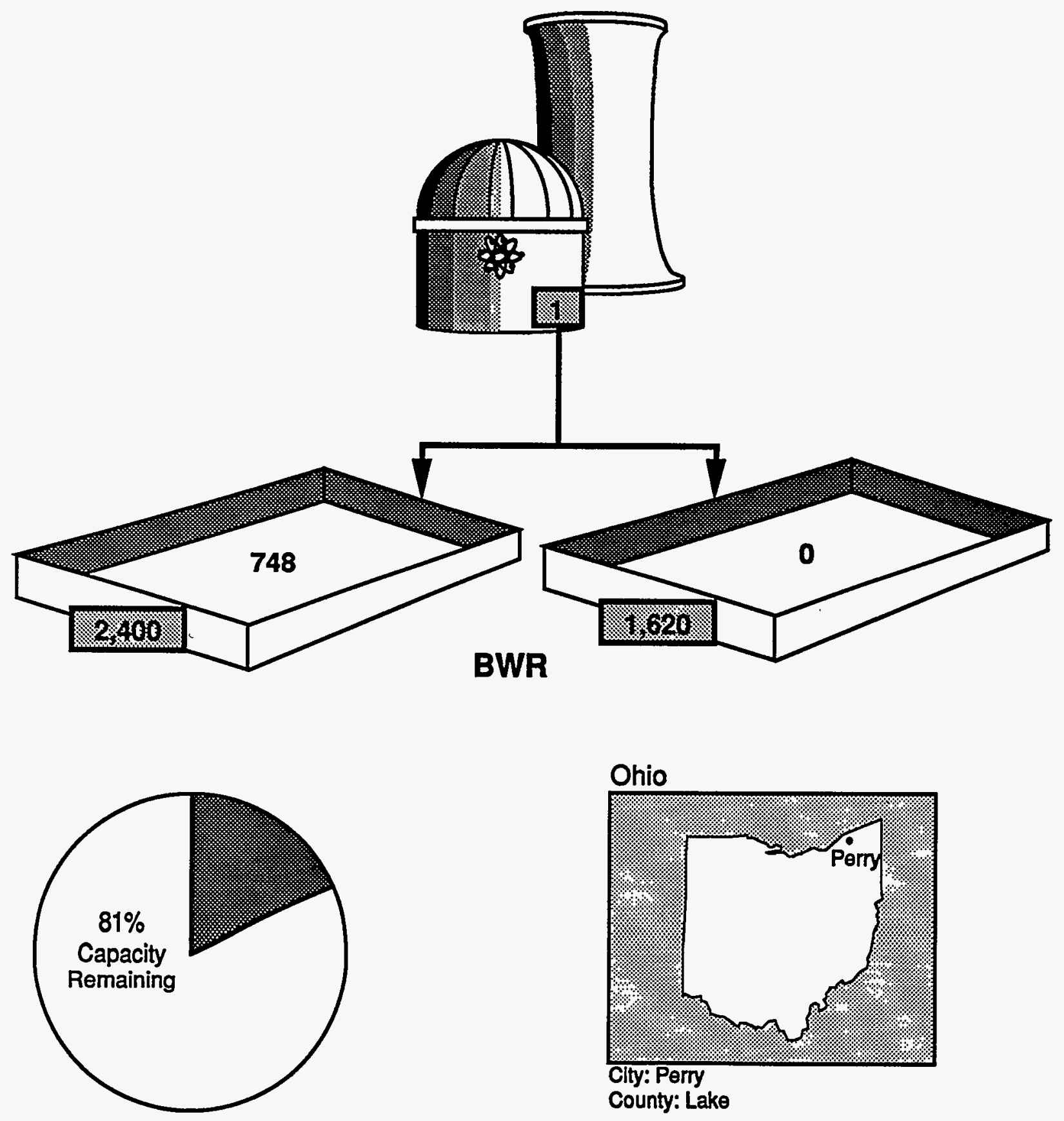


\section{Commonwealth Edison Company}

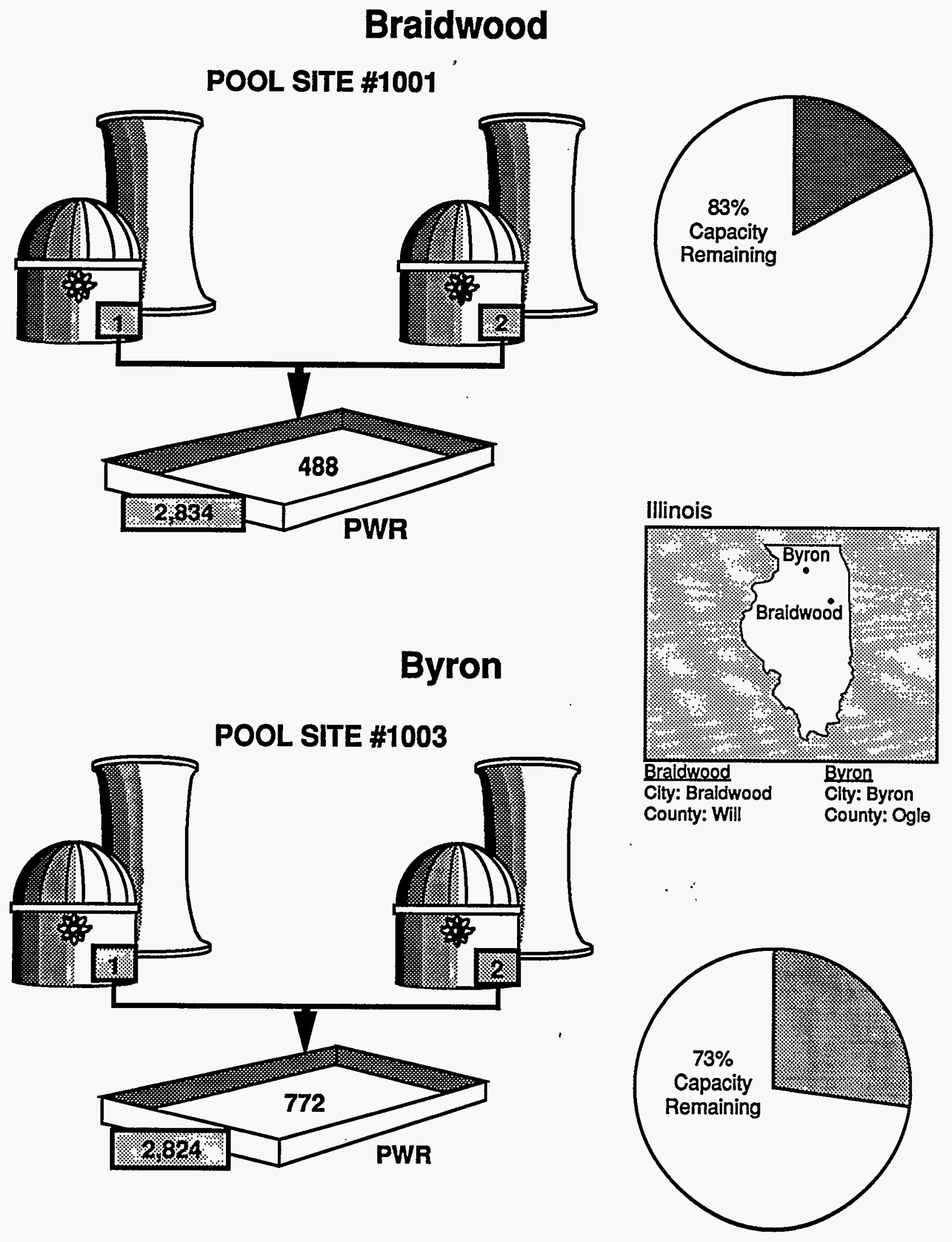




\section{Commonwealth Edison Company}

(Continued)

\section{Dresden}

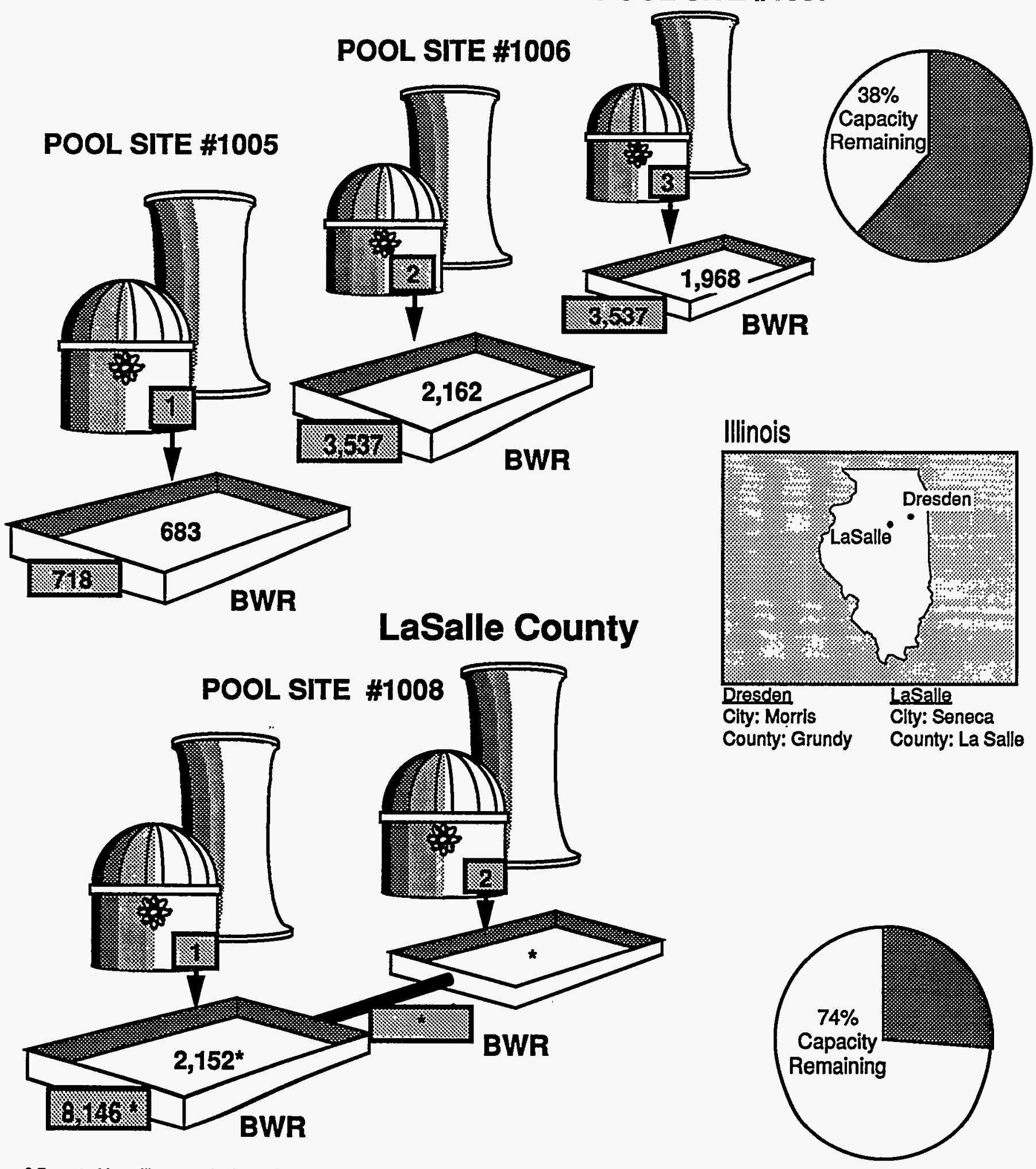

- Reported by utility as a single pool. 


\section{Commonwealth Edison Company (Continued) Quad Cities}
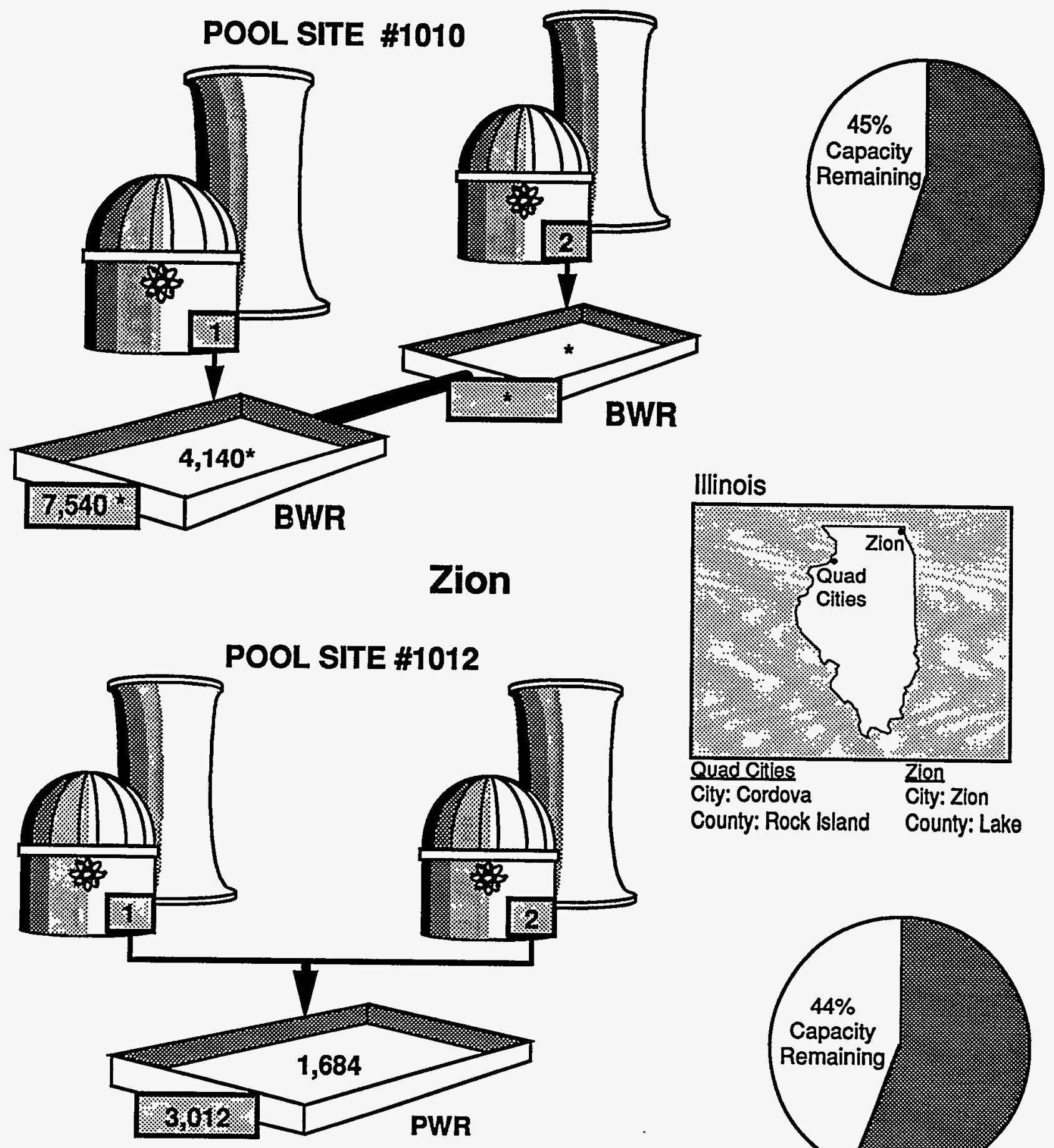

* Reported by utility as a single pool. 


\section{Consolidated Edison Company of New York \\ Indian Point}

POOL SITE \#1102
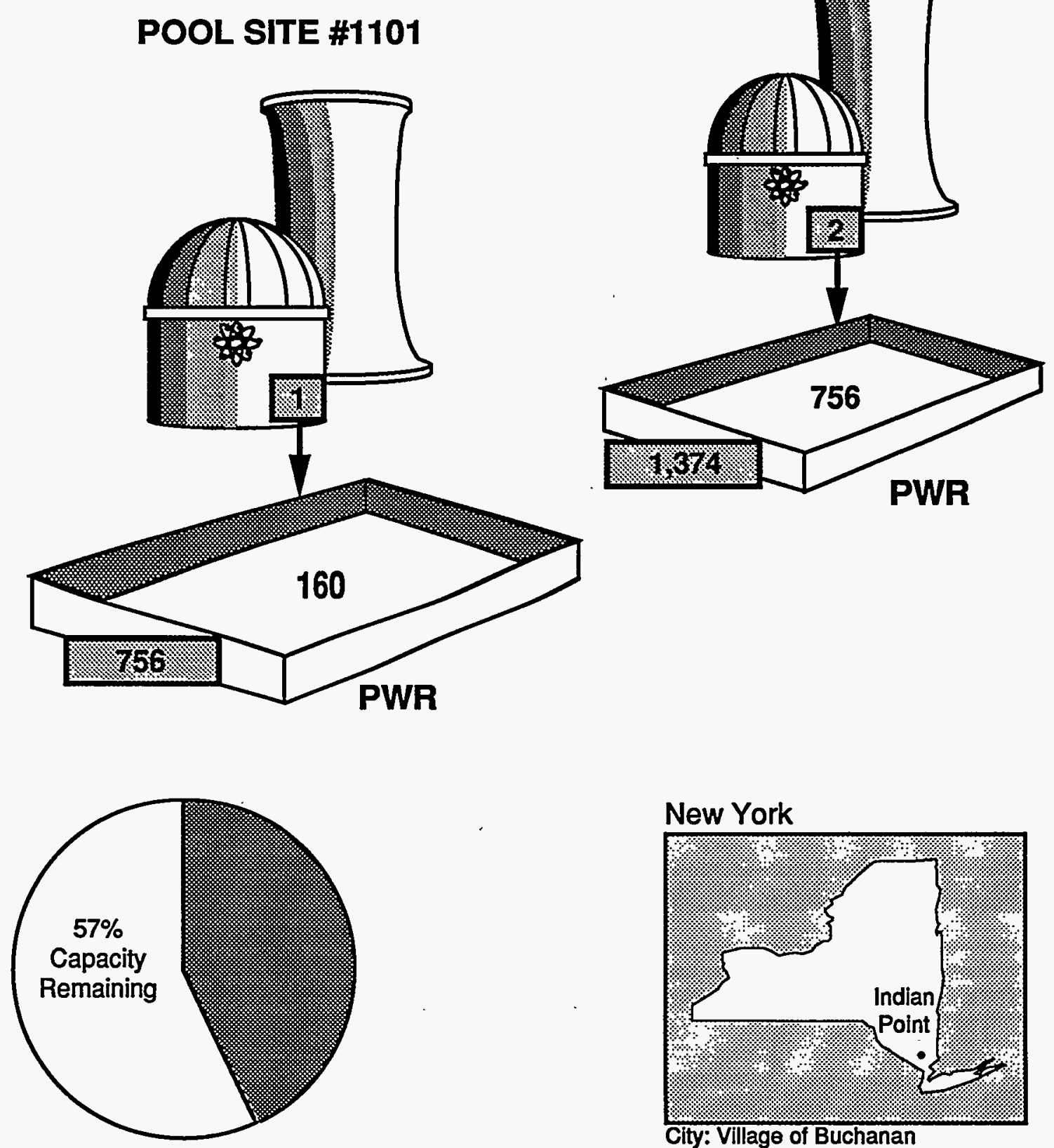

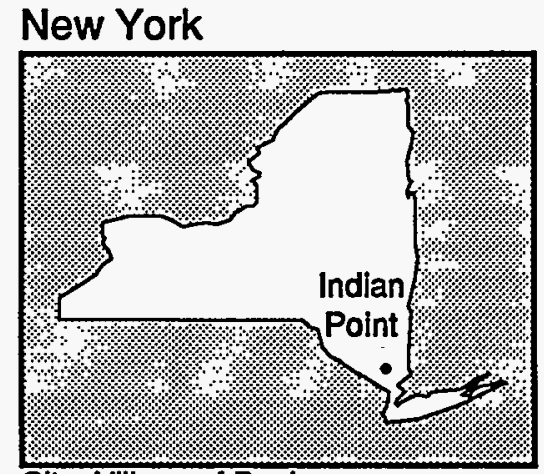

City: Village of Buchanan

County: Westchester 


\section{Consumers Power Company Big Rock Point}
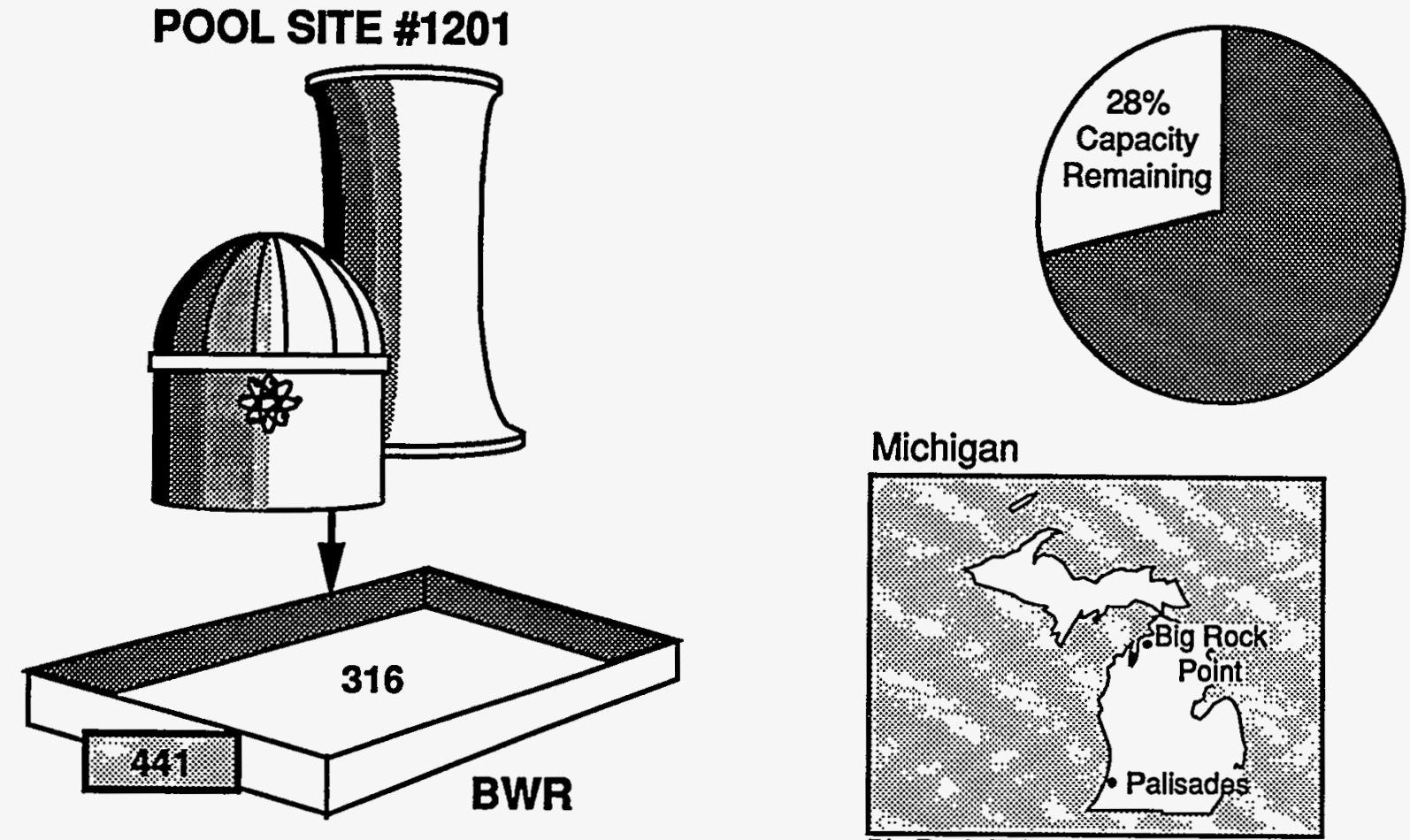

Michigan

POOL SITE \#1204

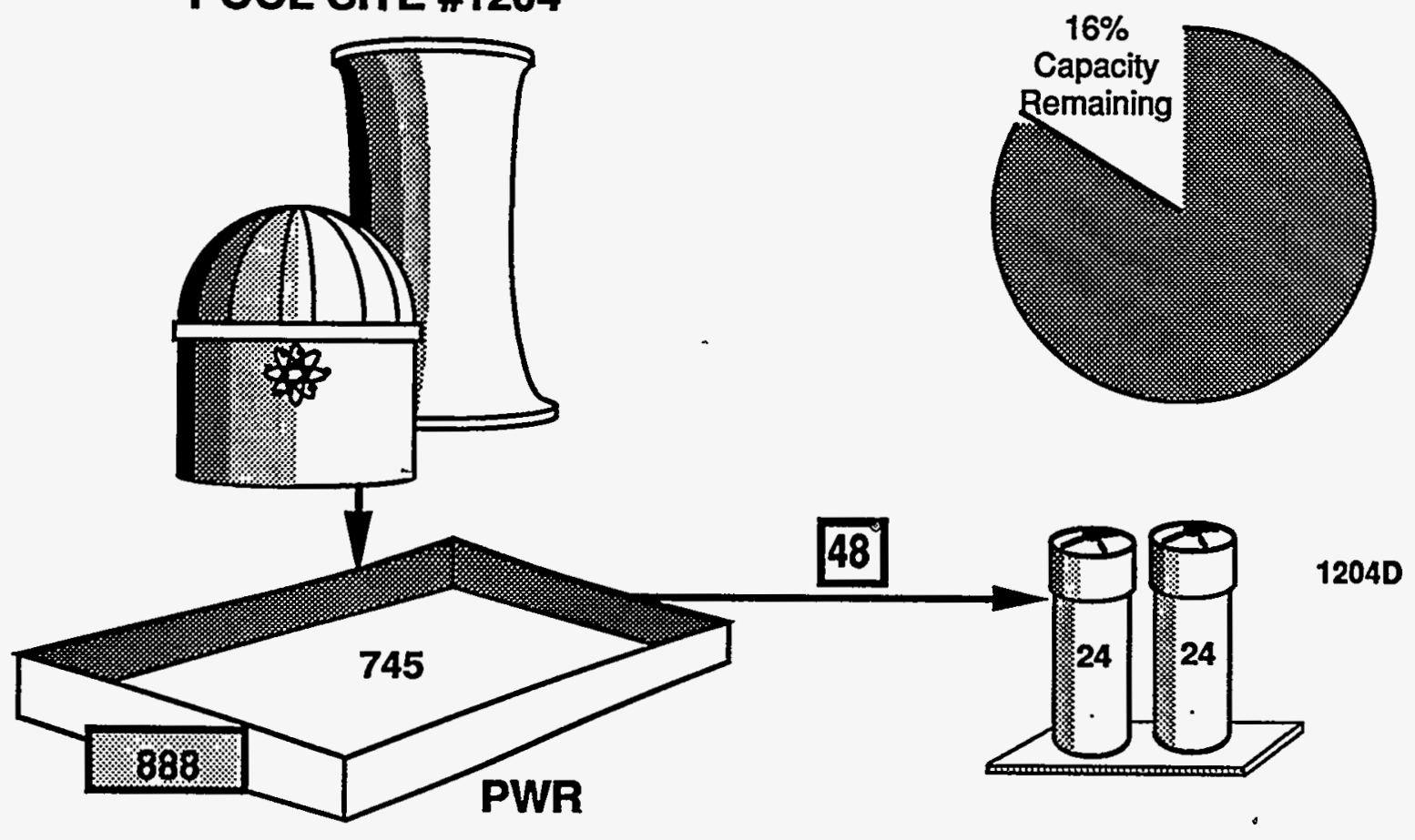




\section{Dairyland Power Cooperative LaCrosse}
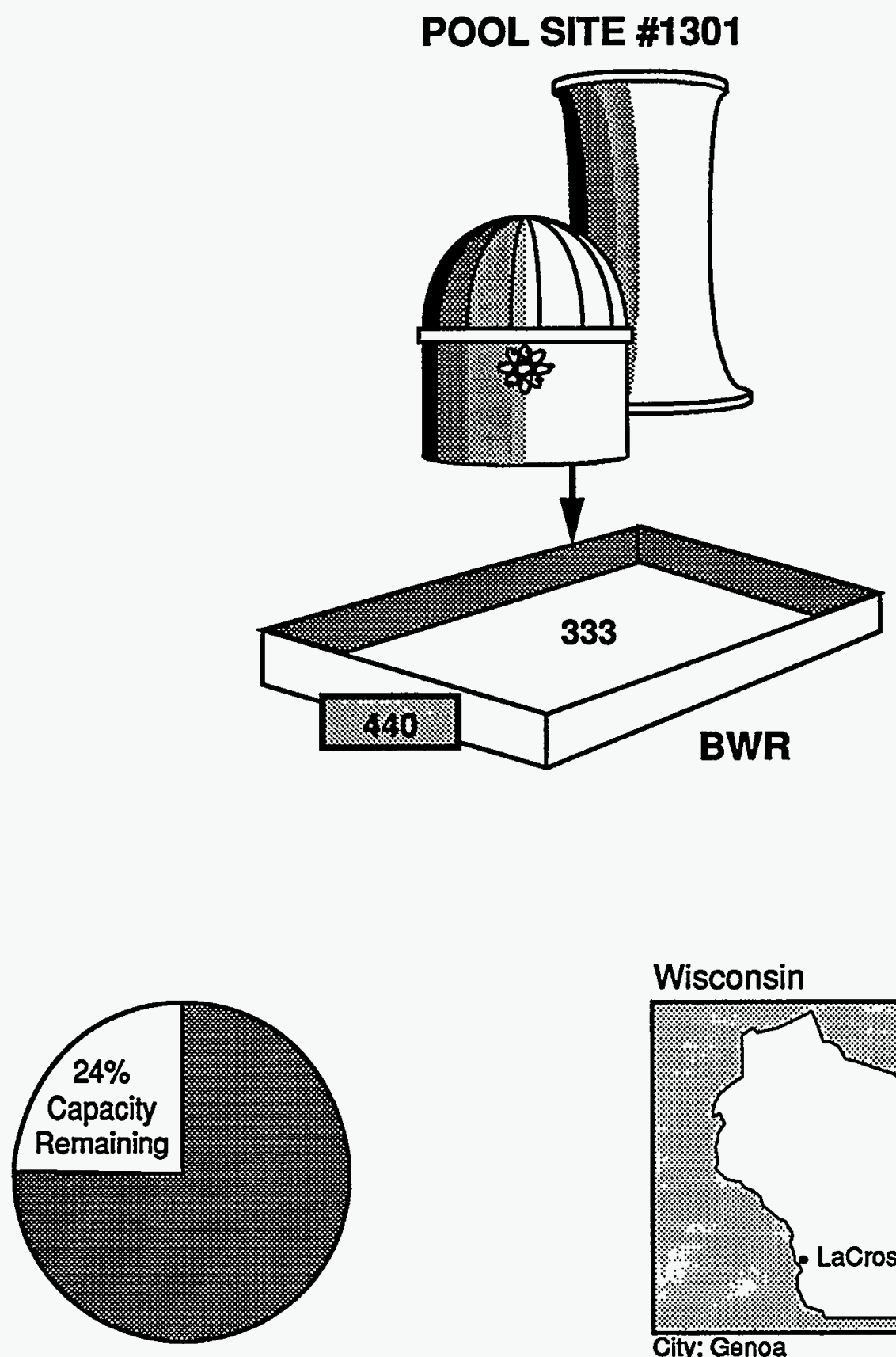

Wisconsin

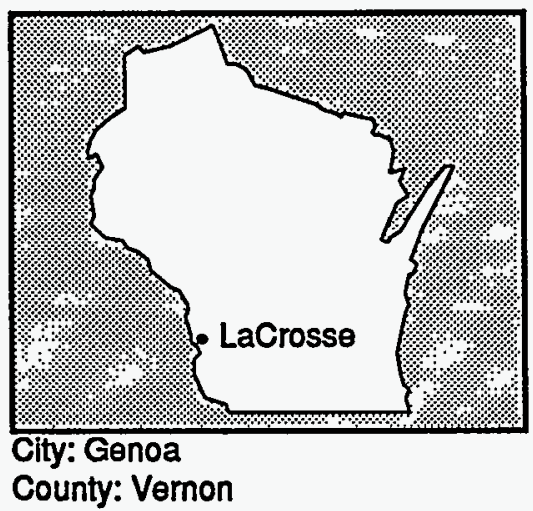




\section{Detroit Edison Company Enrico Fermi}
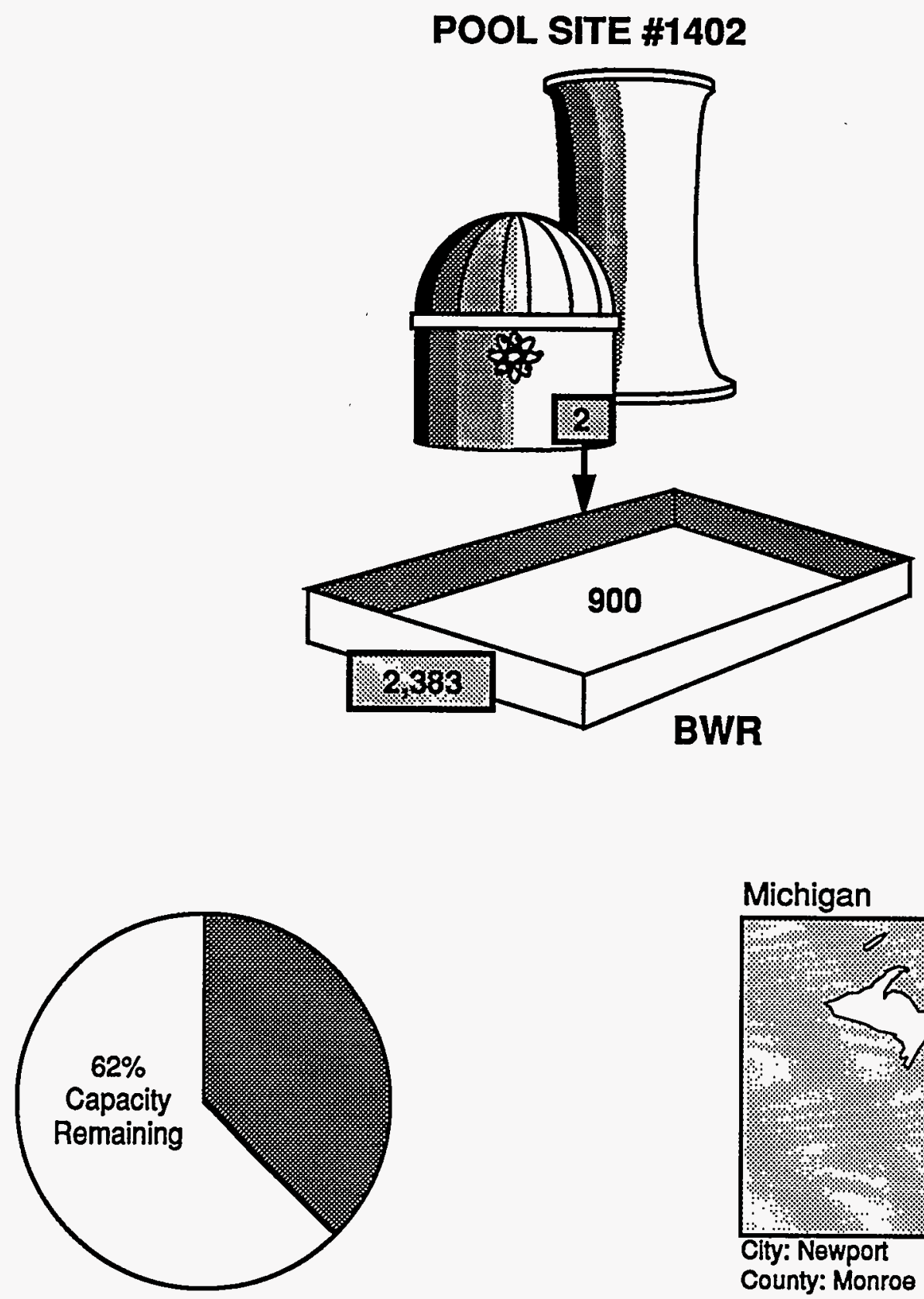

Michigan

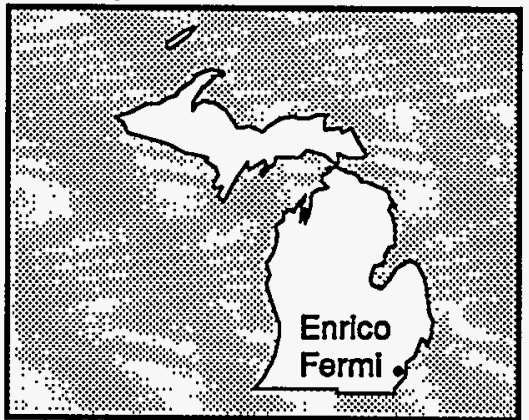

City: Newport

County: Monroe 


\section{Duke Power Company \\ Catawba}

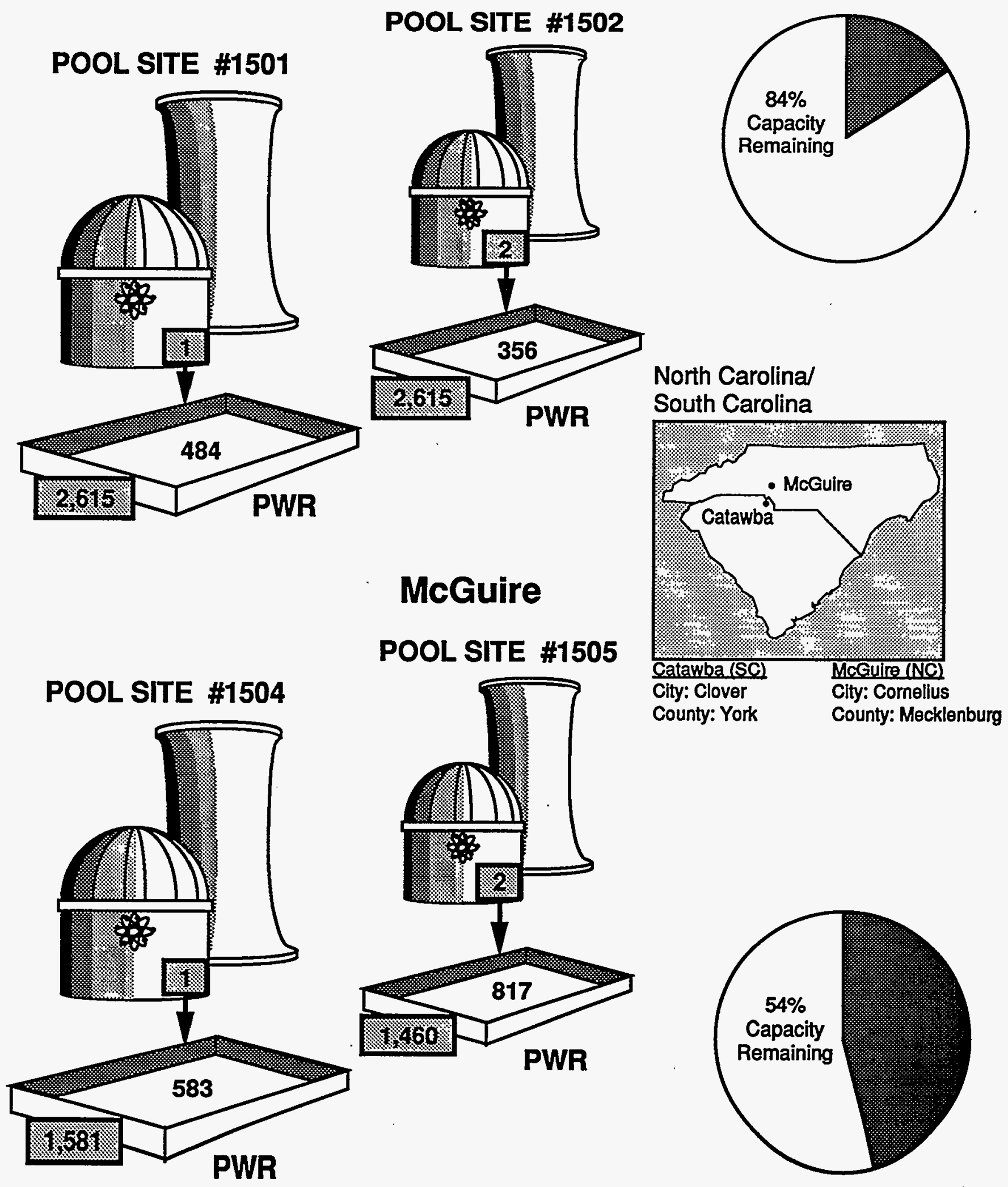




\section{Duke Power Company \\ (Continued) \\ Oconee}

POOL SITE \#1506
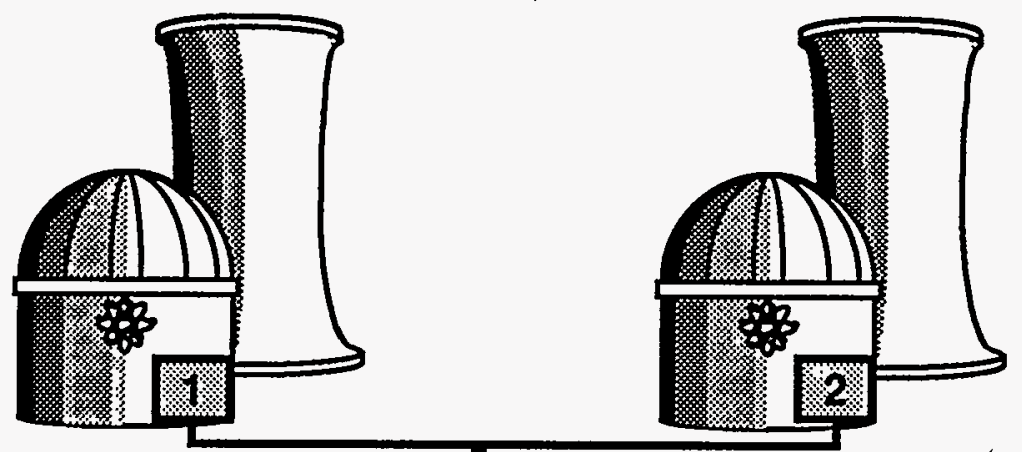

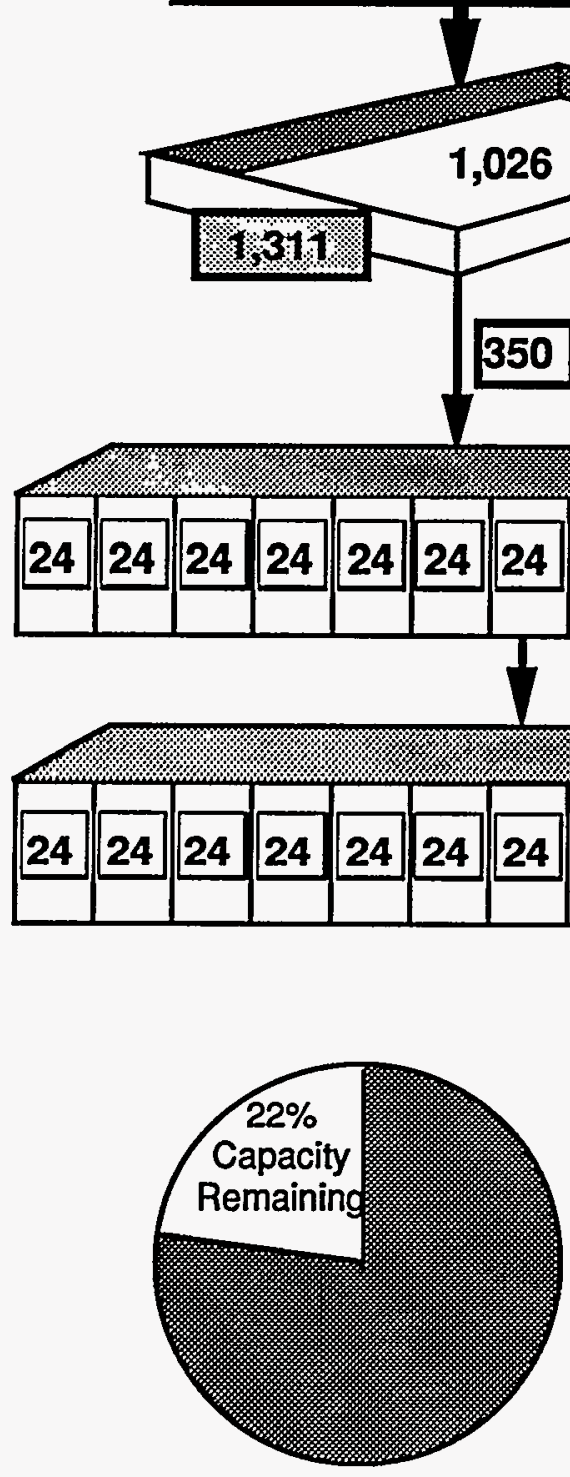

Oconee $1 \& 2$

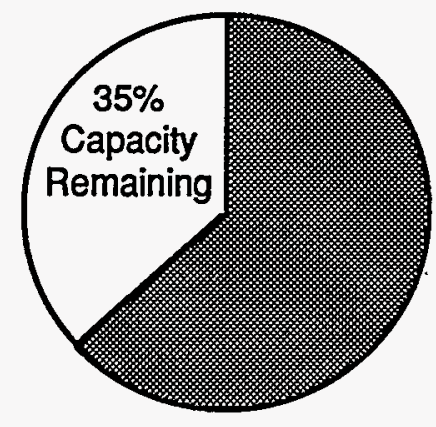

Oconee 3
South Carolina

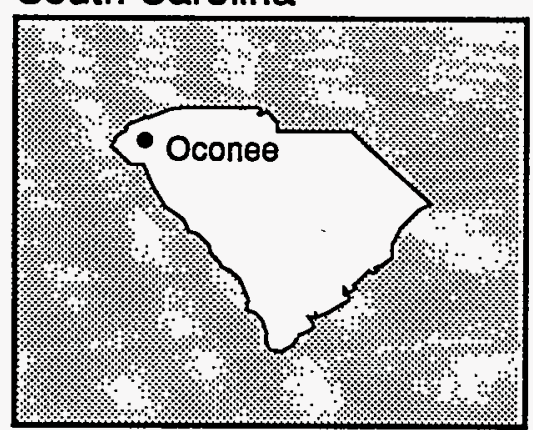

City: Seneca

County: Oconee 


\section{Duquesne Light Company Beaver Valley}
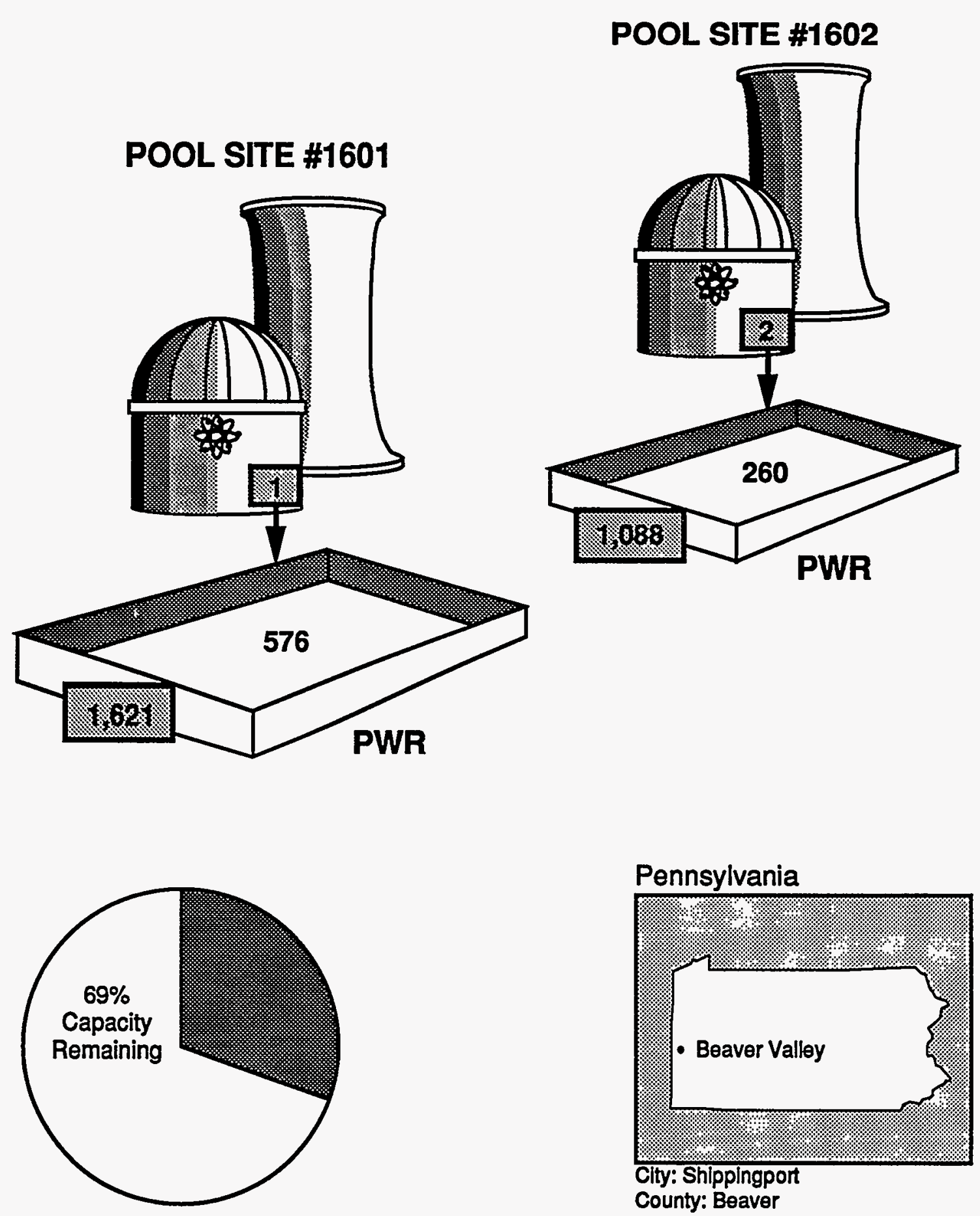


\section{Florida Power Corporation Crystal River}

\section{POOL SITE \#1701}
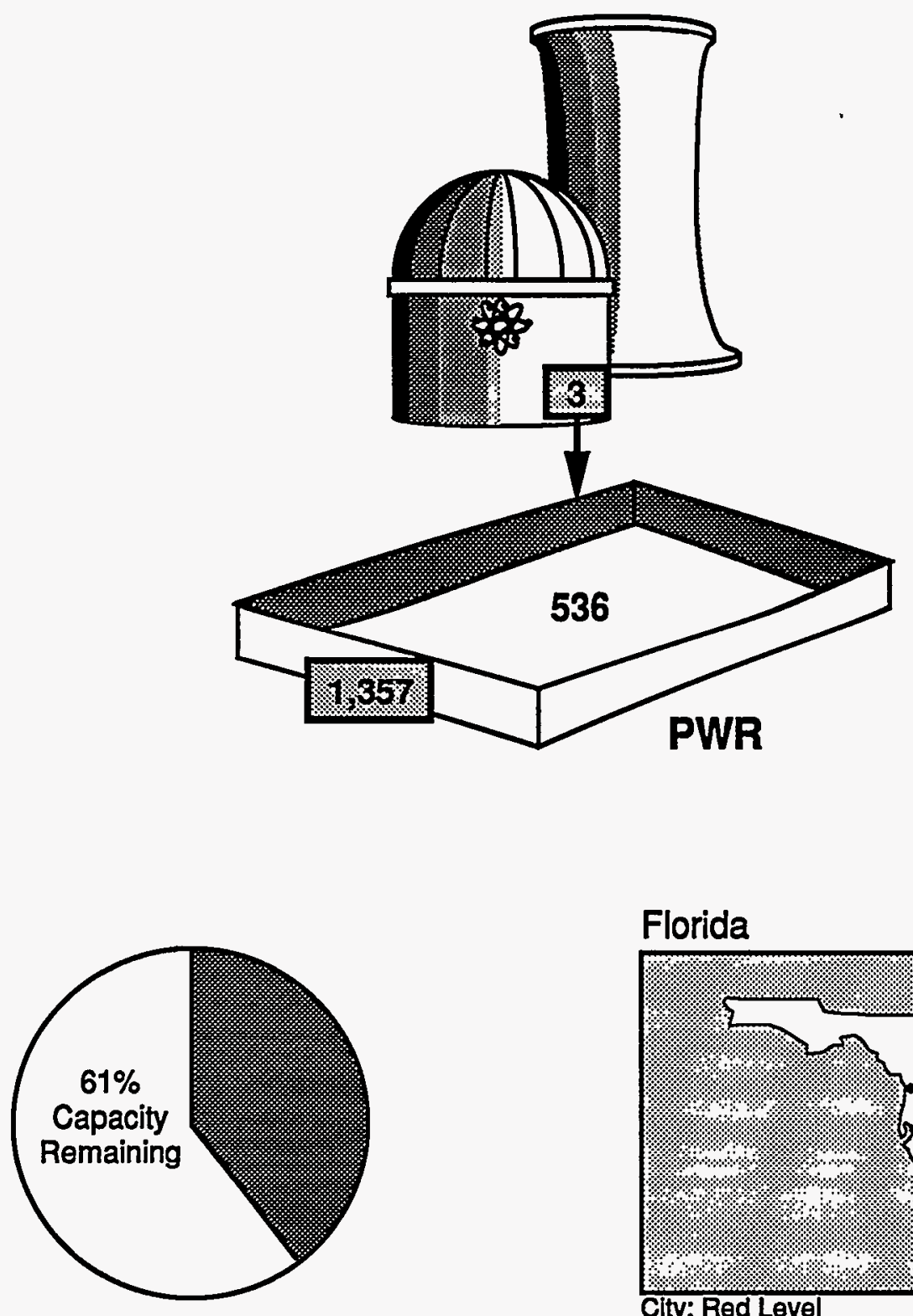

Florida

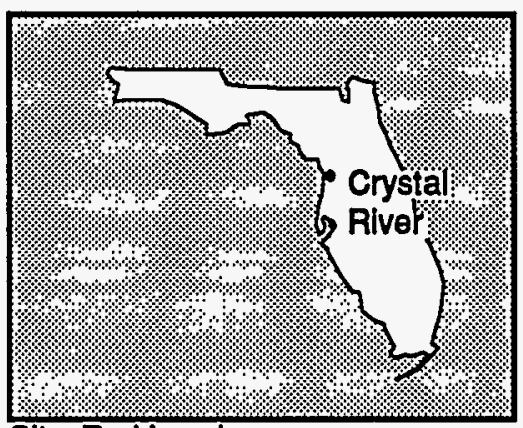

Clty: Red Level

County: Citrus 


\section{Florida Power and Light Company St. Lucie}
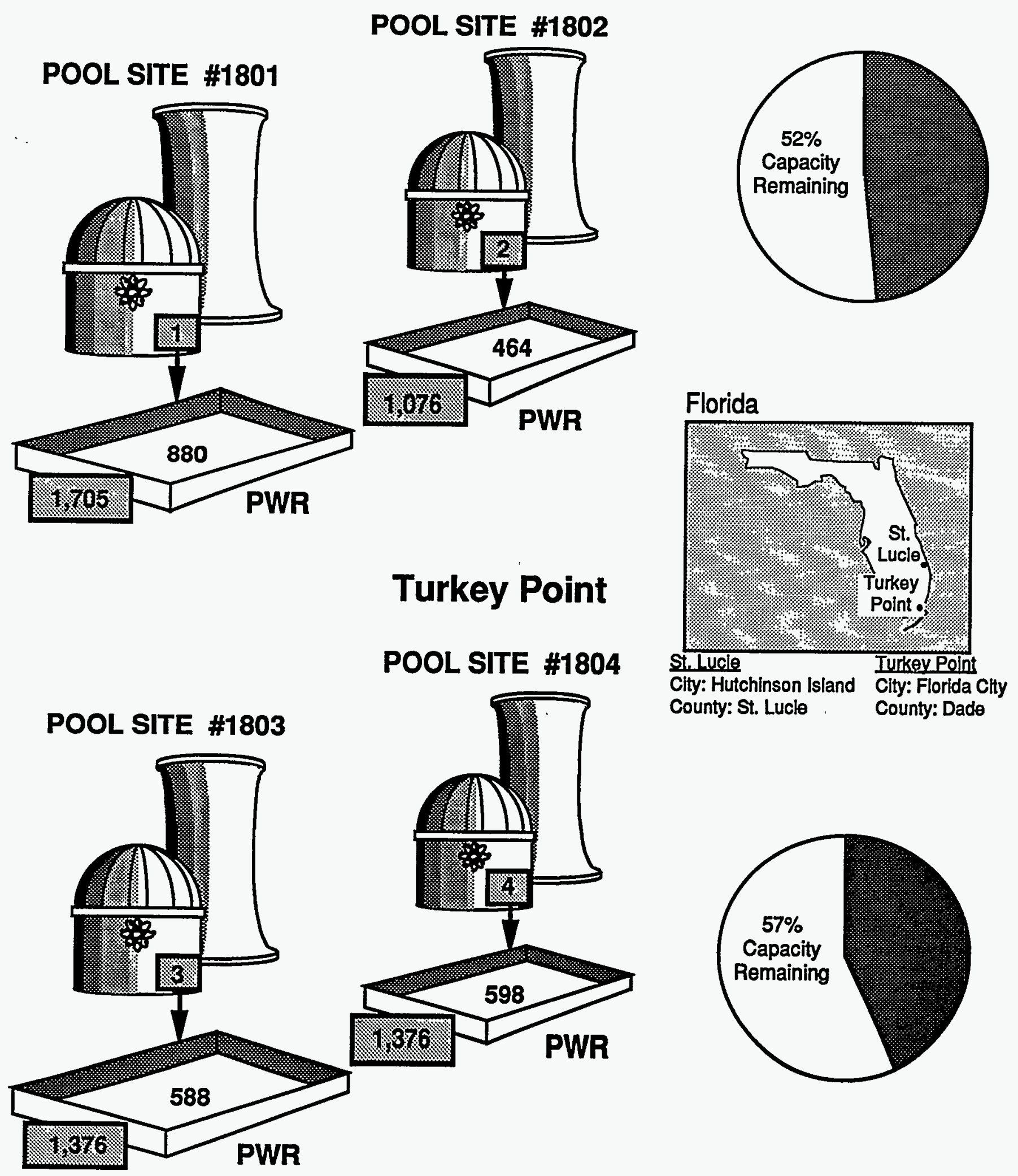


\section{Georgia Power Company}

\section{Hatch}
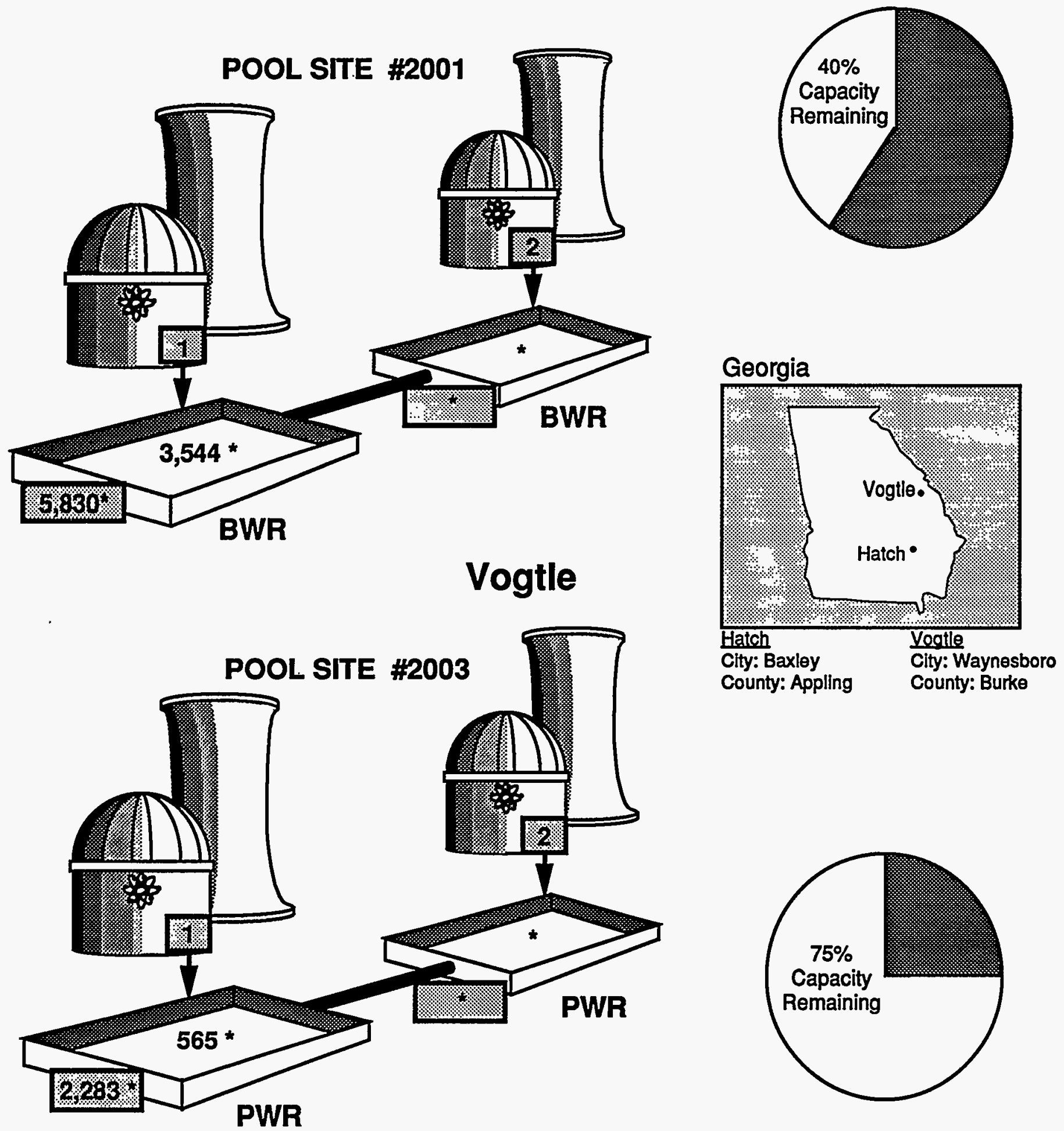

- Reported by utillty as single pool.

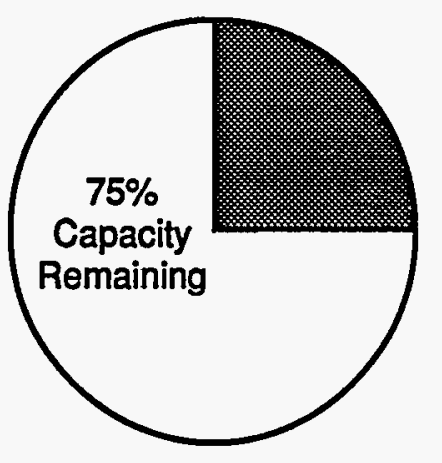




\section{GPU Nuclear Corporation}

\section{Three Mile Island}
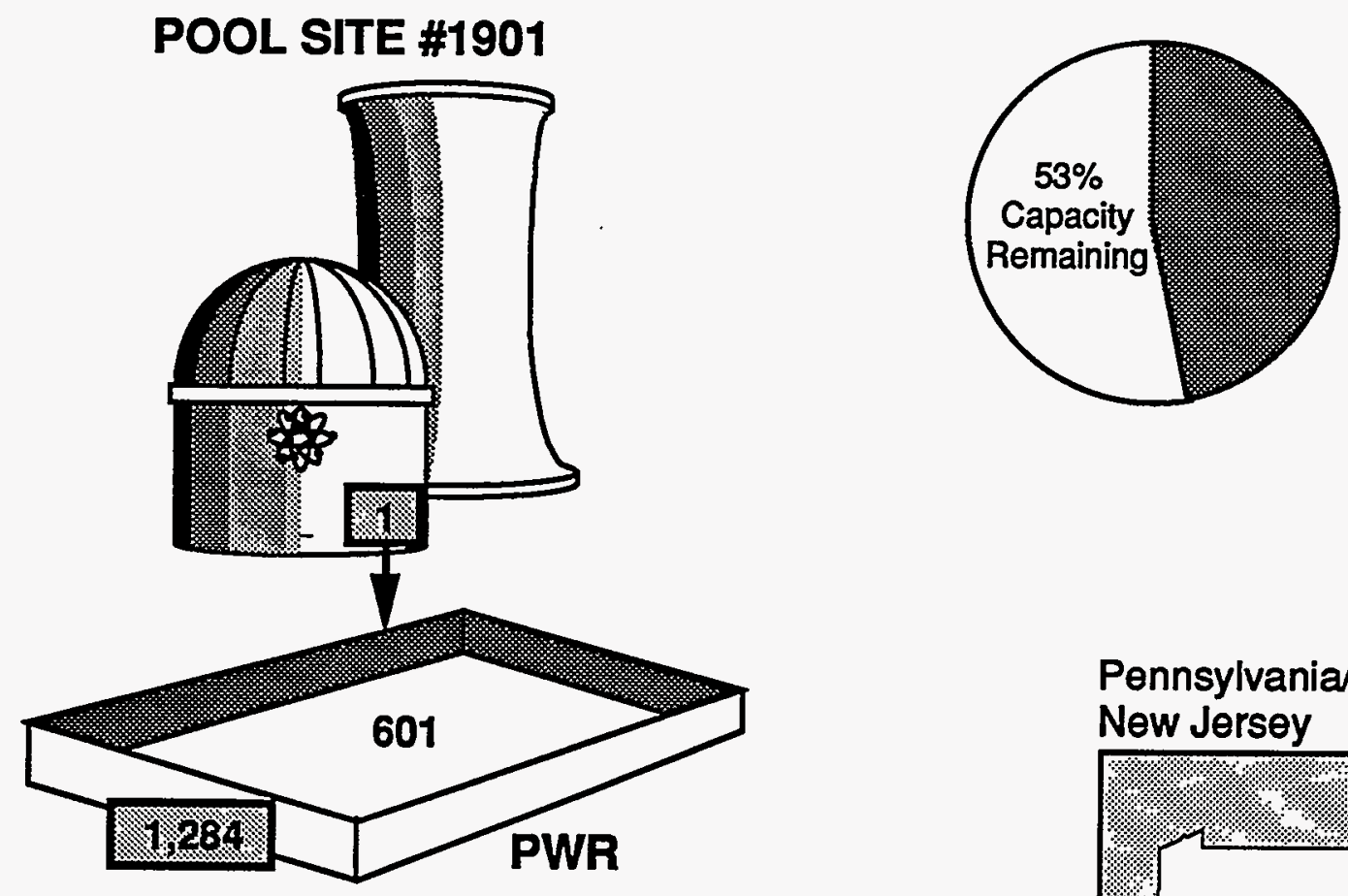

\section{Oyster Creek}

\section{POOL SITE \#1903}

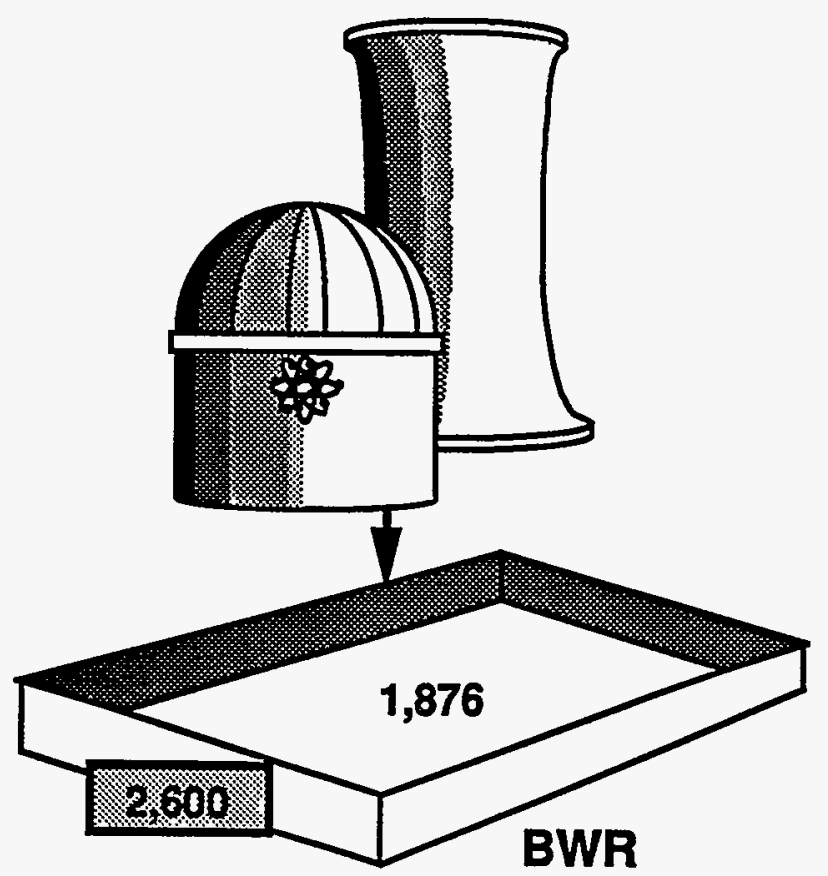

Pennsylvanial New Jersey

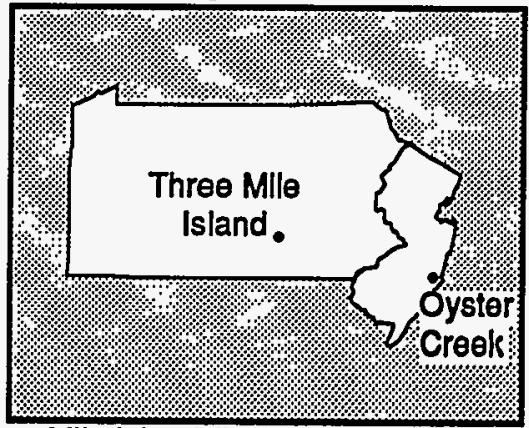

Three Mile /sland (PA) Ovster Creek (NU) Clty: Londondenry Twp. City: Forked River County: Dauphin County: Ocean

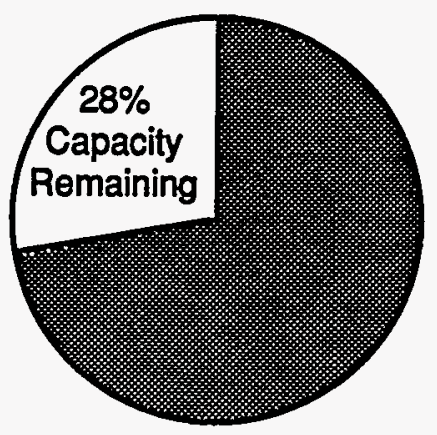




\section{Gulf States Utilities Company River Bend}
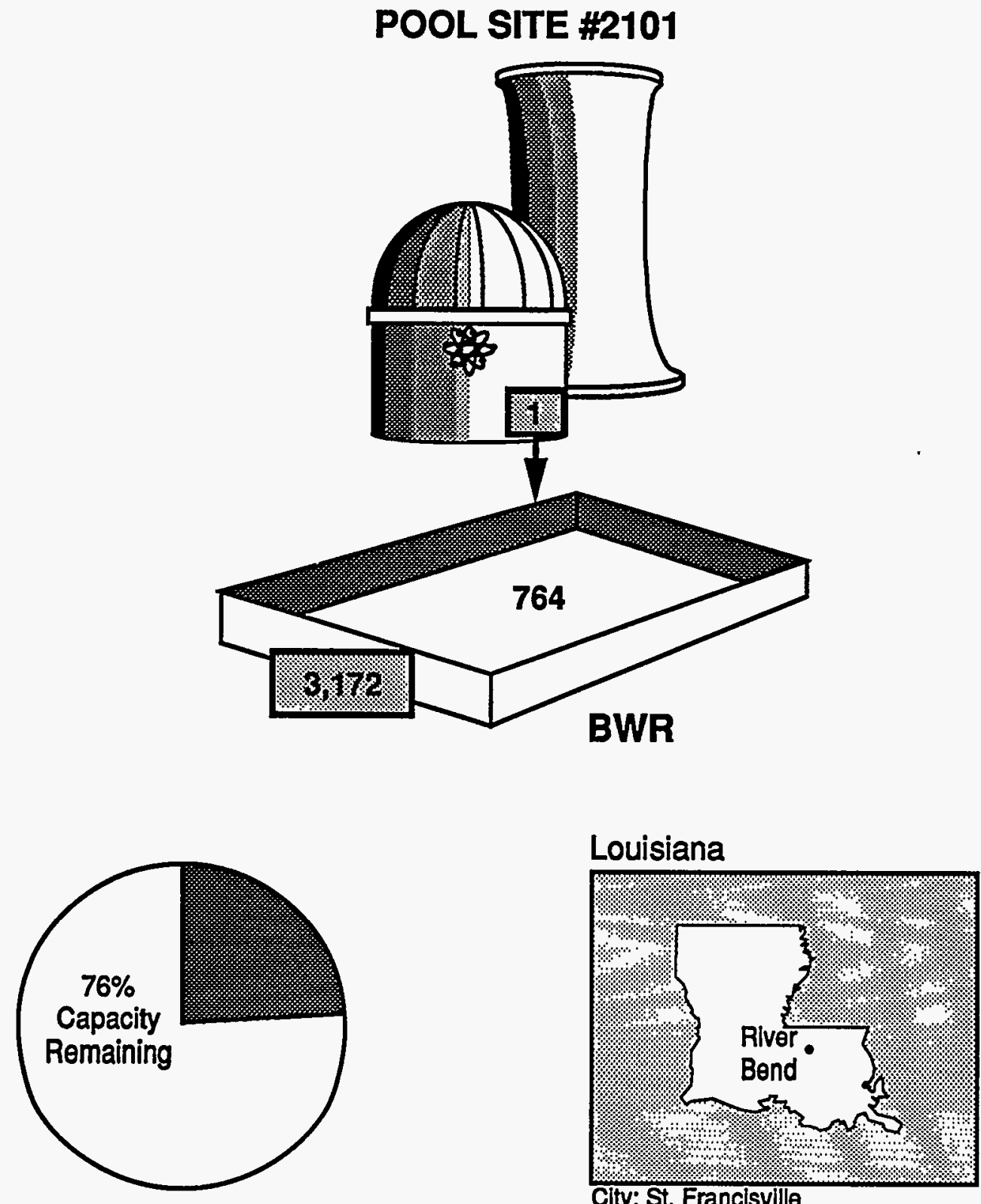

Clty: St. Francisville

County: West Felliciana 


\section{Houston Lighting and Power Company South Texas}
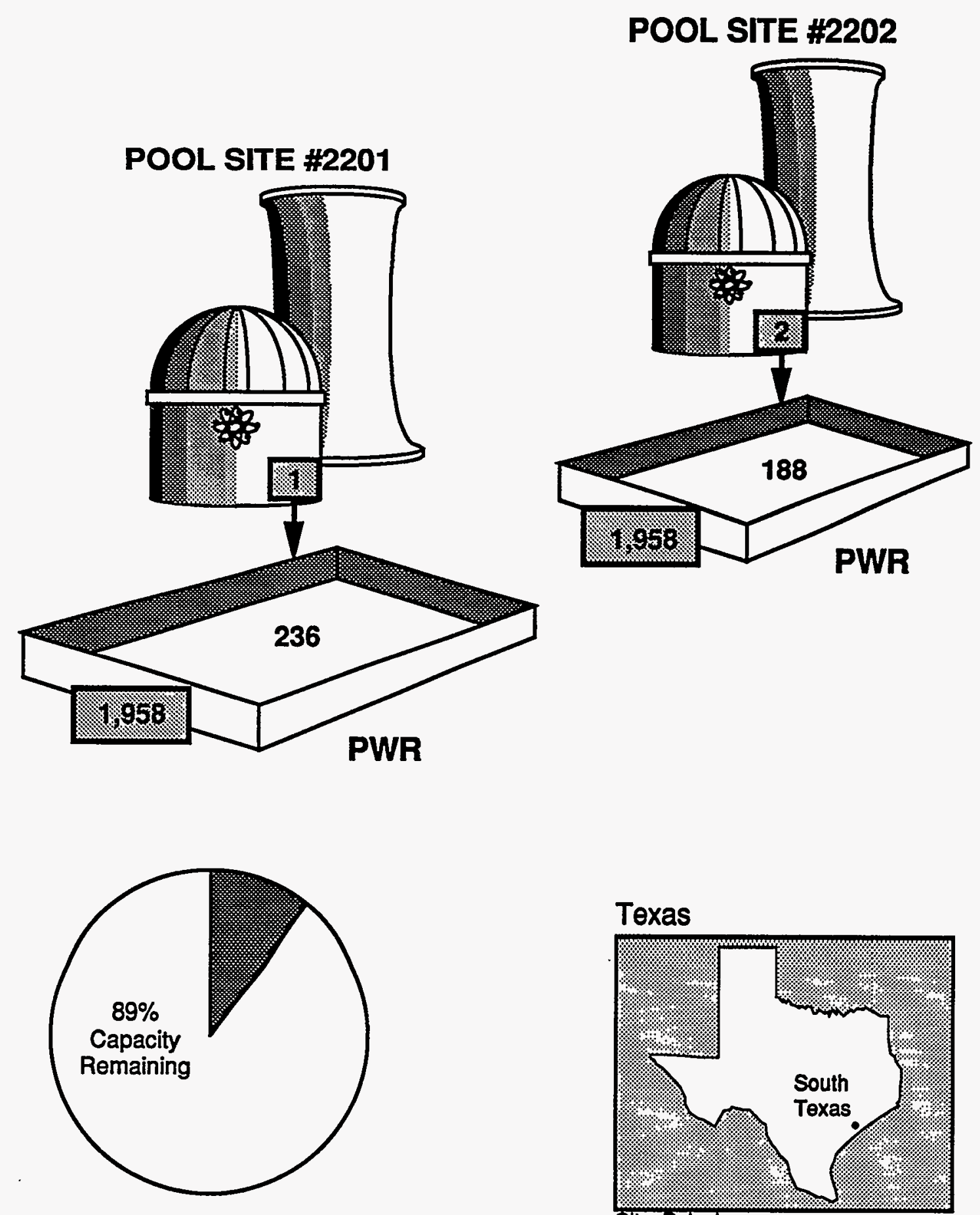

City: Palaclos

County: Matagorda 


\title{
IES Utilities, Inc.
}

\author{
Duane Arnold
}
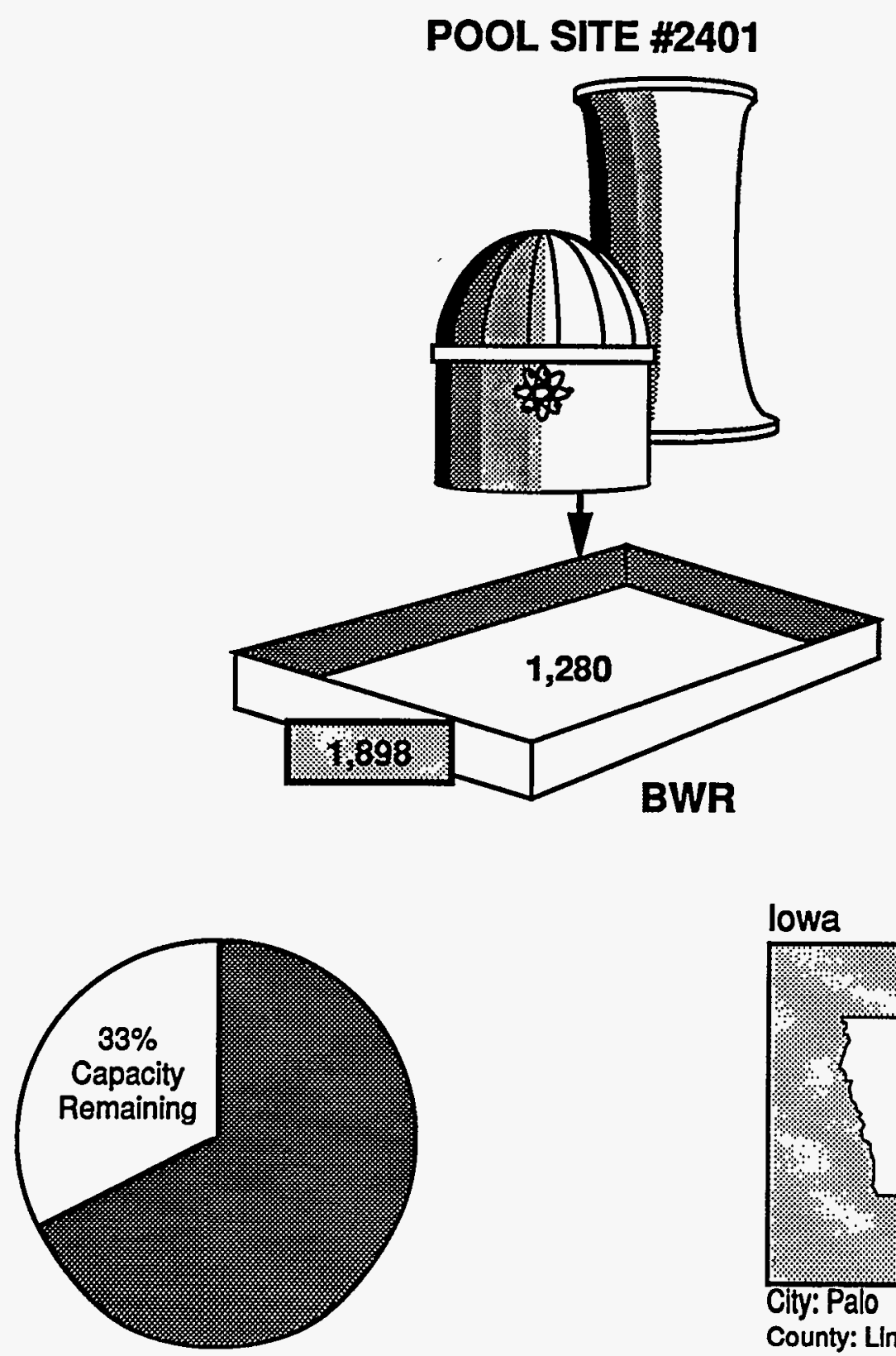

lowa

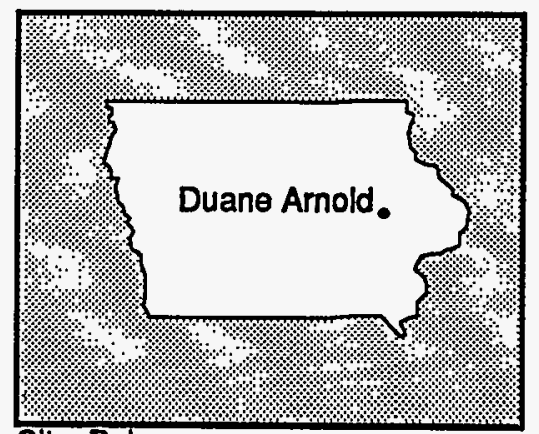

City: Palo

County: Linn 


\section{Illinois Power Company}

Clinton
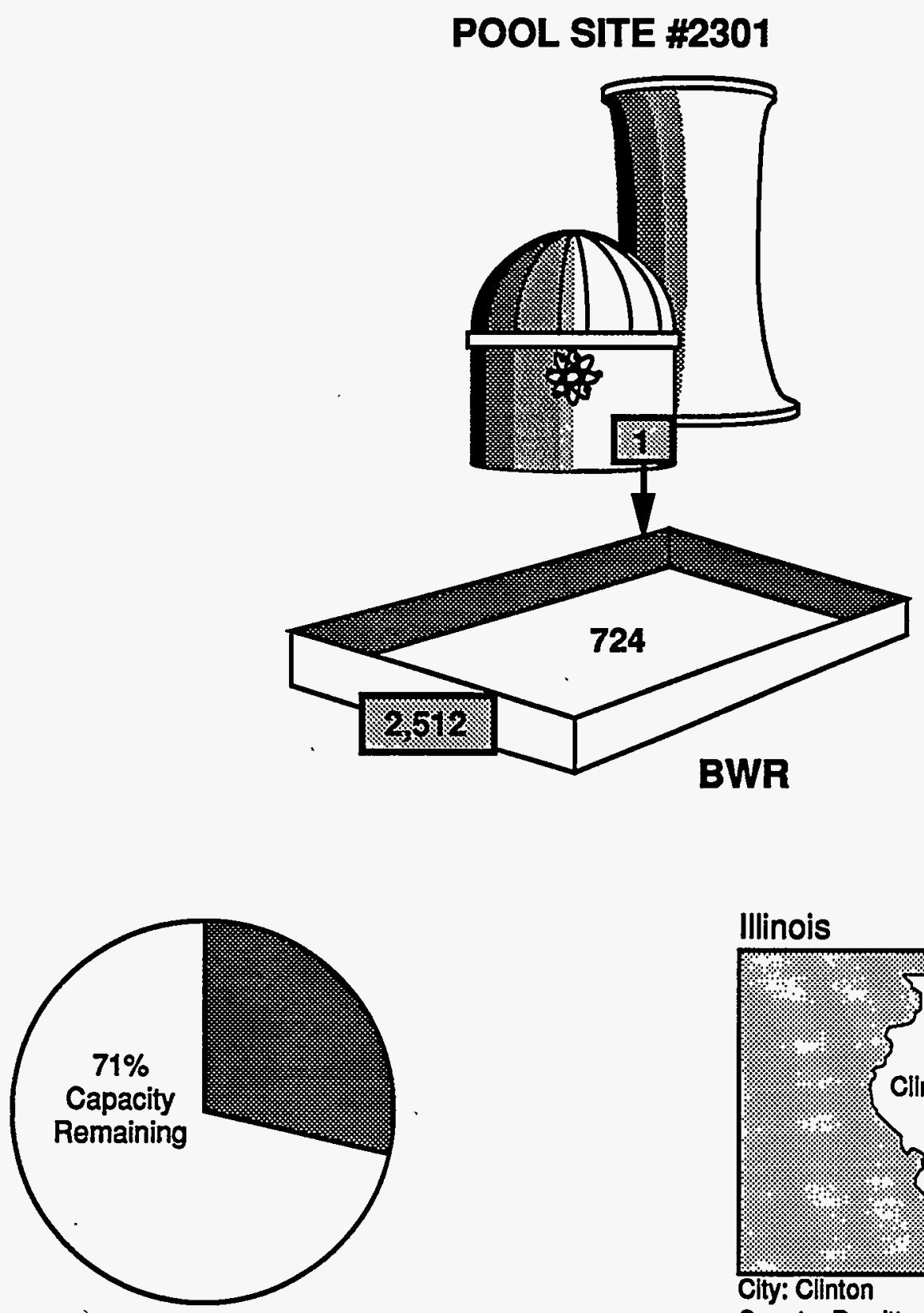

Illinois

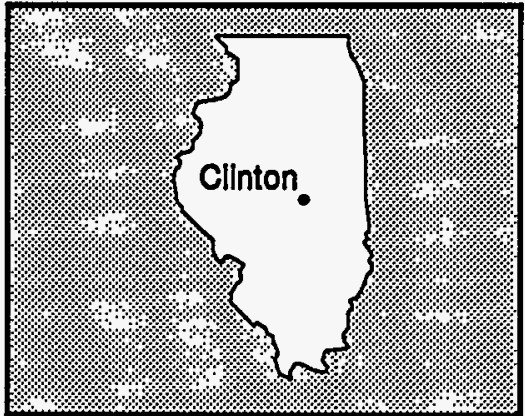

City: Clinton

County: Dowitt 


\section{Indiana Michigan Power Company}

Cook
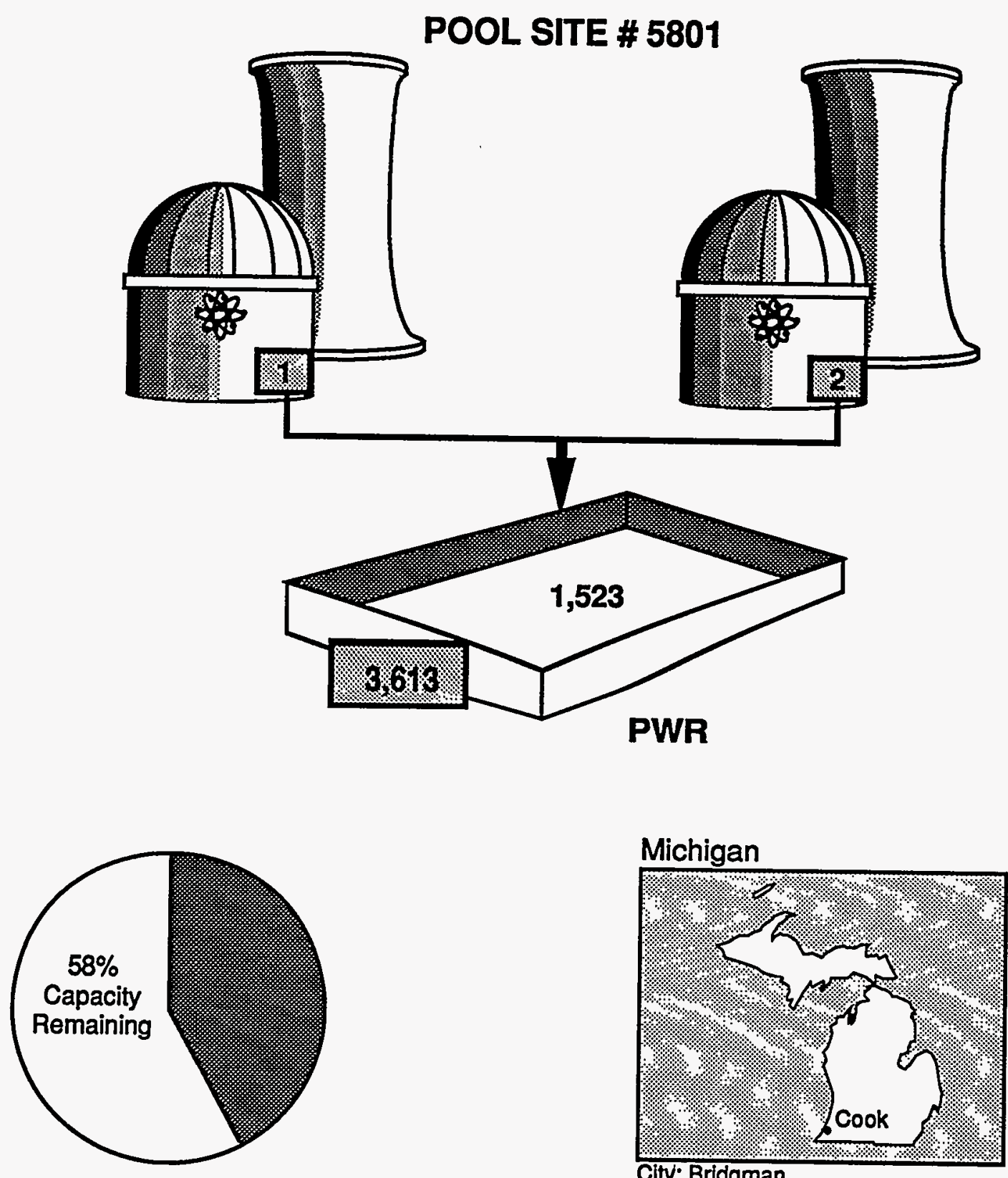

City: Bridgman

County: Berrien 


\section{Kansas Gas and Electric Company Wolf Creek}
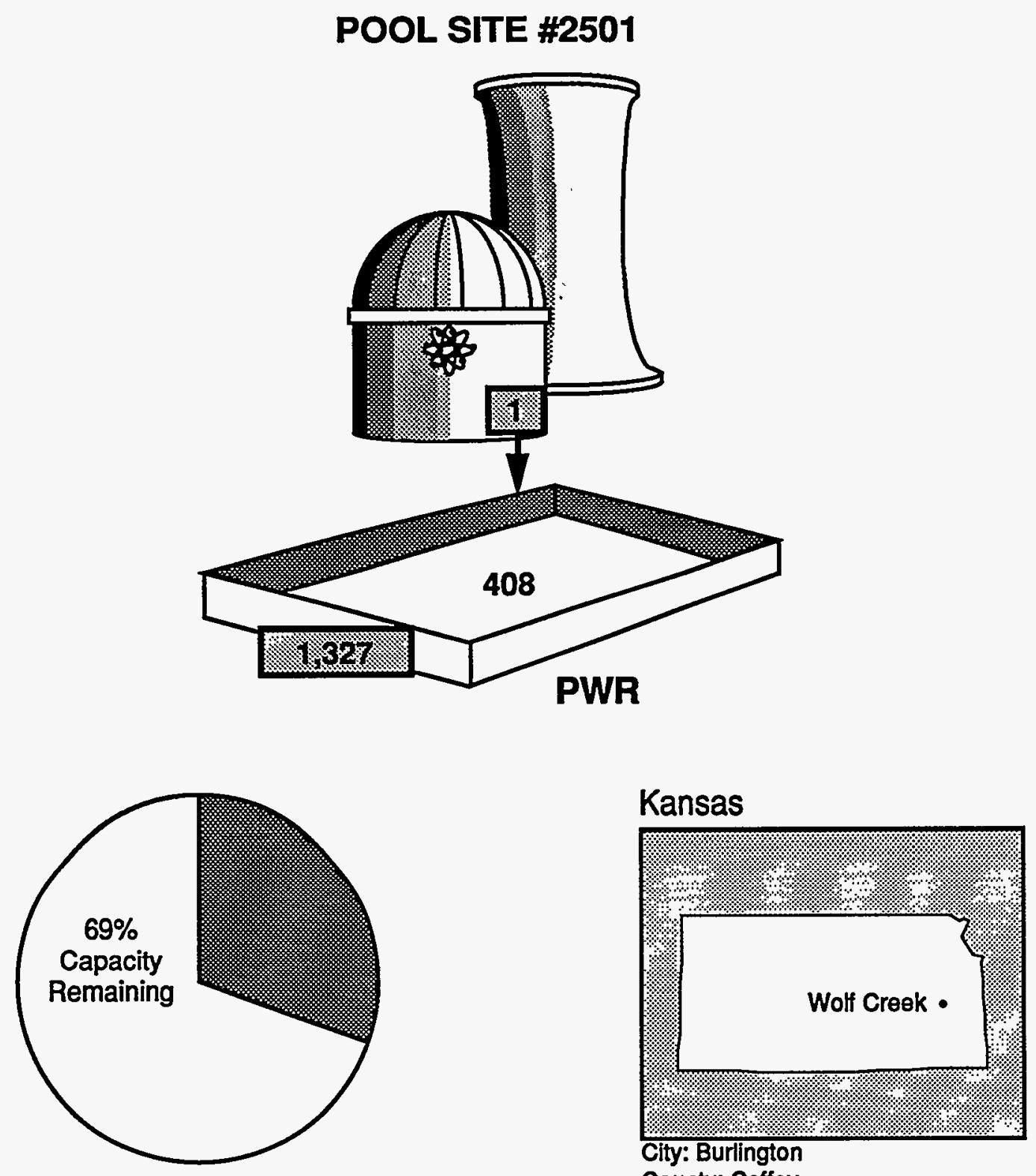

City: Burlington

County: Coffey 


\section{Long Island Power Authority . Shoreham}
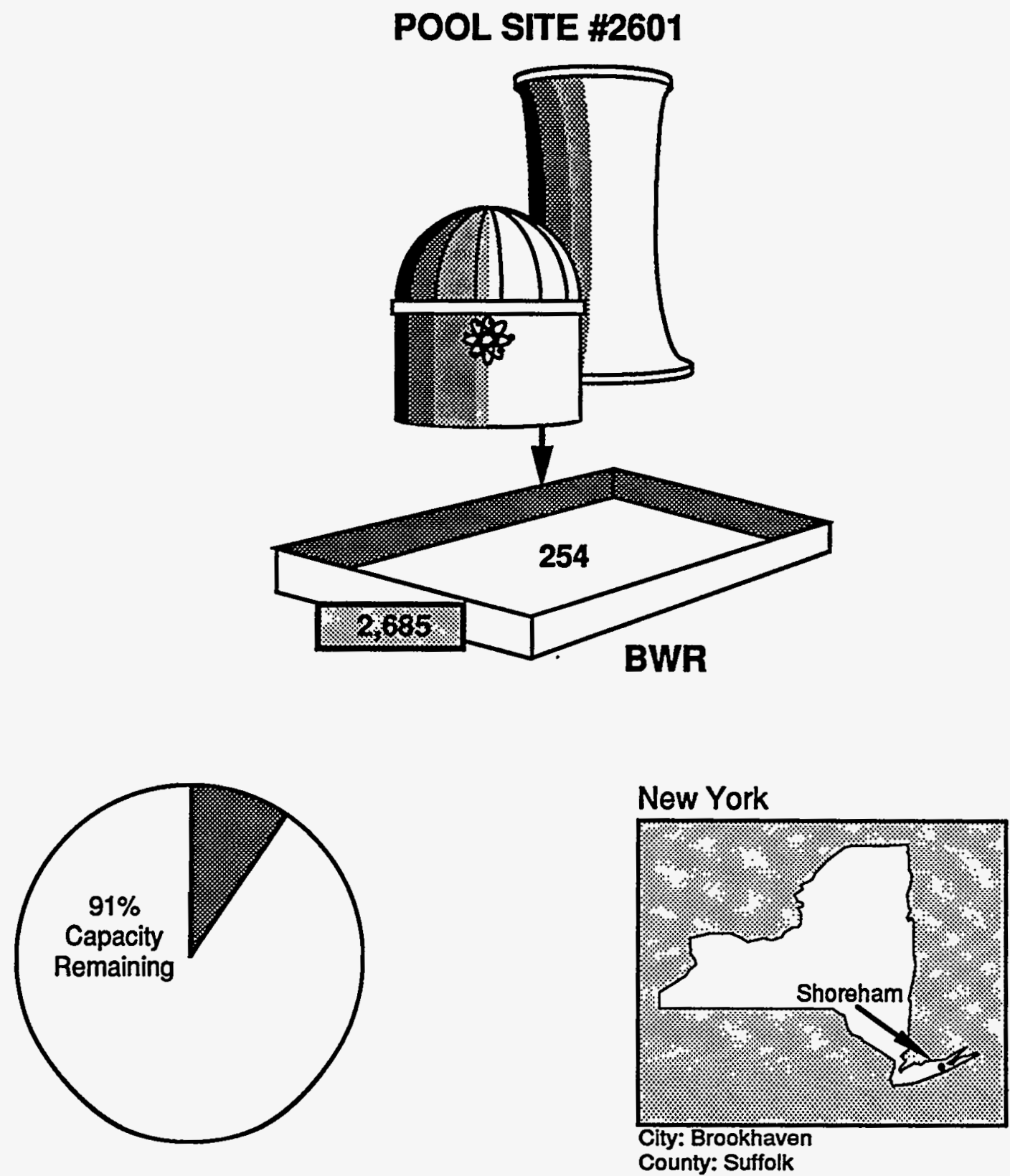


\title{
Louisiana Power and Light Company
}

\author{
Waterford 3
}

POOL SITE \#2701
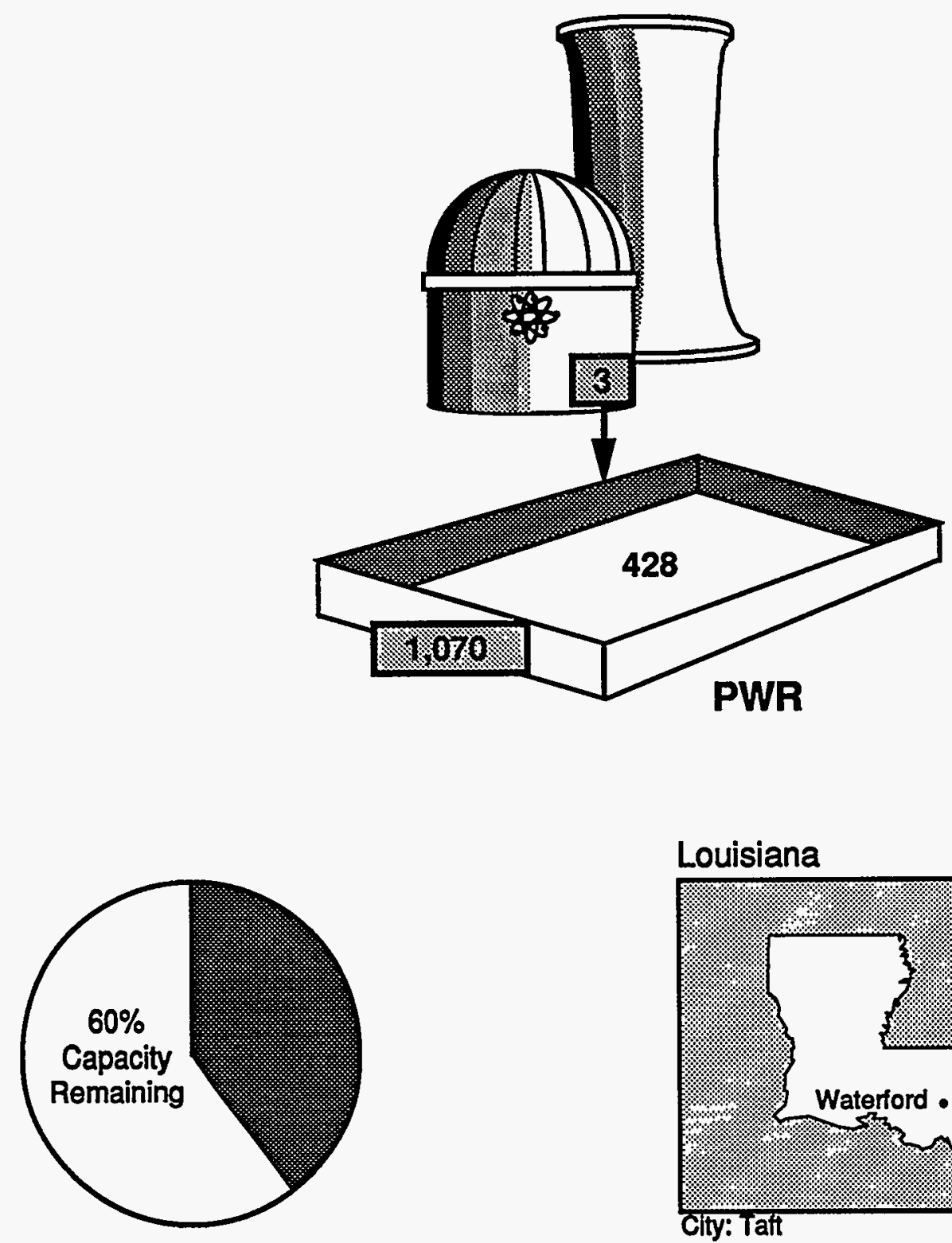

Louisiana

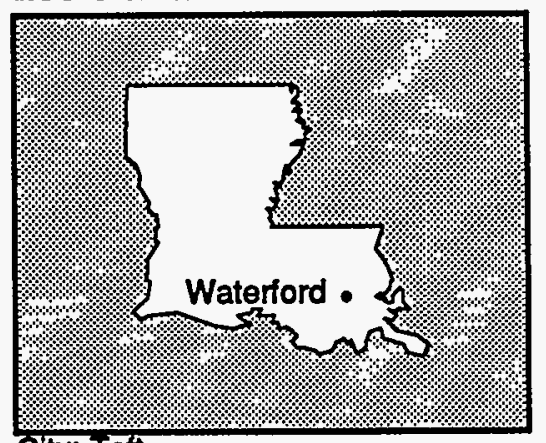

City: Taft

County: St. Charles 


\title{
Maine Yankee Atomic Power Company
}

\author{
Maine Yankee
}

POOL SITE \#2801
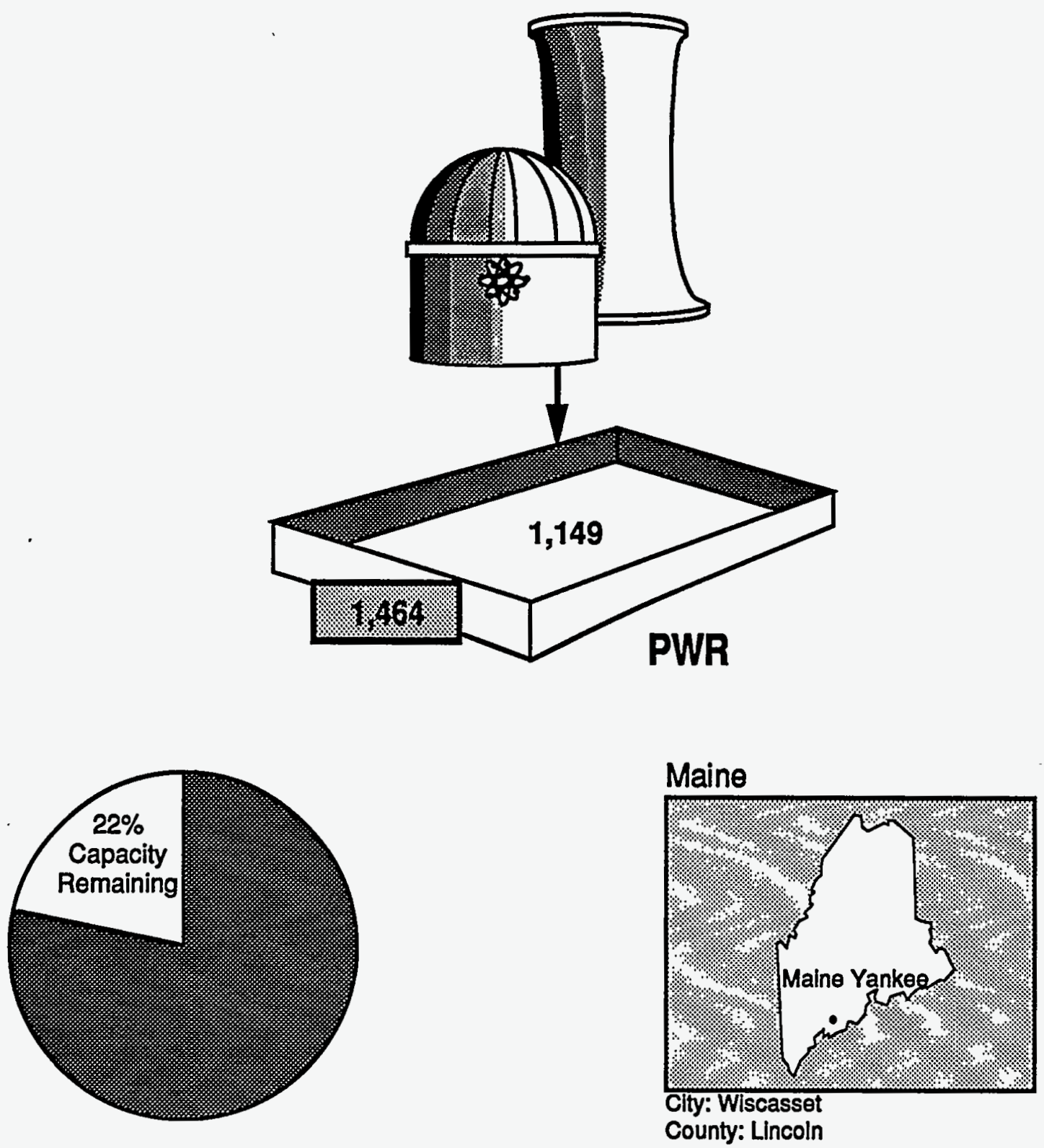


\section{Nebraska Public Power District Cooper Station}
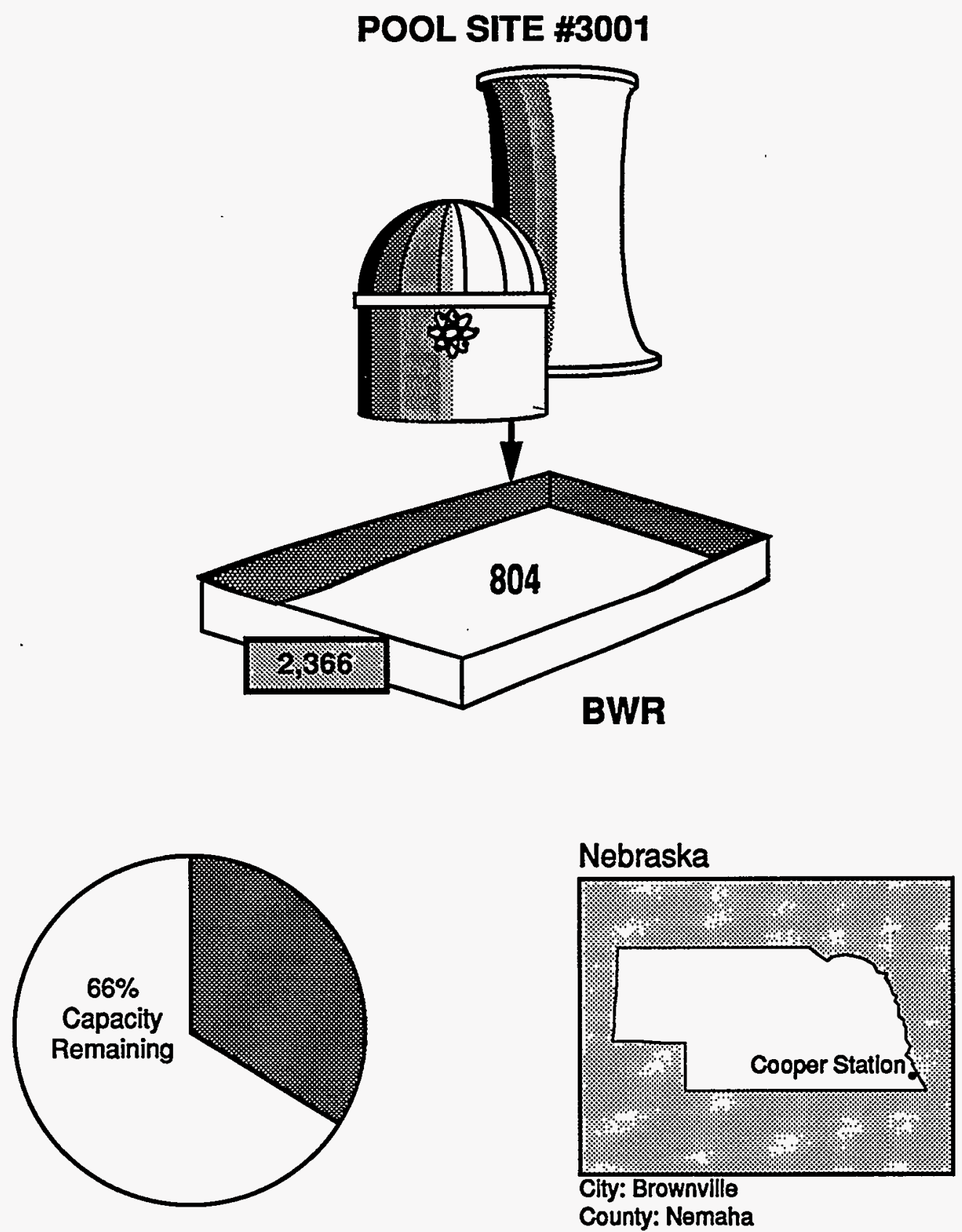


\section{New York Power Authority}

\section{FitzPatrick}
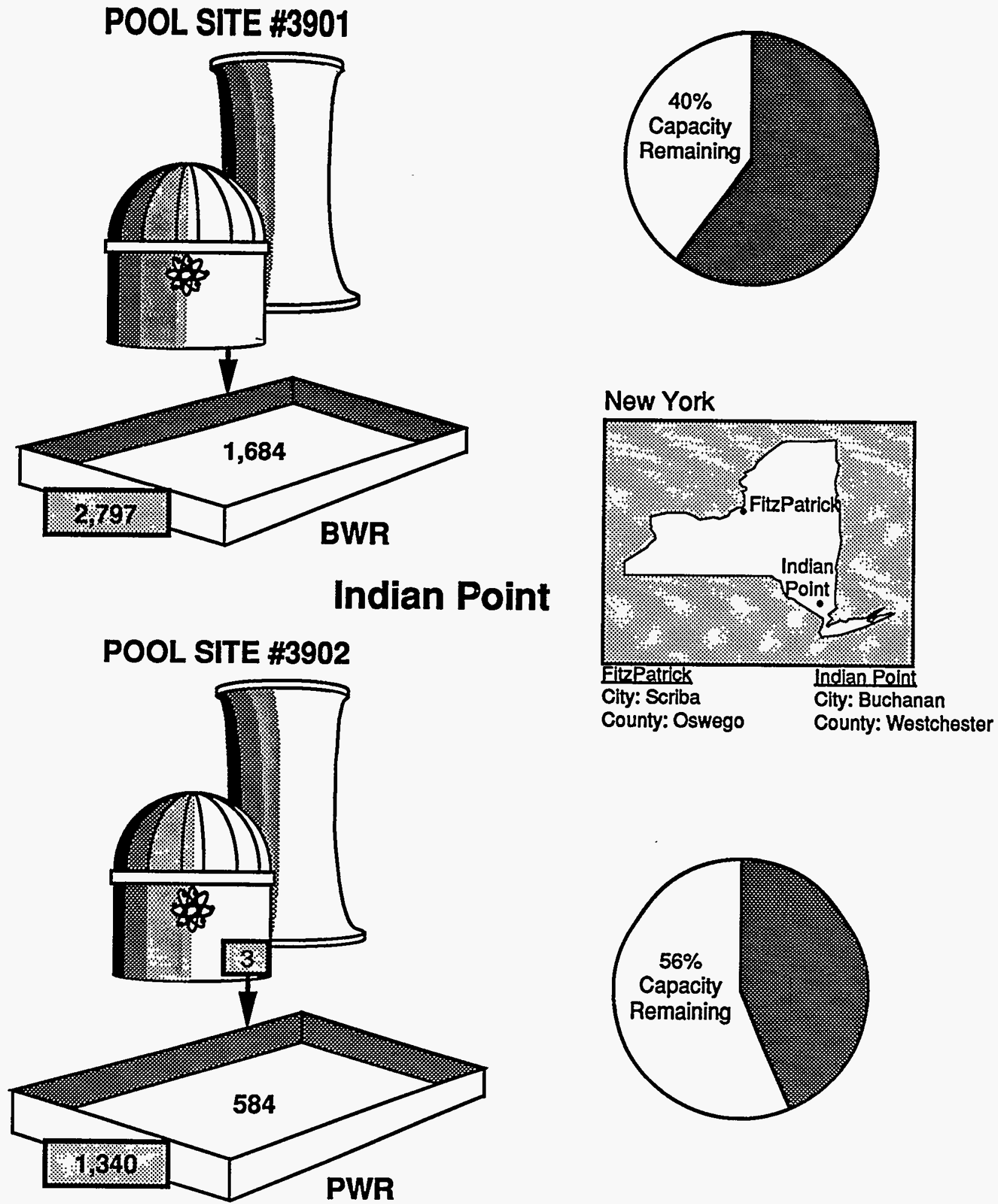


\title{
Niagara Mohawk Power Corporation
}

\author{
Nine Mile Point
}
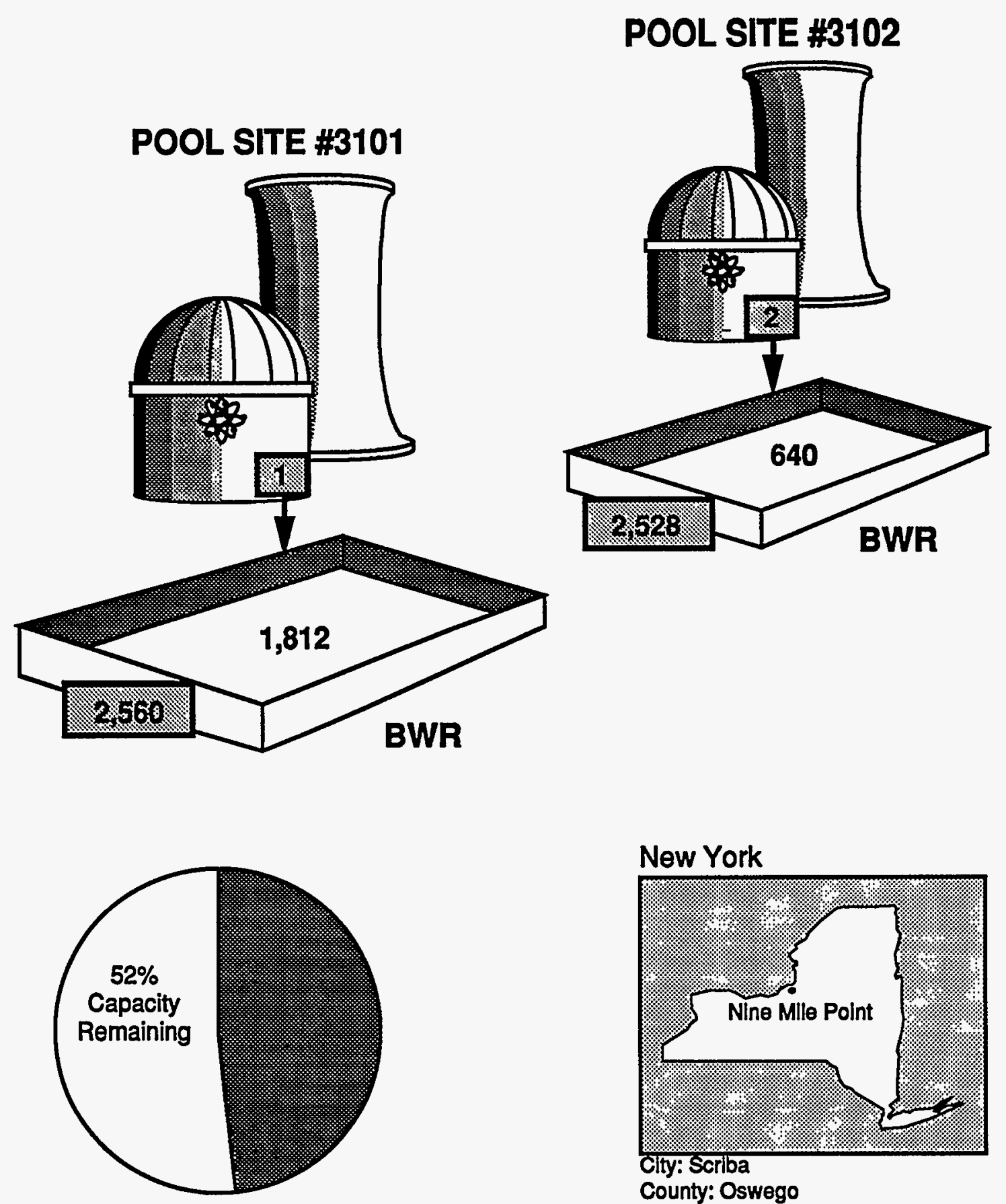

New York

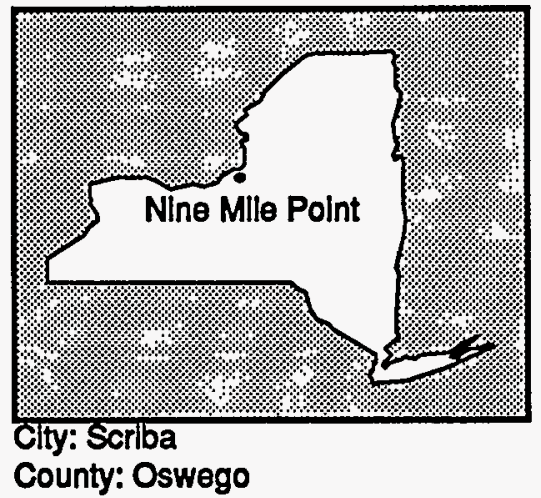




\section{North Atlantic Energy Service Corporation \\ Seabrook}

POOL SITE \#5901
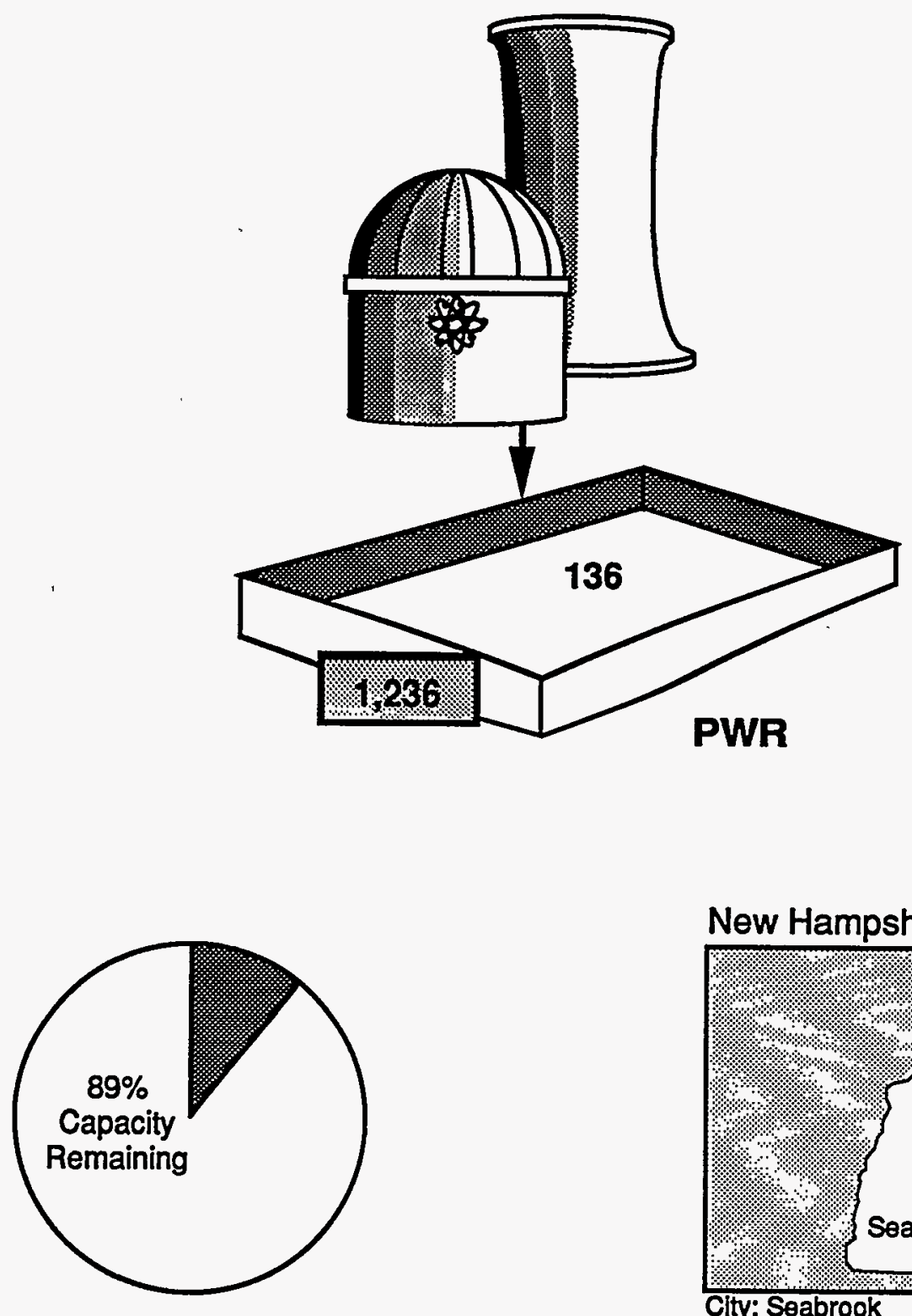

New Hampshire

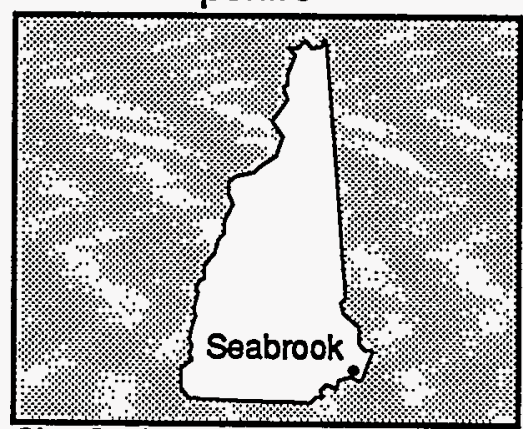

Clty: Seabrook

County: Rockingham 


\section{Northeast Utilities Service Company}

\section{Millstone}

POOL SITE \#3202

\section{POOL SITE \#3201}

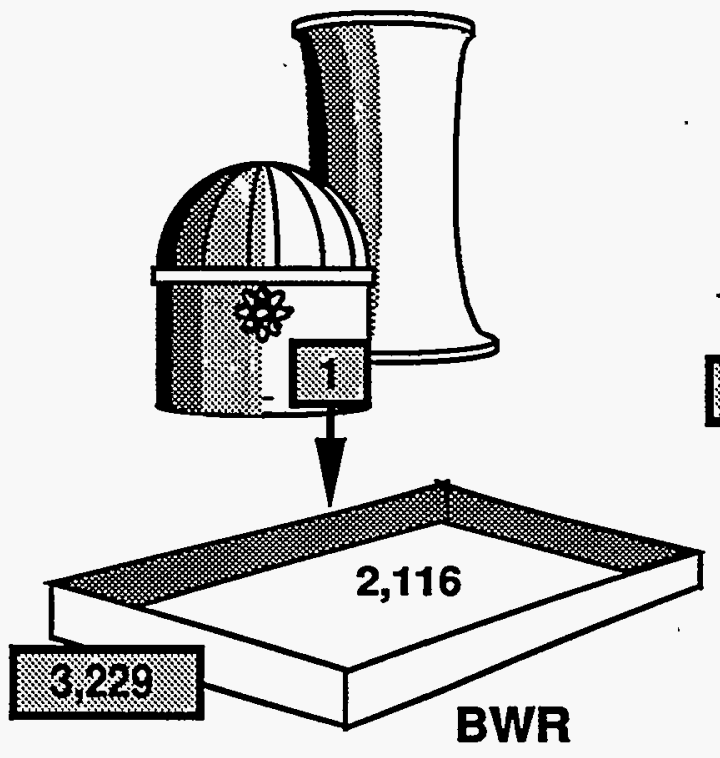

Haddam Neck
POOL SITE \#3203
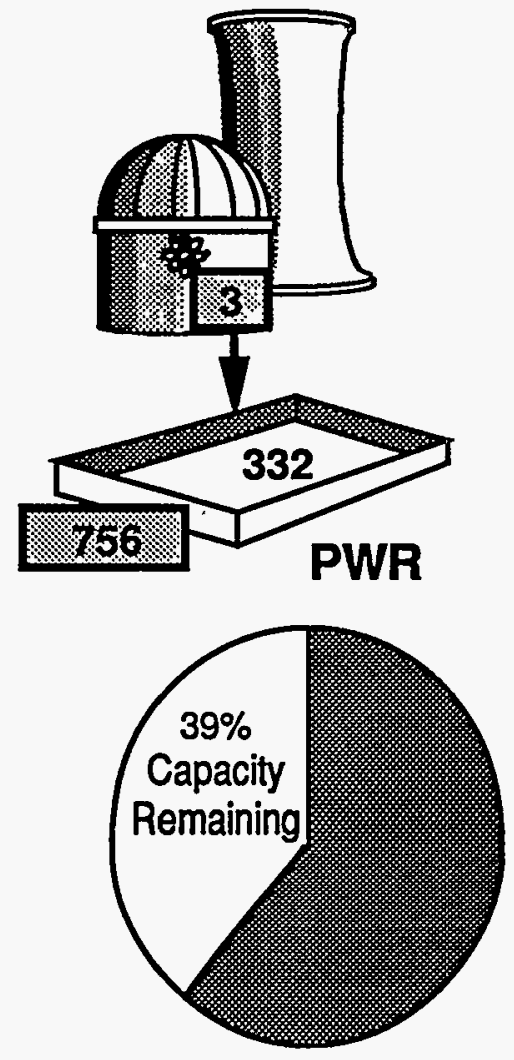

Connecticut
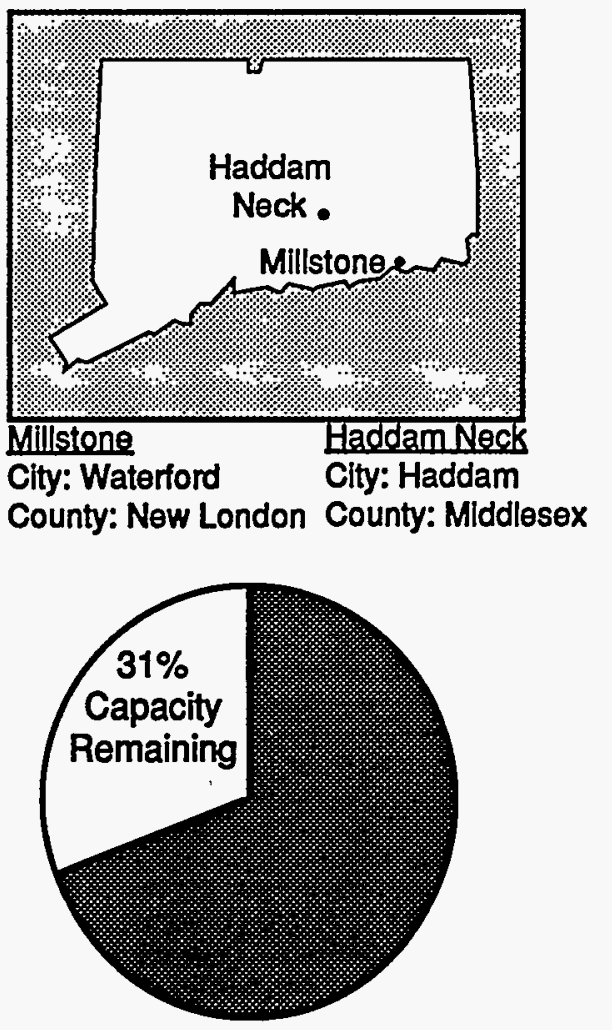


\section{Northern States Power Company}

\section{Monticello}

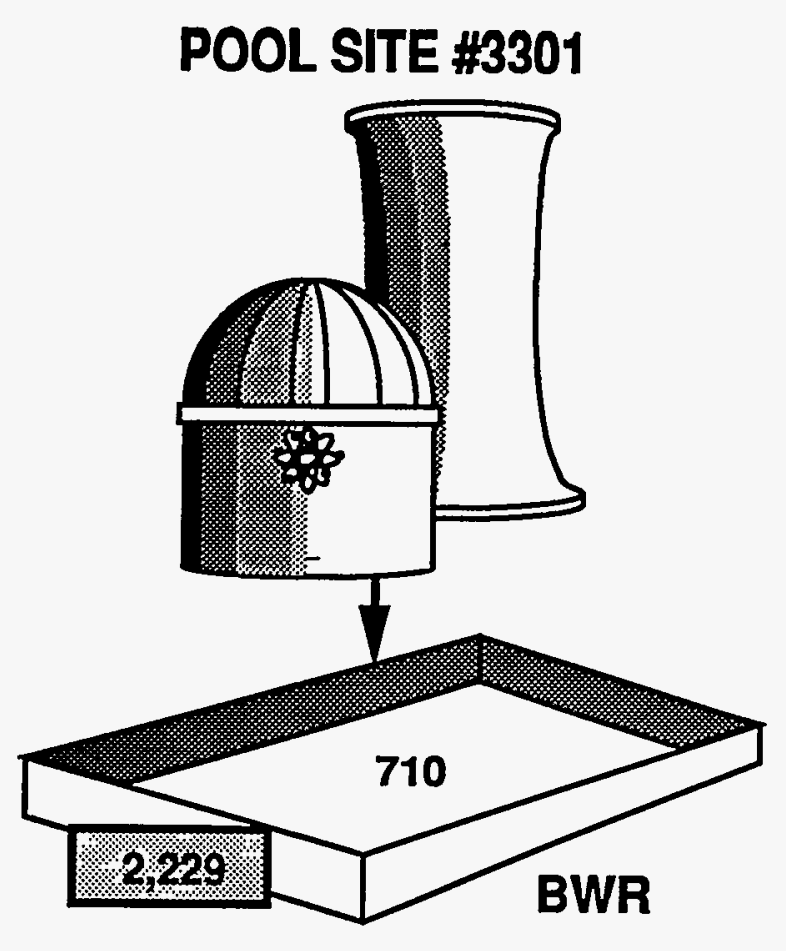

\section{Prairie Island}

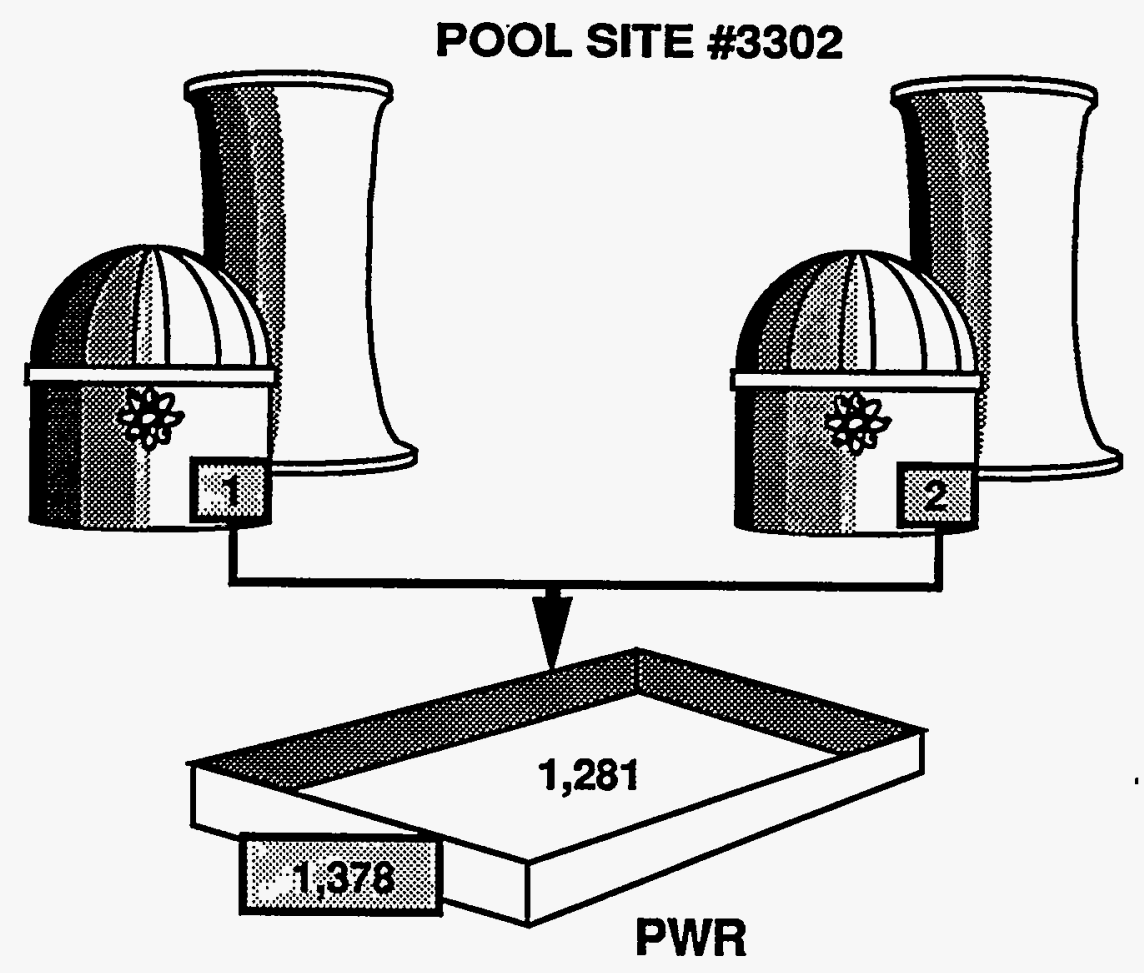

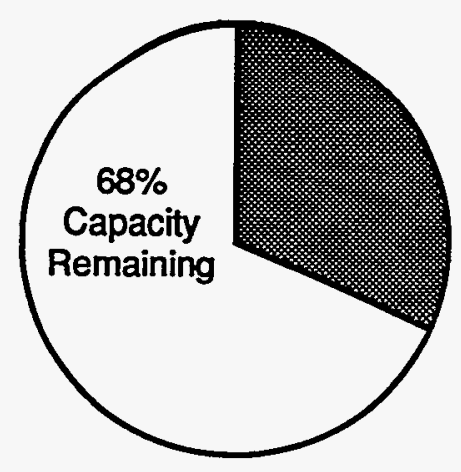

Minnesota

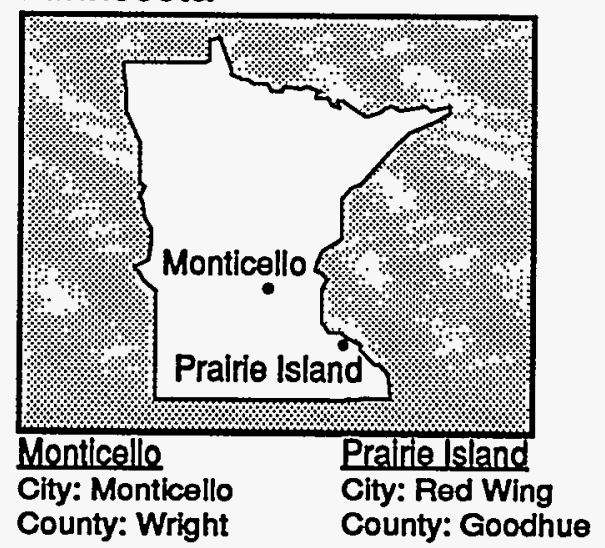




\title{
Omaha Public Power District Fort Calhoun
}

\author{
POOL SITE \#3401
}
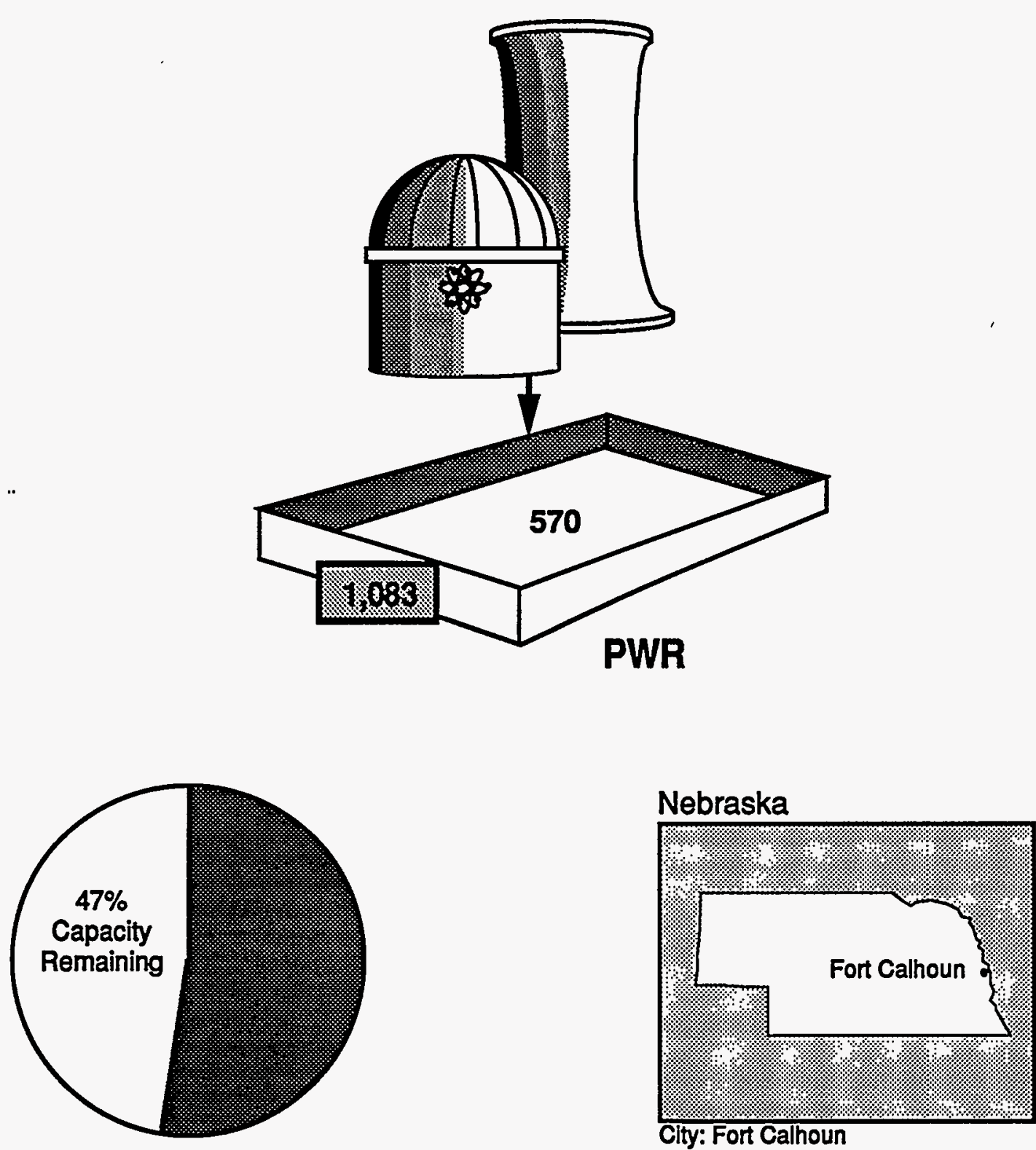

City: Fort Calhoun

County: Washington 


\section{Pacific Gas and Electric Company}

\section{Diablo Canyon}
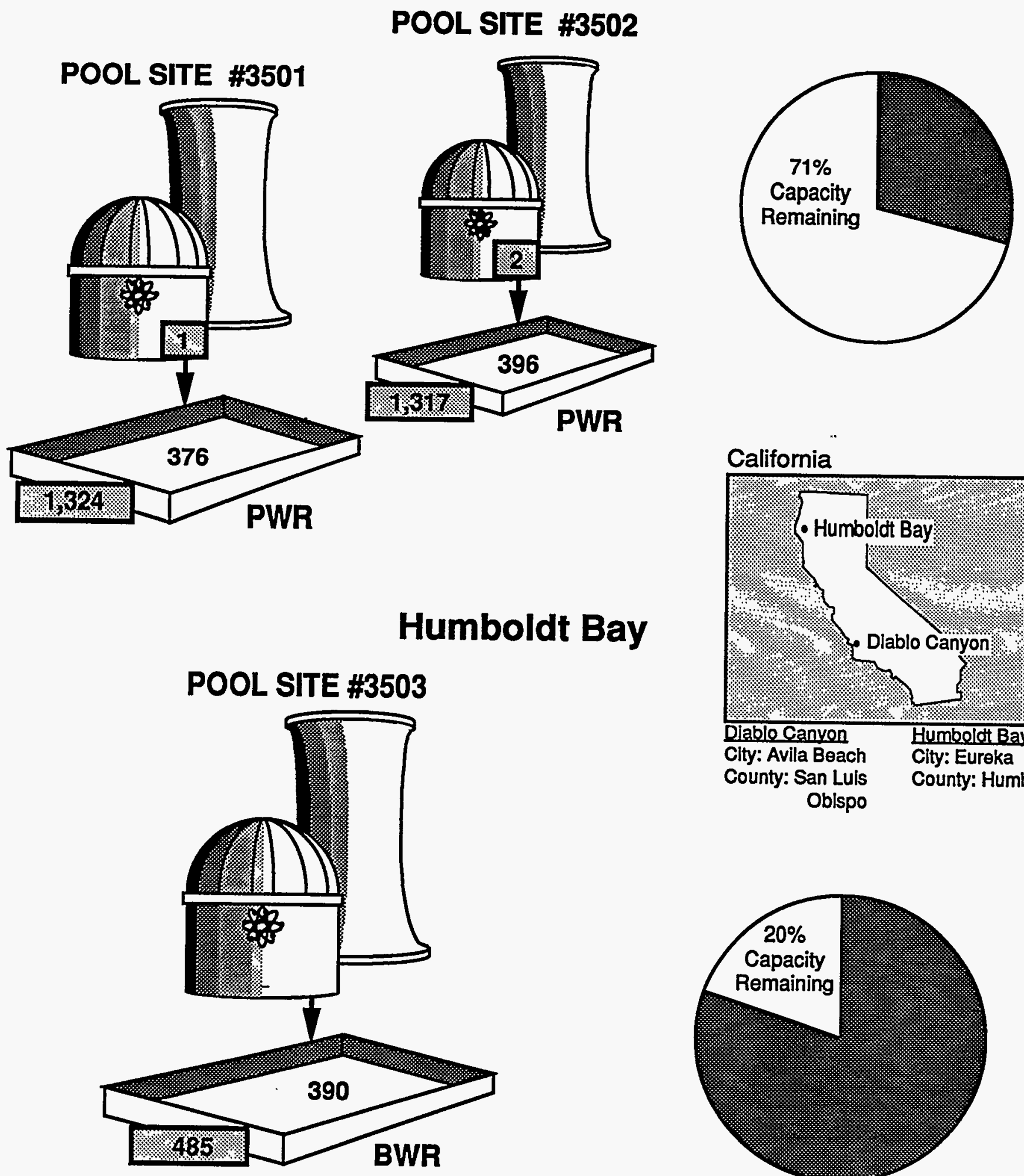

California

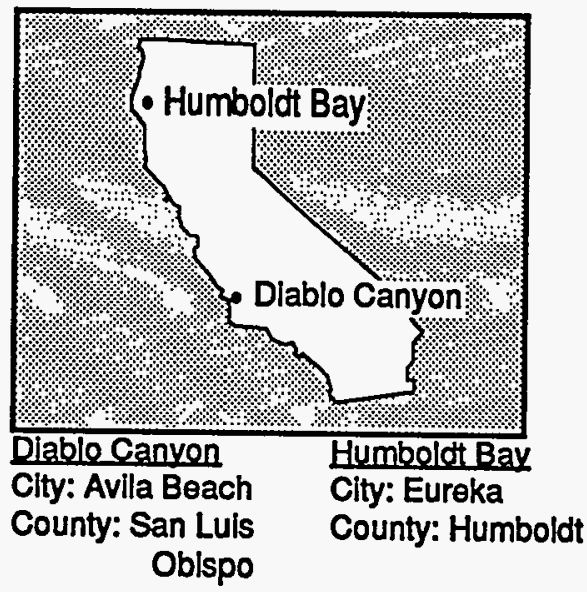




\section{PECO Energy Company}

\section{Limerick}

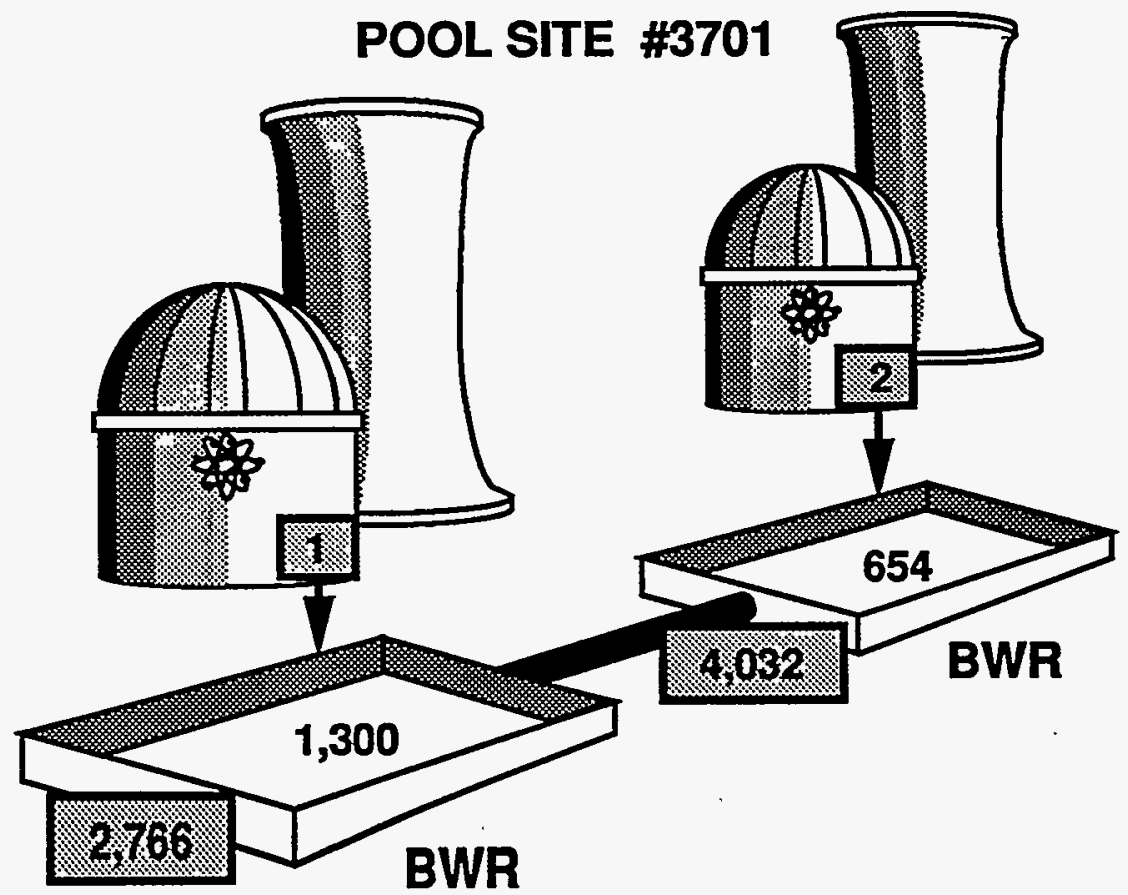

Peach Bottom

POOL SITE \#3704

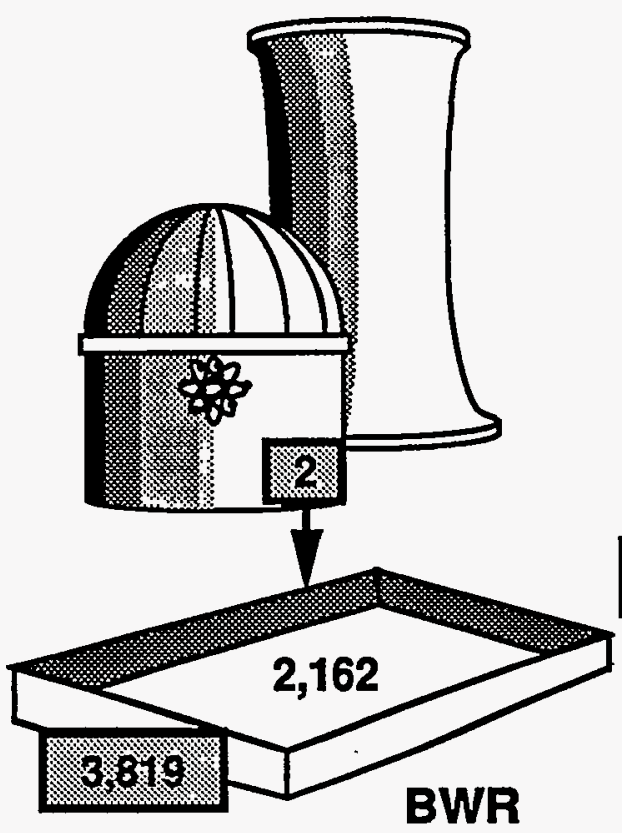

POOL SITE \#3705
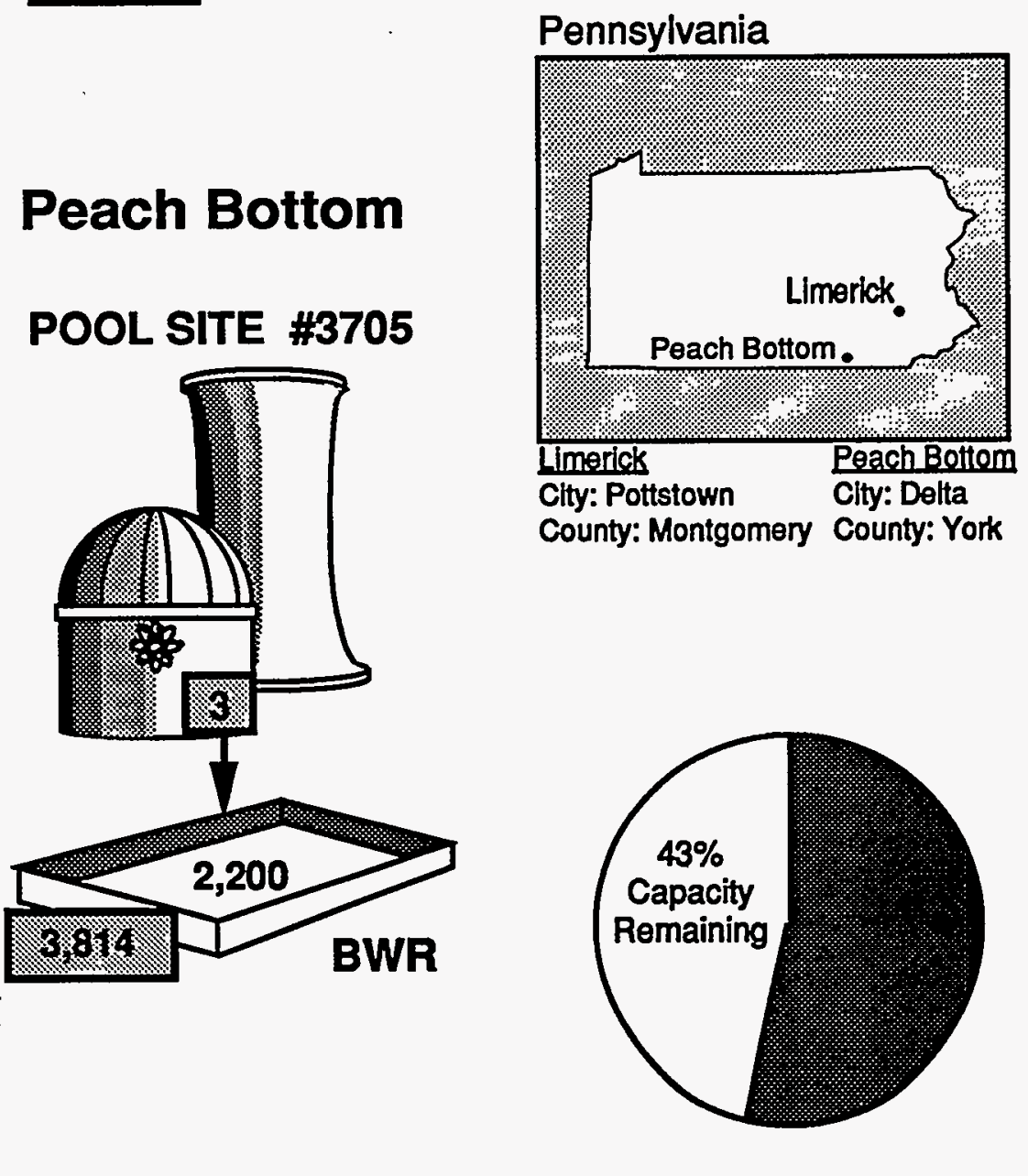


\section{Pennsylvania Power and Light Company}

\section{Susquehanna}
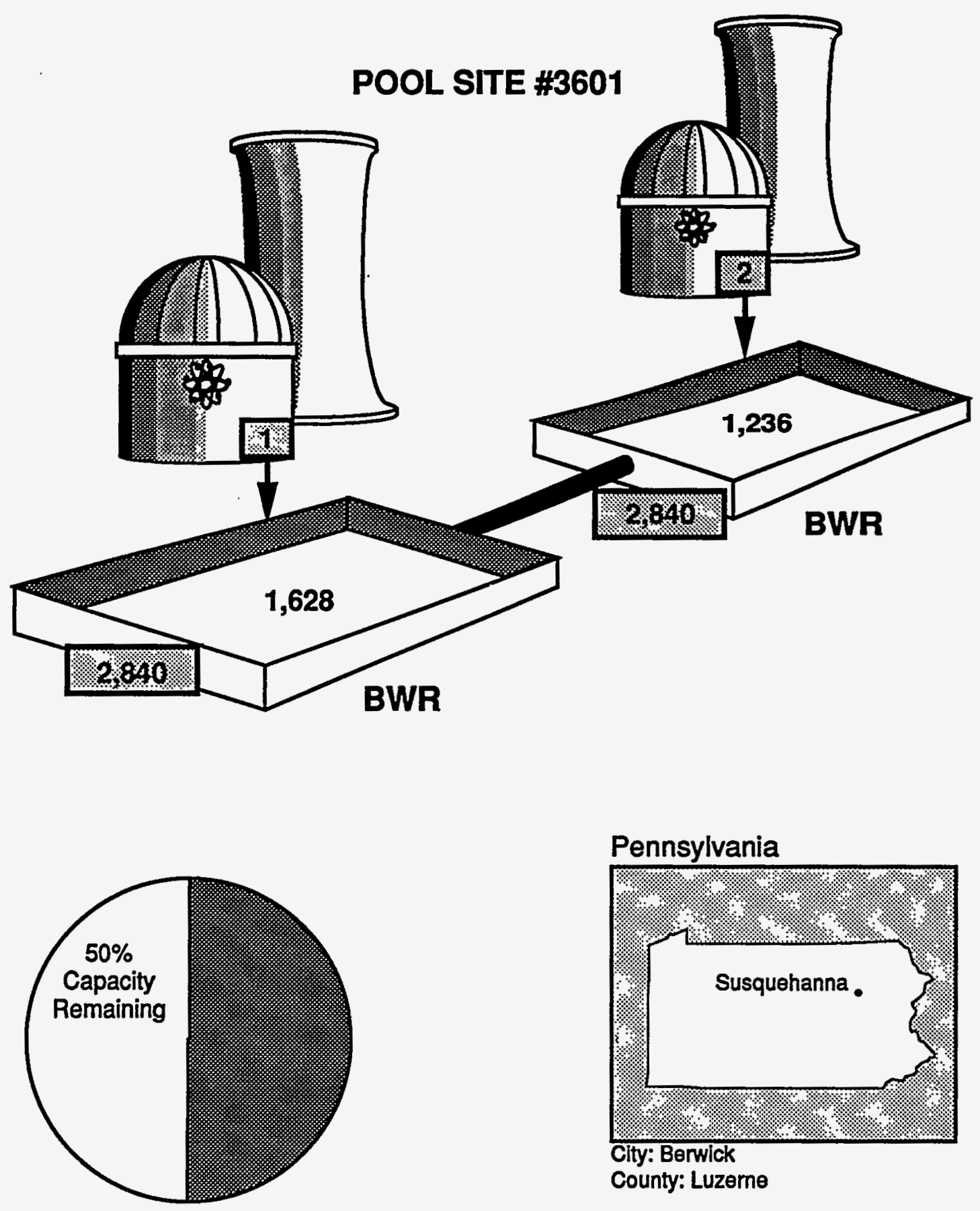

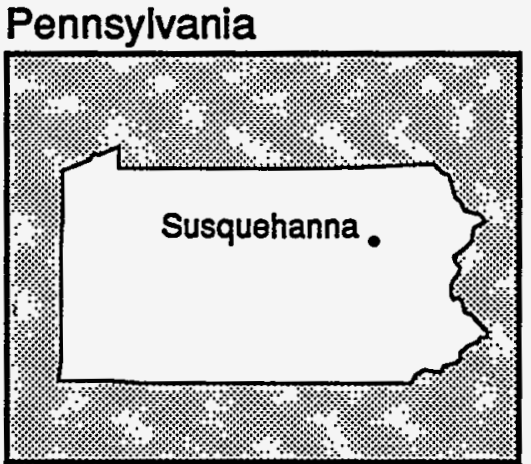

City: Berwick County: Luzerne 


\title{
Portland General Electric Company
}

\author{
Trojan
}

POOL SITE \#3801
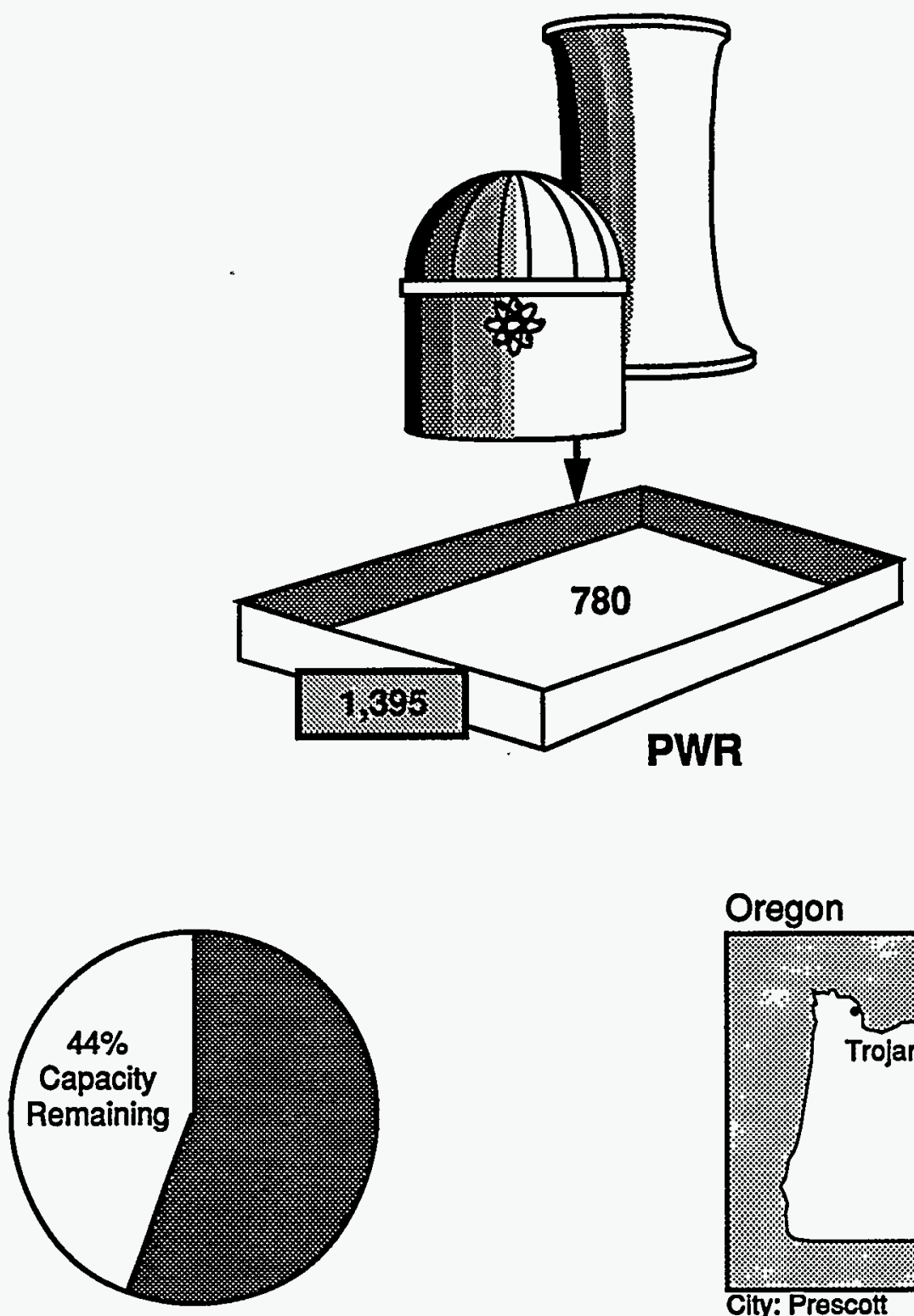

Oregon

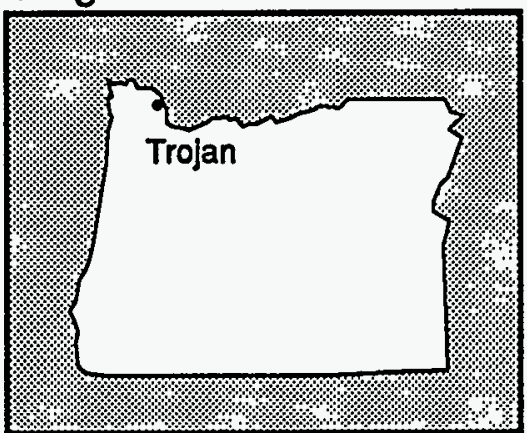

City: Prescott

County: Columbla 


\section{Public Service Electric and Gas Company}

POOL SITE \#4201

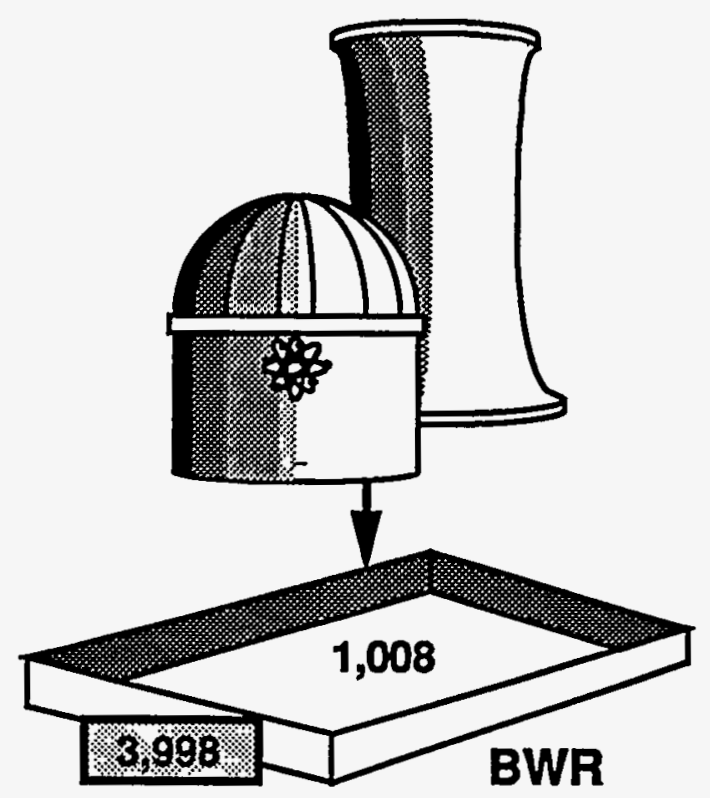

\section{POOL SITE \#4202}

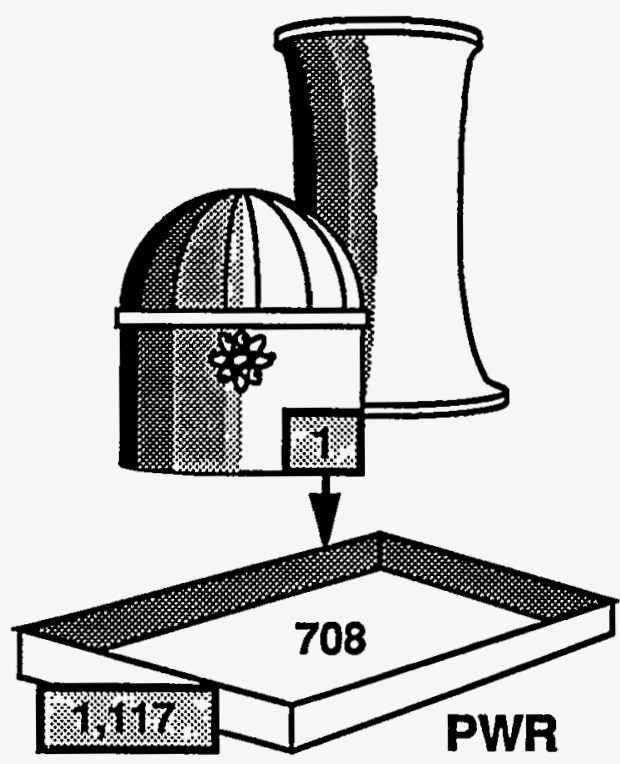

\section{Salem}

\section{Hope Creek}

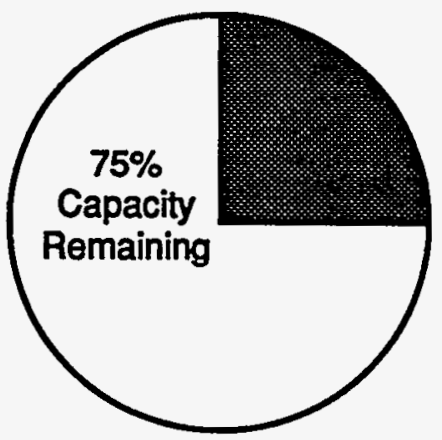

New Jersey

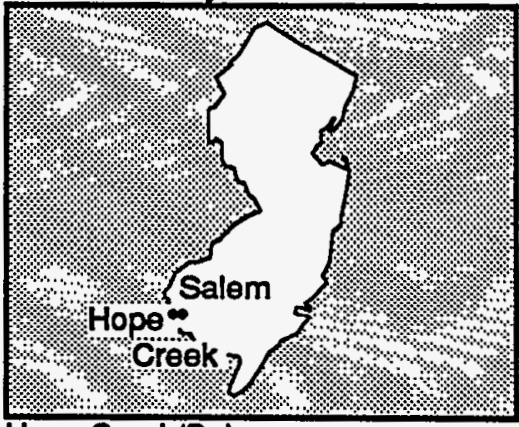

Hope Creek/Salem

City: Lower Alloways

County: Salem

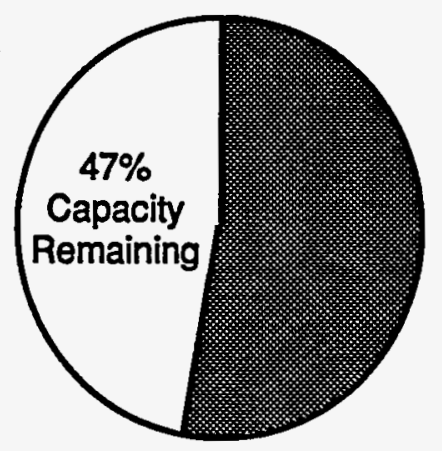




\title{
Rochester Gas and Electric Corporation
}

\author{
Ginna
}
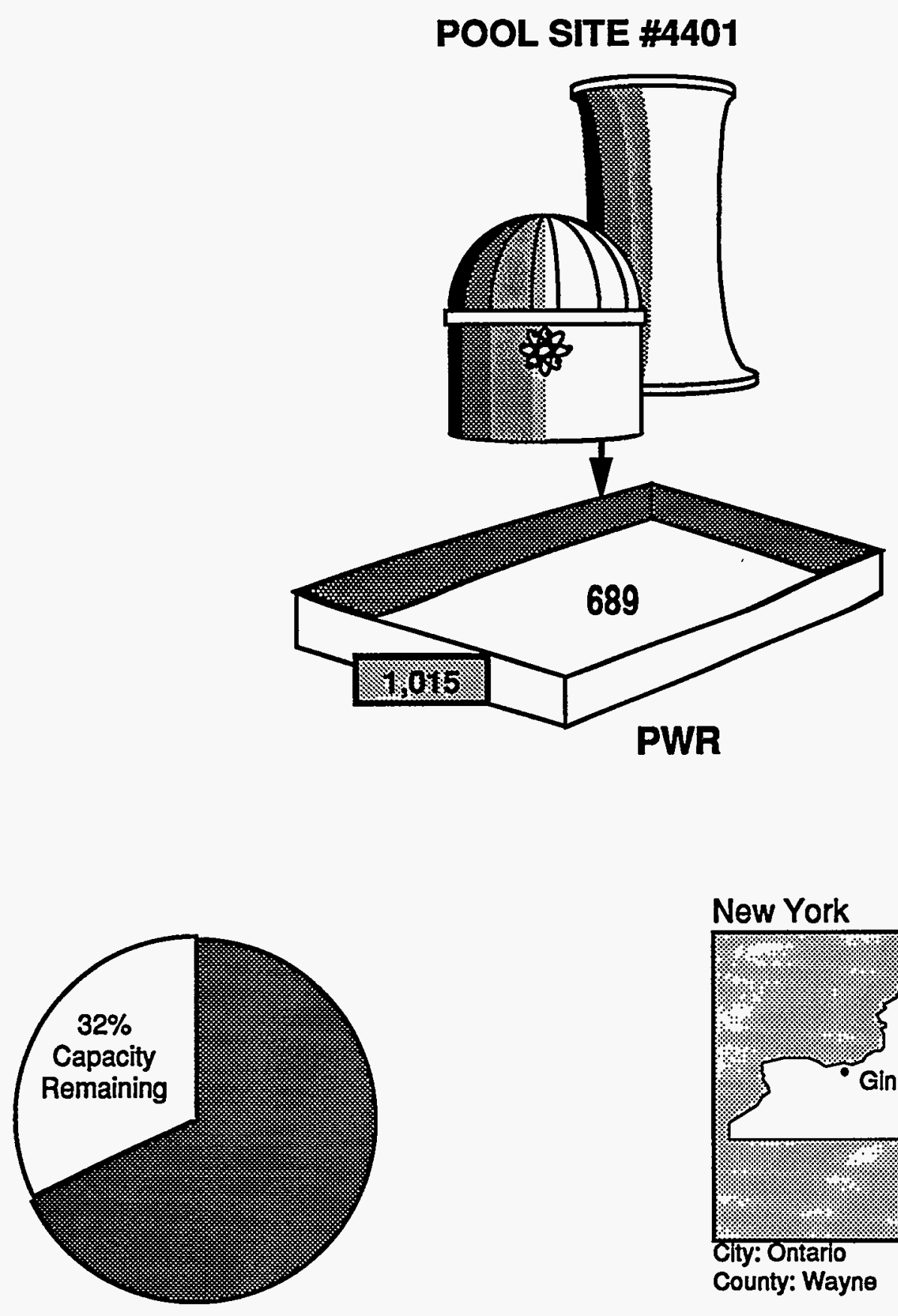

New York

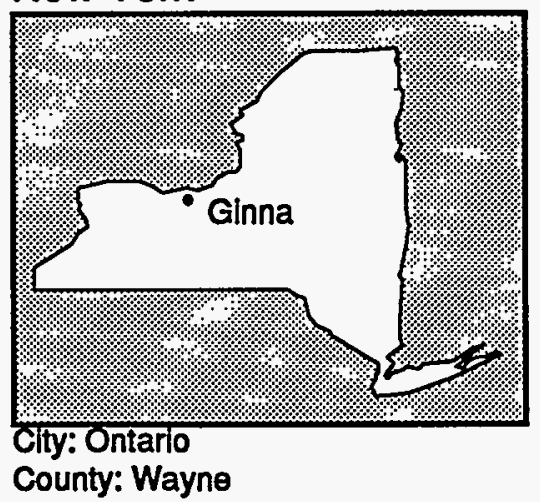




\section{Sacramento Municipal Utility District \\ Rancho Seco}
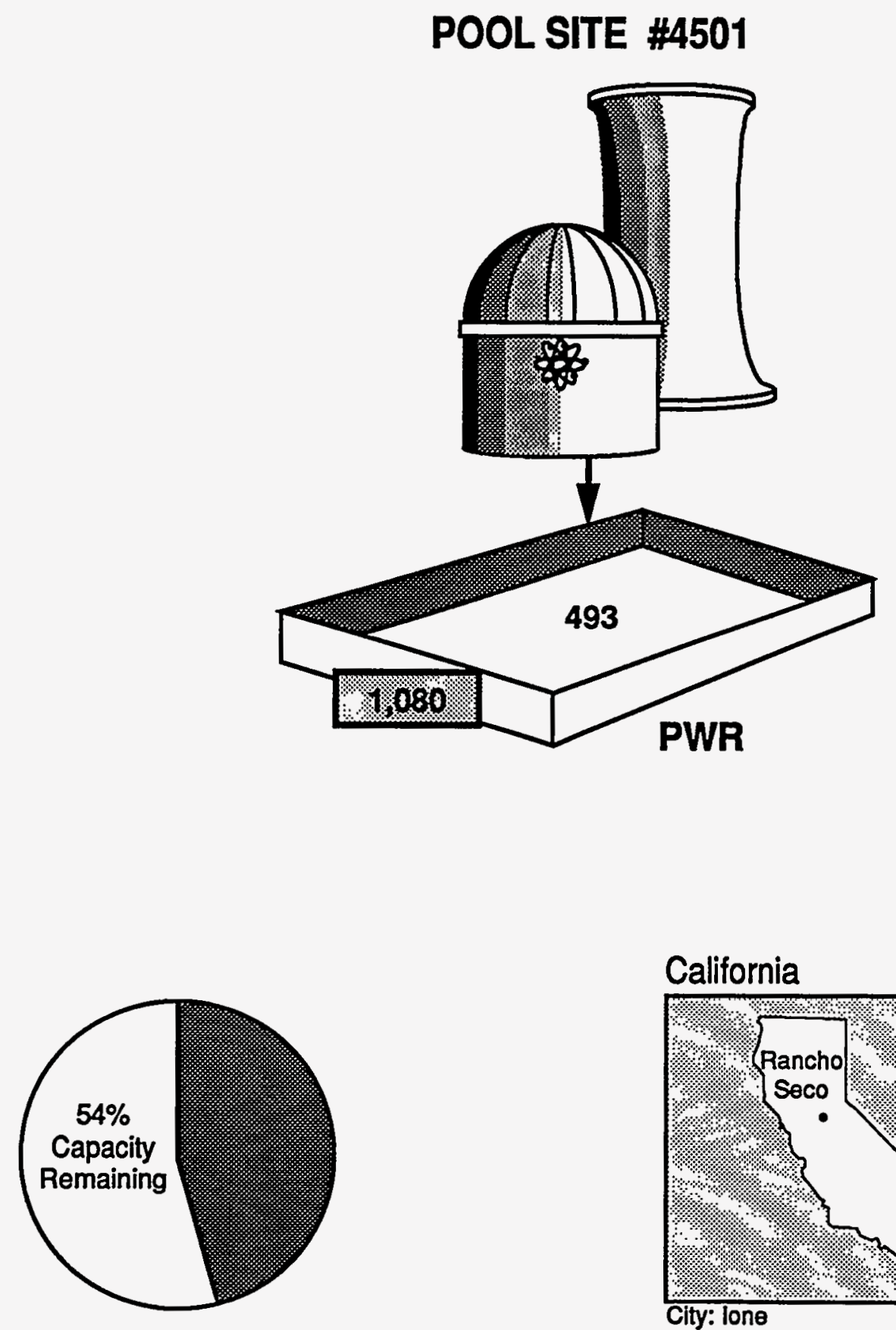

California

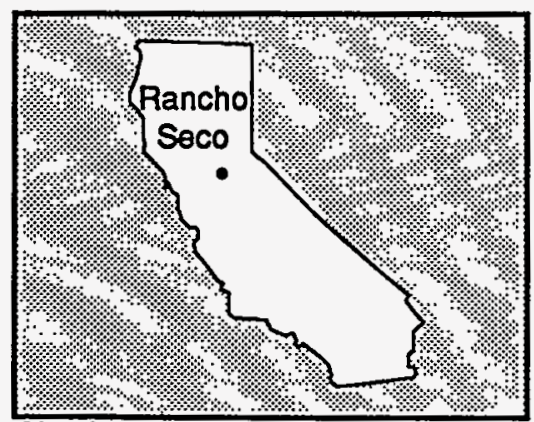

City: Ione

County: Sacramento 


\title{
South Carolina Electric and Gas Company
}

\author{
Summer
}
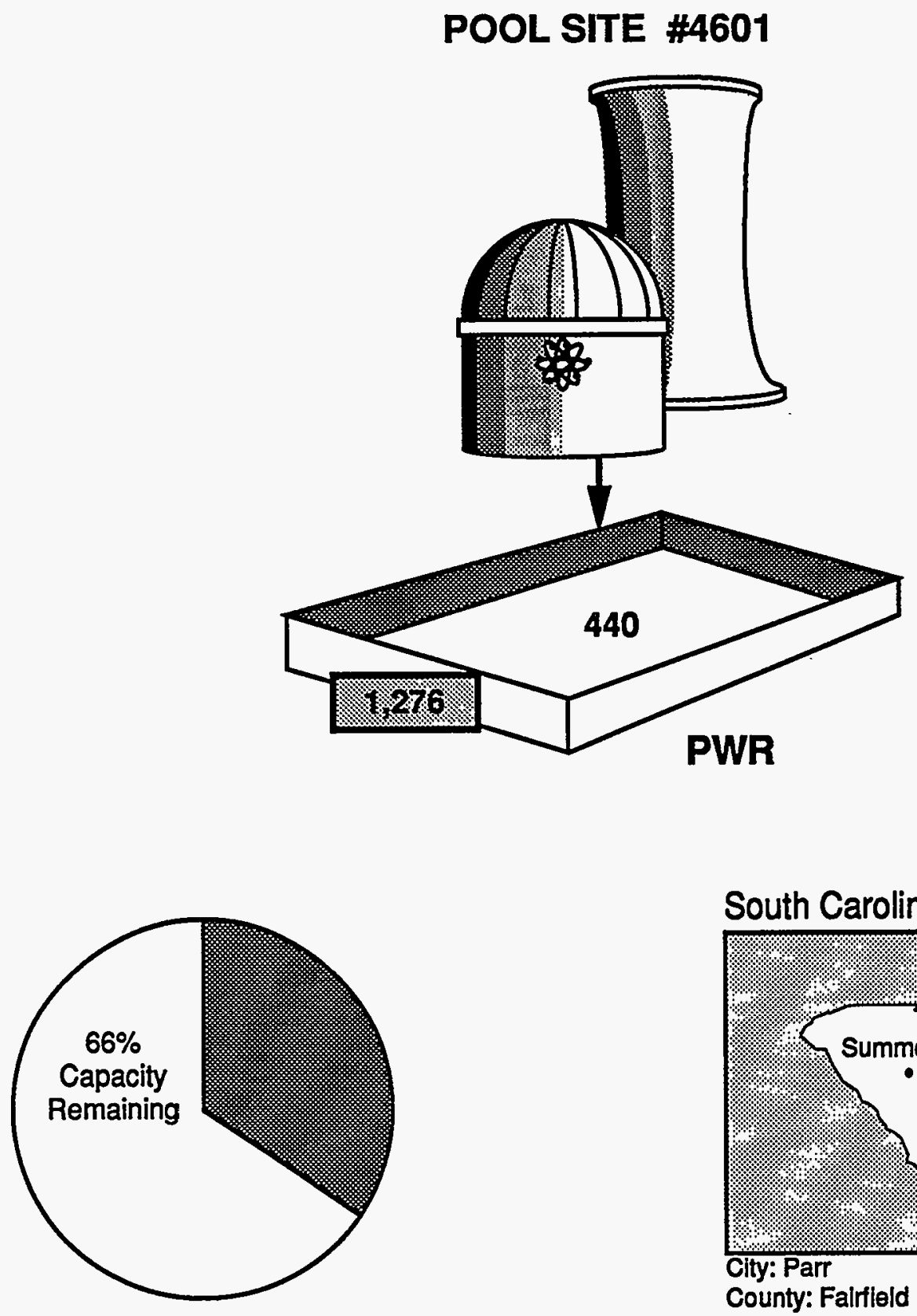

South Carolina

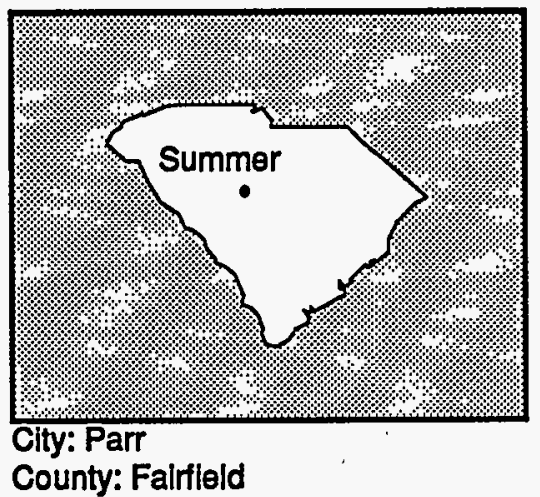




\section{Southern California Edison Company}

\section{San Onofre}
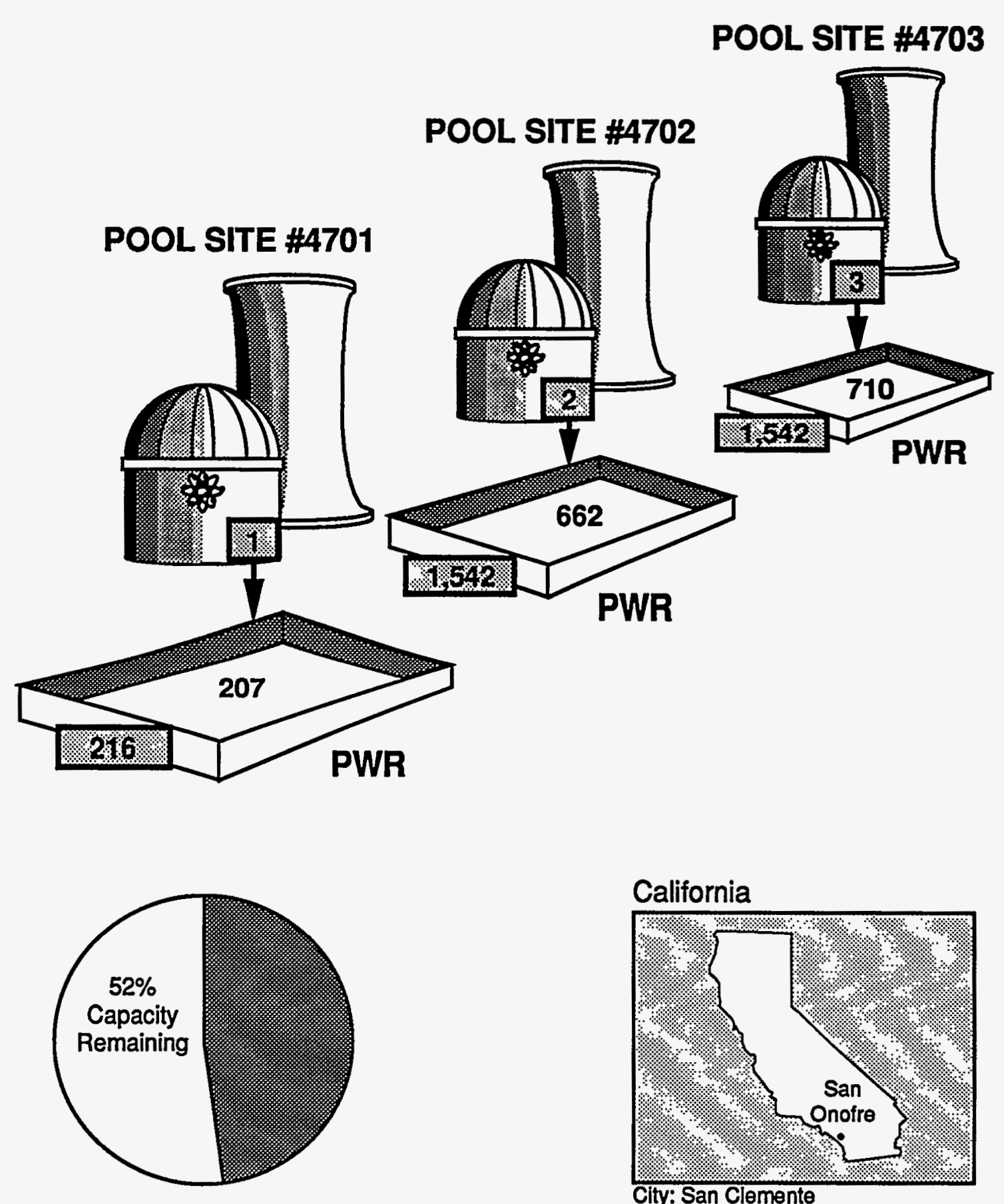

California

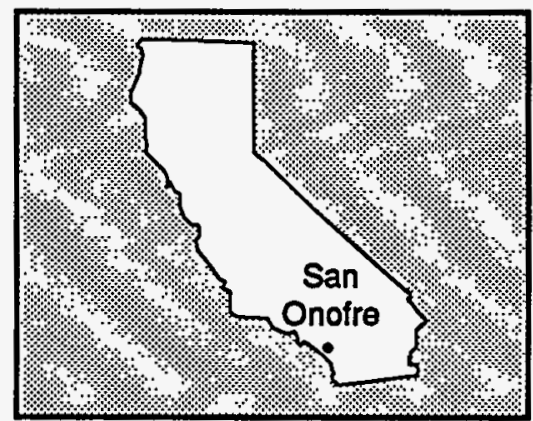

City: San Clemente

County: San Diego 


\title{
System Energy Resources, \\ Inc.
}

\author{
Grand Gulf 1
}
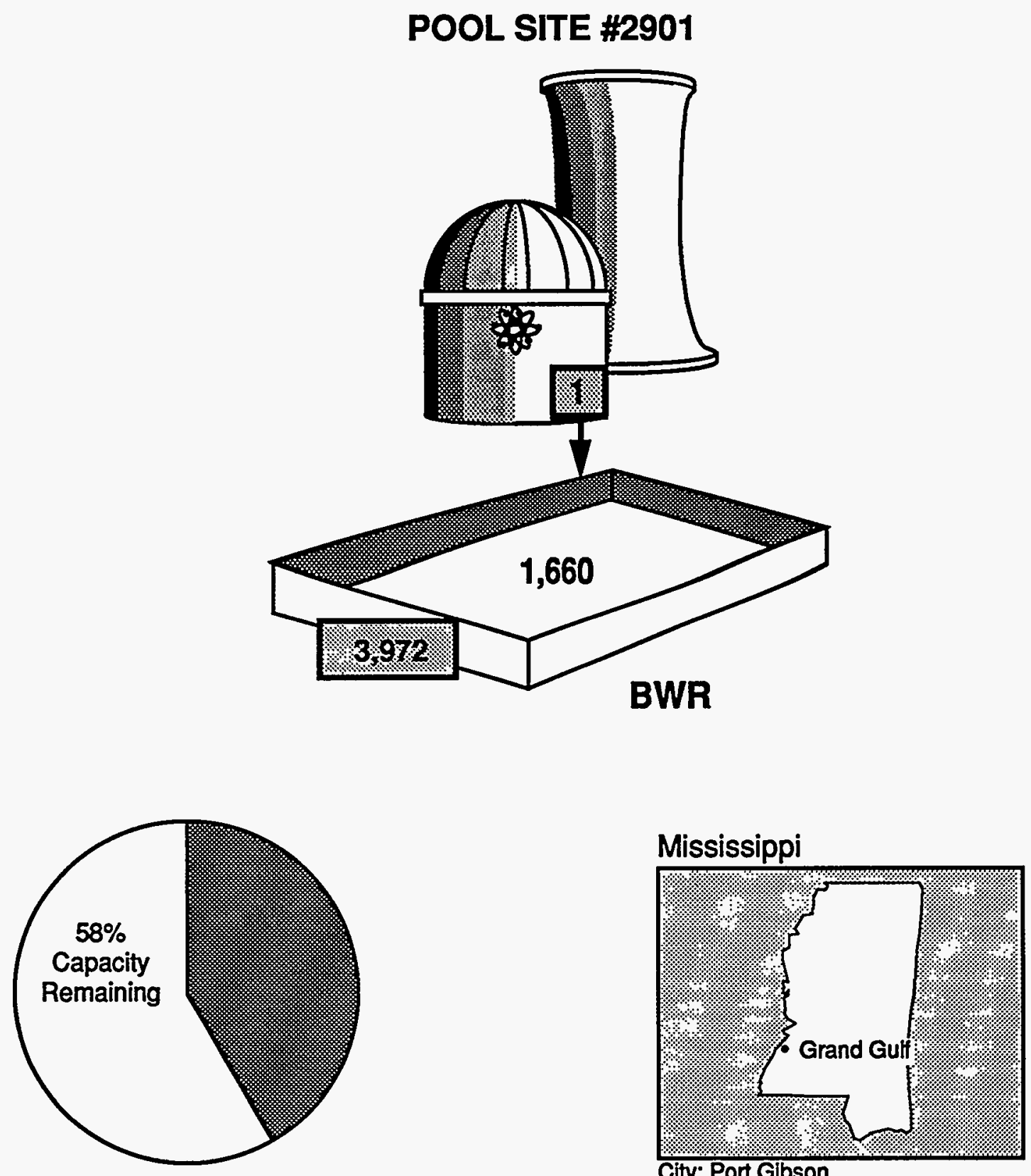

City: Port Gibson

County: Clairborne 
This page is intentionally blank. 


\section{Tennessee Valley Authority Browns Ferry}

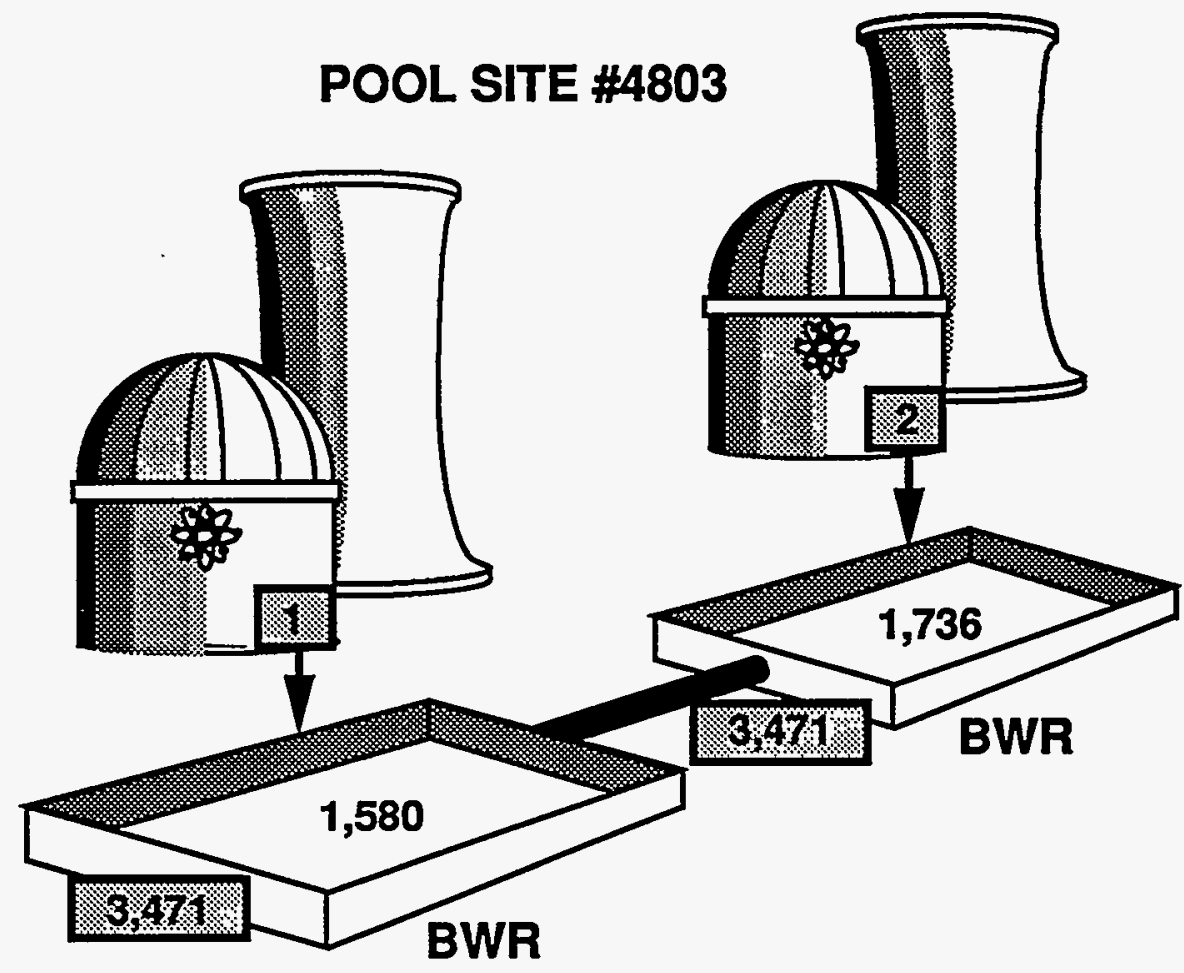

POOL SITE \#4805

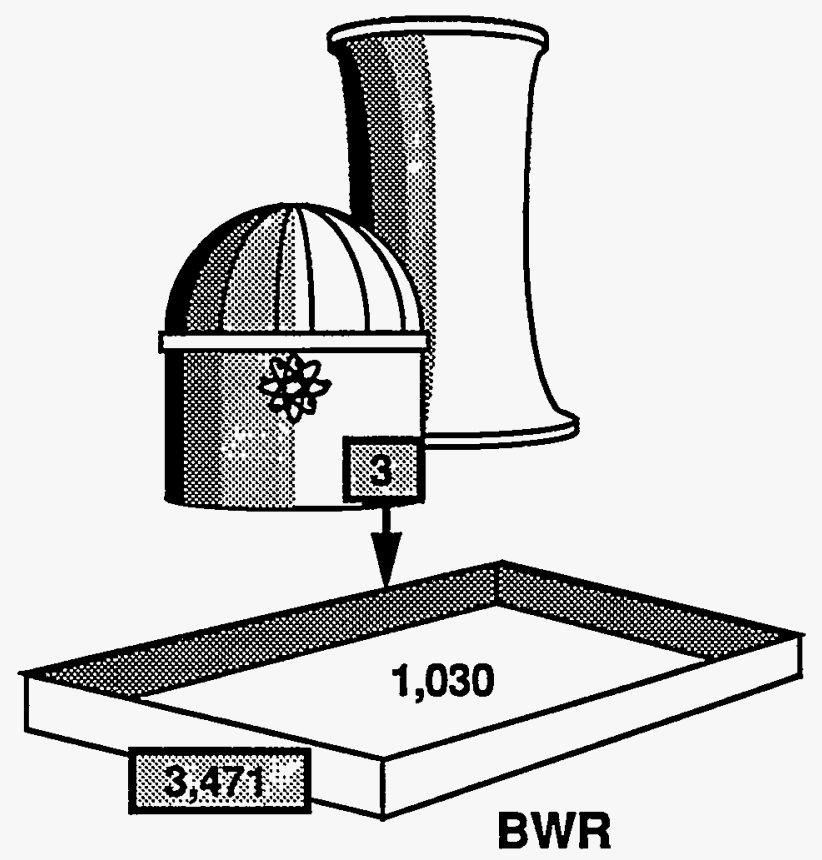

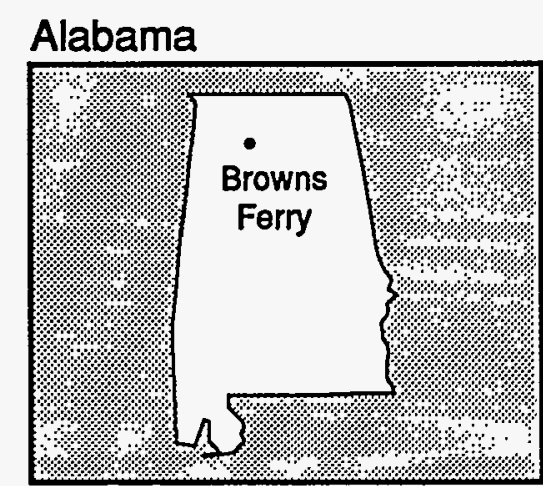

City: DeCatur County: Limestone

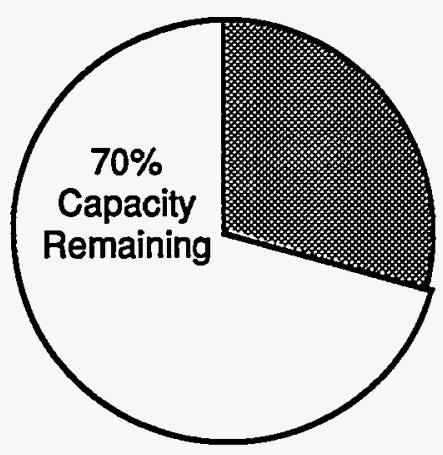




\section{Tennessee Valley Authority \\ (Continued)}

\section{Sequoyah}

POOL SITE \#4808

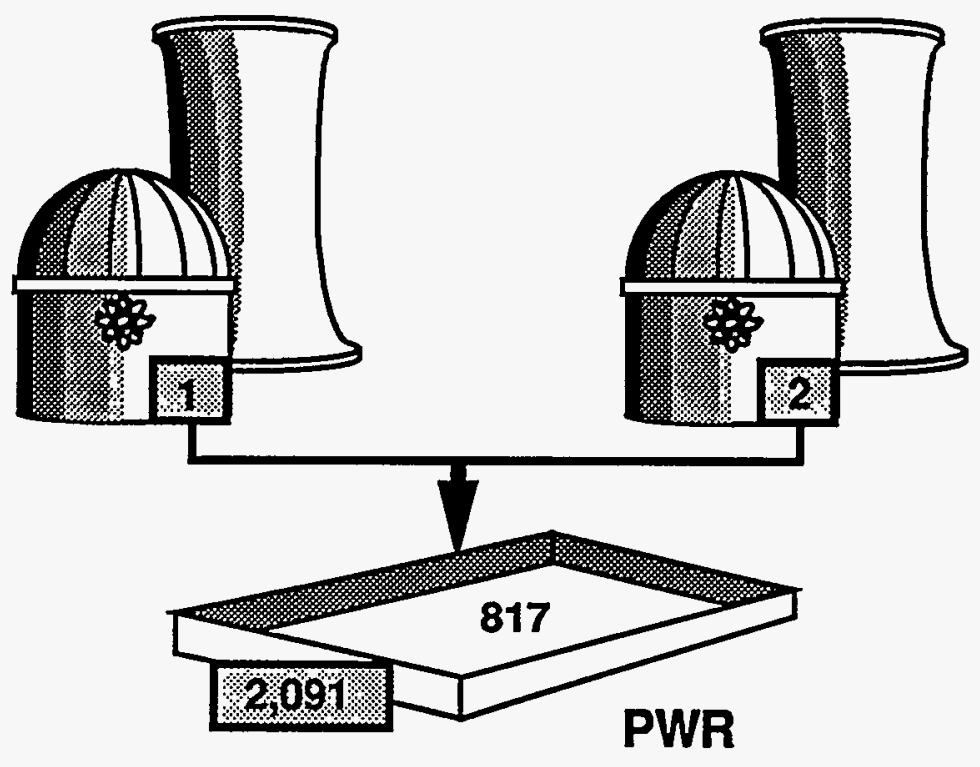

Watts Bar
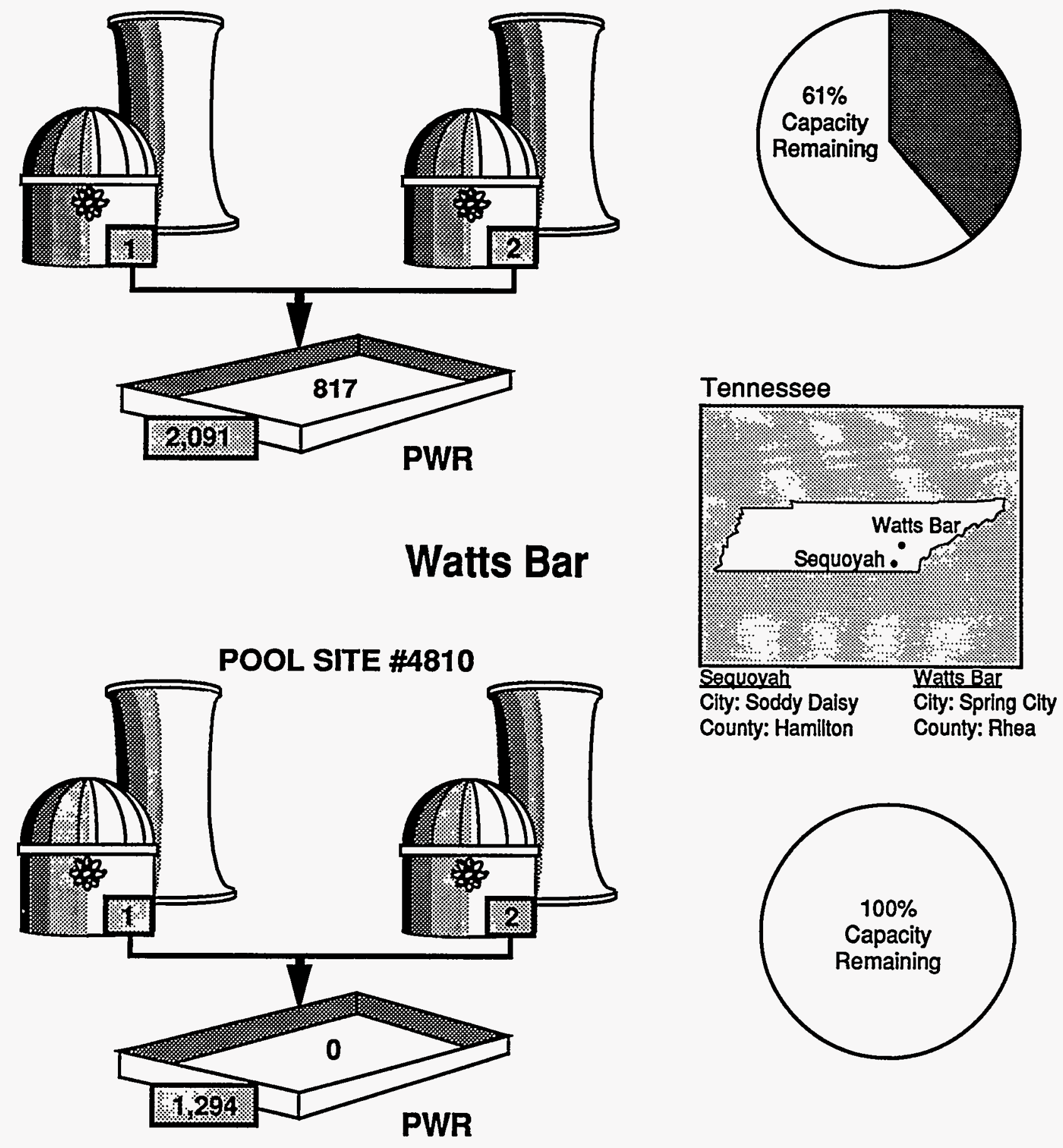


\title{
Toledo Edison Company \\ Davis-Besse
}

\author{
POOL SITE \#5001
}
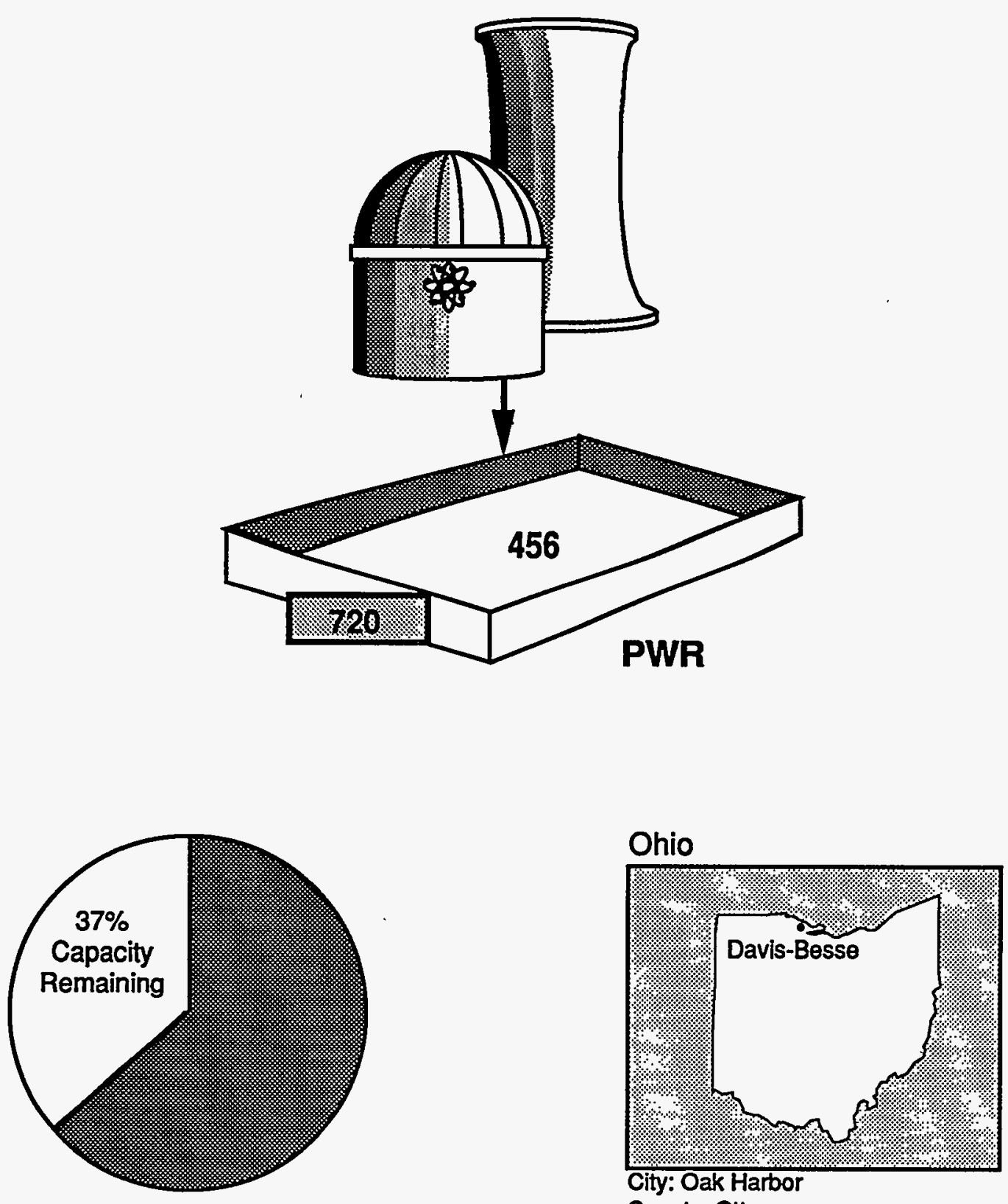

City: Oak Harbor

County: Ottawa 


\section{TU Electric \\ Comanche Peak}
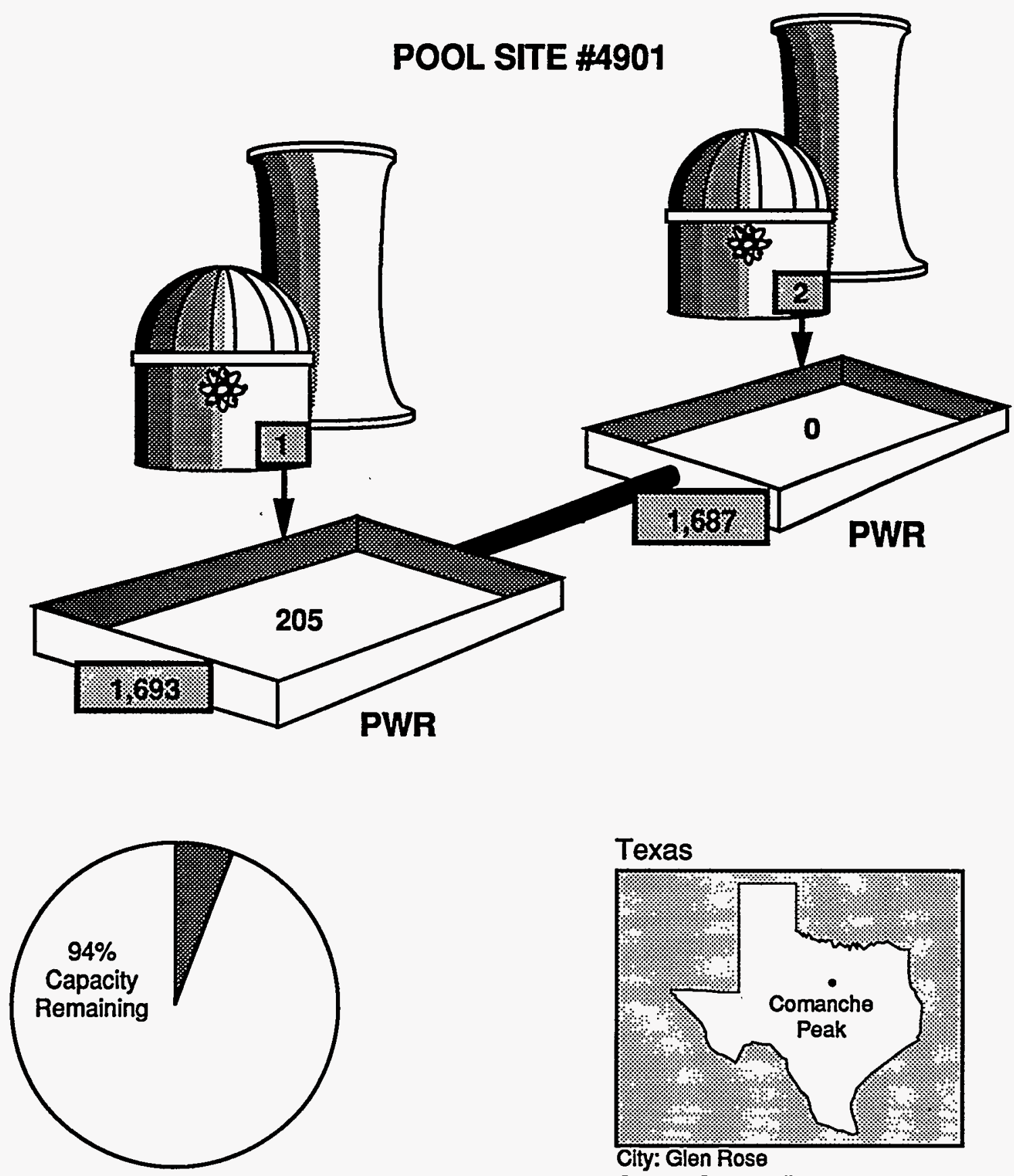

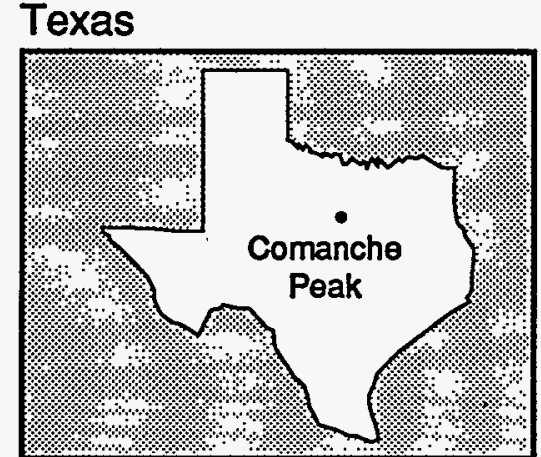

City: Glen Rose

County: Somervell 


\section{Union Electric Company Callaway}
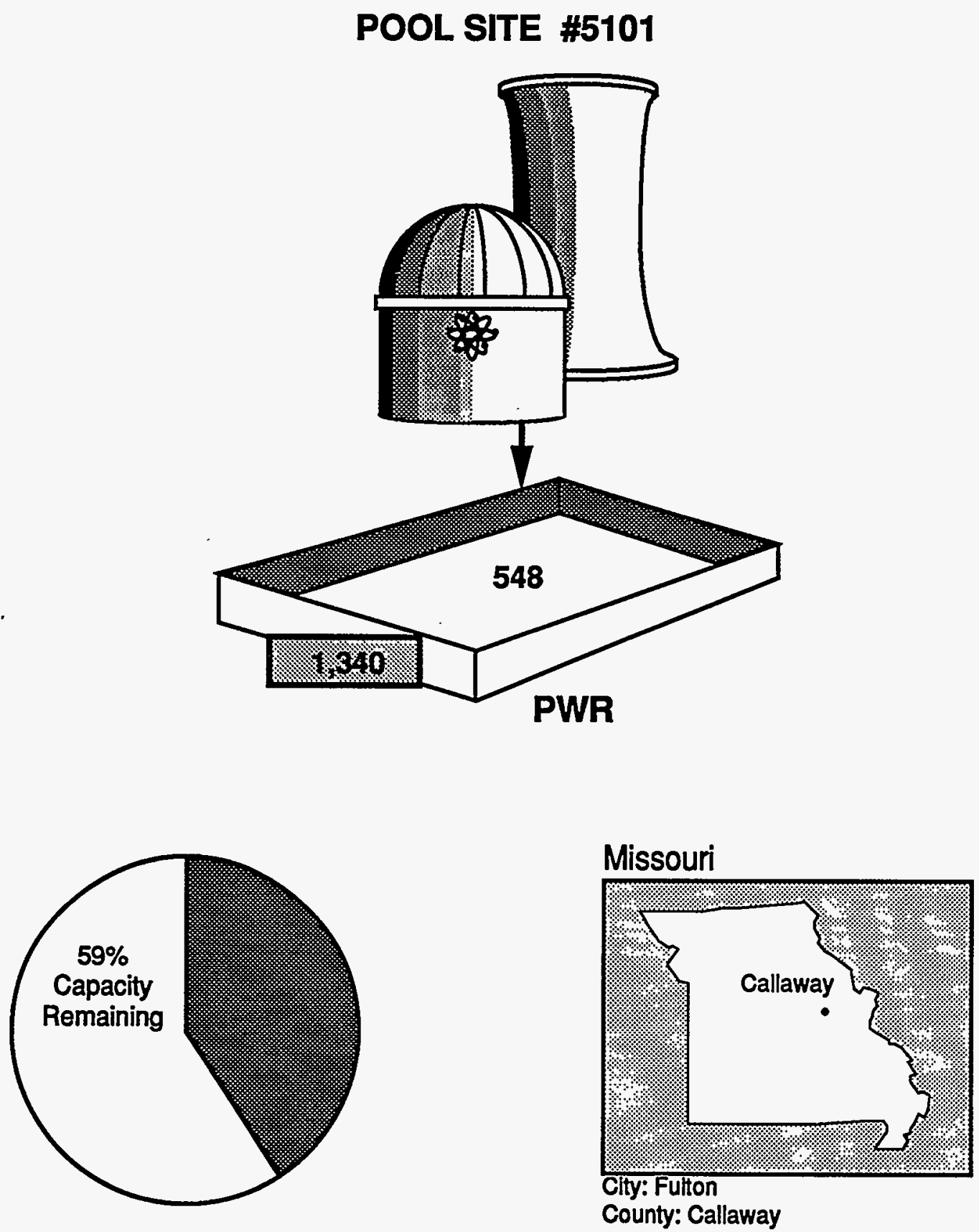


\title{
Vermont Yankee Nuclear Power Corporation
}

\author{
Vermont Yankee
}
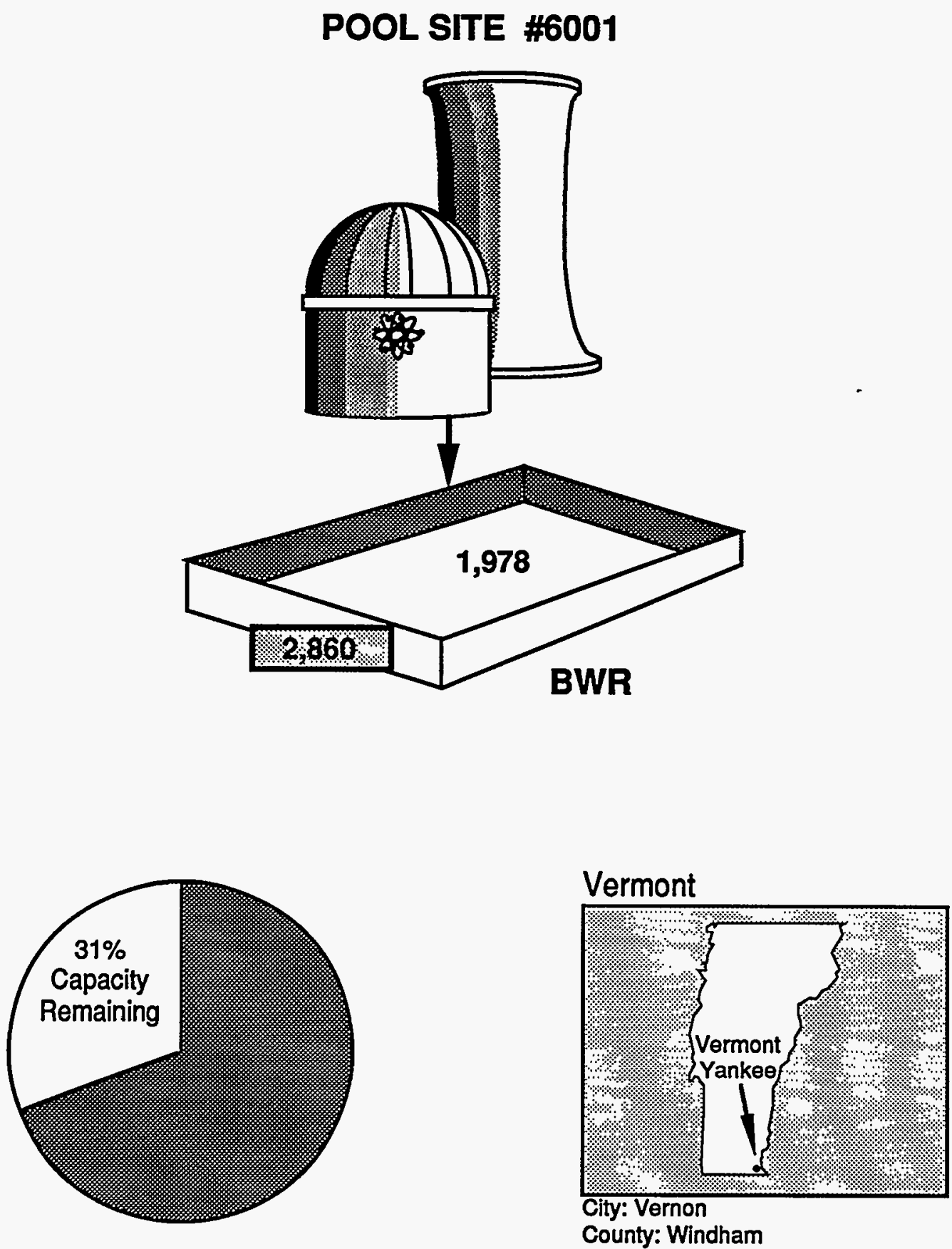


\title{
Virginia Power
}

\author{
North Anna
}

POOL SITE \#5201
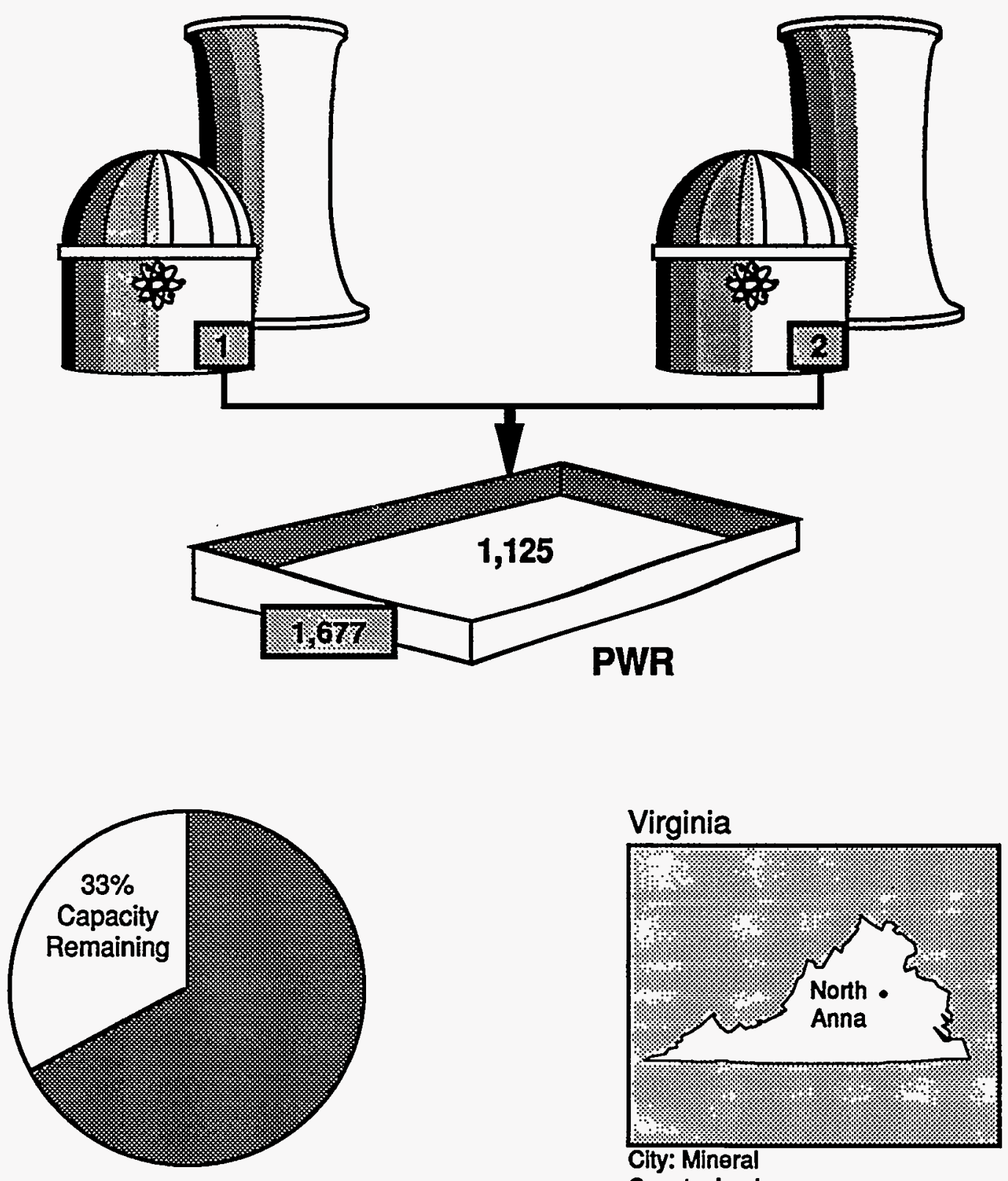

City: Mineral

County: Loulsa 


\section{Virginia Power \\ (Continued) \\ Surry}

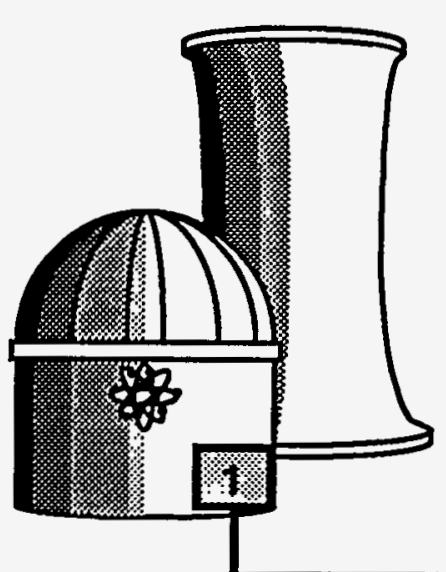

POOL SITE \#5203
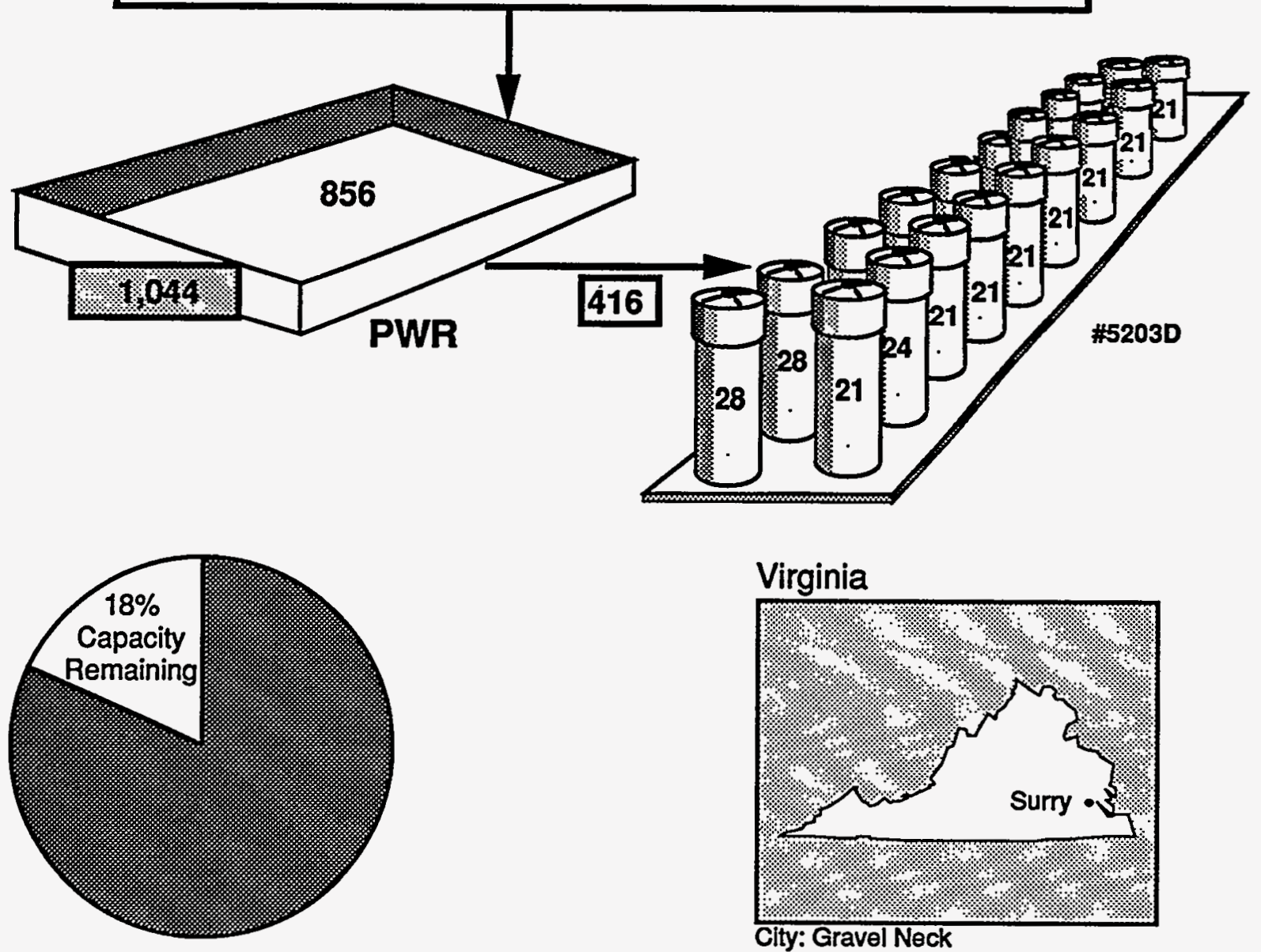

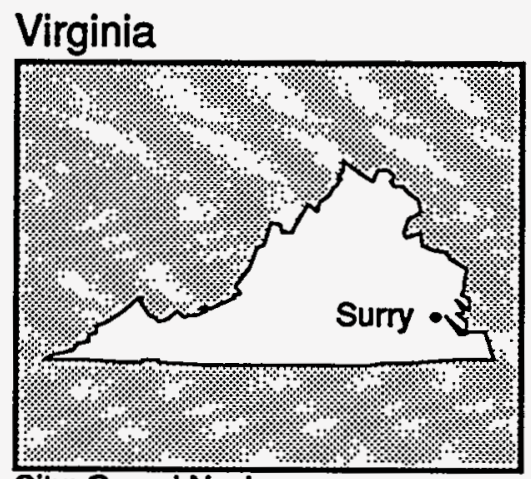

City: Gravel Neck

County: Surry 


\title{
Washington Public Power Supply System
}

\author{
Washington Nuclear
}
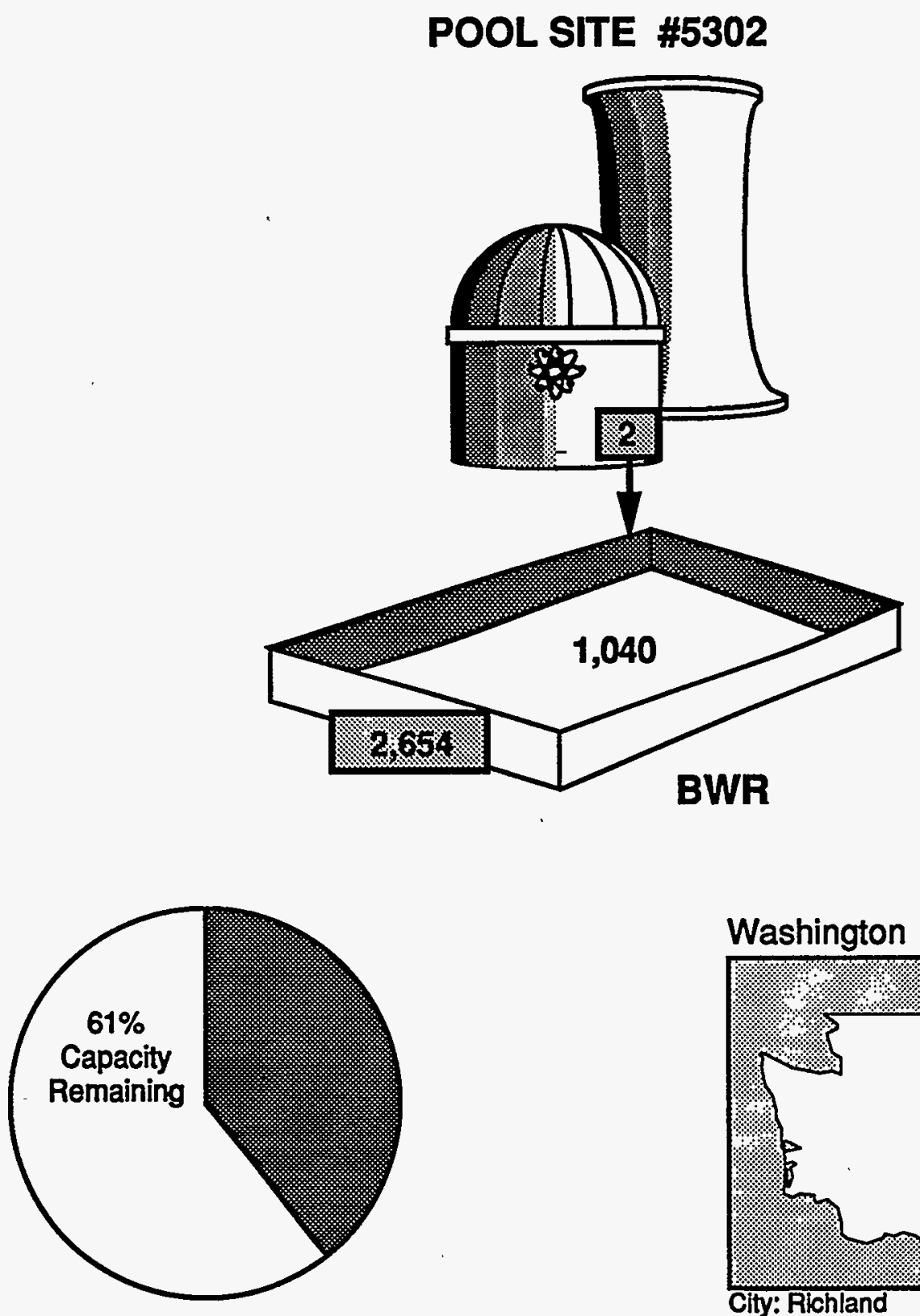

Washington

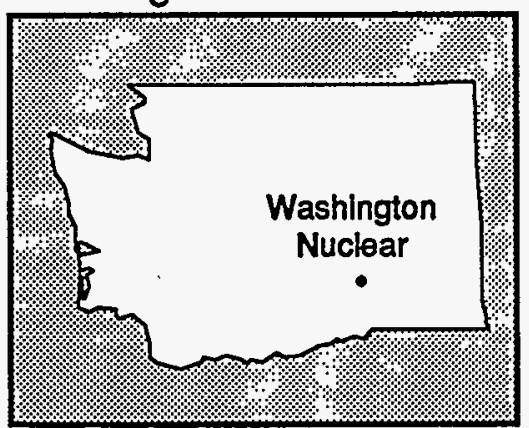

City: Richland

County: Benton 


\section{Wisconsin Electric Power Company Point Beach}

POOL SITE \#5401
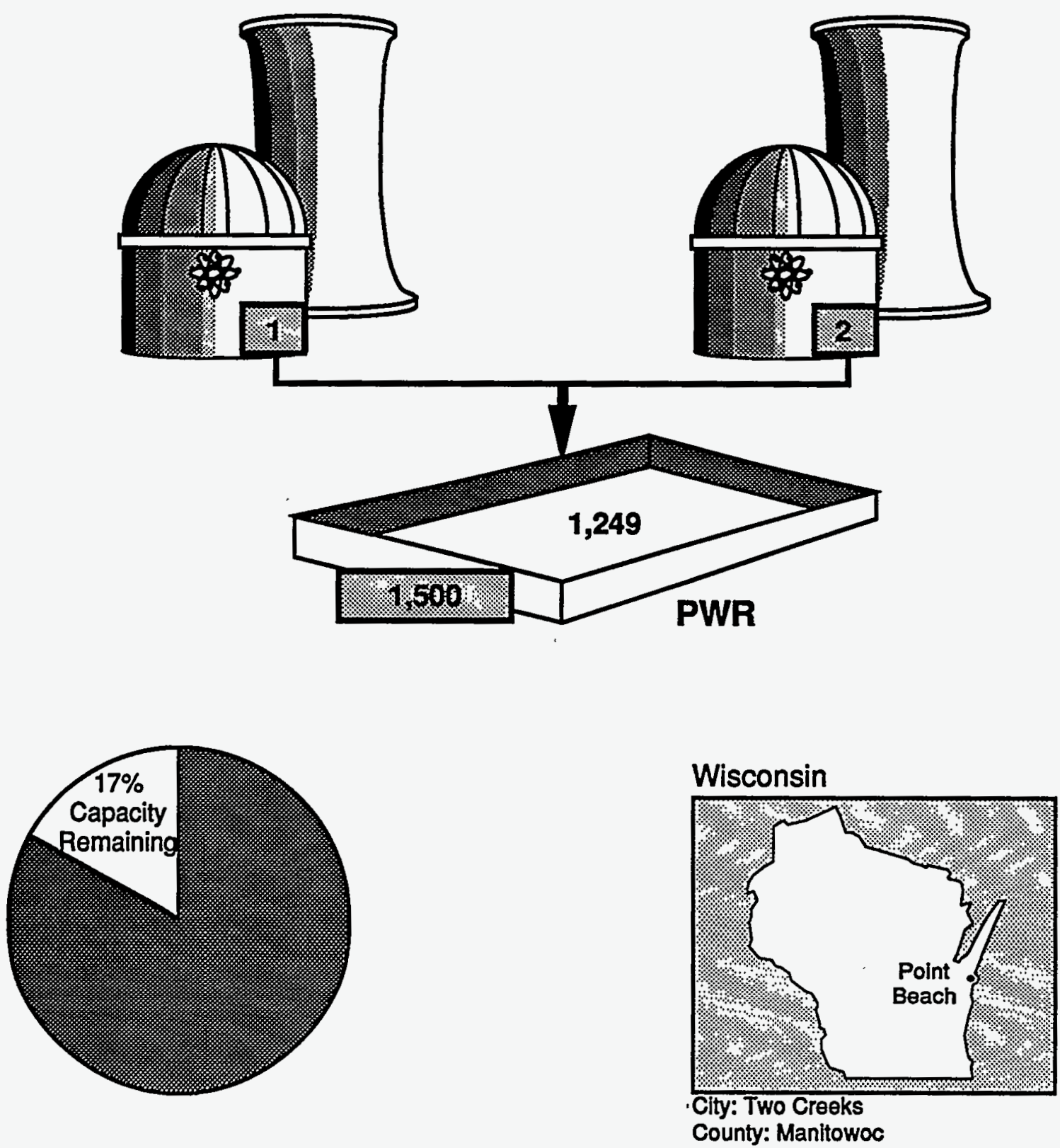


\section{Wisconsin Public Service Corporation Kewaunee}
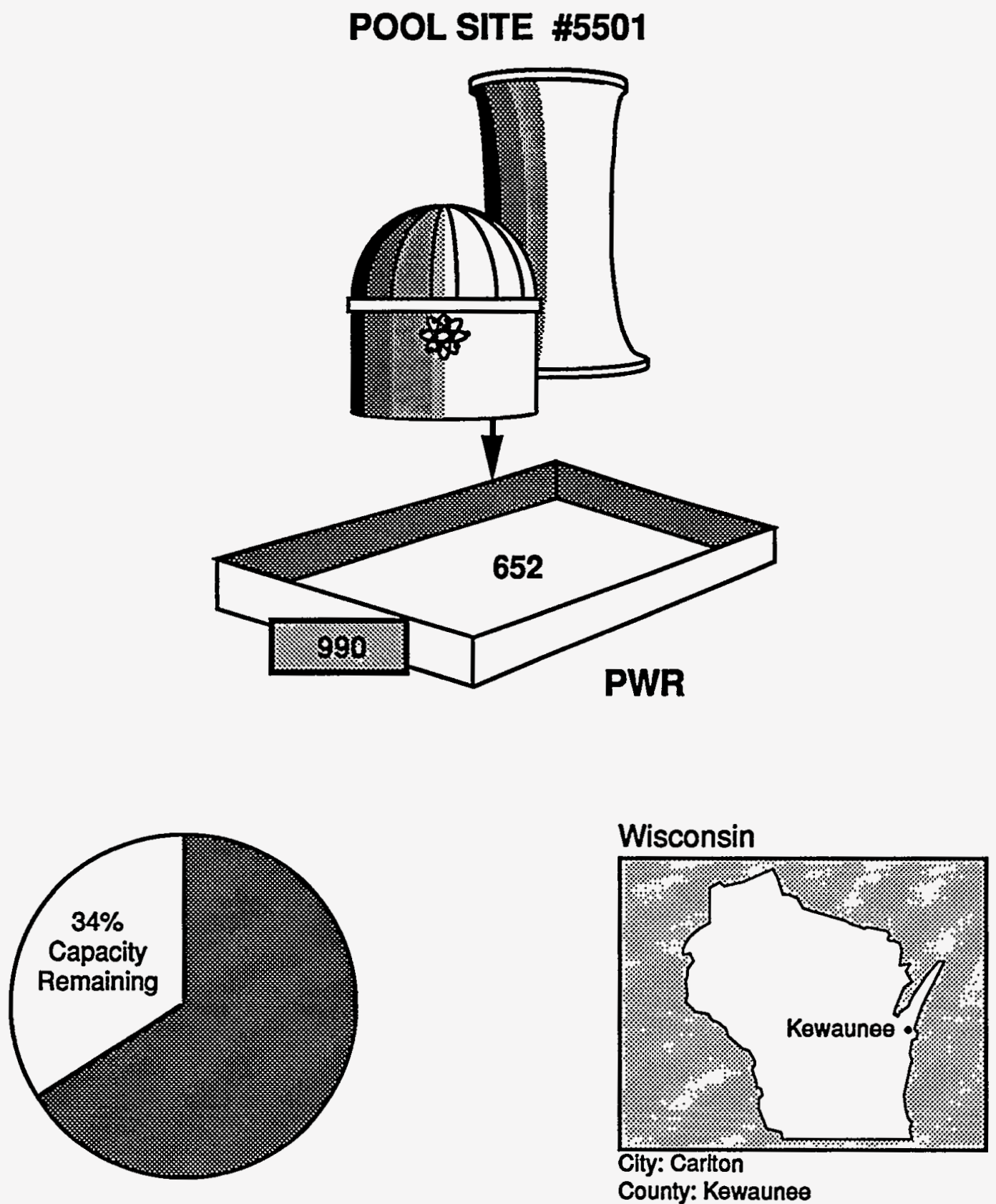


\section{Yankee Atomic Electric Company Yankee Rowe}
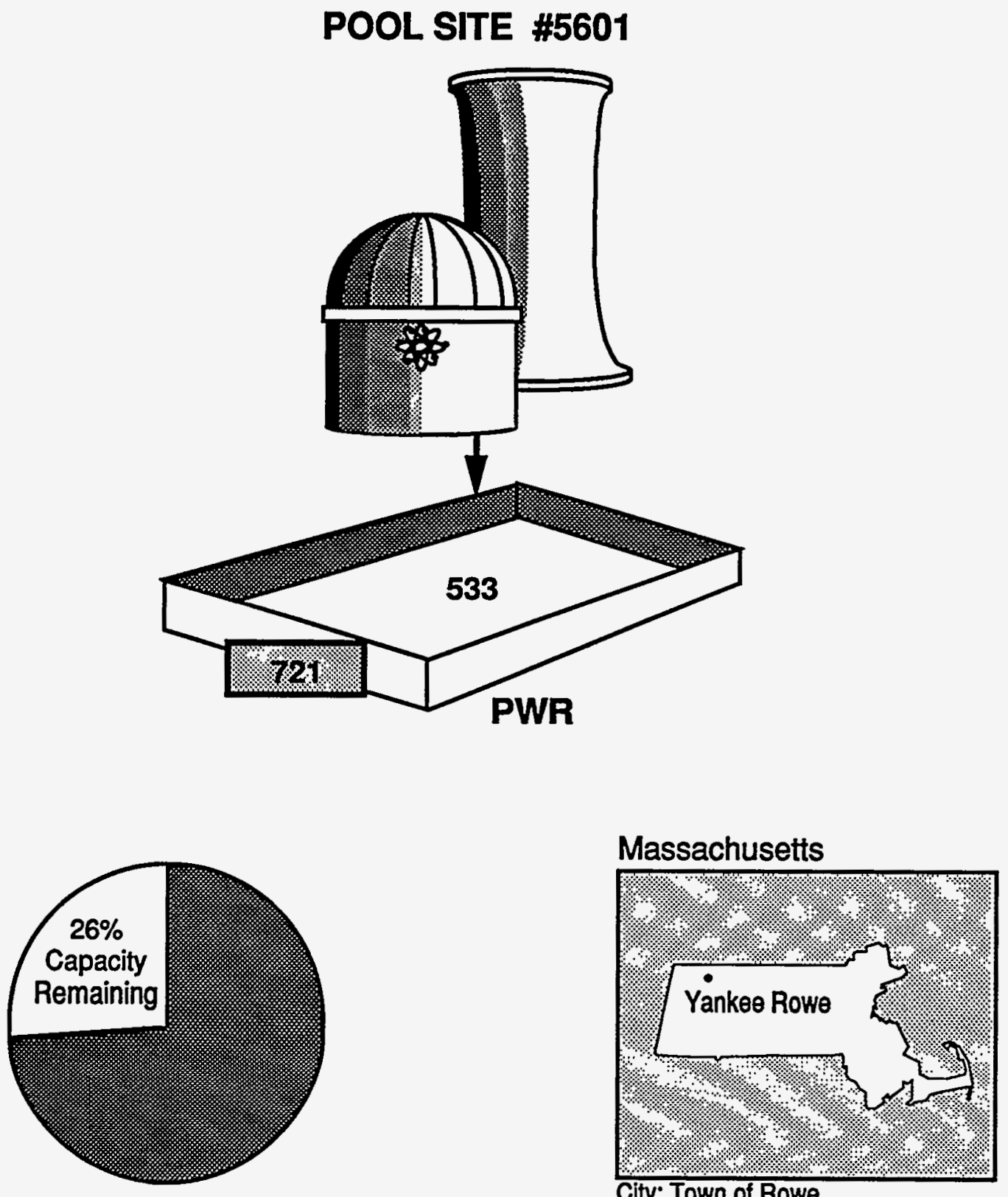

City: Town of Rowe

County: Franklin 


\section{General Electric Company}

\section{Morris Operation \\ Away-from-reactor Spent Fuel Storage Facility}

POOL SITE \#6601

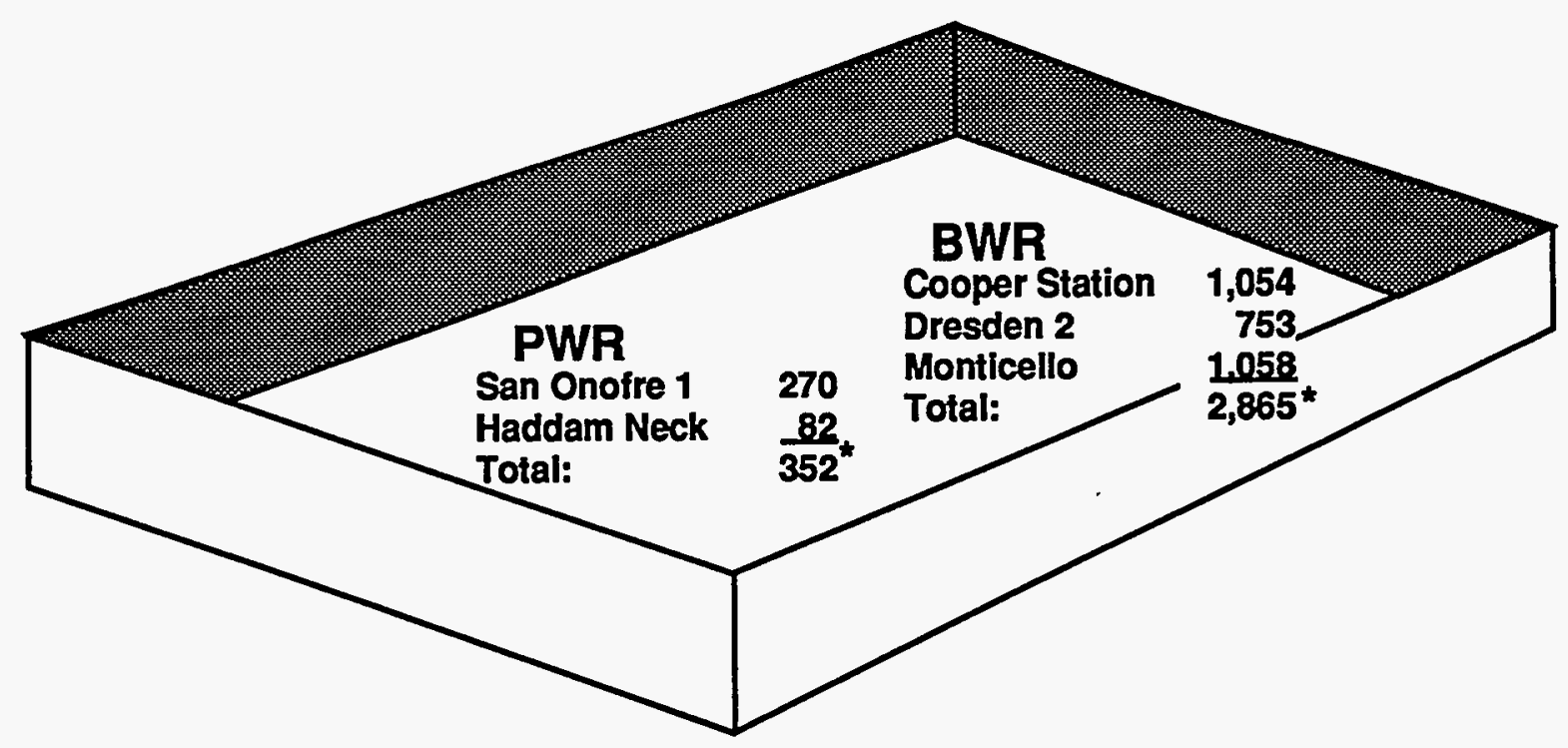

Illinois

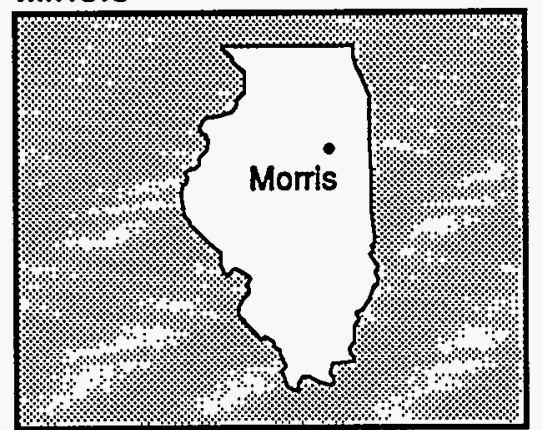

City: Morris

County: Grundy

-There are 7 slots still avallable for use. If PWR assemblies are stored, 4 assemblies will fit in each of the 7 slots totaling 28 assemblies. If BWR assemblies are stored, 9 assemblies will fit in each of the 7 slots totaling 63 assemblies. 


\section{West Valley Demonstration Project \\ Away-from-reactor Spent Fuel Storage Facility}

POOL SITE \#7005
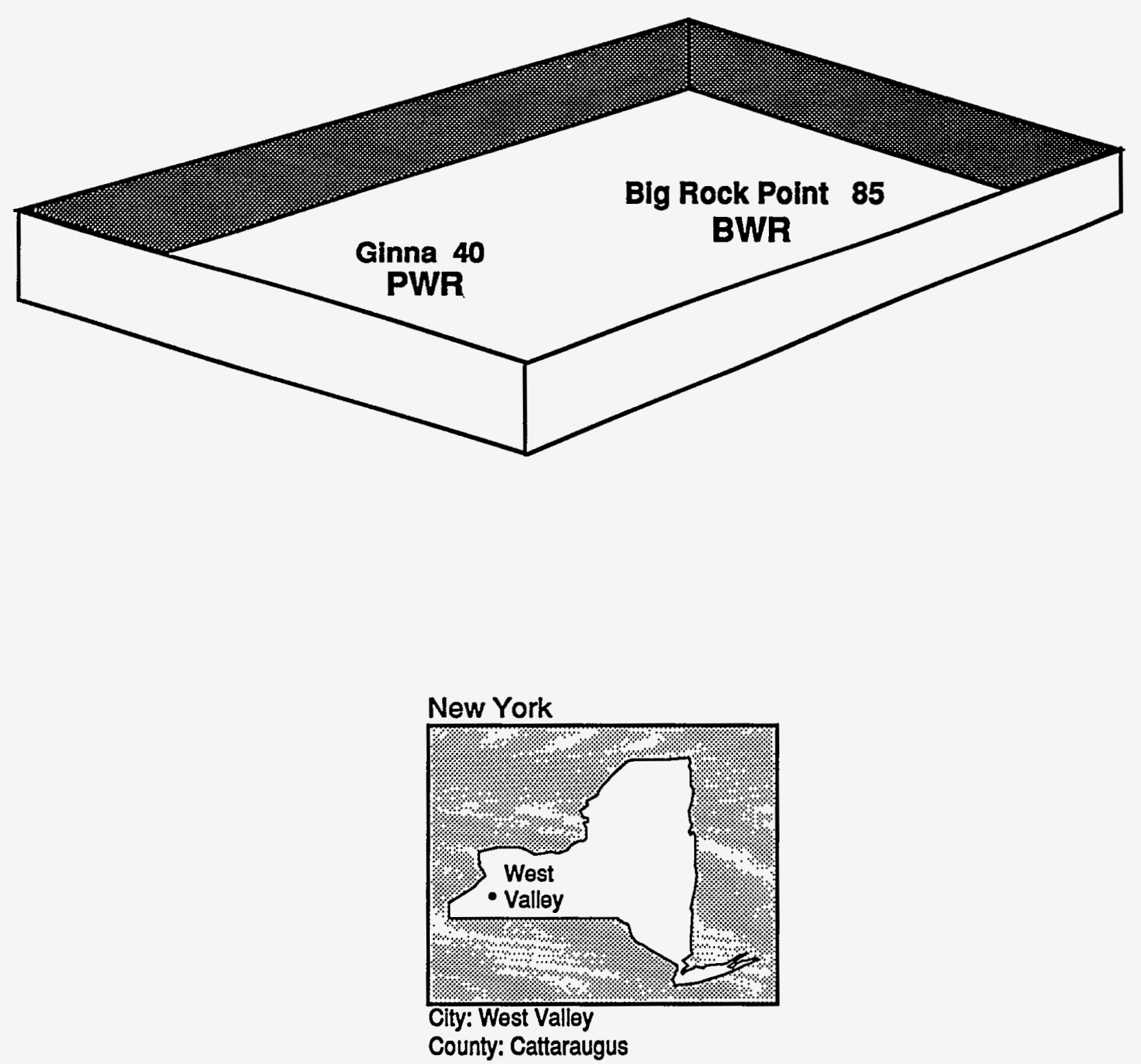
Appendix D

\section{Quality Assurance}

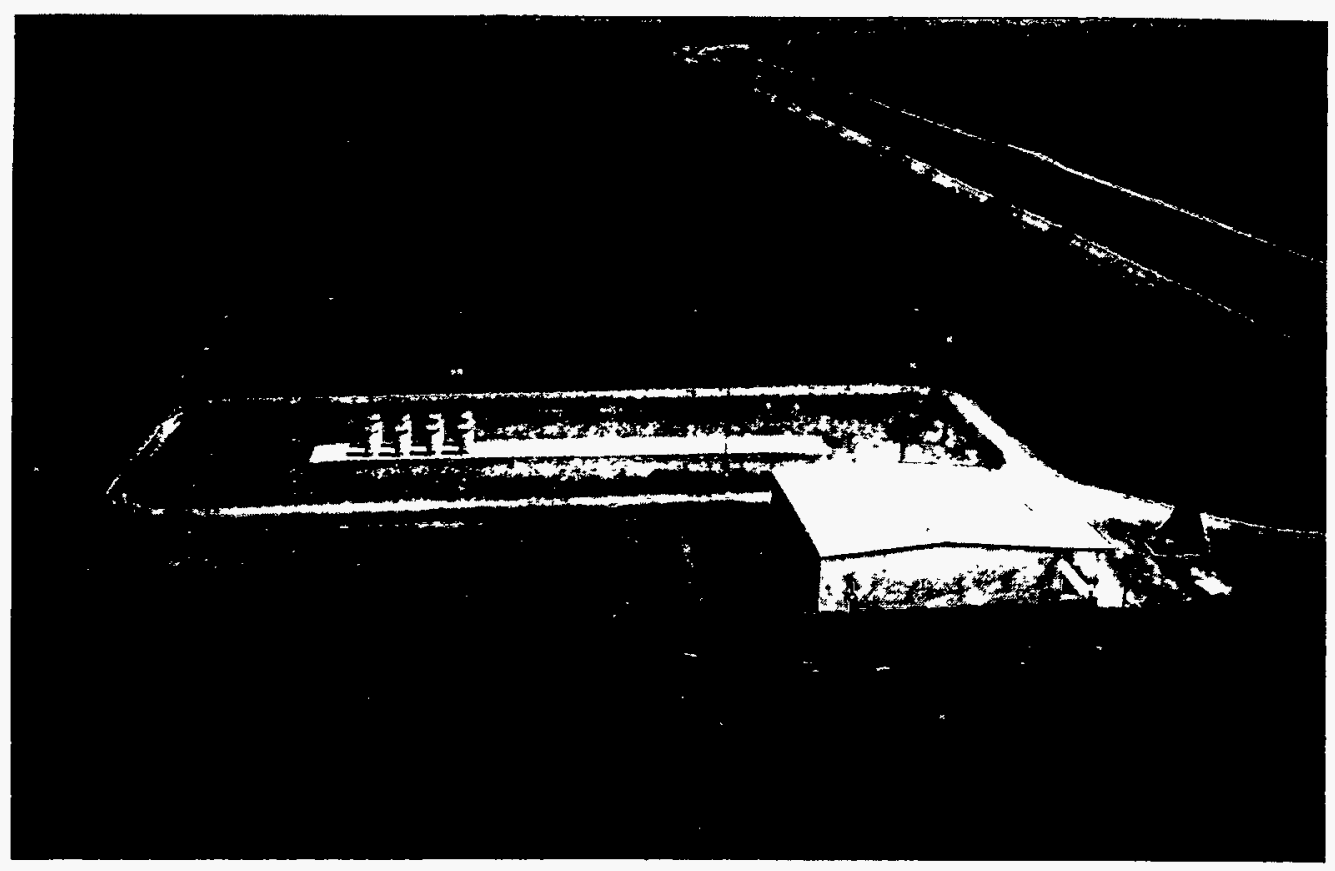

Virginia Power's Surry Independent Spent Fuel Storage Installation site. 


\section{Appendix D}

\section{Quality Assurance}

Data submitted by respondents to the Form RW-859 Nuclear Fuel Data survey are subject to extensive Energy Information Administration (EIA) quality assurance (QA) procedures to ensure their accuracy and completeness. A mainframe EIA QA program, written in Pascal, generates a nuclear fuel cycle matrix and summary reports for each reactor. These reports identify and highlight errors in assembly and fuel batch data submitted by nuclear fuel utilities on Form RW-859. Analysts review the EIA QA matrix report produced by the program to check that the data submitted are consistent with data obtained from previous surveys.

A sample EIA QA report for one reactor on the Form $\mathrm{RW}-859$ data base is presented in Figure D1. The QA report is divided into three parts. The first part of the report is composed of a nuclear fuel cycle matrix generated from assembly data aggregated to the batch level, which presents the fuel batches through their cycle history. The format helps to identify and highlight corrections needed for data on assemblies and batches of irradiated and unirradiated fuel. The sum of the number of assemblies in the fuel batches in the reactor core during each fuel cycle are compared with the reactor's full core capacity. In addition, assemblies permanently or temporarily discharged (as indicated by their status codes) are aggregated and compared with totals of assemblies discharged and currently not in cycle. The matrix extends over all reactor cycles from reactor startup to the fifth cycle past the current reporting period. Other key data displayed in the matrix include utility $\mathrm{D}$, cycle discharge dates, batch status, initial uranium content, burnup, assay (enrichment percent), assembly type codes, and an indicator for defective fuel.

The second part of the report contains a discharge/storage summary page, which is composed of three groups of data. Group 1 represents a count of the assemblies discharged at the end of each cycle. Group 2 represents the net cumulative total number of assemblies in storage during each cycle. Group 3 represents net cumulative totals of initial uranium content for assemblies stored during each cycle. The cycle number and end date for the reactor's next $(n+1)$ cycle also appears on the page.

The final part of the report, the storage pool inventory report, lists storage pool inventories (and reactors contributing to the pool) as of the end of the survey report period. Inventories are subdivided into permanent and temporary discharges. Storage pool inventories by pool ID, based on assembly status codes, are compared with those based on the reactor's reported total inventory. The number of canisters in storage and the number of assemblies contained in these canisters are also reported. Assembly type and pool type (wet or dry) are also indicated. 
Figure D1. Energy Information Administration Quality Assurance Report

Utility ID $=23$

Nuclear Fuel Matrix

Reactor ID $=01$

Utility Name: Illinois Power Company

Reactor Name: Clinton 1

Entries in cycle matrix are number of assemblies.

\begin{tabular}{|c|c|c|c|c|c|c|c|c|c|c|c|c|c|c|c|c|}
\hline \multirow[b]{2}{*}{ Batch } & \multirow[b]{2}{*}{ Stat } & \multirow[b]{2}{*}{$\begin{array}{c}\text { Assmb } \\
\text { Type } \\
\text { Codes }\end{array}$} & \multirow[b]{2}{*}{ Assmbs } & \multirow{2}{*}{$\begin{array}{l}\text { Initial } \\
\text { Uranium } \\
\text { Content } \\
\text { (MTU) }\end{array}$} & \multicolumn{2}{|c|}{ Discharge Date $\longrightarrow$} & \multirow{2}{*}{\multicolumn{2}{|c|}{$\begin{array}{r}01 \\
8901\end{array}$}} & \multirow[t]{2}{*}{$\begin{array}{r}02 \\
9010\end{array}$} & \multirow[t]{2}{*}{$\begin{array}{r}03 \\
9203\end{array}$} & \multirow[t]{2}{*}{$\begin{array}{r}04 \\
9309\end{array}$} & \multirow[t]{2}{*}{$\begin{array}{r}05 \\
9503\end{array}$} & \multirow[t]{2}{*}{$\begin{array}{r}06 \\
9610\end{array}$} & \multirow[t]{2}{*}{$\begin{array}{r}07 \\
9803\end{array}$} & \multirow[t]{2}{*}{$\begin{array}{r}08 \\
9910\end{array}$} & \multirow[t]{2}{*}{$\begin{array}{r}09 \\
0103\end{array}$} \\
\hline & & & & & $\begin{array}{c}\text { Bumup } \\
\text { (MWD/MTU) }\end{array}$ & $\begin{array}{l}\text { Assay } \\
(\%)\end{array}$ & & & & & & & & & & \\
\hline 01A01 & D & G4608GP & 76 & 13.992 & 3000 & 0.711 & & 76 & & & & & & & & \\
\hline 01B01 & D & G4608GP & 92 & 16.918 & 10000 & 1.540 & & 92 & & & & & & & & \\
\hline 01B02 & D & G4608GP & 96 & 17.654 & 15000 & 1.538 & & 96 & 96 & & & & & & & \\
\hline $01 \mathrm{CO}$ & D & G4608GP & 120 & 22.011 & 19000 & 2.004 & & 120 & 120 & & & & & & & \\
\hline $01 \mathrm{CO2}$ & D & G4608GP & 184 & 33.751 & 22000 & 2.004 & & 184 & 184 & 184 & & & & & & \\
\hline $01 \mathrm{C03}$ & D & G4608GP & 56 & 10.272 & 25000 & 2.004 & & 56 & 56 & 56 & 56 & & & & & \\
\hline $02 A 01$ & D & G4808GB & 56 & 10.430 & 28000 & 2.839 & & & 56 & 56 & 56 & & & & & \\
\hline $02 B 01$ & D & G4608GB & 44 & 8.183 & 29000 & 2.839 & & & 44 & 44 & 44 & & & & & \\
\hline${ }^{*} B$ & 1 & & 68 & 0.0 & 0 & 0.0 & & & 68 & 68 & 68 & 68 & & & & \\
\hline${ }^{\star} \mathrm{C}$ & 1 & & 216 & 0.0 & 0 & 0.0 & & & & 216 & 216 & 216 & & & & \\
\hline 04AYY & 1 & G4608G8 & 184 & 33.790 & 0 & 3.005 & & & & & 184 & 184 & & & & \\
\hline 05AYY & I & G4608G10 & 156 & 27.766 & 0 & 3.222 & & & & & & 156 & & & & \\
\hline $03 A 02$ & & & 48 & 8.907 & 33000 & 2.989 & & & & & & & 48 & & & \\
\hline $04 A 01$ & & & 124 & 22.771 & 34000 & 3.005 & & & & & & & 124 & & & \\
\hline $03 A 03$ & & & 32 & 5.938 & 35000 & 2.989 & & & & & & & & 32 & & \\
\hline 04A02 & & & 60 & 11.018 & 37000 & 3.005 & & & & & & & & 60 & & \\
\hline $05 A 01$ & & & 92 & 16.375 & 35000 & 3.222 & & & & & & & & 92 & & \\
\hline 05A02 & & & 64 & 11.391 & 38000 & 3.222 & & & & & & & & & 64 & \\
\hline $06 A 01$ & & & 92 & 16.366 & 37000 & 3.246 & & & & & & & & & 92 & \\
\hline 06B01 & & & 28 & 4.972 & 37000 & 3.241 & & & & & & & & & 28 & \\
\hline 06A02 & & & 56 & 9.962 & 38000 & 3.246 & & & & & & & & & & 56 \\
\hline 06802 & & & 28 & 4.972 & 38000 & 3.241 & & & & & & & & & & 28 \\
\hline 07A01 & & & 52 & 9.251 & 35000 & 3.246 & & & & & & & & & & 52 \\
\hline 07B01 & & & 48 & 8.524 & 37000 & 3.241 & & & & & & & & & & 48 \\
\hline Number & or of $A$ & Assemblies if & n Core & & & & & 624 & 624 & 624 & 624 & 624 & 172 & 184 & 180 & 184 \\
\hline Differen & nce frc & from Core Siz & & & & & & 0 & 0 & 0 & 0 & 0 & -452 & -440 & -444 & .440 \\
\hline
\end{tabular}

Status Codes "D," "T": record type 13 historical data.

Status Code "I": record type 15 in core data.

Status Code " ": record type 14 projected data. 
Figure D1. Energy Information Administration Quality Assurance Report (Continued)

Utility ID $=23 \quad$ Utility Name: Illinois Power Company

Discharge/Storage Summary

Reactor ID $=01$ Reactor Name: Clinton 1

\begin{tabular}{|c|c|c|c|c|c|c|c|c|c|c|}
\hline & Discharge Date $\longrightarrow$ & $\begin{array}{r}01 \\
8901\end{array}$ & $\begin{array}{r}02 \\
9010\end{array}$ & $\begin{array}{r}03 \\
9203\end{array}$ & $\begin{array}{r}04 \\
9309\end{array}$ & $\begin{array}{r}05 \\
9503\end{array}$ & $\begin{array}{r}06 \\
9610\end{array}$ & $\begin{array}{r}07 \\
9803\end{array}$ & $\begin{array}{r}08 \\
9910\end{array}$ & $\begin{array}{r}09 \\
0103\end{array}$ \\
\hline Assemblies Discharged from Cycle (Gross) ${ }^{\mathrm{a}}$ & & 168 & 216 & 184 & 156 & 624 & 172 & 184 & 184 & 184 \\
\hline Permanently Discharged & & 168 & 216 & 184 & 156 & 624 & 172 & 184 & $\begin{array}{l}184 \\
184\end{array}$ & $\begin{array}{l}184 \\
184\end{array}$ \\
\hline Temporarily Discharged (per logic) & & 0 & 0 & 0 & 0 & 0 & 0 & 0 & 0 & 0 \\
\hline Total Assemblies Stored (Net of Discharges) ${ }^{b}$ & & 0 & 168 & 384 & 568 & 724 & 1348 & 1520 & 1704 & 1888 \\
\hline $\begin{array}{l}\text { Permanently Discharged } \\
\text { Temporarily Discharged (per flag) }\end{array}$ & & 0 & 168 & $\begin{array}{r}384 \\
0\end{array}$ & 568 & 724 & 1348 & 1520 & 1704 & 1888 \\
\hline $\begin{array}{l}\text { Temporarily Discharged (per logic) } \\
\text { Difference }\end{array}$ & & 0 & 0 & $\begin{array}{l}0 \\
0\end{array}$ & 0 & 0 & 0 & 0 & 0 & 0 \\
\hline Total Assemblies MTIHM (Net of Discharges) ${ }^{c}$ & & 0.0 & 30.9 & 70.6 & 104.3 & 133.2 & 194.8 & 226.4 & 259.8 & 292.5 \\
\hline $\begin{array}{l}\text { Permanently Discharged } \\
\text { Temporarily Discharged (per flag) }\end{array}$ & & 0.0 & 30.9 & 70.6 & $\begin{array}{r}104.3 \\
0.0\end{array}$ & 133.2 & 194.8 & 226.4 & 259.8 & 292.5 \\
\hline $\begin{array}{l}\text { Temporarily Discharged (per logic) } \\
\text { Difference }\end{array}$ & & 0.0 & 0.0 & 0.0 & $\begin{array}{l}0.0 \\
0.0\end{array}$ & 0.0 & 0.0 & 0.0 & 0.0 & 0.0 \\
\hline
\end{tabular}

${ }^{a}$ Counts of assemblies that were discharged after the end of each cycle. The number can be understood by inserting them into the sentence, "After cycle _there were _assemblies that were in storage during each cycle. The number can be understood by inserting them into the sentence, "After cycle _there were _assemblies permanently/temporarily discharged."

'Net cumulative totals of assemblies that were in storage during each cycle. The numbers can be understood by inserting them into the sentence, "During cycle _. there was a cumulative total of permanently/temporarily stored irradiated assemblies."

"Net cumulative totals of weights of assemblies that were stored during each cycle. The numbers can be understood by inserting them into the sentence, "During cycle - the cumulative weight of the permanently/temporarily stored assemblies was .."

Cycle $\mathrm{N}+1=05$

End of cycle date $=$ MAR-12-1995 
Figure D1. Energy Information Administration Quality Assurance Report (Continued)

Storage Pool Inventory

Utility ID $=23$ Utility Name: Illinois Power Company

Reactor ID = 01 Reactor Name: Clinton 1

Storage Pool Inventory as of $12 / 31 / 93$

Assemblies Discharged a

Into Pool: 2301

Into Pool:

Into Pool:

Into Pool:

Into Pool:

Into Pool:

Into Pool:

Total

Missing

Assemblies Stored

Into Pool: 2301

Into Pool:

Into Pool:

Into Pool:

Into Pool:

Into Pool:

Into Pool:

Pool Contribution -2301

From Reac: 2301

From Reac:

From Reac:

From Reac:

From Reac:

From Reac:

From Reac:

Total

\begin{tabular}{|c|c|c|}
\hline & $\begin{array}{l}\text { Pool } \\
\text { Type }\end{array}$ & $\begin{array}{l}\text { Cani- } \\
\text { sters }\end{array}$ \\
\hline (BWR) & Wet ( & \\
\hline ( ) & & 0 \\
\hline ) & & 0 \\
\hline ) & & $0 /$ \\
\hline ) & & $0 /$ \\
\hline ) & & 0 \\
\hline & & \\
\hline
\end{tabular}

Assmb.

in Cans.

0)

0)

0)

0)

$\begin{array}{rrr}\text { Initial } & \\ \text { Total Uranium Perm. Temp. } & \text { Tumber } & \text { Content Number Number }\end{array}$

Number Content Number Number

$\begin{array}{rrrr}724 & 133.211 & 724 & 0 \\ 0 & 0.000 & 0 & 0\end{array}$

$\begin{array}{llll}0 & 0.000 & 0 & 0 \\ 0 & 0.000 & 0 & 0\end{array}$

$\begin{array}{llll}0 & 0.000 & 0 & 0\end{array}$

$\begin{array}{llll}0 & 0.000 & 0 & 0\end{array}$

$\begin{array}{llll}0 & 0.000 & 0 & 0\end{array}$

$\begin{array}{llll}0 & 0.000 & 0 & 0\end{array}$

$\begin{array}{rrrr}724 & 133.211 & 724 & 0 \\ 0 & 0.000 & & \end{array}$

Weight

724 (MFU)

0

0

0
0

0

$724 \quad 133.216$

0.000

0.000

0.000

0.000

0.000

0.000

133.216

The number of assemblies discharged and their total weight for the life of the reactor ordered by the storage pools at which these assemblies are stored. The total number of assemblies equals the number of permanently discharged assemblies (status code $=D$ ) plus the number of temporarily discharged assemblies $($ status code $=T$ ).

The number of assemblies currently stored in each storage pool. Reactor type (BWR = Boiling-water reactor, PWR $=$ Pressurized-water reactor) is indicated in parentheses. Pool type indicates Wet (storage pool) or Dry (dry cask) storage. The number of assemblies contained in canisters are shown in parentheses (canisters/assemblies).

The number of assemblies stored in the pool indicated along with their total weight ordered by discharging reactor.

Source: Energy Information Administration, Form RW-859, "Nuclear Fuel Data" (1993). 
Appendix E

\section{Technical Notes}

Carolina Power and Light's Robinson Independent Spent Fuel Storage Installation.

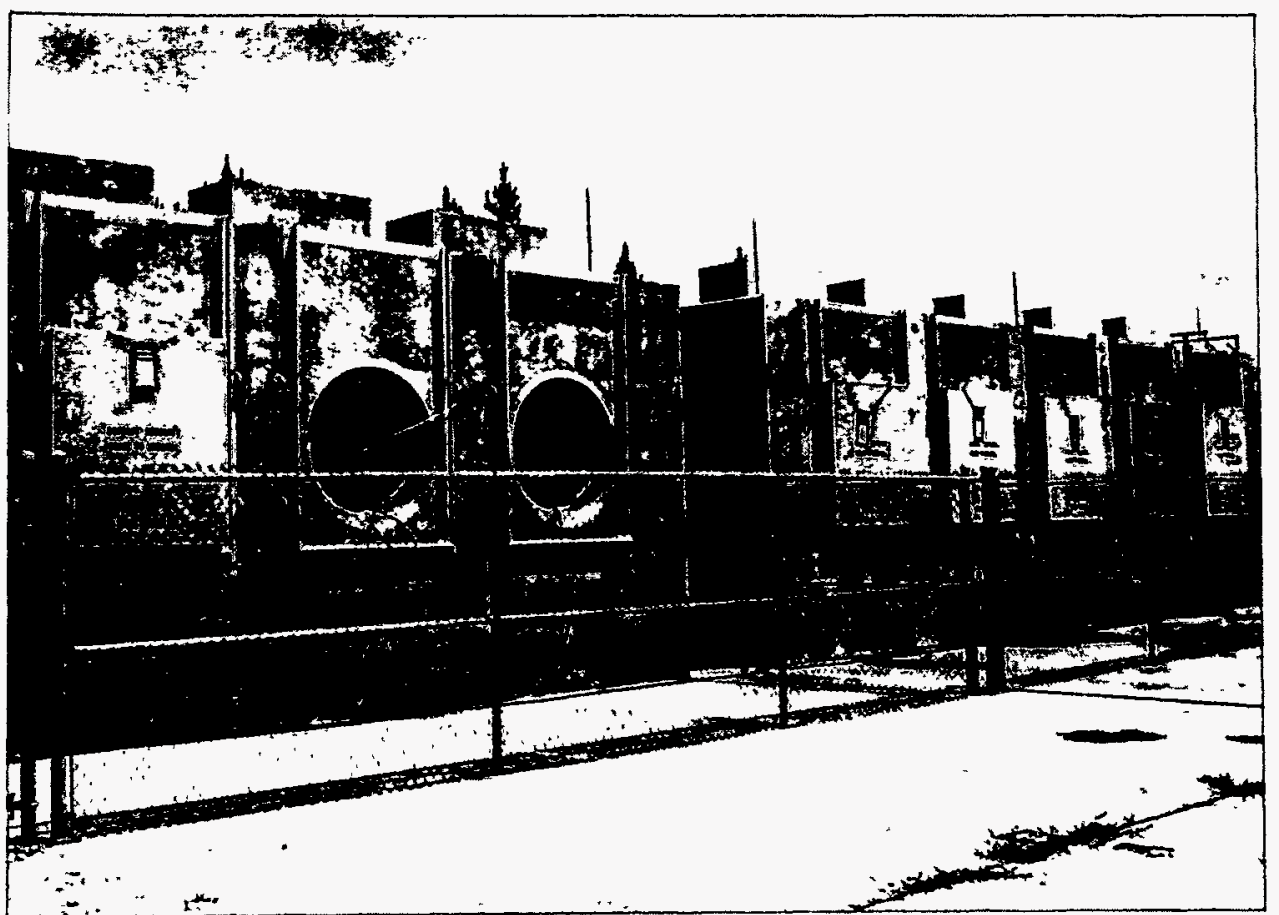


$\ldots$ 


\section{Appendix E}

\section{Technical Notes}

\section{Note 1: Reactor Status}

Data for a total of 120 commercial light-water reactors have been reported on the Form RW-859 survey. The total includes 118 reactors which have been, will be, or are discharging and/or storing nuclear fuel assemblies. Reports are also required for two reactors that are under construction and have not discharged spent nuclear fuel (Watts Bar 1 and 2). For three reactors that were reported on previous surveys (Bellefonte 1 and 2, which have been indefinitely deferred, and Fort St. Vrain, which has been shut down and is not a light-water reactor), reports are no longer required. Nine reactors that have either shut down or been retired (Dresden 1, Humboldt Bay, Indian Point 1, LaCrosse, Shoreham, Rancho Seco, San Onofre 1, Trojan, and Yankee Rowe) are maintained on the data base for their historical information.

$\begin{array}{lrrr} & \underline{1991} & \underline{1992} & \underline{1993} \\ \text { Operating Reactors } & 110 & 108 & 109 \\ \text { Shut Down Reactors } & 7 & 9 & 9 \\ \text { Under Construction } & 3 & 3 & 2 \\ \text { Total } & 120 & 120 & 120\end{array}$

Note: Totals do not include Bellefonte 1 and 2, which are indefinitely deferred, and Fort St. Vrain, which is an HTGR and was shut down in 1989. Totals also do not include four storage-only facilities.

Two reactors that have discharged spent nuclear fuel are not reported on Form RW-859, and their data are not included in this report. Hanford- $N$ was placed in cold standby status by the DOE in 1988 . Hanford-N spent fuel is used in DOE's Defense Programs. Three Mile Island 2 was shut down in 1979 because of an accident. Three Mile Island 2 spent fuel now belongs to a DOE experimental research program. Five other reactors have begun construction but have been indefinitely deferred. These reactors are Perry 2, Bellefonte 1 and 2, and Washington Nuclear Power 1 and 3.
On December 12, 1994, the Tennessee Valley Authority (TVA) made an announcement to halt construction of three nuclear power units - Bellefonte 1 and 2, and Watts Bar 2. Changes in reactor status will be reflected in next year's report.

\section{Note 2: Storage Facilities}

In addition to the spent nuclear fuel assemblies stored at reactor sites, a number of assemblies are stored at away-from-reactor storage facilities throughout the United States. Four of these facilities, General Electric Company (Morris Operation), West Valley Demonstration Project, Babcock \& Wilcox Company (Lynchburg), and General Electric Company (Vallecitos Nuclear) report on the Form RW-859 survey. Data for these facilities are obtained directly from their survey submissions and verified by checking shipment data from the contributing reactors (Table 14). Data for an additional four storage facilities [Idaho National Engineering Laboratory, Ohio (Battelle), South Carolina (Savannah River Site), and Washington (Hanford)] are compiled from shipment data submitted by the contributing reactors.

Table 14 contains complete data on assemblies stored at away-from-reactor storage facilities. These totals are also included in Table ES1, Figures ES1 and ES2, and the State totals in Table 15 . Table 12 presents a compilation of data on shipments from utilities to away-from-reactor storage facilities. See Technical Note 7 on reprocessed assemblies and Technical Note 12 on data adjustments for more information.

\section{Note 3: Pool Site Configurations}

The format for presenting data in Table 13 relies on the individual reactor/pool site configurations. The configurations are shown graphically in the pictograms in Appendix $C$ and include the presence of transfer canals, multiple pools servicing a single reactor, and multiple reactors sharing a single pool site. Each case is presented below. 
- When two pools are joined by a transfer canal, only one pool site ID is assigned since there is only one loading area. Such is the case at Baltimore Gas and Electric Company's Calvert Cliffs 1 and 2. In Table 13 only one pool site ID (0501) is listed, but separate capacity and inventory data are listed for each reactor.

- Only two reactors have multiple pools. Cleveland Electric Illuminating Company's Perry 1 is assigned two pool site ID's (0901 and 0902) with separate capacity and inventory data for each. Carolina Power and Light Company's Harris 1 has four pools which share the same pool site ID (0703). Of these four pools, two are currently licensed and two are not usable at this time. While Harris 1 is a PWR reactor, the storage pools have the capability of storing both PWR and BWR fuel assemblies. In Table 13, separate capacity and inventory data are listed for each reactor type.

- Where two or more reactors share a single pool, as with Commonwealth Edison Company's Byron 1 and 2, only one pool site ID (1003) is listed, and the multiple reactors' data are combined and listed with that pool site.

\section{Note 4: Changes in Reactor Ownership and Utility Names}

During the 1993 reporting year, the ownership of one reactor and the names of two utilities changed. In February 1993, Long Island Lighting Company reached an agreement with the state of New York to transfer ownership of the Shoreham reactor to Long Island Power Authority. In December 1993, Philadelphia Electric Company's name was changed to PECO Energy Company and Iowa Electric Light and Power Company became IES Utilities, Inc. These changes are reflected in this report, including tables in which utility names are listed in alphabetical order.

\section{Note 5: Alternative Data Sources}

The majority of data in this report come directly from a compilation of data on the Form RW-859 survey. Additional data were obtained from the EIA publication, World Nuclear Outlook 1994 (Table 4), and the Nuclear Regulatory Commission publication, Information Digest 1994 (Tables B2 and B3). The following data were obtained from these publications:
- World Nuclear Outlook 1994

- Summer capability rating (net MWe) for all reactors;

- Startup year for Watts Bar 1 and 2; and,

- License expiration year for Watts Bar 1 and 2.

- Information Digest 1994

- Nuclear steam system supplier and design type for all reactors.

\section{Note 6: HTGR Fuel Elements}

One Form RW-859 reactor, Fort St. Vrain, is a high-temperature, gas-cooled reactor (HTGR). An HTGR uses an inert gas (helium) as the primary coolant and a graphite moderator. Highly enriched uranium is used as initial fuel and thorium as a source of new fuel.

This HTGR has spent fuel wells instead of a pool. Each well holds one element (6 inches long, 1 inch in diameter). Data for Fort St. Vrain are listed in Tables 15, 16, and 17, and are not included in totals on those tables.

Fort St. Vrain shut down in 1988 for repairs and will not restart. A total of 726 spent fuel elements (including 6 test elements) were discharged prior to this shutdown and shipped to Idaho National Engineering Laboratory (INEL). An additional 18 spent fuel elements were shipped to INEL in October 1991. The remaining spent fuel elements are stored in an Independent Spent Fuel Storage Installation (ISFSI). The ISFSI was licensed on November 4, 1991, by the U.S. Nuclear Regulatory Commission. The license authorizes storage in the ISFSI of up to 1,482 spent fuel elements, 37 reflector control rod elements, and 6 neutron source elements. A total of 1,464 elements currently reside in the ISFSI.

\section{Note 7: Reprocessed Assemblies}

Spent fuel discharged from light-water reactors contains appreciable quantities of fissile (U-235, $\mathrm{Pu}-239)$, fertile (U-238), and other radioactive materials. These fissile and fertile materials can be chemically separated and recovered from the spent nuclear fuel. 
The recovered uranium and plutonium can, if economic and institutional conditions permit, be recycled for use as nuclear fuel. However, the Nuclear Nonproliferation Treaty of 1978 and other federal laws have prohibited this recovery.

A number of assemblies were discharged and reprocessed prior to 1972. Data on these reprocessed assemblies are not included in this report, except for 962 reprocessed assemblies reflected in Table 12 as shipments.

\section{Note 8: Reactor Dates}

Key dates in the life of a reactor are presented in Table 4. While the majority of the information presented in this table was obtained from Form RW-859 data, other data sources were used in cases where Form RW-859 data were not available (See Technical Note 5: Alternative Data Sources). Definitions for each of the key dates are as follows:

- Startup date-year in which electricity was first generated from the reactor.

- License expiration date-year listed on the reactor's Nuclear Regulatory Commission operating license.

- Loss of ability to operate-year estimated by the utility on Form RW-859 in which the utility would not continue reactor operation because of a lack of storage space for discharged fuel, absent spent fuel pickup by DOE. This also takes into consideration the current licensed capacity (slots) and the total maximum established site capacity.

- Projected retirement year-utility estimate, either the license expiration date or a date based on a license extension.

\section{Note 9: Burnup Ranges}

Burnup is defined as the amount of thermal energy produced per unit mass of fuel irradiated or "burned." Burnup levels are generally expressed in units of gigawattdays thermal per metric ton of uranium (GWDt/MTU). Average burnups are presented in Tables 5, 6, 7, 8, and 11, Figure 4, and Appendix B.
The burnup ranges represent the burnup values of associated batch average discharges. In this report, burnup ranges are grouped in following manner: 0-4.999 GWDt/MTU; 5.000-9.999 GWDt/MTU; 10.000-14.999 GWDt/MTU; etc.

\section{Note 10: Missing Data}

Most Form RW-859 respondents submit complete and accurate data. All Form RW-859 data are subject to extensive EIA quality assurance procedures. As of the final frozen file date of September 6, 1994, a limited number of data items were missing. In these instances, the missing data items involved temporarily discharged assemblies. Utilities are not required to report complete data until these assemblies are permanently discharged. These items, listed below, are omitted from the tables in this report.

- The following 108 assemblies, all temporarily discharged, are missing assembly type codes, initial uranium content, burnups, and enrichment (weight percent).

$\begin{array}{llr}\text { Davis-Besse } & \text { PWR } & 6 \\ \text { Indian Point 2 } & \text { PWR } & 7 \\ \text { Indian Point 3 } & \text { PWR } & 2 \\ \text { Kewaunee } & \text { PWR } & 4 \\ \text { Maine Yankee } & \text { PWR } & 4 \\ \text { North Anna 1 } & \text { PWR } & 8 \\ \text { North Anna 2 } & \text { PWR } & 1 \\ \text { Perry 1 } & \text { BWR } & 4 \\ \text { Salem 1 } & \text { PWR } & 22 \\ \text { Salem 2 } & \text { PWR } & 32 \\ \text { South Texas 1 } & \text { PWR } & 3 \\ \text { Three Mile Island 1 } & \text { PWR } & 15\end{array}$

- The following 459 assemblies, all temporarily discharged, are missing burnups.

$\begin{array}{llr}\text { Browns Ferry 2 } & \text { BWR } & 80 \\ \text { Callaway } & \text { PWR } & 2 \\ \text { Enrico Fermi 2 } & \text { BWR } & 40 \\ \text { Indian Point 2 } & \text { PWR } & 1 \\ \text { Limerick 1 } & \text { BWR } & 306 \\ \text { Maine Yankee } & \text { PWR } & 4 \\ \text { North Anna 1 } & \text { PWR } & 8 \\ \text { North Anna 2 } & \text { PWR } & 5 \\ \text { Salem 1 } & \text { PWR } & 1 \\ \text { South Texas 1 } & \text { PWR } & 4 \\ \text { Three Mile Island 1 } & \text { PWR } & 8\end{array}$




\section{Note 11: Rounding}

All initial uranium contents presented in the tables of this Service Report are presented in metric tons of uranium (MTU) and are rounded to the nearest one-tenth of a MTU. Totals may not exactly equal the sum of components because of independent rounding. All average burnups in the tables.of this report are presented in gigawattdays thermal per initial metric ton of uranium (GWDt/MTU) and are rounded to the nearest one-tenth. Average burnup totals are weighted averages and, as such, are not averages of individual table entries.

\section{Note 12: Data Adjustments}

The Form RW-859 survey is a total universe survey of all commercial nuclear reactors. Respondents usually submit complete data. As such, data adjustments are not usually necessary. In cases where errors or omissions are identified during the quality assurance process, EIA adjusts the data only after the respondent has been contacted to verify the change. A footnote has been added to tables where data for previous years have been revised. The note also states that current-year data may be revised in future publications. When utilities reinsert assemblies discharged in previous years, historical totals change.

\section{Note 13: Storage Capacities}

Form RW-859 respondents are required to report their licensed storage capacity and their maximum established spent fuel storage capacity.

- Licensed storage capacity represents the number of spent nuclear fuel assemblies and canisters to be stored at a given site or spent nuclear fuel pool, as licensed by the Nuclear Regulatory Commission.

- Maximum established spent fuel storage capacity represents the maximum number of intact assemblies that will be able to be stored at some point in the future (between the reporting date and the reactor's end of life) considering any established or current studies or engineering evaluations at the time of submittal for licensing approval from the U.S. Nuclear Regulatory Com- mission. Utilities adjust their maximum established spent fuel storage capacity by adding capacity based on reracking or other engineering improvements or decreasing capacity by subtracting unusable pool locations.

\section{Note 14: Shipment of Shoreham Nuclear Fuel to Limerick}

In early 1993, PECO Energy Company (formerly Philadelphia Electric Company) entered into a threeparty agreement with Long Island Power Authority (LIPA) and General Electric (GE) to reuse the nuclear fuel stored in the spent fuel pool at the Shoreham reactor since mid-1988. It was determined that PECO would be able to use the partially spent fuel at its Limerick plant. LIPA was involved in the agreement due to its role as the new owner of the spent fuel stored at Shoreham [ownership was previously transferred from Long Island Lighting Company (Lilco)]. General Electric, as the original manufacturer of the spent fuel, was required to inspect, repackage, and warranty the fuel assemblies for use at the Limerick plant.

On September 23, 1993, the first of 33 scheduled fuel shipments departed from the Shoreham reactor for delivery to the Limerick plant. Through December 31, 1993, 18 shipments of spent fuel were completed, a total of 306 assemblies delivered. These assemblies are included in the Limerick storage data throughout this report, as PECO assumed ownership of the fuel once the assemblies passed through Shoreham's gate. The balance of the fuel (254 assemblies) was shipped to the Limerick plant in 1994 and will be reflected in next year's report.

\section{Note 15: Assembly Weights}

The weight of assemblies in storage at utilities and away-from-reactor facilities is calculated to a higher degree of precision for presentation in this report. In previous years, the weight of assemblies stored were calculated using rounded average weights. This resulted in a slight difference between the calculated storage weight and the reported discharged assembly weight. The use of more precise weights of discharged assemblies results in a more accurate presentation of assembly storage weights. 


\section{Note 16: Dry Storage at Utilities}

A critical factor in the continued operation of some nuclear power plants in the disposal of spent nuclear fuel. In order to meet the demand for storage space, several utilities have opted to increase their spent fuel storage capacity through the use of dry storage at Independent Spent Fuel Storage Installations (ISFSI's). The majority of data in this report came directly from a compilation of data on Form RW-859. In addition, several utilities contributed supplementary dry storage information regarding license status, vendor, storage type, model, and planned storage capacity (Table 16). Updated yearly projections of spent fuel assemblies scheduled to be stored in ISFSI's (Table 17) were also provided. These supplementary data are included throughout this report to present the most current and accurate data available.

\section{Note 17: Canisters and Nonfuel Components}

The Form RW-859 "Nuclear Fuel Data" survey was revised in 1991 to collect information on all canisters and nonfuel components for each storage pool site. These data are found in Tables 18 through 26 . It should be noted, however, that limitations exist within these data. A number of errors and inconsistencies were discovered in reported canister and nonfuel component data during the processing and verification of the Form RW-859 surveys. Many of the errors were caused by the respondents misinterpreting or misreporting on the questions posed on the survey. Many of the inconsistencies found within the data, however, were corrected during verification telephone calls to respondents. Due to the large number of errors, some but not all have been corrected. 
$\Rightarrow$ 


\section{Glossary}

Transfer Cask loading spent fuel into Oconee's Independent Spent Fuel

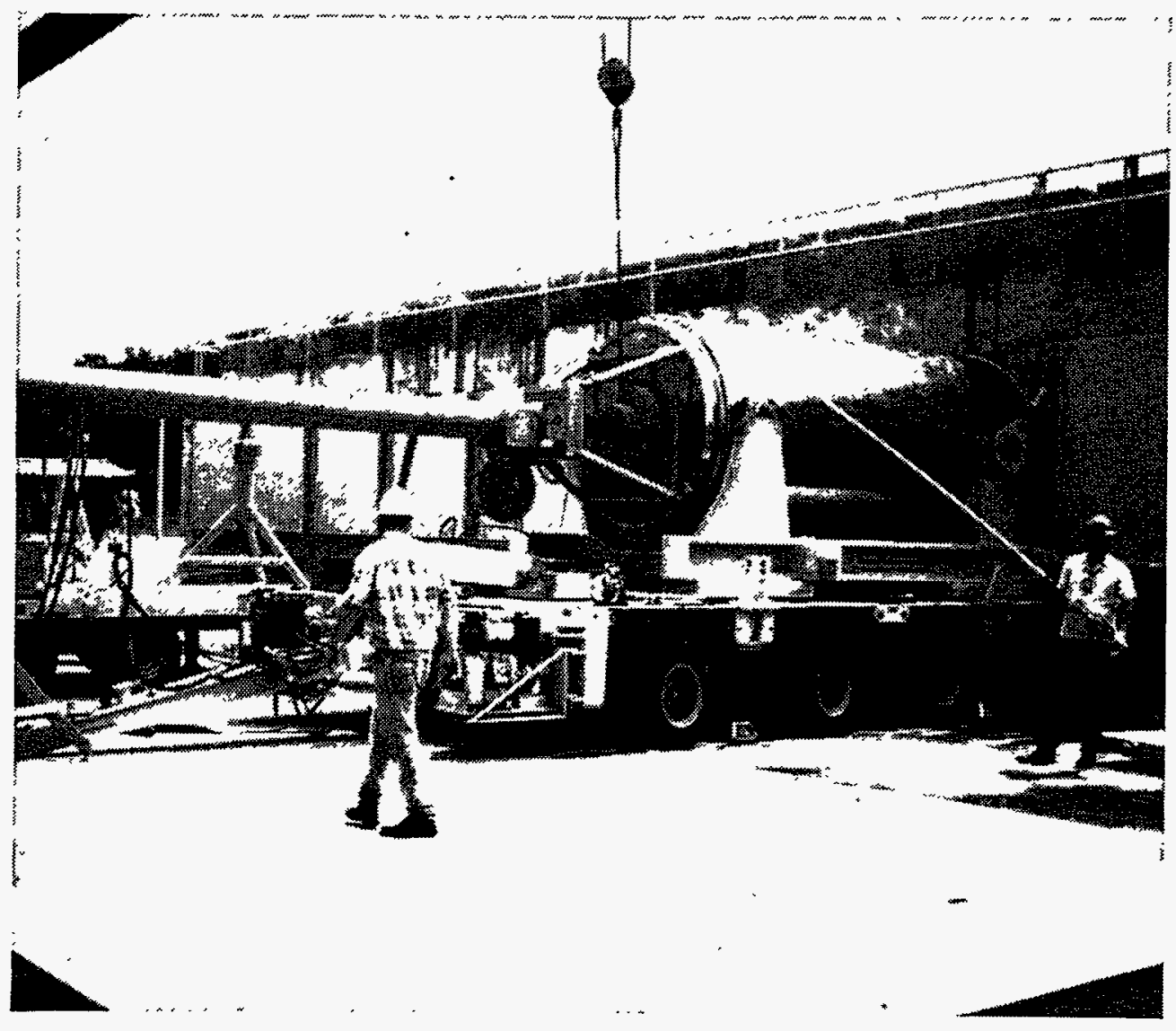




\section{Glossary}

Activity or Radioactivity: The rate at which radioactive material emits radiation, stated in terms of the number of nuclear disintegrations occurring in a unit of time; the common unit of radioactivity is the curie.

\section{Assembly: (See Fuel Assembly.)}

Assembly Class: A generic grouping for individual reactors based on the core configuration. The assembly class for a particular reactor is primarily a function of overall assembly size (length and transverse width). For pressurized water reactors, assembly class is also a function of fuel rod array configuration. A key attribute of the assembly class scheme is that fuel assemblies in an assembly class are only used in reactors belonging to the same assembly class. Codes representing the assembly class are used as the first three characters of the eight-character assembly type codes. Codes for multiple-reactor assembly classes consist of a single-character code representing the reactor vendor and a two-character code identifying the particular assembly class. Codes for single-reactor assembly classes consist of the letter " $X$ " and a two-character code representing the specific reactor. The assembly class also serves as the basis for the characterization of nonfuel components.

\section{Assembly Identifier: (See Fuel Assembly Identifier.)}

Assembly Type: Each assembly is characterized by a fabricator, rod-array size, and model type. An eightdigit assembly type code is assigned to each assembly type based on certain distinguishing characteristics, such as the number of rods per assembly, fuel rod diameter, cladding type, materials used in fabrication, and other design features.

Average Discharge Burnup: The average amount of energy produced by each assembly in a batch of spent fuel assemblies that have been discharged from a nuclear reactor, reported in gigawattdays thermal per metric ton of uranium (GWDt/MTU).

Average Initial Enrichment Assay: Average enrichment for a fresh fuel assembly as specified and ordered in fuel cycle planning. This average includes axial blankets, and axially and radially zoned enrichments.

Average Initial Uranium Content: Average weight in kilograms $(\mathrm{kg})$ of uranium of fresh fuel assemblies in a batch before they are initially inserted into the reactor core. To be included in a batch the initial uranium content of each assembly must be within $3 \mathrm{~kg}$ of the average for boiling water reactor assemblies and within $5 \mathrm{~kg}$ of the average for pressurized water reactor assemblies.

Axial Power Shaping Assemblies: Assemblies used at Babcock \& Wilcox-designed pressurized-water reactors to provide control over the power level of the reactor core during operation. They are very similar to control rod assemblies, but have less neutron absorbing material. Gray axial power shaping assemblies use a weaker neutron absorber, but serve an identical function.

Basket: A container used to store nonfuel components, nonfuel disassembly hardware, and/or other nonfuel materials. Baskets are usually defined as rodlet or garbage and debris containers with dimensions less than that of an intact fuel assembly.

Batch: A logical grouping of assemblies with similar characteristics. All assemblies in a batch have the same assembly type, approximate initial loading weight, initial average enrichment, in core cycle history and discharge date, and approximately the same burnup after final discharge.

Boiling-Water Reactor (BWR): A light-water reactor in which water, used as both coolant and moderator, is allowed to boil in the core. The resulting steam can be used directly to drive a turbine.

Burnable Poison Rod Assemblies (BPRA's): Assemblies are inserted and locked into the guide tubes of pressurized-water reactor fuel assemblies in Babcock \& Wilcox and Westinghouse Electric designed reactors to provide additional reactivity control. Both borosilicate glass and a boron carbide/aluminum oxide mixture have been used as the absorber for these assemblies. Because these absorbing materials are 
rapidly depleted, BPRA's are useful in limiting power output in the higher enriched assemblies that are now being placed into service. The use of burnable poison rod assemblies varies greatly from utility to utility.

Burnup: A measure of the amount of energy obtained from fuel in a reactor. Typically, burnup is expressed as the amount of energy produced per unit weight of fuel irradiated or "burned." Burnup levels are generally measured in units of gigawattdays thermal per initial loading weight of uranium, in metric tons (GWDt/MTU).

Burnup Range: A data structure that represents assemblies, once and only once, by their associated batch average discharge burnup. As a batch is defined as all assemblies having identical reactor and cycle histories, identical nominal initial enrichments, and a burnup within 10 percent of the final mean average burnup, some assemblies placed within a range may not actually fall within that range. For this report, burnup ranges are grouped in the following manner: 0-4.999 gigawattdays thermal per initial loading weight of uranium, in metric tons (GWDt/MTU); 5.000-9.999 GWDt/MTU; 10.000-14.999 GWDt/MTU; etc.

Canister: The outermost container into which vitrified high-level waste, intact spent fuel assemblies, consolidated spent fuel, or spent fuel rods are to be placed. Made of stainless steel or an inert alloy.

Cladding: A corrosion-resistant tube, commonly made of zirconium alloy or stainless steel, surrounding the reactor fuel pellets which provides protection from a chemically reactive environment and containment of fission products.

Commercial Operation: The phase of reactor operation that begins when power ascension ends and the operating utility formally declares the nuclear power plant to be available for the regular production of electricity. This declaration is usually related to the satisfactory completion of qualification tests on critical components of the unit.

Consolidation: The process whereby fuel rods are removed from an assembly and placed into a canister in a grid with spacing closer than that of an intact assembly. Consolidation maximizes density, lowers criticality, and improves heat transfer characteristics.

Control Blades: Blades (often called cruciforms) that control the nuclear chain reaction in boiling-water reactors by limiting the number of neutrons in the core. Made of strongly neutron-absorbing materials, control blades are inserted into the reactor core at planned shutdowns and during scrams. Particular groups of control blades are also used to control the power level of the reactor. Several pressurized-water reactors use(d) control blades.

Control Element Assemblies (CEA's): Assemblies used at Combustion Engineering (CE) designed pressurized-water reactors to provide control over the nuclear chain reaction by limiting the number of neutrons in the core. Made of strong neutron-absorbing materials, $\mathrm{CEA}^{\prime} \mathrm{s}$ are inserted into the reactor core at planned shutdowns and during scrams. At most $C E$ designed reactors, they consist of 4 or 5 control elements connected together by a control rod spider and fit into the guide tubes of a single assembly. At CE System 80 reactors, they consist of either 4 or 12 control elements connected together by a control rod spider. The 4-element CEA's fit into the guide tubes of a single assembly, but the 12-element CEA's fit across 5 fuel assemblies in the core of the reactor.

Control Element Assembly Flow Plugs: Plugs used at some Combustion Engineering designed reactors in the same manner that thimble plug assemblies and orifice rod assemblies are used at Westinghouse Electric and Babcock \& Wilcox designed reactors, respectively.

Control Rod Assemblies (CRA's): Assemblies used at Babcock \& Wilcox designed pressurized-water reactors to provide control over the nuclear chain reaction by limiting the number of neutrons in the core. Made of neutron-absorbing materials, CRA's are inserted into the reactor core at planned shutdowns and during scrams. They consist of 16 control rods connected together by a control rod spider and fit into each guide tube of a single assembly.

Coolant: Material used to conduct heat away from the reactor core to the steam cycle of a nuclear power plant. Liquids, gases, and liquid metals can be used as coolants. Water is used as both the coolant and the moderator in light-water reactors.

Core: The place in the reactor in which the nuclear fuel is irradiated and thermal energy is generated.

Core Size: The fixed number of fuel assemblies that can be irradiated at any one time in the reactor core.

Current Inventory: Number of spent nuclear fuel assemblies stored at a given site or spent nuclear fuel pool, at a given point in time. 
Cycle: The time period running from the startup of one reactor cycle to the startup of the following cycle.

Cycle End Date: The date of reactor shutdown for fuel discharge and refueling.

Decay or Radioactive Decay: The transition of a nucleus from one energy state to a lower one, usually involving the emission of a photon, electron, or neutron.

Design Electrical Rating (Capacity) Net: The nominal net electrical output of a nuclear unit, as specified by the utility for the purpose of plant design.

Discharge Cycle: The sequential numbering of a reactor's refueling, from the start of reactor fuel loading to subsequent refueling. Initial loading, first operation, and first refueling is cycle 01.

Discharged Fuel: Irradiated fuel removed from a nuclear reactor during refueling. (See Spent Nuclear Fuel.)

Disposal Contract: (See Standard Contract.)

Dry Shielded Canister (DSC): The dry shielded canister is a cylindrical stainless steel pressure-retaining component which contains an internal basket assembly to maintain the spent fuel assemblies in a safe geometrical pattern during loading, transfer, storage, and postulated cask drop events.

Dry Storage Facilities: Shielded mobile or stationary containers, silos, modules, vaults, or dry wells filled with an inert gas or with air, as appropriate, in which spent fuel assemblies or canisters of highly radioactive material may be stored.

End of Cycle Date: (See Cycle End Date.)

Enrichment: A nuclear fuel cycle process in which the concentration of fissionable uranium is increased above its natural level of 0.71 percent. Enrichment is the process that changes the isotopic ratio in a material. The process currently utilized in the United States to increase uranium-235 to uranium-238 is gaseous diffusion of uranium hexafluoride $\left(\mathrm{UF}_{6}\right)$.

Enrichment Weight Percent: The isotopic percentage of U-235, by weight, that is present in fuel pellets.

Equilibrium Cycle: An analytical term that refers to fuel cycles that occur after the initial one or two cycles of a reactor's operation. For a given type of reactor, equilibrium cycles have similar fuel characteristics.

Fabricator: The manufacturer or supplier of a particular nuclear fuel assembly. The fabricator of an assembly for a given reactor is not necessarily related to the reactor vendor or to the original fuel fabricator.

Fuel Assembly: The basic unit of nuclear fuel. Uranium dioxide pellets are encased in cladding to form a fuel rod. Fuel rods are structurally connected to form a fuel assembly.

Fuel Assembly Identifier: A unique string of alphanumeric characters that identifies an assembly, bundle, or canister for a specific reactor in which it has been irradiated.

Fuel Channels: Metal (usually zircaloy) box-like items which are used in boiling-water reactors (BWR's) to control the flow of water through the core. BWR fuel bundles are inserted into the fuel channels to form the fuel assembly.

Fuel Cycle: The length of time a reactor is operated between refuelings (typically 14 to 24 months), including the refueling time, usually measured from the startup of one cycle to the startup of the following cycle.

Fuel Rods: Tubes of high-grade zircaloy or stainless steel that contain pellets of ceramic-grade uranium dioxide.

Gigawattday: A unit of measure equal to 1 billion wattdays.

High-Level Waste (HLW): Either of the following are considered to be high-level waste: (1) the highly radioactive material resulting from the reprocessing of spent nuclear fuel, including liquid waste produced directly in reprocessing and any solid material derived from such liquid waste that contains fission products in sufficient concentrations; and (2) other highly radioactive material that the Nuclear Regulatory Commission, consistent with existing law, determines by rule to require permanent isolation.

High-Temperature, Gas-Cooled Reactor (HTGR): A reactor that is cooled by helium and moderated by graphite.

Horizontal Storage Module (HSM): The horizontal storage module is a steel reinforced concrete structure. The HSM may be constructed in-place at the 
Independent Spent Fuel Storage Installation (ISFSI) location or it may be prefabricated off-site and positioned on a reinforced concrete pad at the ISFSI. The principal design functions of the HSM are to provide radiation shielding for the fuel/dry shielded canister during storage and to provide a passive mechanism for the removal of decay heat by natural circulation of ambient air.

Incore Instrumentation: Instrumentation used to monitor the neutron flux within the core. In pressurized-water reactors, this instrumentation is inserted into an instrument tube in the fuel assembly.

Independent Spent Fuel Storage Installation (ISFSI): Dry storage facility located at reactor site in which spent fuel assemblies may be stored. (See Dry Storage Facilities.)

Integral Fuel Burnable Absorbers (IFBA's): Westinghouse Electric design neutron absorbers that are integral to the fuel assembly. IFBA's consist of a zirconium diboride coating on the exterior of selected fuel pellets.

Interim Storage: The temporary holding of wastes on or away from the generator's site when disposal space is not available. Monitoring and human control are provided, and subsequent action involving treatment, transportation, or final disposition is expected.

Irradiated Nuclear Fuel: Nuclear fuel that has been exposed to radiation in the reactor core at any power level.

Licensed Storage Capacity: Maximum number of spent nuclear fuel assemblies and canisters to be stored at a given site or spent nuclear fuel pool, as licensed by the Nuclear Regulatory Commission.

Licensed Thermal Power: The maximum thermal power of the reactor as authorized by the Nuclear Regulatory Commission, expressed in gigawatts thermal (GWt).

Light-Water Reactor (LWR): A nuclear reactor that uses water as the primary coolant and moderator, with slightly enriched uranium as fuel. There are two types of commercial light-water reactors--the boiling-water reactor (BWR) and the pressurized-water reactor (PWR).

Local Power Range Monitors: Incore instrumentation used to monitor the power level of General Electric designed reactors during regular operation (at or near full power).

Low-Level Waste (LLW): Radioactive waste not classified as high-level waste, transuranic waste, spent nuclear fuel, or by-product material specified as uranium or thorium tailings and waste.

Maximum Established Storage Capacity: The maximum established spent fuel capacity for the site is defined by the Department of Energy as the maximum number of intact assemblies that will be able to be stored at some point in the future (between the reporting date and the reactor's end of life) taking into account any established or current studies or engineering evaluations, at the time of submittal for licensing approval from the Nuclear Regulatory Commission.

Maximum Initial Pellet Enrichment Assay: The maximum enrichment in uranium-235 weight percent of all pellets contained in the fuel rods in a nuclear fuel assembly.

Metric Tons of Initial Heavy Metal (MTIHM): The weight of the initial fuel loading (in metric tons) used in an assembly.

Metric Tons Uranium (MTU): A measure of weight equivalent to 2,204.6 pounds of uranium and other fissile and fertile materials that are loaded into an assembly during fabrication of the assembly.

Multi-Purpose Canister (MPC): A hybrid of a universal storage cask and a dual purpose cask used to store, transport and dispose of spent nuclear fuel. Also referred to as a dry storage cask.

Net Summer Capability: The steady hourly output that generating equipment is expected to supply to a system load, exclusive of auxiliary power, as demonstrated by tests during summer peak demand.

Neutron Poison: A material (i.e., boron or cadmium) that absorbs neutrons readily. Insertion of one of these poisons results in a decrease in the reactivity (or neutron multiplying property) of the core, and consequently in a decrease of the neutron density.

Neutron Sources: A base source of neutrons that initiate the nuclear chain reaction within the reactor core. Primary neutron sources are typically poloniumor plutonium-beryllium alloys; secondary neutron sources are typically antimony-beryllium alloys. 
Nonfuel Components: Components that are not associated with a particular fuel. These include, but are not limited to, control spiders, burnable poison rod assemblies, control rod elements, thimble plugs, fission chambers, primary and secondary neutron sources, and BWR channels.

Nuclear Fuel: Fissionable materials that have been enriched to such a composition that, when placed in a nuclear reactor, will support a self-sustaining fission chain reaction, producing heat in a controlled manner for process use.

Nuclear Fuel Cycle: The complete series of steps involved in supplying fuel for nuclear reactors, including mining, refining, enrichment, the original fabrication of fuel elements, their use in a reactor, and management of spent fuel and radioactive wastes. A closed fuel cycle includes chemical reprocessing to recover the fissionable material remaining in the spent fuel; an open fuel cycle does not.

Nuclear Power: Electricity generated by an electric power plant whose turbines are driven by steam produced in a reactor by heat from the fissioning of nuclear fuel.

Nuclear Power Plant: A facility in which heat produced in a reactor by the fissioning of nuclear fuel is used to drive a steam turbine.

Nuclear Reactor: An apparatus in which the nuclear fission chain reaction can be initiated, maintained, and controlled so that energy is released at a specific rate. The reactor apparatus includes fissionable material (fuel) such as uranium or plutonium; fertile material; moderating material (unless it is a fast reactor); a heavy-walled pressure vessel; shielding to protect personnel; provision for heat removal; and control elements and instrumentation.

Nuclear Regulatory Commission (NRC): The U.S. Government organization that regulates the nuclear industry, under the Atomic Energy Act of 1954, et seq., to insure public safety.

Nuclear Waste Policy Act of 1982, as Amended: The Act mandates the establishment of repositories for the long-term storage and disposal of high-level radioactive waste and the collection of fees from utilities to finance these repositories. Under this Act the Department of Energy (DOE) was given the responsibility for providing permanent disposal of spent fuel from domestic nuclear power reactors.
Operable (Nuclear): A U.S. nuclear generating unit is considered operable after it completes low-power testing and is issued a full power amendment to its operating license from the Nuclear Regulatory Commission.

Orifice Rod Assemblies: Short (typically 8-10") metal plugs that are inserted into the guide tubes of fuel assemblies in Babcock \& Wilcox-designed reactors to block the flow of coolant through guide tubes. They consist of 20 orifice rods connected together by a spider or similar holddown device. Each orifice rod assembly fits into the guide tubes of a single assembly.

Other Instrumentation: Incore instrumentation, such as source range monitors (SRM's), intermediate range monitors (IRM's), and transverse incore probes (TIP's), that are used to monitor the power level of General Electric designed reactors.

Pool Crane: A machine for raising assemblies and depositing them into or out of a spent fuel storage pool.

Pool Site: One or more spent fuel storage pools that has a single cask loading area. Each dry cask storage area is considered a separate site.

Pressurized-Water Reactor (PWR): A nuclear reactor in which heat is transferred from the core to a heat exchanger via water kept under high pressure, so that high temperatures can be maintained in the primary system without boiling the water. Steam is generated in a secondary circuit.

Radiation: Particles or waves from atomic or nuclear processes and the process in which energy is emitted as particles or waves.

Radioactivity: (See Activity.)

Reactor, Commercial Power: Any unit of a commercial nuclear facility, licensed by the Nuclear Regulatory Commission, which generates electrical power.

Refueling: The process of shutting down a reactor and replacing some of the spent nuclear fuel assemblies. The refueling process can take from several weeks to months to accomplish.

Reinserted Fuel: Irradiated fuel that is discharged in one cycle and inserted in the same reactor during a subsequent refueling. In a few cases, fuel discharged 
from one reactor has been used to fuel a different reactor.

Repository: A permanent disposal facility for high-level or transuranic wastes and spent fuel.

Reprocessing: The dissolution of spent reactor fuel and separation of uranium, transuranic elements, and fission products.

Reracking: The installation of high-density racks to increase the storage capacity of a spent fuel pool.

Rod Array Configuration: Fuel assemblies are constructed from a number of individual fuel rods arranged together. These arrays have been of many different sizes, generally in square arrays. The number of fuel rods on each side of these arrays is referred to as the rod array configuration. Thus, an assembly with a rod array configuration of $14 \times 14$ has a total of 196 positions in a $14 \times 14$ array (most positions contain fuel rods, although some are guide tube locations, etc.).

Rod Cluster Control Assemblies (RCCA's): Assemblies used at Westinghouse Electric designed pressurized-water reactors to provide control over the nuclear chain reaction by limiting the number of neutrons in the core. Made of neutron-absorbing materials, RCCA's are inserted into the reactor core at planned shutdowns and during scrams. They consist of 16,20 , or 24 control rods connected together by a control rod spider. Each RCCA fits into the guide tubes of a single assembly.

Shipment: A generic term meant to cover all the diverse forms of shipments away from the discharging reactor's spent fuel pool as in: shipped down the transfer canal to an adjoining spent fuel pool; shipped in a transport cask to an on-site storage facility; shipped to an off-site storage facility; or shipped in a storage cask to a dry storage facility. Excludes round-trip and intermediate shipments that do not affect inventories of spent nuclear fuel.

Shutdown Date: Year, month, and day of shut down for fuel discharge and refueling. The date should be the point at which the reactor becomes subcritical.

Site: Any location under institutional control within which radioactive wastes are handled, stored, or disposed.

Slot: A physical position in a rack in a storage pool that is intended to be occupied by an intact assembly or equivalent (that is, a canister or an assembly skeleton).

Spent Fuel Storage Pool: A borated water-filled pool in which assemblies are stored.

Spent Nuclear Fuel: Irradiated fuel that is permanently discharged from a reactor at the end of a fuel cycle. Spent or irradiated fuel is usually discharged from reactors because of chemical, physical, and nuclear changes that make the fuel no longer efficient for the production of heat, rather than because of the complete depletion of fissionable material. Except for possible reprocessing, this fuel must eventually be removed from its temporary storage location at the reactor site and placed in a permanent repository. Spent nuclear fuel is typically measured either in metric tons of heavy metal (i.e., only the heavy metal content of the spent fuel is considered) or in metric tons of initial heavy metal (essentially, the initial mass of the uranium before irradiation). The difference between these two quantities is the weight of the fission products.

Standard Contract: The agreement between the Department of Energy (DOE) and the owners or generators of spent nuclear fuel and high-level radioactive waste disposal, under which DOE will make available nuclear waste disposal services to those owners and generators.

Startup: The first date electrical energy is generated by a reactor.

Storage: Operations designed to provide isolation and easy recovery of spent fuel (and other radioactive materials), which rely on continuous human monitoring, maintenance, and protection from human intrusion for a specified period of time.

Thimble Plug Assemblies: Short (typically 8-10") metal plugs that are inserted into the guide tubes of fuel assemblies in Westinghouse Electric designed reactors to block the flow of coolant through guide tubes. Thimble plug assemblies consist of 16,20 , or 24 thimble plugs connected together by a spider or similar holddown device. Each thimble plug assembly fits into the guide tubes of a single assembly.

Transport Cask: Container that provides shielding during transportation of canisters of radioactive materials, available in several sizes for truck and rail use. 
Transshipment: The shipment of spent nuclear fuel assemblies and canisters from one storage site to another using railroads or trucks as the primary mode of transportation.

Uranium (U): A heavy, naturally radioactive, metallic element (atomic number 92). Its two principally occurring isotopes are uranium-235 and uranium-238. Uranium-235 is indispensable to the nuclear industry because it is the only isotope existing in nature to any appreciable extent that is fissionable by thermal neutrons. Uranium-238 is also important because it absorbs neutrons to produce a radioactive isotope that subsequently decays to plutonium-239, an isotope that also is fissionable by thermal neutrons.

Watt: The electrical unit of power. The rate of energy transfer equivalent to 1 ampere flowing under a pressure of 1 volt at unity power factor. 
$\ldots+\ldots$

$\vdots$

$\therefore \because 2 \quad \therefore \quad \therefore \quad \therefore-1$ 


\section{Bibliography and Notes}

Diablo Canyon nuclear power plant, operated by Pacific Gas and Electric, located in Avila Beach, Claifornia.

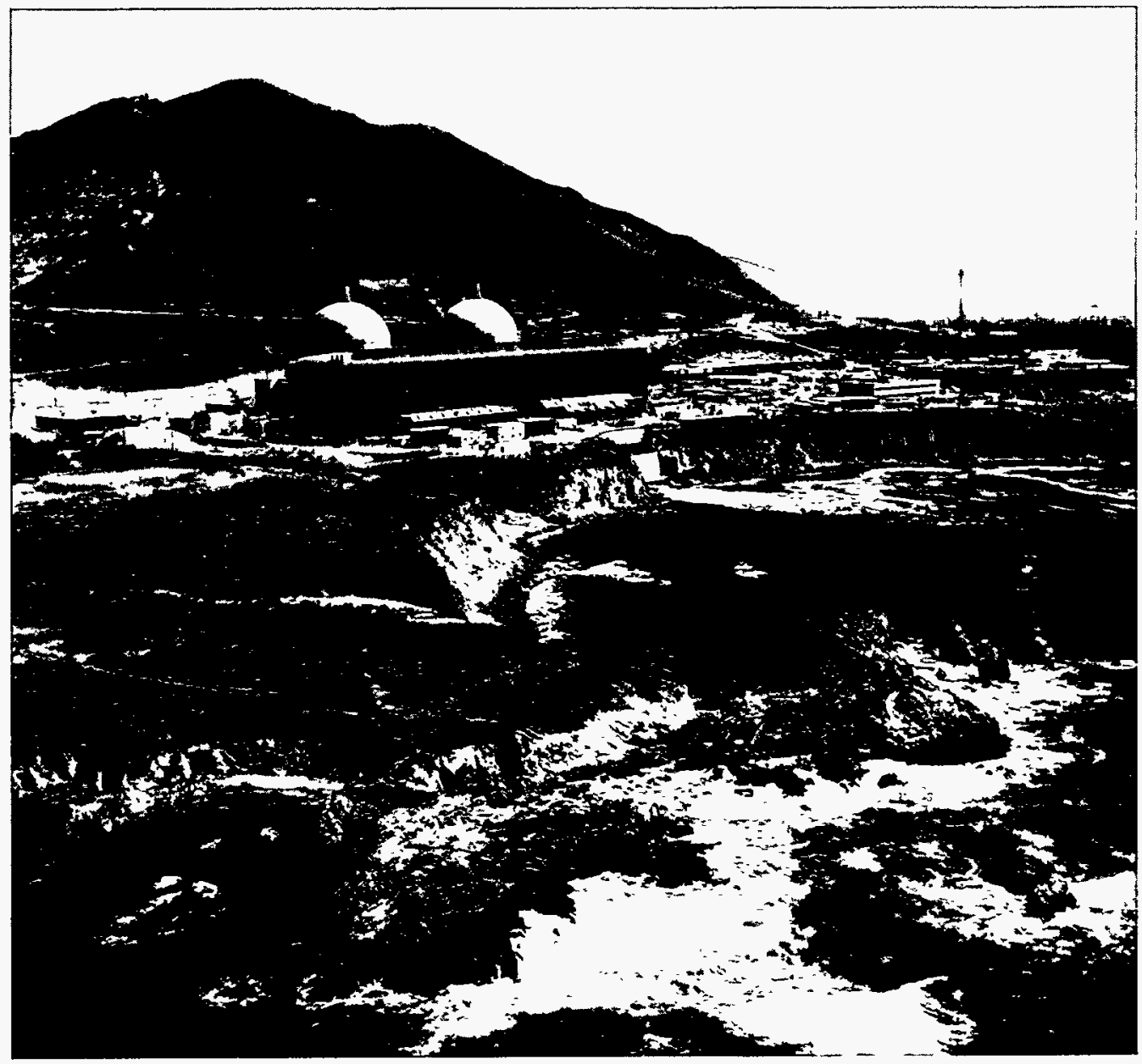




\section{Bibliography and Notes}

This section presents a listing of other publications containing information about nuclear waste along with a brief abstract of each. The most recent version of each report is listed.

Spent Fuel Storage Requirements: 1993 to 2040, Office of Civilian Radioactive Waste Management (DOE/RW-0431).

The source data contained in this report are based on individual reactor information supplied by the reactor owners on the Nuclear Fuel Data survey, Form RW-859. Historical inventories of spent fuel from existing, planned, and projected pressurized-water reactors (PWR's) and boiling-water reactors (BWR's) and one existing high-temperature, gas-cooled reactor (HTGR), and utility estimates of future discharges, are presented through the year 2040. The ultimate needs for additional storage capacity are estimated. These estimates are based on the maximum capacities within current and planned at-reactor facilities and on any planned transshipments of fuel to other reactor sites or facilities.

1994 Acceptance Priority Ranking and Annual Capacity Report, Office of Civilian Radioactive Waste Management.

This document is based on spent nuclear fuel (SNF) discharges as of December 31, 1993. The Standard Contract for Disposal of Spent Nuclear Fuel and/or High-Level Radioactive Waste (10 CFR 961) requires the U.S. Department of Energy to issue an Acceptance Priority Ranking and Annual Capacity Report for planning purposes. As required by the Standard Contract, the acceptance priority ranking is based on the date the spent nuclear fuel was permanently discharged, with the owners of the oldest spent nuclear fuel, on an industry-wide basis, given the highest priority. Projected waste acceptance rates are applied to priority ranking data, resulting in individual allocations for the owners and generators of the spent nuclear fuel. These allocations are listed and summarized in the report.
World Nuclear Outlook 1994, Energy Information Administration (DOE/EIA-0436-94).

This analysis report presents the current status and projections through 2010 of nuclear capacity, generation, and fuel cycle requirements for all countries in the world using nuclear power to generate electricity for commercial use. It also contains information and forecasts of developments in the uranium market. Long-term projections of U.S. nuclear capacity, generation, and spent fuel discharges for three difference scenarios through 2040 are developed for the Department of Energy's Office of Civilian Radioactive Waste Management (OCRWM).

Uranium Industry Annual 1993, Energy Information Administration (DOE/EIA-0478-93).

This report presents data on U.S. uranium raw materials and marketing activities of the domestic uranium industry. It contains aggregated data reported by U.S. companies on the Uranium Industry Annual survey, Form EIA-858, and historical data from prior data collections and other pertinent sources.

Form RW-859 Canister Report, Energy Information Administration Contractor Report (Z, EIA-9308501), $Z$, Inc., Silver Spring, Maryland.

The Nuclear Fuel Data survey, Form RW-859, is now collecting detailed data on canisters and their contents. A canister is a container made of stainless steel or an inert alloy into which radioactive or high-level waste may be placed. The report presents detailed data on the size and contents of canisters stored in pools at commercial utilities that operate nuclear reactors. Each canister may contain an intact spent fuel assembly, consolidated spent fuel assemblies, spent fuel rods or pieces, nonfuel components, nonfuel hardware, or debris. 
Form RW-859 Nonfuel Component Report, Energy Information Administration Contractor Report (ASG/EIA-9308502), Automated Sciences Group, Inc., Oak Ridge, Tennessee.

The Nuclear Fuel Data survey, Form RW-859, is now collecting detailed data on nonfuel components. According to the Standard Contract between the Department of Energy and the nuclear utilities (10 CFR 961), nonfuel components that are contained within the fuel assembly, or which are an integral part of the assembly, may be included as part of the spent nuclear fuel delivered for disposal. Other nonfuel components, generally used within or between assemblies and usually retired from service on a schedule that is different from that of the fuel assemblies are called nonfuel assembly (NFA) hardware. This report focuses on the specific NFA components which are listed in the contract, including control spiders, burnable absorber assemblies, control rod elements, thimble plugs, fission chambers, primary and secondary neutron sources, and fuel channels.

Integrated Data Base for 1994: U.S. Spent Fuel and Radioactive Waste Inventories, Projections, and Characteristics, Office of Civilian Radioactive Waste Management (DOE/RW-0006, Rev. 10).

The Integrated Data Base (IDB) Program contains current data on inventories and characteristics of commercial spent fuel and both commercial and U.S. Government-owned radioactive waste through December 31, 1994. The radioactive materials considered are spent fuel, high-level waste, transuranic waste, low-level waste, commercial uranium mill tailings, remedial action program wastes, commercial reactor and fuel cycle facility decommissioning wastes; highly radioactive materials that may require geologic disposal, and mixed (hazardous and radioactive) low-level waste. For these categories, current and projected inventories are given through the year 2020, and the radioactivity and thermal power are calculated based on reported or estimated isotopic compositions.
Characteristics of Potential Repository Wastes, Office of Civilian Radioactive Waste Management (DOE/RW-0184-R1, Volumes 1-4).

The Characteristics Data Base (CDB) provides the detailed technical characteristics of waste that will, or may, eventually be disposed of in a deep geologic repository. This waste includes spent fuel from commercial light-water reactors (LWR's) and immobilized high-level waste (HLW) from reprocessing. Other sources include spent fuel from non-LWR's such as test and experimental reactors, and various miscellaneous sources such as Greater-Than-Class-Clow-level waste and radioisotope capsules. The technical characteristics of relevant interest are physical descriptions ind measurements, chemical compositions, radiological properties, inventories, and projected quantities. These data are collected, tabulated, organized, placed into computer data files, and made available to users in a range of formats, including hard-copy reports and menu-driven PC data bases.

Information Digest 1994 Edition, Nuclear Regulatory Commission (NUREG-1350, Volume 6).

This digest is a compilation of nuclear and NRC related data and is designed to provide a quick reference to major facts about the agency and the industry it regulates. In general, the data cover 1975 through 1993, with exceptions noted. Information on generating capacity and average capacity factor for operating U.S. commercial nuclear power reactors is obtained from monthly operating reports that are submitted directly to the NRC by the licensee. This information is reviewed by the NRC for consistency only. No independent validation and/or verification is performed. 


\section{COMMENTS CARD AND ORDER FORM FOR SERVICE REPORT}

The EIA solicits your comments on the Service Report, Spent Nuclear Fuel Discharges from U.S. Reactors 1993. Correction of errors and suggestions for improvement are desirable. Please call Kathleen Gibbard at 202/254-5559 (e-mail kgibbard@ eia.doe.gov), or forward a copy of this form or an equivalent memorandum to:

Ms. Kathleen Gibbard

Survey Management Division

Office of Coal, Nuclear, Electric and Alternate Fuels

Energy Information Administration

U.S. Department of Energy

1707 H Street, NW, Room 821

Washington, DC 20006

Category (check all that apply)

$\square$ Order copy(s)

$\square$ Error

口 Improvement

Is there more information attached?

$\square$ Yes. Number of sheets:

$\square$ No

Comments:

Contact Information

Name:

Telephone:

Address: 